\title{
The Effect of Remote
}

\section{Ischaemic Preconditioning on the Immune Response}

\author{
By \\ Jennifer Mae Williams-Spence
}

\begin{abstract}
A thesis
submitted to the Victoria University of Wellington in fulfilment of the requirements for the degree of Doctor of Philosophy
\end{abstract}

Victoria University of Wellington

2015 


\begin{abstract}
Remote ischaemic preconditioning (RIPC) describes the phenomenon where brief intermittent periods of limb ischaemia are used to protect the heart and other organs from subsequent prolonged ischaemic insults. RIPC has been identified as a promising intervention for use during cardiac surgery and has consistently shown a beneficial effect in animal models; however, the results of early clinical trials have not been as successful. The exact mechanisms involved in mediating RIPC have not yet been characterised and a better understanding of the pathways through which RIPC exerts its protective effects will be essential in order to progress the translation of this intervention into the clinical setting. There is increasing evidence that RIPC modifies the inflammatory response, therefore the central aim of the research presented in this thesis was to investigate how RIPC affects the human immune system.
\end{abstract}

We performed a double-blind randomised controlled trial of RIPC in 96 high-risk cardiac surgery patients and found no evidence that the intervention reduced myocardial injury or altered peri-operative expression levels of the key inflammatory cytokines, interleukin (IL)-6, IL-8, and IL-10, during simple or more complex procedures. There was a trend towards higher levels of IL-6 and IL-8 in the preconditioned patients; however, confounding variables in the trial design and the heterogeneous patient population limited our ability to interpret the results.

We next conducted a paired-analysis trial with 10 healthy male volunteers to assess the direct effect of preconditioning on the early immune response, away from any form of ischaemic injury or comorbidities. We found that RIPC directly and significantly decreased serum levels of the chemokines MIP- $1 \alpha$ and MIP-1 $\beta$, but did not increase the serum concentrations of a range of key cytokines or alter the cytokine producing potential of peripheral blood leukocytes. These findings strongly suggest that a cytokine is not likely to be the humoral mediator associated with transmitting the RIPC protective signal. 
RIPC did not alter the immunophenotype or extravasation of peripheral leukocyte populations, or the proliferative and cytokine responses of peripheral blood mononuclear cells (PBMC) to pharmacological, physiological, and antigenspecific stimuli. However, preconditioning did appear to reduce the ability of monocytes and neutrophils to respond to activation signals, as indicated by lower levels of CD11b expression in stimulated cultures, and a significant increase in the basal production of IL-22 was also detected in PBMC cultured for 6 days following preconditioning. These alterations may reduce neutrophil and monocyte tissue infiltration and limit the inflammatory response during the early window of RIPC-induced protection and enhance tissue and wound repair several days later. A multivariate analysis confirmed that there was a significant difference in the response between the control and RIPC treatments and the main contributing factors were identified as changes in neutrophil and $\mathrm{T}$ cell activation, serum levels of MIP-1 $\alpha$ and $\beta$, and production of IL-10 and IL-22 from PBMC cultured for 6 days.

Overall, our results suggest that RIPC has a subtle but direct effect on the systemic innate immune response during the early window of protection in healthy volunteers, whereas the effects on the adaptive immune system seem to be considerably delayed. The changes detected following RIPC are likely to contribute to protection against ischaemia-reperfusion injury but not solely account for the extent of the beneficial effects of RIPC detected in animals. Our findings reinforce the safety profile of this intervention and have defined a number of immune parameters that are altered by preconditioning for focusing future research. 


\section{Acknowledgements}

I owe a great deal of gratitude to a number of important people and organisations that have been invaluable in helping me complete this PhD. First and foremost, I would like to thank my primary supervisor A/Prof Anne La Flamme, whose expert guidance, knack for troubleshooting, and general love of scientific data has been an inspiration throughout this research. It has been a privilege to spend my post-graduate years studying in the LAF lab.

I am also very grateful for the support and feedback from my secondary supervisor, Prof Richard Beasley, and for the additional supervisors of my Honours project, Dr Paul Young and Prof John Miller, who introduced me to clinical research and without whom I would not have become so absorbed in the concept of RIPC.

This research would not have been possible without the generous help of the Medical Research Institute of New Zealand, particularly Dr Janine Pilcher and Mathew Williams, who kindly took care of our study volunteers and collected samples while I raced around the lab trying to do science. A special thank you must also be mentioned to all of the study volunteers who were kind enough to participate in our trials.

The much needed sound statistical advice, analyses, and patience provided by both Prof Mark Weatherall and Dr Dalice Sim were also greatly appreciated.

We were very fortunate to receive project funding from the Lotteries Commission, Wellington Medical Research Fund, New Zealand Heart Foundation, and the Joy McNicoll Postgraduate Award in Biomedical Science. I am also personally grateful to Victoria University for a PhD Scholarship, and to the Wellington Medical Research Fund, Maurice and Phyllis Paykel Trust, and Victoria University for funding to present our research at an overseas conference. 
My time at VUW would not have been the same without the many amazing and talented people I met along the way, especially the original crew from KK601, and the LAF and JHM lab groups. There are too many people to name personally, but I am enormously grateful for all the training, guidance, and support provided by my fellow student scientists. Special mention must go to Dr Laura Green for her support and assistance overcoming the final hurdles in the lab and with proofreading, which were greatly appreciated.

An enormous thank you must go to my beloved family, Mum, Dad, Janne, Kelda, Sandra, and Warwick, for their ongoing support, both personally and financially, throughout this process. Your understanding and good humour were, and always will be, invaluable. I am particularly grateful for my mother's tireless proofreading and all of the late night transport and food deliveries that kept me going.

Last, but by no stretch of the imagination least, I could not have completed this work without the unwavering love, support, inspiration, and sacrifices provided by Si. How I had the good fortune of starting this journey with you as a partner and finishing it with you as a husband, I do not know, but I wouldn't have it any other way. Thank you.

If you will stay close to nature, to its simplicity, to the small things hardly noticeable, those things can unexpectedly become great and immeasurable.

- Rainer Maria Rilke 


\section{Table of Contents}

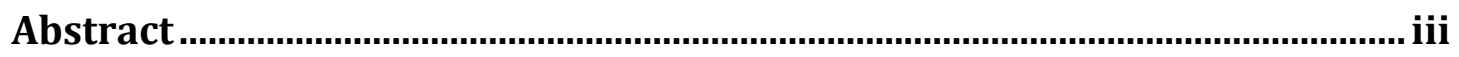

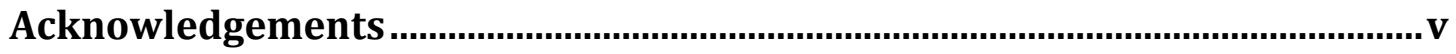

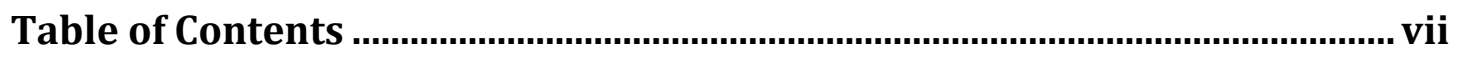

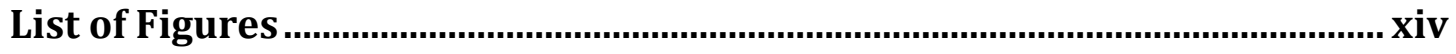

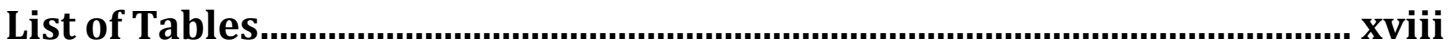

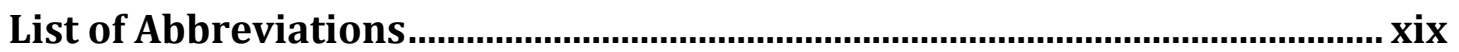

Chapter 1. General introduction...................................................................

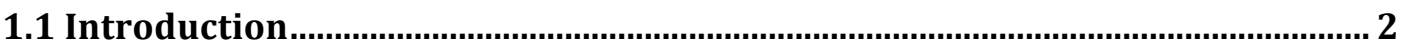

1.2 The need for myocardial protection in cardiac surgery ..................................... 2

1.2.1 Early-phase of inflammation during cardiac surgery............................................ 3

1.2.2 Late-phase of inflammation during cardiac surgery ............................................... 5

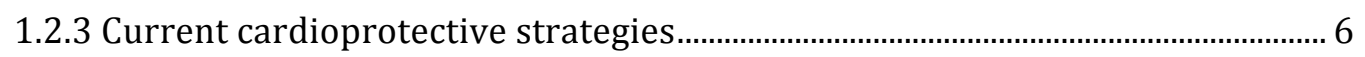

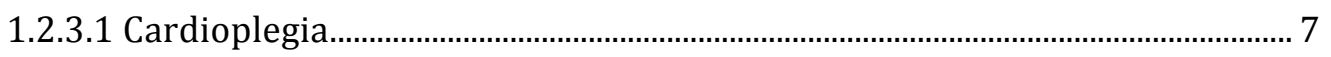

1.2.3.2 Volatile anaesthetics ........................................................................................... 7

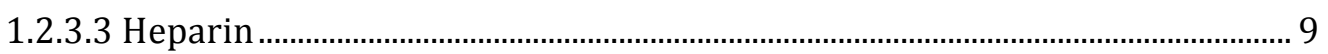

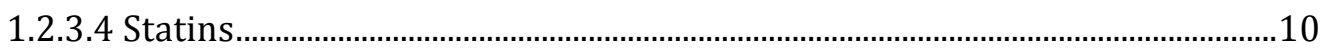

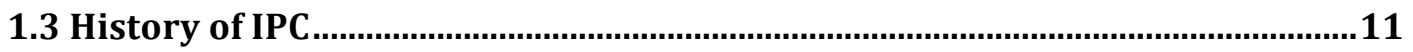

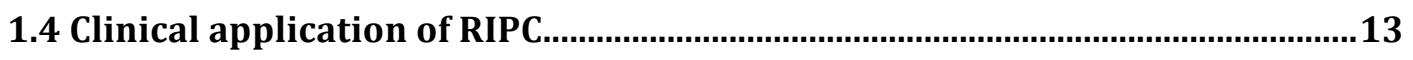

1.4.1 Cardioprotection ..................................................................................................13

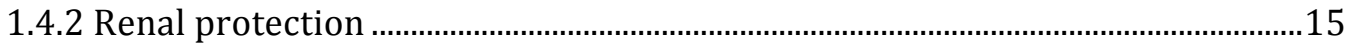

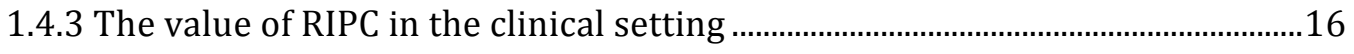

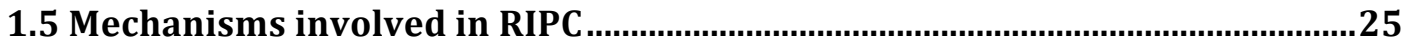

1.5.1 Evidence for humoral, neural, and systemic pathways ………………………....25

1.5.2 Potential key initiators and mediators of RIPC …………………………………...27

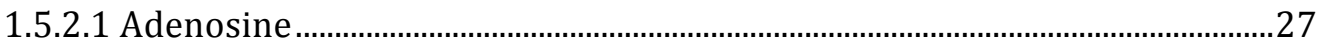

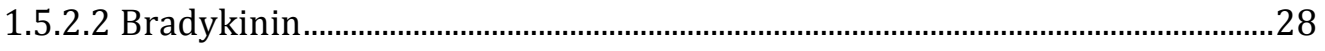

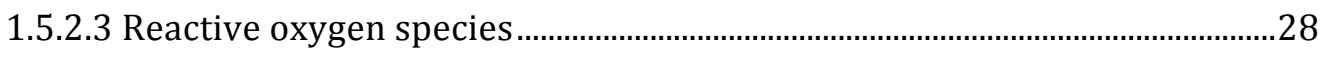

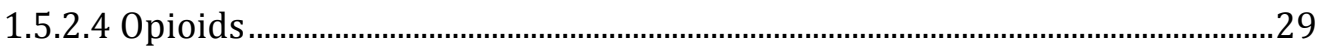

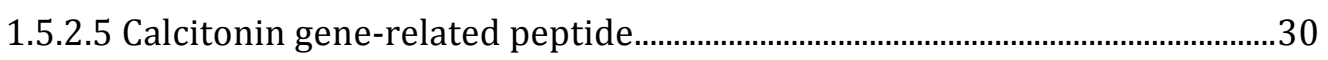

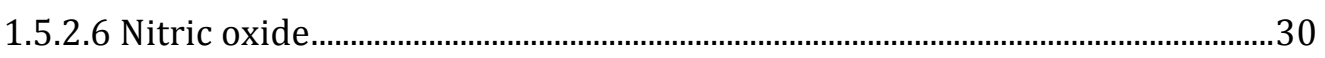


1.5.3 Intracellular signalling during RIPC.

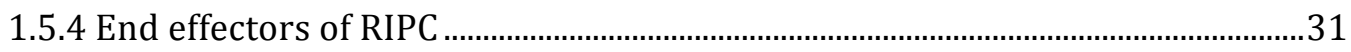

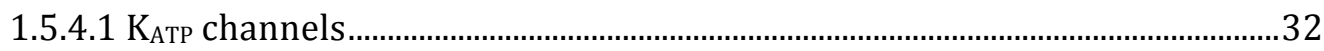

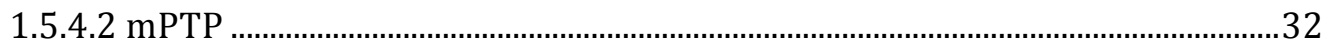

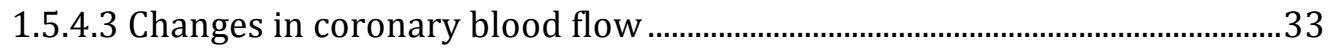

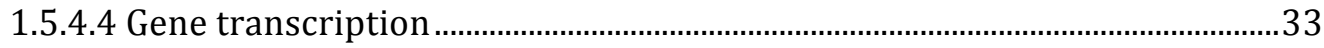

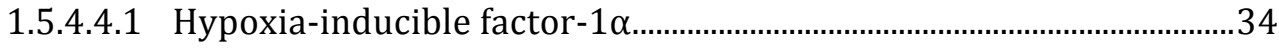

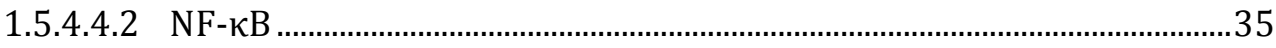

1.5.4.5 Changes to the systemic inflammatory response .............................................36

1.6 Overview of the key immune cell populations...................................................39

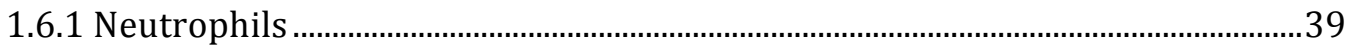

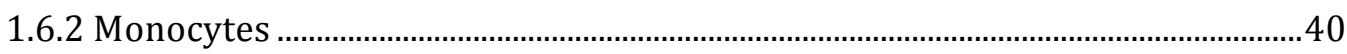

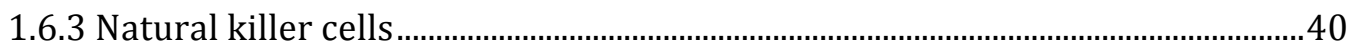

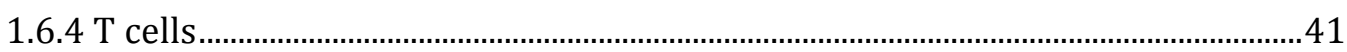

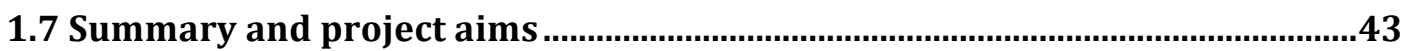

Chapter 2. The effect of RIPC on cytokine production in high-risk cardiac

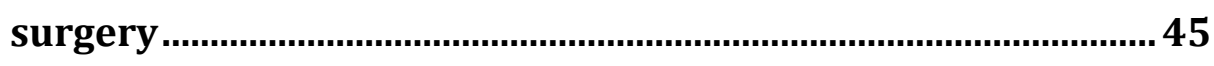

2.1 Introduction

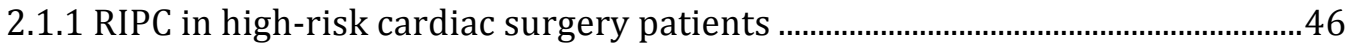

2.1.2 Potential role of cytokines in RIPC ………..............................................................4

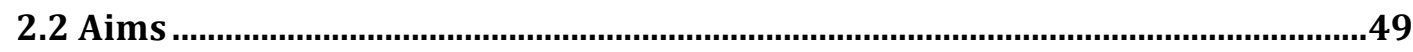

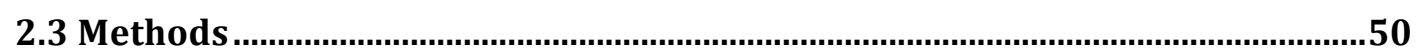

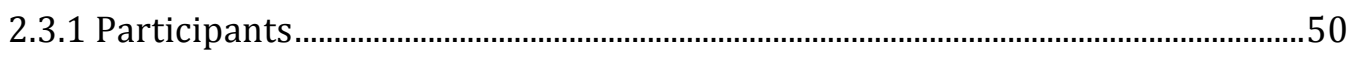

2.3.2 Study blinding and RIPC.................................................................................. 50

2.3.3 Peri-operative management of patients................................................................ 51

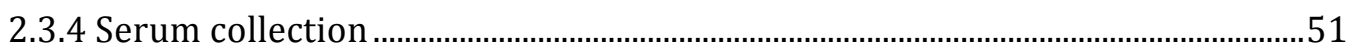

2.3.5 Troponin levels .......................................................................................................... 51

2.3.6 Cytokine quantification .....................................................................................

2.3.7 Comparison of vacutainers for cytokine recovery ..................................................52

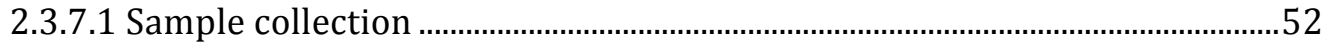

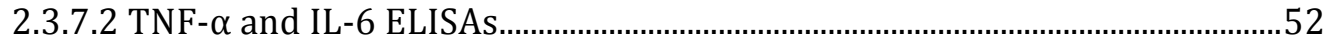

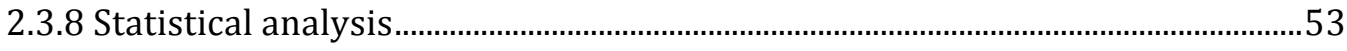

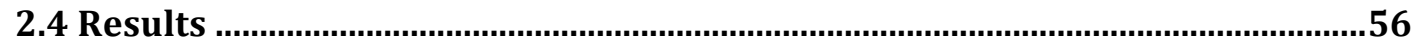

2.4.1 Patient baseline demographic and operative data ...................................................56 
2.4.2 Clinical outcome measures

2.4.3 RIPC and systemic levels of IL-6, IL-8, and IL-10 during high-risk cardiac surgery.

2.4.4 Effect of RIPC on peri-operative cytokine expression in simple versus complex surgery

2.4.5 Relationship between bypass duration and the expression of cytokines .......67

2.4.6 Relationship between statin usage and IL-6 expression .......................................69

2.4.7 Comparison of vacutainers for cytokine recovery ..................................................71

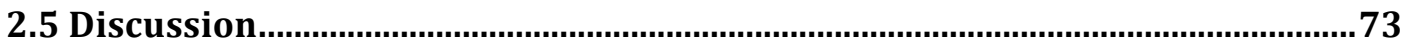

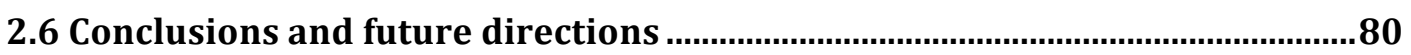

Chapter 3. Development and testing of laboratory techniques for studying human peripheral leukocyte phenotype and function.............. 81

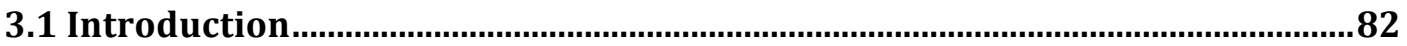

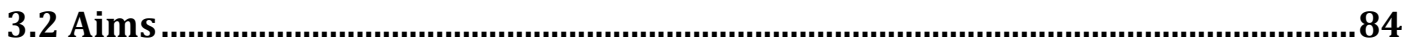

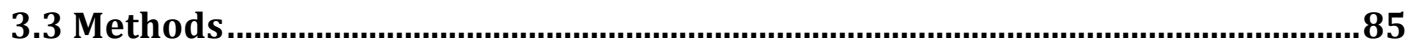

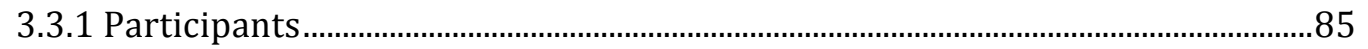

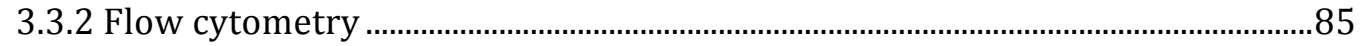

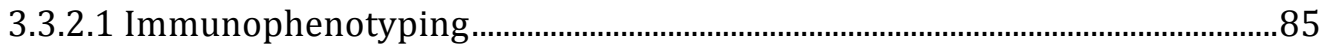

3.3.2.2 Intracellular cytokine staining ……………........................................................

3.3.3 Peripheral blood mononuclear cell cultures ............................................................86

3.3.4 CyQuant proliferation assay …………………………………………………...........

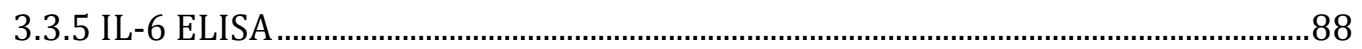

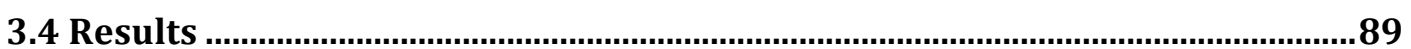

3.4.1 Flow cytometry of whole blood samples ……………………………………....... 89

3.4.1.1 General flow cytometry techniques ……………………………….................92

3.4.1.1.1 Assessing viability ......................................................................................92

3.4.1.1.2 Fc receptor blocking..................................................................................94

3.4.1.2 Testing the immunophenotyping protocol ........................................................96

3.4.1.2.1 Stimulation of neutrophils for measurement of activation.................96

3.4.1.3 Development of ICS protocol ……………............................................................99

3.4.1.3.1 Stimulation of cytokine production and improvements in antibody resolution for intracellular staining ..........................................................99

3.4.1.3.2 Inhibition of protein transport ............................................................. 106

3.4.1.3.3 Examining neutrophil activation state...................................................108 
3.4.1.3.4 Optimisation of flow cytometry application settings 110

3.4.2 Culture of PBMC and assessment of responses................................................... 112

3.4.2.1 Testing the Histopaque-1077 PBMC isolation method ................................113

3.4.2.2 CyQuant proliferation assay ............................................................................ 114

3.4.2.3 Stimulating polyclonal T Cell responses .........................................................115

3.4.2.3.1 PMA and ionomycin .......................................................................... 115

3.4.2.3.2 Anti-CD3/28 beads ................................................................................. 115

3.4.2.4 Stimulating memory T cells with the tetanus antigen ................................ 118

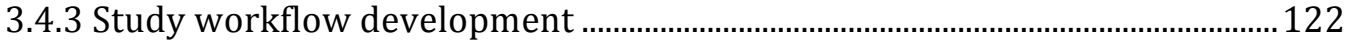

3.4.3.1 Laboratory workflow and patient management ...........................................122

3.4.3.2 Flow cytometry data analysis .................................................................... 125

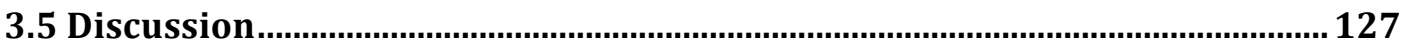

3.6 Conclusions and future directions ..................................................................... 133

Chapter 4. The systemic response to RIPC in healthy volunteers........... 135

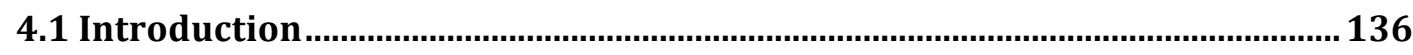

4.1.1 The innate immune system and ischaemia-reperfusion injury .......................137

4.1.2 Evidence that preconditioning alters the innate immune response...............138

4.1.2.1 Leukocyte activation and infiltration .................................................................. 138

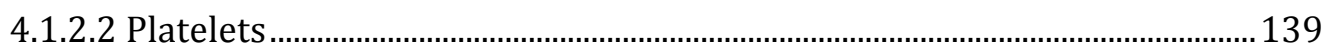

4.1.2.3 Transcription factors .................................................................................. 140

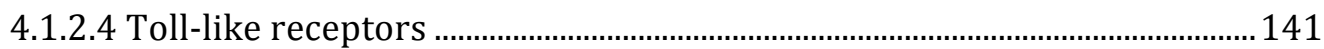

4.1.2.5 Complement ...................................................................................................... 142

4.1.2.6 Matrix metalloproteinases .............................................................................. 143

4.1.2.7 Cytokines ................................................................................................................ 143

4.1.3 Rationale for the study................................................................................... 144

4.2 Aims

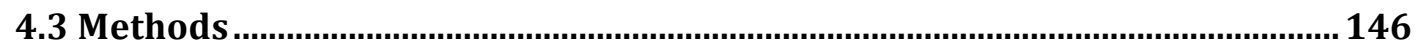

4.3.1 Participants......................................................................................................... 146

4.3.2 Study design \& application of RIPC …………....................................................... 146

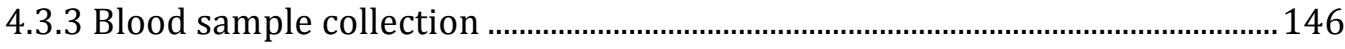

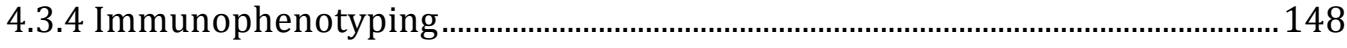

4.3.5 Serum collection ................................................................................................ 148

4.3.6 Serum cytokine quantification ...................................................................... 148

4.3.7 Statistical analysis.............................................................................................. 149 


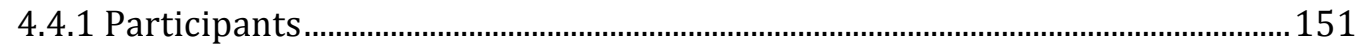

4.4.2 The effect of RIPC on serum cytokine levels ......................................................151

4.4.2.1 Adhesion molecules ....................................................................................... 152

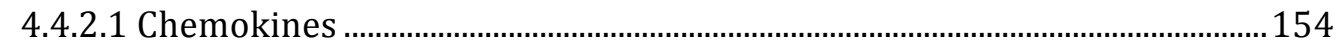

4.4.2.1 T cell cytokines..............................................................................................157

4.4.2.2 General inflammatory cytokines …………………………………………..... 160

4.4.3 Immunophenotyping of peripheral blood cells following RIPC......................... 164

4.4.3.1 Monocytes and neutrophils .............................................................................. 164

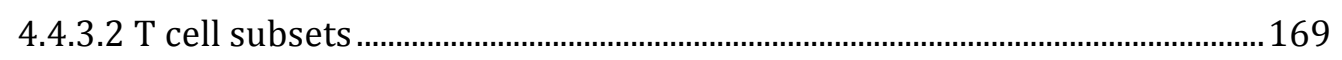

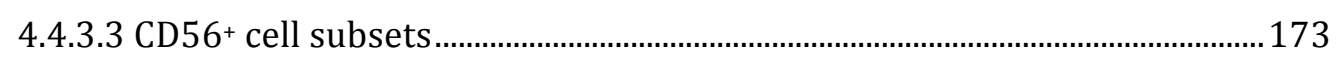

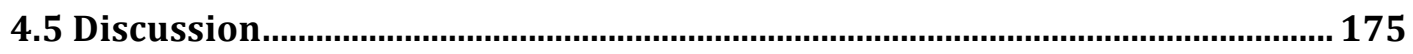

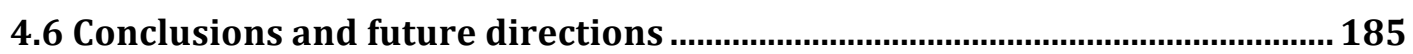

Chapter 5. The effects of RIPC on the function of peripheral leukocytes in

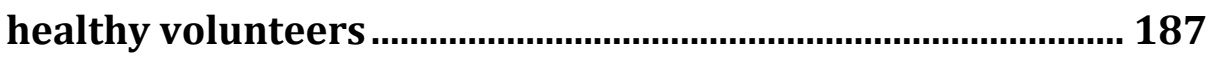

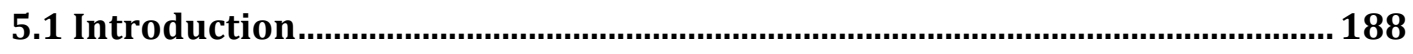

5.1.1 Evidence that preconditioning alters the adaptive immune response..........188

5.1.1.1 Regulatory T cells.......................................................................................... 189

5.1.1.2 Helper and cytotoxic T cell responses ........................................................190

5.1.2 T cells and I/R injury ……………………………........................................... 191

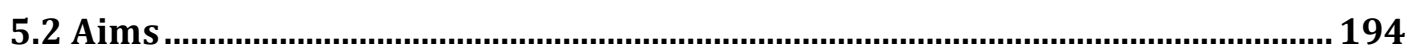

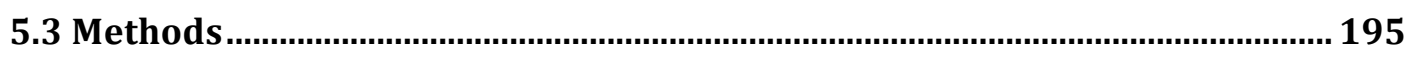

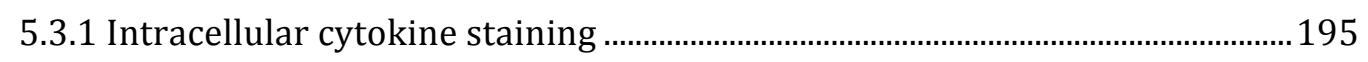

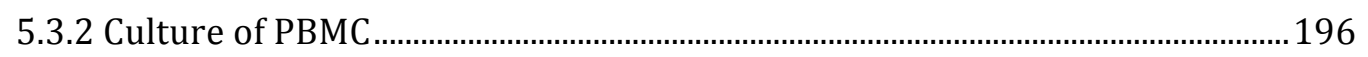

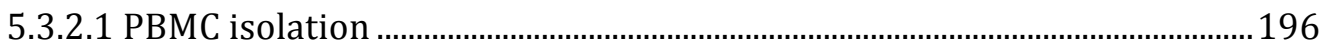

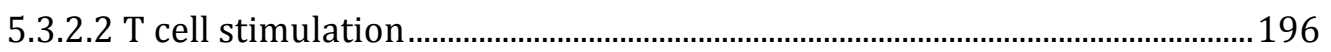

5.3.3 CyQuant proliferation assay ....................................................................................197

5.3.4 Cell culture supernatant cytokine quantification.................................................197

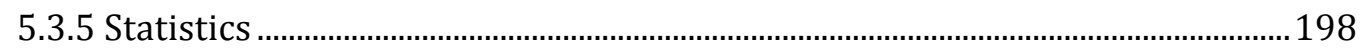

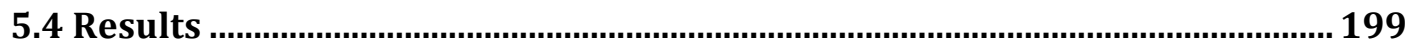

5.4.1 Intracellular cytokine production in whole blood cultures ...............................199

5.4.1.1 Monocytes and neutrophils ............................................................................... 199

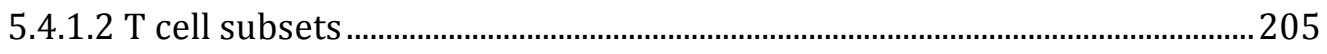

5.4.1.3 CD56 ${ }^{+}$cell subsets......................................................................................209 
5.4.3 PBMC and polyclonal T cell cytokine responses................................................... 214

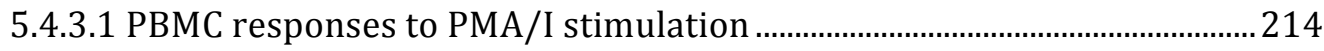

5.4.3.2 Response to anti-CD3/28 dynabead stimulation ......................................... 217

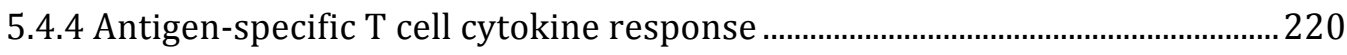

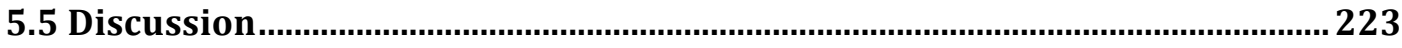

5.6 Conclusions and future directions ..................................................................... 232

Chapter 6. Assessing the global response to RIPC in humans .................. 233

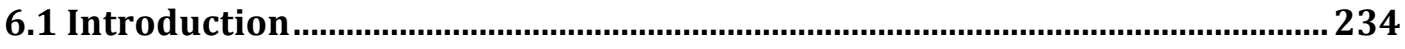

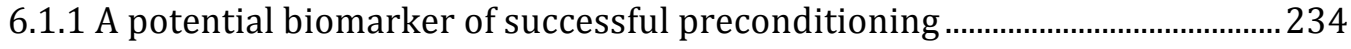

6.1.2 Statistical methods for detecting patterns in the response to RIPC ..............235

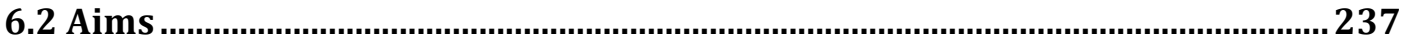

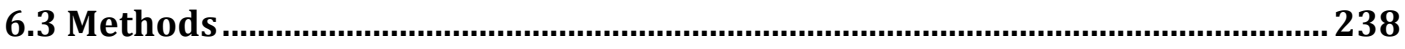

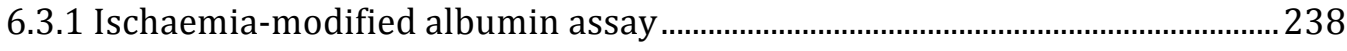

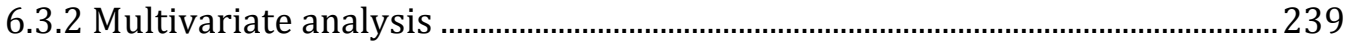

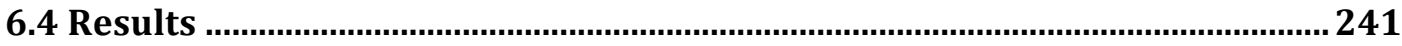

6.4.1 Ischaemia-modified albumin expression following RIPC................................. 241

6.4.2 Multivariate analysis of the response to RIPC ................................................... 244

6.4.2.1 Visual representation of the differences between the treatments and study participants.......................................................................................................... 244

6.4.2.2 Statistical analysis of the differences between the treatments and study

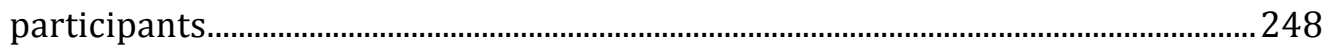

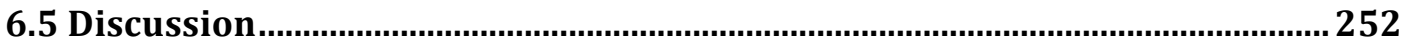

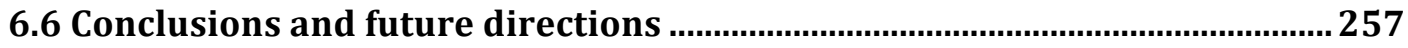

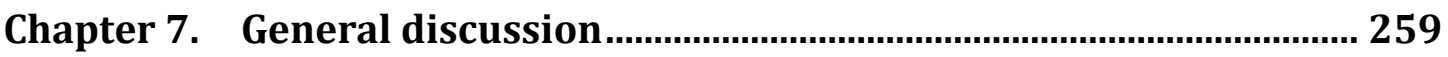

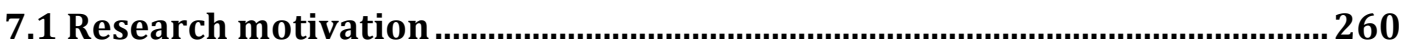

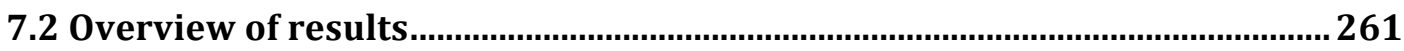

7.3 Alternative mechanisms that may contribute to RIPC ....................................264

7.4 Comparison between animal and human models of RIPC ...............................266

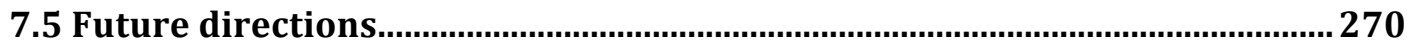

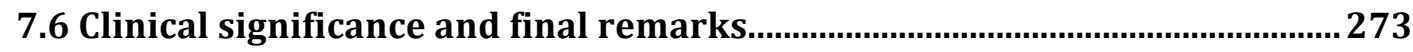




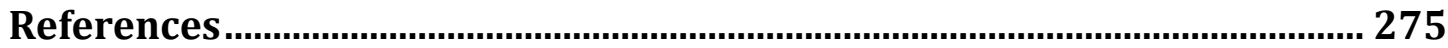

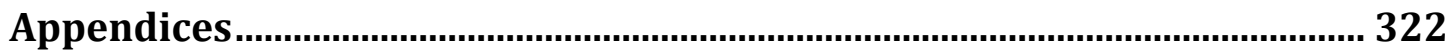

Appendix A. Standardised trial protocols for peri-operative management of cardiac surgery patients .................................................................... 323

Appendix B. Criteria for EuroSCORE and RIFLE Evaluations.............................. 329

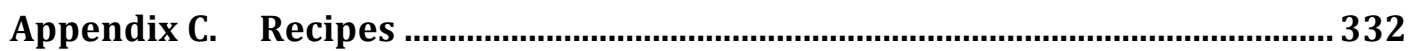

Appendix D. Manuscripts resulting from the trial of RIPC in high-risk cardiac

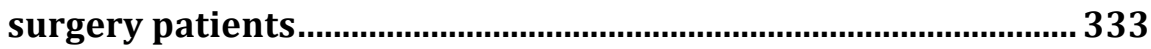

Appendix E. Complete list of flow cytometry antibodies ......................................348

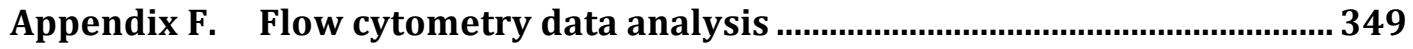

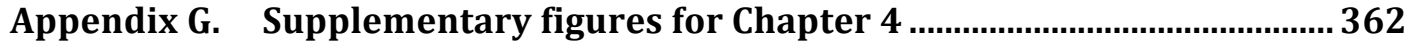

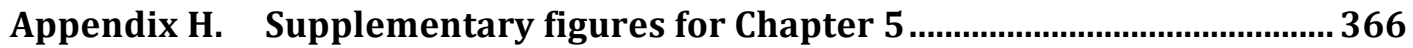




\section{List of Figures}

Figure 1.1 Proposed mechanisms involved in organ protection with RIPC .............38

Figure 2.1 CONSORT diagram of study workflow. 57

Figure 2.2 Levels of IL-6, IL-8, and IL-10 are not affected by RIPC in high-risk cardiac surgery up to 12 hours after cross-clamp removal 62

Figure 2.3 IL-6 and IL-10 levels are influenced by surgery complexity, but not RIPC. 66

Figure 2.4 Correlation between bypass time and cytokine levels in high-risk surgery patients. 68

Figure 2.5 The effect of statins on post-operative IL-6 levels. 70

Figure 2.6 Comparison of TNF- $\alpha$ and IL-6 recovery between commercially available serum vacutainers.

Figure 3.1 Summary of the steps undertaken to test the immunophenotyping protocol 90

Figure 3.2 Summary of work performed to develop the ICS protocol. 91

Figure 3.3 Assessment of cell viability in whole blood samples 93

Figure 3.4 Comparison of Fc receptor-blocking strategies for flow cytometry .... 95 Figure 3.5 fMLP stimulation of neutrophils for activation marker measurement ....

Figure 3.6 PMA/I stimulation of neutrophils activation marker expression 98

Figure 3.7 Stimulation of intracellular cytokine production in 4-hour cultures 102

Figure 3.9 Improvement in IL-8 antibody resolution 103

Figure 3.9 Titration of the IL- 6 antibody for intracellular use 104

Figure 3.10 Stimulation of intracellular cytokine production in 27-hour cultures...

Figure 3.11 Comparison of protein transport inhibitors for intracellular measurement of IL-6, IL-8, and IL-10. 107

Figure 3.12 Activation state of cultured neutrophils 109

Figure 3.13 Optimisation of flow cytometry application settings . 111

Figure 3.14 Summary of the work undertaken to test the methods for PBMC culture and quantification 
Figure 3.15 PBMC purity after Histopaque-1077 isolation

Figure 3.16 Determining the linear detection range for the CyQuant proliferation assay.

Figure 3.17 Stimulation of PBMC with PMA/I and anti-CD3/28 beads 117

Figure 3.18 Stimulation of PBMC with the tetanus vaccine 120

Figure 3.19 Comparison of PBMC cultured with the separated or full tetanus vaccine.

Figure 3.20 Overview of laboratory sample handling schedule 124

Figure 4.1 Overview of treatment administration and blood sample collection during study visits. 147

Figure 4.2 Serum levels of adhesion molecules following RIPC.. 153

Figure 4.3 Serum levels of macrophage inhibitory protein 1 after RIPC 155

Figure 4.4 Post-RIPC systemic chemokine levels 156

Figure 4.5 Serum T cell cytokine expression after RIPC. 159

Figure 4.6 Systemic levels of TNF $\alpha$, IL-1 $\beta$, IFN- $\alpha$, and IL-10 following RIPC .......162

Figure 4.7 Post-RIPC systemic levels of IL-12p70, LAP, and G-CSF 163

Figure 4.8 Size and activation state of circulating neutrophils and monocytes with and without RIPC 167

Figure 4.9 Responsiveness of neutrophils and monocytes to re-stimulation after RIPC treatment 168

Figure 4.10 Proportions of circulating T cell subsets after RIPC 171

Figure 4.11 CD69 expression in T cell subsets following RIPC treatment. 172

Figure 4.12 Proportion and activation of circulating CD56 cells after RIPC...... 174

Figure 5.1 Size and activation state of monocyte and neutrophil populations after 4 or 27 hours of culture

Figure 5.2 Early phase of intracellular IL-6 and IL-8 production in cultured monocytes and neutrophils 203

Figure 5.3 Late stage intracellular IL-6 and IL-8 production in cultured monocytes and neutrophils

Figure $5.4 \mathrm{~T}$ cell subset proportions and activation state after 27 hours culture

Figure 5.5 Late phase intracellular IL- 6 and IL-8 production in cultured T cell subsets. 
Figure 5.6 Intracellular cytokine production in NK cells cultured for 27 hours

Figure 5.7 Cytokine production in NKT cells after 27 hours of culture 211

Figure 5.8 PBMC proliferative responses following RIPC treatment 213

Figure 5.9 Production of Th1 type cytokines in PMA/I stimulated PBMC. 215

Figure 5.10 Levels of Th2 type cytokines in PMA/I stimulated PBMC cultures

Figure 5.11 Th9/Th17/Th22 type cytokines in PMA/I stimulated PBMC cultures.. 216

Figure 5.12 Levels of Th1 type cytokines in anti-CD3/28 dynabead stimulated PBMC

Figure 5.13 Production of Th2 type cytokines in anti-CD3/28 dynabead treated PBMC cultures 218

Figure 5.14 Expression of Th9/Th17/Th22 type cytokines in PBMC cultures stimulated with anti-CD3/28 dynabeads

Figure 5.15 Th1 type cytokine production in tetanus stimulated PBMC. 221

Figure 5.16 Production of Th2 type cytokines in PBMC cultured with the tetanus antigen 221

Figure 5.17 Levels of Th9/Th17/Th22 type cytokines in tetanus stimulated PBMC

Figure 6.1 Levels of ischaemia-modified albumin in study participants 243

Figure 6.2 Total change in ischaemia-modified albumin from baseline to 4 hours after treatment. 243

Figure 6.3 Dendrogram showing relatedness between participants and treatment visits 245

Figure 6.4 Visual representation of the distance between datasets based on multidimensional scaling analysis.

Figure 6.5 Simplified visual representation of the distance between datasets based on multidimensional scaling analysis

Figure A.1 Protocol for haemodynamic management of patients in the first 12 hours following cardiac surgery

Figure F.1 Gating of monocytes and neutrophils in panel 4 350 
Figure F.2 Representative plots of CD63 and CD11b staining in monocytes and neutrophils during immunophenotyping. 351

Figure F.3 Gating of total T cell populations and CD56 ${ }^{+}$cells in panel 3 352

Figure F.4 Gating of CD 4 T cells in panel 1 353

Figure F.5 Gating of CD8 T cells and NK cells in panel 2 354

Figure F.6 Gating of total live leukocytes, monocytes, and neutrophils in panel 7 during ICS 355

Figure F.7 Gating of intracellular cytokines in monocytes and neutrophils.........356

Figure F.8 Gating of intracellular cytokines in CD4 T cells in panel 5. 357

Figure F.9 Gating of intracellular cytokines in CD8 T cells in panel 6 . 358

Figure F.10 Gating of CD69 positive T cells during intracellular cytokine staining. 359

Figure F.11 Gating of NK and CD4+ NKT cells in panel 5 360

Figure F.12 Gating of CD8+ NKT cells and intracellular cytokines in NK and NKT cells in panel 6 361

Figure G.1 Serum cytokine levels for all study participants . 364

Figure G.2 Serum IL-17 concentrations with outliers removed 365

Figure H.1 T cell subset proportions and activation state after 4 hours culture....... 366

Figure H.2 Intracellular IL-6 and IL-8 production in T cells cultured for 4 hours .... 367

Figure H.3 Alternative expressions of intracellular cytokine data in T cells cultured for 27 hours 368

Figure H.4 Intracellular cytokine production in NK cells cultured for 4 hours.. 369

Figure H.5 Cytokine production in NKT cells after 4 hours of culture 370 


\section{List of Tables}

Table 1.1 Clinical trials investigating the cardioprotective effect of RIPC .

Table 1.2 Clinical trials investigating the effect of RIPC on renal outcome measures

Table 2.1 Patient demographic and clinical data 58

Table 2.2 Patient operation details 59

Table 2.3 Statistical comparison of peri-operative IL-6, IL-8, and IL-10 levels between RIPC and control treated patients 63

Table 2.4 Key patient demographic, clinical, and operative details for simple and complex surgery subgroups 65

Table 6.1 Variables included in the multivariate analysis 240

Table 6.2 Baseline demographics of participant groups. 242

Table 6.3 Characteristics of the ACS patient population. 242

Table 6.4 Variables responsible for the difference between control and RIPC treatment responses. 250

Table 6.5 Variables responsible for the differences between study participants

Table B.1 Beta coefficients for the logistic regression model of EuroSCORE...... 330

Table B.2 RIFLE classification criteria 331

Table F.1 Immunophenotyping antibody panels for a Guava easyCyte 8HT ...... 349

Table F.2 Overview of antibody panels used for ICS on the FACSCanto ${ }^{\mathrm{TM}}$ II 349 


\section{List of Abbreviations}

\begin{tabular}{|c|c|}
\hline 8-SPT & 8-( $p$-sulfophenyl)theophylline \\
\hline ACE & Angiotensin converting enzyme \\
\hline ACS & Acute coronary syndrome \\
\hline ADP & Adenosine diphosphate \\
\hline $\mathrm{AF}$ & Atrial fibrillation \\
\hline AF488 & Alexa fluor 488 \\
\hline AKI & Acute kidney injury \\
\hline Akt & Protein kinase B \\
\hline $\mathrm{Al}(\mathrm{OH})_{3}$ & Aluminium hydroxide \\
\hline AMP & Adenosine monophosphate \\
\hline ANOVA & Analysis of variance \\
\hline ANCOVA & Analysis of covariance \\
\hline APC & Allophycocyanin \\
\hline APCs & Antigen presenting cells \\
\hline ASD & Atrial septal defect \\
\hline ATP & Adenosine triphosphate \\
\hline AUC & Area under the curve \\
\hline AVR & Aortic valve replacement \\
\hline BL & Baseline \\
\hline BMI & Body mass index \\
\hline BSA & Bovine serum albumin \\
\hline BV421 & Brilliant violet 421 \\
\hline CABG & Coronary artery bypass graft \\
\hline CBA & Cytometric bead array \\
\hline CD & Cluster of differentiation \\
\hline CGRP & Calcitonin gene-related peptide \\
\hline $\mathrm{CI}$ & Confidence interval / Cardiac index \\
\hline CK-MB & Creatine kinase-MB \\
\hline $\mathrm{CPB}$ & Cardiopulmonary bypass \\
\hline CRP & C-reactive protein \\
\hline CTCM & Complete $\mathrm{T}$ cell media \\
\hline cTnI & Cardiac troponin I \\
\hline cTnT & Cardiac troponin $\mathrm{T}$ \\
\hline CVP & Central venous pressure \\
\hline DAMP & Danger-associated molecular pattern \\
\hline DNA & Deoxyribonucleic acid \\
\hline dPBS & Dulbecco's phosphate buffered saline \\
\hline DTT & Dithiothreitol \\
\hline ECG & Electrocardiogram \\
\hline $\mathrm{EF}$ & Ejection fraction \\
\hline ELISA & Enzyme-linked immunosorbent assay \\
\hline eNOS & Endothelial nitric oxide synthase \\
\hline ERK & Extracellular signal-regulated kinase \\
\hline EuroSCORE & European system for cardiac operative risk evaluation \\
\hline FACS & Fluorescent activated cell sorting \\
\hline $\mathrm{Fc}$ & Fragment crystallizable \\
\hline
\end{tabular}




\begin{tabular}{|c|c|}
\hline FCS & Fetal calf serum \\
\hline fMLP & N-formyl-methionyl-leucyl-phenylalanine \\
\hline FSC & Forward scatter \\
\hline G-CSF & Granulocyte colony-stimulating factor \\
\hline GFR & Glomerular filtration rate \\
\hline HIF- $1 \alpha$ & Hypoxia-inducible factor-1 alpha \\
\hline HOE-140 & Hoechst 140 \\
\hline HSP & Heat shock protein \\
\hline ICAM & Intercellular adhesion molecule \\
\hline ICS & Intracellular cytokine staining \\
\hline ICU & Intensive care unit \\
\hline IFN & Interferon \\
\hline IgG & Immunoglobulin G \\
\hline IL & Interleukin \\
\hline IM & Ionomycin \\
\hline IMA & Ischaemia-modified albumin \\
\hline iNKT & Invariant natural killer T cell \\
\hline iNOS & Inducible nitric oxide synthase \\
\hline IP-10 & Interferon gamma-induced protein 10 \\
\hline IPC & Ischaemic preconditioning \\
\hline IQR & Interquartile range \\
\hline $\mathrm{I} / \mathrm{R}$ & Ischaemia/reperfusion \\
\hline IU & International units \\
\hline JAK & Janus kinase \\
\hline JNK & c-Jun N-terminal kinases \\
\hline LAD & Left anterior descending artery \\
\hline LAP & Latency associated peptide \\
\hline LDH & Lactate dehydrogenase \\
\hline LOD & Limit of detection \\
\hline LPS & Lipopolysaccharide \\
\hline MACE & Major adverse cardiovascular events \\
\hline MAP & Mean arterial pressure \\
\hline MAPK & Mitogen-activated protein kinase \\
\hline MCP & Monocyte chemotactic protein \\
\hline MDA & Malondialdehyde \\
\hline MDS & Multidimensional scaling \\
\hline MFI & Mean fluorescence intensity \\
\hline MHC & Major histocompatibility complex \\
\hline MIP & Macrophage inflammatory protein \\
\hline MPG & Mercaptopropionyl-glycine \\
\hline MPO & Myeloperoxidase \\
\hline mPTP & Mitochondrial permeability transition pore \\
\hline mRNA & Messenger ribonucleic acid \\
\hline MVR & Mitral valve repair or replacement \\
\hline$N F-\kappa B$ & Nuclear factor kappa B \\
\hline NGAL & Neutrophil gelatinase-associated lipocalin \\
\hline NIR & Near-infrared \\
\hline NK & Natural killer \\
\hline NO & Nitric oxide \\
\hline
\end{tabular}




\begin{tabular}{|c|c|}
\hline NSE & Neuron-specific enolase \\
\hline NT-pro-BNP & $\mathrm{N}$ terminal prohormone of brain natriuretic peptide \\
\hline PAMP & Pathogen-associated molecular pattern \\
\hline PAP & Pulmonary artery pressure \\
\hline PBMC & Peripheral blood mononuclear cells \\
\hline PBS & Phosphate buffered saline \\
\hline PCI & Percutaneous coronary intervention \\
\hline PCWP & Pulmonary capillary wedge pressure \\
\hline $\mathrm{PE}$ & Phycoerythrin \\
\hline $\mathrm{PH}$ & Pulmonary hypertension \\
\hline PI3 & Phosphoinositide 3-kinase \\
\hline PMA & Phorbol 12-myristate 13-acetate \\
\hline PMA/I & Phorbol 12-myristate 13-acetate and ionomycin \\
\hline PRR & Pattern recognition receptor \\
\hline RAG-1 & Recombination activating gene 1 \\
\hline $\mathrm{RBC}$ & Red blood cell \\
\hline RCF & Relative centrifugal force \\
\hline RHP & Repetitive hypobaric preconditioning \\
\hline RIFLE & $\begin{array}{l}\text { Risk, injury, failure, loss of kidney function, and end-stage kidney } \\
\text { disease }\end{array}$ \\
\hline RIPC & Remote ischaemic preconditioning \\
\hline RT & Room temperature \\
\hline SA-PE & Streptavidin phycoerythrin \\
\hline SA-HRP & Streptavidin horseradish peroxidase \\
\hline SCID & Severe combined immunodeficiency \\
\hline $\mathrm{SCr}$ & Serum creatinine \\
\hline SD & Standard deviation \\
\hline SEM & Standard error of the mean \\
\hline SIMPER & Similarity percentage analysis \\
\hline SOD & Superoxide dismutase \\
\hline SSC & Side scatter \\
\hline STAT & Signal transducers and activators of transcription \\
\hline SVRI & Systemic vascular resistance index \\
\hline TA & Tricuspid annuloplasty \\
\hline TCR & $\mathrm{T}$ cell receptor \\
\hline TGF & Transforming growth factor \\
\hline $\mathrm{Th}$ & T helper cell \\
\hline TIM-3 & $\mathrm{T}$ cell immunoglobulin domain and mucin domain 3 \\
\hline TLR & Toll-like receptor \\
\hline TNF & Tumour necrosis factor \\
\hline Treg & $\mathrm{T}$ regulatory cell \\
\hline UO & Urine output \\
\hline VSD & Ventricular septal defect \\
\hline WBC & White blood cell \\
\hline WT & Wild type \\
\hline
\end{tabular}



Chapter 1.

\section{General introduction}




\subsection{Introduction}

Worldwide, an estimated one million cardiac surgeries are performed each year, of which 2500 are completed in New Zealand (Auckland District Health Board, 2008). During the majority of these procedures cardiopulmonary bypass (CPB) is employed to replace the roles of the heart and lungs, maintaining the circulation of oxygenated blood to the body and allowing the heart to be operated on. While the utility of CPB is indispensable during these surgeries, its use is associated with periods of oxygen deprivation and a systemic inflammatory reaction, which together escalate the response elicited by the surgical procedure alone (Paparella et al., 2002). As the incidence of diabetes and hypertension increase among an ever-growing aging population a number of cardiac surgery patients are presenting with reduced physiological reserves (Biancari et al., 2009; Etzioni and Starnes, 2011; McGuinness et al., 2008). Taken together, these factors signify an increased risk of morbidity and mortality for many modern cardiac patients and the need for novel strategies to improve clinical outcomes in such cases.

The research presented in this thesis is focused on a potential cardioprotective technique known as remote ischaemic preconditioning (RIPC). This general introduction describes the pathological processes associated with cardiac surgery, the history and clinical development of RIPC, and an overview of what is currently known about the underlying mechanisms involved in this technique. A more detailed summary of the background information pertinent to the research conducted as part of this project, particularly regarding what is known about the impact of RIPC on the immune system, is introduced in the subsequent results chapters.

\subsection{The need for myocardial protection in cardiac surgery}

There are two distinct processes involved in the unfavourable response to CPB. The first of these, termed the early-phase, is initiated when the blood encounters the artificial surface of the extracorporeal bypass circuit, whereas the second process, the late-phase, is induced predominantly by ischaemia-reperfusion (I/R) injury (Warren et al., 2009). 


\subsubsection{Early-phase of inflammation during cardiac surgery}

Interactions between the blood and the negatively charged surface of the CPB tubing results in a protein activation cascade that initiates components of the complement, fibrinolytic, contact, and coagulation pathways, the last two of which lead to thrombin generation (Miller and Levy, 1997). During CPB, thrombin activation of fibrin and platelets is the primary cause of thrombosis, the formation of blood clots within blood vessels. Many of the resulting microemboli re-enter the circulation and block narrow capillaries, leading to small pockets of necrosis in hundreds of loci throughout the body (Abu-Omar et al., 2004; Edmunds, 1998). Introduction of CPB circuits coated with the anticoagulant heparin have been shown to inhibit thrombin formation and reduce activation of the contact system, but not to the extent required to prevent stimulation of the systemic inflammatory response associated with CPB (Baufreton et al., 1998).

The fibrinolytic system functions to offset the coagulation system during CPB by localising clot formation to the site of injury rather than allowing unrestrained systemic thrombosis. The activated endothelium lining the vasculature initiates plasmin production, which breaks down blood clots into soluble products and reduces platelet adhesion and aggregation (van Hinsbergh, 2012). The endothelium thus plays a key role in maintaining the fluidity of the blood by way of the fibrinolytic system; however, there is commonly an imbalance between coagulation and fibrinolysis during CPB which results in poor haemostasis and increased post-operative blood loss (Boyle et al., 1996b).

Complement activation occurs in response to a variety of stimuli, resulting in production of the anaphylatoxins $\mathrm{C} 3 \mathrm{a}, \mathrm{C} 4 \mathrm{a}$, and $\mathrm{C} 5 \mathrm{a}$ that lead to smooth muscle contraction in the airways and increased vascular permeability, both of which result in hypotension and the need for lengthened post-operative ventilatory support (Miller and Levy, 1997). Additionally, C3a and C5a can stimulate transcription of the proinflammatory cytokines tumour necrosis factor (TNF)- $\alpha$, interleukin (IL)-1, and IL-6, thus amplifying the inflammatory response (Miller and Levy, 1997). Complement activation also leads to formation of the 
membrane attack complex (MAC), a transmembrane channel responsible for cell lysis and death in endothelial cells and cardiac myocytes. Together, C5a and MAC are potent activators of neutrophils, promoting chemotaxis, granule release, and the production of toxic oxygen free radicals (Collard and Gelman, 2001; Warren et al., 2009).

Neutrophils are the key effector cells of the inflammatory response and in addition to recognising a broad range of pathogens they can be stimulated by a variety of danger signals from other cells. In the case of CPB these activation signals are diffuse and primarily consist of proinflammatory cytokine release and complement degradation products (Verrier and Boyle, 1996). Further, local activation of endothelial cells at the site of infection or injury by other aspects of the immune response induces the surface expression of neutrophil adhesion molecules, selectins and integrins (Paparella et al., 2002). Together, these responses result in widespread endothelial activation and neutrophil sequestration into the lungs and other organs.

During $\mathrm{CPB}$, neutrophils are chiefly activated by direct interactions with the endothelium and inflammatory mediators such as thrombin, endotoxin and heparin (Boyle et al., 1996a). This activation results in neutrophil degranulation and release of enzymes that damage the structural matrix proteins necessary for normal cell function. Additional non-specific damage occurs with neutrophil secretion of reactive oxygen species (ROS) that can lead to the destruction of cellular membranes, DNA nicking and cross-linking, the degradation of proteins, and further upregulation of endothelial adherence molecules (Korthuis and Granger, 1993). These actions are responsible for much of the CPB-associated damage to the lungs, heart and other tissues, making neutrophils a major target for controlling the inflammatory response.

The endothelium is not passive in these processes but rather assists through production of IL-8 to attract neutrophils and facilitate transendothelial migration and also releases vasoconstrictive agents (Boyle et al., 1996a). A key difficulty with CPB is this impaired performance of the systemically activated endothelium 
post-surgery. The dysfunctional endothelium promotes localised coagulopathy and proinflammatory cytokine production, propagating the inflammation and tissue damage associated with organ dysfunction such as cardiodepression and acute renal injury, which are frequently detected following CPB (Gueret et al., 2009; Verrier and Boyle, 1996).

\subsubsection{Late-phase of inflammation during cardiac surgery}

Ischaemia describes the situation where there is insufficient blood supply to an organ or tissue, thus causing cell death through the resulting reduced oxygen delivery and metabolite washout. Although beneficial in the long term, reperfusion, which is the restoration of blood flow to the ischaemic tissue, initiates both a local and systemic inflammatory response that is associated with more substantial tissue injury than that caused by the initial ischaemia (Abela and Homer-Vanniasinkham, 2003). This phenomenon is known as ischaemiareperfusion (I/R) injury and is the major cause of peri-operative myocardial damage.

Under hypoxic conditions intracellular adenosine triphosphate (ATP) levels are quickly consumed due to decreased oxidative phosphorylation. Cellular energy production thus shifts toward anaerobic metabolism, which results in proinflammatory cytokine production (Abela and Homer-Vanniasinkham, 2003). The lack of oxygen also induces high levels of endothelial trauma, leading to upregulation of leukocyte adhesion molecule expression and consequently neutrophil sequestration following reperfusion, particularly in the lungs (Asimakopoulos et al., 1999). I/R associated tissue damage can result in both local and remote organ pathologies and is largely mediated by the neutrophilendothelial interactions described earlier; however, free radical production and endotoxaemia are additional processes that characterise the late-phase inflammatory response.

The enzymes responsible for breakdown of the free radical precursor hypoxanthine are oxygen-dependent xanthine oxidases (Collard and Gelman, 2001). Under hypoxic conditions, hypoxanthine accumulates and upon 
reperfusion is activated into ROS. ROS generation can lead to cellular structural damage and lipid peroxidation, as well as enhanced leukocyte migration, diapedesis and activation. Additionally, ROS deactivate nitric oxide (NO), which would normally function to clear free radicals, inhibit proinflammatory cytokine production, and facilitate vasodilation (Abela and Homer-Vanniasinkham, 2003). The resulting cellular oedema and endothelial swelling leads to the compression of capillaries and restriction of blood flow, which is further exacerbated by plugging with fibrin, platelets, and leukocyte aggregates. These processes result in what is referred to as the no-reflow phenomenon, which continues to deprive the tissues of oxygen following the initiation of reperfusion (Rezkalla and Kloner, 2002).

Bacterial endotoxins are an additional potent stimulus for the development of systemic inflammation and elevated levels are frequently detected in the circulation of patients as early as twenty minutes after initiation of CPB due to splanchic vasoconstriction (Watarida et al., 1994). The resulting bowel ischaemia and inflammatory conditions increase intestinal permeability, allowing absorption of endotoxins into the portal circulation, which stimulates cytokine production, leukocyte activation, NO release, and systemic inflammation (Swank and Deitch, 1996). The extent of the patient response to endotoxaemia varies significantly, mainly due to each individual's level of anti-endotoxin core neutralising antibodies, but consistently adds to an early response of proinflammatory cytokine production (McGuinness et al., 2008).

\subsubsection{Current cardioprotective strategies}

Since the first successful open heart surgery was performed in 1953 enormous research and technological advances have significantly improved the success rate of these procedures. Despite this progress, post-operative organ damage still causes significant morbidity and mortality in cardiac surgery patients and places a major strain on limited hospital resources (Ministry of Health, 2008). The predominant cause of myocardial damage during cardiac surgery results from the ischaemia caused by cross-clamping the heart that prevents perfusion of the coronary arteries. At present there are a number of cardioprotective 
strategies that are commonly employed in an effort to minimise this ischaemiainduced peri-operative myocardial injury.

\subsubsection{Cardioplegia}

Cardioplegia is defined as an elective, rapid, and reversible paralysis of the heart and is currently the gold standard for cardioprotection during surgery. There are a variety of cardioplegia solutions commonly used to infuse the heart but they all depend on the same basic principle which uses elevated potassium levels to depolarise cardiac cells and prevent repolarisation (Chambers and Fallouh, 2010). This depolarised arrest maintains the heart in a non-beating diastolic state, eliciting protection through limiting metabolic activity in the myocardium, and allowing the surgical procedure to be performed (Ferguson et al., 2014). The solutions can be diluted and infused using either an isotonic crystalloid solution or blood; administered antegrade or retrograde, once, or multiple times; under hypothermic $\left(4-8{ }^{\circ} \mathrm{C}\right)$, tepid $\left(29-33^{\circ} \mathrm{C}\right)$, or normothermic $\left(37^{\circ} \mathrm{C}\right)$ conditions. Despite this wide variety of cardioplegic strategies, there is a distinct lack of clinical research comparing their effectiveness, and the most beneficial combination of factors remains elusive (Ferguson et al., 2014).

Although now a part of common clinical practice, the high levels of potassium involved in depolarising arrest have been associated with a range of adverse effects including, but not limited to, arrhythmias and conduction disturbances, contractile stunning, endothelial injury, and vasoconstriction (Dobson et al., 2013). These effects also further enhance the peri-operative inflammatory response. In recent years the concept of inducing a more electrically stable polarised form of arrest using adenosine and lidocaine has been proposed as an alternative to hyperkalaemic cardioplegia and has shown promising results in early clinical studies, but further trials are necessary (Dobson et al., 2013; Onorati et al., 2013).

\subsubsection{Volatile anaesthetics}

Volatile anaesthetics (VA) are administered as inhaled gases and can be used alone or in combination with intravenous anaesthetics. Several commonly used 
VA, including isoflurane and sevoflurane, have been shown to confer renal, myocardial and neural protection during surgical procedures and clinical ischaemic events (Altay et al., 2012; M. Kim et al., 2007; Landoni et al., 2007). VA reduce myocardial contractility and oxygen demand, but a number of additional pathways have also been identified as contributing to these protective effects. For instance, VA have been shown to stimulate opening of the mitochondrial adenosine triphosphate-sensitive potassium (K $\left.\mathrm{K}_{\mathrm{ATP}}\right)$ channels in both rat trabeculae and human ventricular muscle cells, and delay opening of the mitochondrial permeability transition pore (mPTP), both of which increase cellular resistance to ischaemia (de Ruijter et al., 2003; Jiang et al., 2007; Piriou et al., 2004). Other studies have also implicated the Akt/PI3k anti-apoptotic pathway and regulation of the transcription factor, nuclear factor (NF)- $\kappa \beta$, in VAinduced organ protection (Raphael et al., 2006; Wang et al., 2010). Interestingly, many of these mediators appear to overlap with those involved in classic ischaemic preconditioning (IPC).

At present it is unclear whether the use of volatile anaesthetics with IPC or RIPC can have additive protective effects during cardiac surgery. Many studies have reported a lack of cardioprotection when RIPC is used in conjunction with inhaled anaesthetics and a meta-analysis by Zhou et al. (2013) found that RIPC was less effective when used alongside volatile anaesthesia (Karuppasamy et al., 2011; Rahman et al., 2010). In contrast, more recently Kottenberg et al. (2012) reported that RIPC was effective during anaesthesia with isoflurane but not propofol in coronary artery bypass graft (CABG) patients. Substantial overlap between the pathways activated by VA and IPC could result in competitive inhibition of one or both strategies; therefore a greater understanding of the mechanisms involved in both interventions will be essential to determine their utility as a combined cardioprotective strategy.

Although numerous studies have reported beneficial effects with the use of VA during cardiac surgery, a number of studies have failed to detect an improvement (Bignami et al., 2012; De Hert et al., 2009; Piriou et al., 2004). Further, the use of VA has been associated with myocardial depression and 
vasodilation, and use in patients with severe preoperative myocardial ischaemia or cardiovascular instability was linked with worse clinical outcome when compared to the use of intravenous anaesthetics (Jakobsen et al., 2007; Larach et al., 1990; Van Allen et al., 2012). Overall, despite the widespread adoption of VA for use during cardiac surgery, they appear to have several limitations in the clinical setting.

\subsubsection{Heparin}

Heparin is essential as an anti-coagulant during cardiac surgery but has also been shown to exert additional benefits that are separate from this activity. Numerous animal-based studies have demonstrated that both heparin and Nacetyl heparin, a derivative that lacks the anti-coagulant properties, promote myocardial protection during I/R injury by reducing infarct size and myocardial dysfunction, whereas administration of the heparin-degrading enzyme, heparinase, reverses this effect (Friedrichs et al., 1994; Kilgore et al., 1999; Kouretas et al., 1999; Park et al., 1999). This protection was associated with a reduction in myocardial neutrophil accumulation and complement-mediated cell lysis, and improved preservation of coronary endothelial function.

It is now well established that heparin also has significant anti-inflammatory properties, although the mechanisms through which these effects occur are not completely understood. It appears that heparin is capable of binding and neutralising numerous inflammatory mediators including acute phase proteins, complement, and chemokines (Kuschert et al., 1999; Pangburn et al., 1991; Young et al., 1997). Heparin also reduces neutrophil adhesion to the endothelium, most likely through binding and blocking P-selectin present on activated endothelial cells and platelets (Koenig et al., 1998; Lever et al., 2000; Young et al., 1999). Lastly, it has been shown that uptake of heparin by numerous cell types including the endothelium and myocytes, prevents translocation of the transcription factor NF- $\mathrm{KB}$ into the nucleus, thus suppressing the expression of numerous inflammatory genes (Hochart et al., 2006; Manduteanu et al., 2003; Thourani et al., 2000). Although the cardioprotective effects of heparin have not been studied in-depth in the clinical environment, the 
use of heparin coated CPB circuits has been associated with significantly improved outcomes following cardiac surgery (Aldea et al., 1997, 1996).

\subsubsection{Statins}

Numerous medications including potassium channel activators, general antiinflammatory treatments, and the cholesterol lowering drugs, statins, have also been investigated for use during cardiac surgery due to their potential cardioprotective effects. Statins are the most well researched of these treatments and while they are predominantly recognised for their cholesterol-lowering properties, they also exert pleiotropic effects that promote cardioprotection, such as increased production of nitric oxide (NO), which stimulates coronary vasodilation, improved thrombogenic balance, and anti-inflammatory activity (Ma and Han, 2005; reviewed by Williams and Harken, 2008).

Accordingly, trials have reported atorvastatin treatment to be associated with a nearly $70 \%$ reduction in the combined relative risk of cardiac-related death, peri-operative myocardial infarction (MI), unstable angina, and stroke in vascular surgery patients, and use of simvastatin in CABG patients reduces postoperative thrombocytosis and the incidence of MI (Christenson, 1999; Durazzo et al., 2004). A recent meta-analysis comparing outcome data for patients that were either peri-operative statin recipients or statin-naïve determined that statin use was associated with decreased risk of atrial fibrillation in cardiac surgery patients, lower risk of MI, and reduced length of hospital stay with both cardiac and non-cardiac procedures (Chopra et al., 2012). In contrast, two large prospective trials of statin use in patients with heart failure did not detect a benefit in clinical outcomes with the use of rosuvastatin (GISSI-HF investigators, 2008; Kjekshus et al., 2007). Like many of the other cardioprotective strategies described, the existing use of statins is widespread among patients with chronic heart disease; therefore these treatments are unlikely to significantly improve on current clinical practice, and novel cardioprotective interventions are required. 


\subsection{History of IPC}

Classic or local ischaemic preconditioning (IPC) refers to the phenomenon by which exposure to a brief period of ischaemia followed by reperfusion confers protection from a subsequent sustained ischaemic injury in the same tissue. This effect was first demonstrated when Murry et al. (1986) reported that dogs that underwent four cycles of five-minute circumflex artery occlusion with intervening periods of reperfusion prior to a forty-minute sustained ischaemia suffered only $25 \%$ of the level of damage seen in the animals that were not preconditioned. Yellon et al. (1993) performed the first study in humans, demonstrating that two three-minute periods of aortic cross-clamping followed by a two-minute reperfusion during CABG slowed the rate of ATP depletion in the myocardium, relative to the controls. However, the benefits seen in such initial studies that utilised short periods of aortic cross-clamping and reperfusion as the preconditioning stimulus were offset by the increased risk of ventricular dysfunction and generation of atherosclerotic emboli (Kloner, 2009; Teoh et al., 2002).

An instrumental study by Przyklenk et al. (1993) demonstrated that in a canine model transient occlusion of the circumflex artery could reduce the extent of damage to the myocardium perfused by the left anterior descending artery (LAD) in response to a subsequent sustained ischaemia via the latter vessel. This finding, termed 'regional ischaemic preconditioning', established that the benefits of preconditioning were not restricted to the myocytes in the region directly subjected to the conditioning ischaemia. This study formed the foundation for subsequent research evaluating the potential of using alternative tissue sites for IPC.

In 1996, Gho et al. established that in a rodent model a 15 minute period of intestinal ischaemia induced by occlusion of the anterior mesenteric artery limited myocardial infarct size to the same extent as direct myocardial preconditioning in response to a 60 minute coronary artery occlusion. This protection was abolished when the mesenteric artery was permanently occluded, demonstrating the dependence of the protection on reperfusion of the 
ischaemic tissue. Further, a comparable level of protection was afforded by occlusion of the left renal artery under hypothermic conditions. This type of stimulus was termed 'remote ischaemic preconditioning'.

The inception of RIPC as a clinically relevant phenomenon was largely the result of a study by Birnbaum et al. (1997) that determined that skeletal muscle ischaemia could be used to remotely precondition the myocardium. Using rabbits, it was shown that a 55-65\% reduction of the femoral artery blood flow, coupled with electrical stimulation of the gastrocnemius muscle to increase oxygen demand in the hind limb, afforded a protective response consistent with that achieved through direct coronary artery conditioning. Studies in rats, canines, and pigs provided further evidence that the skeletal muscle could be used as the remote organ for preconditioning and the first application of RIPC in humans followed (Gunaydin et al., 2000; Kharbanda et al., 2002; Liauw et al., 1996; Oxman et al., 1997).

Gunaydin et al. (2000) used two cycles of 3-minute periods of upper limb ischaemia separated by two minutes of reperfusion to induce RIPC in a small pilot study with eight CABG patients. This research provided the first biochemical evidence of RIPC through increased lactate dehydrogenase levels in the preconditioned patients, relative to the controls. Subsequently, Kharbanda et al. (2002) developed the non-invasive form of RIPC that has been routinely adopted for the majority of clinical trial work to date. This intervention targets the skeletal muscle of the upper limb using three five-minute cycles of inflation of a blood pressure cuff to $200 \mathrm{mmHg}$, followed by five-minute periods of reperfusion. It has been demonstrated in humans that this stimulus attenuates $\mathrm{I} / \mathrm{R}$ induced endothelial dysfunction in the contralateral arm, and in a porcine model of MI it reduces both ventricular dysfunction and infarct size (Kharbanda et al., 2002, 2001).

RIPC has been shown to provide two windows of organ protection, which appear to be mediated by different processes. The first occurs immediately, lasting between two and four hours and involving the release or modification of pre- 
formed substances (Kuzuya et al., 1993; Loukogeorgakis et al., 2005). The delayed phase, also referred to as 'late' or 'second window' preconditioning, appears 24 hours later and is generally reported as lasting up to 48 - 72 hours although recently it has been suggested the effects could persist for much longer (Baxter et al., 1997; Loukogeorgakis et al., 2005; Neckár et al., 2004; Park et al., 2003). This second window appears to be dependent on changes in gene transcription (Rizvi et al., 1999).

\subsection{Clinical application of RIPC}

Although the first pilot study of RIPC in humans was performed in 2000, the first full clinical trial was not reported until 2006, when Cheung et al. (2006) investigated the effects of RIPC in children undergoing repair of congenital heart defects and reported that RIPC significantly decreased the release of a marker of cardiac damage, troponin I. Two trials in adult CABG patients followed in 2007 and 2009, both demonstrating a $43 \%$ reduction in troponin $\mathrm{T}$ levels in the preconditioned patients, compared to the controls (Hausenloy et al., 2007; Venugopal et al., 2009). These initial, highly promising results attracted worldwide attention and, consequently, there has been a surge of clinical trials and publications investigating RIPC in the past two years. The results of these recent studies have been varied, with some showing significant protection against myocardial and renal damage from RIPC and others reporting no benefit.

The variation in the methods and sheer volume of the human studies involving RIPC means it is not possible to describe each individually in detail. Instead, we have restricted our overview to trials involving cardiac surgery patients and presented the results in the Tables 1.1 and 1.2. The description in the text will be limited to summarise the current status of RIPC research regarding clinical endpoints pertaining to the organs most well studied to date: the heart and kidneys.

\subsubsection{Cardioprotection}

A review of the literature identified 32 studies of RIPC in both paediatric and adult cardiac surgery patients that included a myocardial injury biomarker, most 
commonly troponin I and T or creatine kinase MB (CK-MB), as an endpoint (Table 1.1). It should be noted that myocardial injury was not the primary outcome measure for all of these studies and therefore some may not have been deliberately powered for this endpoint. The results of these studies were split evenly, with 16 showing a positive result with respect to cardioprotection in patients treated with RIPC and 16 finding no such benefit. Numerous metaanalyses, which have increased statistical power compared to the smaller separate trials, have reported that RIPC is associated with an overall reduction in the peri-operative release of cardiac injury biomarkers, supporting the results of the positive studies (Alreja et al., 2012; Brevoord et al., 2012; D’Ascenzo et al., 2012; Haji Mohd Yasin et al., 2014; Pilcher et al., 2012; Takagi and Umemoto, 2011; L. Yang et al., 2014).

The majority of the listed trials individually lack the design and power required to determine the impact of RIPC on rare and more clinically relevant outcomes, although several publications have recently addressed this. Meta-analyses authored by Brevoord, Yang, Haji Mohd Yasin, Zhang, and the Remote Preconditioning Trialists' Group all reported that RIPC does not reduce the mortality rate in cardiac surgery patients or the length of intensive care unit (ICU) and hospital stays (Brevoord et al., 2012; Haji Mohd Yasin et al., 2014; Remote Preconditioning Trialists' Group et al., 2014; L. Yang et al., 2014; Zhang et al., 2014). Brevoord et al. found that RIPC decreased the incidence of periprocedural MI in patients undergoing cardiac and vascular surgeries or percutaneous coronary intervention (PCI), but did not reduce the rate of major adverse cardiovascular outcomes (MACE), or atrial fibrillation. The remaining meta-analyses reported that RIPC had no impact on a variety of clinical endpoints, including peri-operative MI.

The impact of RIPC on cardioprotection has also been investigated during other clinical applications. Multiple trials and meta-analyses have reported that RIPC reduces the incidence of peri-procedural myocardial infarction during PCI and also protects against haemodialysis-induced myocardial injury, both of which 
were indicated through measurement of troponin levels (D'Ascenzo et al., 2014; Park et al., 2014; Zografos et al., 2014).

Currently, there is no evidence confirming that the reduction in cardiac injury markers frequently detected following RIPC translates into an improved clinical outcome with respect to the heart; however, it is difficult to draw definitive conclusions from the body of literature currently available. There is enormous heterogeneity between study designs, which significantly limits the power of meta-analyses. In the future, larger, double-blinded, randomised, controlled studies of RIPC will be required to conclusively determine whether the intervention can improve myocardial function following cardiac procedures, compared to current strategies.

\subsubsection{Renal protection}

Kidneys have a high energy demand and are particularly vulnerable to ischaemic damage. The incidence of acute kidney injury (AKI) following cardiac surgery has been estimated at levels as high as $30 \%$, depending on the exact definition used, and is associated with a greatly increased risk of mortality (Rosner and Okusa, 2006). Numerous RIPC clinical trials have incorporated endpoints examining post-operative renal damage and function; however, only a small number of studies have been explicitly designed for this purpose. Four of the 11 RIPC cardiac surgery studies identified as including markers associated with renal function showed a benefit from the intervention, and only one of the four studies specifically designed and powered to evaluate RIPC on renal endpoints was positive (Table 1.2). Two meta-analyses by Li et al. (2013) and Yang et al. (2014), which broadened the population of interest to include both cardiac and vascular surgeries, did find that RIPC significantly reduced the incidence of AKI but did not alter renal injury markers such as creatinine and estimated glomerular filtration rate (GFR), or the incidence of renal replacement therapy.

A renal protective effect with RIPC has more clearly been observed during other applications. Ali et al. (2007) were the first to report that RIPC could induce renal protection in humans by demonstrating that the intervention decreased 
the incidence of AKI by 23\% during elective abdominal aortic aneurysm repair, although a smaller subsequent study by Walsh et al. (2009) found RIPC attenuated the increase in urinary biomarkers of renal injury but did not affect the rate of renal impairment. Ischaemia also appears to contribute to the AKI frequently observed following the administration of contrast during procedures involving imaging. Accordingly, remote conditioning during angioplasty has been associated with a sustained improvement in estimated GFR, and RIPC in patients with an increased risk of renal damage has been shown to significantly reduce the incidence of AKI during elective coronary angiography (Er et al., 2012; Whittaker and Przyklenk, 2011).

As appears to be the paradigm with the current status of RIPC research, the precise effect of preconditioning on renal outcome measures has not been established by the trials performed to date. Larger scale studies specifically powered for clinical indicators of renal function will be essential to clarify whether RIPC is capable of protecting the kidneys during cardiac surgery and other clinical procedures that involve renal ischaemia.

\subsubsection{The value of RIPC in the clinical setting}

RIPC has been described as one of the most promising cardioprotective strategies identified to date, and is attractive not only due to its potentially significant impact on clinical outcomes, but also because of the practical aspects associated with its use in the clinical setting (Rahman and Bonser, 2009). Applying RIPC is extremely straightforward and inexpensive, requiring no specialised training or new devices. The equipment used to apply RIPC is already present in the clinics, hospitals and ambulances where it would be utilised. In comparison to the development of pharmaceutical treatments, RIPC can be fasttracked into the clinical environment without the need for a lengthy and expensive approval process. Further, it has a solid safety profile with no reports of adverse affects from any of the numerous trials performed thus far and is not subject to the same potential interaction issues that may be encountered with a pharmaceutical therapy. Preconditioning using the limbs is a particularly safe 
strategy as skeletal muscle is far more robust to ischaemia than visceral organs, which are highly susceptible to irreversible injury (Kaur Randhawa et al., 2014).

In addition, although preconditioning has most commonly been tested in cardiac surgery patients there is a vast range of potential clinical applications for this intervention. At present there are numerous trials registered to investigate the impact of RIPC in further surgical scenarios including plastic surgery and transplantation, cervical decompression, and laparoscopic colorectal procedures. The utility of RIPC is also being explored as an adjunct therapy in patients with ischaemic cerebrovascular conditions such as subarachnoid haemorrhage, stroke, and symptomatic intracranial arterial stenosis. Despite the varied results of small, early trials investigating this intervention in humans, RIPC clearly holds enormous potential to improve current clinical practice. 


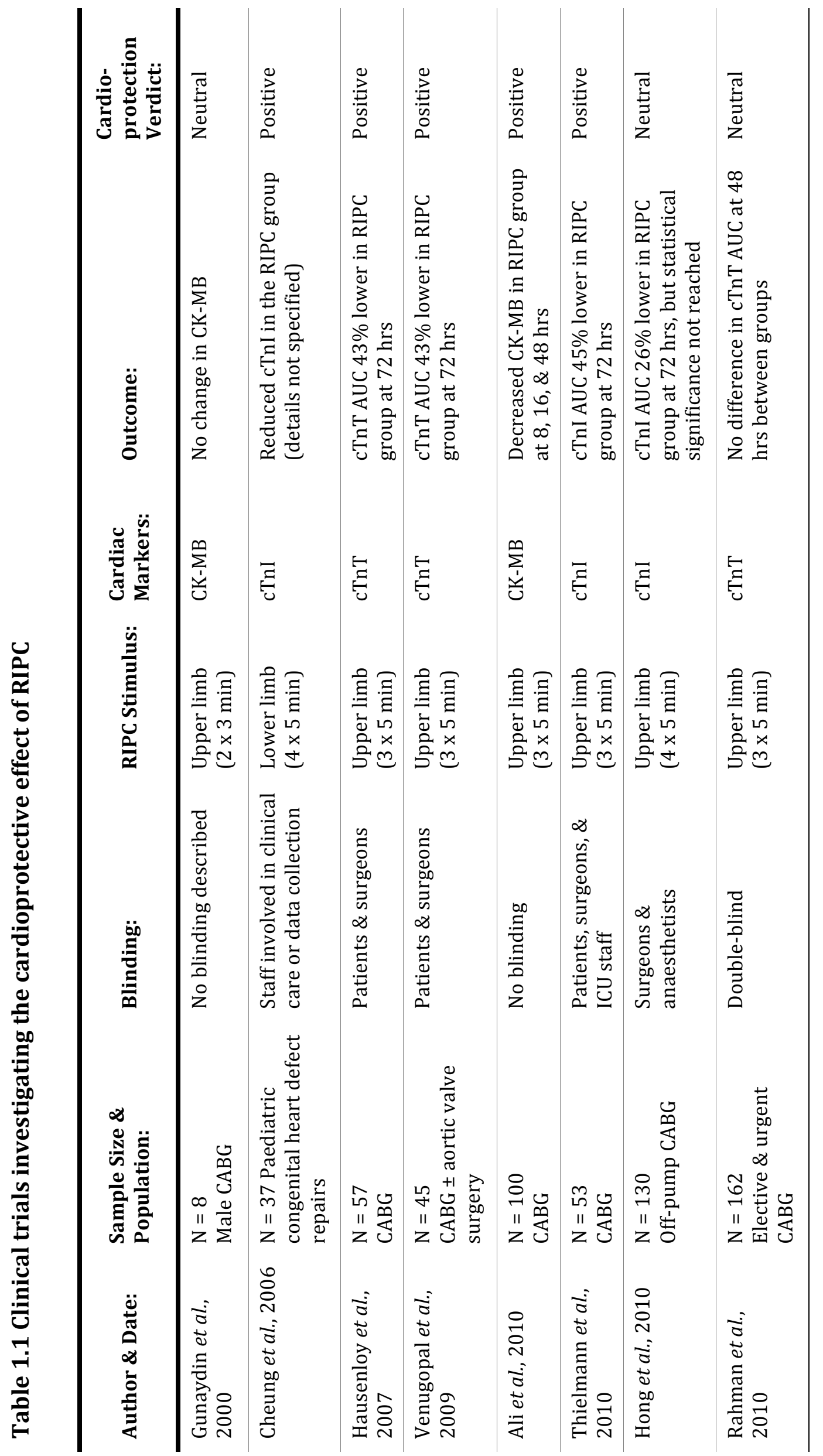




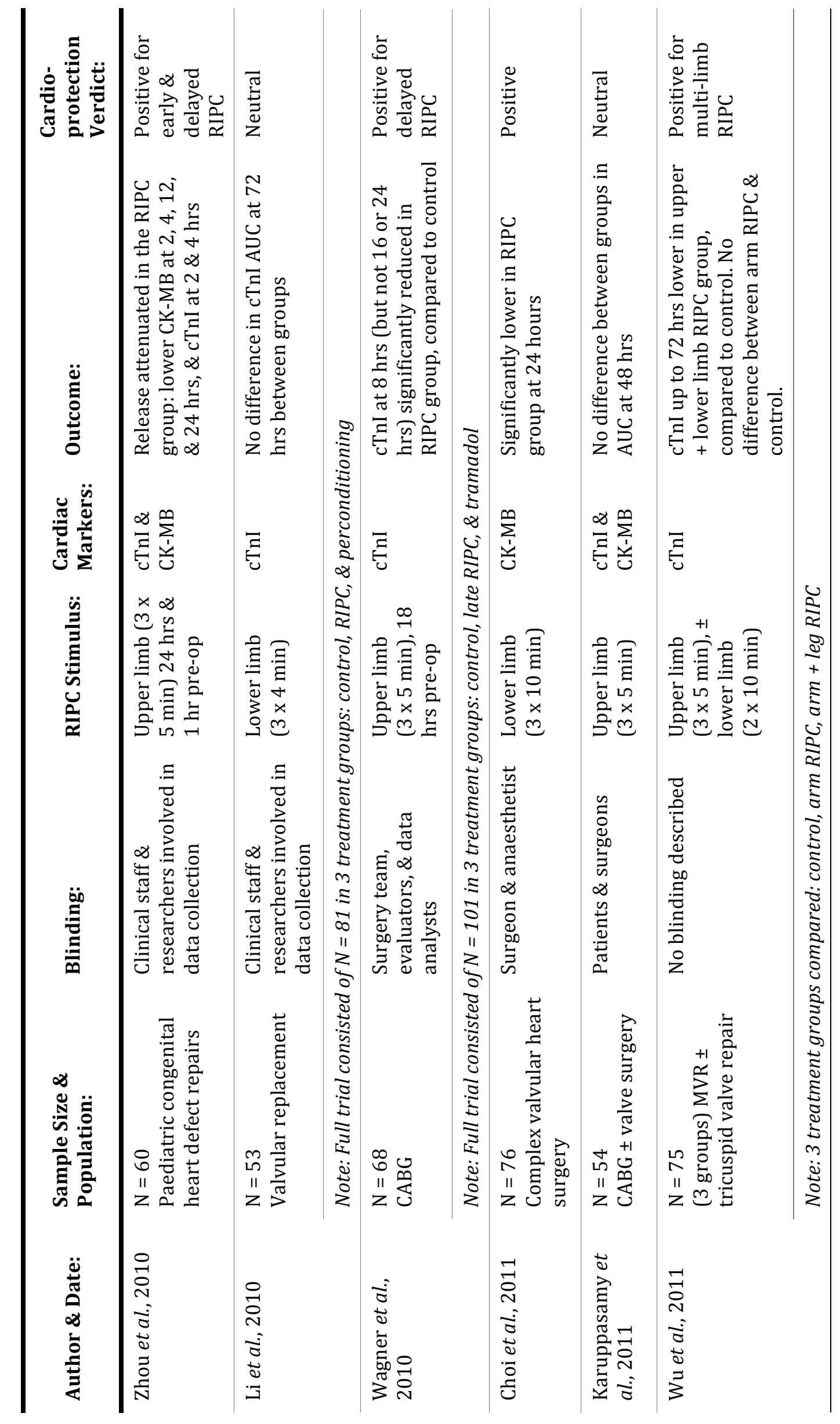




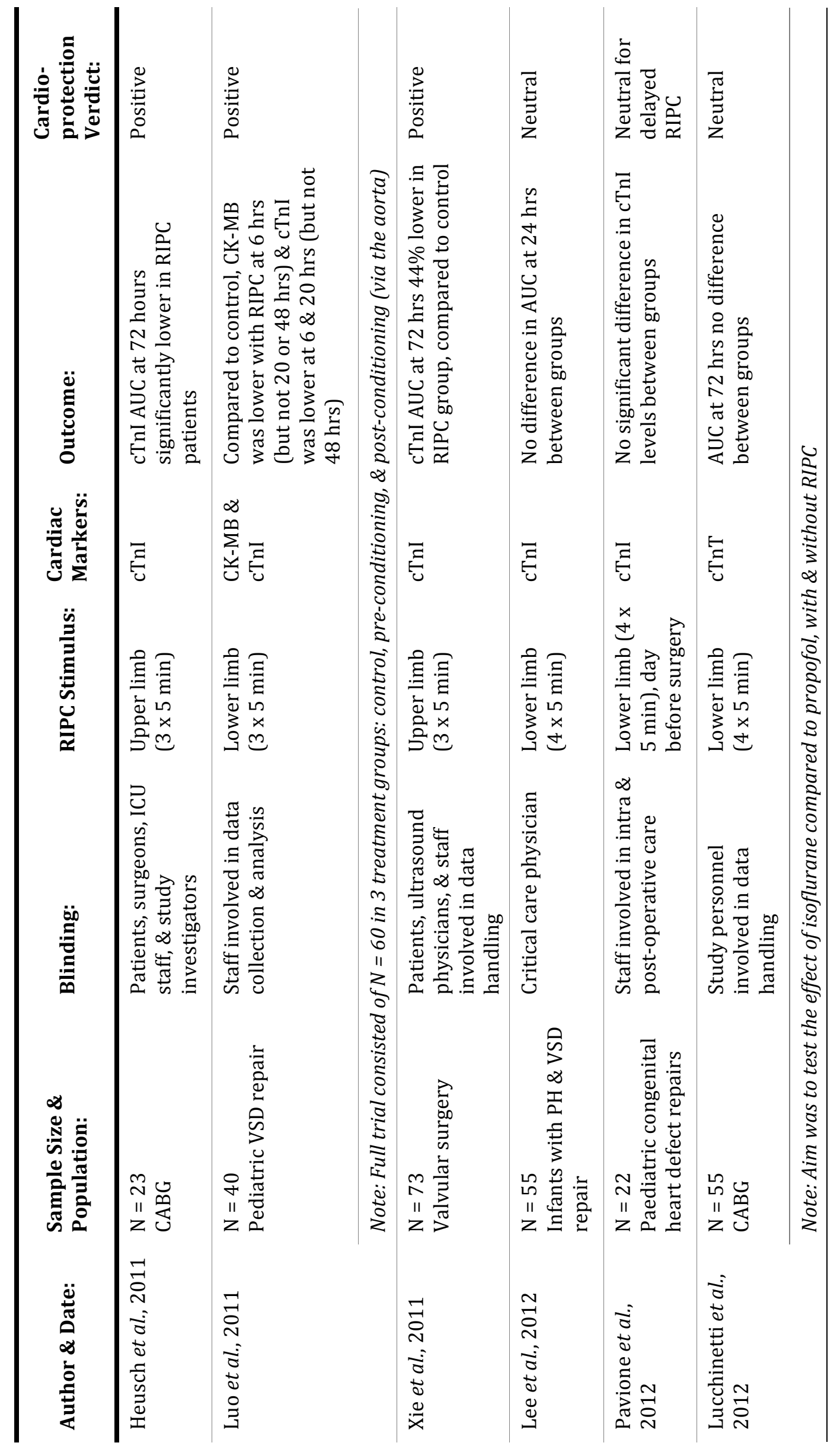




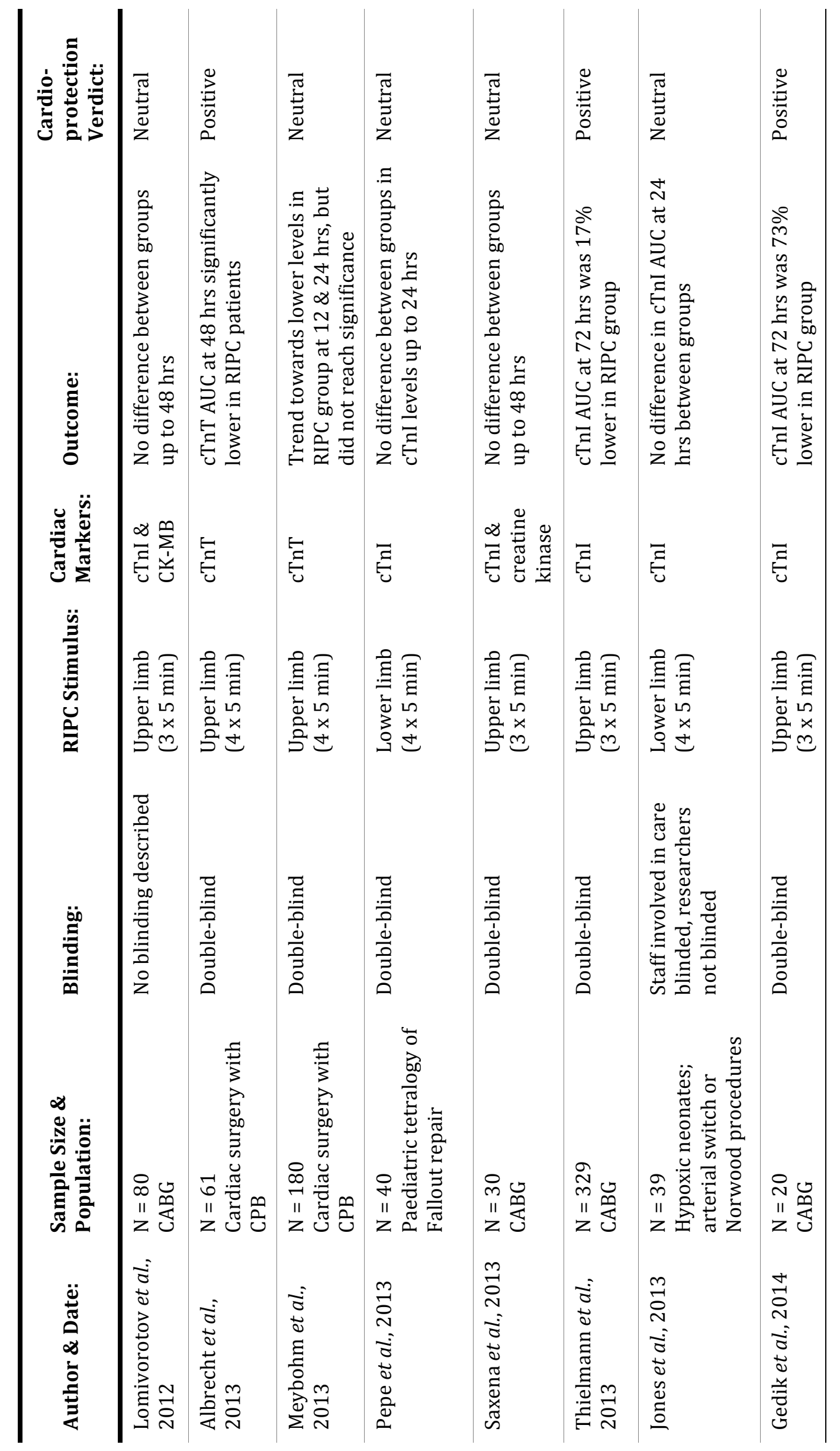




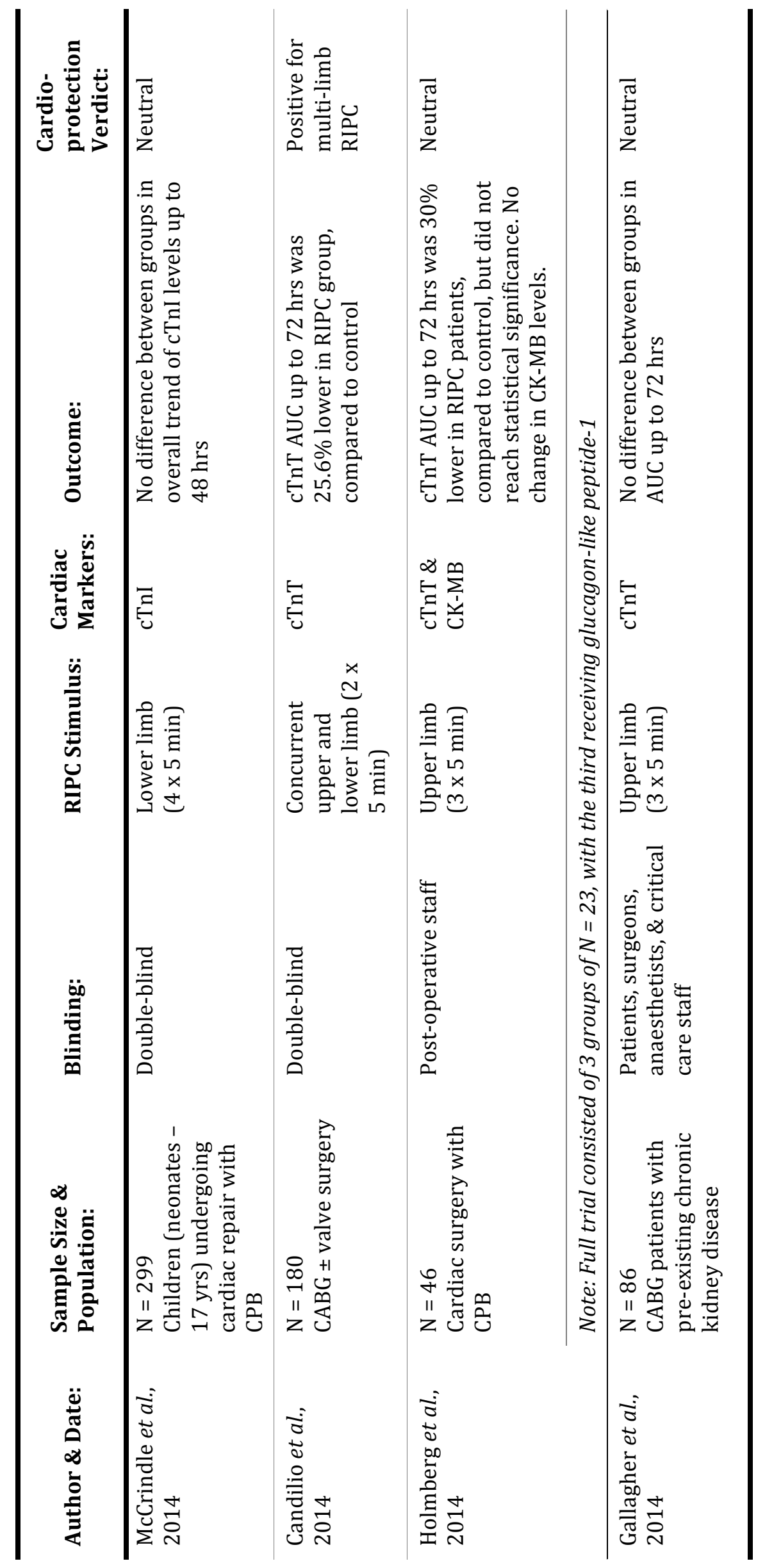




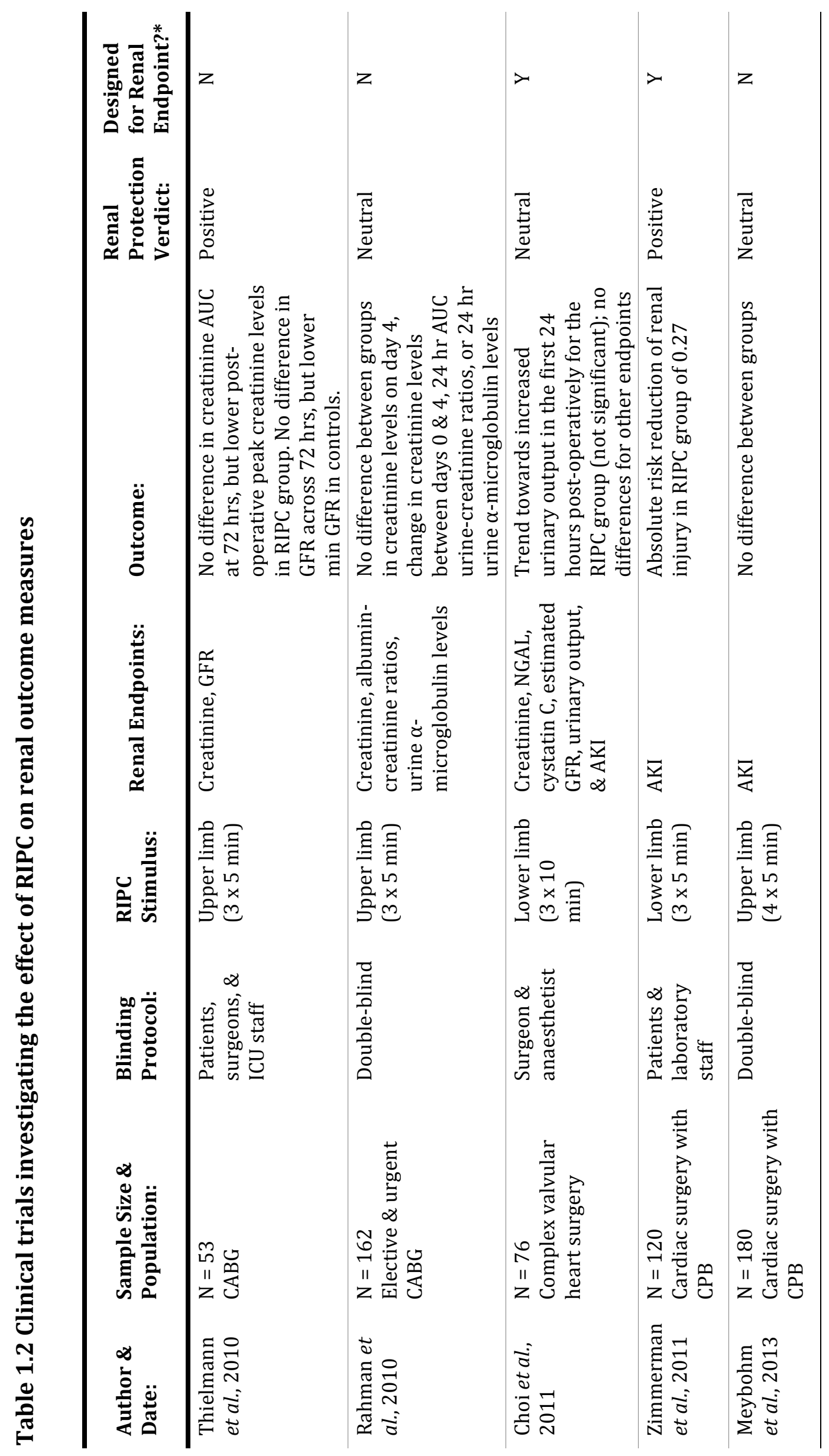




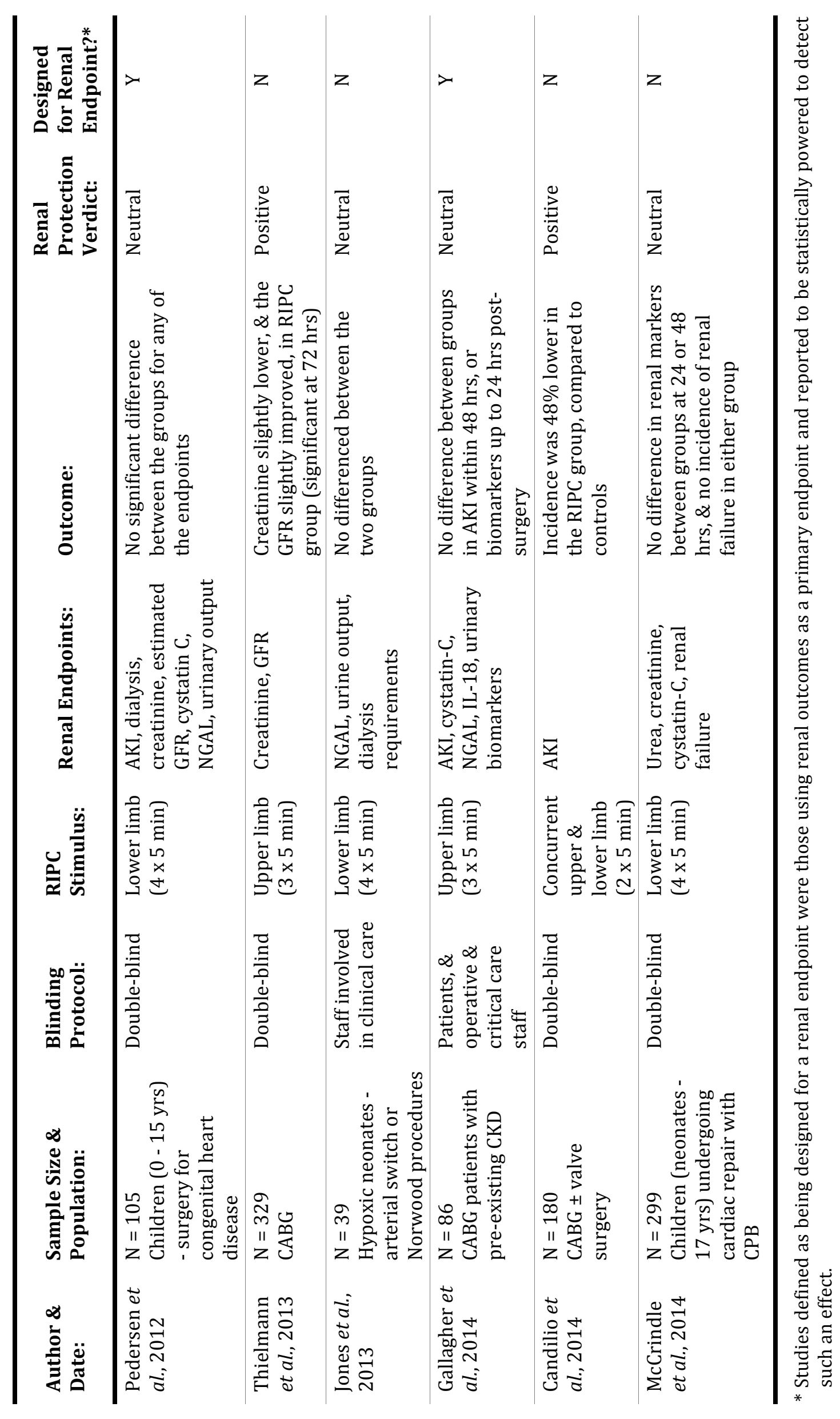




\subsection{Mechanisms involved in RIPC}

While it appears that RIPC functions to prophylactically activate the body's processes for responding to stressful conditions, the exact underlying mechanisms have yet to be fully characterised. Research investigating the pathways involved in RIPC has been very limited in the clinical setting, therefore the majority of what is understood about preconditioning stems from the results of pre-clinical animal work. It is beyond the scope of this thesis to present a complete background of the vast research carried out in these models, therefore the overview will be limited to the aspects that are the most well supported in the literature.

\subsubsection{Evidence for humoral, neural, and systemic pathways}

The least well understood aspect of RIPC is how the protective signal is communicated from the preconditioned tissue to the other organs of the body. Originally there was much debate as to whether the mechanism involved a humoral factor or a neural pathway, although it is now widely accepted that both are likely to play a role, along with systemic anti-inflammatory changes.

The key evidence to suggest the involvement of the peripheral nervous system in RIPC is provided by a number of studies that have established the ability of ganglionic blockers, such as hexamethonium and trimetaphan, and the afferent sensory nerve blocker, capsaicin, to abrogate the protective effects of preconditioning (Tomasz Brzozowski et al., 2004; Gho et al., 1996; Liem et al., 2002; Loukogeorgakis et al., 2005; Schoemaker and van Heijningen, 2000; Tang et al., 1999; Wolfrum et al., 2002). Similarly, resection of afferent nerves from the preconditioned tissue, or vagotomy, have been shown to inhibit the ability to induce RIPC (T. Brzozowski et al., 2004; Ding et al., 2001; Dong et al., 2004; Lim et al., 2010). Interestingly, Lim et al. found that resection of the femoral or sciatic nerves only partially reversed the effects of preconditioning, whereas combined resection completely abolished the protection, suggesting multiple neural pathways may contribute. 
On the other hand, reperfusion of the remotely preconditioned tissue is an essential component of the RIPC stimulus, implicating the involvement of a humoral factor (Gho et al., 1996; Weinbrenner et al., 2002). Further, transfer of whole blood or coronary effluent from renal preconditioned rabbits or isolated rabbit hearts, respectively, into a naïve system significantly reduces the infarct size associated with subsequent myocardial ischaemia (Dickson et al., 1999a, 1999b). These findings are consistent with a study using a porcine model in which Konstantinov et al. (2005) found that a transplanted heart could be protected by the prior application of RIPC in the recipient. Despite the evidence that a circulating humoral factor is critical for induction of RIPC and the identification of numerous substances that are involved in the early response to preconditioning, the exact mediator responsible is still unclear. However, studies using dialysed plasma from preconditioned animals to confer cardioprotection in isolated perfused hearts have established that the factor is likely to be hydrophobic with a molecular weight between 3.5 and $15 \mathrm{kDa}$ (Lang et al., 2006; Serejo et al., 2007; Shimizu et al., 2009).

Lastly, RIPC has been associated with significant changes to circulating leukocyte populations, including alterations in gene transcription, as well as neutrophil adhesion, phagocytosis, and cytokine production (Konstantinov et al., 2004; Shimizu et al., 2010). Overall, these changes are associated with an antiinflammatory response; however, it is unclear whether these are end effector mechanisms of RIPC or if they represent an additional systemic pathway through which the RIPC signal is communicated.

Regardless of the exact pathway through which the initial signal is transmitted, RIPC-induced organ protection is evoked through three crucial steps: the release of key initiating molecules from the preconditioned ischaemic tissue; activation of cell signalling pathways in target cells; and changes to the end effectors of RIPC that directly limit damage or preserve function in cells subjected to subsequent ischaemia. 


\subsubsection{Potential key initiators and mediators of RIPC}

Several molecules released from ischaemic tissue have been found to play a role in the induction of RIPC, but the extent of their respective importance seems to vary between models with respect to both species, and the tissue sites examined. Among the main candidates are adenosine, bradykinin, opioids, NO, calcitonin gene-related peptide (CGRP), and ROS, but studies have also touched on the possible involvement of noradrenaline, prostaglandins, heme oxygenase- 1 , cannabinoids, angiotensin, and O-linked $\beta$-N-acetylglucosamine (O-GlcNAc) (Hausenloy and Yellon, 2008; Kaur Randhawa et al., 2014; Tapuria et al., 2008). These substances may travel through the bloodstream and directly bind receptors on the myocardium, or stimulate afferent nerves in the preconditioned tissue that transmit the signal to efferent nerve fibres that terminate on the heart.

\subsubsection{Adenosine}

Adenosine is a nucleoside that has been implicated as a trigger and mediator during IPC through both initiating and propagating the preconditioning-induced signal cascade, respectively. During ischaemia, adenosine is released by the breakdown of ATP and exerts a variety of functions including dilation of blood vessels and attenuating increases in contractility, thereby both improving oxygen delivery and reducing oxygen demand in the myocardium (Hori and Kitakaze, 1991). Evidence for the involvement of adenosine in RIPC was first provided by studies that demonstrated non-specific blocking of adenosine receptors with 8-( $p$-sulfophenyl)theophylline (8-SPT) abolished the cardioprotective effect afforded by renal or mesenteric RIPC in animals (Ding et al., 2001; Liem et al., 2002; Pell et al., 1998; Takaoka et al., 1999). This protection could be inhibited by either afferent nerve resection or administration of hexamethonium, indicating adenosine activated a neural pathway (Ding et al., 2001; Dong et al., 2004; Liem et al., 2002). However, post-RIPC treatment with 8SPT, but not hexamethonium, could abrogate the protective effects, suggesting adenosine may also directly bind to receptors on the myocardium (Liem et al., 2002). Interestingly, 8-SPT does not affect the renal protection induced by hind 
limb RIPC in rats, suggesting that preconditioning in some organs may operate through adenosine-independent pathways (Wever et al., 2011).

\subsubsection{Bradykinin}

Schoemaker and Heijningen (2000) were the first to show an essential role for bradykinin during RIPC using the rat mesentery. Administration of the bradykinin $\mathrm{B}_{2}$ receptor antagonist, Hoechst 140 (HOE-140), was shown to inhibit RIPC-induced protection, whereas intramesenteric infusion of bradykinin mimicked RIPC. This protective effect could be abolished by hexamethonium, suggesting the involvement of a neural pathway. Similarly, Wolfrum et al. (2002) found that RIPC was associated with an increase in protein kinase C (PKC) activation, which was abrogated by pretreatment with HOE-140 or hexamethonium. In humans, decreased kinin receptor expression on circulating neutrophils following RIPC suggests that preconditioning involves kinin receptor activation and internalisation; however, HOE-140 does not appear to inhibit the effect of RIPC on vasomotor dysfunction in humans (Christian M. Pedersen et al., 2011; Saxena et al., 2010b). Given these conflicting findings, whether bradykinin contributes to RIPC in humans remains unclear.

\subsubsection{Reactive oxygen species}

ROS are generated during ischaemia and are typically associated with tissue damage; however, in 1989 it was determined that although major oxidative stress was associated with inactivation of the critical cellular messenger, PKC, mild levels of oxidative stress actually protected the catalytic site and increased the PKC enzyme activity (Gopalakrishna and Anderson, 1989). Further, ROS have been shown to activate $\mathrm{G}$ proteins independently of $\mathrm{G}$ protein-coupled receptor binding (Nishida et al., 2000). Both PKC and G protein-coupled receptors are critical mediators of RIPC, thus, ROS are capable of indirectly stimulating a number of beneficial processes through these effects.

Consistent with these reports, studies using renal and skeletal muscle RIPC in animal models have demonstrated that treatment with a free radical scavenger, mercaptopropionyl-glycine (MPG), abolishes the cardioprotective effect of 
preconditioning (Chen et al., 2005; Weinbrenner et al., 2004). Interestingly, Weinbrenner et al. found that MPG could sufficiently inhibit the protection afforded by a single cycle of RIPC, but not when three cycles were used, suggesting the involvement of additional mediators in triggering preconditioning using the standard stimulus with 3 cycles. Further, Tritto et al. (1997) established that administration of a low concentration of oxygen radicals could mimic RIPC in isolated reperfused rabbit hearts, improving post-ischaemic recovery of contractile function and reducing the infarct size.

\subsubsection{Opioids}

The involvement of opioids in the protection afforded by RIPC in animal models has been demonstrated by a number of studies that established the renal, neural, and myocardial benefits could be abolished by administration of the nonselective opioid receptor antagonist, naloxone (Patel et al., 2002; Rehni et al., 2007; Wever et al., 2011). Dickson et al. (2002) found that the mesenteric protection conferred by transfer of coronary effluent from preconditioned rat hearts could also be inhibited by naloxone, and Addison et al. (2003) reported that RIPC-induced protection of skeletal muscle could be reversed by treatment with naloxone, but not hexamethonium. Taken together, these studies strongly suggest that opioids produced endogenously in response to RIPC do not operate via a neural pathway but instead directly bind receptors in the target tissues.

Exactly which receptors are involved in this pathway is unclear, with some studies finding the $\delta 1$-opioid receptors to be essential and others implicating solely the א-opioid receptors (Addison et al., 2003; Weinbrenner et al., 2004; S. Zhang et al., 2006). More recently, Surendra et al. (2013) reported that activation of both the $\delta$ and $\kappa$-opioid receptors was necessary to induce cardioprotection with RIPC in isolated cardiomyocytes from rabbits. In CABG patients, however, administration of tramadol, which binds the $\mu$-opioid receptors, failed to induce a preconditioning type of effect and was actually associated with increased myocardial injury (Wagner et al., 2010). While opioids appear to be essential for the induction of RIPC in animals, their relevance during RIPC in humans is thus currently less clear. 


\subsubsection{Calcitonin gene-related peptide}

CGRP is a neurotransmitter released from sensory nerves that has been shown to increase following RIPC in numerous animal studies (T. Brzozowski et al., 2004; Hu et al., 2002; Tang et al., 1999; Wolfrum et al., 2005; Xiao et al., 2001). It appears that a RIPC-induced increase in NO stimulates CGRP release from these nerves that is then transported to the heart, where it stimulates cardioprotection through the activation of PKC (Wolfrum et al., 2005; Xiao et al., 2001). Accordingly, several studies have established that administration of a CGRP receptor antagonist or the sensory nerve blocker, capsaicin, can abrogate the protective effects of RIPC in various animal models (T. Brzozowski et al., 2004; Rehni et al., 2007; Tang et al., 1999; Wolfrum et al., 2005; Xiao et al., 2001). At present there is no literature addressing the role of CGRP during RIPC in humans.

\subsubsection{Nitric oxide}

NO exhibits a diverse array of protective effects that are relevant during I/R injury including scavenging of free radicals, inhibiting apoptosis, promoting vasodilation, and reducing leukocyte adhesion and platelet aggregation (Furchgott and Zawadzki, 1980; Kim et al., 2000; Lefer and Lefer, 1996; Wink et al., 1993). Not surprisingly, countless studies have implicated NO as both a trigger and mediator of RIPC using inducible nitric oxide synthase (iNOS) knockout mice or blockade of iNOS and NO, which abolish the protection induced by both early and late preconditioning (Abu-Amara et al., 2011; Li et al., 2004; Tapuria et al., 2008; Tokuno et al., 2002; Wang et al., 2001). It appears that NO functions through different pathways during early and late RIPC, with endothelial NOS in the myocardium important during the former, and NF- $\mathrm{BB}$ and PKC stimulated transcription of iNOS in the latter (Tapuria et al., 2008). However, a study by Petrishchev et al. (2001) using the intestine for RIPC found no change in the level of protection following nitric oxide synthase inhibition, suggesting the involvement of NO may be tissue dependent. 


\subsubsection{Intracellular signalling during RIPC}

Despite the wide range of mediators implicated in establishing RIPC, there is significant redundancy in the pathways once they reach the target cells. Adenosine, bradykinin, opioids, and cannabinoids all bind G protein-coupled receptors, resulting in the activation of numerous intracellular signalling kinases. Although stimulation of the mitogen associated protein kinases (i.e. JNK, ERK1/2, and p38 MAPK) and the PI3/Akt pathway have been implicated in contributing to RIPC, the central mediator appears to be PKC (Heidbreder et al., 2008; Li et al., 2011; Simkhovich et al., 2013). A comprehensive discussion of the complex and incompletely understood interactions associated with each of the kinases is beyond the scope of this thesis, but a simplified overview of the proposed signalling pathways is presented in Figure 1.1, and several articles have reviewed the topic in-depth (refer to Hausenloy and Yellon, 2006; Sadat, 2009).

Overall, activation of the PKC, MAPK, and PI3/Akt pathways appear to converge to activate opening of the sarcolemmal and mitochondrial $\mathrm{K}_{\mathrm{ATP}}$ channels, inhibit opening of the mPTP, reduce apoptosis, and activate key transcription factors associated with production of cytoprotective genes (Li et al., 2000; Ohnuma et al., 2002; Okamura et al., 1999; S. Zhang et al., 2006). The exact order of the signalling cascade is not fully understood, however, and PKC also appears to be activated by ROS released from the mitochondria, therefore it is possible that PKC may actually be activated downstream of the effects on mitochondria before feeding back into the system (Pain et al., 2000). Changes in gene transcription are also facilitated by activation of the JAK/STAT pathway in animal models of IPC, presumably by cytokine production; however, only one study has implicated STAT involvement in humans during RIPC and further research in this area is needed (Dawn et al., 2004; Hattori et al., 2001; Heusch et al., 2011). The combined effect of these changes to the mitochondria and gene transcription significantly promote cellular survival during I/R.

\subsubsection{End effectors of RIPC}

There are a number of cytoprotective effects well established to be induced by RIPC. During the early response to preconditioning, the major contribution to 
cardioprotection appears to stem from effects on the mitochondria and coronary blood flow, whereas the second window of protection involves changes to the inflammatory response and apoptosis brought about by alterations in gene transcription.

\subsubsection{K KTP channels}

One of the major cytoprotective effects of RIPC involves alterations to the ATP sensitive potassium channels present in the plasma membrane of cardiomyocytes and the inner mitochondrial membrane (Beavis et al., 1993; Loukogeorgakis et al., 2007). Through the intracellular loss of ATP, I/R appears to inhibit the opening of these $\mathrm{K}_{\mathrm{ATP}}$ channels, leading to accumulation of calcium and a loss of cellular or mitochondrial integrity (Costa et al., 2013). Activation of the channels during RIPC appears to slow the rate of ATP depletion, preserve intracellular $\mathrm{pH}$ and phosphocreatinine levels in the myocardium, and stimulates vasodilation, thereby increasing the resistance of cells to ischaemic conditions (Costa et al., 2013; Dos Santos et al., 2002; Kaur Randhawa et al., 2014; Takaoka et al., 1999).

Numerous studies have established the importance of changes to these channels by demonstrating that administration of the mitochondrial $\mathrm{K}_{\text {ATP }}$ channel blocker, 5-hydroxydecanoate, abolishes the protective effects of RIPC (Kristiansen, et al., 2005; Moses, et al., 2005; Pell, Baxter, Yellon, \& Drew, 1998). Although the effects of RIPC on $\mathrm{K}_{\mathrm{ATP}}$ channels are generally associated with the early phase of preconditioning, they have also been shown to play a role in the late stage of myocardial protection in a rat model (Y.-N. Wu et al., 2011). Further, opening of the $\mathrm{K}_{\text {ATP }}$ channels may also act as part of the RIPC trigger mechanism, as well as a mediator, through the production of ROS and subsequent activation of protein kinases (Pain et al., 2000).

\subsubsection{MPTP}

During $I / R$, increases in mitochondrial calcium levels, ROS, and NO, combined with a reduction in the inner mitochondrial membrane potential, lead to opening of the mPTP, which is closed under physiological conditions (recently reviewed 
by Ong et al., 2014). Opening of the MPTP, which is a large-conductance channel, results in the uncoupling of oxidative phosphorylation, thereby further reducing ATP levels. In addition, the leakage of mitochondrial proteins such as cytochrome $\mathrm{C}$ and changes in ion homeostasis activate apoptotic pathways and cause mitochondrial swelling, leading to rupture of the plasma membrane and cell death (Ong et al., 2014). PKCE appears to physically interact with the mPTP and significantly reduce mitochondrial swelling, strongly suggesting that RIPC may prevent mPTP opening during I/R (Baines et al., 2003). Indeed, effects on the pore are well established in models of local IPC, and early studies have shown similar results with RIPC (Halestrap et al., 2007; S. Zhang et al., 2006).

\subsubsection{Changes in coronary blood flow}

Changes to blood flow following RIPC also contribute to the organ protection observed, but do not account for the full effects of preconditioning (Saxena et al., 2010a). Shimizu et al. (2007) found that in a porcine model of myocardial I/R, RIPC significantly decreased resistance in the LAD and increased blood flow. In healthy human volunteers, stimulating a minor I/R injury in the forearm significantly reduces flow-mediated dilation (FMD), whereas RIPC prior to injury preserves peak radial artery FMD at similar levels to those observed at baseline (Kharbanda et al., 2001). Further, RIPC also increases flow velocity in the LAD during early reperfusion in healthy volunteers, and this effect appears to be the result of increased coronary blood flow and myocardial contraction, without a change in heart rate (Zhou et al., 2007).

\subsubsection{Gene transcription}

One of the major effects of RIPC appears to involve changes in the expression of a range of inflammatory and cytoprotective genes. The importance of gene transcription for inducing a beneficial effect during preconditioning was clearly demonstrated by Strohm et al. (2002) who reported that IPC could be abolished by administration of the non-specific transcription inhibitor, actinomycin-D. Subsequent studies have implicated a number of key transcription factors, most notably hypoxic inducible factor (HIF)-1 $\alpha$ and NF- $\kappa B$, in the pathway of IPC, but less research has been performed in RIPC models to date (reviewed by Kaur 
Randhawa et al., 2014; Tapuria et al., 2008). However, the primary stimuli involved in the activation of these factors are common to both methods of preconditioning; therefore it seems likely that these findings can be extended to RIPC.

Alterations in gene expression are predominantly associated with the delayed window of preconditioning; however, changes in mRNA levels in circulating leukocytes have been detected 15 minutes following RIPC in humans, suggesting it may also contribute to the early phase of protection (Konstantinov et al., 2004). These changes involve the upregulation of key cytoprotective mediators such as heat shock proteins, iNOS, inhibitors of apoptosis, and free radical scavengers, and the downregulation of many inflammatory genes involved in leukocyte chemotaxis, adhesion, and cytokine synthesis (Huda et al., 2005; Igor E. Konstantinov et al., 2005; Konstantinov et al., 2004; Li et al., 2004). The net effect of changes in gene expression during IPC thus dampens the inflammatory response and promotes cell survival.

\subsection{Hypoxia-inducible factor-1 $\alpha$}

The hypoxia-inducible factor (HIF)-1 $\alpha$ subunit is targeted for degradation under normoxic conditions but accumulates during periods of oxygen deficiency and forms a dimer with the constitutively produced HIF-1 $\beta$ subunit to make HIF (Kaelin and Ratcliffe, 2008). HIF is a transcription factor that facilitates adaption to ischaemic conditions by activating key genes involved in oxygen metabolism, angiogenesis, cell survival, and metabolic protection, such as vascular endothelial growth factor and glucose transporters (Aragonés et al., 2008; Forsythe et al., 1996; Kaelin and Ratcliffe, 2008). Not surprisingly, treatment of mice with HIF-1 $\alpha$ prior to myocardial I/R has a cardioprotective effect, reducing infarct size and improving ventricular function (Czibik et al., 2011). Increases in HIF-1 $\alpha$ have been frequently associated with organ protective effects in IPC and more recently have also been implicated in the mechanism of RIPC (Albrecht et al., 2013; Cai et al., 2008; Eckle et al., 2008). 
In rats, inhibition of the enzymes that label HIF-1 $\alpha$ for destruction (i.e. HIF-P4Hs) mimics the effect of renal RIPC, whereas administration of the HIF-P4Hs activator, $\alpha$-ketoglutarate, abolishes the cardioprotective benefit from RIPC (Kant et al., 2008). This study also suggested that HIF-1 may stimulate NF- $\kappa B$ signalling. Similarly, HIF-1 $\alpha$ heterozygous knockout mice have been shown to be incapable of responding to RIPC, but the protective effects, which include increased plasma IL-10 levels, can be recovered by injection of a recombinant adenovirus expressing a constitutively active form of HIF-1 $\alpha$. In the same study, HIF-1 was found to bind the IL-10 gene in isolated murine myocytes exposed to repeated intermittent hypoxia (Cai et al., 2013). More recently, Albrecht et al. (2013) demonstrated that HIF-1 $\alpha$ levels were upregulated in the right atrial tissue of cardiac surgery patients following RIPC and prior to commencing bypass. Taken together, these studies strongly suggest HIF-1 is essential for RIPC and in animal models may exert effects, in part, through inducing IL-10 production.

\subsection{NF- $\kappa B$}

The inactive form of NF- $\kappa$ B is stored bound to its inhibitory Ік $\beta$ proteins in the cytoplasm and is readily activated in response to a number of mediators including ROS and inflammatory cytokines. Signals resulting from these stimuli lead to degradation of the Iк $\beta$ proteins, freeing the NF- $\kappa B$ to translocate to the nucleus, bind to DNA, and regulate gene expression (Valen et al., 2001). NF- $\kappa B$ is significantly upregulated during cardiac surgery and induces transcription of numerous proteins associated with the inflammatory response, including cytokines and adhesion molecules (Valeur and Valen, 2009). It is possible that $\mathrm{NF}-\kappa \mathrm{B}$ contributes to cardioprotection through upregulation of manganese superoxide dismutase and inhibition of apoptosis; however, there is overwhelming evidence that the net effect of NF- $\kappa B$ activation is detrimental during I/R injury (Valeur and Valen, 2009).

Maulik et al. (1998) were the first to report on the involvement of NF- $\kappa B$ in preconditioning when they found that IPC in isolated perfused rat hearts was associated with increased nuclear translocation of NF- $\kappa B$ and resistance to I/R 
injury. Two subsequent studies in rabbits and mice supported this finding, demonstrating that IPC was associated with increased activation of NF- $\kappa B$ and that the resulting cardioprotection could be abolished by administration of the NF- $\kappa B$ inhibitor, diethyldithiocarbamate, or use of animals with deletion of the NF-кB p105 subunit (Li et al., 2004; Xuan et al., 1999).

Morgan et al. (1999) determined that the increase in NF- $\kappa B$ activity during IPC in rabbit hearts was followed by inhibition of NF- $\kappa B$ during subsequent I/R. The authors proposed that the mild level of oxidative stress associated with preconditioning led to NF- $\kappa$ B activation of cytoprotective genes, which in turn resulted in the suppression of NF- $\kappa \mathrm{B}$ during I/R. Interestingly, NF- $\kappa \mathrm{B}$ increases cytoplasmic levels of its own inhibitor, Iк $\beta-\alpha$, which may also explain how a preconditioning-induced increase in NF- $\kappa B$ activity can lead to inhibition of NF$\kappa B$ during later prolonged ischaemic insults (Tähepõld et al., 2003). In support of this possibility, Jiang et al. found that delayed renal IPC in rats suppresses I/Rinduced NF- $\kappa$ B activation by reducing Iк $\beta-\alpha$ degradation (Jiang et al., 2007).

Further work in murine models confirmed that early IPC attenuated NF-кB activation during I/R injury in both the liver and small intestine (Funaki et al., 2002; Li et al., 2006; Takeshita et al., 2010). These studies extended the findings to show that the decrease in NF- $\kappa B$ activation was associated with a reduction in IL-1 $\beta$, TNF- $\alpha$, and intercellular adhesion molecule-1 (ICAM-1) mRNA levels, supporting the theory that inhibition of NF- $\kappa B$ contributes to organ protection by reducing proinflammatory gene expression. In the clinical setting, unstable angina has been shown to activate NF- $\kappa \mathrm{B}$, therefore it is likely that IPC in humans utilises the same NF-kB pathway elucidated in the animal studies (Wang et al., 2007).

\subsubsection{Changes to the systemic inflammatory response}

Overall, RIPC appears to have a significant suppressive effect on the inflammatory response to $\mathrm{I} / \mathrm{R}$, particularly during the late phase. These changes seem to be predominantly associated with changes to gene transcription, with Konstantinov et al. (2004) demonstrating that genes involved in leukocyte 
chemotaxis, adhesion and migration, exocytosis, signalling pathways of the innate immune response, and apoptosis are suppressed in circulating leukocytes 15 minutes following RIPC, and further reduced at 24 hours. Accordingly, other studies in healthy human volunteers have shown that RIPC alters key functional properties of neutrophils, including adhesion and cytokine production (Kharbanda et al., 2001; Shimizu et al., 2010). A more detailed overview of what is known about the involvement of the immune response in I/R injury and RIPC is presented in the introductory sections of the following results chapters.

While it is generally accepted that RIPC modifies the inflammatory response, very little is known about the exact processes and end results involved. It is not clear whether these changes constitute a part of the trigger mechanism involved in establishing RIPC or whether they significantly contribute to the protective effects themselves. In particular, there is a significant gap in the literature regarding the involvement of the immune system in RIPC in humans, and during the early phase of protection. 


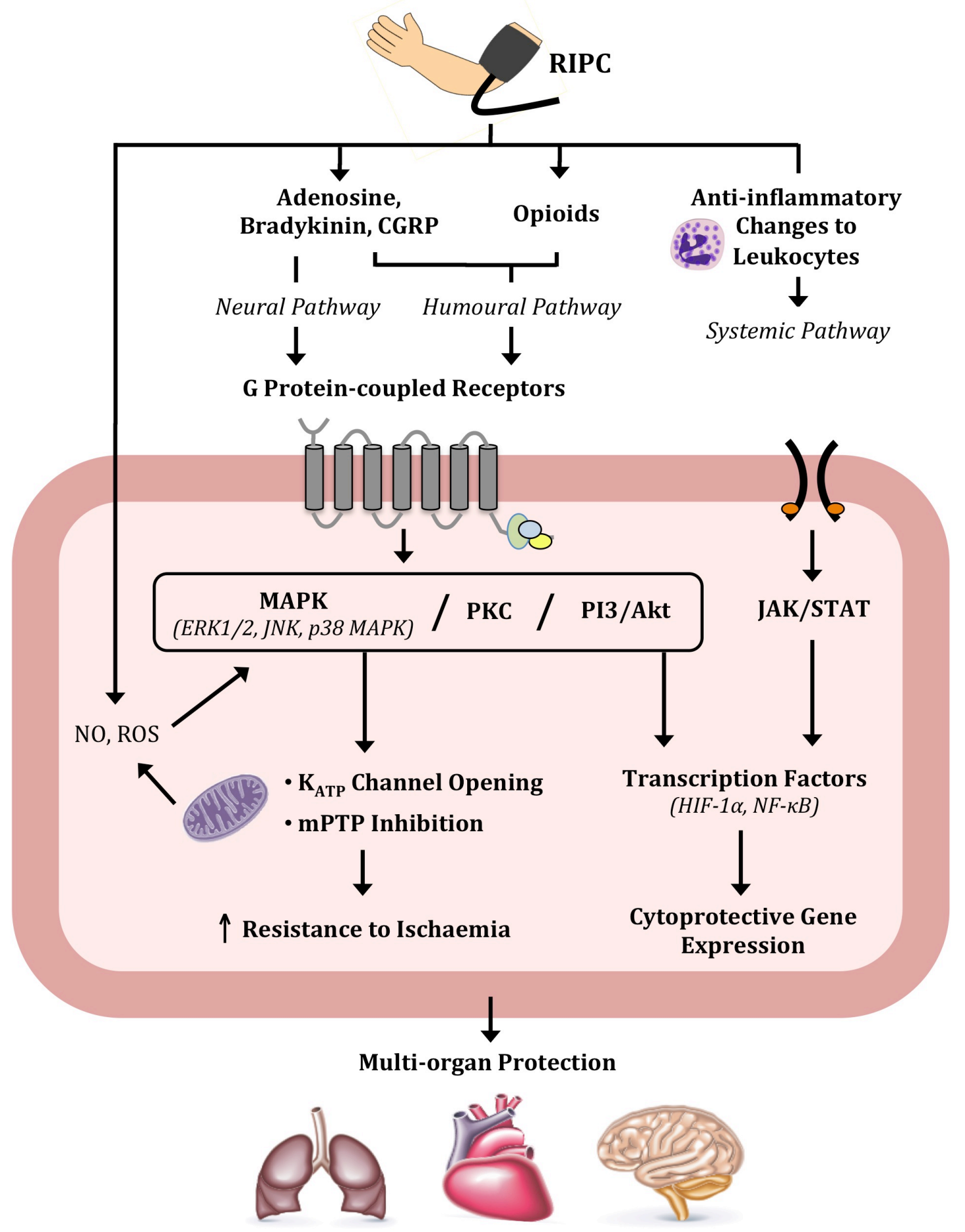

Figure 1.1 Proposed mechanisms involved in organ protection with RIPC

Summary of the key signalling pathways and end effector mechanisms reported to be involved in organ protection following RIPC. While the activation of G protein-couple receptors and changes to mitochondria and gene transcription are agreed on as the beginning and end of the pathways, respectively, the exact order of the signalling kinases in the cascade in between is currently less clear. 


\subsection{Overview of the key immune cell populations}

The immune system encompasses the innate immune response, which provides a non-specific first line of defence against invading pathogens, and the adaptive immune response, which is characterised by antigen-specific protection and a memory for previously encountered pathogens. The cells that mediate these responses, particularly the neutrophils, monocytes, and T cells, are key players in both the pathology of I/R injury and, to a lesser extent, the response to RIPC.

\subsubsection{Neutrophils}

Neutrophils, which are also referred to as polymorphonuclear cells owing to their lobulated nuclei, make up approximately $70 \%$ of circulating white blood cells and therefore are the most abundant of the peripheral leukocytes. During infection or inflammation the neutrophil population often rapidly and markedly increases in size (Amulic et al., 2012). Neutrophils are notoriously difficult to study ex vivo due to their extremely short life span of approximately $7-10$ hours, and sensitivity to activation in artificial circumstances (Dancey et al., 1976). In the experimental setting, neutrophils can be identified by their high cell membrane expression levels of CD16 and the key adhesion factor, CD11b, and low levels of CD14 (Amulic et al., 2012).

The primary function of neutrophils is to circulate in the blood and monitor for signs of infection or host distress, which are normally detected by pattern recognition receptors (PRR) that bind a broad range of pathogen-associated molecular motifs (Amulic et al., 2012). Upregulation of key adhesion molecules, selectins and integrins, on the endothelium during inflammation facilitates the binding and transmigration of neutrophils into the tissues (Gearing and Newman, 1993). Numerous inflammatory mediators such as chemokines and bacterial products direct neutrophil migration and function during pathological processes. Binding of these agents to neutrophil cellular receptors, such as IL-8 to its $G$ protein-coupled receptor, initiates intracellular signalling cascades that stimulate the major effector functions of neutrophils including the oxidative burst, cytokine production, exocytosis of anti-microbial compounds stored in cytoplasmic granules, and phagocytosis (Amulic et al., 2012; Zarbock and Ley, 
2008). These mechanisms are critical to pathogen destruction and clearance, but are non-specific and can also cause extensive damage to host tissues.

\subsubsection{Monocytes}

Monocytes constitute approximately $10 \%$ of the nucleated blood cells in healthy adults, with populations pooled in the lung and spleen ready for mobilisation on demand (Ginhoux and Jung, 2014). There are two major subsets of monocytes in the circulation, which both significantly increase during times of infection. Classical monocytes express moderate to high levels of the cell surface marker $\mathrm{CD}_{14}{ }^{+}$and have a half-life of around 20 hours in the blood, whereas a much smaller population of non-classical CD14 ${ }^{+} \mathrm{CD} 16^{++}$monocytes have a half-life of up to 5 days (Ginhoux and Jung, 2014). These subsets appear to exert slightly different but complementary effects in pathological states, such as during the response to MI (Nahrendorf et al., 2007).

Like neutrophils, monocytes respond to a broad range of pathogens and chemokines, and use cellular adhesion molecules to bind the endothelium and infiltrate tissues at sites of inflammation or infection (Shi and Pamer, 2011). Monocytes exert direct antimicrobial effects by phagocytosing pathogens that are recognised by their PRR or opsonin receptors, and by producing numerous inflammatory mediators including ROS, complement, and cytokines such as IL-6, IL-10, and TNF- $\alpha$ (Auffray et al., 2009). Following extravasation, some monocytes differentiate into macrophage or dendritic-like cells, and others can enter the lymph nodes and present antigens to $\mathrm{T}$ cells, stimulating the adaptive immune response (Ginhoux and Jung, 2014; Shi and Pamer, 2011). Monocytes are thus valuable mediators of the response to infection and function in a variety of manners to maintain tissue homeostasis.

\subsubsection{Natural killer cells}

Natural killer (NK) cells are present as only a small population in healthy adults; they reside in both lymphoid and non-lymphoid tissue sites and survive for approximately two weeks (Vivier et al., 2008; Zhang et al., 2007). NK cells have traditionally been considered a part of the innate immune response due to their 
response to a broad range of non-antigen-specific stimuli; however, they also share many characteristics common to the adaptive immune response such as the formation of immunological memory (Vivier et al., 2011).

NK cells are a type of lymphocyte predominantly recognised for their ability to kill tumour or infected cells in response to expression of stress signals. They are differentiated from other cells and between the different subsets by their expression level of CD56, along with CD16. The majority of the circulating NK cells are $\mathrm{CD} 56^{\mathrm{dim}} \mathrm{CD} 16^{+}$, which produce interferon (IFN)- $\gamma$ and exert cytotoxic effects through the expression of perforin (Vivier et al., 2008). NK cells also act in a regulatory fashion, significantly influencing the responses of other key cells including dendritic cells, B and T lymphocytes, and endothelial cells through killing, or suppressing or enhancing their function (Vivier et al., 2008). While the majority of NK cell activity appears to be protective, NK cells have also been implicated in tissue injury associated with certain disease states; therefore can also have detrimental effects.

\subsubsection{T cells}

$\mathrm{T}$ cells are the major subset of lymphocytes and recognise specific antigens through their unique $\mathrm{T}$ cell receptor (TCR). The majority of $\mathrm{T}$ cells have receptors made up of $\alpha \beta$ chains, although approximately $5 \%$ of cells express the $\gamma \delta$ chains instead and are associated with protection of the skin and mucosal surfaces (Ciofani and Zúñiga-Pflücker, 2010). The $\alpha \beta$ TCR T cells can be broadly divided into two lineages based on the cell surface expression of the co-receptor molecules CD4 and CD8, and circulate through the blood and lymphatic system.

Most commonly, $\mathrm{CD} 4^{+} \mathrm{T}$ cells are activated through interactions with antigen presenting cells (APCs) such as dendritic cells, macrophages, and B cells. APCs phagocytose and digest extracellular pathogens and display the resulting antigenic peptides on their cell surface via major histocompatibility complex (MHC) II molecules (Broere et al., 2011). Upon recognition of these antigens, CD4 cells, also known as the $\mathrm{T}$ helper cell population, are activated from naïve to effector cells and produce cytokines that function to both destroy infected target 
cells and mediate the response of other effector T and B cells, such as through the promotion of antibody production (Broere et al., 2011).

There are multiple subsets of $\mathrm{T}$ helper cells, which are distinguishable by their cytokine profile following activation. Th1 cells are generally regarded as proinflammatory and produce high levels of IFN- $\gamma$ and, to a lesser extent, IL-2 and TNF- $\alpha$, in response to intracellular bacteria, whereas Th2 cells are commonly associated with parasitic infections and the secretion of IL-4, IL-5, IL6, IL-10, and IL-13 (Broere et al., 2011; J. Zhu et al., 2006). The more recently identified Th17 cells appear to respond to extracellular bacteria and fungi, and produce IL-17 and IL-22, while Th22 cells can release IL-22 in isolation (Eyerich et al., 2009; Louten et al., 2009).

The $\mathrm{CD}^{+}$cytotoxic $\mathrm{T}$ cells recognise antigens displayed on MHC I molecules, which are expressed on all nucleated cells and present peptides from intracellular pathogens. Upon activation, $\mathrm{CD}^{+} \mathrm{T}$ cells rapidly proliferate and destroy infected cells through the release of cytolytic granules or the initiation of FAS-mediated apoptosis, and also secrete inflammatory cytokines such as IFN- $\gamma$ and TNF- $\alpha$ (Broere et al., 2011; Fong and Mosmann, 1990).

Regulatory T cells (Treg) encompass several slightly varied cell types that can occur naturally or be induced, but all share the common function of regulating the immune response and preserving immune tolerance (Triplett et al., 2012). The majority of Treg are $\mathrm{CD}^{+}$and express high levels of the alpha chain of the IL-2 receptor, CD25. The exact mechanisms through which Treg mediate immune responses are not fully understood, but they appear to express inhibitory molecules such as cytotoxic T-lymphocyte associated protein 4 (CTLA4), which dampen down the effector responses of other $\mathrm{T}$ cells; secrete immunosuppressive cytokines such as TGF- $\beta$ and IL-10; and decrease the expression of MHC and co-stimulatory molecules on APCs (Broere et al., 2011).

Following clearance of the antigenic agent, the immune response resolves and the majority of the $\mathrm{T}$ cells disappear; however, a small population of cells are 
preserved as memory $\mathrm{T}$ cells. These cells persist in the lymphoid organs for years and are capable of mounting an immediate effector response should the antigen be encountered again (Broere et al., 2011). It is this ability of the adaptive immune system to 'learn' to recognise and quickly respond to specific pathogens that forms the basis for vaccination.

\subsection{Summary and project aims}

RIPC is a novel organ protective technique that has the potential to significantly improve clinical practice; however, successfully translating this intervention from animal studies into clinical applications has been limited by inconsistent results and a lack of understanding of the pathways through which RIPC operates. In particular, despite mounting evidence of the involvement of a systemic anti-inflammatory pathway, there is a distinct lack of information regarding the impact of RIPC on the immune response in humans, especially during the early phase of protection. Clarifying the pathways through which RIPC functions will greatly facilitate the identification of the clinical indications and limitations of this technique. The primary goal of the research conducted for this thesis was to address this critical gap in the literature, with the following specific aims:

1. To investigate the effect of RIPC on inflammatory mediator expression and clinical outcome during high-risk cardiac surgery.

2. To develop and test a series of laboratory assays capable of detecting subtle alterations in the phenotype and function of human immune cells.

3. To utilise the tested assays to characterise the direct effects of RIPC on the immune response in healthy volunteers. 


\section{Chapter 2.}

\section{The effect of RIPC on cytokine \\ production in high-risk cardiac \\ surgery}




\subsection{Introduction}

\subsubsection{RIPC in high-risk cardiac surgery patients}

Recent years have seen a significant change in the demographics of the average cardiac surgery candidate, leading to rising numbers of high-risk patients. The factors behind this shift include patients being older and increases in the occurrence of co-morbidities such as diabetes, left ventricular impairment, and the rate of previous myocardial infarctions (Biancari et al., 2009; Hausenloy and Yellon, 2012). In New Zealand alone, the incidence of heart failure in people aged 65 and over increased by $14 \%$ between the 1970s and 1990s, and the prevalence of Type 2 diabetes has risen by 35\% between 2001 and 2010 (Barker et al., 2006; Diabetes New Zealand, 2010). These factors significantly reduce the patients' physiological reserves and increase the risk of post-operative morbidity and mortality (Hausenloy and Yellon, 2012; Partridge et al., 2012).

Certain types of cardiac surgery, such as re-operative (commonly referred to as 'redo') and valve procedures, also pose a higher risk for patients in comparison to straightforward CABG surgery (Bridgewater et al., 2008; Wauthy et al., 2003). These operations are typically associated with prolonged bypass times and a greater level of structural injury to the heart muscle (Swaanenburg et al., 2001; van Geene et al., 2010). Using brief periods of ischaemia in the form of RIPC to prime the body's natural protective responses to ischaemia is therefore a particularly attractive cardioprotective strategy for use in these high-risk patients. Further, one of the key advantages of RIPC is the lack of side effects or potential negative interactions with medications, which is especially advantageous within this complex population.

Animal studies have repeatedly demonstrated the ability of RIPC to reduce myocardial infarct size by up to $50 \%$ in cardiac I/R injury (Birnbaum et al., 1997; Gho et al., 1996; Kharbanda et al., 2002); however, trials of RIPC in humans undergoing cardiac surgery have not shown such reproducible results (Karuppasamy et al., 2011; Lomivorotov et al., 2012; Meybohm et al., 2013; Rahman et al., 2010). In addition, aspects of the design of these studies such as 
the use of incomplete blinding and varied anaesthesia and bypass protocols have made it difficult to interpret the outcomes. Further research using carefully controlled and considered trial designs will be essential to determine the clinical efficacy of RIPC.

Selecting the most appropriate and informative endpoints for trials in cardiac surgery patients can be challenging. The average CABG surgery patient has a predictive operative mortality risk in the range of 3 to 4\%; however, the currently available assessment tools, such as the logistic European system for cardiac operative risk evaluation (EuroSCORE), commonly overestimate these rates (Kalavrouziotis et al., 2009; Parolari et al., 2009; van Straten et al., 2010). The incidence of post-operative major adverse cardiac events (MACE) are similarly low, making these obvious endpoints less than ideal outcome measures for small or moderately sized studies. The majority of RIPC clinical trials, including the one described in this chapter, have therefore used surrogate endpoints such as troponin or other markers of damage, rather than direct clinical outcome data.

Troponin is a protein that exists predominantly as a three unit complex composed of the different isoforms, I, T, and C (Barry et al., 2008). This trimeric complex is located between the actin filaments of cardiac muscle and a small fraction of the unbound isoforms can also be found in the cytoplasm of myocytes. When the myocytes are injured, both the complex and unbound forms of troponin are released into the serum where they can be readily quantified using commercial assays (Barry et al., 2008). Currently, troponin I and $\mathrm{T}$ are considered the preferred biomarkers of myocardial injury due to their highly cardiac specific and sensitive nature (Jaffe et al., 2000; Thygesen et al., 2007). Although post-operative troponin levels have previously been correlated with increased mortality and MACE, the magnitude of reduction in troponin levels required to improve patient outcome has not been established (Adabag et al., 2007; Domanski et al., 2011; Nesher et al., 2008). This limits the usefulness of troponin as an indicator of the effectiveness of cardioprotective techniques. 
The following trial was designed to assess the impact of RIPC in a population of high-risk cardiac surgery patients. This patient population was selected for a number of reasons. First, at the time our study was designed there were no reported applications of RIPC in high-risk patients, despite their increasing presentation for cardiac surgery. Second, the compromised physiology of these individuals prior to surgery coupled with the greater anticipated burden associated with the procedures being performed identified them as having the most to gain from the potential protection afforded by the intervention. Third, the expected high levels of troponin release from the surgeries undergone by these patients provided a greater opportunity to measure any RIPC-induced benefits. Likewise, the higher estimated frequency of adverse events allowed the possibility to detect a difference in superior endpoint measures, such as AKI or death.

\subsubsection{Potential role of cytokines in RIPC}

The inconsistencies between animal and human studies, and variation amongst the early clinical trials, have prompted a call for further research to investigate the underlying mechanisms involved in RIPC in order to define its clinical indication and limitations (Morita, 2011). There is mounting evidence that RIPC modulates the inflammatory response, suppressing proinflammatory gene expression in human leukocytes and activation of the key effector cells of postoperative tissue damage, neutrophils (Kharbanda et al., 2001; Konstantinov et al., 2004; Shimizu et al., 2010). Furthermore, the inflammatory cytokines IL-6, IL10 , and TNF- $\alpha$ have been established as essential for preconditioning-induced cardioprotection in mice (Cai et al., 2012; Dawn et al., 2004; Smith et al., 2002). In rats, the protective effects of late IPC can also be abolished by concurrent administration of antibodies to IL-1 $\beta$ and TNF- $\alpha$ (Yamashita et al., 2000). These studies suggest that cytokines could be the as yet unidentified humoral mediator that transmits the protective signal from the preconditioned tissue to the heart and other organs of the body.

Despite reports of specific cytokines being obligatory for inducing preconditioning, the animal study literature overwhelmingly suggests that 
preconditioning attenuates the $\mathrm{I} / \mathrm{R}$ induced release of key inflammatory cytokines. Several studies in rat and pig models have demonstrated that IPC significantly reduces systemic levels of IL-6 and macrophage inflammatory protein (MIP)-2 following I/R injury (Harkin et al., 2002; Jiang et al., 2003; Peng et al., 2011; Zhang et al., 2013). Likewise, IPC consistently reduces both mRNA and serum levels of IL-1 $\beta$ and TNF- $\alpha$, and mRNA levels of monocyte chemoattractant protein (MCP)-1, in pig, rat, and mouse models of ischaemic damage (Funaki et al., 2002; Simón et al., 2012; Takeshita et al., 2010; Yang et al., 2013; Yu et al., 2010).

In cardiac surgery, high levels of IL-6 and IL-8 have been associated with numerous post-operative complications including increased myocardial damage and AKI, yet the impact of RIPC on early expression of these biomarkers in human adults has not previously been characterised (Liu et al., 2009; Wei et al., 2001a). IL-6, IL-8, and other cytokines may have a direct role in the initiation of RIPC or function as indirect markers of preconditioning. Alternatively, RIPC may attenuate cytokine levels as part of the protective mechanism, or indirectly through a reduction in myocardial injury. Higher systemic levels of these mediators are associated with increasing duration and invasiveness of surgery, suggesting our high-risk patients provide an ideal setting to examine changes in expression levels (Takayama et al., 2007; Whitten et al., 1998). In this study, we therefore investigated whether RIPC alters cytokine expression in the perioperative period in patients undergoing high-risk cardiac surgery.

\subsection{Aims}

- To determine the impact of RIPC on cardiac and renal endpoints in highrisk cardiac surgery patients.

- To investigate the effect of RIPC on inflammatory cytokine production during high-risk cardiac surgery.

- To explore which surgical factors may impact inflammatory cytokine production following cardiac procedures. 


\subsection{Methods}

A double-blind, randomised, controlled trial of RIPC was performed in 96 adult high-risk cardiac surgery patients recruited over 13 months at Wellington Regional Hospital, New Zealand. The study was registered on the Australian New Zealand Clinical Trials Registry (ACTRN 12609000965202) and received ethics approval from the Central Regional Ethics Committee (CEN/09/12/096).

\subsubsection{Participants}

Patients over 18 years were invited to participate if they were undergoing highrisk cardiac surgery defined as double, triple or mitral valve replacement, coronary artery bypass graft surgery $(C A B G)$ with ejection fraction $(E F)<50 \%$, $\mathrm{CABG}+$ valve(s), or any redo cardiac operation. These surgeries were considered high-risk because they are generally associated with extended bypass times or are performed in patients with significantly impaired cardiac function. For the study overall, patients with peripheral vascular disease affecting the upper limbs, or requiring deep hypothermic circulatory arrest or radial artery conduit harvesting were excluded. Additionally, for the cytokine analyses, patients receiving systemic immunosuppressives were also excluded. Written informed consent was obtained from all patients.

\subsubsection{Study blinding and RIPC}

Patients were permuted-block randomized in groups of 8 by a third party using an online randomisation sequence generator with an allocation ratio of 1:1 to either RIPC or control. Treatment group allocation was concealed in sequentially numbered opaque envelopes until an anaesthetic technician applied the intervention. Each participant had one tourniquet placed on their upper limb and a second tourniquet wrapped around a towel next to them on the operating table. The tourniquet set-up was concealed from the theatre staff by the patient draping. RIPC was applied beginning with the first surgical incision using an ATS 750 electric tourniquet system (Zimmer, Inc., Warsaw, IN, USA) to inflate the cuff to $200 \mathrm{mmHg}$ for 5 minutes, followed by 5 minutes of deflation. This process was performed a total of 3 times, consecutively. For the control group the same intervention was applied to the tourniquet wrapped around the towel. Prior to 
the undraping of the patient, the tourniquet was disconnected by the anaesthetic technician. Patients, theatre staff (with the exception of the anaesthetic technician), ICU staff, and investigators were masked as to treatment allocation until data collection was completed.

\subsubsection{Peri-operative management of patients}

All patients received pre-medication, cardiac anaesthesia involving the use of isoflurane, tepid cardiopulmonary bypass with blood cardioplegia, and postoperative hemodynamic management according to standardised study protocols described in appendix A.

\subsubsection{Serum collection}

Blood samples were collected from the radial artery into BD SST II Advance vacutainers (Becton, Dickinson and Company, Franklin Lakes, NJ, USA) preoperatively, and at 1, 2, 3, 6 and 12 hours following cross-clamp removal. The samples were incubated at room temperature (RT) for 30 minutes, centrifuged, and the serum collected for storage at $-80{ }^{\circ} \mathrm{C}$ until analysis.

\subsubsection{Troponin levels}

High sensitivity troponin $\mathrm{T}$ levels were measured as the samples were collected according to standard clinical procedures at the Wellington Hospital Laboratory Services.

\subsubsection{Cytokine quantification}

The cytokines were quantified using a human inflammatory cytokine cytometric bead array (CBA) kit (\#551811; BD Biosciences, San Jose, CA, USA). The CBA kit consists of six bead populations with distinct fluorescence intensities that can be resolved in the red channel via allophycocyanin (APC). Each population is coated with capture antibodies specific for IL-1 $\beta$, IL-6, IL-8, IL-10, IL-12p70 or TNF- $\alpha$. After these beads are incubated with test samples or standards, phycoerythrin (PE)-conjugated cytokine-specific detection antibodies are added to enable the cytokine-bound beads to be distinguished from unbound beads. The PE fluorescence intensity of the resulting sandwich complexes can then be detected 
in the yellow channel during flow cytometry and compared to a range of standards to ascertain the cytokine concentrations. The cytometric assay beads were mixed, centrifuged at $200 \mathrm{x}$ g for 5 minutes, the supernatant removed, and the beads resuspended in an equal volume of serum enhancement buffer. After a 30-minute incubation at RT, the beads were diluted 2-fold with assay diluent. Following bead preparation, $25 \mu \mathrm{L}$ of undiluted sample and duplicate standards ranging from $20 \mathrm{pg} / \mathrm{mL}$ to $5000 \mathrm{pg} / \mathrm{mL}$ (prepared according to the manufacturer's instructions) were incubated in a V-bottom 96-well plate with 25 $\mu \mathrm{L}$ of the mixed beads for 1.5 hours at RT, protected from light. At the end of the incubation the samples were washed twice by the addition of wash buffer, centrifugation at $400 \mathrm{x}$ g for 5 minutes, and aspiration of the supernatant. After the final wash all but $50 \mu \mathrm{L}$ of supernatant was removed and $25 \mu \mathrm{L}$ of the PEconjugated anti-cytokine antibody mix diluted 2-fold in assay diluent was then added to each sample. After a 1.5-hour incubation, the samples were washed twice, resuspended in $200 \mu \mathrm{L}$ wash buffer, and a minimum of 1800 singlet bead events per sample were acquired on a FACSCanto ${ }^{\mathrm{TM}}$ II flow cytometer (BD Biosciences). The data was analysed with BD FACSDiva and FCAP Array software (BD Biosciences).

\subsubsection{Comparison of vacutainers for cytokine recovery}

\subsubsection{Sample collection}

Cytokine recovery was compared between the BD SST II Advance and Plastic Plus Serum tubes (Becton, Dickinson and Company) by collecting human blood into the vacutainers and adding either a low $(200 \mathrm{pg} / \mathrm{mL})$ or high $(1500 \mathrm{pg} / \mathrm{mL})$ concentration of recombinant murine TNF and human IL-6 proteins. The samples were incubated at RT for 60 minutes, centrifuged, and the serum collected for storage at $-80{ }^{\circ} \mathrm{C}$ until analysis by enzyme-linked immunosorbent assay (ELISA).

\subsubsection{TNF- $\alpha$ and IL-6 ELISAs}

TNF- $\alpha$ and IL-6 levels were measured using a sandwich ELISA in accordance with the manufacturer's instructions (BD Biosciences). For IL-6 detection, 96- 
well ELISA plates (Thermo Fisher, Waltham, MA, USA) were coated with $1 \mu \mathrm{g} / \mathrm{mL}$ purified mouse-anti human IL-6 capture antibody in $0.1 \mathrm{M} \mathrm{Na}_{2} \mathrm{HPO}_{4}(\mathrm{pH} 9)$ and incubated overnight at $4{ }^{\circ} \mathrm{C}$. The capture solution was discarded, and the plates blocked with 5\% blocking solution (5\% FCS in 1x PBS) at RT for 2 hours. The plates were then washed 4 times in ELISA wash buffer $0.05 \%$ Tween 20 (Sigma Aldrich, St Louis, MO, USA) in 1x PBS) and $50 \mu \mathrm{L}$ of standards and samples (diluted 2-fold in 5\% blocking solution) were added and incubated overnight at $4{ }^{\circ} \mathrm{C}$. The samples and standards were discarded and the plates washed 4 times before $0.5 \mu \mathrm{g} / \mathrm{mL}$ biotin anti-human IL-6 antibody (BD Biosciences) diluted in $5 \%$ blocking solution was added and incubated at RT for 1 hour. After the secondary antibody solution was removed and the plates washed 6 times, $50 \mu \mathrm{L}$ of streptavidin horseradish peroxidase (SA-HRP; 1:2000; BD Biosciences) was added and incubated at RT for 1 hour. The SA-HRP solution was then discarded and the plates washed 8 times. Equal volumes of OptEIA TMB A and TMB B solutions (BD Biosciences) were mixed and $100 \mu \mathrm{L}$ immediately added to each well. After sufficient colour development, the reaction was stopped by the addition of $100 \mu \mathrm{L} /$ well of $0.18 \mathrm{M} \mathrm{H}_{2} \mathrm{SO}_{4}$ (stop solution, see appendix $\mathrm{C}$ ). The optical density of the plates at $450 \mathrm{~nm}$ was measured on an EnSpire 2300 multilabel plate reader (PerkinElmer, Wellesly, MA, USA) and the EnSpire software was used to generate a standard curve to determine the sample concentrations. The same protocol was used for TNF- $\alpha$ detection, with $4 \mu \mathrm{g} / \mathrm{mL}$ of rat anti-mouse TNF- $\alpha$ antibody (BD Biosciences) in $0.1 \mathrm{M} \mathrm{Na}_{2} \mathrm{HPO}_{4}$ (pH 6) for capture, a 5 -fold dilution of samples in 5\% blocking solution, and $1 \mu \mathrm{g} / \mathrm{mL}$ biotin anti-mouse TNF- $\alpha$ antibody (BD Biosciences) for detection.

\subsubsection{Statistical analysis}

The primary clinical outcome measures for the trial were post-operative high sensitivity troponin $\mathrm{T}$ levels measured at 6 and 12 hours after cross-clamp removal, duration of noradrenaline use amongst ICU survivors, and worst postoperative renal injury as determined by risk, injury, failure, loss of kidney function, and end-stage kidney disease (RIFLE) criteria (Bellomo et al., 2004). For the cytokine analysis the primary endpoints were the serum levels of the cytokines measured at 1 and 12 hours following cross-clamp removal. 
The power calculation for the trial was based on troponin levels from a previous study of RIPC in CABG patients (Hausenloy et al., 2007). Hausenloy et al. reported a mean troponin $\mathrm{T}$ concentration in the control group at 12 hours of $0.69 \mu \mathrm{g} / \mathrm{L}$ with a standard deviation of $0.48 \mu \mathrm{g} / \mathrm{L}$ which, in a sample size of 96 participants split evenly between two treatments, would enable detection of an absolute difference in troponin T levels of $0.28 \mu \mathrm{g} / \mathrm{L}$. This level of change would correspond to a difference in troponin $\mathrm{T}$ between treatment groups of $40 \%$, which is less than that observed in previous trials, with $80 \%$ power and $5 \%$ significance (Hausenloy et al., 2007; Venugopal et al., 2009).

Professor Mark Weatherall completed the technical aspects of the statistical analysis for the trial in collaboration with the study investigators. Continuous baseline demographic variables were compared between treatment groups using t-tests, and categorical baseline variables were analysed with the Chi-square test. The cytokine, vasopressor support, and troponin data distributions were skewed to the right and normality assumptions were better met with natural logarithm transformation. The log transformed cytokine concentrations were compared using t-tests and analysis of covariance (ANCOVA). The exponent of the difference in logarithms shown in the results is equivalent to the ratio of mean values for the two groups. These exploratory analyses were each carried out with a type I error rate of $5 \%$ and no adjustment was used for multiple comparisons.

For the primary analyses of troponin levels and noradrenaline duration comparisons between treatment groups were made using t-tests. The incidence of post-operative renal injury was analysed using contingency table analysis and the Chi-square test. A Bonferroni correction was used to account for multiple comparisons, therefore the adjusted p-value for accepting statistical significance for these clinical outcome variables was 0.0083 and the corresponding confidence intervals were estimates at the 99.17\% level. A secondary analysis using ANCOVA was also performed to account for the effect of potential confounding variables on troponin levels. The pre-specified confounding 
variables were sulphonylurea and statin use, EuroSCORE, cross-clamp and total bypass times, and the number of procedures performed.

Lastly, the correlation between bypass time and cytokine expression was analysed using two-tailed Pearson's correlation coefficients, and the effect of statin use on IL-6 production was examined using an independent samples t-test. The data analysis was performed using R (R Development Core Team, New Zealand) or SPSS Statistics (IBM Corporation, Armonk, NY, USA), and the graphs produced using GraphPad Prism 5 (GraphPad Software Inc., La Jolla, CA, USA). 


\subsection{Results}

\subsubsection{Patient baseline demographic and operative data}

Peri-operative serum samples were collected from a total of 96 patients; however, one individual was excluded from the final cytokine data analysis due to the use of an immunosuppressive therapy. The final dataset consisted of 47 patients who received the RIPC treatment, and 48 patients in the control group (Figure 2.1).

The randomisation appeared successful, with a similar distribution of key baseline characteristics between the two treatment groups. There were no statistically significant differences between the RIPC and control treated groups with respect to age, sex, ethnicity, or medication use (Table 2.1). A set of clinical variables were collected and used to calculate the EuroSCORE for each patient as a predictive measure of cardiac operative risk (Nashef et al., 1999; Roques et al., 2003). Again, there were no differences between the groups with respect to the overall mean EuroSCORE, or the distribution of key contributing factors (Table 2.1).

While the two treatment groups were well matched with respect to the baseline characteristics, there were some notable differences with the operative details. There was a trend towards a more complex set of surgeries being performed in the RIPC patients, with 5 out of 47 patients undergoing 3 or more procedures, compared to 0 out of 48 patients in the control group ( $p=0.057$; Table 2.2). In addition, the durations of bypass and aortic cross-clamping were longer in the preconditioned patients, although this difference did not reach statistical significance (cross-clamp time: $117 \pm 50.8$ vs. $105 \pm 40.6$ minutes in the control group, $p=0.193$; bypass time: $151 \pm 59.0$ vs. $135 \pm 49.1$ minutes in the control group, $\mathrm{p}=0.185$; Table 2.1). 


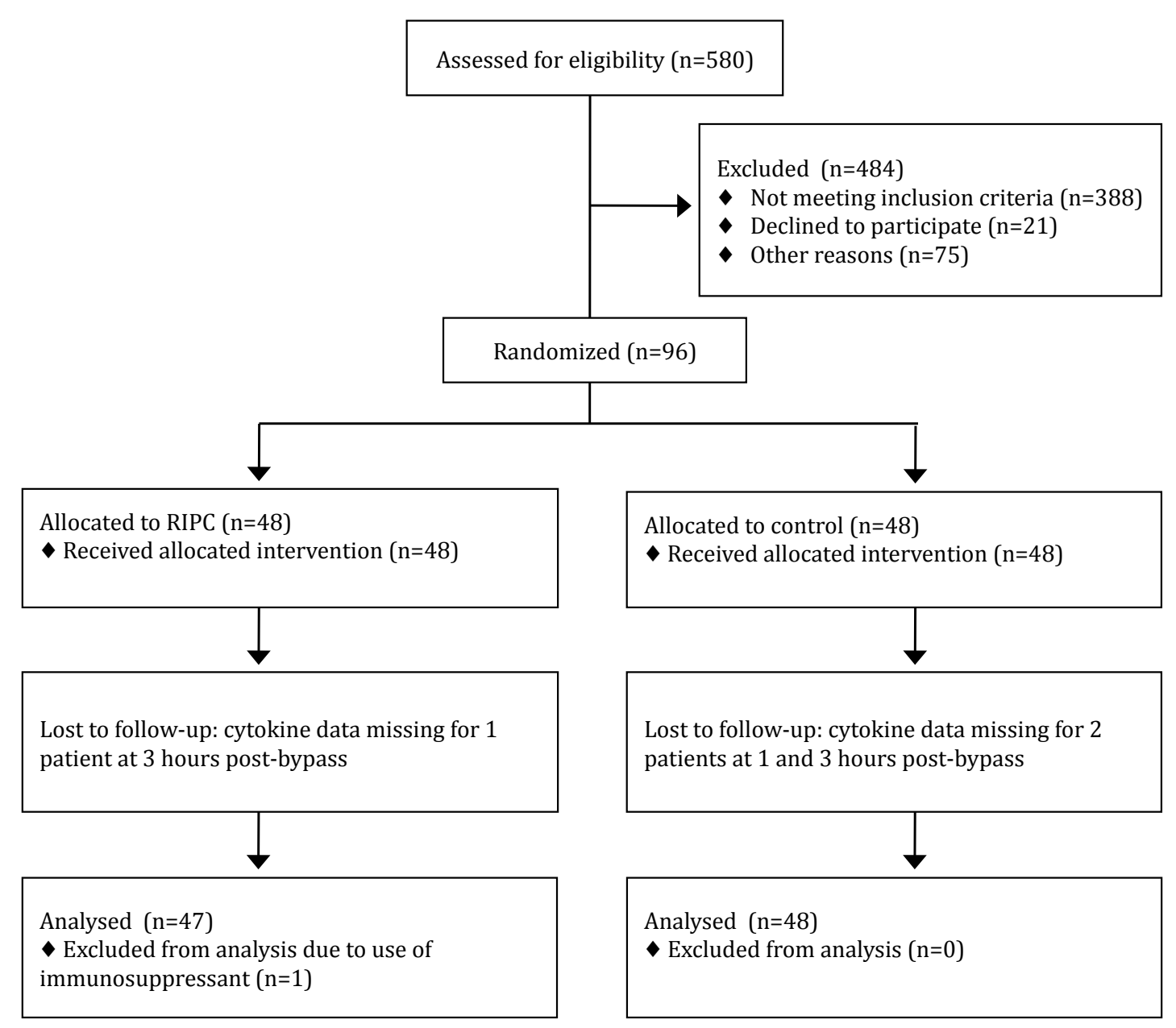

Figure 2.1 CONSORT diagram of study workflow

Flow chart showing the number of patients assessed and recruited into the study, the missing data points, and the reason for patient exclusion from the analysis. 


\begin{tabular}{|c|c|c|c|}
\hline & $\begin{array}{l}\text { RIPC } \\
\mathrm{n}=47\end{array}$ & $\begin{array}{c}\text { Control } \\
\mathrm{n}=48\end{array}$ & P-value \\
\hline \multicolumn{4}{|l|}{ Demographics } \\
\hline Age (mean, years) & $65.3 \pm 12.8$ & $64.3 \pm 14.5$ & 0.726 \\
\hline Female sex & $18(38)$ & $17(35)$ & 0.771 \\
\hline BMI (mean, $\mathrm{kg} / \mathrm{m}^{2}$ ) & $28.5 \pm 5.2$ & $28.6 \pm 5.9$ & 0.869 \\
\hline \multicolumn{4}{|l|}{ Ethnicity } \\
\hline European & $31(66)$ & $38(79)$ & \multirow{3}{*}{0.157} \\
\hline Maori & $7(15)$ & $7(15)$ & \\
\hline Other & $9(19)$ & $3(6)$ & \\
\hline \multicolumn{4}{|l|}{ Clinical details } \\
\hline \multicolumn{4}{|l|}{ Medications } \\
\hline Beta blockers & $34(72)$ & $29(60)$ & 0.219 \\
\hline ACE inhibitors & $24(51)$ & $25(52)$ & 0.921 \\
\hline Statins & 27 (57) & $30(63)$ & 0.615 \\
\hline Sulfonylureas & $4(9)$ & $1(2)$ & 0.161 \\
\hline Digoxin & $5(11)$ & $10(21)$ & 0.173 \\
\hline \multicolumn{4}{|l|}{ EuroSCORE variables } \\
\hline \multicolumn{4}{|c|}{ Left ventricular function } \\
\hline $\mathrm{EF}>50 \%$ & $28(60)$ & $30(63)$ & \multirow{3}{*}{0.090} \\
\hline EF $30-50 \%$ & $19(40)$ & $14(29)$ & \\
\hline $\mathrm{EF}<30 \%$ & $0(0)$ & $4(8)$ & \\
\hline Unstable angina & $0(0)$ & $0(0)$ & \\
\hline EuroSCORE (mean) & $7.07 \pm 6.10$ & $6.58 \pm 6.08$ & 0.694 \\
\hline
\end{tabular}

Table 2.1 Patient demographic and clinical data

Values are reported as mean \pm standard deviation or number (\%). Continuous variables were analysed using t-tests and categorical variables were compared using the Chi-square test. $\mathrm{BMI}=$ body mass index, $\mathrm{ACE}=$ angiotensin converting enzyme, $\mathrm{EF}=$ ejection fraction. 


\begin{tabular}{|c|c|c|c|}
\hline & $\begin{array}{l}\text { RIPC } \\
n=47\end{array}$ & $\begin{array}{c}\text { Control } \\
n=48\end{array}$ & P-value \\
\hline \multicolumn{4}{|l|}{ Operation } \\
\hline AVR & $4(9)$ & $2(4)$ & \multirow{11}{*}{0.571} \\
\hline MVR & $8(17)$ & $10(20)$ & \\
\hline AVR, MVR & $4(9)$ & $6(13)$ & \\
\hline MVR, TA & $2(4)$ & $5(10)$ & \\
\hline AVR, MVR, TA & $2(4)$ & $0(0)$ & \\
\hline AVR, CABG & $11(23)$ & $14(29)$ & \\
\hline MVR, CABG & $4(9)$ & $2(4)$ & \\
\hline AVR, MVR, CABG & $1(2)$ & $0(0)$ & \\
\hline AVR, MVR, TA, CABG & $1(2)$ & $0(0)$ & \\
\hline AVR, MVR, TA, ASD repair & $1(2)$ & $0(0)$ & \\
\hline CABG with $\mathrm{EF}<50 \%$ & 9 (19) & 9 (19) & \\
\hline \multicolumn{4}{|l|}{ Number of procedures } \\
\hline One & $21(45)$ & $21(44)$ & \multirow{3}{*}{0.057} \\
\hline Two & $21(45)$ & $27(56)$ & \\
\hline Three or more & $5(11)$ & $0(0)$ & \\
\hline \multicolumn{4}{|l|}{ Bypass variables } \\
\hline Total bypass time (mean, minutes) & $151 \pm 59.0$ & $135 \pm 49.1$ & 0.185 \\
\hline Cross-clamp time (mean, minutes) & $117 \pm 50.8$ & $105 \pm 40.6$ & 0.193 \\
\hline Cross-clamp fibrillation & $2(4)$ & $2(4)$ & 0.983 \\
\hline
\end{tabular}

\section{Table 2.2 Patient operation details}

Values are reported as mean \pm standard deviation or number (\%). Continuous variables were analysed using t-tests and categorical variables were compared using the Chi-square test. AVR $=$ aortic valve replacement, $\mathrm{MVR}=$ mitral valve repair or replacement, $\mathrm{TA}=$ tricuspid annuloplasty, $\mathrm{CABG}=$ coronary artery bypass graft, $\mathrm{ASD}=$ atrial septal defect, $\mathrm{EF}=$ ejection fraction. 


\subsubsection{Clinical outcome measures}

The principal investigator of the study, Dr Paul Young, was responsible for the overall study design and analysis of the clinical data. The detailed clinical results of the trial, including the secondary endpoints as analysed and presented by $\mathrm{Dr}$ Young are included in full in appendix D. Reproduction of the clinical results in this thesis will be limited to the three primary outcomes measured in the study; high sensitivity troponin $\mathrm{T}$, duration of noradrenaline use, and the incidence of AKI as determined by RIFLE criteria (Bellomo et al., 2004).

The results from the clinical endpoints did not indicate any protective benefit from RIPC for the patients in our study (Young et al., 2012). The point estimates showed non-significantly higher troponin levels in the RIPC group at both 6 and 12 hours following cross-clamp removal (6 hours: 1533 (SD 1784) vs. 828 ng/L (540), ratio of means 1.41, p = 0.035; 12 hours: 1608 (2361) vs. 715 ng/L (586), ratio of means $1.56, \mathrm{p}=0.014$ ) and adjustment for baseline troponin levels, sulphonylurea and statin use, EuroSCORE, cross clamp and total bypass times, and the number of procedures performed reduced both the degree of the difference and the $\mathrm{p}$ values for the troponin levels at 6 and 12 hours ( 6 hours: ratio of means 1.23 (99.17\% CI 0.88 to 1.72$), p=0.10 ; 12$ hours: ratio of means 1.30 (0.92 to 1.84 ), $\mathrm{p}=0.05$ ). Overall, the RIPC patients demonstrated higher requirements for post-operative ICU support, with an increased duration of noradrenaline treatment (21 (IQR 7 - 45) vs. 9 hours (3 - 19), ratio of means 1.70 (99.17\% CI 0.86 to 3.34), p = 0.04) and use of vasopressin (17 vs. $0 \%$, absolute risk difference of $17 \%$ (95\% CI 6 to 28), $\mathrm{p}=0.006)$. There was no difference in the incidence of AKI between the two treatment groups (raw data not shown, $p=0.82$ ).

\subsubsection{RIPC and systemic levels of IL-6, IL-8, and IL-10 during high-risk cardiac surgery}

My Honours project in 2010 involved developing a protocol for assessing inflammatory biomarker expression in high-risk cardiac surgery patients for this trial (Williams, 2010). This project involved selecting the markers and time points of interest, collecting serum samples, and optimising a cytometric bead 
array (CBA) with appropriate controls to quantify the cytokines. The majority of the trial patients were recruited throughout the following year; therefore aside from the initial optimisation and validation of the CBA assay, almost all of the experimental work and all of the analyses were conducted during my $\mathrm{PhD}$ studies. Only the work arising from my PhD studies are included in this thesis.

The serum IL-6, IL-8, and IL-10 levels increased considerably from baseline in all patients following surgery. In contrast, IL-1 $1 \beta$, IL-12p70 and TNF- $\alpha$ concentrations did not increase and were below the level of detection of the CBA (7.2, 1.9, and $3.7 \mathrm{pg} / \mathrm{mL}$, respectively; data not shown). The inflammatory cytokines, IL- 6 and IL-8, peaked between 1 and 3 hours post-reperfusion and remained increased at 12 hours (Figure 2.2A and B). To reduce the incidence of type I error, statistical testing of the cytokine data was limited to the 1 and 12 hour time points, which were deemed the most informative. The point estimates were consistent with the hypothesis that there were higher levels of the proinflammatory mediators present in the RIPC patients, compared to the controls. However, the confidence intervals were wide and there were no statistically significant differences in mean log IL-6 and IL-8 levels between the RIPC and control groups at 1 or 12 hours (Table 2.3) or in the total area under the curve (AUC; IL-6: ratio of means 1.32 [95\% CI 0.84-2.05], p = 0.22; IL-8: 1.17 [0.79-1.72], $\mathrm{p}=0.43$ ).

An ANCOVA model was used to determine if accounting for potential confounding variables altered the difference between the two treatment groups. Adjustment for baseline interleukin levels, age, EuroSCORE predicted mortality, bypass and cross-clamp times, surgery type (CABG only versus all other procedures), and statin use did not alter the absence of a statistically significant difference seen with the univariate analysis (Table 2.3; Roques et al., 2003).

Levels of the immunosuppressive cytokine, IL-10, peaked at 1 hour postreperfusion and then steadily declined (Figure 2.2C). IL-10 levels were similar in the RIPC and control groups (AUC: ratio of means 1.13 [0.70-1.80], $\mathrm{p}=0.62$ ) and this was not affected by adjustment for confounding variables (Table 2.3). 

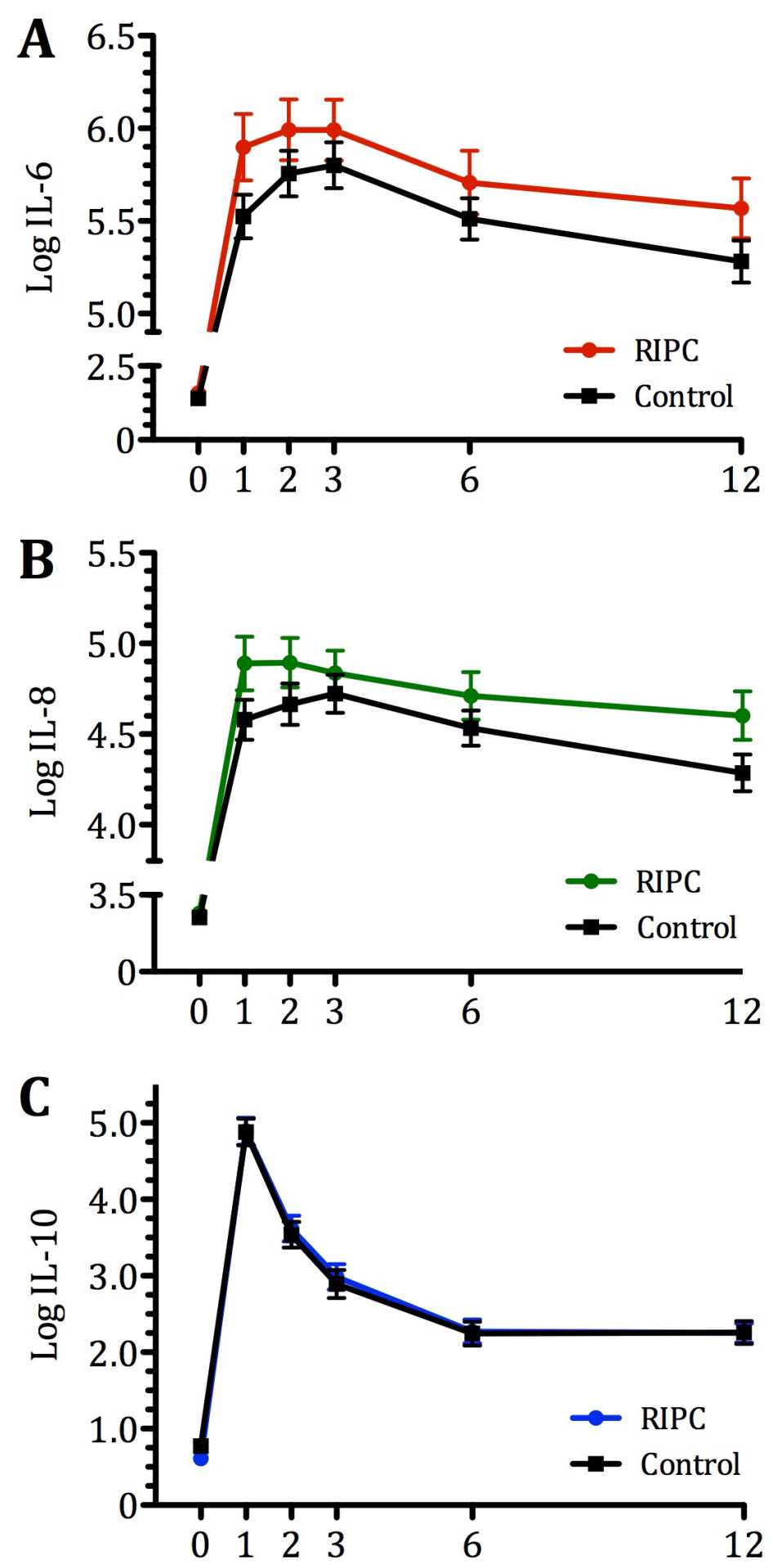

Hours post-x-clamp removal

Figure 2.2 Levels of IL-6, IL-8, and IL-10 are not affected by RIPC in highrisk cardiac surgery up to 12 hours after cross-clamp removal

Serum samples were isolated from blood collected pre-operatively and 1, 2, 3, 6, and 12 hours after cross-clamp removal. A CBA and flow cytometry were used to quantify the serum levels of (A) IL-6, (B) IL-8, and (C) IL-10. The data was natural log transformed to meet normality assumptions and shown are the mean and SEM of $n=48$ controls and $n=47$ RIPC patients. 


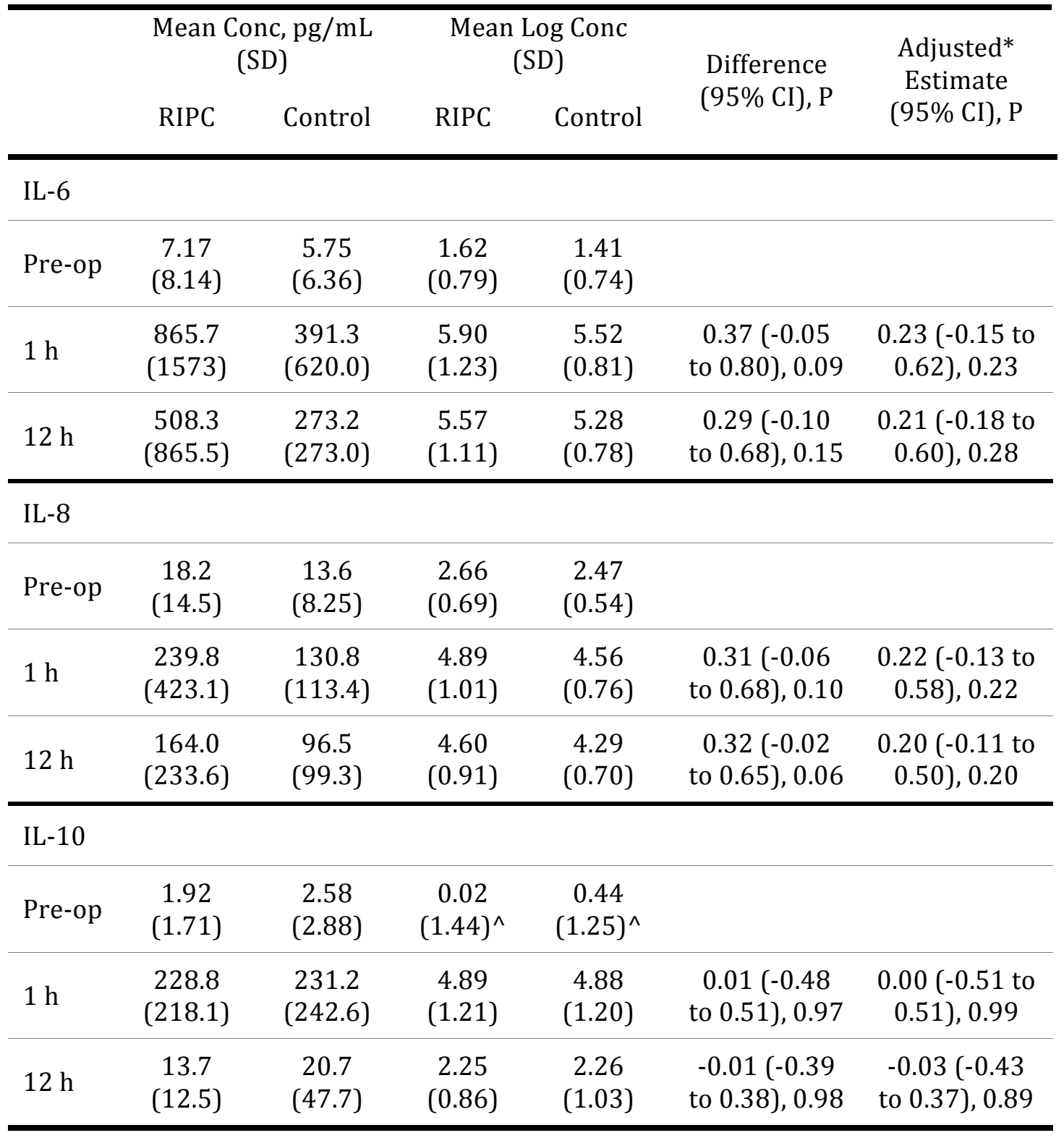

Table 2.3 Statistical comparison of peri-operative IL-6, IL-8, and IL-10 levels between RIPC and control treated patients

Serum samples were isolated from blood collected pre-operatively and after cross-clamp removal and cytokine levels were quantified using a CBA and flow cytometry. Statistical testing was limited to the 1 and 12 hour time points to reduce type I error from repeated measures. The data was natural log transformed to meet normality assumptions and shown are the results from $\mathrm{n}=$ 48 controls and $n=47$ RIPC patients. $\mathrm{CI}=$ confidence interval. $*$ Adjusted for baseline interleukin levels, age, EuroSCORE predicted mortality, total bypass and cross-clamp times, type of surgery, and statin use. ${ }^{\wedge} 12 / 47$ RIPC and 7/48 control patients had concentrations of zero, therefore these were given a value of 0.1 to allow for logarithm transformation. 


\subsubsection{Effect of RIPC on peri-operative cytokine expression in simple versus complex surgery}

A post-hoc analysis investigated whether RIPC differentially modulates cytokine expression depending on the complexity of the surgery undergone. In this study and in previous work, we observed troponin release and bypass duration varied with surgical procedure (Swaanenburg et al., 2001; van Geene et al., 2010). Both Swaaneburg et al. and van Geene et al. reported substantially lower postoperative levels of troponin in patients undergoing straightforward CABG $\pm A V R$ surgery, compared to procedures involving the mitral valve. Participants in our study were therefore categorised and divided based on whether they were having either simple surgery: $\mathrm{CABG}$ with $\mathrm{EF}<50 \%$ or $\mathrm{CABG} \pm$ aortic valve surgery; or complex surgery: MVR \pm any other procedure. ANCOVA with baseline interleukin levels and treatment randomisation as co-variates was used to compare cytokine levels.

As expected, there were notable differences in the baseline and operative variables for the two surgery subgroups (Table 2.4). The patients undergoing complex surgery had significantly longer bypass durations and were more often prescribed beta blockers and/or statins, compared to patients in the simple procedure group. The mean age of the patients in the simple surgery group was significantly higher and the number of female patients significantly lower. There was no difference between the two groups with respect to treatment group allocation or the mean EuroSCORE (Table 2.4).

Complex surgery was associated with significantly higher levels of IL-6 (ratio of means 2.14 [95\% CI 1.45-3.16]; $\mathrm{P}<0.001$ ) and IL-10 (1.82 [1.05-3.19]; $\mathrm{p}=$ 0.033 ) at 1 hour post-bypass and in the total AUC (IL-6: ratio of means 1.54 [95\% CI 1.02-2.34], $\mathrm{P}=0.04$; IL-10: 1.97 [1.16-3.35], $\mathrm{P}=0.012$; Figure 2.3A and B). By 12 hours, there was no evidence of a difference in the estimates (IL-6: 0.91 [0.641.31], $\mathrm{P}=0.62$; IL-10: 1.08 [0.72-1.63], $\mathrm{P}=0.69$ ). RIPC did not alter expression of these cytokines in either simple or complex surgeries (Figure 2.3C - F). There was no difference in IL-8 levels between the simple and complex surgery groups (data not shown). 


\begin{tabular}{|c|c|c|c|}
\hline & $\begin{array}{l}\text { Simple } \\
(\mathrm{n}=49)\end{array}$ & $\begin{array}{c}\text { Complex } \\
(n=46)\end{array}$ & $P$ \\
\hline \multicolumn{4}{|l|}{ Treatment allocation } \\
\hline RIPC & $24(49)$ & $23(50)$ & \multirow{2}{*}{0.92} \\
\hline Control & $25(51)$ & $23(50)$ & \\
\hline \multicolumn{4}{|l|}{ Demographics } \\
\hline Age (mean, yrs) & $69.3 \pm 9.75$ & $60.1 \pm 15.5$ & $<0.01$ \\
\hline Female sex & $10(20)$ & $25(54)$ & $<0.01$ \\
\hline \multicolumn{4}{|l|}{ Ethnicity } \\
\hline European & $42(85)$ & $27(59)$ & \multirow{3}{*}{0.01} \\
\hline Maori & $4(9)$ & $10(22)$ & \\
\hline Other & $3(6)$ & $9(20)$ & \\
\hline \multicolumn{4}{|l|}{ Medications } \\
\hline Beta blockers & $10(20)$ & $22(48)$ & 0.01 \\
\hline ACE inhibitors & $22(45)$ & $24(52)$ & 0.48 \\
\hline Statins & $8(16)$ & $30(65)$ & $<0.01$ \\
\hline EuroSCORE (mean) & $6.76 \pm 6.24$ & $6.90 \pm 5.95$ & 0.91 \\
\hline \multicolumn{4}{|l|}{ Operative details } \\
\hline Bypass time (mean, mins) & $120 \pm 29.4$ & $168 \pm 63.7$ & $<0.01$ \\
\hline
\end{tabular}

Table 2.4 Key patient demographic, clinical, and operative details for simple and complex surgery subgroups

Values are reported as mean \pm standard deviation or number (\%). ACE = angiotensin converting enzyme. 

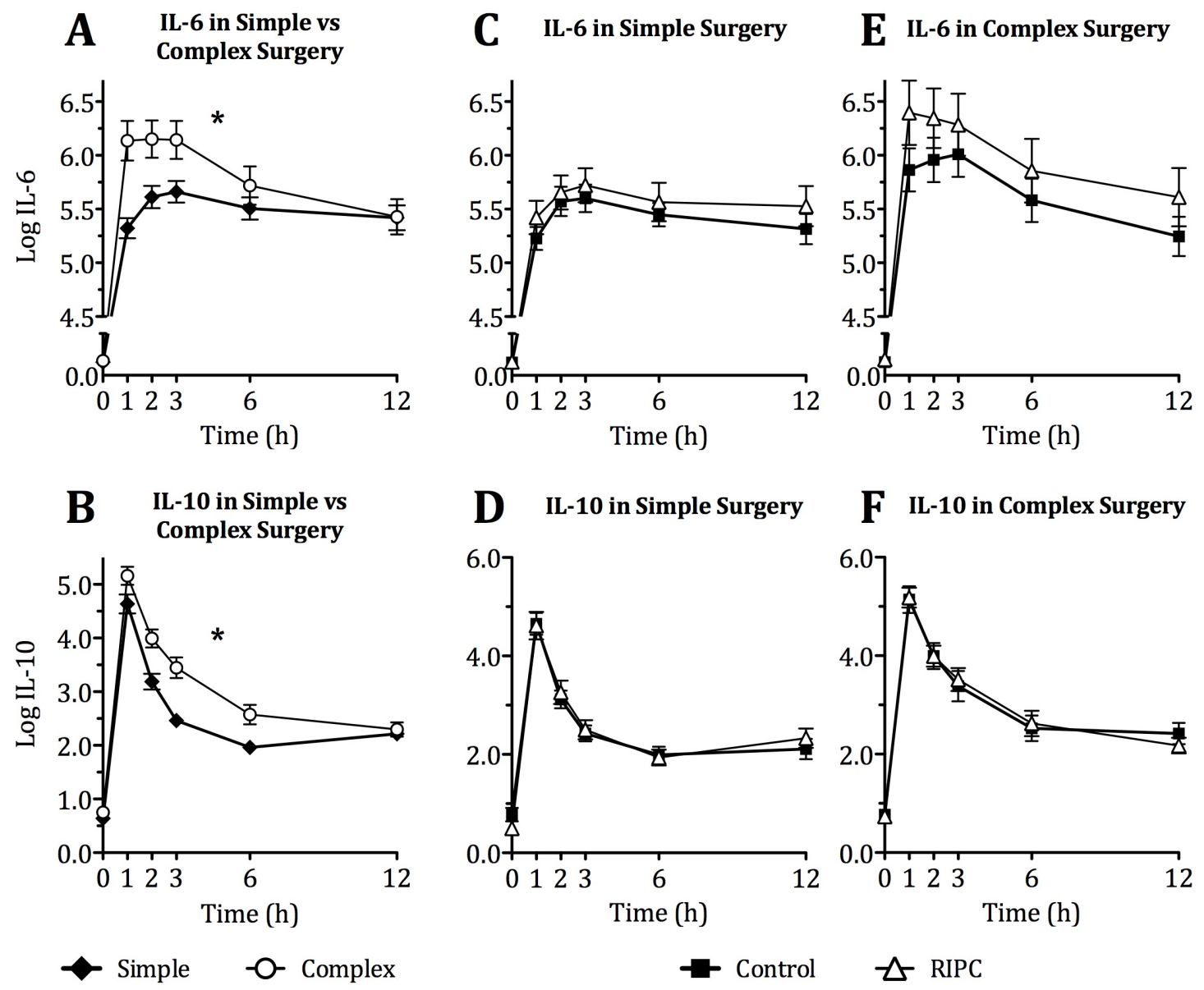

Figure 2.3 IL-6 and IL-10 levels are influenced by surgery complexity, but not RIPC

Serum samples were isolated from blood collected pre-operatively and after cross-clamp removal and cytokine levels were quantified using a CBA and flow cytometry. (A) IL-6 and (B) IL-10 levels, in simple $(n=49)$ versus complex $(n=$ 46) surgeries. (C \& E) IL-6 levels by treatment group in simple and complex surgery patients, respectively. (D \& F) IL-10 levels by treatment group in simple and complex surgery patients, respectively. Data are shown as group means with SEM. ${ }^{*} \mathrm{p}<0.05$ for differences in the total AUC using ANCOVA with baseline interleukin levels and treatment randomisation as covariates. 


\subsubsection{Relationship between bypass duration and the expression of cytokines}

Given the non-significantly longer mean bypass time in our preconditioned patients compared to the controls $(\mathrm{p}=0.185)$, we sought to determine if this might account for the increased cytokine levels detected in these patients. In our study IL-6 levels were weakly correlated with the total bypass time in the early reperfusion period (Figure 2.4A - C). This relationship was more significant in the patients from the RIPC treatment group ( $1 \mathrm{hr}$ : Control $\mathrm{r}=0.304, \mathrm{p}<0.05$ vs. RIPC $r=0.474, p<0.01 ; 2$ hrs: Control $r=0.136, p>0.05$ vs. RIPC $r=0.367, p<$ 0.05). In contrast, the strongest relationship between IL-8 and bypass duration was seen at the 6 and 12-hour time points (Figure 2.4D - F). This positive correlation was low overall, but appeared slightly stronger in the preconditioned patients (6 hrs: Control $r=0.163, \mathrm{p}>0.05$ vs. RIPC $\mathrm{r}=0.446, \mathrm{p}<0.01 ; 12$ hrs: Control $\mathrm{r}=0.308, \mathrm{p}<0.05$ vs. RIPC $\mathrm{r}=0.429, \mathrm{p}<0.01$ ). Interestingly, we also detected a correlation between IL-10 levels at 3 and 6 hours, and the total bypass time (Figure 2.4G - I). The correlation was weak but significant and did not differ between the two treatment groups ( 3 hrs: Control $\mathrm{r}=0.334, \mathrm{p}<0.05$ vs. RIPC $\mathrm{r}=$ 0.390, $\mathrm{p}<0.01 ; 6$ hrs: Control $\mathrm{r}=0.385, \mathrm{p}<0.01$ vs. RIPC $\mathrm{r}=0.391, \mathrm{p}<0.01$ ).

The higher mean bypass time in the RIPC patients may have contributed to the increased levels of IL-6 and IL-8 detected in the point estimates for these patients; however, our adjusted estimates using the ANCOVA model with bypass time as a variable would have successfully accounted for such weak correlations. 

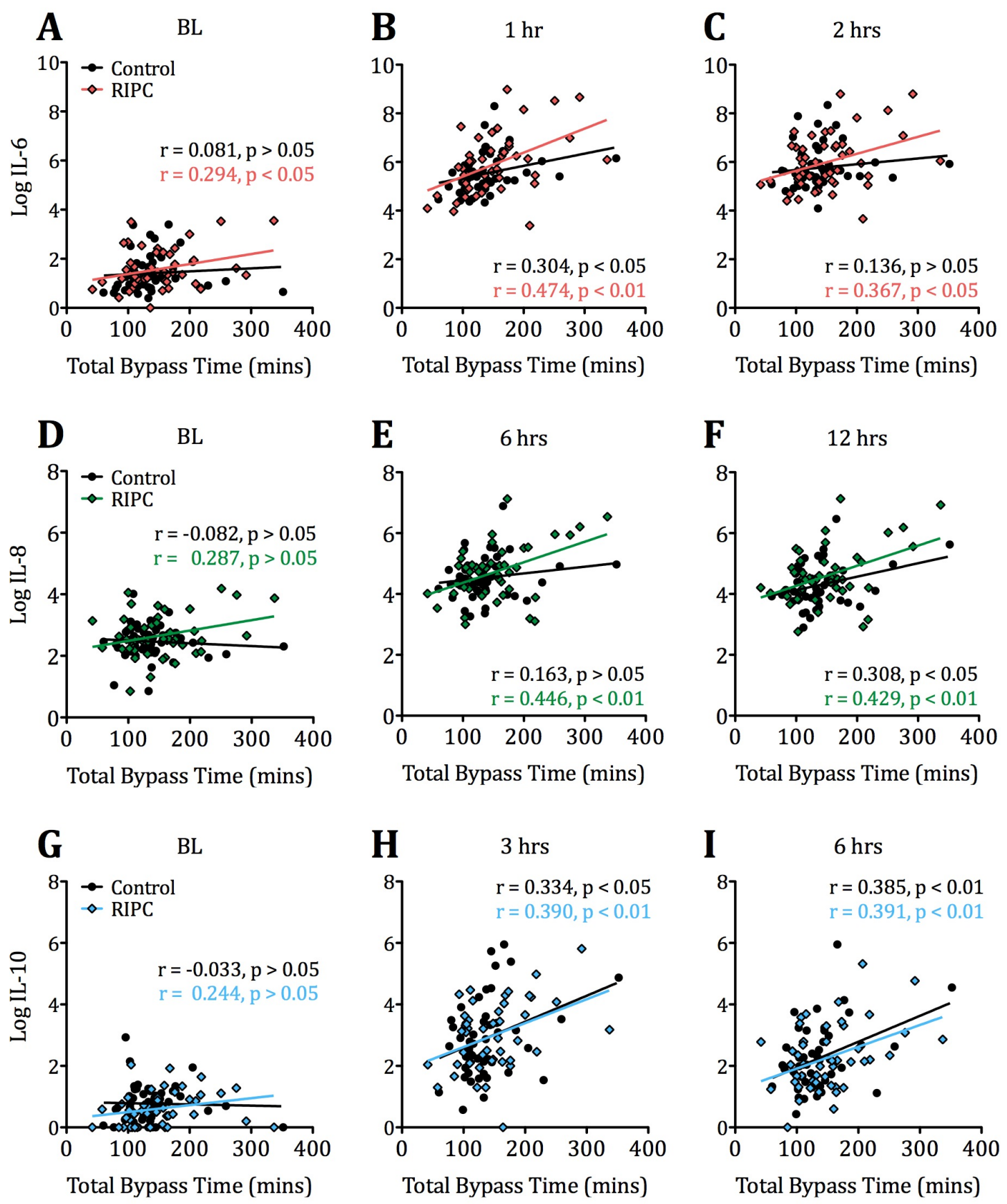

Figure 2.4 Correlation between bypass time and cytokine levels in highrisk surgery patients

Serum samples were isolated from blood collected pre-operatively and after cross-clamp removal and cytokine levels were quantified using a CBA and flow cytometry. (A - C) IL- 6 vs. total bypass duration at baseline, and 1 hour and 2 hours after cross-clamp removal, respectively. (D - F) IL-8 vs. total bypass time at baseline, and 6 and 12 hours after cross-clamp removal, respectively. (G - I) IL-10 vs. total bypass time at baseline, and 3 and 6 hours after cross-clamp removal, respectively. Shown are the Pearson's correlation coefficients from a two-tailed analysis of $n=48$ controls and $n=47$ RIPC patients at baseline and 2, 6 , and 12 hours after cross-clamp removal, $n=47$ controls and $n=47$ RIPC patients at 1 hour, and $n=47$ controls and $n=46$ RIPC at 3 hours. 


\subsubsection{Relationship between statin usage and IL-6 expression}

In a preliminary analysis performed with the data from the first 32 patients as part of my Honours project, it was observed that statin usage in our patients was associated with a significant reduction in the post-operative IL-6 levels measured at 1,2 , and 3 hours after cross-clamp removal. We therefore investigated the association between the use of statins and IL-6 levels using the full dataset.

In the pooled patient population there was no significant difference in the expression levels of IL-6 between those who were and those who were not taking statins, at any time point (Figure 2.5A). In the control group patients alone, there was a significant reduction in IL-6 expression at 1 hour post-bypass in the patients who were prescribed a statin compared to those that were not (log IL-6 at 1 hour: 5.87 (SD 0.92) vs. 5.33 (0.68), p = 0.024), whereas no such differences were detected in the preconditioned patients (Figure 2.5B and C).

Any effect of statins on IL-6 production in our study appeared to be very limited. In the event that statin use did influence IL-6 levels, the distribution of patients taking these medications was even across the two treatment groups (refer to Table 2.1) and the adjusted estimates using the ANCOVA model with statin use as a variable would have accounted for this modest affect. It is therefore highly unlikely that statin use contributed to a difference in IL-6 levels between our two treatment groups. 
A All Patients

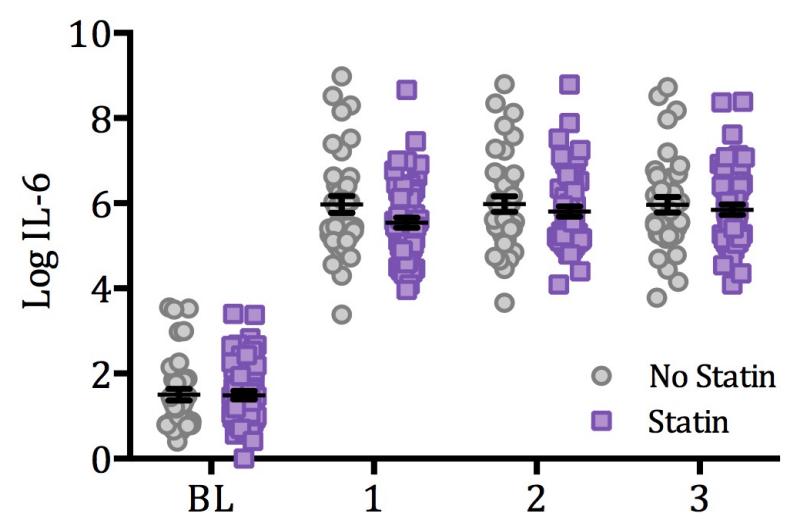

B Control Patients

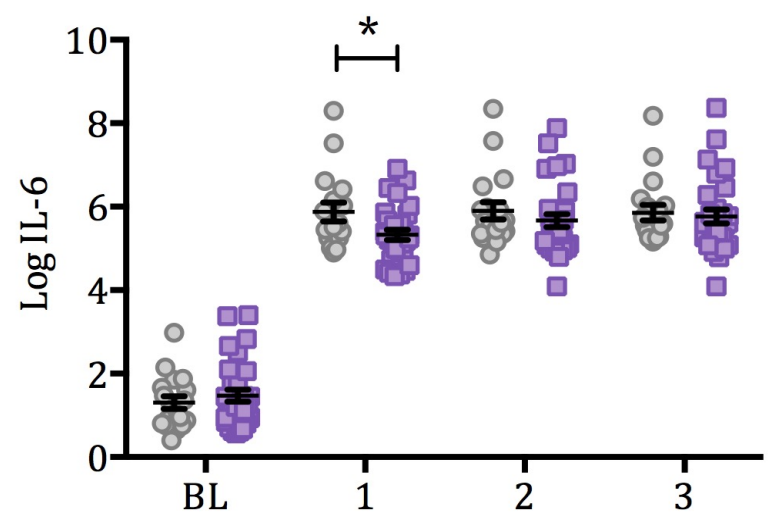

C

RIPC Patients

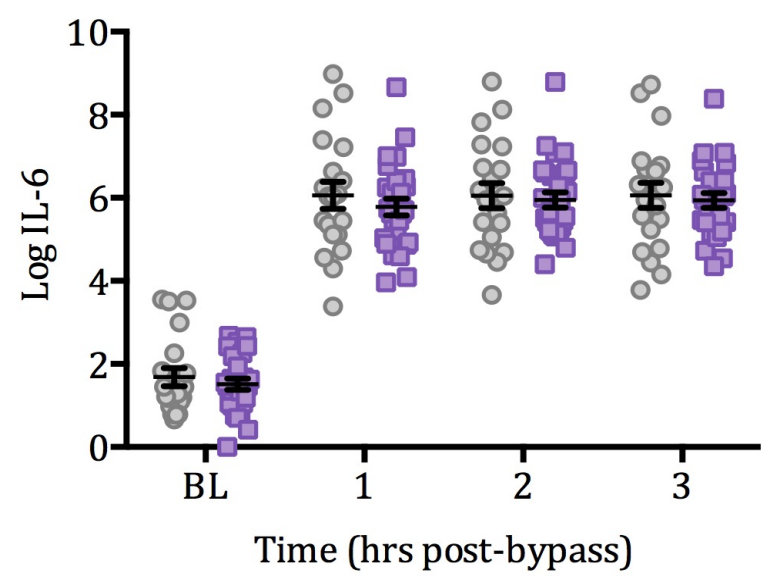

Figure 2.5 The effect of statins on post-operative IL-6 levels

Serum samples were isolated from blood collected pre-operatively and after cross-clamp removal and IL-6 levels were quantified using a CBA and flow cytometry. Log IL-6 levels for patients that were and were not taking statins are shown for (A) all patients, (B) control group patients, and (C) RIPC group patients. Shown are the means and SEM for $n=47$ controls and $n=48$ RIPC patients. Comparisons were made using an independent samples t-test. ${ }^{*} \mathrm{p}<$ 0.05 . 


\subsubsection{Comparison of vacutainers for cytokine recovery}

There are two types of commercially available vacutainers used for human serum collection. The first are the BD SST II Advance Serum tubes, which contain a barrier polymer gel that migrates to the serum-clot interface during centrifugation, and silica particles to aid sample clotting. The alternative, the BD Plus Serum tubes, contain the silica particle coating without a gel additive. In the clinical setting the BD SST II Advance Serum tubes are the most commonly used vacutainers for serum collection and were selected for use in our high-risk cardiac surgery study.

Because TNF- $\alpha$ was not detected in any of the patient samples we wanted to confirm that our methodology was capable of detecting this cytokine. The biologically active form of TNF consists of a bulky trimer with a molecular weight approximately twice that of IL-6, allowing for the possibility that the gel barrier present in our sample collection tubes may have filtered the TNF- $\alpha$ out of the samples (Smith and Baglioni, 1987). We therefore collected blood samples from one individual into vacutainers with and without the barrier gel and then added known concentrations of recombinant mouse TNF- $\alpha$ and human IL- 6 to the samples. After centrifugation the resulting serum cytokine levels were quantified by ELISA.

Comparable levels of both TNF- $\alpha$ and IL- 6 were recovered in the serum of samples collected with both types of vacutainers (Figure 2.6A and B). In fact, slightly lower levels of TNF- $\alpha$ were measured in the samples collected from the vacutainers without the gel, strongly suggesting that the inclusion of the gel barrier in the SST II Advance tubes did not adversely affect recovery of either cytokine from the serum samples. 


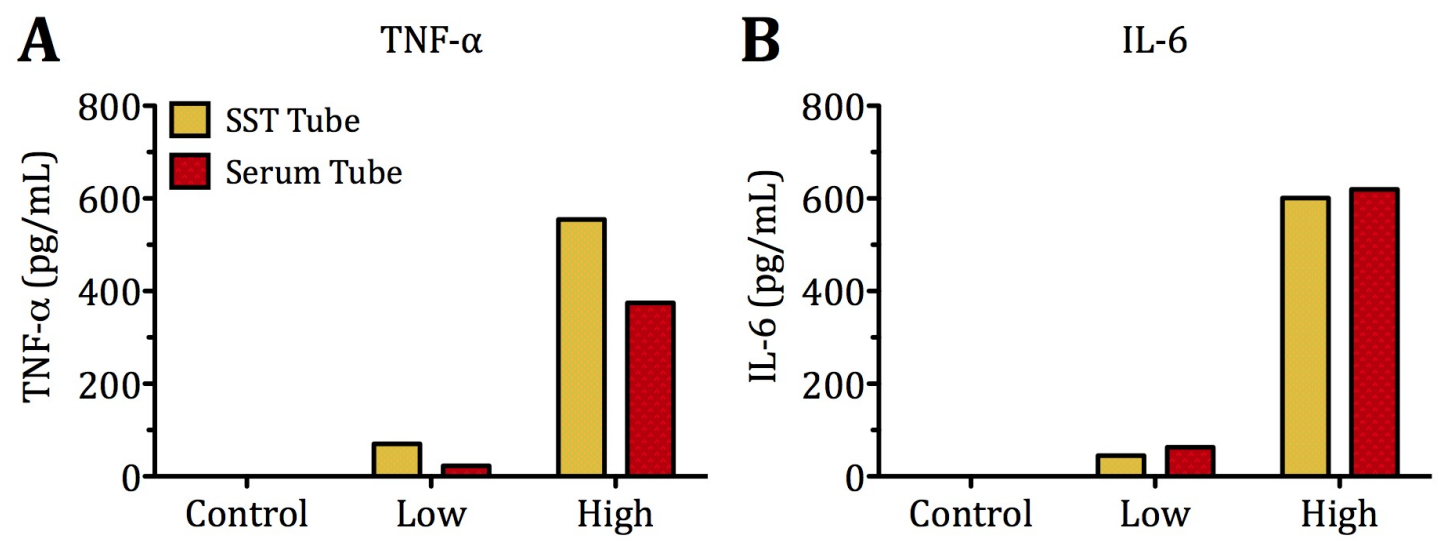

Figure 2.6 Comparison of TNF- $\alpha$ and IL-6 recovery between commercially available serum vacutainers

Blood samples were collected into BD SST II Advance (yellow) or Plastic Plus Serum tubes (red) and spiked with low (200 pg/mL) or high $(1500 \mathrm{pg} / \mathrm{mL})$ concentrations of cytokines. Cytokine levels in the resulting serum samples were determined by ELISA and are shown for (A) murine TNF- $\alpha$ and (B) human recombinant IL-6. Shown are the results from one individual and one experiment. 


\subsection{Discussion}

This study was the first to show that RIPC does not reduce troponin levels in high-risk cardiac surgery patients, or alter expression of a panel of inflammatory cytokines during the first 12 hours post-bypass. Although the point estimates favoured the hypothesis that there were higher levels of IL- 6 and IL-8 in the RIPC group, the differences were not statistically significant even after adjustment for confounding variables such as statin use, EuroSCORE, and total bypass time. Consistent with previous research, we confirmed that peri-operative IL-6 levels were higher with complex valvular surgery compared to straightforward CABG and AVR procedures, and both IL- 6 and IL-8 were weakly correlated to the duration of bypass (Kawahito et al., 2000). In addition we found that the release of the anti-inflammatory cytokine, IL-10, was also enhanced. A post-hoc analysis indicated that RIPC did not alter IL-6 and IL-10 expression in patients undergoing simple procedures or the more complex surgeries that are associated with higher cytokine levels. As this was an exploratory study, one limitation is that multiple comparisons were performed and therefore it is possible that some of the statistically significant differences may represent type I errors.

The precise mechanism of RIPC has yet to be fully elucidated; however, there are data supporting the hypothesis that an unidentified humoral factor transmits the protective signal from the remote preconditioned tissue to the myocardium (Dickson et al., 1999b; Gho et al., 1996; I. E. Konstantinov et al., 2005). Cytokines are a prime candidate for such a factor given their function as endocrine signalling peptides; however, our finding that RIPC does not modify expression of the key cytokines IL-6, IL-8, and IL-10, suggests this mechanism is unlikely. This extends previous research by Karuppasamy et al. (2011) and Saxena et al. (2013), which showed that systemic concentrations of these cytokines were not altered following each cycle of ischemia during RIPC application, or directly after the full preconditioning stimulus, respectively. In contrast, a trial by Albrecht et al. (2013) detected subtle but significant increases in IL-1 $\beta$, IL-8, and TNF- $\alpha$ levels of cardiac surgery patients after preconditioning, although the high level of inter-patient variability limited the power and interpretation of these results. 
One possible explanation for our findings is that the systemic cytokine levels induced by high-risk cardiac procedures are too high to be significantly altered by the RIPC treatment in this setting.

Four additional studies have reported on cytokine expression following RIPC in children undergoing repair of congenital heart defects. Three applied lower limb RIPC and reported IL-6, IL-8, IL-10, and TNF- $\alpha$ levels were not significantly different from the controls (Cheung et al., 2006; McCrindle et al., 2014; Pavione et al., 2012). The fourth, which applied RIPC both one day and one hour preoperatively, found that levels of proinflammatory and anti-inflammatory cytokines were weakly attenuated post-operatively (Zhou et al., 2010). All four studies differ substantially from our own in that bypass times were dramatically shorter, the earliest samples corresponded to time points after detection of the peak period of cytokine expression in our study, and the RIPC interventions were fundamentally different to the standard stimulus applied in the majority of trials, including our own, making it difficult to relate this earlier work to the present findings.

TNF- $\alpha$ is reportedly expressed in patients with heart failure and unstable angina; however, we did not detect measurable levels in our cardiac patients before or after surgery with CPB (Levine et al., 1990; Wang et al., 2007). To confirm that our methodology was capable of detecting TNF- $\alpha$ we included a plasma sample with known levels of TNF- $\alpha$ in each assay run and confirmed the vacutainers used achieved adequate recovery of TNF- $\alpha$ into serum samples. Having performed these additional checks we were confident that there were no measurable levels of TNF- $\alpha$ present in the serum of our surgery patients.

The literature regarding TNF- $\alpha$ expression during cardiac surgery in humans is conflicting. A small number of studies have demonstrated an increase in TNF- $\alpha$ levels following removal of the aortic cross-clamp, and Wang et al. (2007) have reported that TNF- $\alpha$ levels are higher in unstable angina patients, of which there were none in our study (Risnes et al., 2003; Wan et al., 1996). The two RIPC studies that detected measurable TNF- $\alpha$ levels following cardiac surgery 
described low concentration peaks at less than $70 \mathrm{pg} / \mathrm{mL}$ (Cheung et al., 2006; Kim et al., 2012). In contrast, numerous other trials have noted only very low or unmeasurable peri-operative serum levels of TNF, and Borgermann et al. (2002) found CPB caused a 78\% reduction in leukocyte TNF- $\alpha$ mRNA production in response to an ex vivo endotoxin challenge (Karuppasamy et al., 2011; Kawamura et al., 1993; McBride et al., 1995; Saxena et al., 2013; Steinberg et al., 1993; Wan et al., 1999; Wei et al., 2001a; Zhou et al., 2010).

On balance, the weight of the literature appears to be consistent with our finding that the systemic levels of TNF- $\alpha$ produced in response to cardiac surgery with $\mathrm{CPB}$ are below the level of detection or clinical relevance, which is intriguing given that in animal studies preconditioning-induced protection from I/R injury has been frequently associated with significant decreases in plasma levels of TNF- $\alpha$ (Garab et al., 2014; Xiong et al., 2011; Zhang et al., 2013). The myocardium is known to be a major source of TNF- $\alpha$ release following reperfusion, with higher levels detected in human samples collected from the coronary sinus compared to arterial blood (Wan et al., 1996). Models using small animals may not be subjected to this potential site sampling bias or, alternatively, preconditioning may operate through a different pathway in animals.

RIPC appears to afford two windows of protection that may involve different pathways. The first occurs immediately, lasting less than four hours, whereas the second presents 24 hours after the initial conditioning (Loukogeorgakis et al., 2005). RIPC is most feasibly applied in surgery patients post-anaesthesia; therefore most trials to date, including our own, have investigated the efficacy of early-phase RIPC. Biomarkers of myocardial damage peak six to eight hours post-bypass, rendering the first 12 hours following surgery the most relevant period for examining factors that may modify or mediate myocardial injury (Holmvang et al., 2002; Swaanenburg et al., 2001). Our time points were thus carefully selected to allow us to determine whether a RIPC-induced change in cytokine expression could contribute to the protection against myocardial damage previously described in the literature. We were able to capture the 
period of peak peri-operative cytokine release and have shown no change in systemic cytokine levels or clinical outcome measures following RIPC in the pathophysiologically relevant period.

Related studies by Karuppasamy et al. (2011) and Saxena et al. (2013) determined that RIPC does not alter levels of IL-6, IL-8 or IL-10 at 24 and 48 hours following uncomplicated CABG. These studies and our own demonstrate that IL-6 and IL-10 levels are not affected during early or late-phase RIPC in humans; however, there is a large body of evidence forming that suggests cytokines are essential for inducing preconditioning in animal models. Using knockout mice, studies by Dawn et al. (2004), Cai et al. (2012), and Smith et al. (2002) have established that IL-6, IL-10, and TNF- $\alpha$ production, respectively, are obligatory for preconditioning-induced cardioprotection. Further, the benefits of late IPC in rats can be abolished by the simultaneous administration of antibodies to TNF- $\alpha$ and IL-1 $\beta$. Intriguingly, in humans there is also evidence that upregulation of proinflammatory cytokines in unstable angina patients is sufficient to induce a late preconditioning effect (Wang et al., 2007).

Taken together, the results of these studies have two key implications. First, it is possible that the mechanism of RIPC may differ between species, and to that of classic local ischemic preconditioning. This possibility is supported by the recent suggestion that the cytokine-activated transcription factors associated with cardioprotection may differ between animals and humans (Heusch et al., 2011). Second, contrary to the hypotheses of previous research, the studies by Dawn et al. (2004) and Wang et al. (2007) suggest preconditioning would be expected to induce protection through an increase in IL-6 and IL-8 levels, rather than a decrease. Indeed, while RIPC may not alter systemic IL-6 and IL-8 concentrations, it is well established that the reperfused myocardium is a major source of inflammatory cytokines (Wan et al., 1996). The possibility that the local inflammatory milieu in the myocardium is modulated by RIPC cannot be excluded and, thus, sampling blood from the coronary sinus may be of interest in this regard for future studies. 
Our trial adds to a growing number that have not shown a clinical benefit from RIPC in adult cardiac surgery patients (Hong et al., 2010; Karuppasamy et al., 2011; Li et al., 2010; Lomivorotov et al., 2012; Lucchinetti et al., 2012; Meybohm et al., 2013; Rahman et al., 2010; Saxena et al., 2013). It is possible that the absence of a clinical effect in our trial was due to the lack of change in the cytokine levels. If an increase in cytokines is essential to induce RIPC, factors that alter cytokine production such as the use of anti-inflammatory or statin therapies, the latter of which have been shown to reduce cellular IL-6 expression both at rest and in response to LPS challenge, may influence the ability to precondition patients (Node et al., 2003; Rosenson et al., 1999).

Alternatively, a preconditioning effect may have been activated in all patients through secondary pathways. Our study used a standardised anaesthesia protocol that included the use of volatile anaesthetics, which have attracted attention for their ability to induce cardiac preconditioning and decrease inflammatory cytokine expression (Belhomme et al., 1999; Haroun-Bizri et al., 2001; Kawamura et al., 2006). Thus, our control group may also have been, to some extent, preconditioned. This interpretation would suggest that the marginal effect of RIPC in patients already treated with volatile anaesthetics as per our study protocol is negligible, which is supported by a recent metaanalysis that suggested cardioprotection with RIPC is attenuated in patients treated with these agents (Zhou et al., 2013). Furthermore, two recent animal studies have suggested the anticoagulant, heparin, is also capable of limiting ischaemia-induced organ damage; however, when used in conjunction with IPC, heparin appears to abolish the protective effects of preconditioning (Saurim et al., 2014; Warzecha et al., 2012). The authors postulated that clot formation may be essential for inducing preconditioning or that heparin may compete for the pathways targeted by IPC. Several instrumental proof-of-concept preconditioning studies in animals were performed without the administration of heparin, highlighting a potential key difference between many of the animal and human studies (Birnbaum et al., 1997; Gho et al., 1996; Murry et al., 1986; Przyklenk et al., 1993). Given the widespread adoption of these anaesthetic 
agents and use of heparin in cardiac surgery, future research will be essential to clarify their relationship with RIPC.

A particular strength of our study was the use of double blinding to ensure the patient and all staff involved in their care were not privy to the treatment group allocation. At the time it was designed our trial was the first application of RIPC in humans to use such a protocol, whereas the majority of early positive RIPC clinical trials had incomplete or no blinding procedures. In the absence of complete blinding, surgical randomised controlled trials are vulnerable to both performance and ascertainment bias, which can significantly alter the trial results (Karanicolas et al., 2010). A meta-analysis found that RIPC studies without full blinding reported a larger estimate of troponin reduction compared to those with complete blinding, suggesting more weight should be given to the results of trials including an appropriate blinding design (Pilcher et al., 2012).

In addition to the challenges experienced by all trials attempting to translate RIPC from highly controlled animal models into a complex clinical setting, there were a number of limitations to our study. Our definition of high-risk was broad, encompassing patients that were undergoing complex and long procedures, redo surgeries, or having impaired cardiac function prior to the operation. This lead to recruitment of a very heterogeneous patient population and, in turn, resulted in a high level of variability in the responses and a reduction in statistical power. Despite these criteria, the average EuroSCORE of our patients represented a predicted operative mortality risk of 7\%, which is significantly lower than the $20 \%$ 'high-risk' cut-off used by other studies (Kalavrouziotis et al., 2009; Vahanian et al., 2008). The actual rate of mortality in the present trial was 2\%; further suggesting our participant population may not have been particularly high-risk.

An additional consequence of our 'high-risk' inclusion criteria was the extended duration of the surgeries performed. The first phase of RIPC-induced protection is reported to last between two and four hours (Loukogeorgakis et al., 2005; Yang et al., 2010). Given the average bypass time in our trial was 143 minutes, 
and the RIPC intervention was completed anywhere between five and 80 minutes prior to commencing bypass, it is possible that many of our patients underwent reperfusion outside of the first window of protection afforded by the preconditioning. If this contributed to the lack of clinical effect of RIPC in our study, it would reinforce the outcome that preconditioning does not benefit highrisk cardiac surgery patients due to the prolonged surgical times encountered in this population.

The cardioprotective effects of RIPC stem from the ability of this technique to protect against damage resulting from ischaemia. The most common endpoint used in these trials, troponin, is released during cardiac surgery in response to all forms of myocardial damage, including mechanical injury (Barry et al., 2008). In straightforward CABG procedures the majority of the myocardial injury is induced by ischaemic episodes; however, valve surgeries require dissection of the heart muscle itself. Valve repair and replacement procedures are therefore associated with much higher levels of troponin release, although only a small portion of this is attributable to the ischaemic injury that RIPC may be capable of blunting. We proposed that patients undergoing high-risk surgeries may have more to gain from RIPC and while this may be the case, using a surrogate marker of clinical outcome such as troponin levels likely does not provide the sensitivity required to detect such a benefit in the case of the more complex procedures. Larger scale future studies that measure the impact of RIPC on direct clinical outcome measures, such as major adverse cardiovascular and cerebrovascular events or mortality, will be necessary for more conclusive results.

Despite the extensive application of RIPC in clinical trials, the optimal preconditioning stimulus has not yet been defined. The majority of trials have used three five-minute cycles of upper limb ischaemia, but increasing the length of the ischaemic episodes, the number of cycles, or the size of the preconditioned tissue by using the lower limb could potentially improve the RIPC stimulus. Indeed, Wu et al. (2011) found that although standard RIPC did not significantly reduce troponin release in adult MVR surgery patients, combining the stimulus with two ten-minute cycles of upper leg ischaemia was protective, suggesting the 
preconditioning stimulus may need to meet a certain threshold. Prior to completing our high-risk cardiac surgery trial, we designed a second study in 96 straightforward CABG patients to compare the efficacy of two, three, or four cycles of upper limb ischaemia as part of the RIPC regimen. Regrettably, on completion of the analysis of the present study we determined that we would not have the statistical power required to draw any conclusive results from the doseresponse study and therefore did not proceed with our plans. This does leave a notable gap in the literature and, again, larger scale studies will be required to investigate and define the optimal RIPC stimulus in humans.

\subsection{Conclusions and future directions}

This study provides evidence that RIPC does not reduce myocardial injury or alter peri-operative cytokine concentrations in patients treated with the volatile anaesthetic, isoflurane, irrespective of whether the surgeries performed are simple or complex. This suggests that the cytokines studied are not likely the missing humoral mediator involved in transmitting the protective RIPC signal; however, confounding variables in our high-risk cardiac surgery population and the lack of a clinical effect from RIPC limit our ability to interpret these results.

The priority for future research should be to identify and characterise potential factors that may alter the ability to induce RIPC-mediated cardioprotection in order to facilitate the design and interpretation of further clinical trial work. The challenges of working with highly heterogenic cardiac surgery patients, seen in this study and previous work, necessitate the movement of RIPC research into a more controlled setting. 


\section{Chapter 3.}

\section{Development and testing of}

\section{laboratory techniques for}

\section{studying human peripheral}

\section{leukocyte phenotype and function}




\subsection{Introduction}

Following on from the results of the study in high-risk cardiac surgery patients, it was clear that a greater basic understanding of the mechanistic processes involved in RIPC would be necessary to advance the clinical side of the research. Further, a number of limitations with the previous trial, including the choice of endpoints and the complex characteristics of the high-risk cardiac surgery patient population, made it challenging to interpret the trial results. Our primary goal for the next study was therefore to construct a rigorous and focused investigation that would provide clear and interpretable answers to our research questions.

A common theme in the RIPC literature concerns the heterogeneous nature of the patient populations studied to date, which creates challenges in the data analysis and negatively impacts the statistical power. For this reason, we decided to investigate the effect of RIPC in healthy human volunteers using a strict set of inclusion and exclusion criteria, which would eliminate many of the confounding factors seen in surgical patients. In addition, the study was set up in a pair-wise fashion to allow participants to act as their own control, thereby increasing the statistical power in the face of a highly variable inter-individual dataset.

The primary focus of the study was to determine how RIPC influences the immune response; therefore, we elected to look at systemic cytokine levels and changes in the peripheral blood leukocytes. The latter involved measuring changes in the size, phenotype, and intracellular cytokine production within the different cell subsets as well as examining the proliferative and functional changes in cultured T-cells.

In the absence of an ischaemic injury it was anticipated that the RIPC stimulus might induce only minor, although potentially significant, changes to the immune response. Beyond the usual study design considerations it was therefore of particular importance for this trial that the laboratory procedures were robust, consistent, and reproducible to ensure that subtle changes in the endpoints could be accurately detected and that the data was comparable between both 
treatments and participants. Further, it was necessary that the endpoints and laboratory techniques were carefully selected to make the trial feasible for one individual to carry out.

Although there are existing protocols available in the literature describing intracellular cytokine staining (ICS), these tend to focus on the analysis of specific cell populations. In addition, it is well established that even slight variations in reagents and equipment between laboratories can significantly influence assay performance (Nomura et al., 2008; Welters et al., 2012). To examine cytokine production in T cells, NK cells, monocytes, and neutrophils, the latter of which are poorly accounted for in currently available protocols, we therefore needed to test a system that catered to the requirements of each of these populations in a mixed whole blood sample. Likewise, while broad descriptions of the conditions for culturing peripheral blood mononuclear cells (PBMC) with polyclonal stimulants have been published previously, these procedures required further testing to ensure they were suitable for the purpose of our study.

In contrast to intracellular cytokine staining, methods for stimulating antigenspecific T cell responses in PBMC from healthy volunteers are less well described and standardised. Previous studies have utilised the tetanus toxin or tetanus toxoid to stimulate cells in vitro; however, the former presents safety challenges and the latter is not only difficult to source, but the protocols in the literature differ enormously, even down to the units of measure (Gabrilovich et al., 1997; Landay et al., 1983; Rimaniol et al., 2004). Part of our set-up therefore required developing a novel method for stimulating isolated PBMC using the safe and readily available tetanus booster vaccine.

In summary, a considerable period of the study timeline was devoted to testing each aspect of the laboratory techniques required for the study endpoints in order to collect the highest quality data possible. The following chapter presents an overview of the work undertaken and establishes the foundation for our confidence in the data collected and presented in Chapters 4 and 5. 


\subsection{Aims}

- To test the conditions for immunophenotyping human peripheral blood leukocytes.

- To develop a protocol for detecting intracellular IL-6, IL-8, and IL-10 levels in a range of different leukocytes from a whole blood sample.

- To test the conditions for stimulating and measuring the proliferative response of $\mathrm{T}$ cells to polyclonal and antigen-specific agents.

- To create laboratory and data analysis workflows capable of being consistently and reproducibly performed by one individual throughout the study. 


\subsection{Methods}

\subsubsection{Participants}

Healthy male volunteers were invited to give blood samples for testing of the laboratory techniques if they were 18 years or older, non-smokers, and not taking any regular medications or experiencing any acute illness for one week prior to blood collection. This work received ethics approval from the Central Regional Ethics Committee (CEN/11/06/034), and written informed consent was obtained from all volunteers by the principal investigator. PhD students trained in phlebotomy collected the blood samples.

\subsubsection{Flow cytometry}

Blood samples for flow cytometry were collected in heparinised vacutainers. All antibodies were purchased from BD Bioscience or Biolegend (San Diego, CA, USA) and were first titrated in order to maximise the resolution between cell populations and minimise the background level of fluorescence. Refer to appendix $\mathrm{C}$ for the composition of buffers, and appendix $\mathrm{E}$ for a complete list of antibodies and dilutions used.

\subsubsection{Immunophenotyping}

Where a stimulated sample was indicated, an aliquot of blood was either incubated with fMLP $\left(0.001-10 \mu \mathrm{M}\right.$; Sigma Aldrich) for 10 minutes at $37^{\circ} \mathrm{C}$ or PMA (50 or $100 \mathrm{ng} / \mathrm{mL}$; Sigma Aldrich) and ionomycin (500 ng/mL; Sigma Aldrich) for up to 60 minutes at either RT or $37^{\circ} \mathrm{C}$. Stimulated samples were cooled on ice for 3 minutes prior to staining, and unstimulated blood was kept at RT. Primary antibody mixes were diluted in FACS buffer to a total volume of 25 $\mu \mathrm{L}$, and were then mixed with an equal volume of whole blood. Following a 20 minute incubation at RT, the samples were mixed with 1X FACS lyse (BD Biosciences) to eliminate the red blood cells (RBC), and incubated for a further 10 minutes at RT. The samples were then centrifuged at $300 \mathrm{x} g$ for 5 minutes, the supernatant aspirated, and either a second wash was performed or the cells were resuspended in FACS buffer. Samples were collected on a Guava easyCyte 
8HT using InCyte software (Merck Millipore, Darmstadt, Germany), and the data analysed using FlowJo 7.6.1 software (Tree Star, Ashland, OR, USA).

\subsubsection{Intracellular cytokine staining}

The various steps involved in the ICS procedure were tested individually, in accordance with the following general protocol. Heparinised whole blood was diluted 5:1 with complete $\mathrm{T}$ cell media (CTCM) with or without PMA and ionomycin (PMA/I) ranging from $10-50 \mathrm{ng} / \mathrm{mL}$ or $500-1000 \mathrm{ng} / \mathrm{mL}$, respectively, and incubated for 4 or 27 hours at $37{ }^{\circ} \mathrm{C}$ with $5 \% \mathrm{CO}_{2}$. One of two different protein transport inhibitors, brefeldin A (5 ug/mL; Biolegend) or the monensin-based GolgiStop (in dilutions ranging from 1:266 to 1:1333; BD Biosciences) was added for the final 4 hours of culture. RBC were lysed using a 15 minute incubation with Pharm Lyse (BD Biosciences) at RT followed by centrifugation at $400 \mathrm{xg}$ for 5 minutes, and the samples washed twice in PBS. A viability dye, eFluor 780 (eBioscience, San Diego, CA, USA) was titrated using a serial 2-fold dilution ranging from 1:1000 - 1:32000, staining for 30 minutes on ice in a 96-well round-bottom plate and then washing once with PBS. Samples were then incubated with anti-CD16 antibody or FACS buffer for 20 minutes, Fc receptor blocked with $2 \mathrm{mg} / \mathrm{mL}$ intragam P (CSL, Victoria, Australia) for 10 minutes, then stained with the remaining extracellular marker antibodies for 20 minutes, all at RT, protected from light. Cells were washed once in FACS buffer, and fixed in $4 \%$ paraformaldehyde for 15 minutes. Samples were then washed twice with FACS buffer, resuspended in 0.1 or $0.2 \%$ saponin buffer for 10 minutes, then centrifuged at $400 \mathrm{x}$ g for 5 minutes and the supernatant aspirated. The samples were incubated with intracellular staining mixes in a total volume of $50 \mu \mathrm{L}$ for 20 minutes at RT, washed once with saponin buffer and once with FACS buffer. Samples were resuspended in FACS buffer and filtered through nylon mesh before acquisition on a FACSCanto $^{\text {TM }}$ II with Diva software (BD Biosciences). The data was analysed with FlowJo 7.6.1 software (Tree Star).

\subsubsection{Peripheral blood mononuclear cell cultures}

PBMC were isolated from heparinised blood samples following the manufacturer's instructions for Histopaque-1077 (Sigma Aldrich). The viable cell 
concentration was determined by mixing a sample of cells $1: 1$ with $0.4 \%$ Trypan blue (Sigma Aldrich) and counting on an improved Neubauer haemocytometer with a compound microscope (CX41; Olympus, PA, USA). Isolated PBMC were cultured in duplicate in a final volume of $200 \mu \mathrm{L}$ in 96 -well flat-bottom plates, with or without stimulants. A range of concentrations of cells $\left(0.8-2 \times 10^{6}\right.$ cells/mL), PMA (5 and $10 \mathrm{ng} / \mathrm{mL}$ ), and ionomycin (200 and $500 \mathrm{ng} / \mathrm{mL}$ ), were compared to find the optimal stimulation conditions. Dilutions of the ADT Booster vaccine (containing the tetanus toxoid, ranging from 1:5 - 1:160; CSL) and anti-CD3/CD28 Expander Beads (Life Technologies, Grand Island, NY, USA) to cell ratios $(1: 1,0.5: 1$, or $0.2: 1)$ were also tested. The anti-CD3/CD28 beads were prepared by washing in Dulbecco's phosphate buffered saline (dPBS), pelleting for 1 minute on a magnet, aspirating the supernatant, and resuspending in CTCM. The PMA/I, and anti-CD3/CD28-stimulated samples were incubated for 24 hours at $37{ }^{\circ} \mathrm{C}, 5 \% \mathrm{CO}_{2}$, then centrifuged at $400 \times \mathrm{g}$ for 5 minutes. The supernatant was harvested and stored at $-20^{\circ} \mathrm{C}$, and the pelleted cells at $-80{ }^{\circ} \mathrm{C}$, for further analyses. The tetanus-stimulated samples were cultured for 6 days and in some experiments (as specified in the results) the tetanus vaccine was added to the unstimulated samples immediately prior to the centrifugation at the end of the incubation period. The supernatant and cells were stored as described above.

\subsubsection{CyQuant proliferation assay}

The CyQuant reagent (Life Technologies) was prepared at $1 \mathrm{X}$ and $2 \mathrm{X}$ concentration in cell lysis buffer and nuclease free distilled water, as per the manufacturer's instructions. The cell samples were thawed and $200 \mu \mathrm{L}$ of reagent was added per well, and left to equilibrate in the dark for 3 minutes. A control sample was used to run an optimisation assay on an EnSpire 2300 multilabel plate reader (PerkinElmer) and, based on these results, the fluorescence was measured at excitation of $480 \mathrm{~nm}$ and emission of $520 \mathrm{~nm}$. The background absorbance level (taken from the control wells without cells) was subtracted from the samples and the stimulation index calculated by expressing the mean fluorescence intensity (MFI) of each replicate as a ratio to that of the corresponding control. 


\subsubsection{IL-6 ELISA}

IL-6 levels were measured using a sandwich ELISA, as described previously in section 2.3.7.2. 


\subsection{Results}

The work presented in this chapter was largely designed to test the effect of minor alterations to existing protocols available in the literature. For this reason, the results were obtained from individual experiments that were not repeated or subjected to statistical analyses; therefore the conclusions drawn from this work should be interpreted with caution. Further repeats of the experiments would be necessary in order to use these results to generate fully optimised protocols.

\subsubsection{Flow cytometry of whole blood samples}

Two flow cytometry protocols were examined in parallel; an immunophenotyping procedure for determining changes in the size and activation of the peripheral leukocyte subsets, and an ICS protocol for investigating IL-6, IL-8, and IL-10 production in whole blood samples. Overviews of the steps undertaken for each of these methods are presented in Figures 3.1 and 3.2. The procedures that overlapped between the two protocols are presented first, followed by the specific experiments performed for the testing of the protocols for immunophenotyping and, lastly, ICS. 


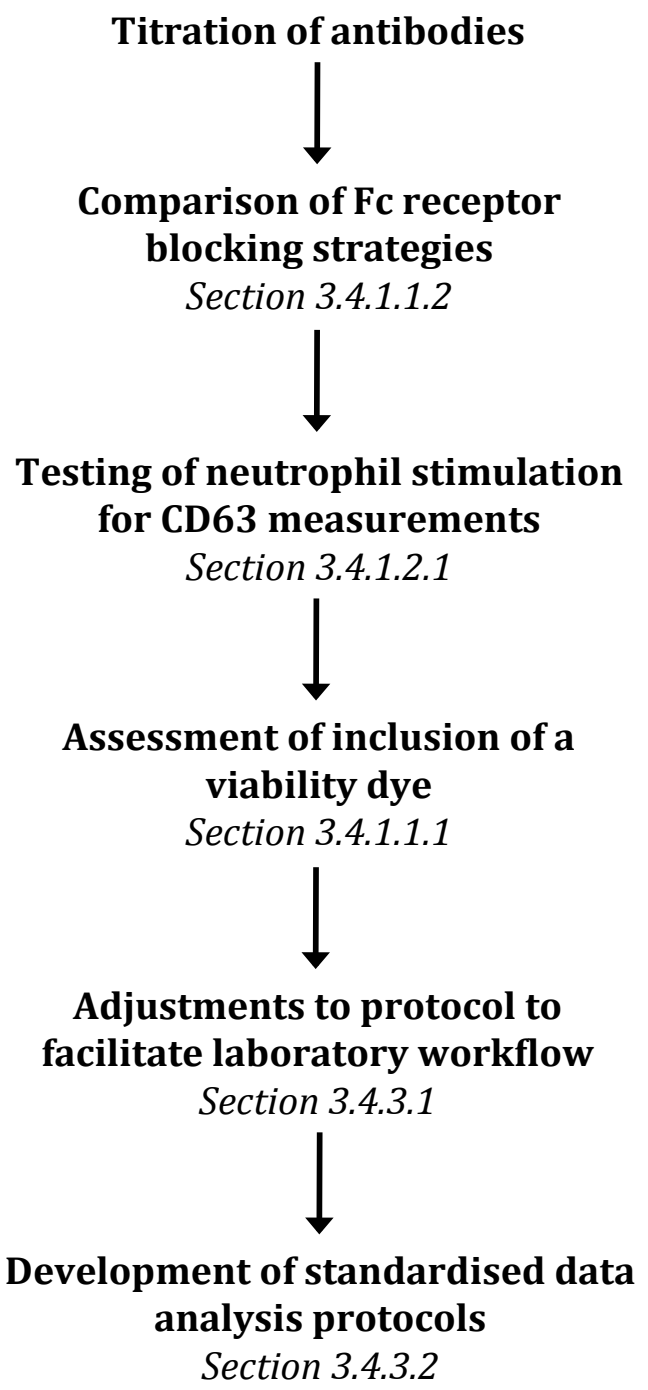

Figure 3.1 Summary of the steps undertaken to test the immunophenotyping protocol

Flowchart demonstrating the order of the steps performed when developing the final immunophenotyping protocol, and the corresponding thesis sections that detail the work. 


\section{Antibody titration}

using existing protocol from the literature

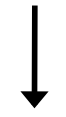

Viability dye test \& titration

Section 3.4.1.1.1<smiles>C[13CH2]</smiles>

Comparison of stimulant concentrations

for inducing cytokine production

Section 3.4.1.3.1

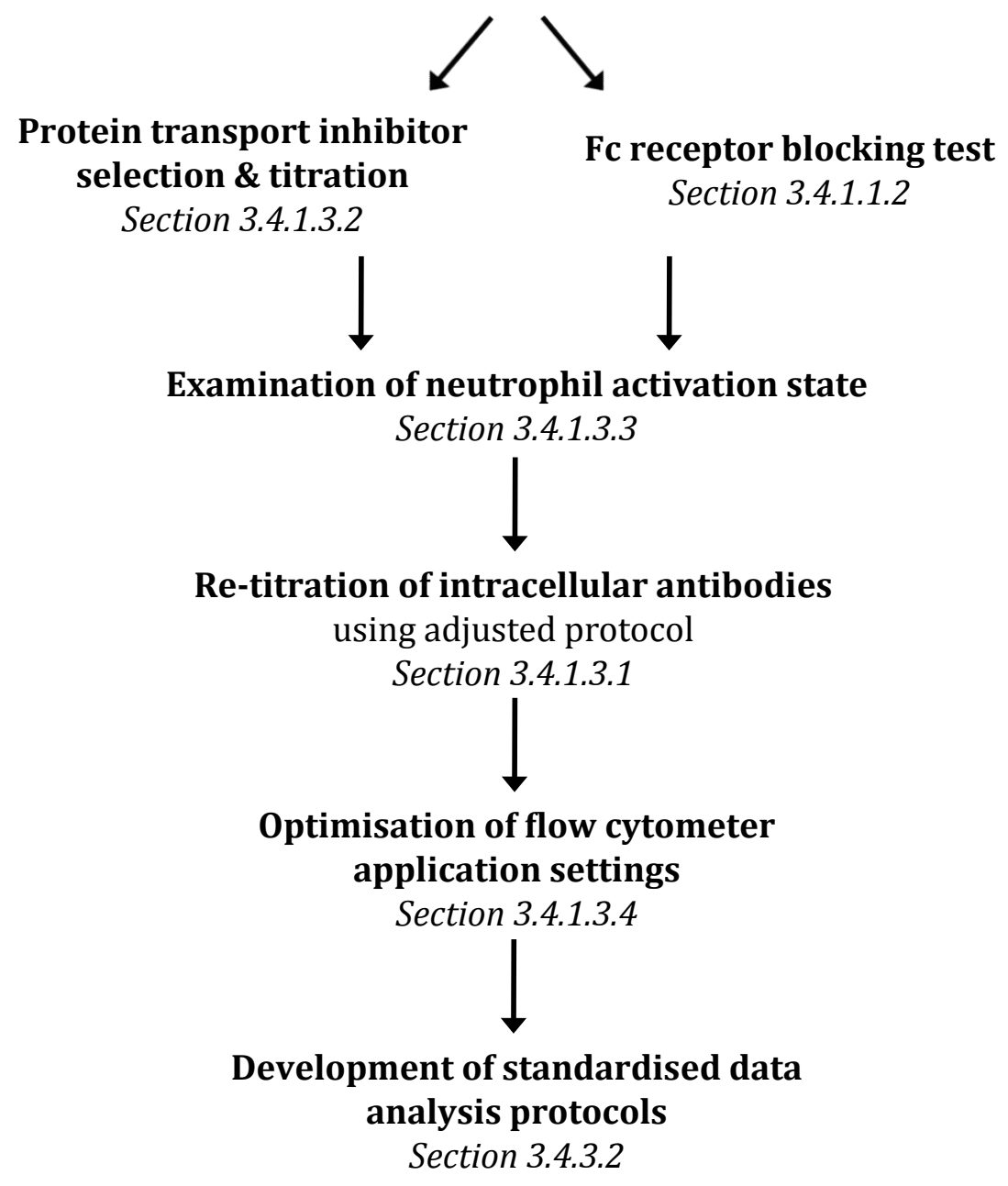

Figure 3.2 Summary of work performed to develop the ICS protocol

Flowchart summarising the order of the work performed while testing a protocol for measuring intracellular cytokine production, and the corresponding thesis sections that detail the results. 


\subsubsection{General flow cytometry techniques}

\subsection{Assessing viability}

Non-specific binding of antibodies to apoptotic and dead cells can create falsepositive data, therefore it was critical to develop protocols that allowed nonviable cells to be excluded from the final flow cytometry analyses (Givan, 2000; O'Brien and Bolton, 1995). We addressed this issue by incorporating a viability marker, eFluor 780, into our staining panels. The eFluor 780 dye was titrated by combining fresh blood with an equal volume that had undergone two cycles of freezing at $-80^{\circ} \mathrm{C}$ for 15 minutes, in order to create a sample with clear live and dead cell populations. At the lowest dilution, corresponding to the manufacturer's recommended concentration for use, the background fluorescence was considerably increased in all cell populations, and a subset of dead cells was only visible as a tail of fluorescence on the major peak (Fig 3.3A). At the higher dilutions, a second peak of dead cells became visible at the end of the axis (Fig 3.3B - F). When gated and viewed on a SSC vs. FSC dot plot, it was clear that these cells were highly fluorescent neutrophils, and the lower peak were lymphocytes (data not shown). The best resolution for detecting both populations of non-viable cells was seen between the 1:8000 and 1:16000 dilutions (Fig 3.3D and E), therefore a dilution of 1:10000 was selected for use in the final ICS protocol.

Given the 23 hour difference between the two time points selected for the ICS protocol, the viability and surface staining had to be performed separately for the two sets of cultures. The viability dye was prepared for the first time point, then stored at $4{ }^{\circ} \mathrm{C}$ overnight and used again for the second time point. To check that this overnight storage did not affect the ability to detect and gate out nonviable cells, a sample of dye prepared one day earlier was compared to freshly made stock. The difference between the two dye stocks was found to be negligible (data not shown).

For the immunophenotyping procedure, whole blood samples were used immediately after collection. Not surprisingly, the viability dye stained only a 
very small population of non-viable cells (less than $0.5 \%$ ) in fresh blood; therefore it was not included in the final immunophenotyping protocol (Fig $3.3 G)$.
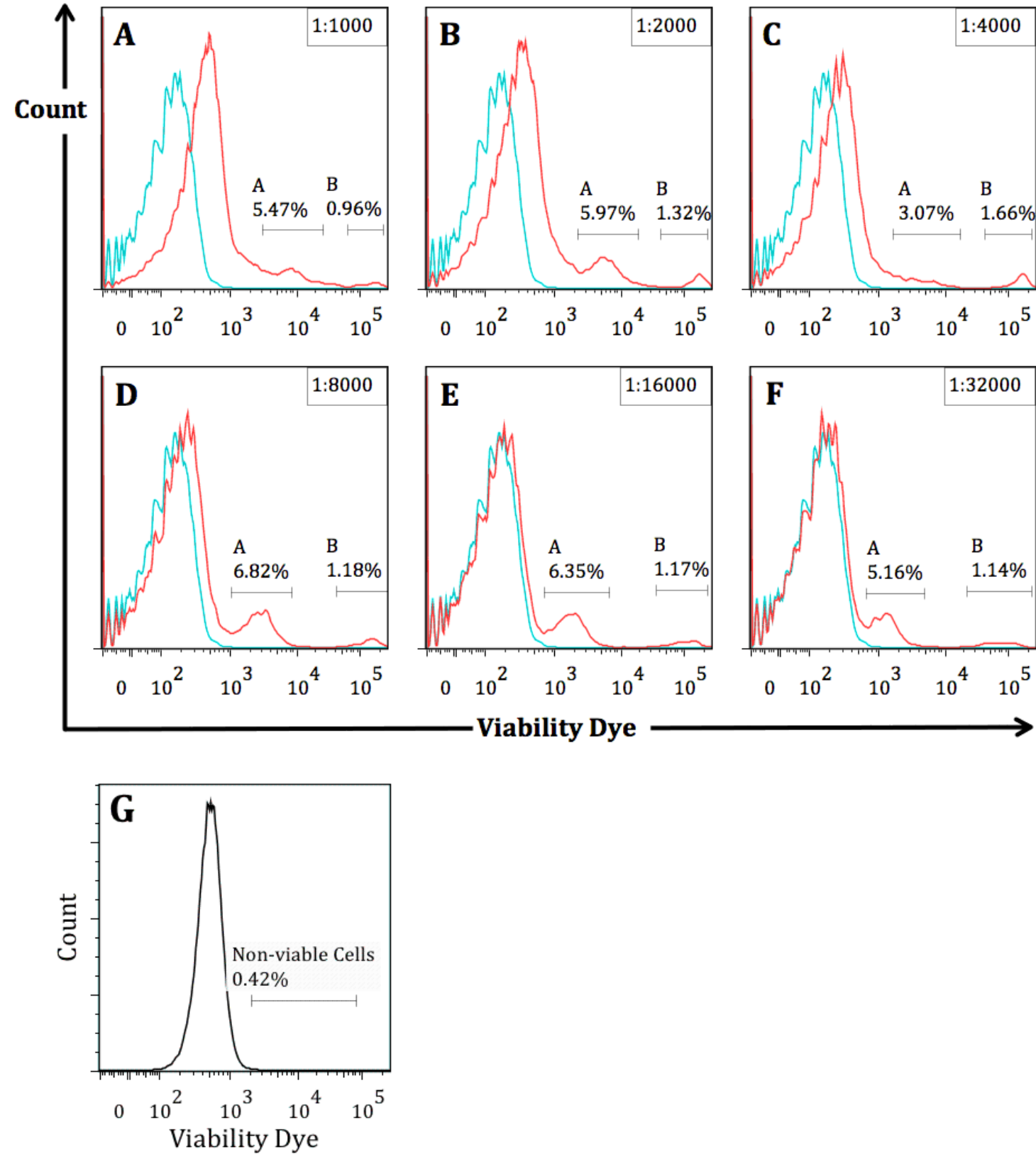

Figure 3.3 Assessment of cell viability in whole blood samples

Freeze-killed and/or fresh blood were combined in equal volumes and the RBC lysed with Pharm Lyse solution. Samples were incubated with the eFluor 780 viability dye at various concentrations for 30 minutes on ice and then acquired on a flow cytometer. (A - F) Titration of the viability dye (red) compared to unstained samples (blue); $(G)$ fresh whole blood stained with viability dye at the concentration deemed optimal from the titration (1:10000). Shown are the data from an individual experiment. 


\subsection{Fc receptor blocking}

Non-specific binding of monoclonal antibodies to cells that express a high level of fragment crystallisable $(\mathrm{Fc})$ receptors on their surface can be mitigated by incubating the cells with serum or an alternative blocking agent prior to staining for flow cytometry; however, care must be taken when CD16 (a low affinity IgG receptor III) is a parameter of interest, as blocking will also prevent the binding of the CD16-specific antibody. To determine whether a blocking step would be necessary while performing our immunophenotyping procedure using fresh whole blood, we tested and compared three common methods; washing the sample with a $0.5 \%$ bovine serum albumin (BSA) buffer prior to staining, incubating the sample with intragam P (human $\operatorname{IgG}$ ), or no blocking.

In the lymphocyte population, where Fc receptor expression is low, no difference in staining and background fluorescence was detected between the three methods (Figure 3.4A). Monocytes have a much higher level of Fc receptor expression and in our neutrophil and monocyte populations it appeared the background fluorescence was slightly lower in the sample treated with intragam P (Figure 3.4B). This subtle difference did not have any impact on the data analysis and results (data not shown).

Including intragam $\mathrm{P}$ in our immunophenotyping protocol would have required pre-staining with CD16, blocking, and then completing the extracellular staining, as three separate steps. A key focus of our laboratory work was to mimic the in vivo environment as best we could, and keep the artificial conditions and stimulations to a minimum. It was felt that this extended method could introduce more neutrophil activation and variability then the act of blocking would prevent, therefore it was decided not to use a blocking agent in the immunophenotyping protocol for the purposes of our study.

For the ICS protocol involving isolated, cultured cells and a larger number of antibodies, it was felt that blocking was necessary to obtain the highest quality data. For these samples, we did pre-stain with CD16 before adding intragam P, and then the remaining surface staining antibodies. 
Lymphocytes

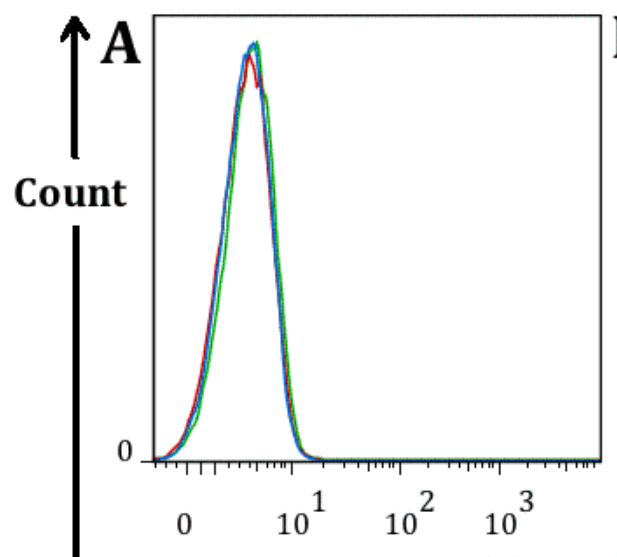

Monocytes \& Neutrophils

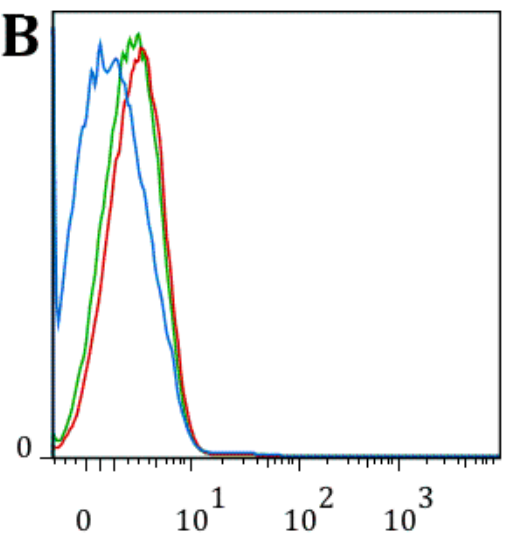

IgG1 PerCP/Cy5.5

Figure 3.4 Comparison of Fc receptor-blocking strategies for flow cytometry

Whole blood samples were washed with $0.5 \%$ BSA buffer (green), pre-incubated with $2 \mathrm{mg} / \mathrm{mL}$ intragam P for 10 minutes (blue), or left unblocked (red) prior to staining with a PerCP/Cy5.5 conjugated isotype control antibody and acquisition on a flow cytometer. The resulting fluorescence in the red channel is shown for (A) lymphocytes and (B) monocytes and neutrophils. Data shown are from a single experiment. 


\subsubsection{Testing the immunophenotyping protocol}

\subsection{Stimulation of neutrophils for measurement of activation}

To stimulate expression of an activation marker of interest, CD63, which is detectable on granulocytes and monocytes, we tested a variety of concentrations of fMLP using a 10 -minute incubation of whole blood at $37^{\circ} \mathrm{C}$. These variables were selected based on comparable studies in the literature (Cham et al., 1994; Shimizu et al., 2010; Simard et al., 2010; van Eeden et al., 1999; Zhang et al., 1995). Concentrations of fMLP from $0.001-10 \mu \mathrm{M}$ all induced substantial increases in an alternative marker of neutrophil activation, CD11b; however, there was no change in CD63 levels, compared to the unstimulated control (Figure 3.5F). The inability of fMLP to induce CD63 expression under these circumstances rendered it inappropriate for use in the study.

Instead, we tested whether stimulating neutrophils with the pharmacological stimulants, PMA/I, provided a more reliable surface expression of CD63. To this end we compared incubations at RT and $37^{\circ} \mathrm{C}$, at four time points ranging from 15 minutes to 1 hour. PMA/I successfully stimulated measurable levels of CD63 in both neutrophils and monocytes. Neutrophil expression levels were highest at the shortest time point, 15 minutes, whereas monocyte levels continued to increase up to 1 hour (Figure $3.6 \mathrm{~K}$ and L). Temperature appeared to have a negligible effect on CD63 expression.

The activation of the neutrophils and monocytes was confirmed by the significant increase in CD11b expression, which was cumulative across the time points for both populations of cells (Figure 3.6M and N). Additionally, elevated CD11b levels were seen in the samples incubated at $37{ }^{\circ} \mathrm{C}$; therefore, it was determined that stimulating the neutrophils with PMA/I for 15 minutes at $37{ }^{\circ} \mathrm{C}$ provided the best conditions for measuring CD63 and CD11b expression in the same sample. 

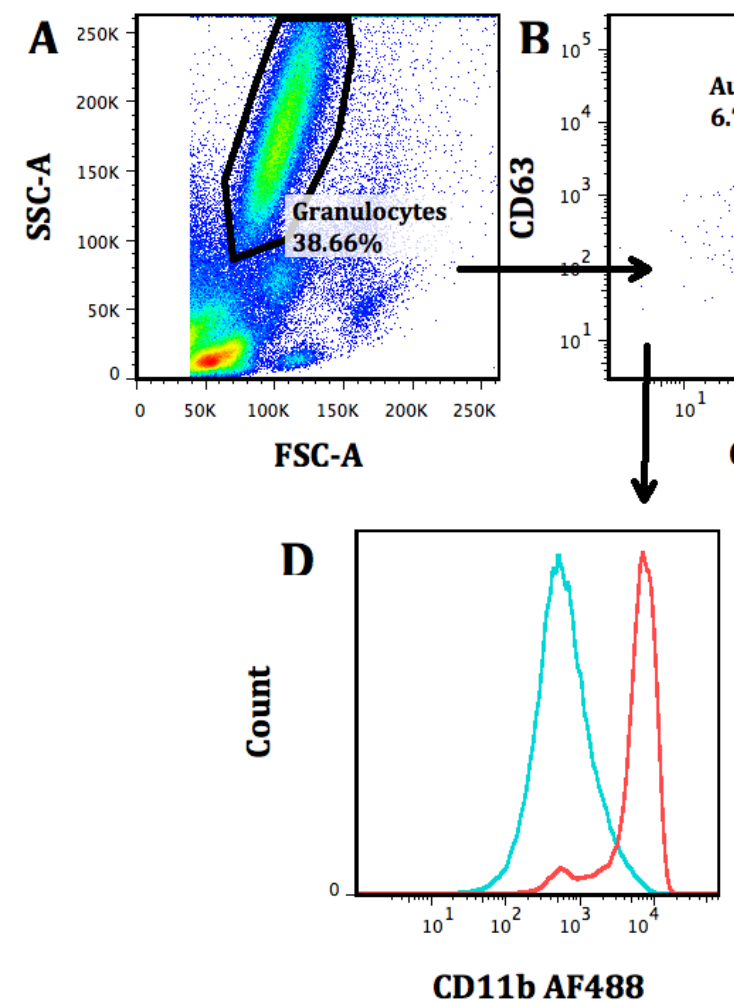

CD11b AF488
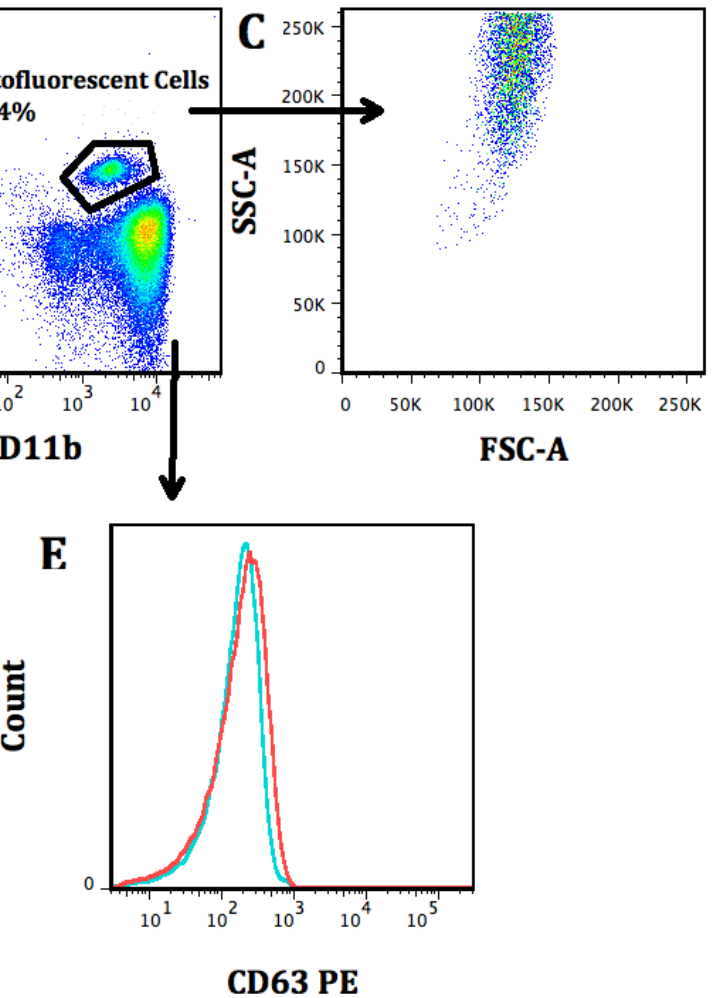

F $\quad$ MLP Stimulation of Neutrophils

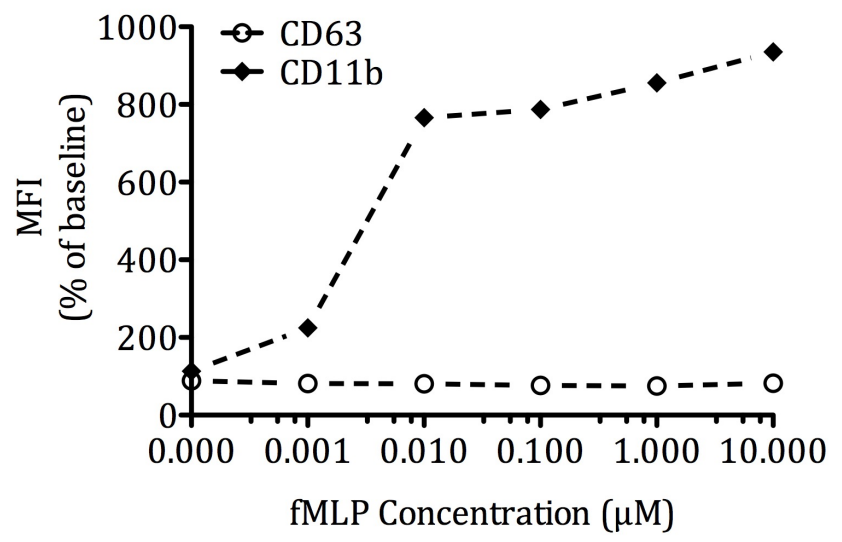

Figure 3.5 fMLP stimulation of neutrophils for activation marker measurement

Whole blood was stimulated with $0.001-10 \mu \mathrm{M}$ fMLP at $37^{\circ} \mathrm{C}$ for 10 minutes before staining for activation markers, RBC lysis, and acquisition on a flow cytometer. (A) Stimulated granulocytes were gated using SSC vs. FSC; (B) MFI was calculated for the PE (CD63) and AF488 (CD11b) channels in the granulocyte population, including a small population of autofluorescent cells that were detected in all samples; (C) SSC vs. FSC of autofluorescent cells; (D) CD11b and (E) CD63 staining in unstimulated (blue) and 0.1 $\mu \mathrm{M}$ fMLP-stimulated nonautofluorescent granulocytes (red); (F) MFI of CD63 and CD11b expression in the granulocytes from the whole blood samples. Shown is data from an individual experiment. 

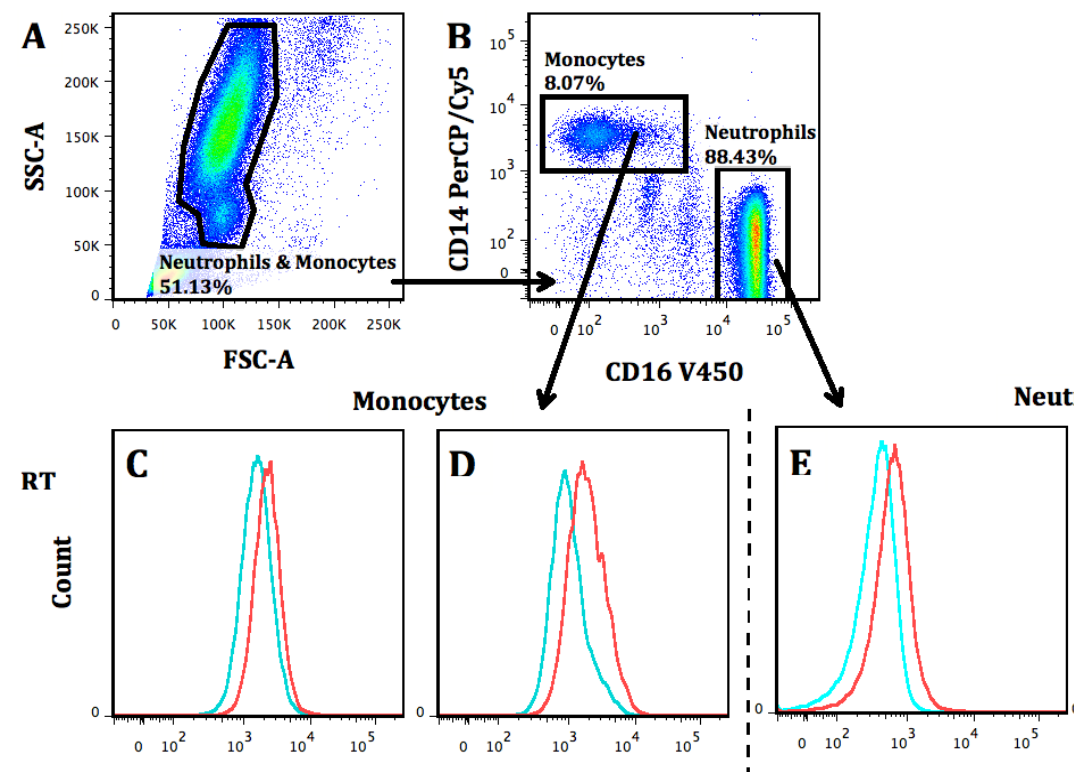

Neutrophils
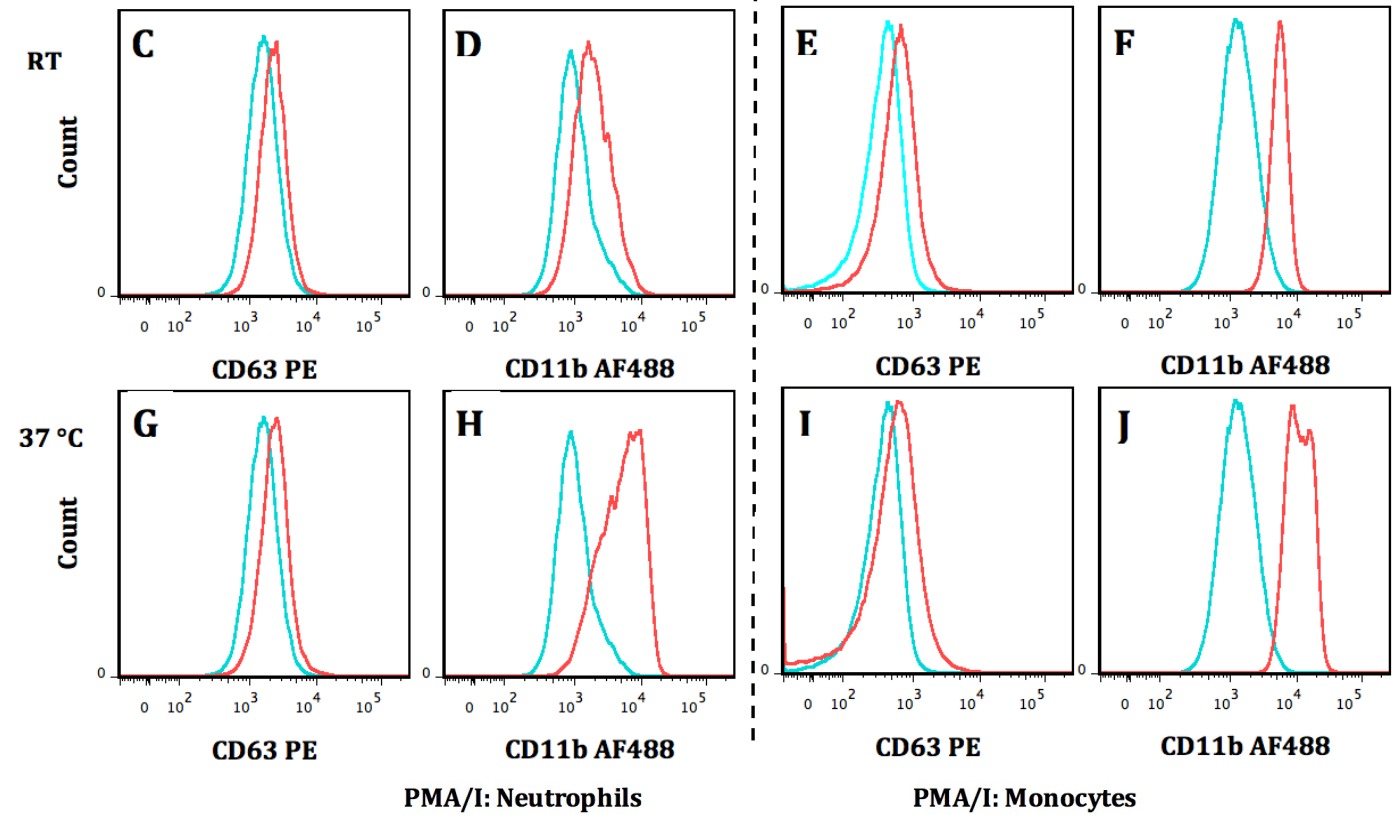

PMA/I: Monocytes
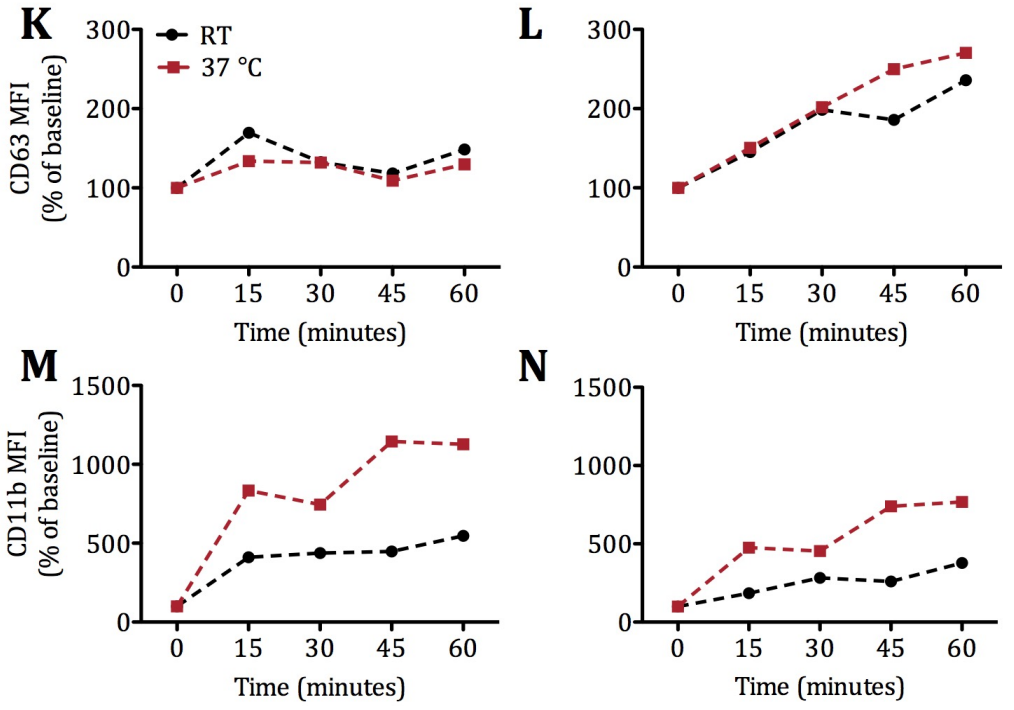

$\mathbf{N}$

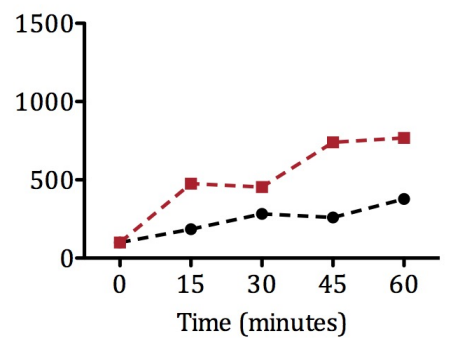

Figure 3.6 PMA/I stimulation of neutrophils activation marker expression Whole blood was stimulated with $100 \mathrm{ng} / \mathrm{mL}$ PMA and $500 \mathrm{ng} / \mathrm{mL}$ ionomycin at RT or $37^{\circ} \mathrm{C}$ before staining for activation markers, RBC lysis, and flow cytometry. (A) Stimulated cells gated using FSC vs. SSC; (B) gating of CD $14^{+}$CD16-/low monocytes and CD14-CD16 ${ }^{\text {high }}$ neutrophils; unstimulated (blue) and stimulated (15 mins; red) staining for CD63 (C, E, G, and I) and CD11b (D, F, H, and J) at RT $(\mathrm{C}-\mathrm{F})$ and $37^{\circ} \mathrm{C}(\mathrm{G}-\mathrm{J})$ in monocytes $(\mathrm{C}, \mathrm{D}, \mathrm{G}$, and $\mathrm{H})$ and neutrophils $(\mathrm{E}, \mathrm{F}, \mathrm{I}$ and J). MFIs of CD63 (K and L) and CD11b (M and N) in PMA/I stimulated neutrophils ( $\mathrm{K}$ and $\mathrm{M}$ ) and monocytes ( $\mathrm{L}$ and $\mathrm{N}$ ). Data is from an individual experiment. 


\subsubsection{Development of ICS protocol}

3.4.1.3.1 Stimulation of cytokine production and improvements in antibody resolution for intracellular staining

Two culture durations were selected for investigating the intracellular cytokine production in peripheral leukocyte populations following RIPC. The first, 4 hours, was designed to provide insight as to how RIPC immediately affects cytokine production during the first window of protection, whereas the second, 27 hours, allowed for any effect of RIPC on gene expression to take effect. The earlier time point was better suited to measuring production of IL-6 and IL-8 in the innate cells and the later time point was targeted towards the monocytes and lymphocytes, and the measurement of IL-10. The culture conditions for these two time points were separately tested to best address the different cell subsets and cytokines relevant to each.

The intracellular antibodies were initially titrated using a general staining protocol based on the existing literature. The relative staining intensities could be distinguished between the monocyte and neutrophil populations by the marker CD45, which is present at a higher density on monocytes than neutrophils (Figure 3.7D and E; Loken et al., 1990). Using the concentration of IL-8 antibody that performed best in our titration experiment, we found a marked increase in IL-8 production in the neutrophils and monocytes stimulated with PMA/I for 4 hours, relative to the unstimulated control (Figure 3.7G). The highest levels of production were detected in the cells treated with $20 \mathrm{ng} / \mathrm{mL}$ PMA and $1 \mu \mathrm{g} / \mathrm{mL}$ ionomycin, but all three conditions similarly increased IL-8 staining beyond the levels detected in the isotype control samples (Figure 3.7G). In contrast, the MFI of IL-8 was significantly below the level of the isotype control antibody in the cells cultured with media alone. Cell viability exceeded 94\% in all of the 4-hour cultures and did not substantially differ between the different concentrations of PMA/I tested (Figure 3.7I).

Over the course of adjusting our ICS protocol, changes to the flow cytometer settings and buffers resulted in a substantial improvement in the resolution of 
intracellular IL-8 staining. Doubling the concentration of saponin used in the permeability buffer from $0.1 \%$ to $0.2 \%$ to facilitate entry of the comparatively bulky PE fluorophore through the cell pores was particularly beneficial. Figure 3.8 shows a comparison of IL-8 staining in samples from the early experiment used to examine stimulation conditions, and our last experiment to test our final ICS protocol. Both samples were cultured with the same concentrations of stimulants and stained with the same antibody dilution. The staining improved from $105 \%$ of the level detected in the isotype control to $419 \%$ following adjustments to the protocol (Figure 3.8A and B).

When we used the IL-6 antibody concentration deemed optimal during our preliminary titration to compare the efficacy of different stimulant concentrations on IL-6 production in neutrophils and monocytes, there was no clear difference between the conditions. The level of IL-6 staining detected in the unstimulated sample was markedly higher than the isotype control, suggesting a level of constitutive IL-6 production in neutrophils and monocytes, which appeared not to be augmented by PMA/I treatment (Figure 3.7H). However, the IL-6 antibody was subsequently re-titrated using our amended ICS protocol, and the use of a higher concentration of antibody was found to detect a clear increase in IL-6 levels in monocytes and neutrophils stimulated with PMA/I, compared to the baseline levels seen in the unstimulated samples (Figure 3.9D). The IL-6 antibody dilution selected for use in the study was 1:25.

Given the minimal difference between the three combinations of PMA/I concentrations that we tested on intracellular IL- 6 and IL-8 production and cell viability, we elected to stimulate the blood with $50 \mathrm{ng} / \mathrm{mL} \mathrm{PMA}$ and $500 \mathrm{ng} / \mathrm{mL}$ ionomycin. These concentrations are the most commonly used in the literature and resulted in a good level of IL-8 production and cell viability in our experiment.

After 26 hours of culture IL-10 production was most clearly measured in leukocytes that were stimulated with 20 or $50 \mathrm{ng} / \mathrm{mL}$ PMA and $500 \mathrm{ng} / \mathrm{mL}$ ionomycin; however, these conditions resulted in a marked decrease in viability 
of the $\mathrm{CD} 45^{+}$cells (Figure 3.10G and H). A closer look at the leukocyte population using FSC vs. SSC revealed a notable reduction in the proportion of granulocytes detected in the samples treated with the lowest dose of stimulants, $10 \mathrm{ng} / \mathrm{mL}$ PMA and $500 \mathrm{ng} / \mathrm{mL}$ ionomycin, and this was further enhanced when the PMA concentration was increased to $50 \mathrm{ng} / \mathrm{mL}$, with no distinguishable neutrophil population (Figure 3.10D - F).

Although the higher concentrations of stimulants increased the level of IL-10 staining, relative to the isotype control, the associated death in particular leukocyte subsets was not acceptable for addressing our research questions. For this reason, we decided to stimulate the 27-hour cultures with $10 \mathrm{ng} / \mathrm{mL}$ PMA and $500 \mathrm{ng} / \mathrm{mL}$ ionomycin during the study. These conditions stimulated IL-10 production while maintaining an overall leukocyte viability of approximately $92 \%$. 


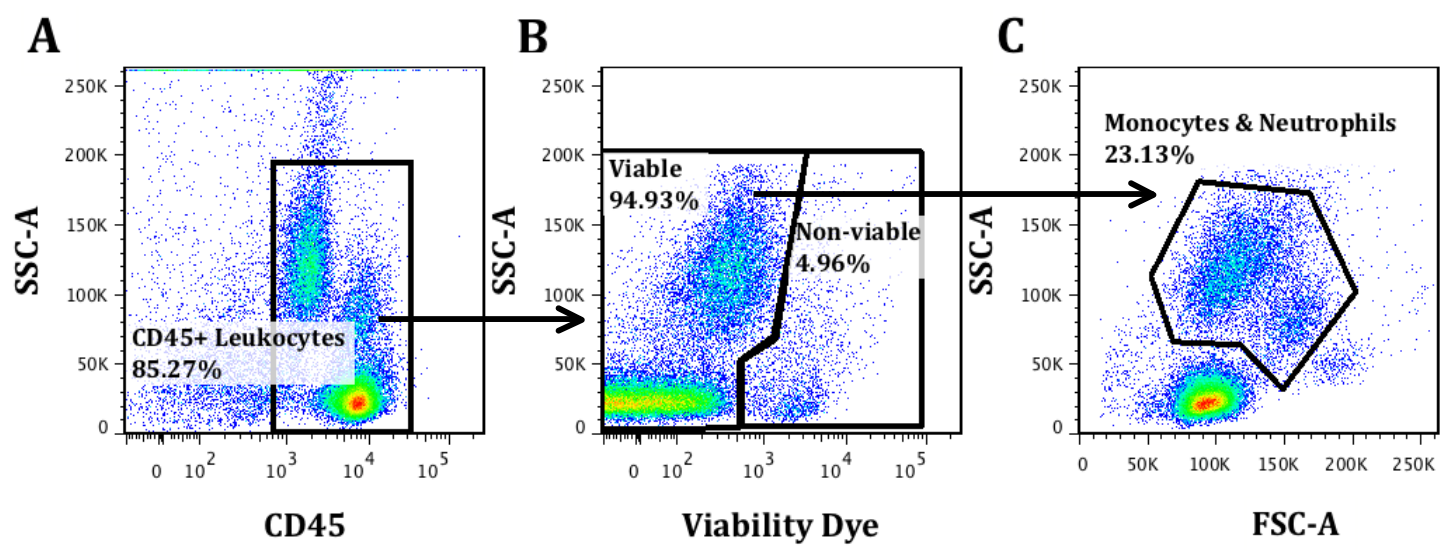

D

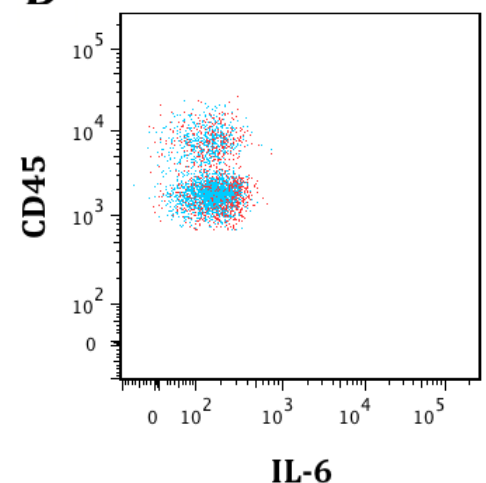

$\mathbf{E}$

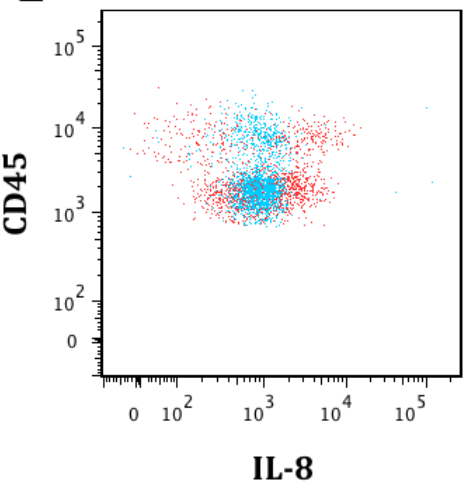

$\mathbf{F}$

\begin{tabular}{|c||c|c|}
\hline \multicolumn{1}{||}{ Sample } & APC MFI \\
\hline & IgG1 APC & 139.38 \\
\hline & IL-6 APC & 174.31 \\
\hline
\end{tabular}

\begin{tabular}{|c|c|c|}
\hline & Sample & PE MFI \\
\hline & IgG1 PE & 837.58 \\
\hline \hline & IL-8 PE & 935.99 \\
\hline
\end{tabular}
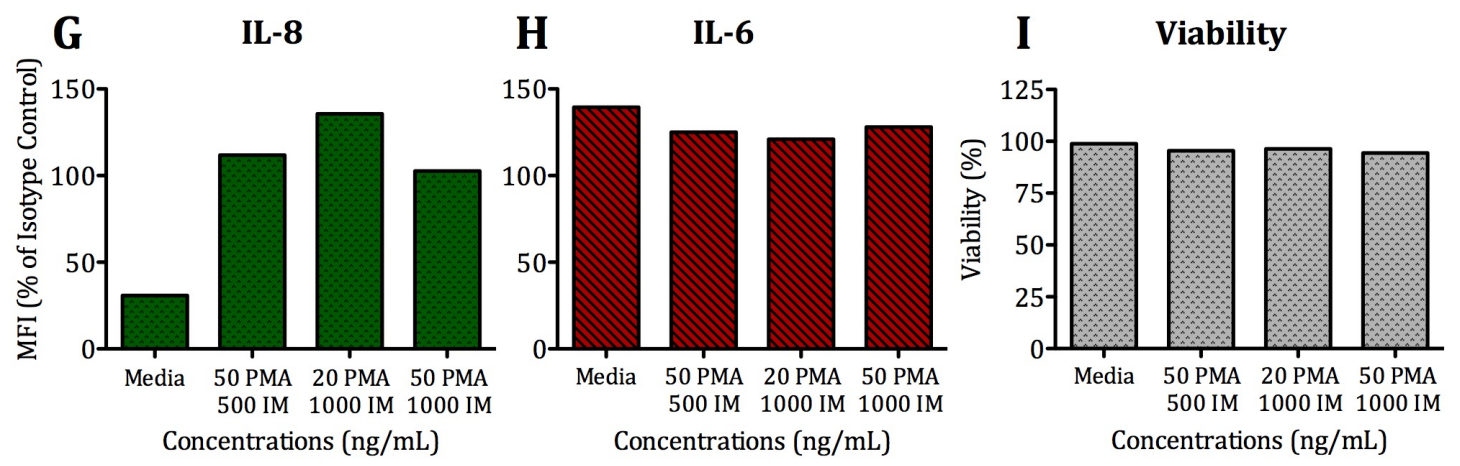

Figure 3.7 Stimulation of intracellular cytokine production in 4-hour cultures

Whole blood was incubated with various concentrations of PMA/I for 4 hours in the presence of GolgiStop (1:1000), then stained for viability and intracellular cytokines and analysed by flow cytometry. (A) CD45+ leukocytes were gated; (B) non-viable $\mathrm{CD} 45^{+}$leukocytes were excluded; (C) neutrophils and monocytes from the viable cells were gated using SSC vs. FSC; IL-6-APC (D) and IL-8-PE (E) stained cells (red), compared to isotype control stained samples (blue) and the MFIs (F) from the combined monocyte and neutrophil populations stimulated with $50 \mathrm{ng} / \mathrm{mL}$ PMA and $500 \mathrm{ng} / \mathrm{mL}$ ionomycin. Production of IL-8 (G), IL-6 (H), and the viability (I) of neutrophils and monocytes stimulated with PMA/I. Cytokine results are presented as MFI expressed as a percentage of the corresponding isotype control stained sample. Shown is data from one experiment. 


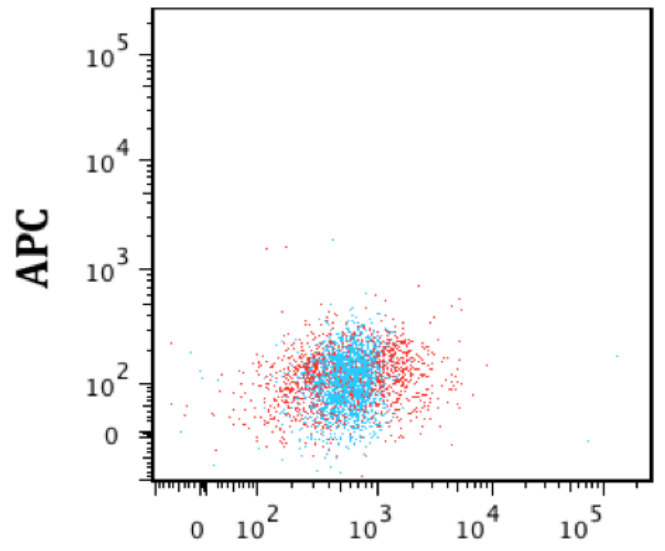

IL-8

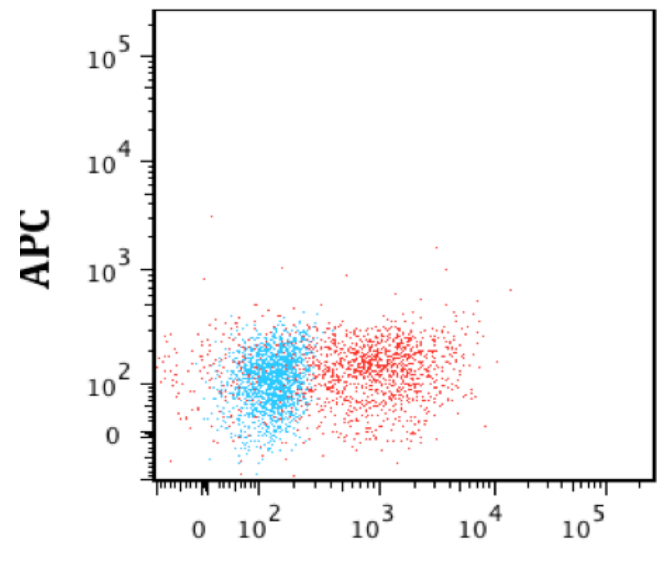

IL-8

(Comp-PE-A) IL-8 : Geom. Mean: 535.49

(Comp-PE-A) IL-8 : Geom. Mean: 126.67

(Comp-PE-A) IL-8 : Geom. Mean: 563.45

Figure 3.8 Improvement in IL-8 antibody resolution

Whole blood samples were cultured for 4 hours with $50 \mathrm{ng} / \mathrm{mL}$ PMA and 500 $\mathrm{ng} / \mathrm{mL}$ ionomycin, then stained with IL-8-PE antibody according to (A) a protocol guided by the literature, or (B) our final adjusted ICS procedure with $0.2 \%$ saponin buffer and optimised flow cytometer settings. Presented are the monocytes and neutrophils gated from SSC vs. FSC. Isotype control samples are shown in blue and IL-8 stained cells in red. Data are taken from individual experiments. 


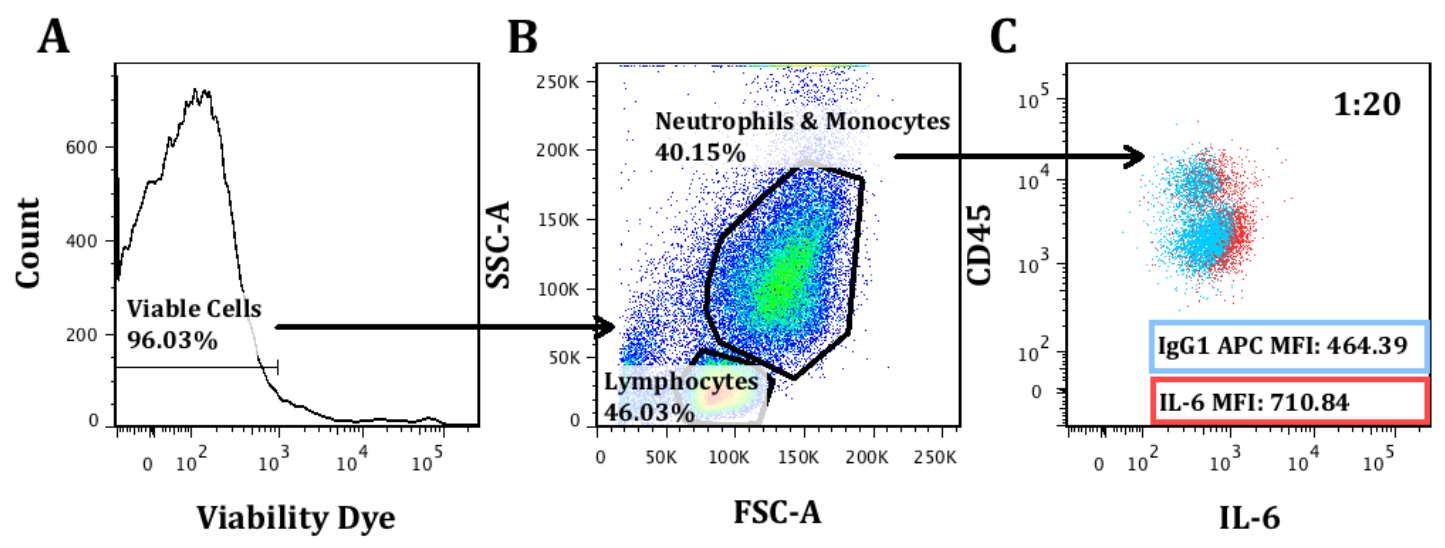

\section{Titration of IL-6 Antibody}

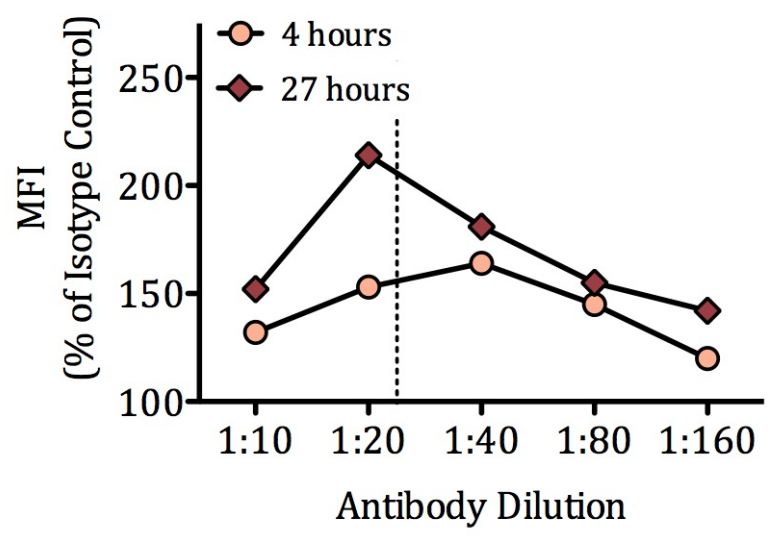

\section{Figure 3.9 Titration of the IL-6 antibody for intracellular use}

Whole blood samples were cultured for 4 hours with $50 \mathrm{ng} / \mathrm{mL}$ PMA and 500 $\mathrm{ng} / \mathrm{mL}$ ionomycin, or 27 hours with $10 \mathrm{ng} / \mathrm{mL}$ PMA and $500 \mathrm{ng} / \mathrm{mL}$ ionomycin, with GolgiStop (1:1000) for the final 4 hours. Samples were then stained with CD45 and IL-6 according to our partially tested ICS protocol and assessed by flow cytometry. (A) Viable leukocytes stimulated for 4 hours were gated; (B) the neutrophil and monocyte populations were gated using SSC vs. FSC; (C) the MFI of IL-6-APC (red) was compared to the isotype control (blue) in the neutrophils and monocytes; (D) titration of the IL-6-APC antibody in neutrophils and monocytes in 4 and 27 hour cultures, expressed as the percentage of the corresponding isotype control sample. Shown are the data from one experiment. 

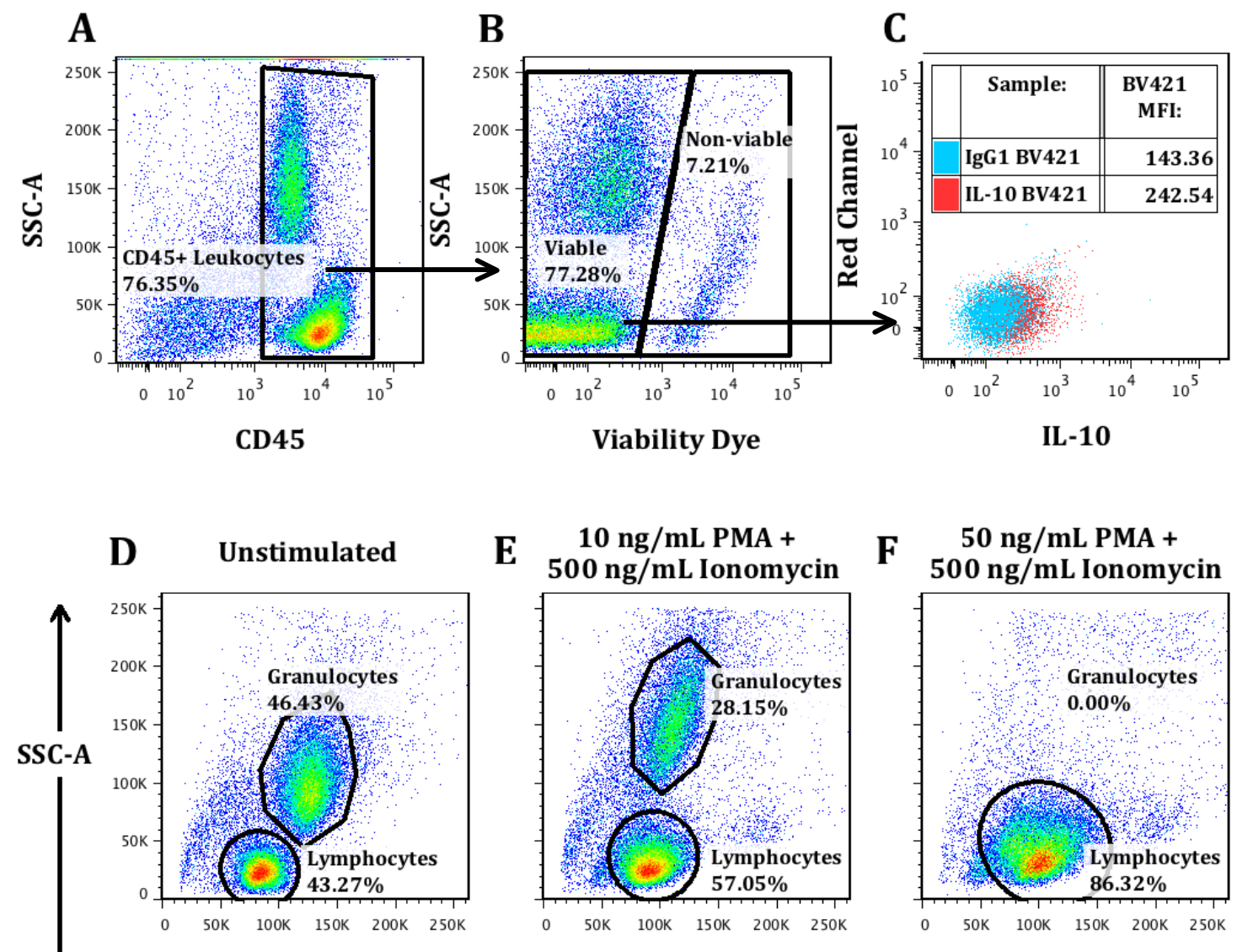

E $\quad \begin{gathered}10 \mathrm{ng} / \mathrm{mL} \text { PMA + } \\ 500 \mathrm{ng} / \mathrm{mL} \text { Ionomycin }\end{gathered}$
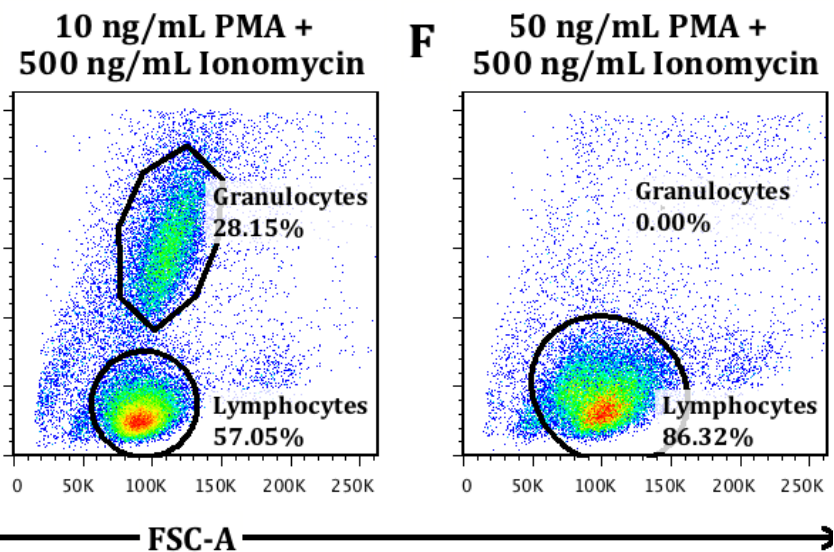

G

IL-10 MFI

$\mathbf{H}$

Viability
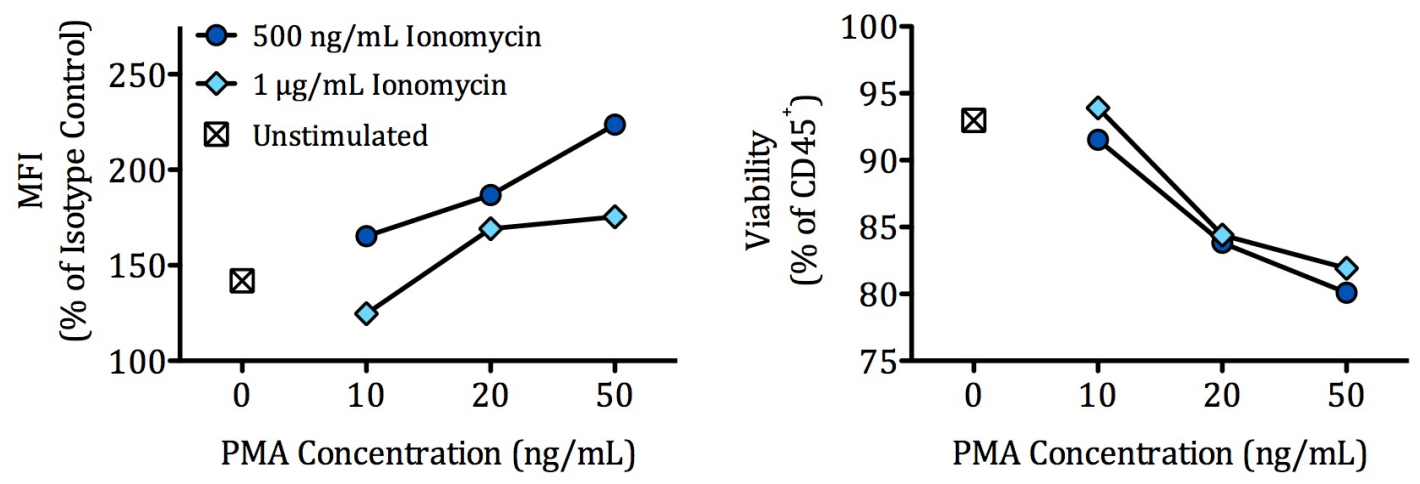

Figure 3.10 Stimulation of intracellular cytokine production in 27-hour cultures

Whole blood was incubated with various concentrations of PMA/I for 27 hours in the presence of GolgiStop (1:1000) for the final 4 hours of culture, then stained for viability and intracellular cytokines and analysed by flow cytometry. (A) $\mathrm{CD}_{4} 5^{+}$leukocytes were gated; (B) non-viable $\mathrm{CD} 45^{+}$leukocytes were excluded; (C) the MFI of IL-10 BV421 (red) was compared to the isotype control (blue) in the live $\mathrm{CD}^{2} 5^{+}$leukocytes. Changes in the leukocyte populations were observed on SSC vs. FSC in samples that were unstimulated (D), or treated with $10 \mathrm{ng} / \mathrm{mL} \mathrm{PMA}$ and $500 \mathrm{ng} / \mathrm{mL}$ ionomycin (E) or $50 \mathrm{ng} / \mathrm{mL} \mathrm{PMA} \mathrm{and} 500 \mathrm{ng} / \mathrm{mL}$ ionomycin (F). Production of IL-10 (G) and the viability (H) in cultured leukocytes were expressed as the percentage of the corresponding isotype control sample. Shown are the data from one experiment. 


\subsection{Inhibition of protein transport}

A key step in staining for intracellular cytokines is the use of a protein transport inhibitor to effectively trap the cytokines within the cells being examined. The two most commonly used agents for this purpose are brefeldin A (BFA) and monensin, which have differential effects on cell viability and cytokine capture, depending on the particular cell population and cytokines being examined. There was little information in the literature to suggest which is preferable when measuring IL-6, IL-8, or IL-10; therefore we tested both with our ICS protocol.

After 4 hours of culture there was a comparable level of IL-6 production detected in the neutrophils and monocytes between the samples treated with BFA and the monensin based product, GolgiStop. However, higher levels of IL-8 were measured in the cells cultured with BFA, although both protein transport inhibitors were associated with increased IL-8 staining, relative to the isotype control (Figure 3.11A). In contrast, leukocytes cultured for 26 hours with GolgiStop added for the last 4 hours showed markedly higher intracellular levels of IL-10, compared with the use of BFA (Figure 3.11B). Viability in the CD $45^{+}$ leukocytes was comparable between both protein transport inhibitors at both time points; however, it should be noted that the percentage of $\mathrm{CD}^{4} 5^{+}$cells differed between the two treatments (4 hours: BFA 82.80\% (SD 0.67) vs. GolgiStop 85.80\% (0.42); 26 hours: 69.28\% (0.58) vs. 58.19\% (0.83); Figure 3.11C). Overall, the transport inhibitors performed similarly; therefore we elected to continue with the agent used for the previous experiments, GolgiStop.

There was little information available regarding the optimal concentration of GolgiStop to use in whole blood cultures. Based on the manufacturer's notes for isolated cells in culture, a range of dilutions between 1:266 and 1:1333 were tested. At the lower dilutions the GolgiStop appeared to be toxic, with no IL-6 antibody fluorescence detected above the level of the isotype control (Figure 3.11D). At the highest dilution, GolgiStop did not inhibit protein transport and, again, the IL-6 levels were not markedly different from the control. The clearest population of IL-6 producing cells was detected with a GolgiStop dilution of 1:1000, so this concentration was used in our final protocol (Figure 3.11D). 
A

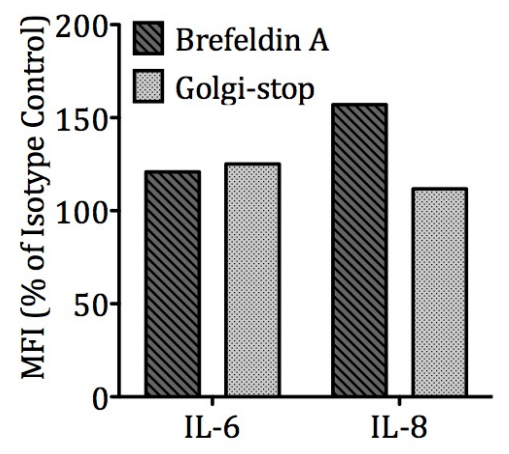

B

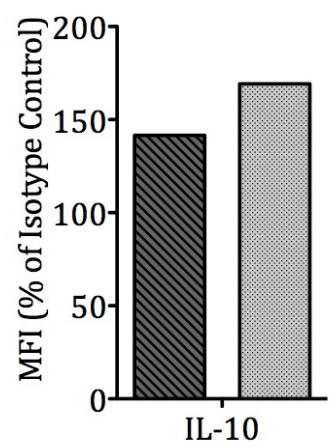

C

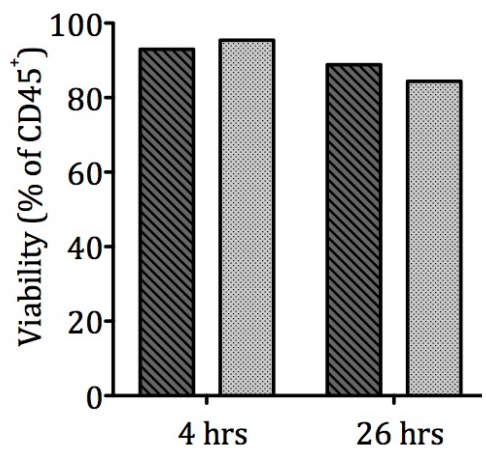

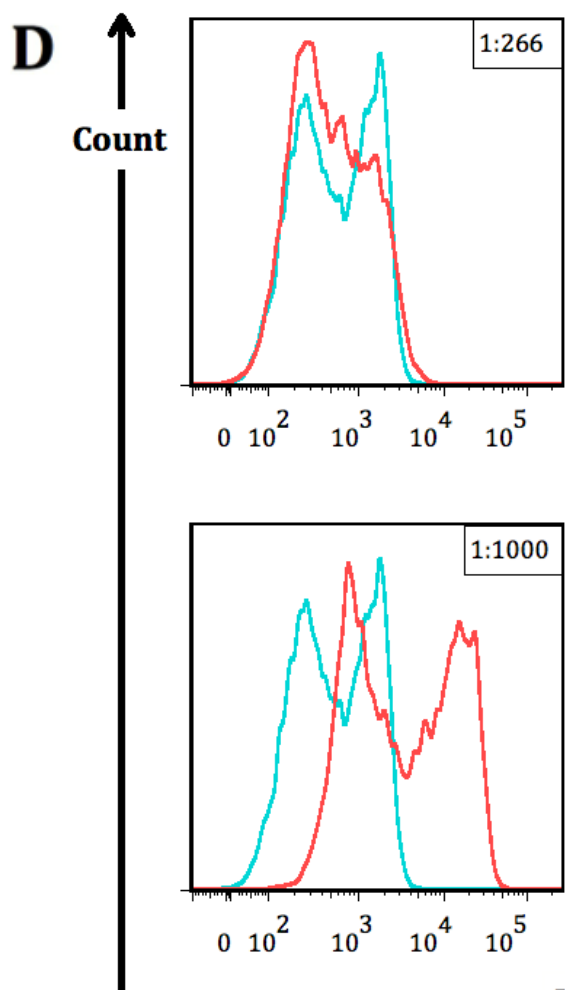
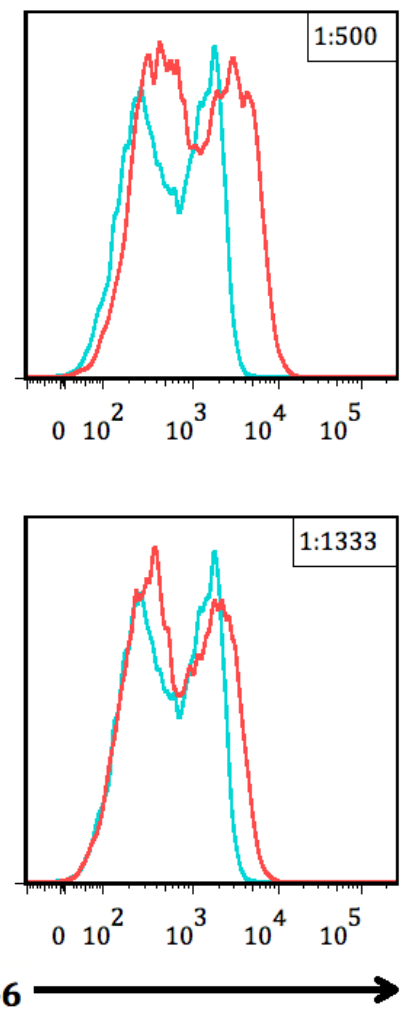

Figure 3.11 Comparison of protein transport inhibitors for intracellular measurement of IL-6, IL-8, and IL-10

Whole blood was stimulated in the presence of either brefeldin A (5 $\mu \mathrm{g} / \mathrm{mL})$ or GolgiStop (1:1000) for the final 4 hours of culture. Samples were stained for intracellular cytokines and viability, and analysed by flow cytometry. (A) IL-6 and IL-8 production in 4-hour cultures stimulated with PMA (50 ng/mL) and ionomycin (500 ng/mL); (B) 26-hour cultures stimulated with PMA $(20 \mathrm{ng} / \mathrm{mL})$ and ionomycin (1 $\mu \mathrm{g} / \mathrm{mL})$, and assessed for IL-10; (C) cell viability at the end of culture; (D) IL-6 levels (red) compared to isotype control (blue) in 4-hour cultures stimulated with PMA $(50 \mathrm{ng} / \mathrm{mL})$ and ionomycin $(500 \mathrm{ng} / \mathrm{mL})$ in the presence of varied dilutions of GolgiStop. Cytokine results presented as MFI are expressed as a percentage of the corresponding isotype control stained sample, and viability as the percentage of $\mathrm{CD} 45^{+}$cells. Data is from a single experiment. 


\subsection{Examining neutrophil activation state}

Neutrophils are highly sensitive, short-lived cells that can become activated in response to a multitude of stimuli, including temperature fluctuations, mechanical disturbances, and contact with anti-coagulants (Repo et al., 1995; van Eeden et al., 1999). Particular care was taken to minimise such sources of activation during our experimental procedures; however, it was necessary to confirm that the neutrophils in our unstimulated samples were both expressing the resting phenotype that we expected and were still responsive to stimulation.

Whole blood samples were collected and processed according to our final ICS protocol. The samples were stained for CD11b and assessed with flow cytometry. As expected, after 4 hours in culture our unstimulated samples showed only low CD11b expression in neutrophils, and these levels were clearly upregulated upon stimulation (MFI 937 in the unstimulated vs. 1909 in the stimulated; Figure $3.12 \mathrm{~A}$ ). After 27 hours in culture the baseline level of CD11b expression in the unstimulated samples increased, relative to the 4-hour samples; however, stimulation with PMA/I still elicited a further, small increase in CD11b (1338 vs. 1912; Figure 3.12B). This suggested that our laboratory procedures alone did not activate or inhibit neutrophils to the extent that they could not respond as expected to subsequent stimulation in vitro. 


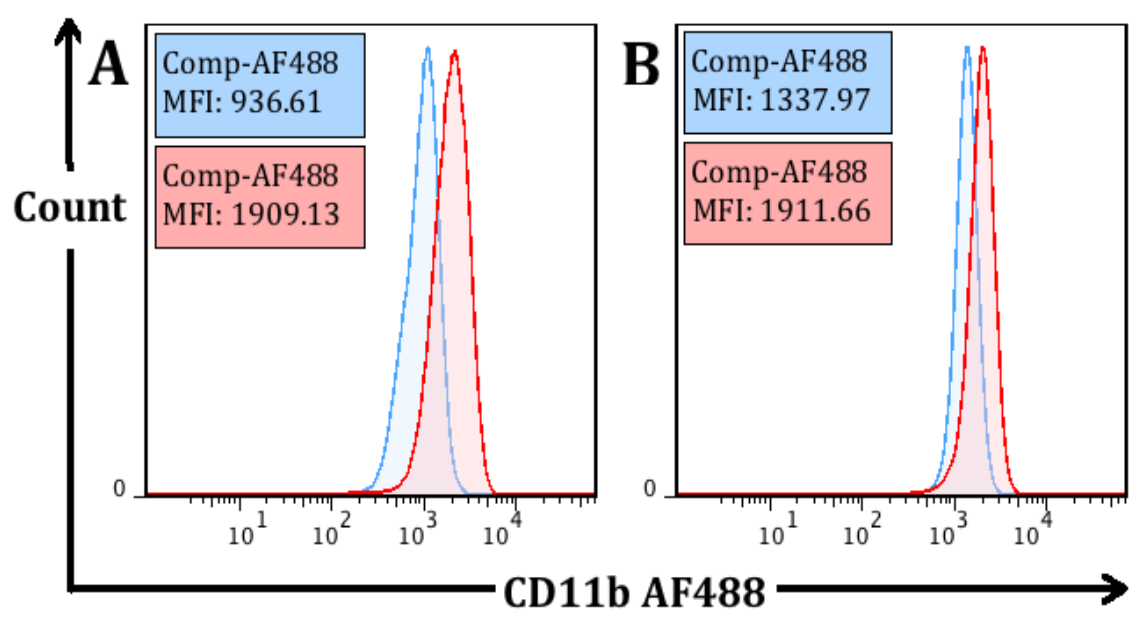

Figure 3.12 Activation state of cultured neutrophils

Whole blood was cultured with or without stimulants and GolgiStop (1:1000), then processed according to our ICS protocol in duplicate to assess CD11b expression. (A) Neutrophils from 4-hour cultures were either unstimulated (blue) or PMA/I treated $(50 \mathrm{ng} / \mathrm{mL} / 500 \mathrm{ng} / \mathrm{mL}$; red); (B) 27-hour cultures of unstimulated neutrophils (blue) or PMA/I treated neutrophils $(10 \mathrm{ng} / \mathrm{mL} / 500$ $\mathrm{ng} / \mathrm{mL}$; red). Data is shown for a representative replicate from a single experiment. 


\subsection{Optimisation of flow cytometry application settings}

Experiments that depend on measures of MFI or low resolution markers are particularly vulnerable to the day to day variability inherent to flow cytometers. Our immunophenotyping samples were not subject to such issues as they were collected on a Guava easyCyte flow cytometer that utilises fixed voltages; however, this was a concern with our ICS samples acquired on a BD FACSCanto ${ }^{\mathrm{TM}}$ II. To combat this issue we created and saved flow cytometry application settings using DIVA software, following the set-up procedure recommended by BD Bioscience. In addition to accounting for daily equipment variation, this procedure is designed to maximise the resolution between negative and positive populations for each channel. This could have been performed at any stage of our work, but we elected to complete it as the final step in our preparation for the study, once all of the steps in our ICS protocol had been tested and finalised, and closest to the time when we would be acquiring the actual patient data.

Briefly, the most recent flow cytometer baseline report was used to determine $2.5 \mathrm{x}$ the electronic noise robust standard deviation $\left(\mathrm{rSD}_{\mathrm{EN}}\right)$ for each channel, and these were defined as the target values. An unstained, unstimulated sample processed according to our ICS protocol was then run on the cytometer, and the lymphocytes, monocytes, and granulocytes were gated both separately (Figure 3.13A) and under a total leukocyte gate (Figure 3.13B) using FSC vs. SSC. During acquisition the minimum acceptable voltages were set by adjusting the PMT voltages for each channel until the $\mathrm{rSD}_{\mathrm{EN}}$ of the total leukocyte population was equal to the target value identified using the baseline report (Figure 3.13C). For channels that were only used for a particular subset of cells, such as the green channel off the violet laser (which was limited to markers of lymphocytes) the $\mathrm{rSD}_{\mathrm{EN}}$ was optimised for that particular subset, rather than the total leukocytes. An unstained, stimulated sample was also run to verify that the stimulation did not alter the sample $\mathrm{rSD}_{\mathrm{EN}}$ (Figure 3.13C). Single stained samples were then used to confirm that highly positively stained cell populations were still clearly visible within the linear detection range for each channel (Figure 3.13D - K). Lastly, the settings were saved and then used to set the voltages for each experiment relative to the daily flow cytometer performance check with $\mathrm{C}, \mathrm{S}$, and $\mathrm{T}$ beads. 
A

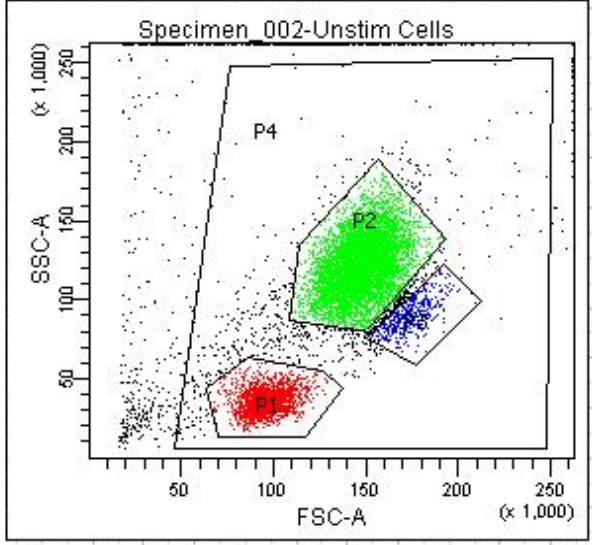

B

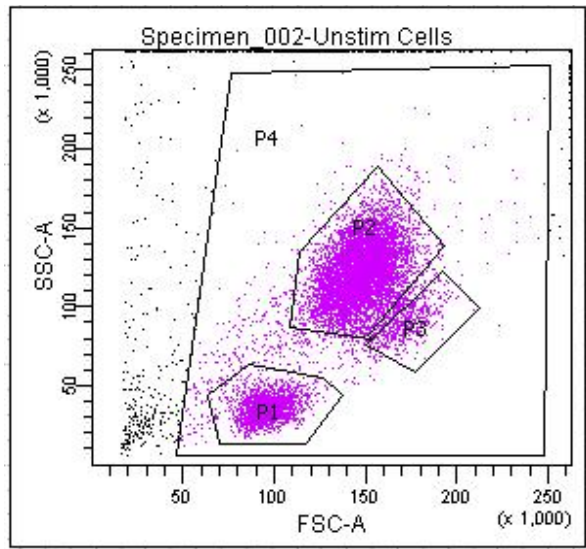

\begin{tabular}{|l|c|c|c|c|c|c|c|c|}
\cline { 2 - 9 } \multicolumn{1}{c|}{ C } & \multicolumn{2}{|c|}{ Violet $(405 \mathrm{~nm})$} & \multicolumn{4}{c|}{ Blue $(488 \mathrm{~nm})$} & \multicolumn{2}{c|}{ Red (633 nm) } \\
\cline { 2 - 9 } \multicolumn{1}{c|}{} & $\begin{array}{c}\text { V450 \& } \\
\text { BV421 }\end{array}$ & V500 & AF488 & PE & $\begin{array}{c}\text { PerCP \& } \\
\text { PerCP/Cy5.5 }\end{array}$ & PE/Cy7 & APC & APC/H7 \\
\hline Target Value & 60 & 61 & 70 & 75 & 75 & 69 & 56 & 56 \\
\hline $\begin{array}{l}\text { Unstimulated } \\
\text { Leukocytes }\end{array}$ & 57 & 57 & 66 & 73 & 70 & 68 & 54 & 56 \\
\hline $\begin{array}{l}\text { Stimulated } \\
\text { Leukocytes }\end{array}$ & 57 & 57 & 70 & 68 & 66 & 64 & 59 & 56 \\
\hline
\end{tabular}

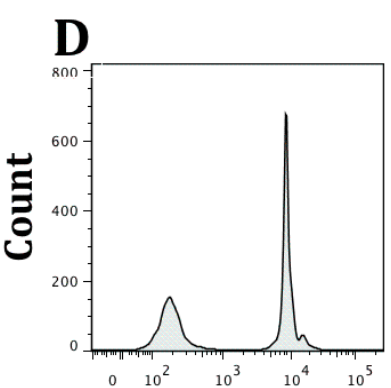

IL-10 BV421

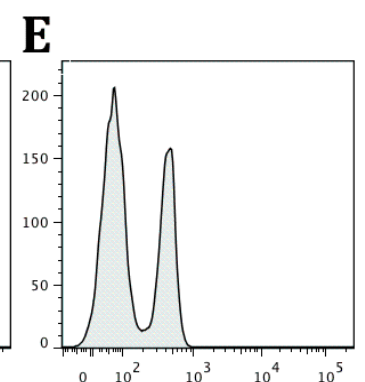

CD4 V500

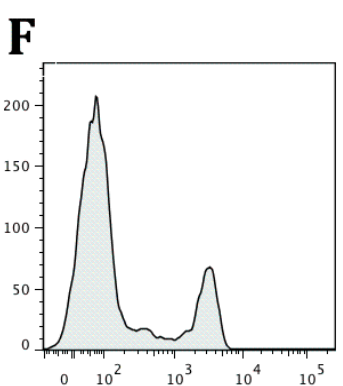

CD8 AF488

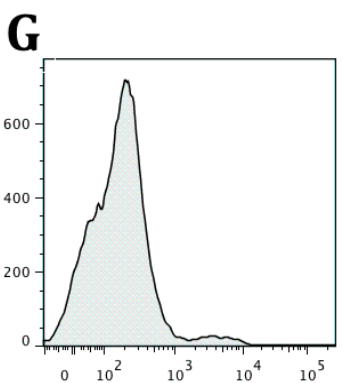

IL-8 PE

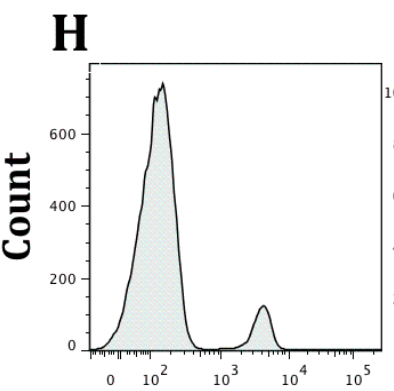

CD14 PerCP

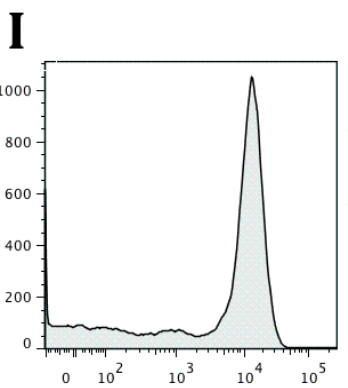

CD16 PE/Cy7

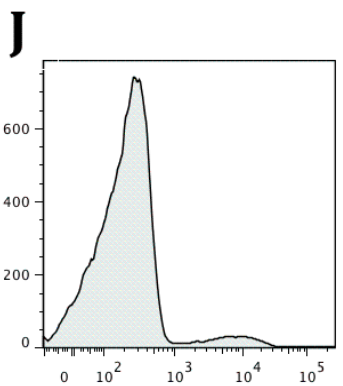

IL-6 APC
$\mathbf{K}$

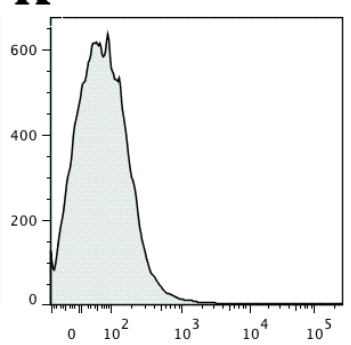

Viability (APC/H7)

Figure 3.13 Optimisation of flow cytometry application settings

Blood was cultured for 4 hours with media or PMA/I (50 ng/mL / $500 \mathrm{ng} / \mathrm{mL})$ in the presence of GolgiStop (1:1000), then stained intracellularly and acquired on the flow cytometer. (A) Unstimulated cells gated as lymphocytes (red), neutrophils (green), and monocytes (blue); (B) unstimulated cells gated using a total leukocyte gate (purple); (C) $\mathrm{rSD}_{\mathrm{EN}}$ in the unstimulated and stimulated total leukocytes, compared to target values; (D - K) histograms showing detection of brightest antibodies for each channel. Data is from a single experiment. 


\subsubsection{Culture of PBMC and assessment of responses}

Techniques for stimulating $\mathrm{T}$ cells in culture and measuring the resulting changes in proliferation and cytokine production were tested for use in the study to assess the effect of RIPC on the acquired immune response. A summary of this work is presented in Figure 3.14.

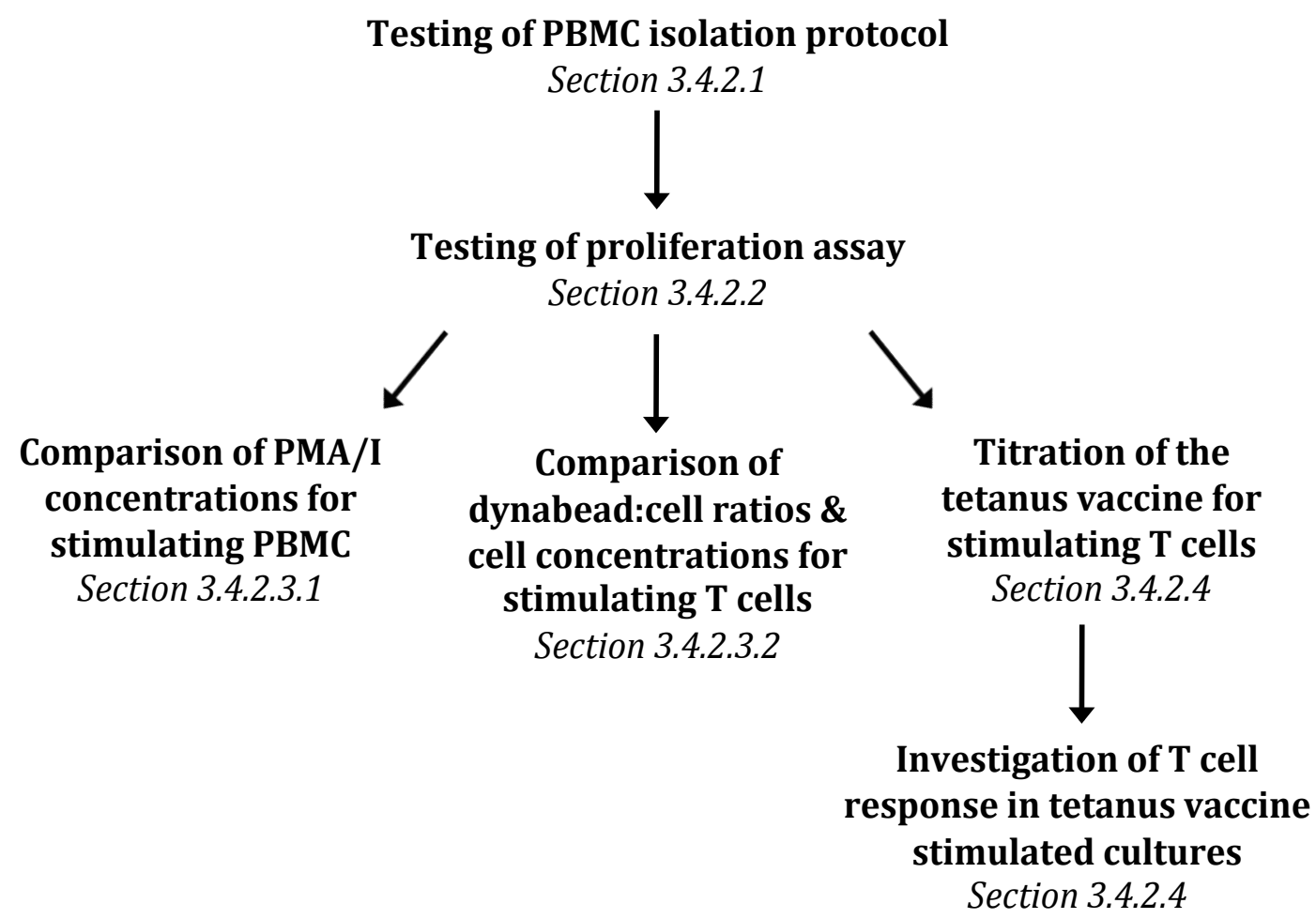

Figure 3.14 Summary of the work undertaken to test the methods for PBMC culture and quantification

Flowchart depicting the work performed for testing the conditions for culturing PBMC, and the corresponding thesis sections that detail the work. 


\subsubsection{Testing the Histopaque-1077 PBMC isolation method}

PBMC were isolated from heparinised whole blood using Histopaque-1077, according to the manufacturer's instructions. Following isolation the cells were stained with cell surface markers and analysed with flow cytometry to validate the purity. As expected, the sample was predominantly composed of $\mathrm{CD}^{+} \mathrm{T}$ cells, with a smaller population of $\mathrm{CD} 14^{+}$monocytes (Figure 3.15B). There was no clear neutrophil population visible on the SSC vs. FSC plot; however, a proportion of the CD14-CD3- cells did stain positively for CD16 (Figure 3.15A and B). The low position of these cells on the SSC axis was not consistent with neutrophils, and these cells were most likely $\mathrm{CD}_{16}{ }^{+} \mathrm{NK}$ cells, and a small population of $\mathrm{CD} 14^{\text {low }} \mathrm{CD} 16^{+}$monocytes (Figure 3.15C). The remaining CD3CD14- events were consistent with the characteristics expected of B cells and CD16-CD56 ${ }^{++}$NK cells. Overall, the Histopaque-1077 method of isolation provided a sufficiently pure PBMC population, with little evidence of neutrophil contamination.

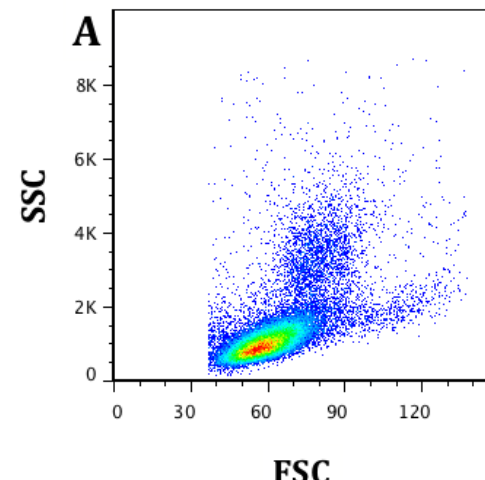

FSC

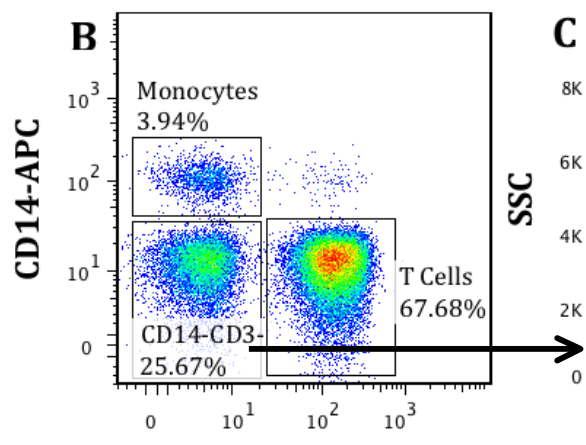

CD3-PE

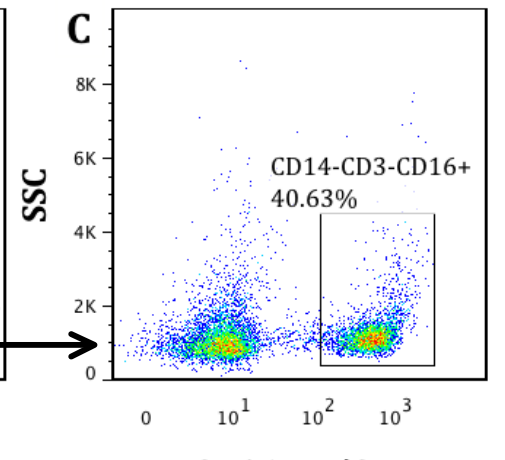

CD16-PE/Cy7

Figure 3.15 PBMC purity after Histopaque-1077 isolation

PBMC were isolated from heparinised whole blood using the Histopaque-1077 density gradient centrifugation method, then stained in duplicate with cell surface markers and analysed by flow cytometry. (A) Total isolated cells; (B) CD14 vs. CD3 in total isolated cells; (C) CD16 expression in the CD3-CD14- cell population. Shown are data from a representative replicate for a single experiment. 


\subsubsection{CyQuant proliferation assay}

The CyQuant proliferation assay utilises a dye that exhibits enhanced fluorescence when bound to cellular nucleic acids in order to quantify cell numbers. When used according to the manufacturer's instructions, the assay has a reported linear detection range from 50 to $5 \times 10^{4}$ cells, and increasing the concentration of the dye can extend this span. To determine the number of cells that could be accurately quantified in our PBMC samples we tested the ability of the CyQuant assay to detect up to $2 \times 10^{5}$ cells when used with $1 \mathrm{X}$ or $2 \mathrm{X}$ the recommended dye concentration. As expected, both concentrations of dye showed a linear relationship between fluorescence and the number of cells, up to $5 \times 10^{4}$ cells per sample. Beyond $5 \times 10^{4}$ cells, the fluorescence in the $1 \mathrm{X}$ dye samples began to plateau and was best modelled by non-linear regression (goodness of fit $=0.9727$ vs. 0.9338 for linear regression), whereas the samples incubated with the $2 \mathrm{X}$ dye had a reasonably linear relationship up to the highest number of cells tested, $2 \times 10^{5}$, with a linear regression goodness of fit measure of 0.9680 (vs. 0.9744 for non-linear regression; Figure 3.16). For the study, we opted to use the dye at $2 \mathrm{X}$ concentration, and plate the cells no higher than $1 \mathrm{x}$ $10^{5}$ cells/sample, in order to allow the final cell number to remain within the linear range of detection following stimulation of proliferation.

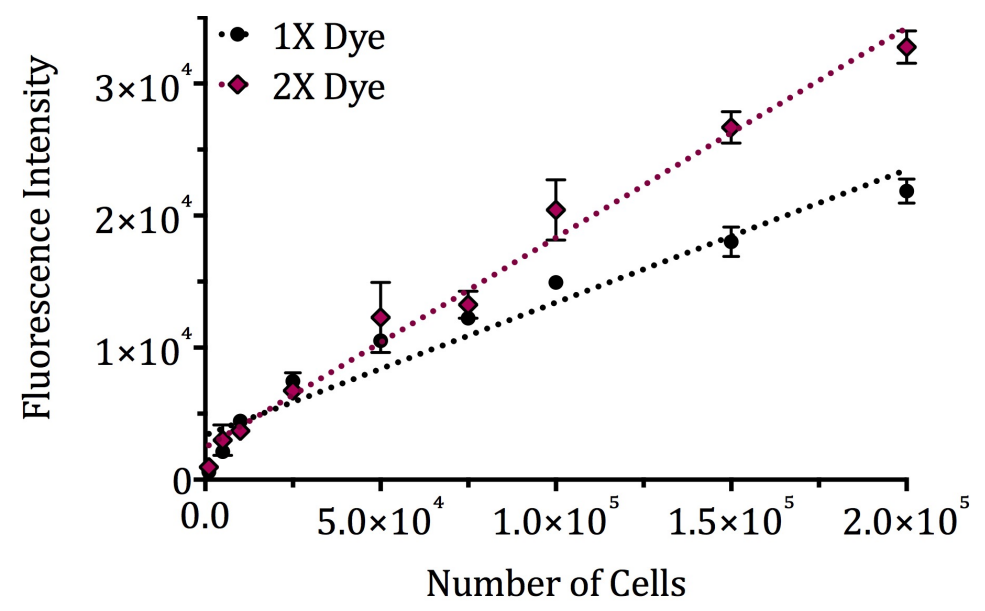

Figure 3.16 Determining the linear detection range for the CyQuant proliferation assay

PBMC were isolated using Histopaque-1077 and set-up in duplicate in a 96-well plate at cell numbers between $1 \times 10^{3}$ and $2 \times 10^{5}$ per well. The CyQuant assay was performed using $1 \mathrm{X}$ (black) or $2 \mathrm{X}$ (pink) dye, and the resulting fluorescence measured at 480/520 $\mathrm{nm}$. Shown are the means, SEM, and linear regression lines from one experiment. 


\subsubsection{Stimulating polyclonal T Cell responses}

\subsection{PMA and ionomycin}

Non-specific cellular activation can be induced using the pharmacological agents, PMA/I. In the literature, the most commonly used concentrations of PMA/I for stimulating cells in culture are 5 or $10 \mathrm{ng} / \mathrm{mL}$, and 200 or $500 \mathrm{ng} / \mathrm{mL}$, respectively (Kruisbeek et al., 2004). We compared all four combinations of these concentrations with cells plated at $5 \times 10^{4}$ per well and found no significant proliferation in any of the conditions after 24 hours of culture. In fact, all of the stimulated samples had lower CyQuant values than the unstimulated samples (Figure 3.17A). This was not unexpected, as the recommended incubation duration for measuring significant levels of $\mathrm{T}$ cell proliferation is normally in the range of 3 to 6 days (Kruisbeek et al., 2004). For this particular study we were focused on the short-term impact on $\mathrm{T}$ cell function, particularly cytokine production, therefore elected to use a shorter culture period. Indeed, high levels of IL-6 production were detected in the supernatants from all four cultures and the specific concentration of stimulants used did not substantially impact these levels (Figure 3.17B). Based on these results, the concentrations of $5 \mathrm{ng} / \mathrm{mL}$ PMA and $200 \mathrm{ng} / \mathrm{mL}$ ionomycin were selected for use in the cultures, and cells were plated at $1 \times 10^{5} /$ well to improve the accuracy of the CyQuant assay.

\subsection{Anti-CD3/28 beads}

Polyclonal yet physiologically relevant $\mathrm{T}$ cell activation can be achieved using beads with antibodies specific for the TCR-CD3 complex and the co-stimulatory molecule CD28. The manufacturer's instructions recommend stimulating T cells with a ratio of anti-CD3/28 dynabeads to cells of 1:1. Given we were working with a mixed population of PBMC (comprised of approximately $60 \% \mathrm{~T}$ cells) rather than isolated $\mathrm{T}$ cells, we tested three ratios of beads to cells, and two concentrations of cells, to determine the best method of dynabead stimulation.

The highest proliferative response was detected in the sample plated with $8 \mathrm{x}$ $10^{4}$ cells, and there was negligible difference between the $1: 1,0.5: 1$, and $0.2: 1$ bead to cell ratios in the samples plated at $1.33 \times 10^{5}$ cells/well (Figure 3.17C). 
As expected, there were higher levels of IL-6 detected in the samples with 133,000 cells per well, and a dose-response relationship was seen with the dilution of the beads. The sample with fewer cells also produced significant levels of IL-6 (Figure 3.17D). The best combination of proliferation and IL-6 production was seen when $8 \times 10^{4}$ PBMC were plated with beads at a 1:1 bead to cell ratio, and these were the conditions chosen for use in the study. 
A

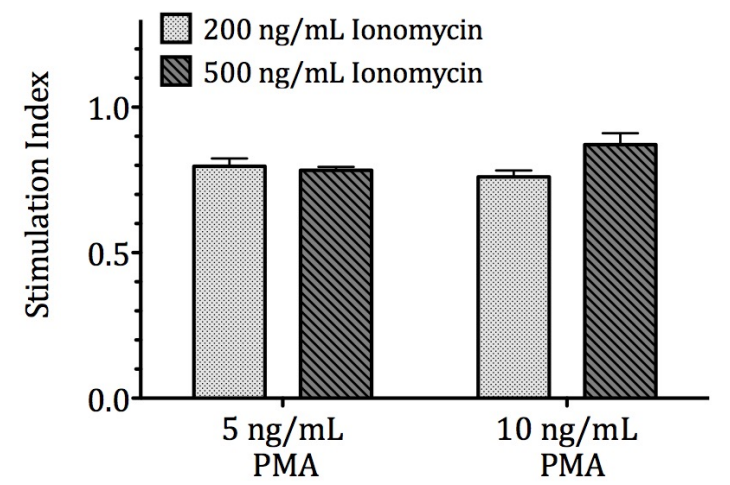

C

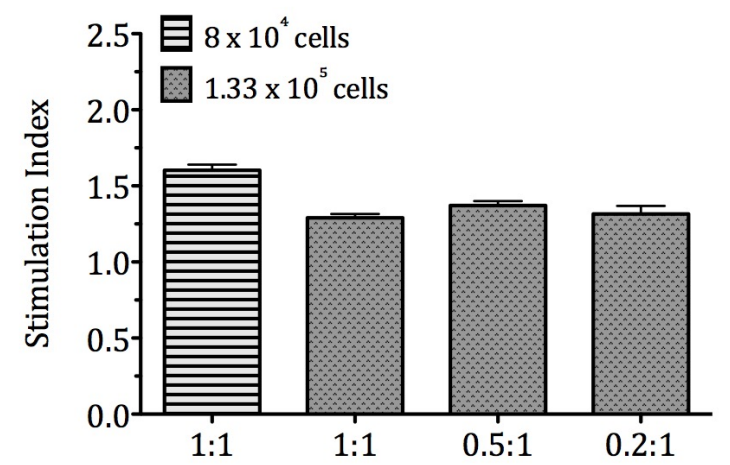

B

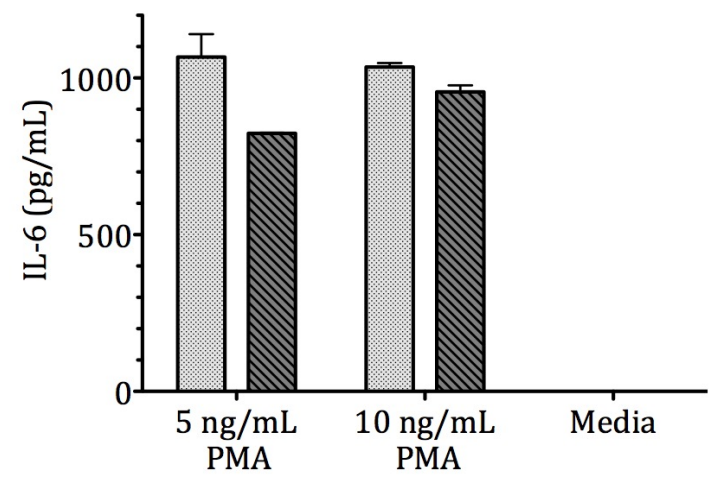

D

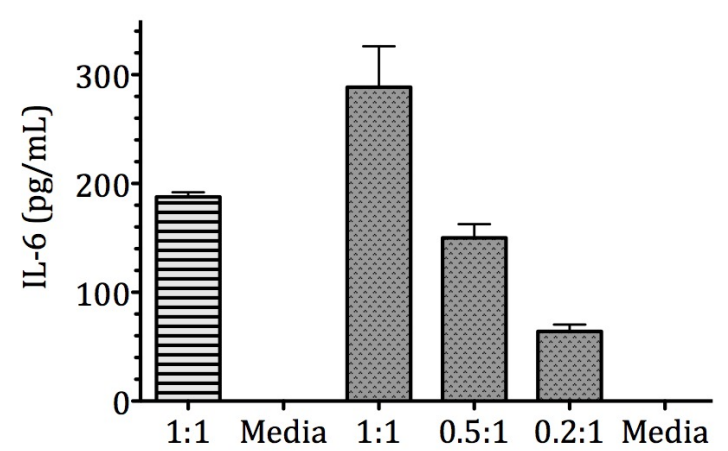

Figure 3.17 Stimulation of PBMC with PMA/I and anti-CD3/28 beads

Isolated PBMC were cultured for 24 hours with or without stimulants, then proliferation was measured with the CyQuant assay and culture supernatant IL-6 levels quantified by ELISA. (A) Stimulation index and (B) IL-6 levels in $5 \times 10^{4}$ cells/well stimulated with various concentrations of PMA/I. (C) Stimulation index and (D) IL-6 levels for cells plated at differing concentrations and antiCD3/28 bead:cell ratios. Stimulation indices were calculated relative to the unstimulated controls. Shown are the means and SEM for duplicates from one experiment. 


\subsubsection{Stimulating memory $T$ cells with the tetanus antigen}

In order to determine whether RIPC affected the memory response, we developed an assay to assess antigen-specific responses to tetanus using the ADT Booster vaccine. This antigenic target was selected because it is part of the childhood vaccination schedule in New Zealand and booster vaccinations are recommended at 11 years of age, resulting in a high proportion of individuals with the ability to recognise and mount an immunological response to the tetanus toxin. We chose to use the booster vaccine to stimulate our cells, as this was readily available, safe, and provided the exact antigen recognised by immunised individuals.

There was no previously described protocol for using the ADT booster vaccine to stimulate cells available in the literature. Therefore, the vaccine was first titrated in a volunteer who had recently (in the last 10 years) had a tetanus booster and an individual who had not received a recent tetanus booster. A consistent proliferative response was detected in both individuals, which was lost once the vaccine was diluted beyond 1:20 (Figure 3.18A). It was possible that the memory response was still significant in the participant that had not recently been immunised; however, it was noted that the vaccine had a slightly cloudy appearance, and that this may have impacted the CyQuant assay readings.

To determine whether the vaccine itself may be influencing the CyQuant assay, a comparison was made between using the full vaccine, or a 'separated' vaccine, where the vaccine was left for 10 minutes to allow the particulate matter to settle and the remaining clear solution was aspirated for use. With the samples treated with the full vaccine, the vaccine was also added to the control wells at the end of the culture period, immediately prior to centrifugation and freezing of the cells, in order to keep the opacity of the samples comparable.

There was no proliferation detected in the samples cultured with the separated vaccine, and addition of the vaccine to the control wells used with the full vaccine samples prior to running the proliferation assay altered the background level in the control wells but did not affect the conclusions drawn from the CyQuant 
results (Figure 3.18B). Further, microscopic examination of the cultured cells showed clustering only in the samples incubated with the full vaccine, and isolated cells in the samples with the separated vaccine or media alone (3.19 A C). Clustering of the cells is consistent with a recall response, suggesting that separating the vaccine removed the tetanus antigen (Chtanova et al., 2009). Taken together, these results indicated that the CyQuant levels detected in the samples cultured with the full vaccine were the result of a legitimate proliferative response, rather than simply an increase in background due to the vaccine opacity.

To further test that the cells cultured with the tetanus vaccine were being stimulated as expected, the level of IL- 6 in the culture supernatants was measured after 6 days of culture. When cells were plated at a high concentration of $2 \times 10^{5}$ cells per well, consistent levels of IL-6 were measured, regardless of the tetanus vaccine dilution (Figure 3.18C). Notably in this data, there was a pronounced difference in the response between the individual that did have a recent tetanus booster, and the volunteer who did not. When the number of cells was reduced to $1 \times 10^{5}$ per well, an improved resolution of IL-6 production could be seen, and showed a dose-response relationship with the concentration of vaccine (Figure 3.18D). This concentration of cells also ensured that following proliferation, the CyQuant assay would still be operating well within the linear range previously identified (refer to section 3.4.2.2).

Based on these results, it was determined that the best conditions for stimulating PBMC with the tetanus vaccine were at a final dilution of $1: 5$, with $1 \times 10^{5}$ cells per well, and that adding the vaccine to the control wells prior to performing the CyQuant assay was necessary to ensure the background was comparable between the samples. 

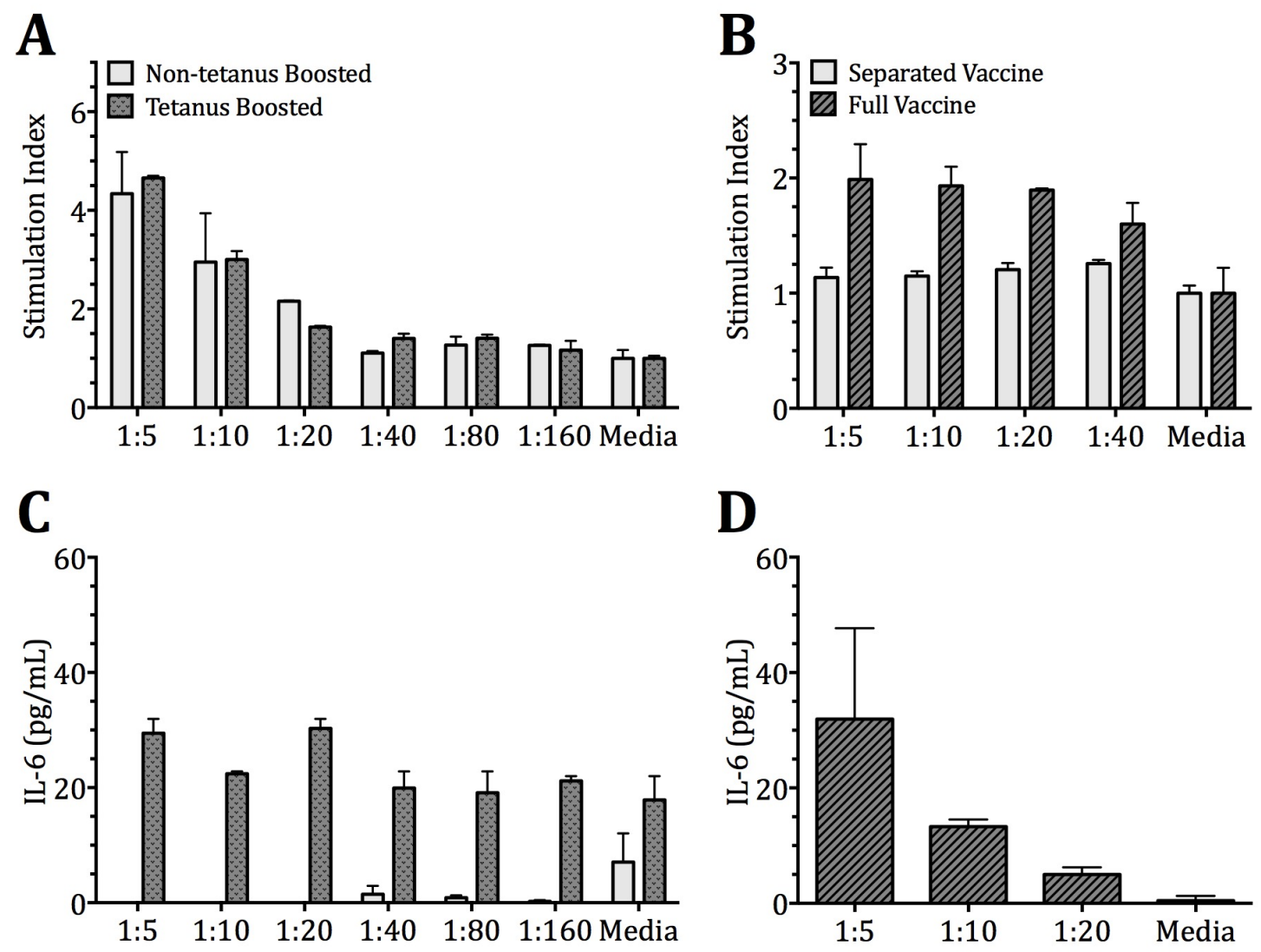

Figure 3.18 Stimulation of PBMC with the tetanus vaccine

Isolated PBMC were plated with various dilutions of the tetanus vaccine and cultured for 6 days. Cell proliferation was measured using the CyQuant assay, and supernatant IL-6 levels quantified by ELISA. Left: comparison and titration of (A) tetanus-induced proliferation and (C) IL-6 production between 'nontetanus boosted' and 'tetanus boosted' individuals with cells plated at $2 \mathrm{x}$ $10^{5}$ /well. Right: cells were plated at $1 \times 10^{5}$ cells/well and shown are (B) the comparison of proliferative responses to the separated and full versions of the vaccine, and (D) IL-6 production in the full vaccine stimulated PBMC from a tetanus-boosted individual. Shown are the means and SEM of duplicates from one experiment. 

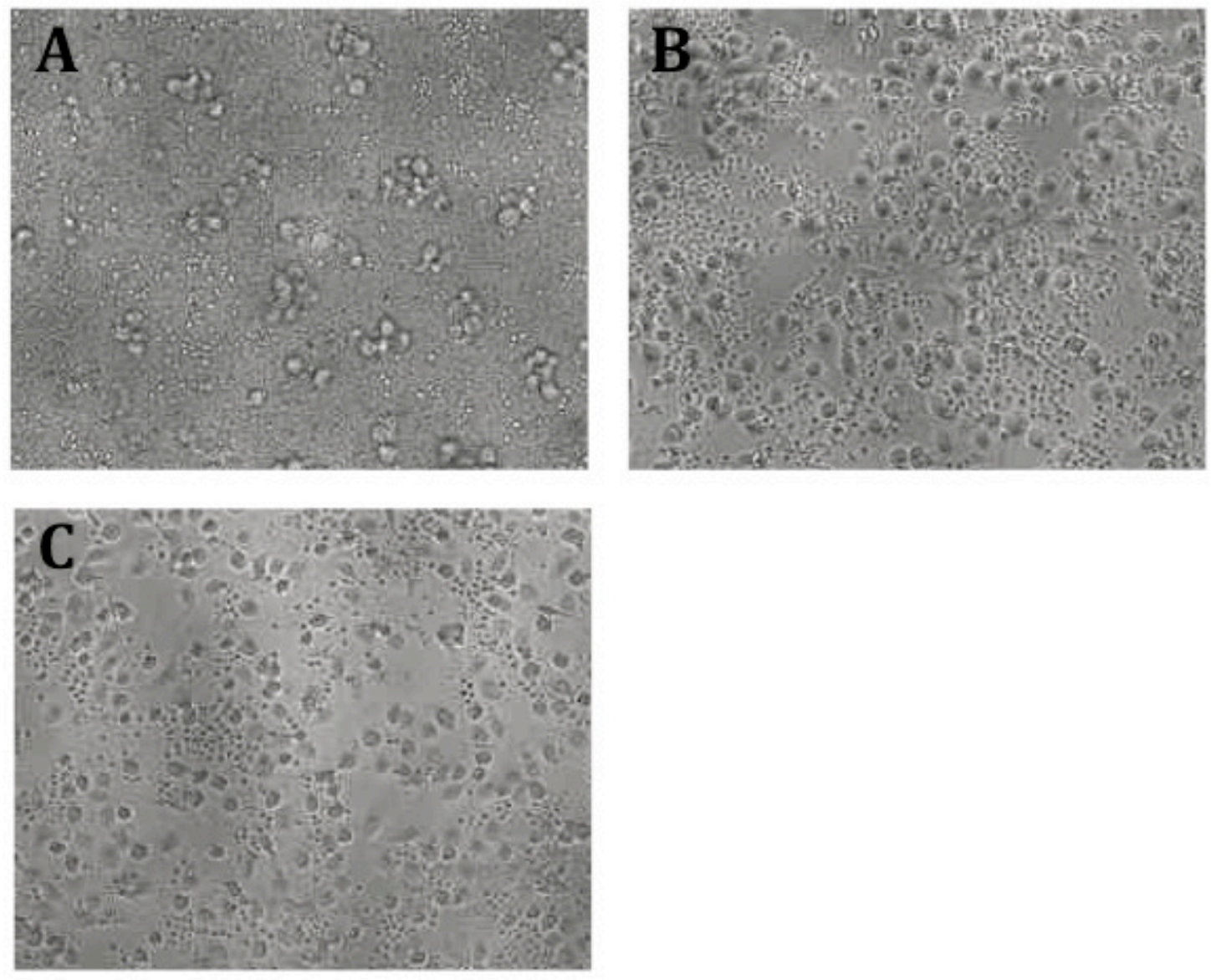

Figure 3.19 Comparison of PBMC cultured with the separated or full tetanus vaccine

Histopaque-1077 isolated PBMC were plated in duplicate at $2 \times 10^{5}$ cells/well and cultured for 6 days with (A) full tetanus vaccine diluted 1:5; (B) separated tetanus vaccine diluted 1:5, (C) media. Shown are representative photos from one of two experiments. 


\subsubsection{Study workflow development}

\subsubsection{Laboratory workflow and patient management}

A critical element in designing the study in healthy volunteers was creating a laboratory workflow that could be reasonably and repeatedly performed by a single individual. In the initial phase of study design, the endpoints and time points were carefully selected for this purpose; however, after we performed a pilot run testing the finalised laboratory protocols, problematic aspects of timing involved with the workflows were further amended, as necessary.

Initially, we proposed to examine neutrophil activation by stimulating the blood samples collected at baseline and 20 minutes after treatment, prior to the staining for immunophenotyping. It became clear that performing this extra step with the 20-minute blood samples would create numerous clashes between subsequent laboratory time points. Instead, it was deemed more achievable to perform the post-treatment stimulation using the 1-hour samples, with the 40minute difference between these two time points unlikely to impact the ability to detect any clinically significant changes.

Likewise, performing two final wash steps on the flow cytometry samples was time intensive and resulted in a back up of the laboratory work. An experiment was run to compare samples that were handled according to our standard protocol, involving two washes, with samples that were washed only once. Despite a slightly higher level of debris being present in the latter samples, reducing the number of wash steps had no impact on the final data measurements (data not shown). The immunophenotyping protocol was therefore adjusted to include only one wash at the final step, keeping the laboratory workflow attainable.

Performing the treatment (control or RIPC) immediately after the baseline blood sample collection created complications with the sample processing due to the short gap between the first and second blood samples. This issue was alleviated by inserting a 10-minute 'stand down' period between the baseline bloods and 
applying the treatment, allowing sufficient time to process the bloods to a consistent standard.

After the changes to the laboratory techniques were made, a final laboratory overview was produced and is presented in Figure 3.20. With multiple samples being handled at one time, and the strong likelihood that any differences in our endpoints following RIPC would be subtle, it was essential that the laboratory procedures were as consistent as practically achievable, between both participants and visits. To address this, a detailed ordered laboratory checklist and protocol were created to ensure that all samples were handled in exactly the same manner during each study visit. In addition to specifying which samples should be prioritised at which steps, these documents ensured that equipment and buffers were calibrated and pre-warmed at the correct times, and were an essential part of our strategy to obtain the highest quality data. Further, all study sessions were scheduled for the same time of day to eliminate any variation in the results that might be caused by circadian rhythms.

Lastly, due to the heavy workload in the laboratory, it was necessary for a second individual to manage the patient and collect the blood samples. To ensure that the patient handling and blood sample collection were performed consistently, a patient checklist form was created and used during each study visit. 


\begin{tabular}{|c|c|c|c|c|c|c|c|c|}
\hline \multirow{2}{*}{\begin{tabular}{|l|} 
Time \\
$9: 00$ \\
\end{tabular}} & \multirow{2}{*}{$\begin{array}{l}\text { Volunteer } \\
\text { Bi Bloods }\end{array}$} & \multicolumn{2}{|c|}{$\begin{array}{c}\text { Immuno- } \\
\text { phenotyping }\end{array}$} & \multirow{2}{*}{$\begin{array}{c}\text { CD63 } \\
\text { Analysis } \\
\text { Stmpulation }\end{array}$} & \multirow[t]{2}{*}{ ICS } & \multirow[t]{2}{*}{\begin{tabular}{|c|} 
PBMC \\
Cultures
\end{tabular}} & \multicolumn{2}{|c|}{ Serum Collection } \\
\hline & & \multirow{3}{*}{ stant } & & & & & \multirow{3}{*}{ cotting } & \\
\hline $\begin{array}{l}9: 10 \\
9: 20 \\
9: 30\end{array}$ & $\begin{array}{c}\text { Control / RIPC } \\
\text { Treatment } \\
\end{array}$ & & & \multirow{2}{*}{${ }^{s t c \text { with }}$} & & & & \\
\hline $\begin{array}{l}9: 40 \\
9: 50\end{array}$ & & & & & & & & \\
\hline $10: 00$ & $20 \mathrm{~min}$ Bloods & \multirow{6}{*}{$\begin{array}{l}\text { Wash } 8 \\
\text { Resusp }\end{array}$} & \multirow{3}{*}{ Stain } & \multirow{6}{*}{ stamiratom } & \multirow{6}{*}{ mptwa } & & \multirow{6}{*}{$\begin{array}{l}\text { Centrifüue } \\
\text { Aliquiot }\end{array}$} & \multirow{6}{*}{$\begin{array}{l}\text { Clotting } \\
\text { time }\end{array}$} \\
\hline $10: 10$ & & & & & & & & \\
\hline $10: 20$ & & & & & & & & \\
\hline 10:30 & & & Lyse & & & & & \\
\hline $10: 40$ & 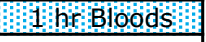 & & Wash \& & & & & & \\
\hline 10:50 & & & & & & Sedle & & \\
\hline $\begin{array}{l}11: 00 \\
11: 10\end{array}$ & & \multirow{2}{*}{ sem } & & \multirow{5}{*}{ 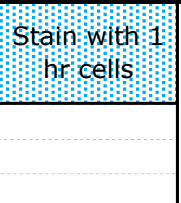 } & & \multirow{2}{*}{ 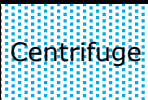 } & & $\frac{\text { Centrifuge }}{\text { Aliquot }}$ \\
\hline $\begin{array}{l}11: 10 \\
11: 20\end{array}$ & & & & & & & cotting & Aliquot \\
\hline $11: 30$ & & Lysel & & & & $10 y$ est & & \\
\hline $11: 40$ & & Wash 8 & & & & $p B M G s$ & Cettifuge & \\
\hline $11: 50$ & & Resspp & & & & & 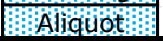 & \\
\hline $12: 00$ & & & & & & & & \\
\hline $12: 10$ & & & & & & 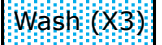 & & \\
\hline $12: 20$ & & & & & & & & \\
\hline $12: 30$ & & & & & & Codot & & \\
\hline $12: 40$ & & & & & & Biafe Gels & & \\
\hline $12: 50$ & & & Acquire & & & & & \\
\hline 13:00 & & & samples & & & & & \\
\hline Break & & & & & & & & \\
\hline $13: 40$ & $4 \mathrm{hr}$ Bloods & & & & & & & \\
\hline 13:50 & & slatin: & & & & & & \\
\hline $14: 00$ & & & & & & & & \\
\hline $14: 10$ & & Lyse: & & & & & & Clotting \\
\hline $14: 20$ & & Wash \& & & & & & & time \\
\hline $14: 30$ & & Resusp: & & & & & & \\
\hline $14: 40$ & & & & & & & & Centrifuge \\
\hline $14: 50$ & & & & & & & & Aliquot \\
\hline 15:00 & & & & & & & & \\
\hline $15: 10$ & & & & & Exysersing & & & \\
\hline $15: 20$ & & Acquire & & & Wash $\times 2$ & & & \\
\hline $15: 30$ & & samples & & & 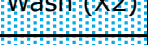 & & & \\
\hline $15: 40$ & & & & & & & & \\
\hline $15: 50$ & & & & & 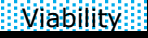 & & & \\
\hline $16: 00$ & & & & & stailia & & & \\
\hline $16: 10$ & & & & & & & & \\
\hline $16: 20$ & & & & & Was & & & \\
\hline $16: 30$ & & & & & & & & \\
\hline $\begin{array}{l}16: 40 \\
16: 50\end{array}$ & & (2) & & & 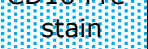 & & & \\
\hline$\frac{10: 50}{17: 00}$ & & & & & & & & \\
\hline $17: 10$ & & & & & Bock & & & \\
\hline $17: 20$ & & Clean \& & & & & & & \\
\hline $17: 30$ & & Shut & & & tch & & & \\
\hline $\begin{array}{l}17: 40 \\
17: 50\end{array}$ & & down & & & & & & \\
\hline 17:50 & & Guava & & & Wash & & & \\
\hline $\begin{array}{l}18: 00 \\
18: 10\end{array}$ & & & & & 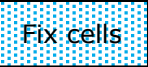 & & & \\
\hline $18: 20$ & & & & & Was hi & & & \\
\hline $18: 30$ & & & & & & & & \\
\hline
\end{tabular}

Figure 3.20 Overview of laboratory sample handling schedule

Schedule depicting the parallel handling of study blood samples for each of the assays performed. 


\subsubsection{Flow cytometry data analysis}

A substantial volume of flow cytometry data was collected during the study. Intracellular cytokine staining results are particularly vulnerable to poor gating strategies or inconsistencies during data analysis, necessitating the development of standardised protocols (Nomura et al., 2008; Welters et al., 2012). This task was tackled by generating pre-saved analysis workspace templates for the software, FlowJo, and creating accompanying step-by-step guides used to gate the data in the manner shown in appendix F. These guides were based on the following considerations.

The Guava easyCyte 8HT is a unique bench top microcapillary flow cytometry system that works with fixed voltages, therefore compensation controls are not technically required for each individual experiment. Given the particular importance of accurate compensation when working with autofluorescent cells such as neutrophils, and because a minor amount of 'drift' may occur in the machine settings over time, we elected to run a set of single stained samples during the first study visit for each participant. These samples were then used to produce compensation matrices that were used for the immunophenotyping flow analysis for all samples from each participant. When the FACSCanto ${ }^{\mathrm{TM}}$ II was used to acquire the intracellularly stained samples, pre-saved application settings were applied (as described in section 3.4.1.3.4) and compensation controls were run with every experiment.

For all analyses, doublets were first gated out using a plot of FSC-height (FSC-H) vs. FSC-area (FSC-A). In the ICS samples, non-viable cells were also excluded prior to any further analysis using the viability dye in the infrared channel. The gating hierarchy was optimised for each panel in order to maximise the accuracy and resolution. For example, in panel 2, which focused on immunophenotyping the $\mathrm{T}$ cell subsets, gating on the $\mathrm{CD}^{+}$cells first, as was done in other panels, was compromised by autofluorescence from the neutrophils in the near-infrared (NIR) channel. Instead, a broad FSC vs. SSC lymphocyte gate was applied prior to using a histogram gate to isolate the $\mathrm{CD}^{+}$events, which maintained a more pure population (refer to appendix F, Figures F.4 and F.5). 
Isotype controls for markers of activation and naivety (e.g. CD62L) were used on samples stained with the necessary cell surface markers to provide an accurate negative staining control for the corresponding population of interest. This negative control for each activation marker was then used to set default histogram and quadrant gates for this population. The analysis protocols divided these gates into those that should be individually adjusted to best suit each individual sample, such as the $\mathrm{CD}^{+}$histogram gates, or those that should only be altered for an entire analysis, such as the IL-6 and IL-8 quadrant gates, since these markers had a lower resolution and were far more sensitive to small changes in gating. The type of gate and view used was carefully selected to maximise the accuracy, such as using a quadrant gate with a contour view for delineating the boundaries with CD8 vs. CD62L (refer to appendix F, Figure F.5E and F).

Where data was expressed relative to a control sample, the population was compared to samples prepared in an identical fashion (i.e. unstimulated or stimulated and gated on the same population) but stained with isotype control antibodies. This was particularly important when analysing neutrophils, as stimulation alone can increase the baseline level of autofluorescence, and therefore comparison to an unstimulated control can create data artefacts.

For endpoints described by measures of fluorescence intensity (e.g. intracellular cytokine and activation marker levels), the data was expressed as the geometric mean of the population. We opted to use the mean, rather than the median, due to the increased sensitivity this provided for our data. Although the median is less affected by outliers or skewed data and therefore considered more robust for certain applications, we found only small populations of cells were activated during some assays, and using the median failed to detect and represent such subtle changes. 


\subsection{Discussion}

The work conducted in this chapter was focused on testing a series of laboratory assays and designing workflows to facilitate sensitive and accurate measurements of changes to the immune response. We found PMA/I induced measurable neutrophil degranulation where the traditionally used stimulant, fMLP, was ineffective in our laboratory. We tested stimulation protocols and protein transport inhibitors to finalise a protocol for the intracellular detection of IL-6 and IL-8 in cells from a whole blood sample. It was found that the use of a blocking agent and viability dye in immunophenotyping flow cytometry samples provided no additional value for data analysis; however, both were deemed important when performing intracellular staining. The CyQuant assay was adjusted to allow for the detection of proliferation in more concentrated cell samples, and was used to test culture conditions for polyclonal stimulation of $\mathrm{T}$ cells, and a novel protocol for using the tetanus vaccine to initiate an antigenspecific memory $\mathrm{T}$ cell response. Lastly, we developed laboratory, patienthandling, and data analysis workflows to minimise the effect of any potential experimental variation. One important limitation of this work was that the experiments were not repeated, therefore it is not possible to conclusively state whether the parameters selected for inclusion in the final assay protocols were the optimal choice.

We opted to perform ICS in our study in order to extend our investigation of RIPC on cytokine production beyond looking at isolated serum levels. Intracellular staining has the advantage of providing the means not only to detect changes in the amount of cytokines, but also discern which cell subsets are producing them. Neutrophils are a major source of tissue damage and proinflammatory mediators following cardiac surgery, therefore adjusting the ICS protocol to include these cells was a priority (Cassatella, 1995; Warren et al., 2009).

There is limited information regarding ICS in granulocytes available in the literature. Neutrophils are highly sensitive and capable of responding to numerous types of stimuli, making them vulnerable to activation during isolation 
and purification procedures. Previous work has confirmed that isolation protocols lead to dramatic increases in cell surface markers indicative of activation, including $\mathrm{C} 3 \mathrm{~b}$ receptors, which likely alter the in vitro responses of these cells (Fearon and Collins, 1983; Freitas et al., 2008). For this reason, we tested an ICS protocol that bypassed cell purification and instead utilised whole blood samples.

At the other end of the spectrum, the common anti-coagulants, EDTA and citrate, appear to reduce the responsiveness of neutrophils to in vitro stimulation (Engstad et al., 1997; Freitas et al., 2008; Repo et al., 1995). Both EDTA and citrate are calcium chelators, binding free calcium ions and thus removing a key element of multiple neutrophil signalling pathways (Pfister et al., 1984). This has a particularly significant impact on the ability of the cells to respond to stimulation with PMA. Typically, EDTA is the anti-coagulant of choice for neutrophil studies in part due to this inhibitory effect, which can be protective during purification procedures; however, the consequent artificial inhibition of neutrophil function was not suited to address our research question.

Although heparin has been shown to upregulate CD11b expression, it does not appear to inhibit neutrophil responses to stimulation (Freitas et al., 2008). We therefore used heparinised blood samples in our work, and sought to minimise any additional potential sources of neutrophil activation, such as temperature fluctuations and mechanical disturbances from repeated pipetting (Freitas et al., 2008). The low level of CD11b expression detected in our unstimulated samples, compared to those that were deliberately stimulated, suggested the heparintreated, non-purified neutrophils processed using our ICS protocol were preserved in a resting state. This combination of heparin, consistent temperature, and minimal manipulation appeared to provide us with the greatest ability and sensitivity for measuring neutrophil responses to RIPC.

We found PMA/I to be sufficient stimulators of IL-6, IL-8, and IL-10 production in all of the leukocyte subsets examined. LPS is the most commonly recommended stimulant of cytokine production in monocytes; however, our aim 
was to use ICS to assess the direct effect of RIPC on cytokine production, rather than looking at the way it modifies the response to an ischaemic injury. In order to assess changes in intracellular cytokine production, it was essential to stimulate the cells; therefore we opted to use the non-specific pharmacological activators, PMA/I, rather than LPS, which mimics a pathophysiological form of activation. LPS-stimulated cells were used as a positive control for IL-6 and IL-8 production in monocytes during each experiment; however, we found PMA/I stimulation appeared to be capable of inducing a comparable level of cytokine production.

While we were able to detect intracellular IL-10 in monocytes and T cells, our final ICS protocol required a high concentration of antibody in order to discriminate a reasonable signal. Our experience was consistent with the literature, which report only low measurements of intracellular IL-10 in leukocytes. Caraher et al. (2000) found that stimulating rat splenocytes for less than 24 hours with PMA/I did not induce any intracellular IL-10 production in CD4 $\mathrm{T}$ cells. Instead, a 5 day in vitro priming and re-stimulation protocol was necessary to induce a modest (3.19\%) population of IL-10 producing cells Likewise, Muris et al. (2012) reported only $0.45 \%$ of monensin treated human T cells stained positively for IL-10. Interestingly, this study also found that monensin significantly reduced the number of IL-10 positive cells, and brefeldin A had an even more detrimental effect. In the present work, we also found monensin slightly preferable to brefeldin A for IL-10 staining, but did not compare either to samples that were not treated with a protein transport inhibitor.

Despite monensin reportedly decreasing the overall number of IL-10 producing cells, Muris et al. noted that it appears to increase the amount of IL-10 in each cell, as indicated through higher MFI levels. In the absence of a significant population of cytokine producing cells, MFI is the most sensitive measure of changes in intracellular cytokines. With this in mind, and the knowledge that removal of monensin from the cultures would have a detrimental effect on IL-6 and IL-8 measurements, this slightly reduced IL-10 detection sensitivity was a 
reasonable compromise for the purpose of our study. However, ultimately the expense associated with the amount of antibody required for the full trial prevented us from including it in the final protocol. Instead, we turned to the serum CBA results for a more conclusive indication as to the effect of RIPC on IL10 production. Any future attempts to examine intracellular IL-10 production may require separate detection from other cytokines, using cultures without the addition of a protein transport inhibitor.

Although not fully described in this chapter, significant consideration was also given to the design and validation of the flow cytometry panels used for immunophenotyping the fresh whole blood samples. Antibodies, fluorophores, and markers were selected and pre-titrated using well-established tenets of flow cytometry that have been previously published in the literature, therefore this data was not presented (Baumgarth and Roederer, 2000; Mahnke and Roederer, 2007). One aspect that was described was the comparison of different methods for blocking non-specific antibody binding. The effect of blocking Fc receptors appeared negligible in our immunophenotyping samples, which is not surprising given we were staining in whole blood. Human serum contains sufficient levels of immunoglobulin to bind the Fc receptors present on leukocytes and prevent non-specific antibody binding (Sedlmayr et al., 2001). In contrast, our ICS samples were washed several times prior to extracellular staining, consequently removing the serum and necessitating the incorporation of a blocking step for these samples.

A key aim of our immunophenotyping assay was to measure any RIPC-induced changes in neutrophil activation. CD63 is found in the membrane of azurophilic granules and therefore is only present intracellularly in resting neutrophils and monocytes (Pfistershammer et al., 2004). Following cell stimulation, exocytosis of these granules results in the release of their lysosomal enzyme contents, and expression of CD63 on the cell surface, making it a useful marker of activation (Cham et al., 1994). It was interesting that despite successful activation of the cells, as shown through increased CD11b levels, we were unable to detect any granule exocytosis in neutrophils using fMLP, the agent of choice for such assays 
in the literature. Simard et al. (2010) demonstrated that fMLP stimulation of isolated neutrophils induces a significant, transient increase in CD63 expression, peaking at five minutes and returning almost to baseline levels within 30 minutes. It is possible that we missed the peak expression of CD63 with our 10 minute stimulation although this is unlikely, given we found no increase at all compared to the unstimulated sample levels.

Release of azurophilic granules requires a more potent stimulus than most other functional neutrophil responses (Sengeløv et al., 1993; van Eeden et al., 1999). The majority of neutrophil studies work with isolated cells, raising the possibility that the extra cell handling involved in these experiments could prime the neutrophils, thus reducing the stimulation threshold required for inducing degranulation. Irrespective of the reason for the lack of effect, our results indicated that fMLP was not an appropriate stimulant for use in our study. Using PMA/I treatment, we measured CD63 expression that was comparable to other work, and therefore used these alternative agents for stimulating the study samples (Estensen et al., 1974).

The majority of RIPC mechanistic research has focused on the interaction with components of the innate immune system; however, there is also evidence that RIPC may affect the acquired immune response (Sullivan et al., 2009). To explore this possibility, we wanted to analyse changes in T cell proliferation and cytokine production. Assays for measuring such responses to polyclonal stimulants are well described in the literature; however, testing the $\mathrm{T}$ cell recall response to tetanus required the development of a new stimulation protocol (Kruisbeek et al., 2004; Trickett and Kwan, 2003).

We elected to use the ADT Booster vaccine to stimulate our isolated PBMC. This vaccine consists of the tetanus toxoid adsorbed to an adjuvant, aluminium hydroxide $\left(\mathrm{Al}(\mathrm{OH})_{3}\right)$, and we found it induced a level of proliferation and IL-6 production in our PBMC cultures that was significantly higher than what we would have expected from a minor population of tetanus-specific memory cells. Although $\mathrm{Al}(\mathrm{OH})_{3}$ is not immunogenic itself, it is well established that adsorption 
of the tetanus toxoid onto this adjuvant markedly increases antigen-induced $\mathrm{T}$ cell proliferation (Mannhalter et al., 1985; Ulanova et al., 2001). It has been shown that $\mathrm{Al}(\mathrm{OH})_{3}$ increases the attraction and antigen uptake of APCs, stimulates monocytes to produce key cytokines such as IL-1, and promotes development of monocytes and macrophages into more mature, specialised APCs (Mannhalter et al., 1985; Rimaniol et al., 2004; Ulanova et al., 2001). The net result of these changes is a more pronounced $\mathrm{T}$ cell response compared to stimulation with the soluble tetanus toxoid alone, which could account for the substantial responses we measured. Further, the ADT Booster vaccine also contains the diphtheria toxoid, therefore likely stimulates an additional population of memory T cells in vaccinated individuals.

Interestingly, Ulanova et al. have reported that $\mathrm{Al}(\mathrm{OH})_{3}$ by itself upregulates MHC II molecule expression on monocytes, and induces monocyte and Th1 type cytokine production in human PBMC cultures. Consequently, one caveat of using the tetanus booster vaccine to stimulate memory $\mathrm{T}$ cells is that the resulting cytokine production must be analysed in the context of the enhanced immune response to the adjuvant. Interestingly, we did not detect any IL-6 production in the tetanus-stimulated cells from the individual who had not received a recent tetanus booster in our work, supporting the idea that at least the IL- 6 cytokine response was antigen-specific. However, analysis of IL-6 production from further donors that have not recently received a tetanus booster would be necessary to conclusively determine that the IL- 6 detected is the result of an antigen-specific response.

According to the literature, the potential stimulatory effect of the adjuvant on cytokine production is isolated; it appears $\mathrm{Al}(\mathrm{OH})_{3}$ does not directly elicit proliferation, suggesting any measured changes in cell numbers can be attributed solely to the antigenic response (Ulanova et al., 2001). In order to confirm this in the current work we would have also needed to culture cells with the adjuvant alone, which unfortunately was not available. The combination of the large magnitude of the proliferative response detected and the lack of a vehicle-only control in our testing therefore raises the possibility that the 
proliferative response detected in our PBMC cultures may not have been exclusively antigen-specific. Further work to validate this method, such as through the use of tetramers expressing the tetanus antigen and flow cytometry, would be valuable to confirm the specificity of the response.

A particular challenge of setting up our study in healthy volunteers was the volume of laboratory work associated with each participant, which required they be recruited individually. Great care was taken to ensure consistency in the measurement of endpoints between participants, such as bulk aliquoting and freezing of reagents to remove even the minor variability associated with the use of different pipettes and freeze-thaw cycles of reagents. In addition, lab workflows that specifically described the timing and order of sample handling were developed and tested in a pilot run. These protocols were an essential part of the strategy to minimise experimental variation and guarantee that the data between visits could be appropriately combined and compared.

\subsection{Conclusions and future directions}

Easily the most critical aspect of executing a successful clinical trial is in the study design, particularly in work that requires the accurate detection of subtle differences in endpoints. We undertook a process of assay and workflow testing to ensure we had the consistency and sensitivity necessary to distinguish any subtle alterations to the immune response induced by RIPC in healthy volunteers. The work presented in this chapter was critical to allow us to address the research aims of our next study, and establishes our confidence in drawing conclusions from the trial results presented in the following two chapters. 
Chapter 4.

\section{The systemic response to RIPC in healthy volunteers}




\subsection{Introduction}

Our next line of research utilised the assays tested in the previous chapter to investigate the effects of RIPC on the immune response in healthy volunteers. The results of this study are presented in Chapters $4-6$, and are preceded by an overview of what was known about the relationship between the immune response and preconditioning at the time our study was conceptualised.

Research investigating the mechanisms that contribute to IPC in human studies has been somewhat limited by the feasibility of collecting tissue samples and the variability between participants, compared with animal models. The majority of information regarding the pathways in preconditioning therefore stems from preclinical studies in animals; however, there is evidence that the mediators involved in preconditioning may differ in humans (Heusch et al., 2011). Further, although models of RIPC using limb ischaemia have more recently been developed in rats and mice, classical IPC is simpler to apply in small animals and the majority of the literature has focused on the effects of direct IPC. At present it is not fully understood what differences exist between the processes associated with IPC and RIPC. Despite the variation between the models used for preconditioning research, it is clear that the pathways between species and the different forms of preconditioning overlap in many respects; therefore, we evaluated all of the available literature when considering how RIPC might influence the human immune system (Moskowitz and Waeber, 2011).

The immune system encompasses the innate immune response, which provides a non-specific first line of defence against invading pathogens, and the adaptive immune response, which is characterised by antigen-specific protection and a memory for previously encountered pathogens. The focus of this chapter largely concerns the innate immune response and how preconditioning may induce cardioprotection by altering the course of inflammation, whereas Chapter 5 addresses the effect of RIPC on the functional response of the cells, with a particular focus on cells associated with the adaptive immune system. 


\subsubsection{The innate immune system and ischaemia-reperfusion injury}

The innate immune system forms the body's first protective response to invading pathogens and is largely mediated by monocytes, macrophages, and neutrophils (Janeway and Medzhitov, 2002). These cells are capable of responding to a broad range of stimuli including many that the body may not have previously encountered. This is achieved through the membrane bound and intracellular expression of pattern recognition receptors (PRRs) that detect pathogen-associated molecular patterns (PAMPs) (Chen and Nuñez, 2010). PAMPs utilise motifs that are highly conserved amongst classes of microbes and distinguishable from host molecules. Binding of these PAMPs to PRRs on innate immune cells triggers a signalling cascade that results in transcription of the key genes associated with host defence (Chen and Nuñez, 2010).

Traditionally the immune system was considered to rely on the distinction between foreign material and host molecules when mounting a response; however, it is now accepted that innate immune cells can also be stimulated by endogenous danger-associated molecular patterns (DAMPs) released from damaged host tissues (Matzinger, 2002). For instance, one class of PRRs, toll-like receptors (TLRs), have been shown to respond to heat shock proteins 60 and 70 and high-mobility group box 1 protein, following their release from necrotic cells (Basu et al., 2000; Ohashi et al., 2000; Park et al., 2004; Scaffidi et al., 2002). Such immune cell activation in the absence of an infectious agent is termed 'sterile inflammation', and is the critical process through which organ damage occurs in the setting of I/R injury (Eckle and Eltzschig, 2011).

During cardiac surgery the innate immune system responds to a set of diffuse stimuli resulting from interactions with the artificial surface of the CPB machine circuit and the I/R encountered in the heart and other tissues. This leads to a systemic inflammatory response involving activation of complement, upregulation of adhesion molecules on immune cells as well as myocytes and the endothelium, activation and tissue infiltration of inflammatory cells, and widespread cytokine release. Although monocytes and lymphocytes contribute to these processes, neutrophils are the main effector cells involved and their 
subsequent production of reactive oxygen species and other proinflammatory mediators propagates the detrimental response to surgery (for a comprehensive review refer to Warren et al., 2009). There is mounting evidence to suggest that IPC limits this peri-operative organ damage by modifying the course of the innate immune response.

\subsubsection{Evidence that preconditioning alters the innate immune response}

\subsubsection{Leukocyte activation and infiltration}

The most well characterised effect of IPC on the immune response is its ability to reduce neutrophil infiltration during I/R injury. Extravasation of neutrophils involves several key phases, including rolling of the neutrophil along the endothelium due to low affinity associations with selectin molecules, cell immobilisation through high affinity binding to integrins, and finally transmigration between the endothelial cells into the interstitial fluid (Gearing and Newman, 1993).

In murine and porcine models, classic IPC has consistently been shown to significantly decrease neutrophil infiltration and myeloperoxidase (MPO) activity (an indicator of polymorphonuclear cell accumulation) in the lungs following I/R (Harkin et al., 2002; Kinsey et al., 2010; Olguner et al., 2006; Peng et al., 2011). The reduction in neutrophil extravasation detected in preconditioned animals has been associated with decreased adhesion and increased leukocyte rolling velocity in murine hepatic sinusoidal venules and feline and rat mesentery (Erling et al., 2010; Howell et al., 2000; Kubes et al., 1998; Tapuria et al., 2009).

Davis et al. (1999) have shown that IPC completely attenuates the increase in Pselectin induced following I/R injury in the rat jejunum, and attributed this to a change in expression levels on the vascular endothelium. Likewise, IPC reduces P-selectin expression on endothelial cells and neutrophil accumulation in remote organs following hepatic I/R injury (Linden et al., 2006). Although there is evidence that preconditioning directly alters neutrophil adhesion molecule 
expression, these studies by David et al. and Linden et al. establish that the endothelium also plays a critical role in modulating leukocyte infiltration.

RIPC in healthy volunteers appears to have similar effects on neutrophils to those reported in animal studies of IPC, reducing adhesion, CD11b, and kinin receptor expression levels during the delayed preconditioning window 24 hours later (Konstantinov et al., 2004; Saxena et al., 2010b; Shimizu et al., 2010). Kharbanda et al. (2001) have reported that RIPC also significantly abrogates I/R induced CD11b expression on human neutrophils during the early phase of preconditioning, 15 minutes after reperfusion of a minor ischaemic injury. Further, Shimizu et al. have demonstrated that repeated RIPC suppresses neutrophil phagocytic activity. Taken together these studies strongly suggest that RIPC exerts a protective effect by reducing the ability of neutrophils to become activated and infiltrate tissues in the setting of I/R injury.

The effects of preconditioning on leukocytes are not limited to neutrophils. Delayed IPC has been shown to significantly reduce macrophage infiltration of the kidneys in rats, which was accompanied by a significant decrease in renal expression of the adhesion molecule, ICAM-1 (Jiang et al., 2007). In contrast, Cho et al. (2010) found that the renal protection afforded by delayed preconditioning in mice was associated with an increase in $\mathrm{CD} 11 \mathrm{c}^{+}$macrophages and dendritic cells and that depletion of these cells partially reversed the protective effect of IPC. The reason for the disparity between these two studies is unclear and further research is needed to clarify the role of macrophages and dendritic cells in the response to preconditioning.

\subsubsection{Platelets}

Mass activation of platelets during cardiac surgery contributes to the pathology of I/R injury through both the formation of microemboli that can interrupt the blood supply, and the release of pre-formed proinflammatory mediators stored in granules (Abu-Omar et al., 2004; Weerasinghe and Taylor, 1998). Two studies in canines have established that IPC significantly reduces platelet-mediated thrombosis, platelet-fibrinogen binding, and the formation of neutrophil-platelet 
aggregates in models of coronary stenosis (Hata et al., 1998; Linden et al., 2006). Equally, RIPC in humans attenuates the increase in monocyte-platelet aggregates induced by I/R injury and following ex vivo stimulation of blood from coronary artery disease patients subjected to exercise stress tests (Battipaglia et al., 2011; C. M. Pedersen et al., 2011). The mechanism through which IPC reduces platelet activation and reactivity is unclear, but ultimately appears to contribute by reducing the inflammatory burden following ischaemic injury.

\subsubsection{Transcription factors}

Some of the strongest evidence to suggest preconditioning affects the immune system stems from the repeated finding that both IPC and RIPC influence the activation of key transcription factors associated with the inflammatory response, including NF- $\kappa \mathrm{B}, \mathrm{HIF}-1 \alpha$, and STAT proteins. The classic notion that RIPC-induced changes in gene expression are limited to the delayed phase of preconditioning was challenged by the finding that administration of a nonspecific RNA-synthesis inhibitor, actinomycin-D, completely abolished the cardioprotection induced by early IPC in a porcine model (Strohm et al., 2002). Changes to gene transcription thus appear to be relevant during both windows of protection afforded by IPC.

A comprehensive review of the literature implicating NF- $\kappa B$, HIF- $1 \alpha$, and STAT signalling in IPC was presented in Chapter 1 and strongly suggests that the protective effects of preconditioning are mediated, at least in part, through a reduction in activation of these key factors and the resulting suppression of the inflammatory genes under their transcriptional control. This hypothesis is consistent with a key study performed by Konstantinov et al. in healthy volunteers that demonstrated RIPC suppresses numerous genes associated with cytokine synthesis, leukocyte chemotaxis, adhesion, migration, exocytosis, innate immunity signalling pathways, and apoptosis. These transcriptional changes were significant within 15 minutes of completing the preconditioning, during the early window of protection, and further enhanced after 24 hours, corresponding to the delayed phase of RIPC (Konstantinov et al., 2004). 


\subsubsection{Toll-like receptors}

TLRs are widely expressed on numerous cell types including neutrophils, macrophages, endothelial cells, and myocytes and can be activated by a wide variety of products released from damaged and necrotic cells (Boyd et al., 2006; Faure et al., 2000; Li et al., 2001; Sabroe et al., 2002). The exact role of TLRs in the pathology of I/R injury is unclear, with conflicting reports from studies in animals to date. It appears that the effect of TLR activation may depend on both the tissue examined and nature of the injury sustained (Chao, 2009).

With respect to the heart, TLR signalling results in NF- $\kappa B$ activation and production of the proinflammatory cytokines that contribute to the tissue damage elicited by I/R (F. Arslan et al., 2010; Li et al., 2001). Accordingly, it has repeatedly been shown that deletion of certain TLRs, namely TLR2 and TLR4, or the key TLR signalling molecules, MYD88 and IRAK-4, significantly improves outcome and reduces the infarct size sustained following myocardial I/R injury in mice (Chong et al., 2004; Favre et al., 2007; Feng et al., 2008; Hua et al., 2007; S.-C. Kim et al., 2007; Maekawa et al., 2009; Oyama et al., 2004; Riad et al., 2008; Shishido et al., 2003; Timmers et al., 2008; Zhao et al., 2009). Likewise, inhibition of TLR4 with the antagonist eritoran decreases infarct size and NF- $\kappa B$ activation, and administration of a leukocyte TLR2 signalling inhibitor treatment improves cardiac function during I/R (Fatih Arslan et al., 2010; Shimamoto et al., 2006). The protection detected in these studies seems to be associated with a reduction in tissue infiltration of inflammatory cells, cytokine production, and cardiomyocyte apoptosis (Ha et al., 2011).

Conversely, a number of studies have demonstrated that low-dose LPS stimulation of TLR4 can be protective against a subsequent ischaemic injury in the isolated heart, and TLR4-MyD88 signalling reduces cardiomyocyte apoptosis and improves function (Song et al., 1996; Wang et al., 2011; X. Zhu et al., 2006). Further, administration of TLR2 ligands in mice prior to myocardial I/R decreases infarct size and improves recovery of cardiac function (Ha et al., 2010; Mersmann et al., 2010). These discrepancies may be partially accounted for by limitations in models that involve systemic deletion of TLR genes, as inhibition of 
TLRs on endothelial and immune cells also impairs neutrophil recruitment and the resulting detrimental myocardial inflammatory response (Chao, 2009).

Dong et al. (2010) found the effects of IPC are abolished in TLR2 deficient mice, suggesting preconditioning may be at least partly mediated through TLR2 signalling. Interestingly, this study reported that the cardioprotective effect of IPC was unaltered in animals lacking TLR4, yet Yu et al. (2010) found that IPCinduced protection was associated with a significant decrease in expression levels of TLR4 and the associated cytokines, TNF- $\alpha$ and IL-1 $\beta$, in rats subjected to myocardial I/R. In addition, TLR4-deficient mice show a reduced ability to respond to IPC-induced neuroprotection (Pradillo et al., 2009). Further research is required to clarify the role of TLRs in IPC; however, it seems likely that these receptors may link the stimuli produced by ischaemic tissue to the NF- $\kappa B$ activation associated with preconditioning.

\subsubsection{Complement}

Complement activation during cardiopulmonary bypass contributes to tissue injury predominantly through the activation of neutrophils and platelets, and production of the membrane attack complex (MAC) that causes cell lysis (Miller and Levy, 1997; Warren et al., 2009). Not surprisingly, administration of a soluble form of CR1, which inhibits the key complement proteins C3b and C4b, decreases infarct size by $44 \%$ in rats subjected to myocardial I/R injury. This protection was associated with a reduction in MAC and leukocyte infiltration within the infarct zone (Weisman et al., 1990). Treatment of rats with antibodies against a different complement protein, C5, have also been shown to reduce infarct size, polymorphonuclear cell infiltration, and apoptosis in response to I/R injury (Vakeva et al., 1998). Further, similar antibody treatment in human cardiac surgery patients reduces myocardial damage by $40 \%$ and significantly decreases neutrophil activation (Fitch et al., 1999). Tanhehco et al. have demonstrated that IPC reduces infarct size and mRNA levels of C1q, C1r, C3, C8, $\mathrm{C} 9$, and MAC in the myocardial area at risk in rabbit hearts, both isolated and in vivo, highlighting another potential mechanism through which preconditioning may limit peri-operative tissue damage (Tanhehco et al., 2000, 1999). 


\subsubsection{Matrix metalloproteinases}

Matrix metalloproteinases (MMPs) are a family of enzymes produced by numerous activated cells, including leukocytes, that degrade extracellular matrix proteins (Parks et al., 2004). Levels of MMP-2 have been shown to increase with ischaemia in isolated perfused rat hearts, and inhibition of MMP-2 improves recovery of mechanical function (Cheung et al., 2000). In pigs, I/R has also been associated with a significant increase in MMP-9 activity in the myocardium (Danielsen et al., 1998; Lu et al., 2000).

Zitta et al. (2012) established a role for MMPs in the mechanism of RIPC with an interesting study that used sera collected from preconditioned cardiac surgery patients to treat an intestinal cell line subjected to hypoxia in vitro. It was determined that samples collected one hour after RIPC demonstrated reduced MMP-2 and 9 activity and were able to diminish hypoxia-induced cell damage, compared to samples collected prior to, or directly after, preconditioning. Further, Konstantinov et al. (2004) reported that RIPC significantly upregulates a key inhibitor of MMP-2 and 9, tissue inhibitor of metalloproteinases-1, both 15 minutes and 24 hours later. Taken together, these studies strongly suggest that regulation of MMP activity may contribute to organ protection following preconditioning.

\subsubsection{Cytokines}

Cytokines are the primary signalling molecules used by the innate immune system to direct inflammation, and function in both a paracrine and autocrine fashion to amplify or limit the cellular response (Valeur and Valen, 2009). There has been extensive research implicating cytokines in preconditioning, as was reviewed in Chapter 2. Briefly, animal studies have established that IL-6, IL-10, and TNF- $\alpha$ are essential for inducing preconditioning, yet proinflammatory cytokine levels are generally attenuated during I/R injury after IPC (Cai et al., 2012; Dawn et al., 2004; Ren et al., 2004; Smith et al., 2002; Takeshita et al., 2010; Xu et al., 2011). 
It is possible that induction of cytokine production during the preconditioning ischaemia may stimulate a negative feedback loop that decreases the response to subsequent ischaemic episodes. Alternatively, proinflammatory cytokines induced during the mild ischaemia associated with preconditioning may activate different pathways, compared to those elicited by a sustained ischaemic injury. For instance, TNF- $\alpha$ preconditioning has been shown to selectively modify NF- $\kappa \mathrm{B}$ activity to inhibit transcription of inflammatory genes while maintaining promotion of beneficial genes, such as the antioxidant enzyme, MnSOD (Ginis et al., 2002). Although the literature regarding cytokine expression with RIPC in the clinical setting is limited and variable, these findings from the animal studies suggest that cytokines may be a critical mediator involved in establishing preconditioning.

\subsubsection{Rationale for the study}

The results of trials evaluating RIPC in cardiac surgery patients have not found it to be as protective or consistent as was shown in pre-clinical animal studies. Establishing the pathways involved in the induction of RIPC will be essential in order to identify and address the hurdles faced in the clinical setting and to characterise the patient population that will benefit most from this intervention. Work in animal models has convincingly demonstrated that IPC modifies the innate immune response; however, it remains unclear whether RIPC in humans functions through the same pathways. In addition, it is not known which of these effects may contribute to the induction of preconditioning-induced cardioprotection, and which are simply the indirect results of an overall reduction in tissue damage. Thus, further work in human studies is required to fill this critical gap in the literature.

We designed the present study to investigate the mechanism through which RIPC operates in humans, with a focus on the early immune response. A clear theme has emerged from the previous research that suggests upregulation of components of the immune response during preconditioning results in attenuation of the same, often proinflammatory, mediators during ischaemic injury. In order to detect whether RIPC induces such changes, it was critical the 
study design maximised the ability to measure subtle and direct changes to the immune response. Measurement of inflammatory markers in the typical cardiac surgery patient is confounded by the underlying comorbidities and characteristics in this population that alter both the baseline immune state and the inflammatory response to surgery. We therefore administered the intervention to healthy volunteers to assess the direct effect of RIPC away from any form of ischaemic injury or comorbidities.

\subsection{Aims}

- To determine the effect of the RIPC intervention on serum levels of key inflammatory cytokines and biomarkers.

- To examine changes in the proportions of circulating leukocyte subsets induced by RIPC.

- To investigate whether RIPC alters the activation state of peripheral blood leukocytes. 


\subsection{Methods}

A paired-analysis trial of RIPC was performed with 10 healthy volunteers recruited over 8 months at the Medical Research Institute of New Zealand. The study was registered on the Australian New Zealand Clinical Trials Registry (ACTRN 12611000856910) and received ethics approval from the Central Regional Ethics Committee (CEN/11/06/034).

\subsubsection{Participants}

Healthy male volunteers who had received a tetanus vaccine or booster within the previous 10 years were invited to participate in the study provided they were over the age of 18 years, did not have peripheral vascular disease or an acute illness (specifically within one week of the study visits), were nonsmokers, and not taking any regular medications. Written informed consent was obtained from all participants by a study investigator.

\subsubsection{Study design \& application of RIPC}

In this unblinded, paired-analysis trial all participants received the control and RIPC treatment during two separate visits. Because the effects of RIPC have been reported to persist for several weeks, the trial was designed such that all subjects received the control treatment first and the RIPC during the second visit. A study investigator administered both treatments. On day 0 , participants received the control treatment, which involved a tourniquet being placed on the non-dominant upper arm for 30 minutes, without inflation. On day seven, the participants returned and received the RIPC, which consisted of applying the cuff to the same arm and inflating it to $200 \mathrm{mmHg}$ for 5 minutes (using an ATS 750 electric tourniquet system; Zimmer), followed by 5 minutes of deflation. This cycle was then immediately repeated twice more, and the cuff removed. An overview of treatment application during each study visit is presented in Figure 4.1.

\subsubsection{Blood sample collection}

During both study visits, four blood samples were collected from the contralateral arm at baseline, and 20 minutes, 1 hour, and 4 hours after the 
treatment using separate venepunctures with 23 gauge needles (Figure 4.1, Becton, Dickinson, and Company). Study investigators trained in phlebotomy performed the procedures and particular care was taken to minimise the use of a blood-taking tourniquet during sample collection.

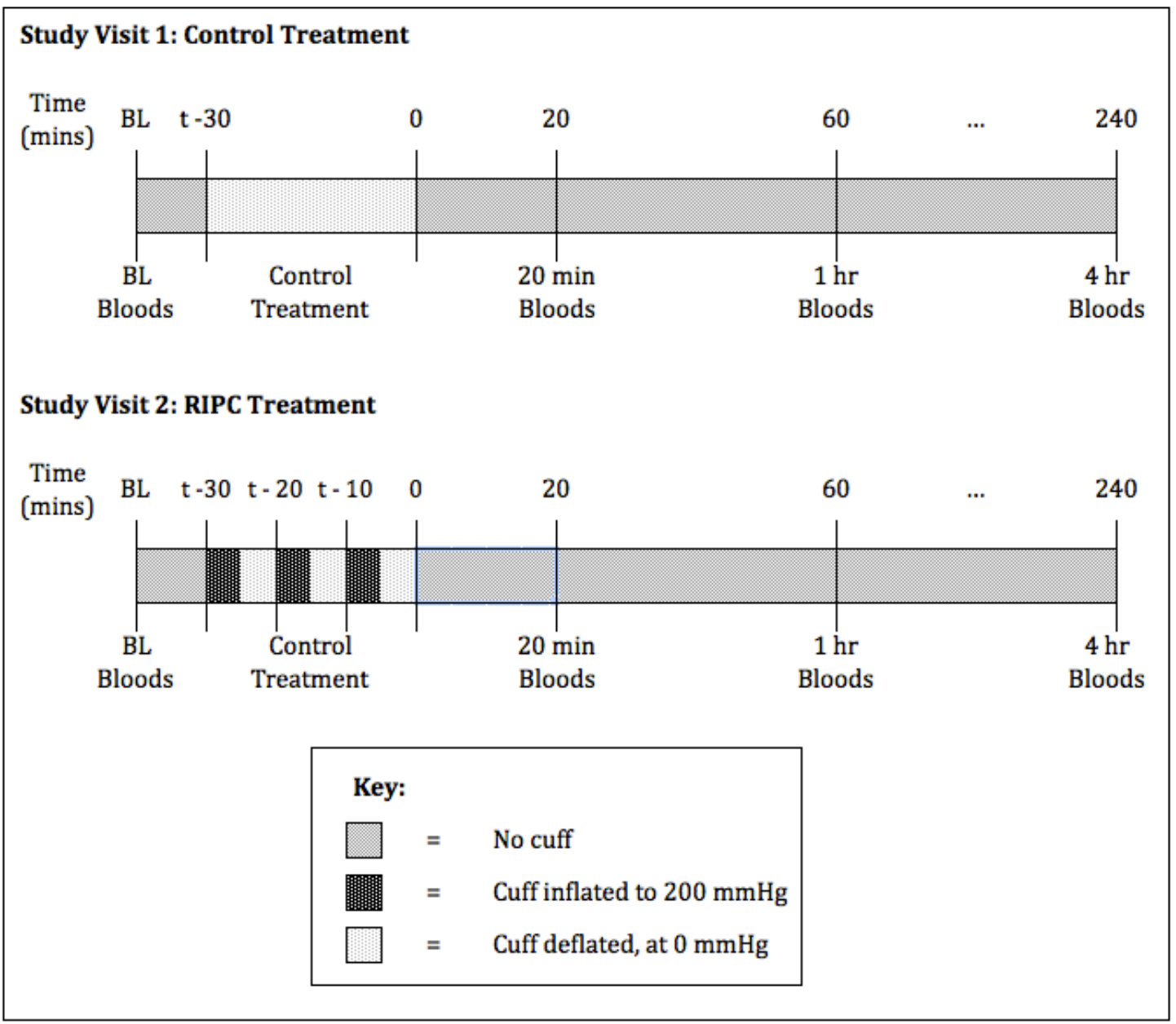

Figure 4.1 Overview of treatment administration and blood sample collection during study visits

Participants had baseline blood samples collected at the start of each session and always received the control treatment during the first visit, and RIPC at the second visit. Both treatments took 30 minutes to apply, including the final 5minute period of reperfusion with the RIPC, and the blood sample times were measured from the completion of the treatment. 


\subsubsection{Immunophenotyping}

Blood samples for flow cytometry were collected in heparinised vacutainers (Becton, Dickinson, and Company). Where a stimulated sample was indicated, an aliquot of blood was incubated with $50 \mathrm{ng} / \mathrm{mL}$ PMA and $500 \mathrm{ng} / \mathrm{mL}$ ionomycin (Sigma Aldrich) for 15 minutes at $37^{\circ} \mathrm{C}$, and cooled on ice for 3 minutes prior to staining. Unstimulated blood was kept at RT. All flow cytometry antibodies were pre-titrated in order to maximise the resolution between cell populations and minimise the background level of fluorescence, and all samples were stained in duplicate. Primary antibody mixes were diluted in FACS buffer to a total volume of $25 \mu \mathrm{L}$, and were then mixed with an equal volume of fresh whole blood. Following a 20 minute incubation at RT, protected from light, the samples were mixed with FACS lyse (BD Biosciences) to eliminate the red blood cells, and incubated for a further 10 minutes at RT. The samples were then centrifuged at $300 \mathrm{x}$ g for 5 minutes, the supernatant aspirated, and cells resuspended in FACS buffer. Samples were collected on a Guava easyCyte 8HT using InCyte software (Merck Millipore), and the data was analysed using FlowJo 7.6.1 software (Tree Star). Refer to appendix C for the composition of buffers, and appendix E for a complete list of antibodies and dilutions used.

\subsubsection{Serum collection}

Blood for serum samples was collected directly into silica-coated vacutainers (Becton, Dickinson, and Company). The tubes were inverted to mix several times, incubated at RT for 60 minutes, then centrifuged at $1500 \mathrm{x}$ g for 12 minutes. The serum was aspirated and stored in aliquots at $-80^{\circ} \mathrm{C}$ until further analysis.

\subsubsection{Serum cytokine quantification}

The serum cytokine levels were quantified using a human inflammation 20plex FlowCytomix kit (\#BMS819FFRTU; eBioscience). The kit consisted of 20 bead populations of two sizes with distinct fluorescence intensities that could be resolved in the red flow cytometer channel. Each population was coated with capture antibodies specific for one of the 20 analytes: E-Selectin, G-CSF, ICAM-1, IFN- $\alpha$, IFN- $\gamma$, IL-1 $\alpha$, IL-1 $\beta$, IL-4, IL-6, IL-8, IL-10, IL-12p70, IL-13, IL-17A, IP-10, LAP, MCP-1, MIP-1 $\alpha$, MIP-1 $\beta$, or TNF- $\alpha$. The beads, together with biotin- 
conjugated secondary anti-analyte antibodies, were incubated with the serum sample. Streptavidin (SA)-PE was then added to distinguish the cytokine-bound beads from the unbound population and the resulting fluorescence in the yellow channel used to determine the analyte concentrations within each sample, relative to a range of known standards. The beads were mixed and diluted 2 -fold with assay buffer and then washed by centrifugation at $3000 \mathrm{x}$ g for 5 minutes, aspiration of the supernatant, and resuspension in the same volume of reagent dilution buffer. The biotin-conjugate mixture was diluted 2-fold with assay buffer. The standards were reconstituted according to the manufacturer's instructions and prepared using a 3-fold serial dilution. Following reagent preparation, $25 \mu \mathrm{L}$ of undiluted serum and duplicate standards were incubated with $25 \mu \mathrm{L}$ of mixed beads and $50 \mu \mathrm{L}$ of biotin conjugate mix in a 96-well Vbottom plate for 2 hours at RT. At the end of the incubation the samples were washed twice by the addition of wash buffer, centrifugation at $400 \mathrm{x} g$ for 5 minutes, and aspiration of the supernatant. After the final wash all but $100 \mu \mathrm{L}$ of supernatant was removed and $50 \mu \mathrm{L}$ of SA-PE solution diluted 1:62.5 in assay buffer was incubated with each sample for a further hour at RT. The samples were then washed twice more, resuspended in assay buffer and acquired on a FACSCanto $^{\mathrm{TM}}$ II flow cytometer (BD Biosciences) set up according to the kit manufacturer's instructions. The data was analysed with BD FACSDiva (BD Biosciences) and FlowCytomixPro (eBioscience) software.

\subsubsection{Statistical analysis}

The number of subjects required for the study was calculated based on the preliminary analysis performed with cytokine data from the first 32 patients in the high-risk cardiac surgery with RIPC trial. Using paired sample t-tests, it was determined that a sample size of 10 would be adequately powered $(5 \%$ significance, $80 \%$ power) to detect differences between the two treatments at levels as low as $1.7 \mathrm{pg} / \mathrm{mL}$ for IL-10, and $4.3 \mathrm{pg} / \mathrm{mL}$ for IL-6 and IL-8. Differences in the serum cytokine concentrations of a lesser magnitude than could be detected using 10 participants would not be of physiological interest. 
The statistical analysis of the trial results was performed under the guidance of Dr Dalice Sim. Serum cytokine concentrations, proportions of immune cell subsets, and activation marker expression levels in unstimulated samples were compared between treatment groups and time points using a repeated measures two-way ANOVA with the Greenhouse-Geisser correction and Bonferroni posthoc tests. CD11b and CD63 expression in stimulated blood samples was compared to unstimulated levels using repeated measures three-way ANOVA.

Graphs were produced using GraphPad Prism 5 (GraphPad Software Inc) and statistical tests were performed using SPSS Statistics (IBM Corporation). Differences corresponding to $\mathrm{p}$ values $<0.05$ were considered statistically significant; however, the high number of statistical tests performed for the study was also taken into consideration when interpreting the results. Comparisons were not found to be significant unless specified. 


\subsection{Results}

\subsubsection{Participants}

10 healthy male volunteers were recruited with a mean age of 23.3 years (range 19.1 - 29.4) and body mass index of $23.4 \mathrm{~kg} / \mathrm{m}^{2}$ (range 18.7 - 30.6). All participants had received a tetanus booster vaccine within the last 1 - 8 years (mean 4.2), and showed no symptoms of acute illness at the time of the study visits. The RIPC intervention was well tolerated by all participants, with no adverse effects.

\subsubsection{The effect of RIPC on serum cytokine levels}

Following on from the results of our cardiac surgery trial, which showed a slight trend towards higher levels of IL- 6 and IL-8 in the preconditioned patients, we wanted to determine if the RIPC intervention directly altered systemic cytokine levels. Furthermore, it is clear that induction of RIPC involves transmission of the protective signal from the preconditioned tissue to the heart and other remote organs by an undefined humoral mediator. One of the primary aims of our study was therefore to screen serum samples for increases in a selection of cytokines and soluble markers that may be relevant to eliciting ischaemic tolerance. This was achieved using a CBA kit to assay 20 key mediators of the inflammatory response in samples collected up to four hours following preconditioning.

There were two participants that had consistently elevated levels of prototypic inflammatory cytokines, including IL-12p70, IFN- $\gamma$, and TNF- $\alpha$, at both the baseline and post-treatment measures during the control and RIPC visits, and this data is presented in appendix G (Figure G.1). Despite screening for symptoms of acute illness at the start of each study session, it was possible that these participants had underlying inflammatory processes occurring at the time of the blood sampling that could not be separated from the effect of the treatment; therefore, they have been excluded from the serum cytokine analyses presented in the following sections. 


\subsubsection{Adhesion molecules}

We found measurable levels of the soluble forms of both adhesion molecules examined, ICAM-1 and E-selectin, in the serum from our healthy volunteers and these did not differ between the control and RIPC treatment samples (Figure 4.2A and C). To account for very subtle differences in the baseline levels between the two groups, the data was also expressed normalised to the baseline concentrations (Figure 4.2B and D). This analysis emphasised the slight decrease in ICAM-1 expression at 4 hours in the preconditioned group, and reductions in all post-treatment measurements of E-selectin; however, these trends were not statistically significant.

It should be noted that one participant had unusually high levels of E-selectin in every sample and this data was excluded from the final analysis for two reasons. First, the complete dataset was considered an outlier, relative to the other participants. Second, the levels exceeded the maximum detection range of the assay, and therefore lacked accuracy. The slight loss of statistical power from this exclusion was of little consequence given that RIPC did not appear to affect serum expression of the cellular adhesion molecules up to four hours posttreatment. The E-selectin data for all participants is presented in appendix G (Figure G.1A). 


\section{ICAM-1}

A

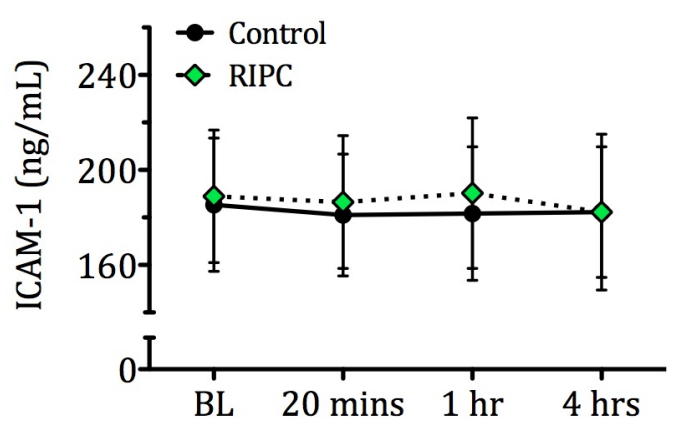

B

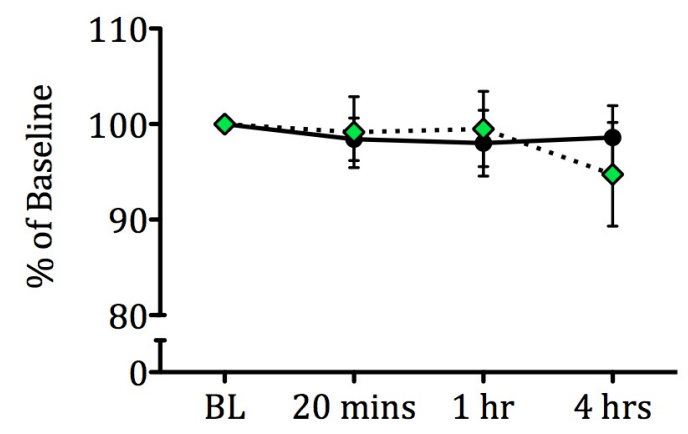

\section{E-Selectin}

C

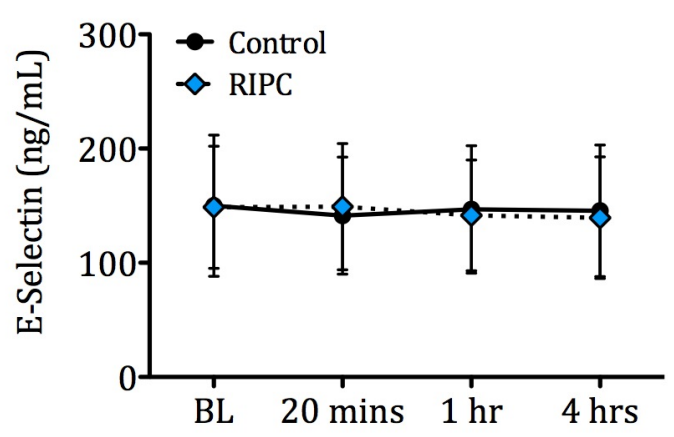

D

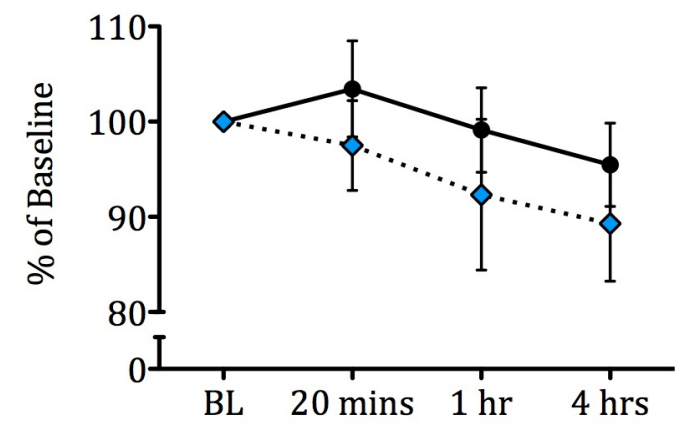

Figure 4.2 Serum levels of adhesion molecules following RIPC

Blood samples were collected from healthy volunteers at baseline, and 20 minutes, 1 hour, and 4 hours after control or RIPC treatment and serum biomarker levels were quantified using a CBA and flow cytometry. (A) Absolute ICAM-1 levels; (B) ICAM-1 levels normalised to baseline expression; (C) absolute E-selectin levels; (D) E-selectin levels normalised to baseline expression. A twoway repeated measures ANOVA was used to compare the treatments and time points. Shown are the means and SEM for $\mathrm{n}=8$ for the ICAM- 1 analysis, and $\mathrm{n}=7$ for E-selectin. 


\subsubsection{Chemokines}

Many of the protective effects of RIPC correspond to activities induced by chemokines; therefore their levels were of particular interest to us in the present study. We detected a small but statistically significant reduction in MIP-1 $\alpha$ expression following preconditioning, compared to the control treatment (Figure 4.3A). The levels appeared to decrease from baseline concentrations as early as 20 minutes, and stabilised between the 1 and 4 hour samples (Figure 4.3B). Looking at the total change between the levels at baseline and 4 hours for each participant whilst comparing the control and RIPC visits, it appeared this change in the pattern of MIP-1 $\alpha$ expression was very subtle. (Figure 4.3C).

No difference in MIP-1 $\beta$ between the control and preconditioning treatments was detected with the absolute levels; however, when the small difference in baseline expression levels between the groups was accounted for by expressing the data relative to the pre-treatment blood samples, there was a significant reduction in MIP-1 $\beta$ after RIPC (Fig 4.3D and E). A closer look at the 4-hour measurements showed that 6 out of 8 participants had increases in MIP-1 $\beta$ levels during the control visit, whereas 7 out of 8 individuals had decreases in MIP-1 $\beta$ following the preconditioning (Figure 4.3F).

Levels of MCP-1 were significantly reduced at 1 and 4 hours post-treatment, compared to baseline, but did not differ between the two treatment groups (Figure 4.4A). Expressing the MCP-1 levels relative to the baseline concentration for each individual emphasised the decrease across the time points and a significant difference was detected as early as 20 minutes after treatment for both groups (Figure 4.4B). Likewise, expression of IP-10 and IL-8 was not affected by preconditioning and maintained relatively consistent levels across the 4-hour sampling period (Figure 4.4C-F). When the IL-8 data was normalised to the pre-treatment levels it appeared that the concentrations during the control visit may have increased at 20 minutes and 1 hour after treatment, compared to during the RIPC visit; however, there was a high level of variability in the response between individuals and this difference was not statistically significant (Figure 4.4F). 

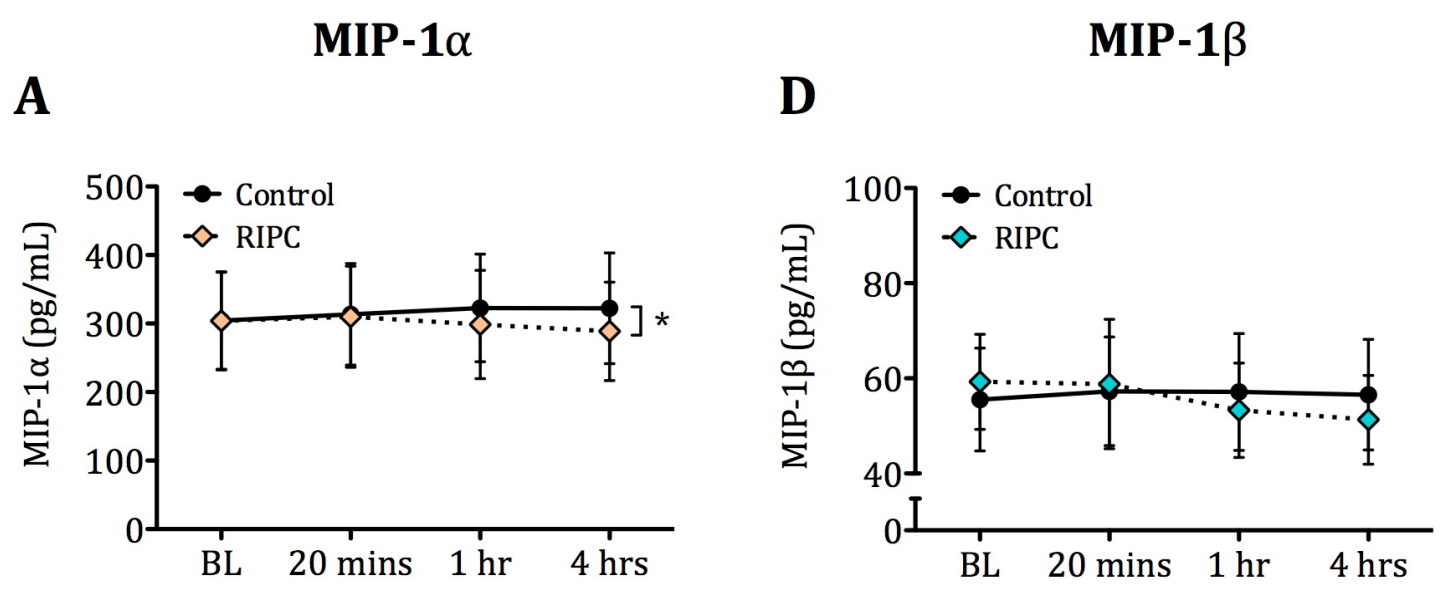

B

$\mathbf{E}$
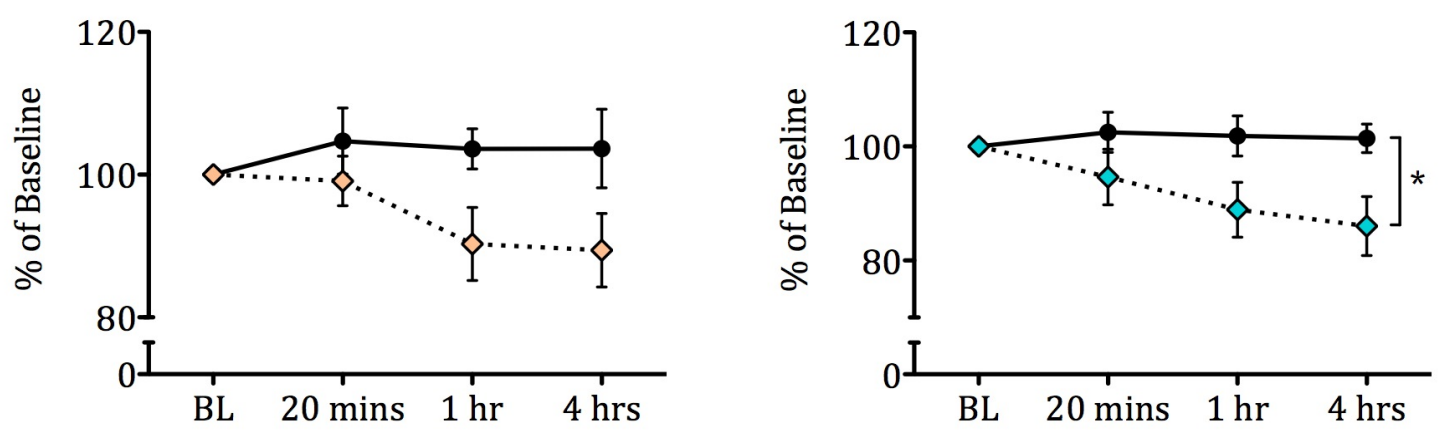

C

$\mathbf{F}$
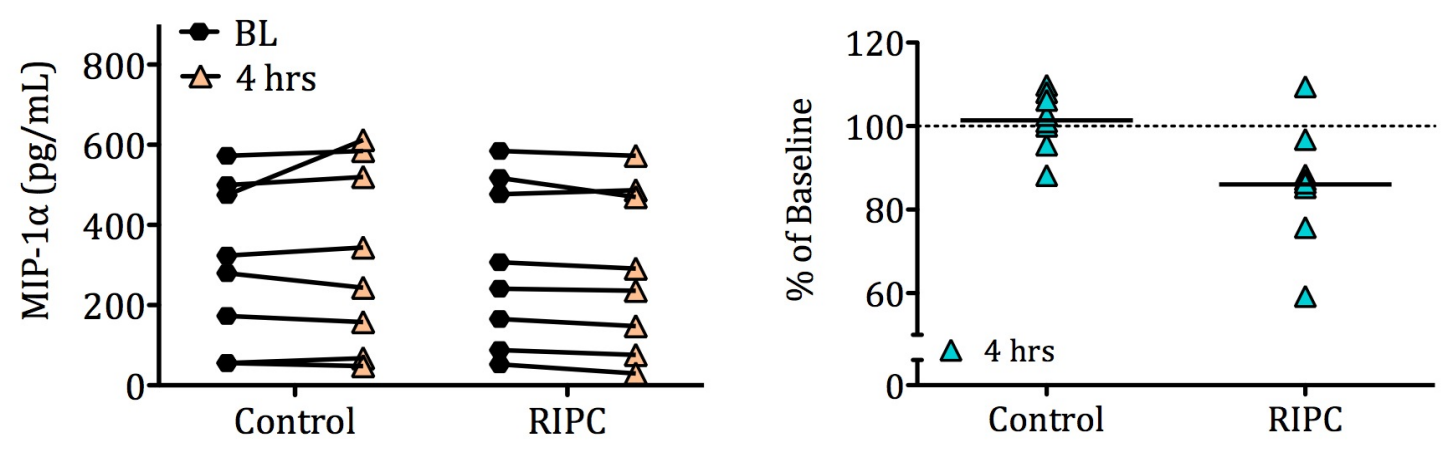

Figure 4.3 Serum levels of macrophage inhibitory protein 1 after RIPC

Blood samples were collected from healthy volunteers at baseline, and 20 minutes, 1 hour, and 4 hours after control or RIPC treatment and serum biomarker levels were quantified using a CBA and flow cytometry. Left: MIP-1 $\alpha$ presented (A) as absolute levels, (B) normalised to baseline expression, (C) as total change from baseline to 4 hours for each participant. Right: MIP-1 $\beta$ presented (D) as absolute levels, (E) normalised to baseline expression, (F) as 4 hour levels expressed relative to baseline for each participant. A two-way repeated measures ANOVA was used to compare the treatments and time points, with Bonferroni post-hoc tests. ${ }^{*} \mathrm{p}<0.05$. Shown are the means and SEM for $\mathrm{n}=$ 8 participants. 


\section{MCP-1}

A

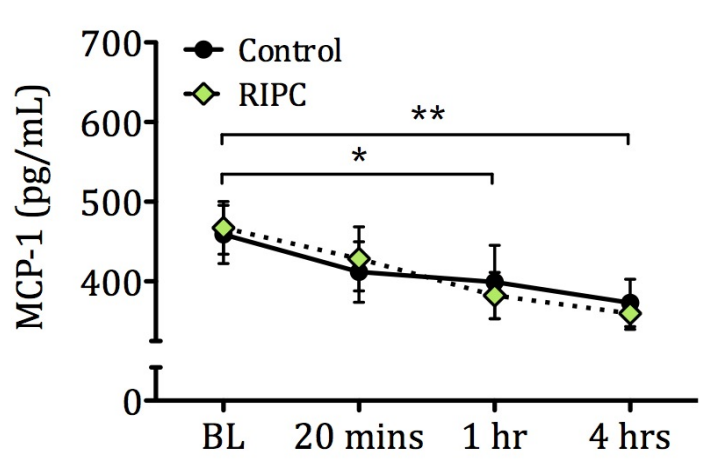

B

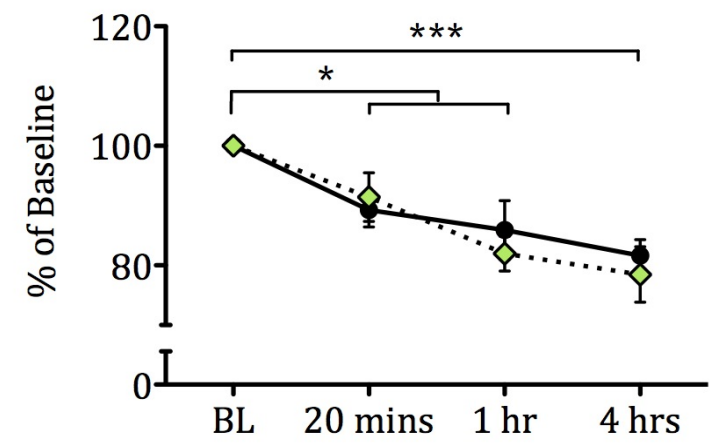

\section{IP-10}

C

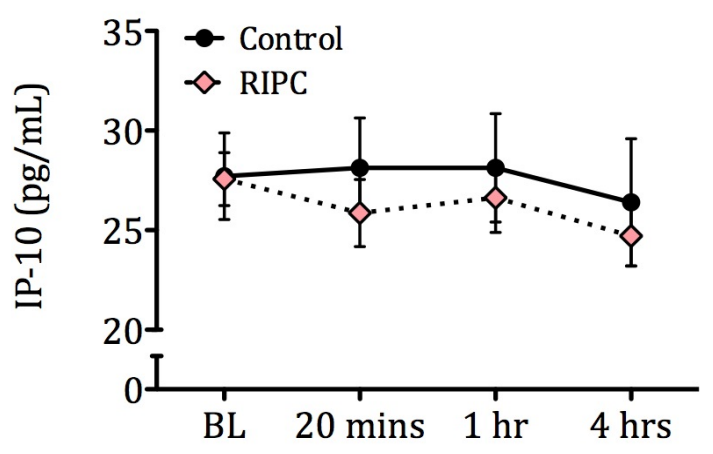

D

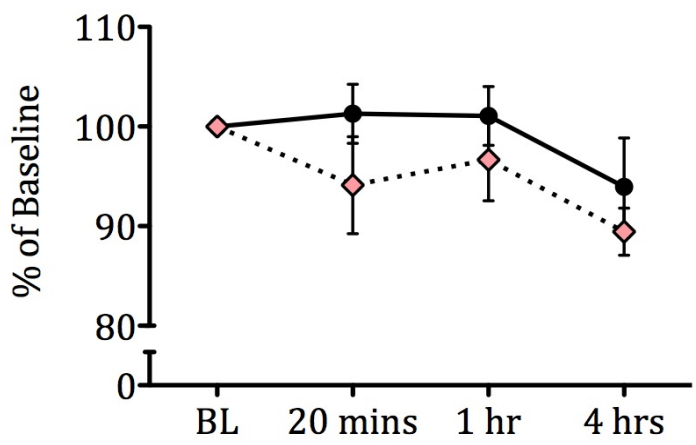

\section{IL-8}

$\mathbf{E}$

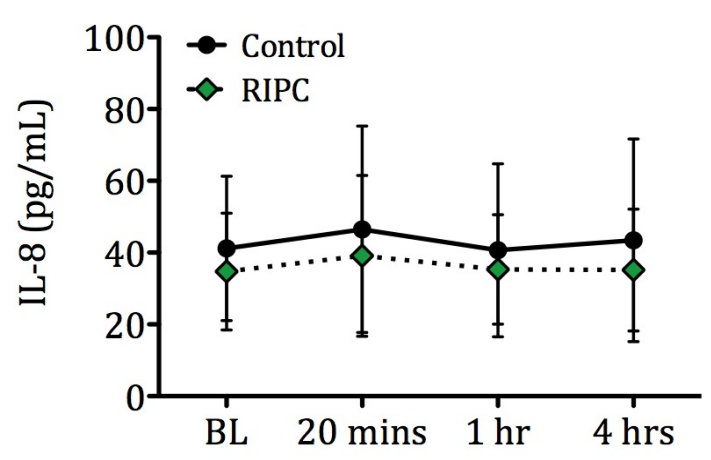

$\mathbf{F}$

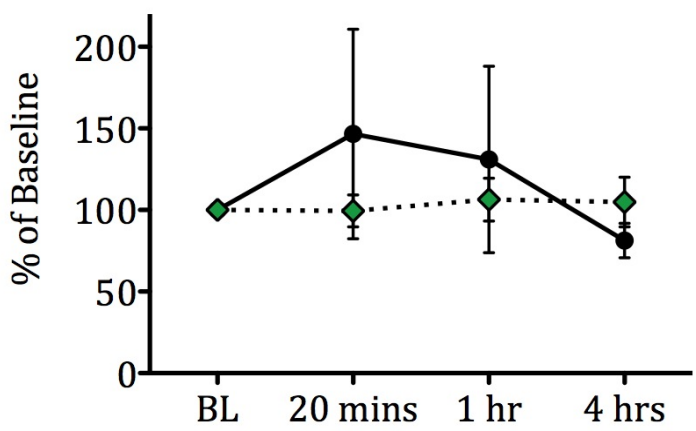

Figure 4.4 Post-RIPC systemic chemokine levels

Blood samples were collected from healthy volunteers at baseline, and 20 minutes, 1 hour, and 4 hours after control or RIPC treatment and serum biomarker levels were quantified using a CBA and flow cytometry. Expression levels are presented for (A and B) MCP-1, (C and D) IP-10, and (E and F) IL-8. Shown are absolute concentrations (A, C, and E), and data normalised to baseline levels (B, D, and F). A two-way repeated measures ANOVA was used to compare the treatments and time points, with Bonferroni post-hoc tests. ${ }^{*} \mathrm{p}<0.05,{ }^{* *} \mathrm{p}<$ 0.01 , and ${ }^{* * *} \mathrm{p}<0.001$. Shown are the means and SEM for $\mathrm{n}=8$ participants. 


\subsubsection{T cell cytokines}

Upon activation, $\mathrm{T}$ cells can produce numerous cytokines to assist and direct the immune response; the exact profile of which depends on the type of response elicited. In measuring cytokines commonly associated with $\mathrm{T}$ cells, we found a general trend towards decreasing serum levels of IL-13 across the time points, but this trend did not differ between the groups or reach statistical significance ( $p=0.087$; Figure 4.5A). Similarly, IFN- $\gamma$ levels decreased between the baseline and 1-hour samples in both groups, but appeared to return to resting levels by 4 hours (Figure 4.5C). Although the theoretical limit of detection for IFN- $\gamma$ with the CBA kit used was $1.6 \mathrm{pg} / \mathrm{mL}$, we observed that any samples with MFI values corresponding to concentrations lower than $44.85 \mathrm{pg} / \mathrm{mL}$ were automatically designated as 'zeros' by the software analysis. The sensitivity of our IFN- $\gamma$ measurements was therefore inadequate for this analysis and the results should be interpreted with caution. Normalising the data to the pre-treatment cytokine levels did not alter the pattern of expression seen with either treatment (Figure $4.5 B$ and D).

IL-17A levels appeared fairly consistent across the time points and treatment groups, with the exception of notably different levels in the baseline samples (Figure 4.5E). When this was accounted for by normalising the data relative to the pre-treatment IL-17A levels, there was a trend towards lower expression in the preconditioned participants at 20 minutes and 1 hour after treatment; however, this trend did not reach statistical significance and the large error bars in the control group data suggested there was a high level of variability between participants ( $\mathrm{p}=0.064$; Figure 4.5E). A closer look at the individual replicates indicated that one participant had remarkably lower baseline IL-17A levels during the control visit and when the data was expressed relative to these baseline levels the values for the subsequent time points thus appeared to be inflated. Removal of this participant markedly reduced both the difference between the groups and the variability in the control visit mean data (refer to appendix G, Figure G.2). Overall, it appeared that RIPC did not alter serum IL-17A levels. 
Minimal levels of IL-4 were measured and most fell below the theoretical limit of detection for the assay (20.8 pg/mL; data not shown). Of notable exception to this were the levels in the two participants that were excluded from the analysis due to heightened baseline inflammatory marker expression, confirming our CBA was capable of measuring IL-4 levels when they were present (refer to appendix G, Figure G.1R). Our results did not suggest the $\mathrm{T}$ cell response was polarised towards any particular Th cell subtype. 


\section{IL-13}

A

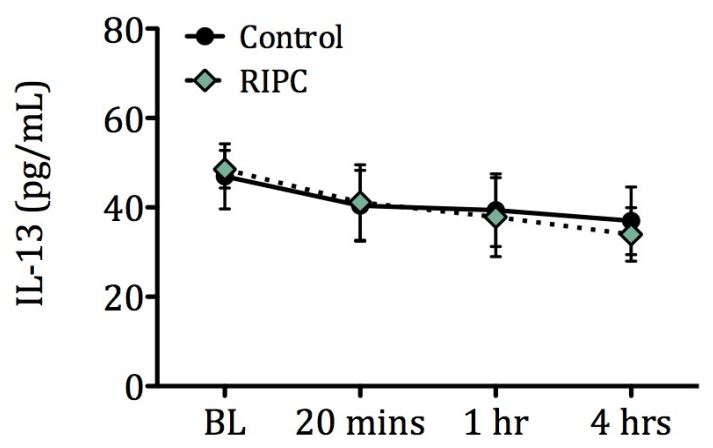

B

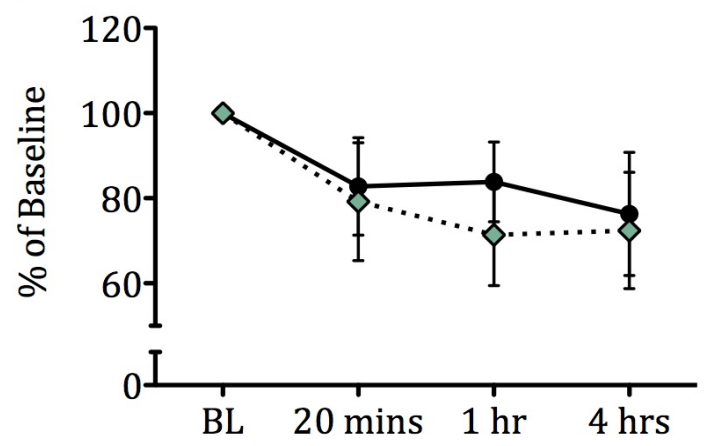

IFN- $\gamma$

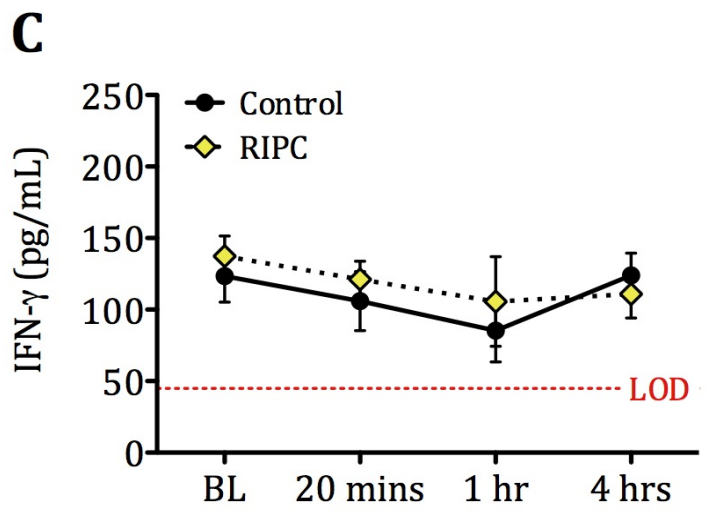

D

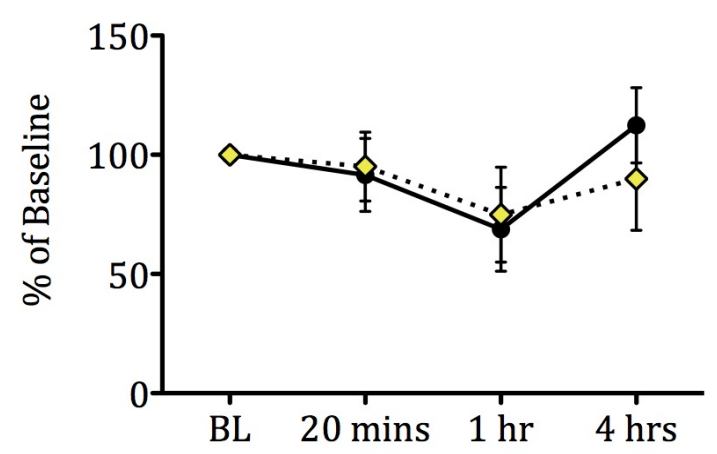

IL-17A

$\mathbf{E}$

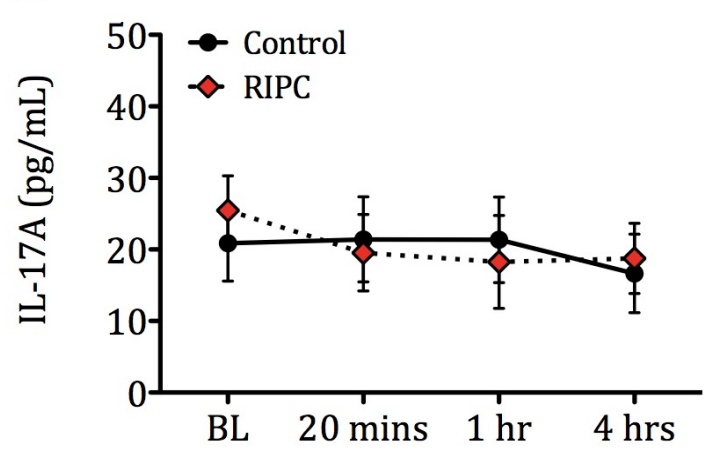

$\mathbf{F}$

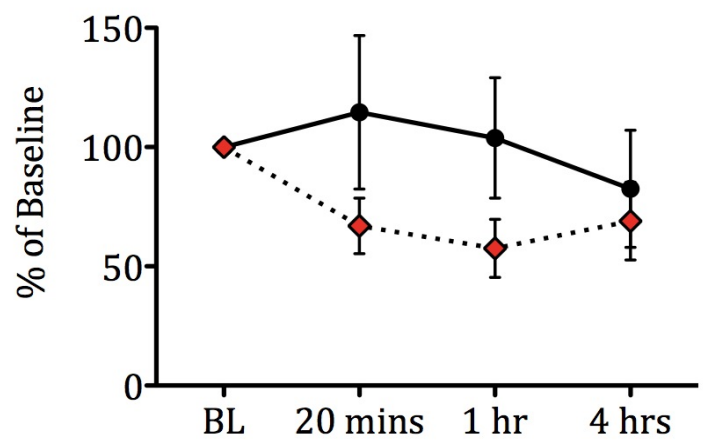

Figure 4.5 Serum T cell cytokine expression after RIPC

Blood samples were collected from healthy volunteers at baseline, and 20 minutes, 1 hour, and 4 hours after control or RIPC treatment and serum biomarker levels were quantified using a CBA and flow cytometry. Expression levels are presented for (A and B) IL-13, (C and D) IFN- $\gamma$ along with the assay limit of detection (LOD), and (E and F) IL-17A. Shown are absolute concentrations ( $\mathrm{A}, \mathrm{C}$, and $\mathrm{E}$ ), and data normalised to baseline levels (B, D, and F). A two-way repeated measures ANOVA was used to compare the treatments and time points. Shown are the means and SEM for $\mathrm{n}=8$ participants. 


\subsubsection{General inflammatory cytokines}

In our participants, serum levels of the generally pro-inflammatory cytokines TNF- $\alpha$ and IL-1 $\beta$, pleiotropic IFN- $\alpha$, and regulatory IL-10 did not significantly change between the treatment visits or time points (Figure 4.6A-E). The data could not be expressed as a percentage of the baseline levels for TNF- $\alpha$, IL-1 $\beta$, or IL-10, as numerous participants had no detectable levels prior to treatment. There was a slight trend towards lower expression of IL-1 $\beta$ in the preconditioned participants at $4 \mathrm{hrs}$; however, this trend did not reach statistical significance and there also appeared to be an equal but opposite difference at the baseline measure ( $p=0.152$; Figure 4.6D). The actual limit of IL-1 $\beta$ detection for our assay run was notably higher than the theoretical limit reported by the manufacturer, and this reduction in sensitivity may have contributed to the high level of variability in the measurement of this cytokine. Of note was that the circulating levels of IL-10 were very low both before and after treatment, supporting a healthy immune status for our participants (Figure 4.5E).

Interestingly, levels of IL-12p70, which is typically associated with proinflammatory effects, appeared to decrease between baseline and 4 hours in the preconditioned participants and increase for the control group (Figure 4.7A). The overall levels of IL-12p70 were low and variable, leading to wide error bars and no statistically significant difference between the two groups. The data could not be expressed relative to the baseline levels, as several participants had no detectable IL-12p70 prior to treatment; however, a closer look at the overall change between the control and RIPC treated patients indicated that the majority of participants individually mirrored the relationship seen in the mean data, with control patients showing an increase in IL-12, and the preconditioned participants showing a reduction (Figure 4.7B). Although the levels were too low to draw conclusive results in this work, these data suggest IL-12p70 may be a cytokine of interest to future RIPC studies.

Serum LAP expression was significantly lower in the 1 and 4 hour samples for both treatment groups (Figure 4.7C). The baseline levels in the RIPC group were slightly higher and when this was adjusted for by expressing the data relative to 
the daily baseline value, there was a larger decrease in LAP expression in the preconditioned participants (Figure 4.7D). Nonetheless, this effect did not reach statistical significance; therefore, overall it appears RIPC did not affect LAP concentrations. Levels of G-CSF, which generally functions to dampen down inflammation, were also similar between the two groups and did not change up to 4 hours post-treatment (Figure 4.7E). Accounting for the higher baseline levels in the RIPC group by normalising the data suggested there was a slight reduction in G-CSF in the preconditioned participants, but this effect was small and again, did not reach statistical significance (Figure 4.7F).

The serum concentrations of IL-1 $\alpha$ and IL-6, which are regarded as proinflammatory and pleiotropic cytokines, respectively, were generally close to, or below, the limits of detection of the assay $(0.5$ and $1.2 \mathrm{pg} / \mathrm{mL}$, respectively; data not shown). However, measurable levels of these cytokines were detected in the participants that were excluded from the analysis, confirming the capability of measuring IL- $1 \alpha$ and IL-6 levels in samples, if present, using our methodology (refer to appendix G, Figure G.1S and T). Overall, our results provided no indication that RIPC directly promoted a proinflammatory or antiinflammatory cytokine response. 
IFN- $\alpha$

A

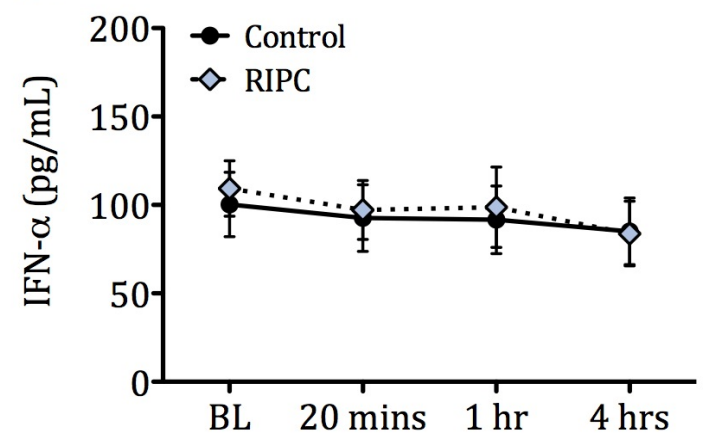

TNF- $\alpha$

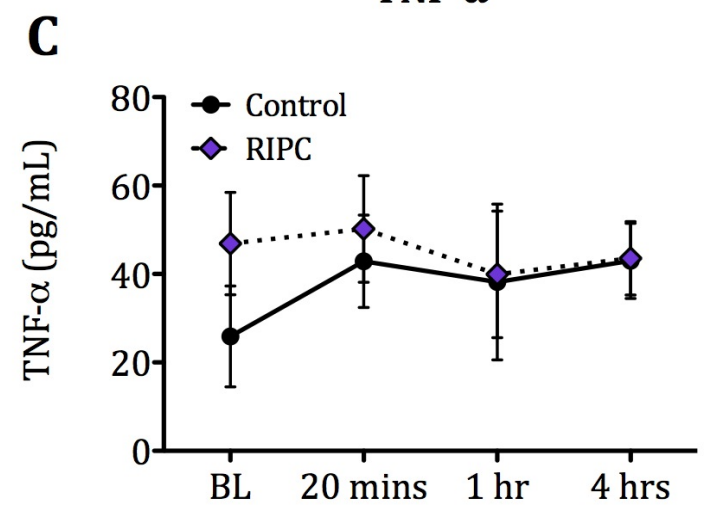

IL-10

$\mathbf{E}$

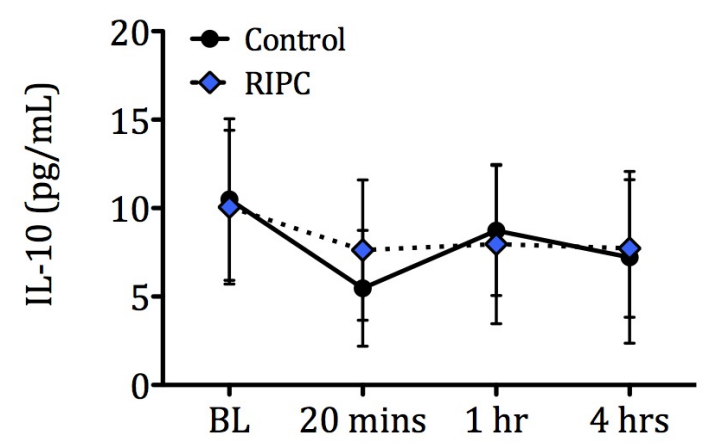

B

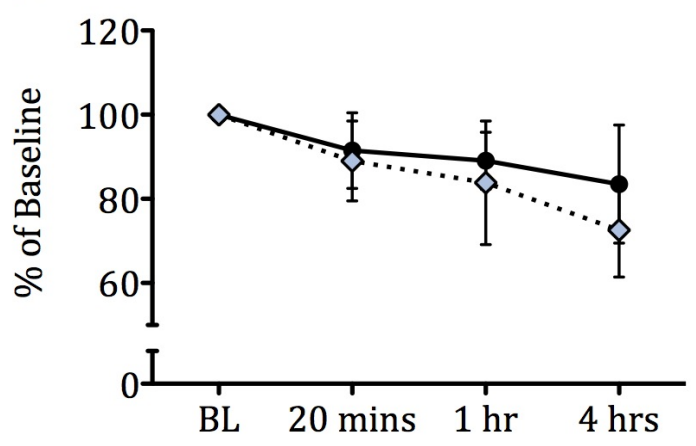

IL-1及

D

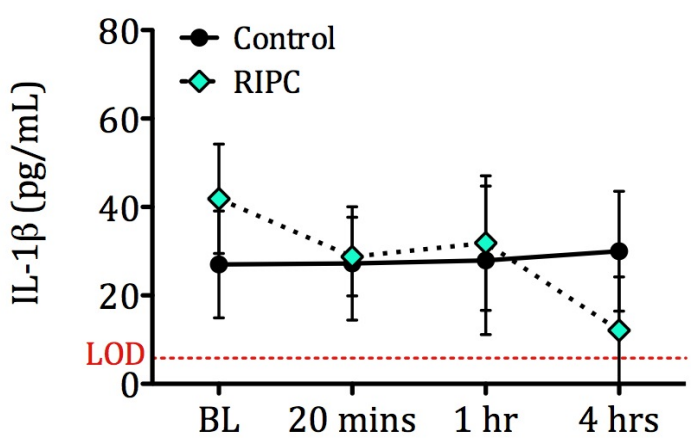

Figure 4.6 Systemic levels of TNF $\alpha$, IL-1 $\beta$, IFN- $\alpha$, and IL-10 following RIPC

Blood samples were collected from healthy volunteers at baseline, and 20 minutes, 1 hour, and 4 hours after control or RIPC treatment and serum biomarker levels were quantified using a CBA and flow cytometry. Shown are (A) the absolute levels of IFN- $\alpha$; (B) IFN- $\alpha$ levels normalised to baseline expression; (C) absolute levels of TNF- $\alpha$; (D) absolute levels of IL- $1 \beta$ and the assay limit of detection (LOD); (E) absolute levels of IL-10. A two-way repeated measures ANOVA was used to compare the treatments and time points. Shown are the means and SEM for $\mathrm{n}=8$ participants. 


\section{IL-12p70}

A

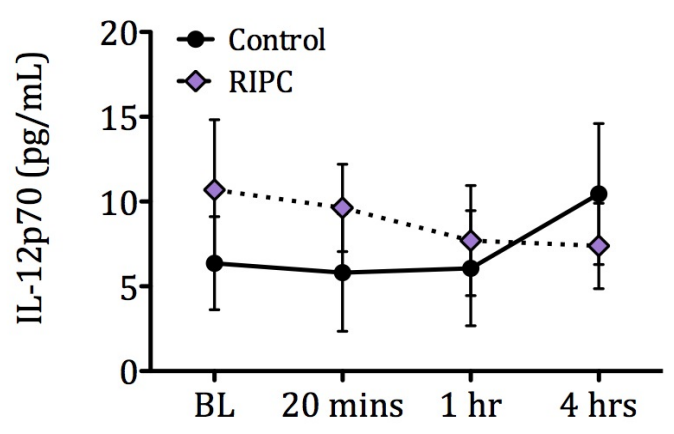

B

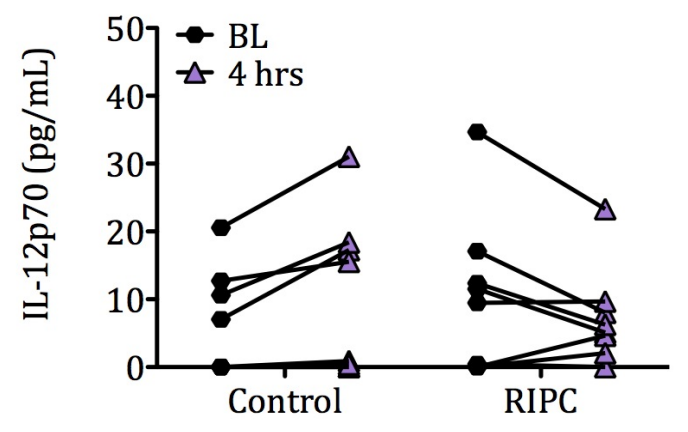

\section{LAP}

C

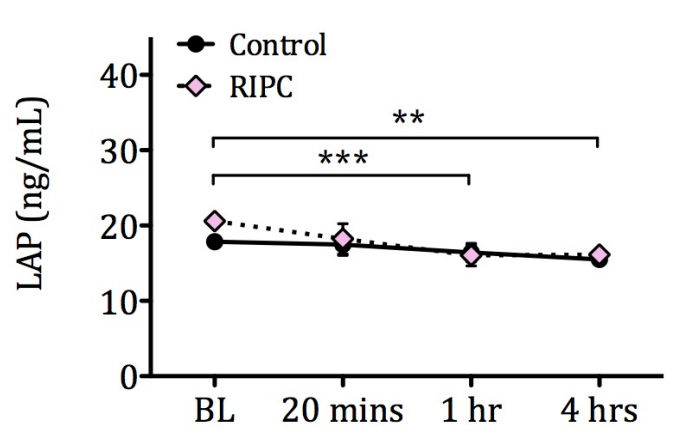

D

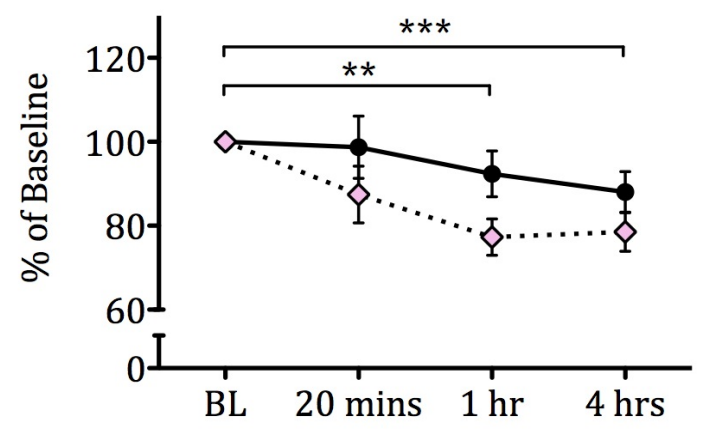

\section{G-CSF}

$\mathbf{E}$

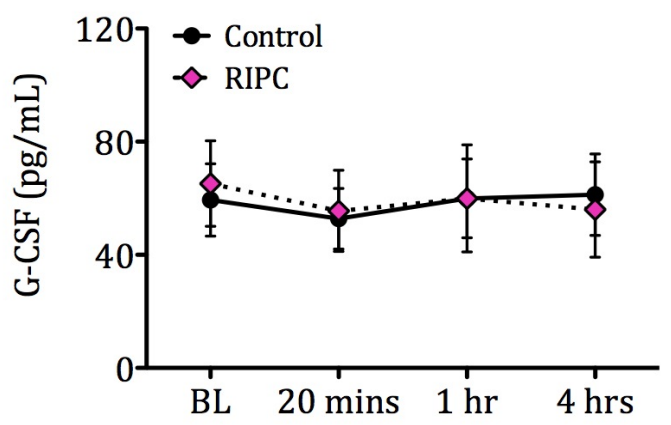

$\mathbf{F}$

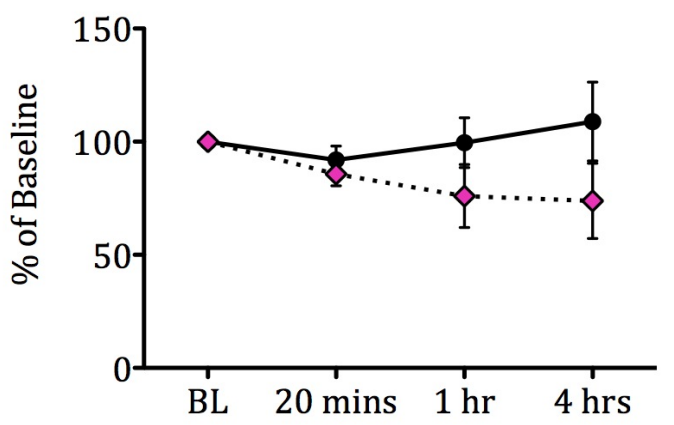

Figure 4.7 Post-RIPC systemic levels of IL-12p70, LAP, and G-CSF

Blood samples were collected from healthy volunteers at baseline, and 20 minutes, 1 hour, and 4 hours after control or RIPC treatment and serum biomarker levels quantified using a CBA and flow cytometry. Expression levels are presented for (A and B) IL-12p70, (C and D) LAP, and (E and F) G-CSF. Shown are absolute concentrations (A, C, and E), and the change in IL-12p70 from baseline to 4 hours for each participant (B), or data normalised to baseline levels (D and F). A two-way repeated measures ANOVA was used to compare the treatments and time points, with Bonferroni post-hoc tests. ${ }^{* *} \mathrm{p}<0.01$ and ${ }^{* * *} \mathrm{p}$ $<0.001$. Shown are the means and SEM for $\mathrm{n}=8$ participants. 


\subsubsection{Immunophenotyping of peripheral blood cells following RIPC}

To determine whether RIPC directly altered the proportions or activation states of circulating peripheral leukocytes, we performed immunophenotyping with flow cytometry on blood samples collected at baseline, and 20 minutes, 1 hour, and 4 hours after treatment.

Having detected high baseline cytokine levels in two participants during our serum analysis, it was necessary to confirm that any underlying inflammatory processes in these individuals did not affect the other endpoints of the study; therefore, the entire immunophenotyping dataset was analysed with and without the inclusion of these two participants. Removing the participants, which equated to a fifth of the total data, significantly reduced the statistical power but had only subtle effects on the statistics with no change to the conclusions. Therefore, because the individual results obtained from these participants were consistent with the remaining dataset and thus did not appear to be outliers, they were included in the final analysis. The only exception occurred with the T cell and $\mathrm{CD}^{2} 6^{+}$subset data. One of the participants with high baseline serum cytokine levels did not stain with a distinct positive population for CD3 with any of the antibodies used in the study and therefore could not be included in these analyses.

\subsubsection{Monocytes and neutrophils}

The immunophenotyping flow cytometry was performed on a Guava easyCyte 8HT (Merck Millipore, Germany). This machine uses a capillary system that requires small volumes of reasonably dilute samples for accurate results. The effectiveness of the red blood cell lysis step varied from person to person; therefore, some participants had a higher level of debris in the samples than others. For this reason, the forward scatter threshold had to be increased for a number of samples to reduce the amount of debris collected and ensure an adequate number of leukocyte events were acquired. The drawback of this method was that expressing the size of the leukocyte populations as a proportion of the total singlet events was not comparable between all participants. Since the neutrophils and monocytes are clearly identifiable on a SSC vs. FSC plot and are 
unaffected by changes in the FSC threshold, we decided to express the proportion of neutrophils and monocytes in each sample relative to a broad total neutrophil and monocyte gate that was constant between all participants.

The proportion of circulating CD14-CD $16^{+}$neutrophils was highly consistent across the time points examined, whereas there was a significant increase in $\mathrm{CD} 14^{+} \mathrm{CD} 16^{-/ \text {low }}$ monocytes at 20 minutes, which appeared to return to the baseline levels by the 4-hour sample (Figure 4.8A and B). There was no difference between the two treatment groups in the proportions of either cell type. The total neutrophils and monocytes detected at each time point accounted for approximately $85-90 \%$ of the cells contained within the broad SSC vs. FSC gate. The unidentified cells did not express any of the markers associated with the subsets examined in our study ( $\mathrm{T}$ cell subsets, NK cells, neutrophils, and monocytes) therefore were most likely eosinophils, and immature neutrophils and monocytes not yet expressing their characteristic cell surface markers.

The expression levels of the activation markers, CD11b and CD63, were also consistent between the two treatment groups in both cell populations (Figure 4.8C - F). There did, however, appear to be a fluctuation in the overall levels between time points, with notably higher CD11b and CD63 detected at baseline and 1 hour. This was most likely attributable to a small difference in the handling procedure for these two time points. A sample of the blood collected at baseline and 1 hour after treatment was stimulated for 15 minutes to examine leukocyte responsiveness. During the stimulation, the remaining blood was kept at room temperature and this extra waiting period or increase in handling may have had a small stimulatory effect on some of the blood cells. Although this makes it difficult to comment on the impact of time and blood sampling on resting CD11b and CD63 levels in neutrophils and monocytes, it is still clear that RIPC had no effect on these markers, compared to the control treatment.

To determine whether RIPC altered the responsiveness of neutrophils and monocytes, we examined activation marker expression in blood samples collected at baseline and 1 hour, and stimulated with PMA/I for 15 minutes. This 
brief stimulation resulted in a significant reduction in the proportion of both CD14-CD16 ${ }^{+}$neutrophils and CD14+CD16-/low monocytes (Figure 4.9A and B). This change most likely reflected an alteration in the phenotypic properties used to gate the cell subsets following stimulation with PMA, as has been previously observed in both monocytes and neutrophils (Bazil and Strominger, 1991; Huizinga et al., 1990). The observed change in proportions of the cell subsets appeared to be independent of RIPC (Figure 4.9A and B).

PMA/I stimulation of the blood significantly increased CD11b and CD63 expression in both neutrophils and monocytes, but there were no differences between the two treatment groups (Figure 4.9C - F). Interestingly, the neutrophil CD63 levels were significantly higher in the samples collected at 1 hour post-treatment, compared to the baseline, irrespective of whether the sample was stimulated or not (Figure 4.9E). Given this effect was present in both treatment groups, it may represent a minor response to the repeated blood sampling and confirms the ability of our methods to detect subtle differences in CD63 expression. 

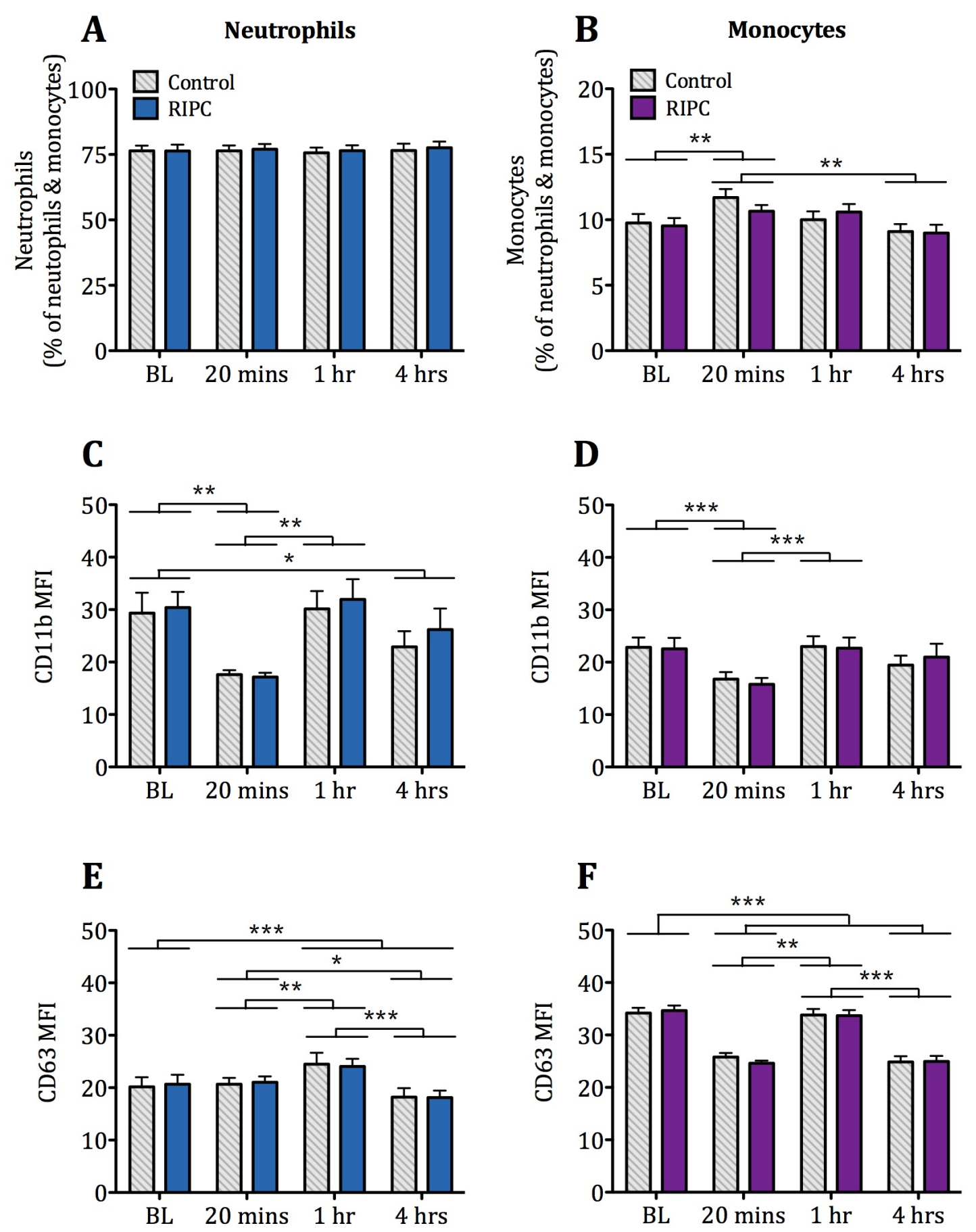

Figure 4.8 Size and activation state of circulating neutrophils and monocytes with and without RIPC

Blood samples were collected from healthy volunteers at baseline, and 20 minutes, 1 hour, and 4 hours after control or RIPC treatment, and peripheral leukocytes were assessed in duplicate by flow cytometry. Left: CD14-CD16 ${ }^{+}$ neutrophils showing (A) total proportion; (C) CD11b MFI; (E) CD63 MFI. Right: CD16-/low CD14 ${ }^{+}$monocytes showing (B) total proportion; (D) CD11b MFI; (F) CD63 MFI. A two-way repeated measures ANOVA was used to compare the treatments and time points, with Bonferroni post-hoc tests. ${ }^{*} \mathrm{p}<0.05,{ }^{* *} \mathrm{p}<$ 0.01 , and ${ }^{* * *} \mathrm{p}<0.001$. Shown are the means and SEM for $\mathrm{n}=10$ participants. For representative data plots refer to appendix F, Figures F.1 and F.2. 


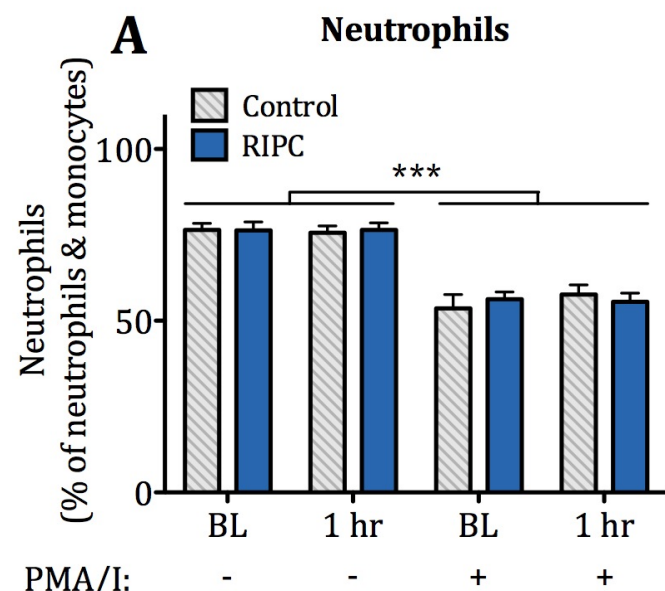

C

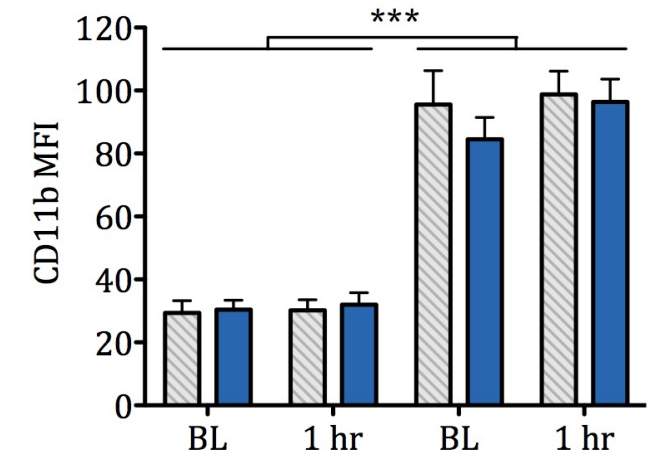

PMA/I:

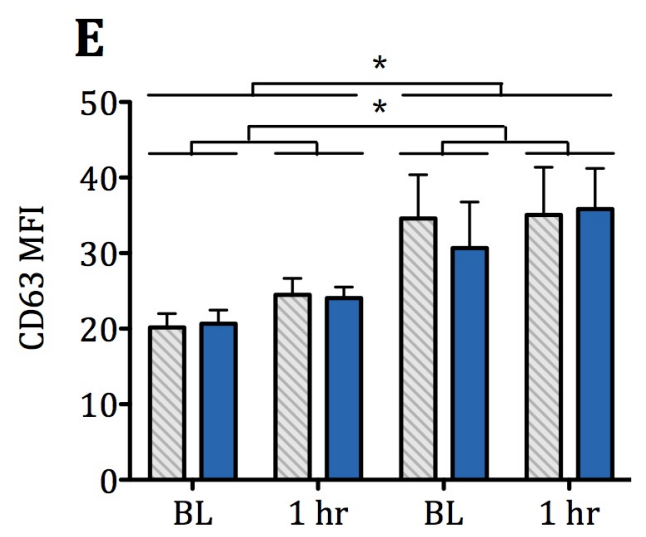

PMA/I:

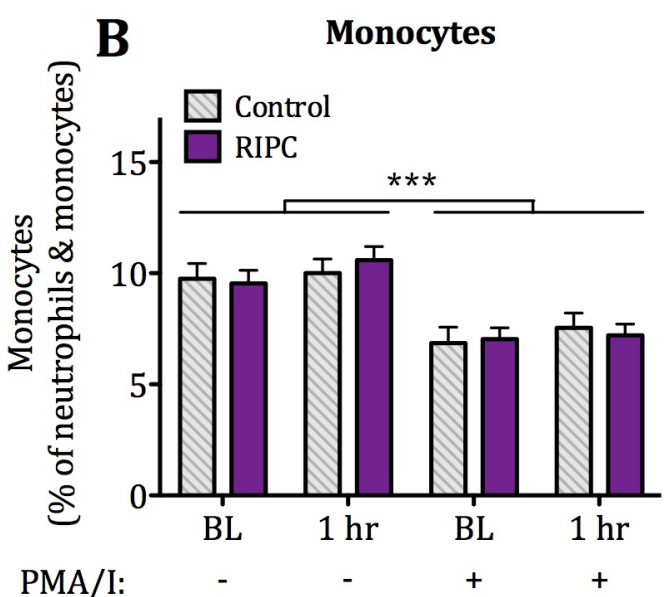

D

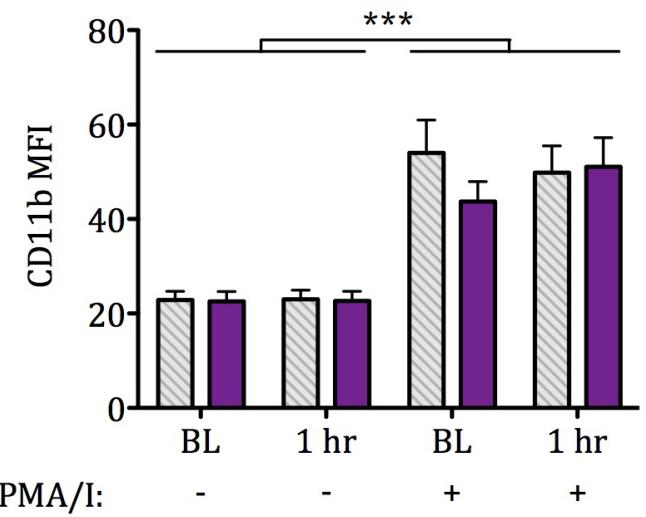

$\mathbf{F}$

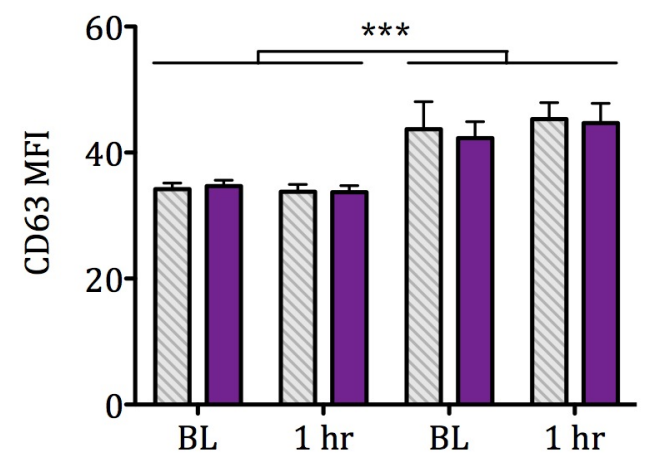

PMA/I:

Figure 4.9 Responsiveness of neutrophils and monocytes to re-stimulation after RIPC treatment

Blood samples were collected at baseline and 1 hour after treatment. Following rest or stimulation with PMA/I (50 and $500 \mathrm{ng} / \mathrm{mL}$, respectively), cells were stained in duplicate and assessed by flow cytometry. Left: CD14-CD16 ${ }^{+}$ neutrophils showing (A) total proportion; (C) CD11b MFI; (E) CD63 MFI. Right: CD16-/lowCD14+ monocytes showing (B) total proportion; (D) CD11b MFI; (F) CD63 MFI. A three-way repeated measures ANOVA was used to compare the treatments and time points, with Bonferroni post-hoc tests. ${ }^{*} \mathrm{p}<0.05$ and ${ }^{* * *} \mathrm{p}<$ 0.001 . Shown are the means and SEM for $n=10$ participants. For representative data plots refer to appendix F, Figures F.1 and F2. 


\subsubsection{T cell subsets}

Due to the variability in the FSC threshold between participant datasets and its close proximity to the lymphocyte population, it was not possible to create a general lymphocyte gate using SSC vs. FSC that was comparable between all individuals. Because of this, we expressed the parent $\mathrm{CD}^{+} \mathrm{T}$ cell population as a proportion of the total singlet events collected, as this measure was far less variable between participants. All subsequent T cell subsets were expressed as a percentage of the total $\mathrm{CD}^{+} \mathrm{T}$ cells.

There was a small but significant reduction in $\mathrm{CD}^{+} \mathrm{T}$ cells after 20 minutes, irrespective of which treatment the participants received, but no change in the circulating levels of $\mathrm{CD}^{+}{ }^{+} \mathrm{CD} 4{ }^{+}(\mathrm{CD} 4)$ and $\mathrm{CD}^{+}{ }^{+} \mathrm{CD} 8{ }^{+}(\mathrm{CD} 8) \mathrm{T}$ cells (Figure 4.10A, $\mathrm{C}$, and F). A small population of double positive $\mathrm{CD} 3^{+} \mathrm{CD} 4{ }^{+} \mathrm{CD} 8^{+} \mathrm{T}$ cells was also detected, and this subset did not appear to be affected by preconditioning (Figure 4.10B). Although our immunophenotyping panels included CD25 as a marker of regulatory cells, the poor resolution of this antibody coupled with the rare expression resulted in less than $3 \%$ of the $\mathrm{CD}^{+}$cells being identified as $\mathrm{CD}^{2} 5^{+}$. This low number of events was insufficient for accurate analysis and therefore was not presented in this thesis.

There was a slight reduction in the proportion of CD4 $\mathrm{T}$ cells that stained positively for the marker of naivety, CD62L, 20 minutes after treatment and this was mirrored by a significant increase in the CD62L- CD4 T cell population in all post-treatment samples (Figure 4.10D and E). The change in proportion of the CD62L positive and negative CD4 T cell subsets was the same with and without preconditioning. The proportions of CD62 $\mathrm{L}^{+}$and CD62L- CD8 cells were highly consistent across both the time points and treatment groups (Figure 4.10G and $\mathrm{H})$.

Expression levels of the early $\mathrm{T}$ cell activation marker CD69 significantly increased in the CD4 T cells 20 minutes post-treatment, and then returned back to baseline levels by the 4 hour time point (Figure 4.11A). Both the CD62 $\mathrm{L}^{+}$and CD62L- CD4 T cell subsets appeared to be affected equally although, as expected, 
the effector population had a slightly higher baseline level of CD69 expression (Figure 4.11C and E). In contrast, CD69 gradually and continually declined across the post-treatment time points in both the CD62 $\mathrm{L}^{+}$and $\mathrm{CD} 62 \mathrm{~L}^{-} \mathrm{CD} 8 \mathrm{~T}$ cell populations (Figure 4.11B, D, and F). There was no difference between the two treatments with respect to the activation states for any of the $T$ cell subsets examined. 

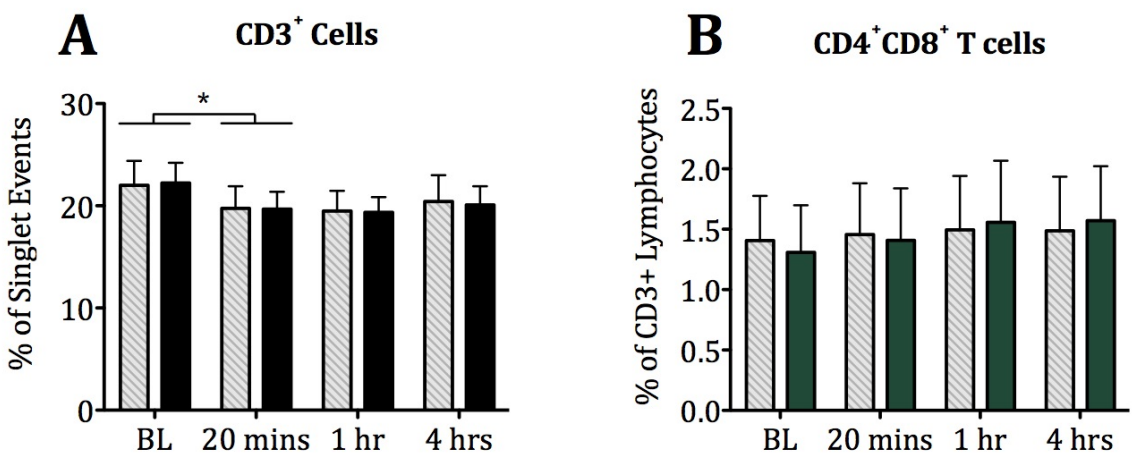

C CD4 T Cells D CD62L CD4 T Tallo

Figure 4.10 Proportions of circulating $T$ cell subsets after RIPC

Blood samples were collected from healthy volunteers at baseline, and 20 minutes, 1 hour, and 4 hours after control (grey bars) or RIPC treatment (black or green bars), and peripheral leukocytes were assessed in duplicate by flow cytometry. (A) Total $\mathrm{CD}^{+}$lymphocytes; (B) double positive $\mathrm{CD}^{+}{ }^{+} \mathrm{CD} 4^{+} \mathrm{CD} 8^{+}$ lymphocytes; (C) total $\mathrm{CD}^{+}{ }^{+} \mathrm{CD} 44^{+} \mathrm{T}$ cells; (D) CD62 $\mathrm{L}^{+} \mathrm{CD} 4 \mathrm{~T}$ cells; (E) CD62 $\mathrm{L}^{-} \mathrm{CD} 4$ T cells; (F) total CD8 ${ }^{+} \mathrm{T}$ cells; (G) CD62 $\mathrm{L}^{+} \mathrm{CD} 8 \mathrm{~T}$ cells; (H) CD62L- CD8 T cells. A two-way repeated measures ANOVA was used to compare the treatments and time points, with Bonferroni post-hoc tests. ${ }^{*} \mathrm{p}<0.05$ and ${ }^{* * *} \mathrm{p}<0.001$. Shown are the means and SEM for $\mathrm{n}=9$ participants. For representative data plots refer to appendix F, Figures F.3, F.4, and F.5. 

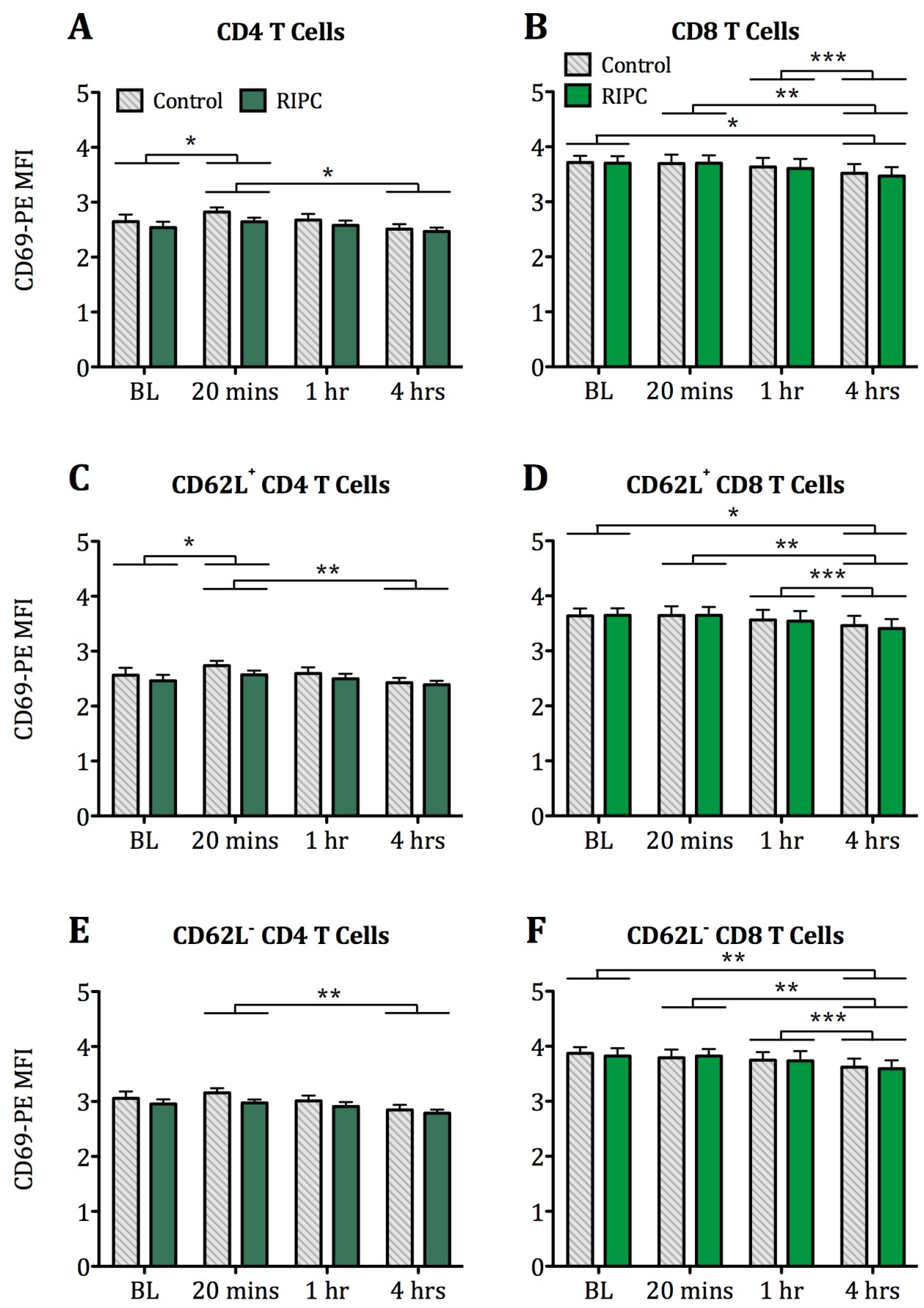

Figure 4.11 CD69 expression in T cell subsets following RIPC treatment

Blood samples were collected from healthy volunteers at baseline, and 20 minutes, 1 hour, and 4 hours after control or RIPC treatment, and peripheral leukocytes were assessed in duplicate by flow cytometry. Left: CD69 MFI in CD4 $\mathrm{T}$ cells for (A) total population; (C) $\mathrm{CD}^{2} 2 \mathrm{~L}^{+}$subset; (E) CD62L' subset. Right: CD69 MFI in CD8 T cells for (B) total population; (D) CD62L $\mathrm{L}^{+}$subset; (F) CD62 $\mathrm{L}^{-}$ subset. A two-way repeated measures ANOVA was used to compare the treatments and time points, with Bonferroni post-hoc tests. ${ }^{*} \mathrm{p}<0.05,{ }^{* *} \mathrm{p}<$ 0.01 , and ${ }^{* * *} \mathrm{p}<0.001$. Shown are the means and SEM for $\mathrm{n}=9$ participants. For representative data plots refer to appendix F, Figures F.4 and F.5. 


\subsubsection{3 $\mathrm{CD}^{2} 6^{+}$cell subsets}

A small population of $\mathrm{CD}^{2} 6^{+}$cells was detected amongst the lymphocytes, comprising approximately $50-60 \%$ CD3-CD56+CD16 ${ }^{+}$NK cells, and $20-30 \%$ $\mathrm{CD}^{+}{ }^{+} \mathrm{CD} 56^{+} \mathrm{CD} 16^{-/+}$NKT cells. Neither population significantly changed in size across the time points, or between the control and RIPC treatments (Figure 4.11A - C). Similarly to what was seen with the CD4 T cells, NK cell CD69 expression significantly increased 20 minutes post-treatment and then returned back to baseline levels by the 4 hour time point in both groups (Figure 4.11D). This change in activation state of the NK cells was consistent with the parallel reduction in the proportion of $\mathrm{CD}^{2} 2 \mathrm{~L}^{+} \mathrm{NK}$ cells and increase in CD62 $\mathrm{L}^{-} \mathrm{NK}$ population (Figure 4.11E and F). 
A CD56 $^{+}$Lymphocytes

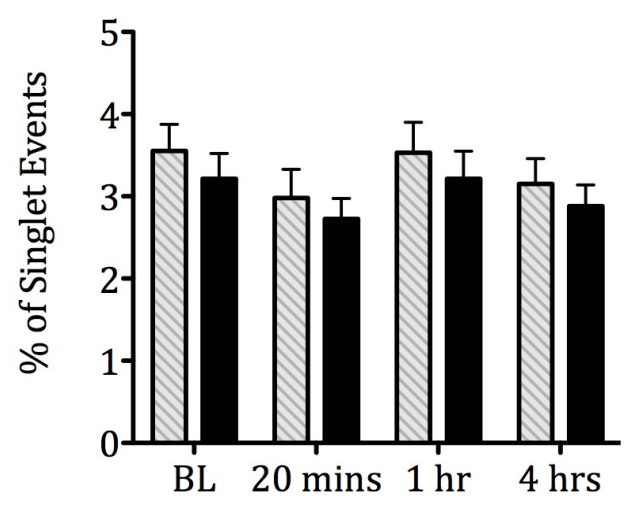

C

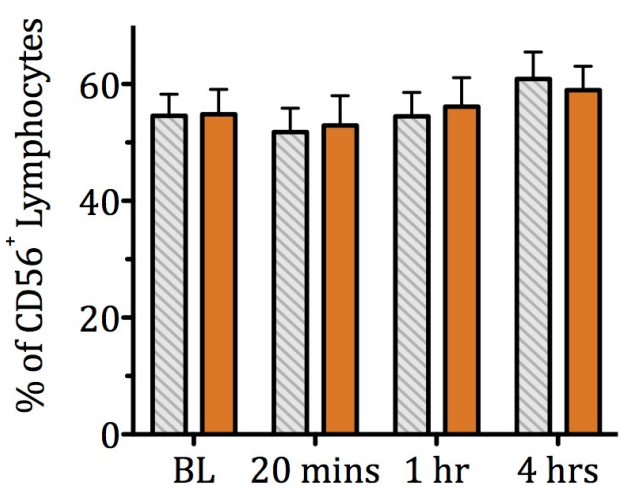

$$
\text { E }
$$

CD62L $^{+}$NK Cells

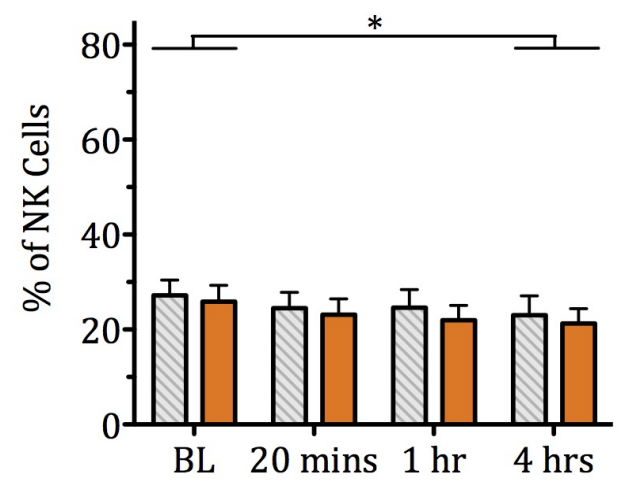

B NKT Cells

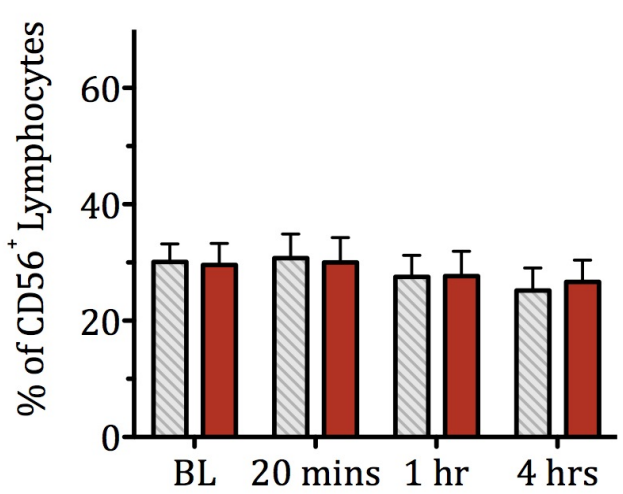

D CD69 in NK Cells

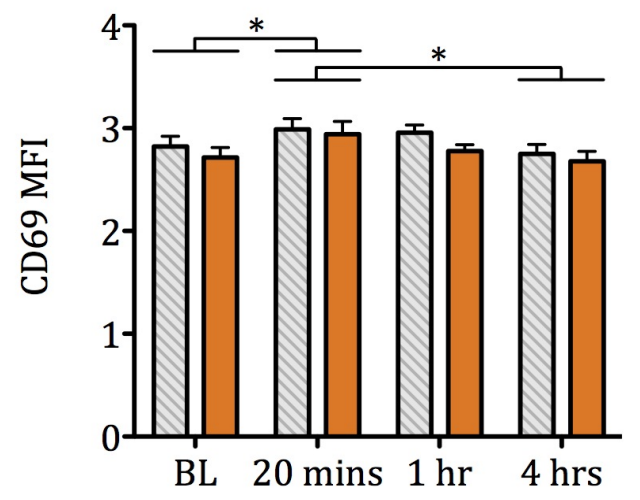

$\mathbf{F}$

CD62L' NK Cells

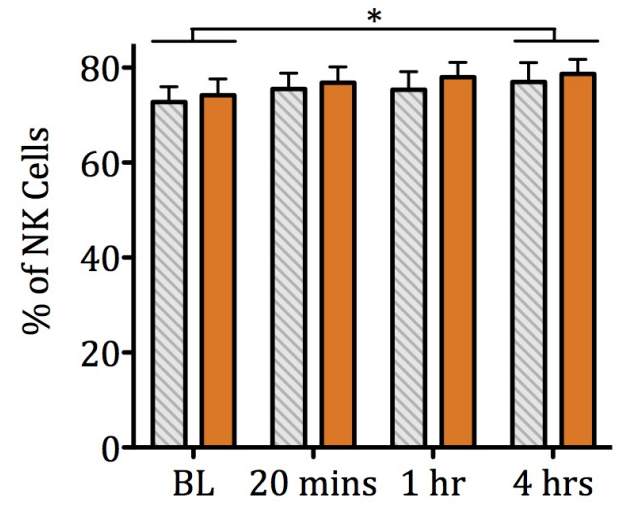

Figure 4.12 Proportion and activation of circulating $\mathrm{CD}^{+} 6^{+}$cells after RIPC Blood samples were collected from healthy volunteers at baseline, and 20 minutes, 1 hour, and 4 hours after control (grey bars) or RIPC treatment (black or coloured bars), and peripheral leukocytes were assessed in duplicate by flow cytometry. (A) Total CD56+ lymphocytes; (B) CD3+CD56 ${ }^{+}$NKT cells; (C) total

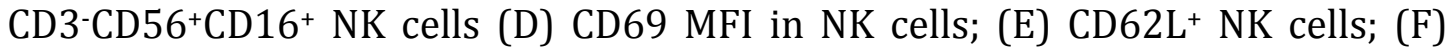
CD62L- NK cells. A two-way repeated measures ANOVA was used to compare the treatments and time points, with Bonferroni post-hoc tests. ${ }^{*} p<0.05$. Shown are the means and SEM for $\mathrm{n}=9$ participants. For representative data plots refer to appendix F, Figures F.3 and F.5. 


\subsection{Discussion}

The aim of this chapter was to investigate the direct effect of RIPC on the systemic immune response in healthy volunteers. We found that preconditioning reduced the expression of both MIP- $1 \alpha$ and MIP-1 $1 \beta$, but did not alter serum concentrations of other key cytokines, including IL-6 and IL-8. RIPC did not change the phenotype of resting immune cells, nor did it affect the ability of neutrophils or monocytes to become activated in response to a brief stimulation. Although the systemic response to preconditioning was minimal, numerous changes in the levels of inflammatory markers were detected across the study time points for both treatments, suggesting the influence of a diurnal rhythm, or that the venepunctures alone elicited a subtle but significant immune response.

Our study is the first to show that RIPC reduces systemic levels of both MIP-1 $\alpha$ and MIP-1 $\beta$. Increased expression of the MIP family of chemokines has been frequently associated with responses to ischaemic conditions and oxidative stress including stroke, myocardial infarction, and cardiac surgery (de Jager et al., 2008; Kim et al., 2009; Vandervelde et al., 2007; Zaremba et al., 2006). The exact role of MIP- $1 \alpha$ and $\beta$ in the setting of ischaemia is not clear, but these proteins are most commonly known for their trafficking effects on $\mathrm{T}$ cells, monocytes, dendritic cells, and NK cells, and have also been implicated in driving a proinflammatory Th1 response through modulating helper $\mathrm{T}$ cell differentiation (Karpus and Kennedy, 1997; Maurer and von Stebut, 2004).

MIP-1 $\alpha$-induced chemotaxis of monocytes may have a beneficial role in wound repair; however, the net effect of MIP- $1 \alpha$ and $\beta$ appears to enhance and facilitate inflammatory processes (DiPietro et al., 1998; Maurer and von Stebut, 2004). It is therefore not surprising that MIP- $1 \alpha$ has been implicated in the pathology of lung I/R injury, and that anti-MIP-1 $\alpha$ therapy significantly decreases vascular permeability and neutrophil infiltration, reducing lung injury by $37 \%$ (Krishnadasan et al., 2004). Further, I/R injury is associated with polarisation towards a Th1 response, which may be facilitated by MIP-1 $\alpha$ (Karpus and Kennedy, 1997; Sullivan et al., 2009). Overall, the literature suggests that a reduction in MIP-1 levels would be expected to be protective in I/R injury, 
potentially reducing immune cell infiltration and lessening the magnitude of the inflammatory response.

Interestingly, we also noted a trend towards a reduction in circulating G-CSF levels following preconditioning. G-CSF is the primary cytokine involved in the regulation of granulocytopoiesis and an absence of G-CSF results in neutropenia (Lieschke et al., 1994). A decrease in circulating G-CSF could result from increased binding to receptors on precursor cells in the bone marrow, or from mechanisms that promote its degradation. Neutrophils are short-lived cells, reportedly surviving between 10 hours and 5 days in the circulation and for an even shorter period following activation and infiltration into the tissues (Dancey et al., 1976; Pillay et al., 2010). Consequently, up to $10^{11}$ new neutrophils are produced daily in healthy individuals, and changes to granulocytopoiesis can significantly alter the circulating pool of cells within a short period of time (Demetri and Griffin, 1991). It is not possible to comment on whether RIPC altered neutrophil production based on the results of our study, but our finding that G-CSF levels may be decreased following preconditioning warrants further investigation in future work.

The impact of RIPC on cytokine expression in humans is a contentious topic, with little agreement between the studies performed to date. Konstantinov et al. (2004) reported that RIPC leads to suppression of the three essential kinases involved in TNF synthesis during both the early and late phases, suggesting preconditioning reduces levels of at least one key inflammatory cytokine. This work was supported by a study of late-RIPC in infants undergoing ventricular septal defect repair that showed a reduction in post-operative levels of IL-6, IL-8, and TNF- $\alpha$ (Zhou et al., 2010). In contrast, a recent trial in adult cardiac surgery patients determined that RIPC not only increased systemic levels of IL-1 $\beta$, IL-8, and TNF- $\alpha$ levels directly after the intervention, but also upregulated postbypass IL-1 $\beta$ levels in right atrial tissue biopsies (Albrecht et al., 2013). Further, Shimizu et al. (2010) demonstrated that repeated RIPC increases the capacity of neutrophils to produce IL-1 $\beta$, IL-6, and TNF- $\alpha$ in response to LPS challenge. 
Our finding that RIPC does not directly increase systemic levels of a range of key cytokines is consistent with our results from the high-risk cardiac surgery patient trial, and further supports our assertion that a cytokine is unlikely to be the humoral factor responsible for transmitting an immediate protective RIPC signal. In particular, the lack of change in IL-6 and IL-8 levels in the present study verify that the slightly increased levels of these cytokines detected in our preconditioned cardiac surgery patients were most likely either unrelated to the RIPC treatment, or the result of an indirect effect of the intervention.

In the absence of a critical role in the initiation of RIPC, any beneficial functions conferred by inflammatory cytokines during I/R injury are likely outweighed by their tissue-destructive effects. Interestingly, MIP-1 is known to stimulate the release of IL-6; therefore, based on the reduced expression of MIP- $1 \alpha$ and $\beta$ detected in our healthy volunteers, it would be reasonable to expect that the IL-6 levels induced during I/R injury would be lower following the successful application of RIPC (Fahey et al., 1992). In addition, many of the pathways implicated in the mechanism of RIPC, such as the reduction in TLR expression, appear to converge to suppress NF- $\kappa \mathrm{B}$ and the associated inflammatory response genes, which include numerous cytokines (Li et al., 2001; Morgan et al., 1999). We believe the results of the present study add weight to the existing literature supporting the argument that RIPC either does not directly alter cytokine levels, or reduce the cytokine response to I/R injury.

There is mounting evidence that RIPC leads to increased accumulation, stabilisation, and activation of the hypoxia-triggered transcription factor subunit HIF-1 $\alpha$ (Cai et al., 2012; Kant et al., 2008; Ke and Costa, 2006). Recently, increased levels of HIF-1 $\alpha$ have even been measured in human atrial tissue biopsies directly after limb RIPC, prior to I/R injury (Albrecht et al., 2013). Activated HIF-1 $\alpha$ stimulates expression of hundreds of genes, including the cytokines IL-1 $\beta$, MCP-1, and IL-10 (Cai et al., 2013; Mojsilovic-Petrovic et al., 2007; W. Zhang et al., 2006). Although the early phase of RIPC is typically associated with the release or modification of pre-formed mediators, it is interesting to note that we found no change in the circulating levels of IL-1 $\beta$ or 
IL-10, and MCP-1 significantly decreased across the time points for both treatment groups in our study.

It has been suggested that because the effects of HIF-1 $\alpha$ depend on gene transcription it may be more relevant to the late phase of RIPC, rather than the early response (Sadat, 2009). We sampled serum levels up to 4 hours following treatment, which is slightly beyond the reported endpoint of the early window of protection, and could theoretically allow sufficient time for cellular signalling, gene transcription, mRNA processing, and translation of a measurable amount of protein. Although total protein levels may be significantly higher 24 hours later, it is possible that subtle changes to systemic cytokine transcription and expression could have been detectable in our 4-hour samples. It therefore seems likely that the impact of HIF- $1 \alpha$ activation on cytokine production may be tissue specific, and/or of a magnitude not strong enough to alter systemic levels during the early window of protection (Stroka et al., 2001). Alternatively, we may not have met the threshold for inducing HIF-1 $\alpha$ with our preconditioning regimen. A last possibility concerns the debate regarding the exact position of HIF-1 $\alpha$ in the RIPC-induced signalling pathway (Heusch, 2012). Although HIF-1 $\alpha$ is known to stimulate cytokine production, the transcription factor can also be induced by cytokines, therefore increased HIF-1 $\alpha$ activity may not precede an increase in IL$1 \beta$, MCP-1, and IL-10 levels during preconditioning. Regardless of the underlying mechanism, we did not find any indirect suggestion that HIF-1 $\alpha$ might contribute to the induction of preconditioning by influencing transcription of these cytokines during the early window of protection, using the standard RIPC stimulus.

Many theories have been proposed regarding how RIPC may affect neutrophils in order to blunt the damage elicited by I/R injury. It is possible that RIPC directly activates neutrophils, leading to their infiltration of the preconditioned tissue and thus limiting the number of cells available to cause damage in organs subsequently exposed to prolonged periods of ischaemia. We found no change in the proportion of circulating neutrophils, or any of the other leukocyte subsets examined, indicating that RIPC did not promote extravasation of any individual 
immune cell populations. Further, we found no change in expression levels of the activation marker, CD11b, in unstimulated or briefly stimulated neutrophils following RIPC treatment. Taken together, these results strongly counter the suggestion that preconditioning activates and sequesters neutrophils.

The lack of leukocyte transmigration following RIPC appeared to be consistent with the unaltered levels of the soluble adhesion factors associated with cellendothelial interactions that were measured in the serum samples. ICAM-1 mRNA levels have been shown to significantly increase one hour after I/R injury in both murine and canine models, and the protective effect detected in ICAM-1 depleted or deficient animals has highlighted the critical role this adhesion factor plays in I/R induced tissue pathology (Kelly et al., 1996; Kukielka et al., 1993; Rabb et al., 1995). ICAM-1 is constitutively expressed on endothelial cells, and the upregulation detected following I/R appears to be predominantly from cardiac myocytes. Extracellular expression of ICAM-1 in myocytes is measurable within six hours of reperfusion; however, soluble ICAM-1 levels are unlikely to mirror this increase until sufficient proteolytic cleavage of the protein into the circulation occurs (Kukielka et al., 1993). Similarly, E-selectin is not stored and therefore must be produced upon stimulation. Although it can be formed within two hours and reaches maximal levels four to eight hours after induction, newly synthesised E-selectin is not shed into the circulation until 18 - 48 hours later (Pigott et al., 1992).

The kinetics of ICAM-1 and E-selectin expression suggest that even if RIPC induced a significant change in the cellular expression of these molecules that contributed to the early window of protection, the time points in our study most likely would not have been able to capture this change through the measurement of serum levels of the soluble forms. Serum samples collected $24-48$ hours following preconditioning would be preferable for capturing peak soluble ICAM1 and E-selectin levels; however, this endpoint would still lack the information to decipher when cellular expression of the adhesion molecules increased, and on which cell types. Future studies where ICAM-1 is a major focus would benefit from the use of flow cytometry to perform a more detailed analysis of leukocyte 
expression levels. From the present study we can, however, rule out the possibility that RIPC induces shedding of ICAM-1 and E-selectin into the circulation within 4 hours of the intervention.

Our finding that preconditioning did not increase CD11b expression on peripheral neutrophils up to four hours following treatment is consistent with three previous studies also performed with healthy volunteers (Kharbanda et al., 2001; Konstantinov et al., 2004; Shimizu et al., 2010). Interestingly, Kharbanda and colleagues followed their preconditioning protocol with a twenty minute sustained inflation of the blood pressure cuff on the upper arm to simulate a mild I/R injury, and found that IPC attenuated the ischaemia-induced increase in CD11b expression. In contrast, when we stimulated RIPC-exposed neutrophils for 15 minutes in vitro to simulate an immune stimulus, we found no change in the ability of neutrophils to become activated after preconditioning. Shimizu et al. also reported a similar lack of change in CD11b expression with fMLP stimulation of neutrophils sampled one day post-preconditioning. Further, Konstantinov et al. found that although CD11b levels were unchanged fifteen minutes after treatment, there was a significant reduction in $\mathrm{CD} 11 \mathrm{~b}$ on circulating neutrophils 24 hours later, yet Shimizu et al. found no such change using the same timeframe.

The reason for the discrepancies between the RIPC studies with respect to the effect on neutrophils is unclear. The ex vivo treatment of neutrophils using pharmacological stimulants (PMA/I) or agents derived from bacterial origins (fMLP) may signal through a different pathway than I/R injury and therefore elicit different results, or minor differences in the design and methodology of the studies may have contributed. While all four studies conclude that RIPC does not directly alter the activation state of circulating neutrophils during the early window of protection, further work is required to clarify how preconditioning impacts these cells following a subsequent stimulation and during the delayed phase of protection. 
After stimulation of whole blood samples as part of our immunophenotyping protocol, we detected a significant decrease in the proportions of neutrophils and monocytes in the samples. Because the assay was performed in vitro, with nowhere for the cells to migrate and insufficient time for cellular disintegration in cases of death, there was no physiological explanation for the decrease in the size of these populations. Instead, the change most likely reflected a difference in the phenotypic properties of the stimulated cells. PMA treatment has previously been shown to result in marked CD14 shedding in monocytes, with a 30 minute stimulation leading to as much as a $60 \%$ reduction in extracellular CD14 expression (Bazil and Strominger, 1991). Our results extend this time range, suggesting that significant PMA-induced CD14 shedding can occur with as little as 15 minutes of treatment. Huizinga et al. (1990) reported a significant reduction also occurs with neutrophil CD16 expression in response to a 10 minute PMA stimulation. The reduction in neutrophils and monocytes identified by flow cytometry in our study following PMA treatment was thus consistent with the existing literature, and appeared to be independent of RIPC.

Increased CD63 expression in neutrophils was detected by Shimizu et al. (2010) 24 hours after RIPC and we were interested in whether this change might take effect during the early window of protection. However, we found no change in CD63 expression on resting or stimulated neutrophils and monocytes up to 4 hours post-RIPC, suggesting that induction of neutrophil exocytosis is limited to the late phase of RIPC-induced protection. Interestingly, there is increasing evidence that the impact of preconditioning on the immune response in general may be more relevant during the second window of protection. Both cardiac surgery and gene expression studies in humans have suggested inflammatory cytokine production is attenuated 20 - 24 hours following RIPC administration, during the late phase (Konstantinov et al., 2004; Wei et al., 2001b; Zhou et al., 2010). Further, the effects of RIPC on neutrophil adhesion and kinin receptor expression have also been detected the day following RIPC treatment (Saxena et al., 2010b; Shimizu et al., 2010). The time points in our study were selected to assess the effect of RIPC on the first phase of protection, and our results suggest that RIPC has only minor, subtle effects on the immediate immune response. 
Further work will be required to determine if changes to the immune response are more predominant during the second window of protection, rather than the early phase that has commonly been explored in cardiac surgery trials.

An unanticipated finding from our study was the significant change in numerous variables, independently of the application of RIPC. Serum levels of MCP-1 were significantly decreased in both treatment groups, along with reductions in the proportion of circulating CD3 and CD8 T cells, and naïve NK and CD4 T cell populations. In contrast, levels of monocytes, CD4 T cells, and CD69 expression in the NK population significantly increased 20 minutes after either treatment, and higher levels of effector NK and CD4 T cell subsets were detected at all posttreatment time points. Altogether, these changes suggest a general inflammatory process occurred following the baseline blood sample collection during both study visits. Given the consistency of the changes between the participants, this most likely reflects a response to the repeated venipunctures or the effects of circadian rhythm. Indeed, it is well established that small changes in cytokine expression and leukocyte levels follow a 24 hour cyclical pattern, and our participants all started their study visits around the same approximate time; however, it is also possible that stress associated with participating in the trial may have contributed (Born et al., 1997).

The significant inflammatory response detected in both treatment groups highlights the necessity of carefully designed and controlled trials. Had we relied on measuring the effects of RIPC by solely comparing post-treatment samples to the baseline results, these changes could have easily been mistaken for the effects of preconditioning. Instead, including an entirely separate control treatment for each participant enabled us to distinguish the legitimate effects of RIPC from the minor inflammatory response to blood collection, circadian rhythm, and/or trial participation. Further, these findings confirmed that our assays had the sensitivity required to pick up subtle changes in our end points, as per the goal of our assay testing process. 
While the clinical efficacy of RIPC remains to be determined, no trials have reported any adverse effects resulting from preconditioning to date. The noninvasive nature of RIPC coupled with its consistent track record means that it is widely accepted as having a solid safety profile, and our finding that RIPC elicits only minor direct responses from the immune system are in keeping with this belief (Camara-Lemarroy, 2014; Candilio et al., 2011). However, the lack of change in systemic cytokine levels and immunophenotype of resting cells does not preclude the possibility that RIPC alters the way the immune system responds to subsequent challenges. To fully characterise the impact of preconditioning, it will be necessary to assess the changes in functional responses of the immune cells, such as would be encountered during I/R injury.

A limitation of our study was the small sample size and high number of statistical tests performed on the data set. The trial was designed as a hypothesisgenerating study, which involved measuring a broad range of endpoints at multiple time points to increase the likelihood of capturing any small changes to the immune response induced by RIPC. Analysing our full dataset from both Chapters 4 and 5 thus necessitated just over 200 individual ANOVAs and t-tests. The probability of encountering a type I error, which involves an incorrect rejection of the null hypothesis, is increased with each test performed. At a $5 \%$ significance level, we would expect to falsely reject the null hypothesis (that there was no difference between the treatment groups or time points) approximately 10 times. We found statistically significant differences in vastly more than 10 variables, most of which were between time points or culture conditions, rather than the control and RIPC treatments. Such significant changes were consistent with our biological expectations for the samples and therefore appeared to be legitimate differences; however, our overall results must be considered within the context of this statistical limitation and we are hesitant to draw definitive conclusions about the direct effect of RIPC from this study. Our primary aim was to identify parameters of interest for future, more focused research, and our analysis was appropriate and successful for this purpose. 
Our sample size was calculated to give us adequate power for detecting subtle changes in serum cytokine levels; however, signs of inflammatory processes in two of our participants led to the exclusion of their data from parts of the analysis. The paired nature of our trial design strengthened our ability to measure subtle differences in the responses and, indeed, we did detect significant differences between the groups even when the dataset was reduced to 8 participants. That said, collecting data from a higher number of individuals when feasible in future studies would be advisable.

Many of our endpoints were assessed using repeated measures ANOVA, which is a statistical test known to be dependent on data sphericity. The assumption of sphericity requires that the variances of the differences between the levels of each variable are equal (Field, 1998). This assumption can be tested using Mauchly's Test of Sphericity; however, this method performs poorly with sample sizes as small as those used in the present study, often failing to detect deviations from sphericity. To ensure that we were not violating the assumptions underlying our ANOVA results, we used the Greenhouse-Geisser correction to lower the degrees of freedom and increase the p-value, thus minimising the risk of a type I error (Greenhouse and Geisser, 1959).

The Greenhouse-Geisser correction is the most conservative method for calculating p-values without assuming sphericity; therefore, one drawback of this approach is that we may have reduced our power to detect significant differences in our dataset (Field, 1998; Greenhouse and Geisser, 1959). Ultimately, finding the balance between maintaining statistical power and offsetting the likelihood of a type I error when analysing data from an exploratory study is challenging and, despite the steps undertaken to appropriately manage the statistical analyses, our results must be interpreted in the context of this issue.

Lastly, the methodology for measuring CD11b and CD63 in both unstimulated and stimulated neutrophils and monocytes using the same samples could be improved for future studies. Either the pipetting associated with removing an 
aliquot of blood for the stimulation, or the twenty minute delay where the 'unstimulated' blood was left at room temperature, resulted in a substantial increase in MFI for both markers, compared to the time points where only unstimulated blood was used and samples were stained immediately. Although we are reasonably confident that RIPC did not alter the expression of CD11b or CD63, this difference did reduce our ability to measure subtle changes. Staining the unstimulated samples immediately after collection and the stimulated cells separately could have circumvented this issue and would be preferable for future experiments. Of note is that the error bars for these measurements were small, confirming that the effect was similar for all participants and that our development of protocols to ensure samples were handled identically between visits was effective.

\subsection{Conclusions and future directions}

We found that in healthy volunteers, RIPC directly and significantly decreased serum levels of the chemokines MIP- $1 \alpha$ and MIP-1 $\beta$, which may be protective during $\mathrm{I} / \mathrm{R}$ injury by reducing immune cell infiltration and limiting the inflammatory response. Systemic levels of a range of key cytokines were not increased by preconditioning, adding weight to the view that a cytokine is not likely to be the humoral mediator associated with transmitting the RIPC protective signal. Further, RIPC did not alter the immunophenotype or extravasation of peripheral leukocytes populations. Taken together, these results suggest that the direct impact of RIPC on the systemic immune response during the early window of protection is very subtle. The effect of RIPC on the functional responses of immune cells may be more relevant to the mechanisms behind the early phase of protection, and requires further study in human subjects. 
Chapter 5.

\section{The effects of RIPC on the function of peripheral leukocytes in healthy volunteers}




\subsection{Introduction}

Although the majority of the literature addressing I/R injury focuses on the damage mediated by the innate immune response, the cells of the adaptive immune system also play a crucial role. The main cells of the adaptive response are the $\mathrm{B}$ and $\mathrm{T}$ lymphocytes, although there is increasing evidence that NK cells also exhibit many of the traits associated with the acquired arm of the immune system (Vivier et al., 2011). The adaptive immune system predominantly responds to specific foreign antigens and develops an immunological memory that enables faster future responses to infection with the same pathogen; however, under certain circumstances including during I/R injury, $\mathrm{T}$ cells can also be activated in an antigen-independent fashion (Strainic et al., 2008; Unutmaz et al., 1994).

\subsubsection{Evidence that preconditioning alters the adaptive immune response}

Studies investigating the time course of RIPC found the protection might persist for several weeks, which seemed unlikely to be mediated by the short-lived cells of the innate immune response and thus highlighted a potential role for the cells of the adaptive system (Neckár et al., 2004; Park et al., 2003). Further, BurneTaney et al. (2006) demonstrated that adoptive transfer of immune cells from a wild type (WT) mouse subjected to renal I/R into a nude mouse, which are deficient in $\mathrm{T}$ cells, was protective against a subsequent ischaemic injury in the recipient. This protection was observed using cells transferred 5 days after the initial insult and was not associated with a reduction in neutrophil and macrophage infiltration in the nude mice, suggesting the effect was mediated by cells of the adaptive immune system. Similarly, Ascon et al. (2006) found transfer of lymphocytes isolated from the kidney 24 hours post-I/R injury into nude mice reduced the extent of damage from I/R induced 24 hours later. Despite these initial promising studies, there was limited literature evaluating the impact of IPC on the adaptive immune response at the time we designed our study; however, what was available suggested there were important links between IPC and the responses of certain $\mathrm{T}$ cell subsets during I/R. 


\subsubsection{Regulatory T cells}

Delayed ischaemic preconditioning-induced increases in Treg populations have been associated with a protective response in two murine studies. Kinsey et al. (2010) found that IPC significantly reduced kidney damage caused by an I/R injury induced 7 days later and this was associated with an increase in Treg and $\mathrm{CD} 4{ }^{+} \mathrm{CD} 25^{+} \mathrm{IL}-10$ producing cells in the outer medulla interstitium of the kidney, which is the region most vulnerable to ischaemic damage. This protective effect was not detected when the I/R was inflicted 3 days after IPC, before the renal Treg accumulation occurred. Antibody depletion of the Treg population partially reversed this beneficial effect and increased neutrophil infiltration, whereas adoptive transfer of Tregs prior to I/R in non-preconditioned mice induced a similar anti-inflammatory protective effect in the kidney to that seen with IPC (Kinsey et al., 2010).

Similar results were seen in a study by Cho et al. (2010), where IPC was associated with an increase in the number of kidney Tregs and a reduction in renal damage and inflammation following $I / R$ induced one week later. Splenocytes from preconditioned animals showed reduced proliferation and inflammatory cytokine production in response to ex vivo T cell receptor (TCR) or LPS stimulation, compared to the sham operated mice. There was also a reduction in the in vivo neutrophil infiltration of the kidney. Depletion and adoptive transfer of Tregs partially attenuated, and then reinstated, the protective effects of the IPC (Cho et al., 2010).

A study investigating the use of an adenosine $A_{1}$ receptor agonist as a means of pharmacologically preconditioning mice against lymphocyte-mediated inflammation reported an inhibitory effect that was associated with an increase in Tregs following ex vivo stimulation of isolated splenocytes (Naamani et al., 2014). Further, IPC has been shown to induce CD39 expression during hepatic protection against I/R (Hart et al., 2010). CD39 is a membrane-bound protein frequently found on Tregs, which hydrolyses extracellular ATP and ADP to AMP (Dwyer et al., 2007). Thus, CD39 contributes to the anti-inflammatory effects of Tregs by both reducing the circulating levels of proinflammatory ATP and 
through generating AMP, which is subsequently degraded into adenosine. Given the reported involvement of adenosine signalling in the induction of IPC, these studies add further weight to research suggesting an increase in Tregs may contribute to the delayed window of protection with preconditioning.

\subsubsection{Helper and cytotoxic T cell responses}

There has been limited research investigating the effect of IPC on CD4 and CD8 T cell responses; however, a clinical study in patients undergoing cruciate ligament surgery has suggested preconditioning modulates helper $\mathrm{T}$ cell responses while having only minor effects on the cytotoxic $\mathrm{T}$ cell population (Sullivan et al., 2009). IPC was associated with a significant reduction in circulating CD4 but not CD8 T cells compared to both baseline and the control group, 4 and 24 hours after reperfusion. Systemic levels of IL-2 were also lower and expression of the death receptor, CD95, on CD4 and CD8 T cells was higher, 24 hours post-surgery in the patients that received IPC. Expression levels of CD45RO, which is indicative of activated and memory T cells, were reduced on CD4 and increased on CD8 cells, following preconditioning (Sullivan et al., 2009). Taken together, these results strongly suggest that IPC may exert a protective effect through suppressing the helper $\mathrm{T}$ cell response, but not cytotoxic T cells.

The idea that IPC exerts a protective effect through inhibition of the Thelper cell response is consistent with a study that found IPC was associated with a trend towards reduced P-selectin expression in dogs (Linden et al., 2006). Upregulation of P-selectin expression during I/R facilitates entry of Th1 and Th2 cells into sites of inflammation, propagating the inflammatory response and ensuing tissue damage (Bonder et al., 2005). In contrast, Wei et al. (2012) found that RIPC inhibited expression of $\mathrm{T}$ cell immunoglobulin domain and mucin domain 3 (TIM-3) and its ligand, galectin-9, which are associated with inducing death in Th1 cells. Although the precise effects of RIPC on the adaptive immune response are not clear, overall these early studies strongly suggest that preconditioning may alter the course of I/R injury through modification of CD4 T cell function. 


\subsubsection{T cells and I/R injury}

Given the limited research addressing the role of the adaptive immune system in RIPC, we looked to what was known about the involvement of lymphocytes in the pathology of I/R to identify further processes that might be relevant to our study. $T$ cells were initially connected to the pathology of I/R by a number of gene deletion studies in mice. Horie et al. (1999) were the first to report that mice with severe combined immunodeficiency (which therefore lack functional B and T cells) showed a blunted inflammatory response to I/R injury in the gut. Similar responses were seen in renal and hepatic I/R models, and Yang et al. (2005) were the first to demonstrate that mice lacking recombination activating gene (RAG)-1 (which results in the loss of mature $B$ and $T$ cells) were also protected from myocardial I/R injury, with a significantly reduced infarct size in the lymphocyte deficient animals (Burne et al., 2001; Day et al., 2006; Geudens et al., 2007; Rabb et al., 2000; Shen et al., 2002; Zwacka et al., 1997).

Importantly, the myocardial, intestinal, renal, and hepatic protection seen in $\mathrm{T}$ cell or lymphocyte deficient animals could be consistently reversed by the transfer of wild type (WT) splenocytes or restoration of the $\mathrm{CD}^{+} \mathrm{T}$ cell population (Horie et al., 1999; Khandoga et al., 2006; Yang et al., 2006; Zwacka et al., 1997). Lappas et al. (2006) found that the protection against hepatic I/R injury in RAG-1 knockout mice could also be reversed by the adoptive transfer of NKT cells. In a murine model of stroke, RAG-1 deficient mice showed increased resistance to neurological injury and this was attributed to the loss of both CD4 ${ }^{+}$ and $\mathrm{CD}^{+} \mathrm{T}$ cells, whereas depletion of $\mathrm{B}$ cells alone did not confer a benefit (Yilmaz et al., 2006). Taken together, these studies clearly indicate a pathogenic role for $\mathrm{CD}^{+}{ }^{+} \mathrm{T}$ cells during I/R injury in multiple organs, and potentially for NKT cells in the liver and CD8 ${ }^{+}$cells in the brain.

Both Savransky et al. (2006) and Hochegger et al. (2007) found deletion of the TCR $\alpha \beta$, or $\gamma \delta$ protein chains reduced renal injury and cytokine expression, suggesting that $\mathrm{T}$ cell activation during $\mathrm{I} / \mathrm{R}$ is TCR-dependent. This was supported by a study that showed adoptive transfer of $\mathrm{T}$ cells with a limited TCR repertoire (i.e. expressing a transgenic ovalbumin-specific TCR) into $\mathrm{T}$ cell 
deficient mice lessened the extent of renal I/R injury compared to animals that received WT cells (Satpute et al., 2009). Furthermore, blockade of the costimulatory CD28-B7 and CD154-CD40 pathways is protective during ischaemia and although these pathways are essential to the activation of naïve $\mathrm{T}$ cells, it should be noted that these molecules exert additional functions beyond solely $\mathrm{T}$ cell activation (De Greef et al., 2001; Shen et al., 2002; Takada et al., 1997). Despite this mounting evidence of antigen-specific activation of $\mathrm{T}$ cells during ischaemia, the particular stimuli responsible have not yet been identified.

While the previous studies demonstrated a role for antigen-specific $\mathrm{T}$ cells in I/R injury, Shen et al. (2009) found that blocking of antigen-specific CD4 T cell responses without depletion of the cells was not sufficient to protect against liver I/R injury, suggesting antigen non-specific CD4 $\mathrm{T}$ cell activation was involved in the I/R injury. Indeed, $\mathrm{T}$ cells have been shown to be activated independently of their TCR through ROS, inflammatory cytokines, chemokines, and complement, and all of these are present in high levels during I/R (Strainic et al., 2008; Unutmaz et al., 1994). Although which of these components drive antigen-independent $\mathrm{T}$ cell mediated I/R injury has not been identified, it seems likely that both antigen-dependent and independent pathways are involved in the activation of $\mathrm{T}$ cells following ischaemia.

As indicated by the benefit seen in studies described above, $\mathrm{T}$ cells are generally associated with exacerbation of I/R injury, and several studies have linked this damage to the release of IL-17. In a murine model of stroke, IL-17 production by $\gamma \delta \mathrm{T}$ cells located in the infarct boundary zone during the late response to injury increases the production of neurotoxic factors such as IL-1 $\beta$, TNF- $\alpha$, and MMPs, which, in turn, promote neuronal apoptosis (Shichita et al., 2009). Similarly, IL17 production from $\mathrm{T}$ cells contributes significantly to intestinal I/R injury, with mice lacking IL-23, and therefore having reduced IL-17, showing lower levels of injury compared to WT animals (Edgerton et al., 2009). Lastly, in the murine lung, targeted deletion of the invariant NKT (iNKT) cell population reduces I/R injury, and this effect is reversed by the adoptive transfer of WT but not IL-17deficient iNKTs (Sharma et al., 2011). 
In addition to IL-17 production, $\mathrm{T}$ cells may contribute to I/R by interacting with various innate immune cells. For example, multiple studies have correlated $\mathrm{T}$ cell depletion with a reduction in neutrophil infiltration of the ischaemic tissue, suggesting $\mathrm{T}$ cells may participate in their recruitment (Khandoga et al., 2006; Lappas et al., 2006; Rabb et al., 2000; Zwacka et al., 1997). Further, Khandoga et al. demonstrated that during hepatic I/R, CD4+ $\mathrm{T}$ cells infiltrate the sinusoids and exacerbate microvascular and hepatocellular damage by increasing plateletendothelial cell interactions. Finally, Shen et al. (2009) found that CD4 T cell expression of the co-stimulatory molecule, CD154, was critical for stimulating CD40 on innate liver cells, resulting in higher levels of injury.

In contrast, there have been a limited number of studies indicating a protective role for certain $\mathrm{T}$ cell subsets during I/R. Lieszi et al. (2009) found Treg production of IL-10 was critical during ischaemic stroke in mice, reducing infarct size by suppressing the proinflammatory cytokines, TNF- $\alpha$, IL- $1 \beta$, and IFN- $\gamma$. Epigenetic modification, in part through histone acetylation, has been associated with the stabilisation of Foxp3 transcription and Treg function (Floess et al., 2007). Accordingly, administration of an inhibitor of histone deacetylase in mice was shown to enhance Treg suppressive activity and improve cardiac graft survival, which may be equally relevant during myocardial I/R in the absence of transplantation (Tao et al., 2007). Selective activation of resident liver NKT cells shortly before an ischaemic insult has also been shown to limit hepatic injury. This protective effect was dependent on NKT-induced increases in IL-13 and the adenosine $A_{2 A}$ receptor expression levels (Cao et al., 2009).

Our knowledge regarding the mechanisms through which $\mathrm{T}$ cells mediate tissue damage during I/R is incomplete but, taken together, the previously described studies suggest that they are likely to be complex and multifactorial. Overall, the literature addressing the role of the adaptive immune system during I/R injury highlights the involvement of $\mathrm{T}$ cells in both pathological and protective functions, defining them as important potential targets for modulation by RIPC. 


\subsection{Aims}

- To determine whether RIPC alters intracellular cytokine production in peripheral blood leukocytes.

- To investigate the effect of RIPC on the activation potential of neutrophils, monocytes, and $\mathrm{T}$ cells cultured in vitro.

- To examine changes in antigen-specific and polyclonal responses of PBMC following RIPC. 


\subsection{Methods}

Participants were recruited and treated as described in Chapter 4. Please refer to sections 4.3.1 - 4.3.3 for full details of the study design, participants, and blood sample collection, and appendices $\mathrm{C}$ and $\mathrm{E}$ for the composition of buffers, and a complete list of antibodies and dilutions used, respectively.

\subsubsection{Intracellular cytokine staining}

Heparanised whole blood was diluted 5:1 with CTCM with or without $50 \mathrm{ng} / \mathrm{mL}$ PMA and $500 \mathrm{ng} / \mathrm{mL}$ ionomycin (4 hour cultures; Sigma Aldrich) or $10 \mathrm{ng} / \mathrm{mL}$ PMA and $500 \mathrm{ng} / \mathrm{mL}$ ionomycin (27 hour cultures; Sigma Aldrich) and incubated at $37{ }^{\circ} \mathrm{C}$ with $5 \% \mathrm{CO}_{2}$. A monensin based protein transport inhibitor, GolgiStop (BD Biosciences), was added for the final 4 hours of culture. The 4 hour culture samples were extracellularly stained and fixed, then stored overnight at $4{ }^{\circ} \mathrm{C}$. The 27-hour samples were processed the following day, with intracellular staining and acquisition carried out at the same time for all samples. RBC were lysed using a 15 minute incubation with Pharm Lyse (BD Biosciences) at RT followed by centrifugation at $400 \mathrm{x}$ g for 5 minutes, and the samples washed twice in PBS. The cells were stained in duplicate with fixable viability dye eFluor 780 (eBioscience) for 30 minutes on ice in a 96-well round-bottom plate and washed once with PBS. Samples were incubated with anti-CD16 antibody or FACS buffer for 20 minutes, FC receptor blocked with $2 \mathrm{mg} / \mathrm{mL}$ intragam P (CSL) for 10 minutes, then stained with the remaining extracellular marker antibodies for 20 minutes, all at RT, protected from light. Cells were washed once in FACS buffer and then fixed in $4 \%$ paraformaldehyde for 15 minutes. Samples were then washed twice with FACS buffer, resuspended in $0.2 \%$ saponin buffer for 10 minutes, then centrifuged at $400 \mathrm{x}$ g for 5 minutes and the supernatant aspirated. The samples were incubated with intracellular staining mixes prepared in saponin buffer in a total volume of $50 \mu \mathrm{L}$ for 20 minutes at RT, washed once with saponin buffer and once with FACS buffer. Samples were resuspended in FACS buffer and filtered before acquisition on a FACSCanto ${ }^{\text {TM }}$ II with Diva software (BD Biosciences). The data was analysed with FlowJo 7.6.1 software (Tree Star). 


\subsubsection{Culture of PBMC}

\subsubsection{PBMC isolation}

PBMC were isolated following the manufacturer's instructions. Briefly, freshly collected heparinised blood was diluted 3:5 with dPBS without calcium or magnesium (Life Technologies), and carefully layered on top of RT Histopaque1077 (Sigma Aldrich). The sample was centrifuged at $400 \mathrm{x} \mathrm{g}$ for 30 minutes at RT, and the upper layer of supernatant discarded. The middle opaque mononuclear cell layer was collected and washed three times in dPBS (centrifuged at $250 \mathrm{x} \mathrm{g}, 5$ minutes) and resuspended in CTCM. The viable cell concentration was determined by mixing a sample of cells $1: 1$ with $0.4 \%$ Trypan blue (Sigma Aldrich) and counting on an improved Neubauer haemocytometer with a compound microscope (CX41; Olympus).

\subsubsection{T cell stimulation}

Isolated PBMC were cultured in triplicate in a final volume of $200 \mathrm{uL}$ in 96 -well flat-bottom plates, with or without stimulants. A total of $1 \times 10^{5}$ cells per well were stimulated with a final concentration of $5 \mathrm{ng} / \mathrm{mL}$ PMA and $200 \mathrm{ng} / \mathrm{mL}$ ionomycin (Sigma Aldrich), or the tetanus toxoid-containing ADT Booster vaccine (CSL) diluted 1:5. Anti-CD3/28 Expander Beads (Life Technologies) were cultured 1:1 with PBMC plated at $8 \times 10^{4}$ per well, and were prepared by washing in dPBS, pelleting for 1 minute on a magnet, aspirating the supernatant, and resuspending in CTCM. The PMA/I, and dynabead stimulated samples were incubated for 24 hours at $37{ }^{\circ} \mathrm{C}, 5 \% \mathrm{CO}_{2}$, then centrifuged at $400 \mathrm{x}$ g for 5 minutes. The supernatant was harvested and stored at $-20{ }^{\circ} \mathrm{C}$, and the pelleted cells at $-80{ }^{\circ} \mathrm{C}$, for further analyses. The tetanus-stimulated samples were cultured for 7 days and, due to the opacity of the tetanus vaccine and the effect of this on the subsequent assay, the tetanus vaccine was added to the control unstimulated samples immediately prior to centrifugation. The supernatant and cells were stored as described above. 


\subsubsection{CyQuant proliferation assay}

The CyQuant reagent (Life Technologies) was prepared at 2X concentration in cell lysis buffer and nuclease free distilled water, as per the manufacturer's instructions. The cell samples were thawed and $200 \mathrm{uL}$ of the CyQuant reagent was added per well, and left to equilibrate in the dark for 3 minutes. The fluorescence was measured at excitation of $480 \mathrm{~nm}$ and emission of $520 \mathrm{~nm}$ on an EnSpire 2300 multilabel plate reader (PerkinElmer), and the background absorbance level (taken from the control wells without cells) was subtracted from the samples. The stimulation index was calculated by expressing the MFI of each replicate as a ratio to that of the corresponding control.

\subsubsection{Cell culture supernatant cytokine quantification}

The culture supernatant cytokine levels were quantified using a human Th1/Th2/Th9/Th17/Th22 13plex FlowCytomix kit (\#BMS817FF; eBioscience). The kit consisted of 13 bead populations of two sizes with distinct fluorescence intensities that could be resolved in the red flow cytometer channel. Each population was coated with capture antibodies specific for one of the 13 analytes: IFN- $\gamma$, IL-1 $\beta$, IL-2, IL-4, IL-5, IL-6, IL-9, IL-10, IL-12p70, IL-13, IL-17A, IL-22, or TNF- $\alpha$. The beads, together with biotin-conjugated secondary antianalyte antibodies, were incubated with the supernatant sample. SA-PE was then added to distinguish the cytokine-bound beads from the unbound population, and the resulting fluorescence in the yellow channel used to determine the analyte concentrations within each sample, relative to a range of known standards. The beads were mixed at a final dilution of 1:80 and then washed by centrifugation at $3000 \mathrm{x}$ g for 5 minutes, aspiration of the supernatant, and resuspension in the same volume of reagent dilution buffer. The biotinconjugates were also mixed and diluted 1:80 with assay buffer. The standards were reconstituted according to the manufacturer's instructions, and prepared using a 3-fold serial dilution. Following reagent preparation, $25 \mu \mathrm{L}$ of undiluted culture supernatant and duplicate standards were incubated with $25 \mu \mathrm{L}$ of mixed beads and $50 \mu \mathrm{L}$ of biotin conjugate mix for 2 hours at RT in a 96-well V-bottom plate. At the end of the incubation the samples were washed twice by the addition of wash buffer, centrifugation at $400 \mathrm{x}$ g for 5 minutes, and aspiration of 
the supernatant. After the final wash, all but $100 \mu \mathrm{L}$ of supernatant was removed and $50 \mu \mathrm{L}$ of SA-PE solution diluted 1:125 in assay buffer was incubated with each sample for a further hour at RT. The samples were then washed twice more, resuspended in assay buffer, and acquired on a FACSCanto ${ }^{\mathrm{TM}}$ II flow cytometer (BD Biosciences) set up according to the kit manufacturer's instructions. The data was analysed with BD FACSDiva (BD Biosciences) and FlowCytomixPro (eBioscience) software.

\subsubsection{Statistics}

The statistical analysis of the trial results was performed under the guidance of Dr Dalice Sim. The intracellular cytokine concentrations and supernatant cytokine levels were compared between treatment groups and conditions using two-way repeated measures ANOVA with the Greenhouse-Geisser correction and Bonferroni post-hoc tests. For the ICS, the 4-hour and 27-hour culture results were analysed separately. PBMC proliferation levels were compared using a twotailed paired t-test. Graphs were produced using GraphPad Prism 5 (GraphPad Software Inc) and statistical tests were performed using SPSS Statistics (IBM Corporation). Differences corresponding to $\mathrm{p}$ values $<0.05$ were considered statistically significant, and comparisons were not found to be significant unless specified. 


\subsection{Results}

\subsubsection{Intracellular cytokine production in whole blood cultures}

In our trial of RIPC in high-risk cardiac surgery patients, we found a general trend towards slightly increased levels of IL-6 and IL-8 in the preconditioned group as early as 1 hour following bypass ( $p=0.09$ and 0.10 for the unadjusted differences for IL-6 and IL-8 1 hour after cross-clamp removal, respectively). We were therefore interested in whether RIPC could directly alter the ability of key immune cells to produce these cytokines, and which cell types might be contributing to the increased levels. To address this question, we performed ICS using whole blood from samples collected one hour after treatment during each visit.

In Chapter 4, we observed that there were two participants with consistently elevated levels of serum cytokines at both the baseline and post-treatment time points during both study visits. The entire ICS dataset was analysed with and without the inclusion of these two participants, and their exclusion was found to have only subtle effects on the statistical analysis that did not change the conclusions drawn from the data. The individual results obtained from these participants were consistent with the remaining dataset and did not appear to be outliers; therefore they were included in the final analysis presented in the following sections.

\subsubsection{Monocytes and neutrophils}

Monocytes and neutrophils are rapidly activated during cardiac surgery and mediate much of the tissue damage sustained in the post-operative period; therefore, their potential contribution to cytokine production during both the early and late phases of RIPC-induced protection were of particular interest. To determine the effect of RIPC on neutrophil and monocyte function, whole blood was stimulated for 4 or 27 hours with PMA/I. This stimulation induced significant changes in the proportion of live cells, CD11b expression, and intracellular cytokine production, as expected. 
Following a 4-hour culture period, neutrophils constituted approximately $60 \%$ of the total live leukocytes assessed from the unstimulated blood samples, and there was a slight reduction in the proportion of viable neutrophils in the samples stimulated with PMA/I (Figure 5.1B). In contrast, the monocyte population represented between $6-8 \%$ of the total leukocytes and there was a slight increase in the proportion following 4 hours of stimulation, which most likely was due to the reduction in neutrophils (the most predominant leukocyte subset) rather than an increase in the absolute number of monocytes (Figure 5.1A). The level of cell survival in neutrophils stimulated for 27 hours was similar to that detected in the shorter cultures; however, there was a slight reduction in viable cells in the unstimulated sample (Figure 5.1F). In contrast, there was a substantial decrease in live monocytes following 27 hours stimulation; whereas, the proportion of cells in the unstimulated cultures seemed largely unchanged (Figure 5.1E). The size of the viable monocyte and neutrophil populations were not affected by preconditioning.

There was a notable trend towards reduced CD11b expression in cultured monocytes, following RIPC. This affect was most pronounced in the 4-hour cultures, although the difference between the treatment groups did not reach statistical significance ( 4 hours: $p=0.094 ; 27$ hours: $p=0.265$; Figure $5.1 \mathrm{C}$ and G). A similar trend of reduced CD11b expression was observed in the neutrophils from preconditioned participants; however, this effect appeared limited to the stimulated samples, suggesting RIPC may reduce the ability of neutrophils to become activated in response to subsequent stimulation (Figure 5.1D and H). The difference between the groups appeared largest after 27 hours of culture, but did not reach statistical significance ( 4 hours: $p=0.06$; 27 hours: $p=0.058$ ).

In both the 4 and 27-hour cultures, monocytes were found to predominantly produce IL-8, and only very low levels of IL-6 (Figure 5.2A and C, and Figure 5.3A and C). Notably, monocyte IL-8 production was higher in the unstimulated samples, suggesting this expression was constitutive. The percentage of unstimulated viable monocytes that stained positively for IL-8 increased from 10 - $20 \%$ at 4 hours, to $30-40 \%$ after 27 hours (Figure 5.2C and Figure 5.3C). The 
pattern of neutrophil cytokine production was similar between the 4 and 27hour cultures, with enhanced responses in the latter samples. Between $5-10 \%$ of neutrophils produced IL-6 constitutively after 4 hours culture, whereas, 10 $15 \%$ of neutrophils stained positively for intracellular IL-8 following stimulation (Figure 5.2B and D). Very little, if any, IL-8 production was detected in the unstimulated neutrophils and only a minor population of monocytes and neutrophils simultaneously produced IL- 6 and IL-8 $(<1 \%$ in the 4 hour cultures and $<10 \%$ in the 27 hour cultures; Figure 5.2E and F, and Figure 5.3E and F). RIPC did not significantly alter IL-6 or IL-8 production in unstimulated or stimulated monocytes and neutrophils, with either duration of cultures. Thus, it appears that monocytes and neutrophils do not produce increased levels of IL-6 or IL-8 following RIPC and may, in fact, be suppressed by the intervention. 

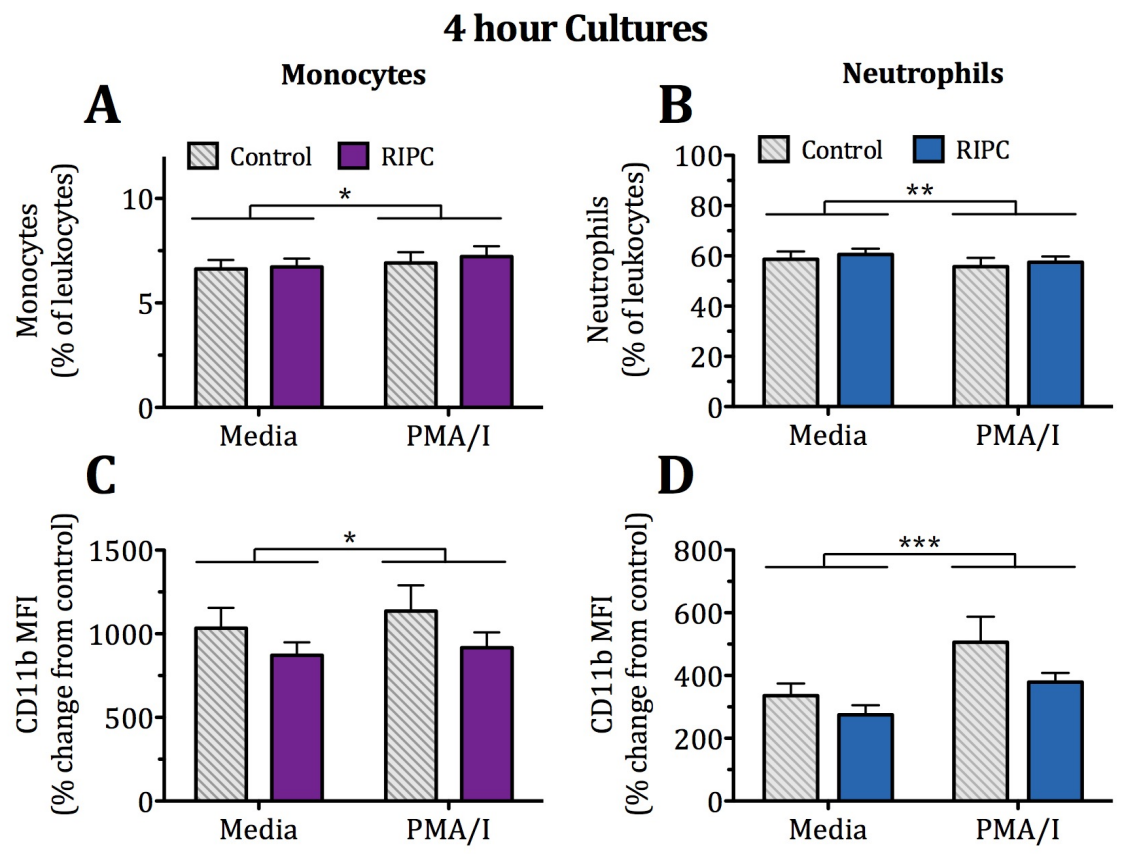

\section{7 hour Cultures}
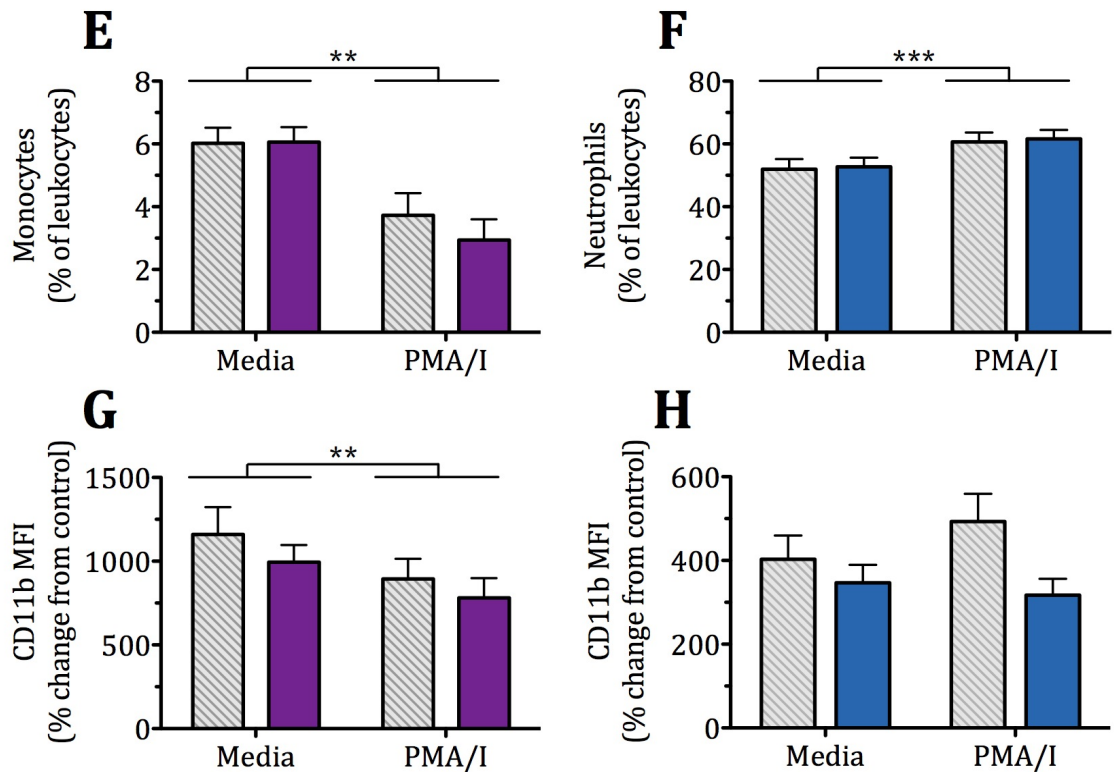

Figure 5.1 Size and activation state of monocyte and neutrophil populations after 4 or 27 hours of culture

Blood was collected 1 hour after treatment, cultured, and assessed for intracellular cytokines with flow cytometry. (A - D) samples cultured for 4 hours with or without $50 \mathrm{ng} / \mathrm{mL}$ PMA, $500 \mathrm{ng} / \mathrm{mL}$ ionomycin, and GolgiStop (1:1000). $(\mathrm{E}-\mathrm{H})$ samples cultured for 27 hours with or without $10 \mathrm{ng} / \mathrm{mL}$ PMA and 500 $\mathrm{ng} / \mathrm{mL}$ ionomycin, with GolgiStop (1:1000) for the final 4 hours. (A \& E) CD14 ${ }^{+}$CD16-/low monocytes; (B \& F) CD14-CD16 ${ }^{+}$neutrophils; (C \& G) CD11b in monocytes; (D \& H) CD11b in neutrophils. MFI is expressed as the percentage change from the isotype control. Two-way repeated measures ANOVA with Bonferroni post-hoc tests were used to compare the treatments and culture conditions. ${ }^{*} \mathrm{p}<0.05,{ }^{* *} \mathrm{p}<0.01$, and ${ }^{* * *} \mathrm{p}<0.001$. Samples were prepared in duplicate and shown are the group means and SEM for $n=10$. For representative data plots refer to appendix F, Figure F.6. 


\section{IL-6 ${ }^{+} /$IL-8 $^{-}$Cells}
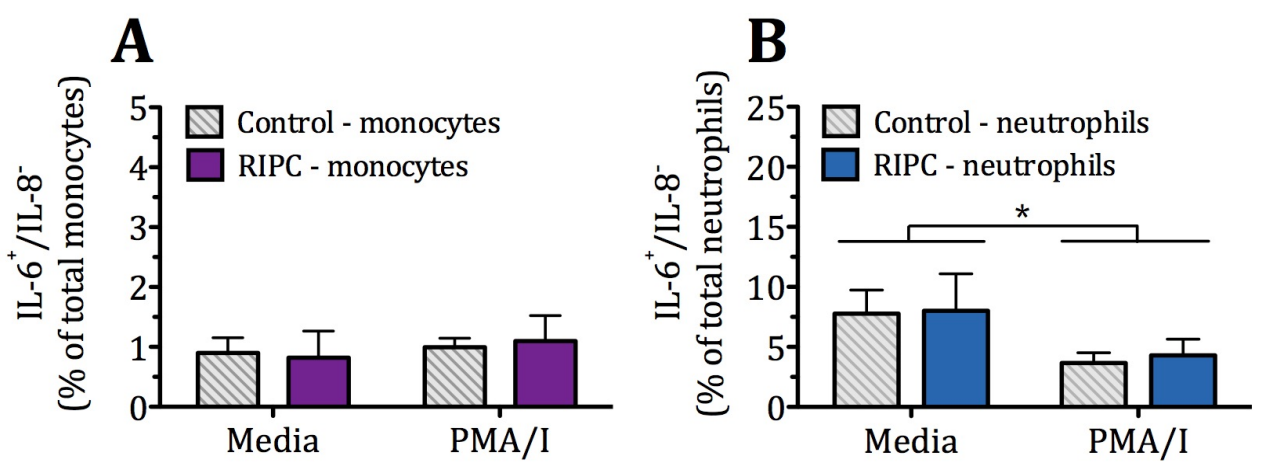

\section{IL-6 $^{-} /$IL-8 $^{+}$Cells}
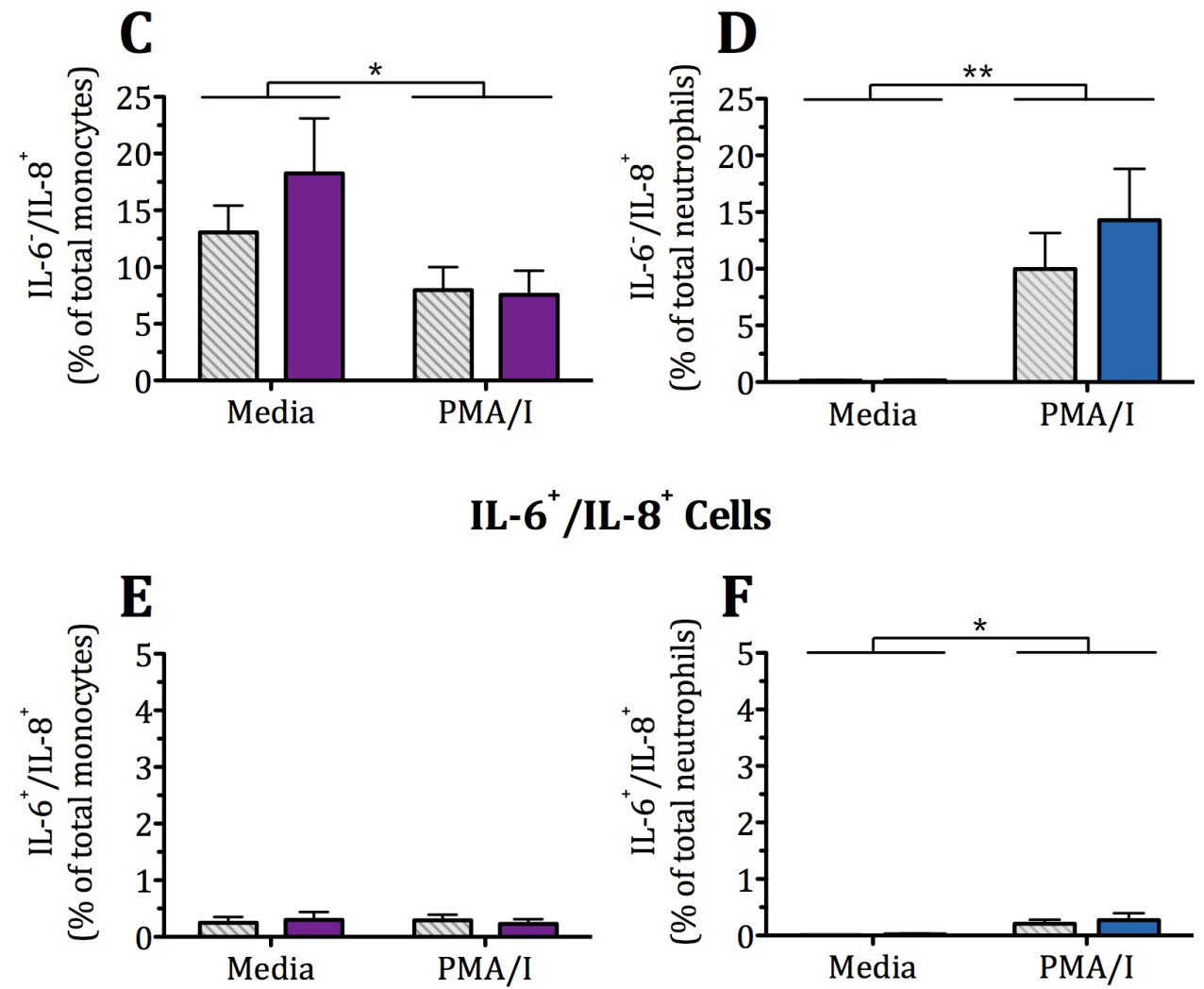

\section{IL-8 ${ }^{+}$Cells}

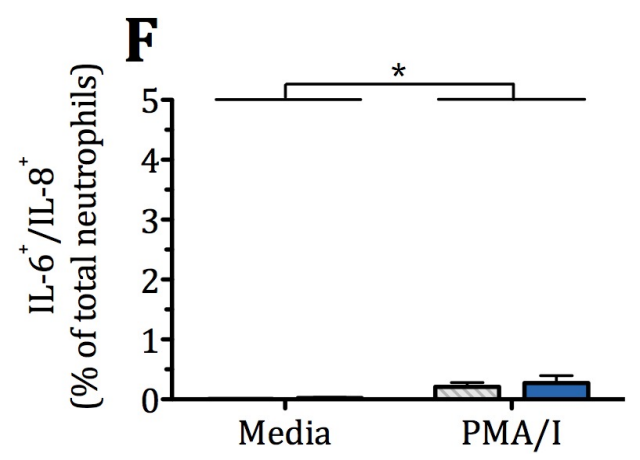

Figure 5.2 Early phase of intracellular IL-6 and IL-8 production in cultured monocytes and neutrophils

Blood was collected 1 hour after treatment; cultured for 4 hours with or without $50 \mathrm{ng} / \mathrm{mL}$ PMA, $500 \mathrm{ng} / \mathrm{mL}$ ionomycin, and GolgiStop (1:1000); then assessed for intracellular cytokines with flow cytometry. (A and B) Percentage of IL- $6^{+} / \mathrm{IL}-$ 8- monocytes (purple) and neutrophils (blue); (C and D) IL-6-/IL-8 ${ }^{+}$cells; ( $\mathrm{E}$ and F) $\mathrm{IL}-6^{+} / \mathrm{IL}-8^{+}$cells. Cells are expressed as the percentage of the total live parent population. Two-way repeated measures ANOVA with Bonferroni post-hoc tests were used to compare the treatments and culture conditions. ${ }^{*} \mathrm{p}<0.05$ and ${ }^{* *} \mathrm{p}$ $<0.01$. Samples were prepared in duplicate and shown are the group means and SEM for $\mathrm{n}=10$. For representative data plots refer to appendix F, Figures F.6 and F.7. 


\section{IL-6 ${ }^{+} /$IL-8 $^{-}$Cells}
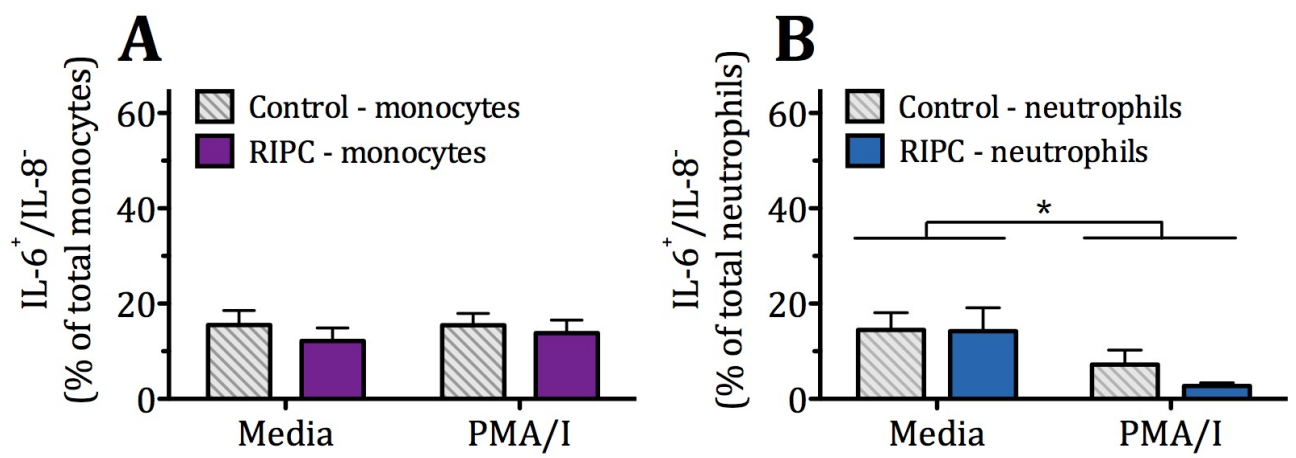

IL-6- /IL-8 ${ }^{+}$Cells
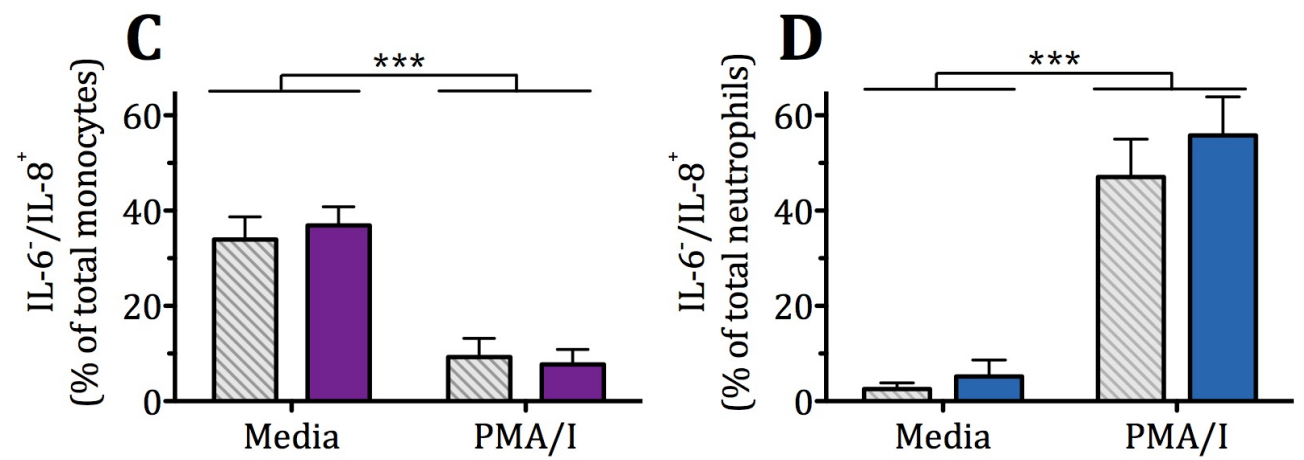

\section{IL-6 $^{+} /$IL-8 $^{+}$Cells}
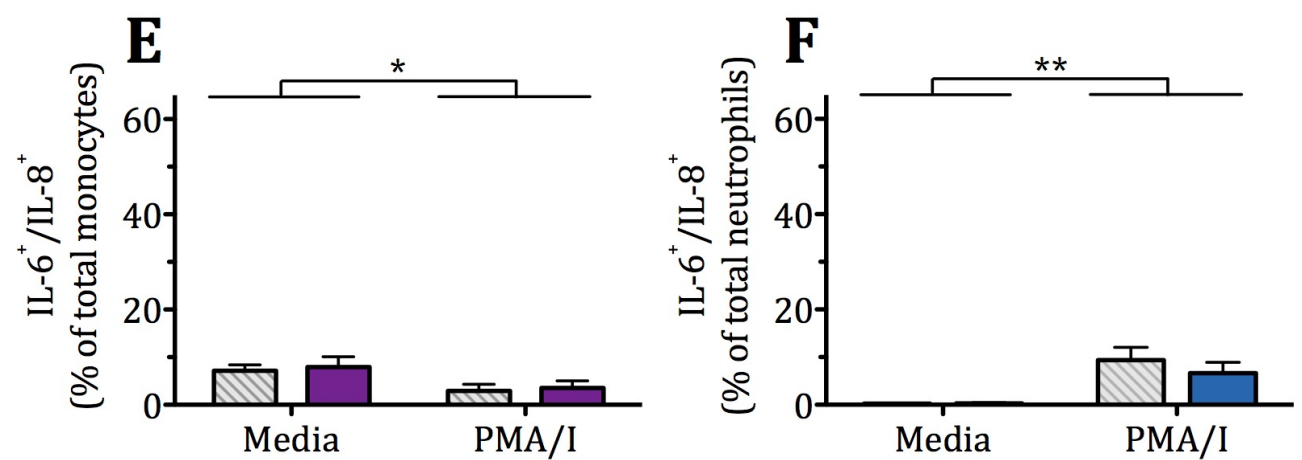

Figure 5.3 Late stage intracellular IL-6 and IL-8 production in cultured monocytes and neutrophils

Blood was collected 1 hour after treatment; cultured for 27 hours with or without $10 \mathrm{ng} / \mathrm{mL}$ PMA, $500 \mathrm{ng} / \mathrm{mL}$ ionomycin, and GolgiStop (1:1000) for the final 4 hours; then assessed for intracellular cytokines with flow cytometry. (A and B) Percentage of IL- $6^{+} /$IL-8 $^{-}$monocytes (purple) and neutrophils (blue); (C and D) IL-6 $-/ \mathrm{IL}^{-} 8^{+}$cells; ( $\mathrm{E}$ and F) $\mathrm{IL}_{-} 6^{+} / \mathrm{IL}-8^{+}$cells. Cells are expressed as the percentage of the total live parent population. Two-way repeated measures ANOVA with Bonferroni post-hoc tests were used to compare the treatments and culture conditions. ${ }^{*} \mathrm{p}<0.05$ and ${ }^{* *} \mathrm{p}<0.01$. Samples were prepared in duplicate and shown are the group means and SEM for $n=10$. For representative data plots refer to appendix F, Figures F.6 and F.7. 


\subsubsection{T cell subsets}

In addition to investigating the effect of RIPC on neutrophil and monocytes in PMA/I-stimulated whole blood samples, the effect on $\mathrm{T}$ cells was also determined. T cell responses are believed to be more relevant during the delayed phase of preconditioning, and in support of this, cytokine production following 4 hours of culture was not altered by preconditioning in our trial (refer to appendix $\mathrm{H}$ for the full dataset; Ascon et al., 2006; Sullivan et al., 2009). Therefore, we focused on the $\mathrm{T}$ cell cytokine responses in the 27-hour cultures. Because the CD4 population could not be clearly resolved for one participant, their data was not included in portions of the analysis, reducing the sample size to 9 for those parameters as noted in the figure legends. Finally, as with the monocyte and neutrophil analyses, PMA/I caused clear changes in the expression of $\mathrm{T}$ cell markers of activation and cytokine production in samples from both control and RIPC-treated participants.

On average, $\mathrm{CD}^{+} \mathrm{T}$ cells constituted approximately $26 \%$ of the viable leukocyte population after 27 hours culture, and this proportion was slightly reduced following stimulation with PMA/I in both groups (Figure 5.4A). The levels of the $\mathrm{CD}^{+}$and $\mathrm{CD}^{+}$subsets were very consistent between visits and culture conditions (Figure 5.4B and C). The $\mathrm{CD}^{+}$cells were the predominant subset, accounting for around $53 \%$ of the total $\mathrm{CD}^{+}$cells, on average. RIPC did not alter the proportions of any of these populations, compared to the control visit.

Although there was a high level of variation between participants, expression levels of CD25 and another $\mathrm{T}$ cell activation marker, CD69, were both substantially increased in the CD4 and total T cell populations, respectively, after stimulation with PMA/I (Figure 5.4D and E). This finding confirmed the successful activation of these cells using our ICS protocol. There was a trend towards an increased level of $\mathrm{T}$ cell activation in the stimulated cultures following RIPC but this difference did not reach statistical significance. CD69 is an early marker of $\mathrm{T}$ cell activation and a significant upregulation of CD69 was also detectable in the PMA/I stimulated cultures after 4 hours; however, the 
difference between the treatment groups was only observed in the 27 hour cultures (refer to appendix H, Figure H.1E).

A notable population of the unstimulated CD4 $\mathrm{T}$ cells were positive for intracellular IL-6, and this proportion was increased following stimulation with PMA/I (Figure 5.5A). Interestingly, the MFI of IL-6 staining did not differ between the unstimulated and stimulated CD4 T cells, suggesting that a greater number of cells were individually producing a lower amount of IL-6 following stimulation, compared to the constitutive production in unstimulated cells (refer to appendix H, Figure H.3A). A small population of CD8 T cells also produced IL6 , with and without stimulation, but to a lesser extent than was detected in the CD4 subset (Figure 5.5C). IL-8 production in both the CD4 and CD8 T cell populations was very low, with less than $1 \%$ of cells staining positively for intracellular levels (Figure 5.5B and D). Expression levels of both IL-6 and IL-8 in T cells did not change with RIPC. Taken together, these results suggest that RIPC may increase the propensity of $\mathrm{T}$ cells to become activated, but that this enhanced activation does not result in increased production of the inflammatory cytokines, IL-6 and IL-8. 

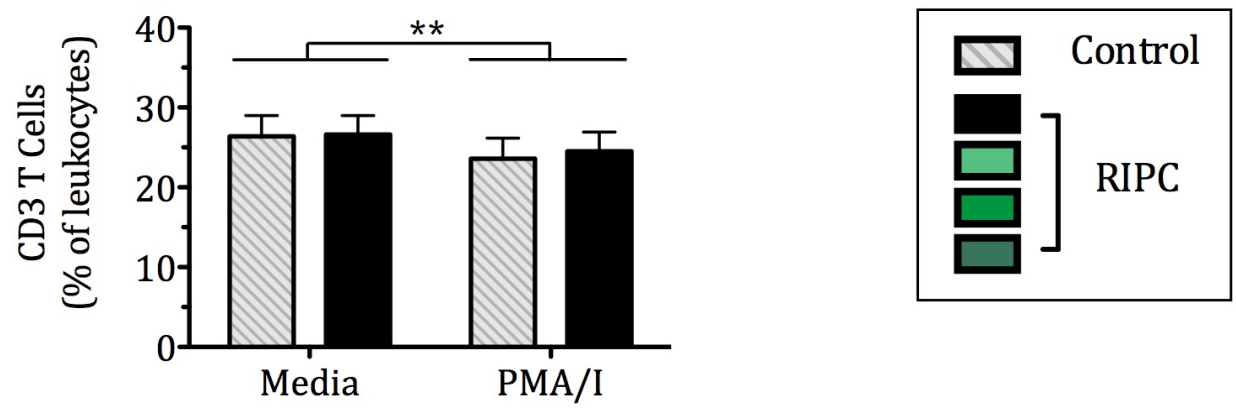

B CD4 T Cells

C CD8 T Cells
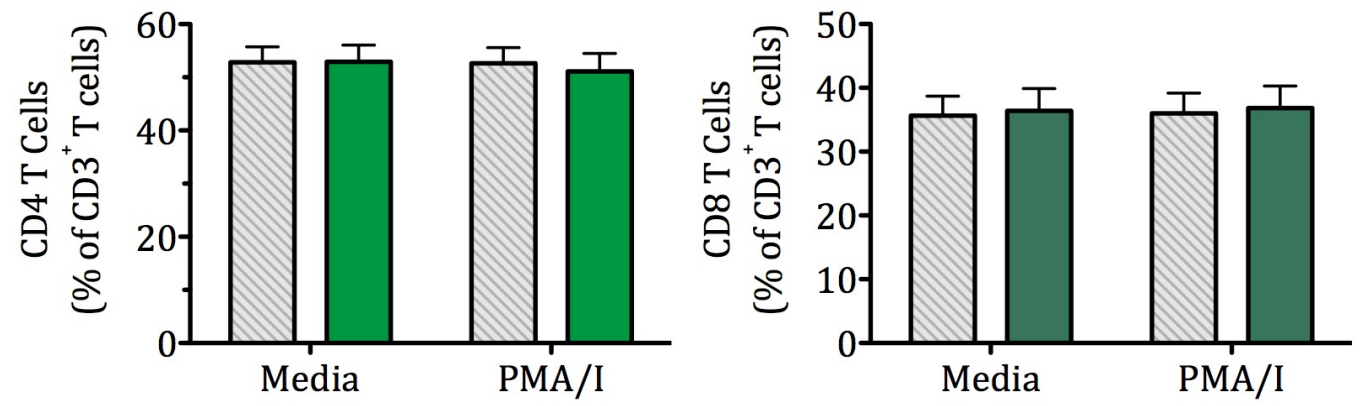

D $\mathrm{CD}^{+}{ }^{\mathrm{T}}$ Cells

$\mathbf{E}$ CD69 $^{+}$T Cells
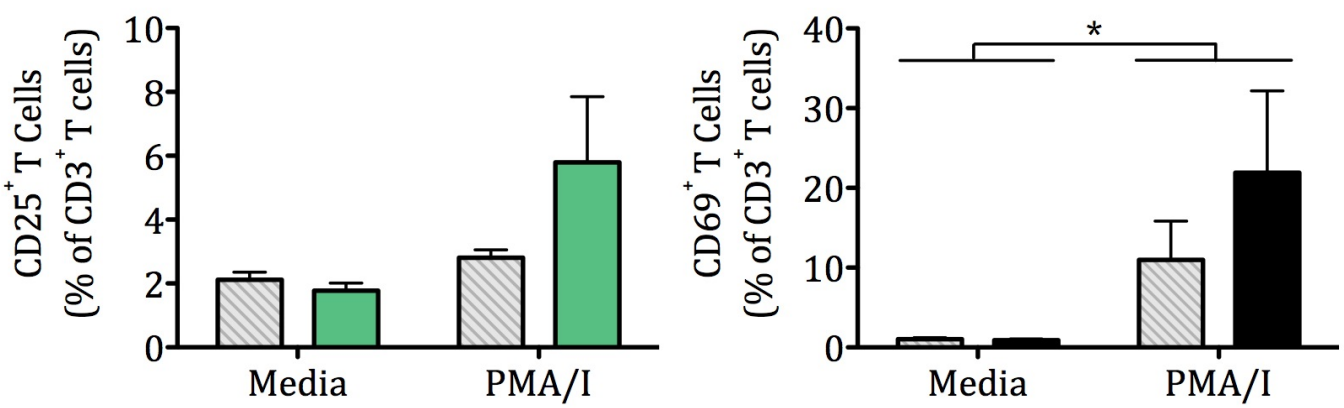

Figure $5.4 \mathrm{~T}$ cell subset proportions and activation state after 27 hours culture

Blood was collected 1 hour after treatment; cultured for 27 hours with or without $10 \mathrm{ng} / \mathrm{mL}$ PMA, $500 \mathrm{ng} / \mathrm{mL}$ ionomycin, and GolgiStop (1:1000) for the final 4 hours; then assessed for intracellular cytokines with flow cytometry. Proportions of cell populations are shown for (A) $\mathrm{CD}^{+}$cells; (B) CD4 ${ }^{+} \mathrm{T}$ cells; (C) CD8 ${ }^{+} \mathrm{T}$ cells; (D) CD4+CD25+ $\mathrm{T}$ cells; (E) CD69+ ${ }^{+}$cells. CD3 ${ }^{+}$cells are expressed as the percentage of the total live leukocytes, and the remaining subsets as a percentage of the $\mathrm{CD}^{+}$parent population. Two-way repeated measures ANOVA with Bonferroni post-hoc tests were used to compare the treatments and culture conditions. ${ }^{*} \mathrm{p}<0.05$ and ${ }^{* *} \mathrm{p}<0.01$. Samples were prepared in duplicate and shown are the group means and SEM for $n=10$ for $A, C$, and $E$, and $n=9$ for $B$ and D. For representative data plots refer to appendix F, Figures F.8, F.9 and F.10. 
A $\quad$ IL-6 $^{+}$Cells $\quad$ B $\quad$ IL-8 $^{+}$Cells
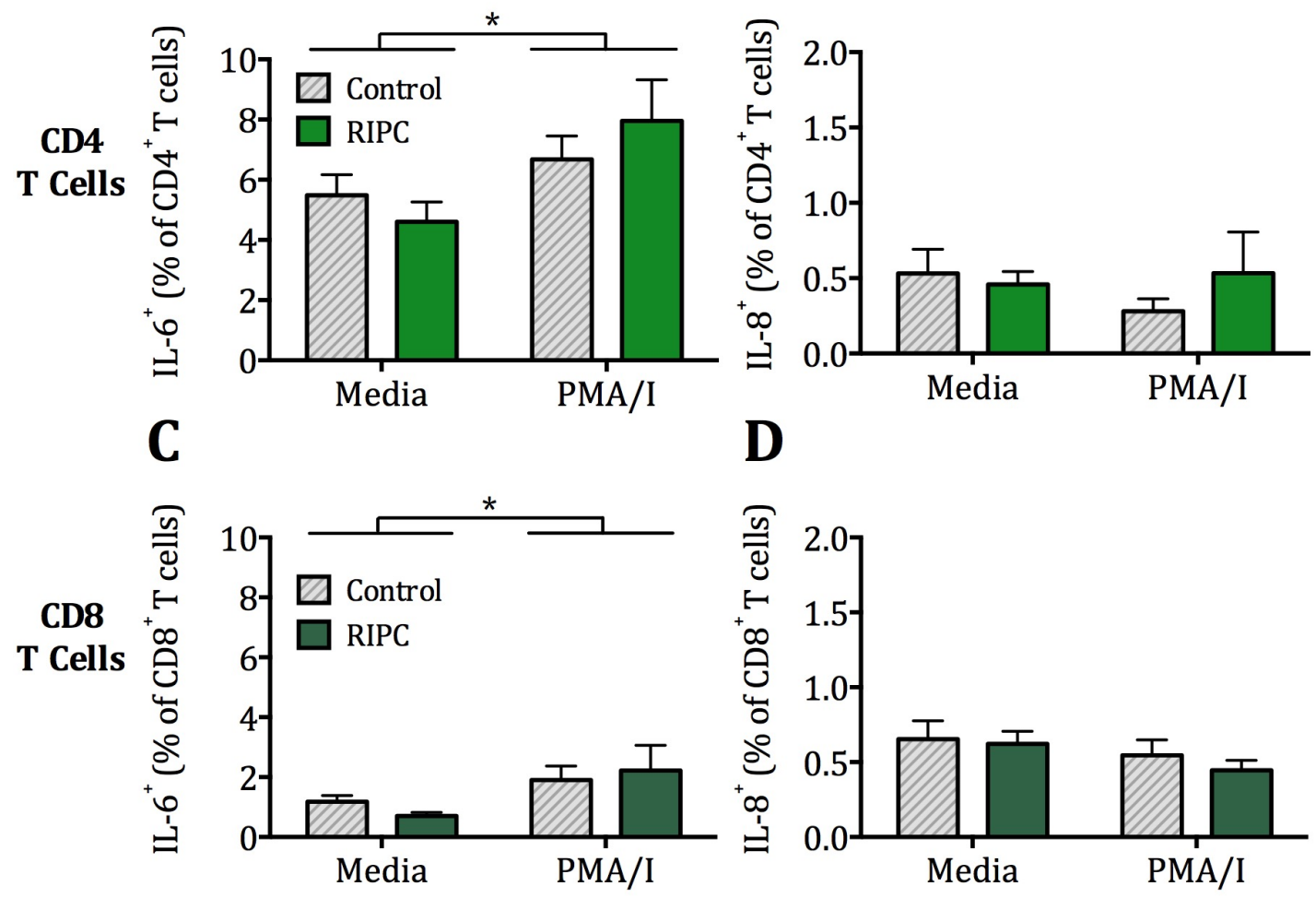

Figure 5.5 Late phase intracellular IL-6 and IL-8 production in cultured T cell subsets

Blood was collected 1 hour after treatment; cultured for 27 hours with or without $10 \mathrm{ng} / \mathrm{mL}$ PMA, $500 \mathrm{ng} / \mathrm{mL}$ ionomycin, and GolgiStop (1:1000) for the final 4 hours; then assessed for intracellular cytokines with flow cytometry. Percentages of CD4 T cells (A and B) and CD8 T cells (C and D) stained positively for IL-6 (A and C) and IL-8 (B and D) are shown. Cells are expressed as the percentage of the total live parent $\mathrm{CD}^{+}$population. Two-way repeated measures ANOVA with Bonferroni post-hoc tests were used to compare the treatments and culture conditions. ${ }^{*} \mathrm{p}<0.05$. Samples were prepared in duplicate and shown are the group means and SEM for $n=9$ for $A$ and $B$, and $n=10$ for $C$ and D. For representative data plots refer to appendix F, Figures F.8 and F.9. 


\subsubsection{CD56 $^{+}$cell subsets}

In the whole blood cultures, $\mathrm{CD} 3^{-} \mathrm{CD} 56^{+} \mathrm{NK}$ cells accounted for approximately $2.7 \%$ of the total viable leukocytes after 27 hours without stimulation, and this was reduced to less than $2 \%$ following treatment with PMA/I (Figure 5.6A). Intracellular levels of IL-6 and IL-8 were very low in this subset, but IL-8 production was induced in $2-3 \%$ of NK cells following stimulation, and IL-6 was also significantly but modestly induced with PMA/I (Figure 5.6B and C).

A very minor population of $\mathrm{CD}^{+} \mathrm{CD}^{2} 6^{+}$NKT cells $(<1 \%$ of the total live leukocytes) was present in the unstimulated samples cultured for 27 hours, and was slightly reduced following stimulation (Figure 5.7A). The majority of these NKT cells expressed CD8, and a smaller population were positive for CD4 (Figure 5.7B and C). Unstimulated NKT cells appeared to be a minor source of IL-6 and IL-8, and the number of cytokine producing cells was increased following PMA/I treatment, although the response was variable between individuals (Figure 5.7D and $\mathrm{E})$.

Cytokine production in the $\mathrm{CD}^{2} 6^{+}$subsets was negligible after 4 hours of culture (refer to appendix H for data, Figures H.4 and H.5). Overall, while PMA/I had a significant effect, RIPC did not alter the size of the NK and NKT populations, or their constitutive or induced production of IL- 6 and IL-8 during either culture period. 

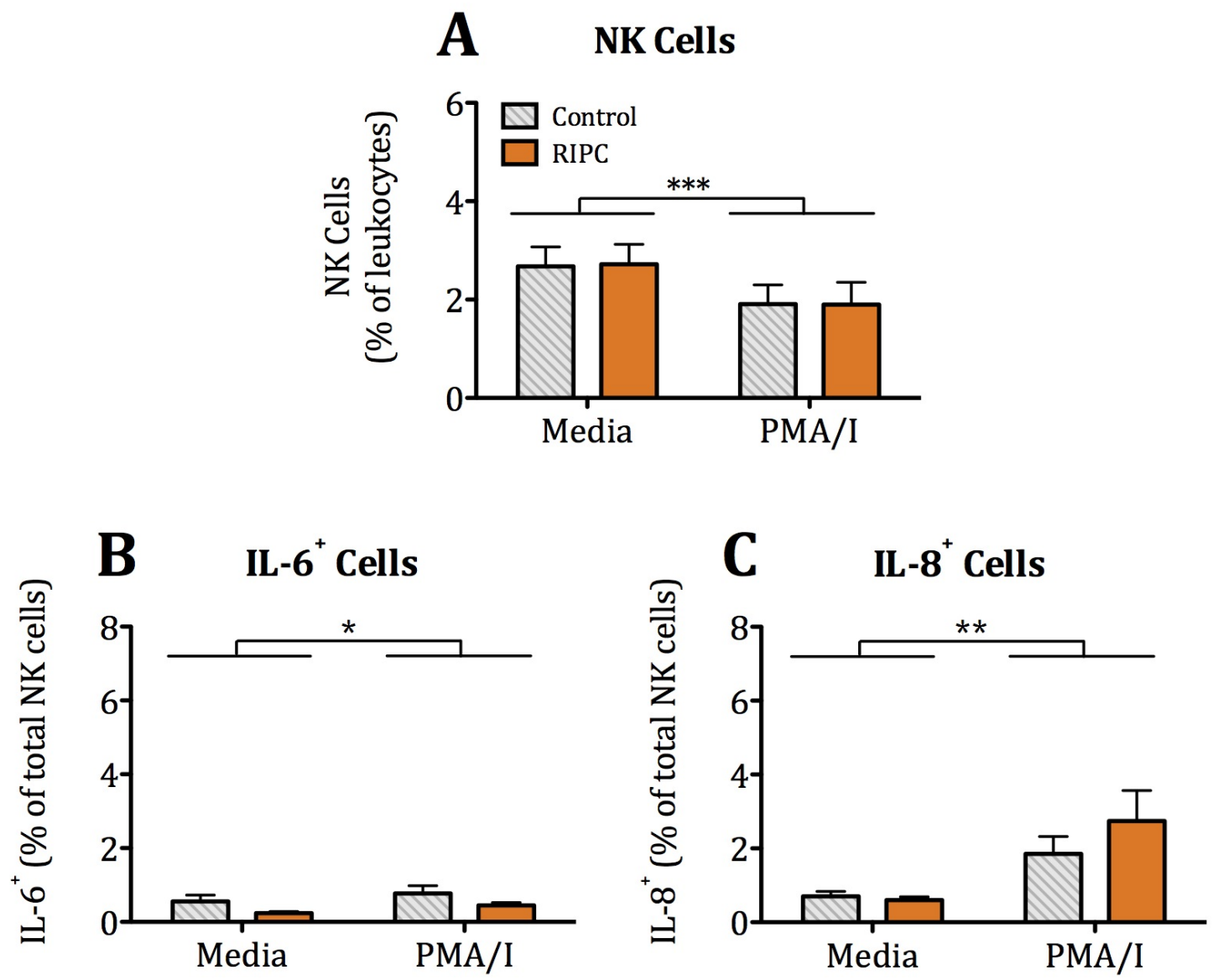

Figure 5.6 Intracellular cytokine production in NK cells cultured for 27 hours

Blood was collected 1 hour after treatment; cultured for 27 hours with or without $10 \mathrm{ng} / \mathrm{mL}$ PMA, $500 \mathrm{ng} / \mathrm{mL}$ ionomycin, and GolgiStop (1:1000) for the final 4 hours; then assessed for intracellular cytokines with flow cytometry. (A) Proportion of $\mathrm{CD}^{-} \mathrm{CD}^{-} 6^{+} \mathrm{NK}$ cells; (B) percentage of $\mathrm{IL}^{-6^{+}}$NK cells; (C) percentage of IL-8 $8^{+} \mathrm{NK}$ cells. Cells are expressed as the percentage of the total live parent population. Two-way repeated measures ANOVA with Bonferroni post-hoc tests were used to compare the treatments and culture conditions. ${ }^{*} \mathrm{p}<$ $0.05,{ }^{* *} \mathrm{p}<0.01$, and ${ }^{* * *} \mathrm{p}<0.001$. Samples were prepared in duplicate and shown are the group means and SEM for $\mathrm{n}=10$. For representative data plots refer to appendix F, Figure F.12. 


\section{A NKT Cells}
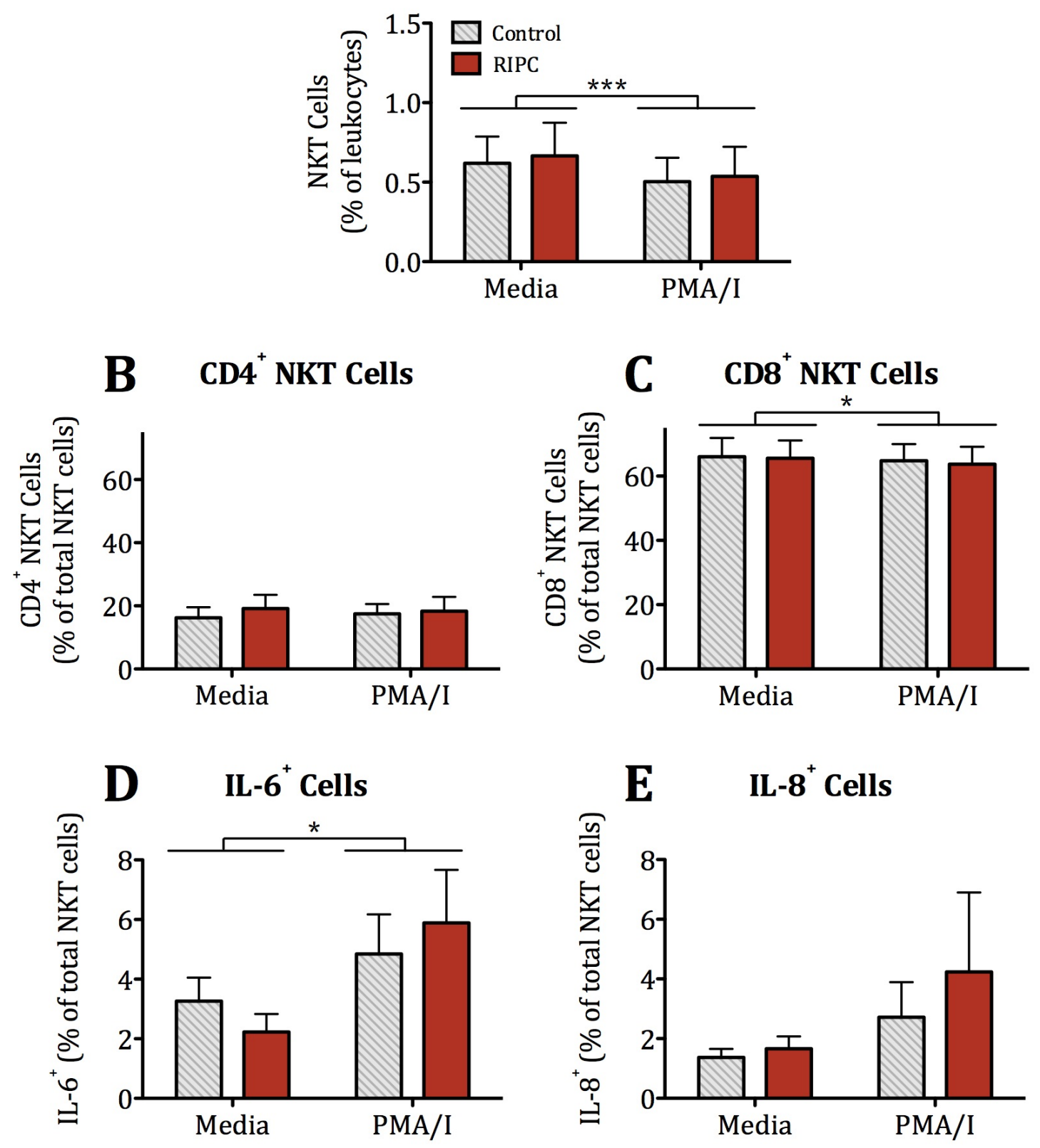

Figure 5.7 Cytokine production in NKT cells after 27 hours of culture

Blood was collected 1 hour after treatment; cultured for 27 hours with or without $10 \mathrm{ng} / \mathrm{mL}$ PMA, $500 \mathrm{ng} / \mathrm{mL}$ ionomycin, and GolgiStop (1:1000) for the final 4 hours; then assessed for intracellular cytokines with flow cytometry. (A) Proportion of $\mathrm{CD}^{+} \mathrm{CD}^{+} 6^{+} \mathrm{NKT}$ cells; (B) CD4 ${ }^{+}$NKT cells; (C) $\mathrm{CD}^{+}$NKT cells; (D) percentage of IL- $^{+}$NKT cells; (E) percentage of IL- $^{+}$NKT cells. Cells are expressed as the percentage of the total live parent population. Two-way repeated measures ANOVA with Bonferroni post-hoc tests were used to compare the treatments and culture conditions. ${ }^{*} \mathrm{p}<0.05$ and ${ }^{* * *} \mathrm{p}<0.001$. Samples were prepared in duplicate and shown are the group means and SEM for $n=10$. For representative data plots refer to appendix F, Figures F.11 and F.12. 


\subsubsection{PBMC proliferative response}

Given $\mathrm{T}$ cells have been implicated in both detrimental and beneficial roles during I/R injury and recovery, we were particularly interested in how RIPC might alter the capacity of this cell population to divide and expand. To investigate proliferation, PBMC were stimulated with PMA/I (non-specific pharmacological stimulus), anti-CD3/CD28 antibodies (antigen-independent T cell receptor stimulus), and the ADT (tetanus and diphtheria) Booster vaccine (antigen-specific $\mathrm{T}$ cell receptor stimulus).

The majority of participants showed a significant increase in lymphocyte proliferation in response to the pharmacological stimulants, PMA/I, after RIPC (Figure 5.8A). This trend did not reach statistical significance, however, as two participants showed a notable decrease in their proliferative response $(\mathrm{p}=$ 0.116). One of these participants showing a reduction in PMA/I-induced PBMC proliferation following RIPC was also one of the participants with high levels of inflammatory baseline serum cytokine levels. Exclusion of the two participants with the high serum cytokine levels reduced the $\mathrm{p}$ value for the difference in PMA/I induced proliferation between treatments to 0.014 ; however, there was no evidence from the remainder of our study that these participants had altered PBMC responses, therefore they were included in the final analysis.

Treatment with antibodies that bind the T cell receptor-CD3 complex and costimulatory CD28 molecule specifically activate $\mathrm{T}$ cells in a physiologically relevant manner. The $\mathrm{T}$ cell proliferative response to $\mathrm{CD} 3 / 28$ stimulation in PBMC cultures was variable between both participants and study visits, with approximately equal numbers of participants showing increases or decreases in proliferation after RIPC treatment (Figure 5.8B). It was not possible to distinguish any effect of the preconditioning from random variability in the proliferative response to polyclonal stimulation of $\mathrm{T}$ cells. Lastly, a strong antigen-specific proliferative memory $\mathrm{T}$ cell response to the tetanus toxin was detected in all participants after 6 days of culture, and did not appear to be altered by RIPC (Figure 5.8C). 


\section{A PMA \& Ionomycin B Anti-CD3/28 Beads C ADT Booster}
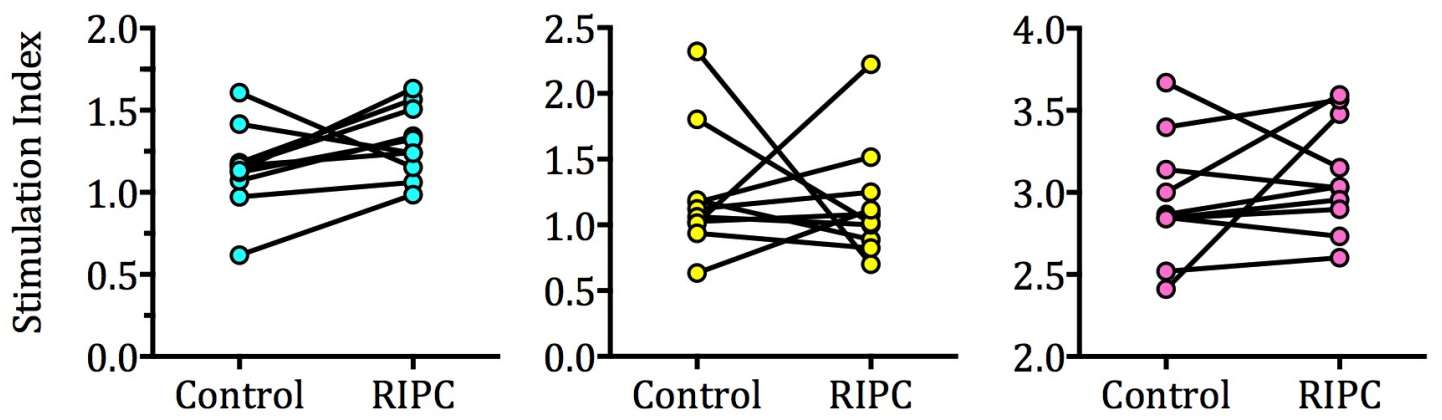

Figure 5.8 PBMC proliferative responses following RIPC treatment

PBMC were isolated from blood collected 1 hour after treatment, and assessed for proliferation with the CyQuant assay following culture with various stimulants. (A) Cells plated at $1 \times 10^{5}$ cells/well with or without $5 \mathrm{ng} / \mathrm{mL}$ PMA and $200 \mathrm{ng} / \mathrm{mL}$ ionomycin for 24 hours. (B) Cells plated $8 \times 10^{4}$ cells/well plated with or without anti-CD3/28 dynabeads at a 1:1 bead to cell ratio for 24 hours. (C) Cells plated at $1 \times 10^{5}$ cells/well with or without a 1:5 dilution of the ADT booster vaccine for 6 days. The stimulation index represents the level of proliferation relative to the unstimulated control sample. Two-tailed paired ttests were used to compare the treatment groups. Shown are the means of triplicates for $\mathrm{n}=10$ participants. 


\subsubsection{PBMC and polyclonal T cell cytokine responses}

We examined the cytokine levels in supernatants from the cell cultures in our proliferative assays to determine whether RIPC altered the general PBMC and T cell cytokine responses, or polarised them towards a particular subset of $\mathrm{T}$ helper cells (e.g. Th1). Because two participants had high baseline serum cytokine levels (Chapter 4), a selection of endpoints were tested with and without the inclusion of their data; however, their results had only subtle effects on the analysis and did not alter the interpretation of the data. Therefore, these participants were included in the final analysis presented in the following sections.

\subsubsection{PBMC responses to PMA/I stimulation}

PMA/I treatment induced significant production of most of the cytokines examined after 24 hours. With respect to the cytokines associated with the typical Th1 response, very high levels of both IL-2 and IFN- $\gamma$ were detected in the stimulated cultures and were absent in the unstimulated samples (Figure 5.9 A and C). IL-12p70 was also present but only at very low concentrations (Figure 5.9B). PMA/I stimulation significantly increased production of the Th2 cytokines, IL-4, IL-5, IL-6, IL-10, and IL-13, and constitutive expression of IL-13 was detected in the unstimulated samples (Figure 5.10A - E). Lastly, pharmacological stimulation with PMA/I also induced significant levels of IL-1 $\beta$, IL-17, and TNF- $\alpha$, which are associated with the proinflammatory Th17 subset (Figure 5.11A, B, and D). A low level of constitutive production of IL-1 $\beta$ was also detected in the unstimulated samples while the moderate constitutive levels of IL-22 were unaltered by stimulation (Figure 5.11A and C). IL-9 was generally below the level of detection (1.5 pg/mL; data not shown). In comparing the two treatment groups, no differences in the levels of cytokine production were detected with or without PMA/I stimulation. Finally, neither RIPC nor PMA/I treatment appeared to bias the Thelper cell response toward Th1, Th2, Th17, Th22, or Th9 cells. 

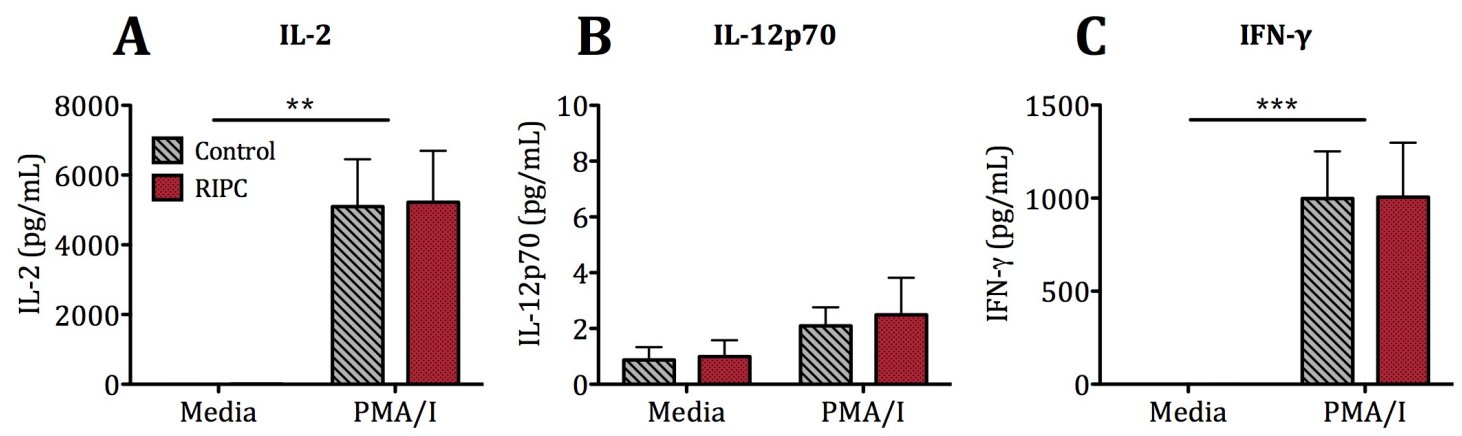

Figure 5.9 Production of Th1 type cytokines in PMA/I stimulated PBMC

PBMC were isolated from blood collected 1 hour after treatment and plated at 1 x $10^{5}$ cells/well, in triplicate, with or without $5 \mathrm{ng} / \mathrm{mL} \mathrm{PMA} \mathrm{and} 200 \mathrm{ng} / \mathrm{mL}$ ionomycin, for 24 hours. Supernatant cytokine levels were assessed with a CBA and flow cytometry. (A) IL-2; (B) IL-12p70; (C) IFN- $\gamma$. Two-way repeated measures ANOVA with Bonferroni post-hoc tests were used to compare the treatments and culture conditions. ${ }^{* *} \mathrm{p}<0.01$ and ${ }^{* * *} \mathrm{p}<0.001$. Shown are the means of triplicates for $\mathrm{n}=10$ participants.
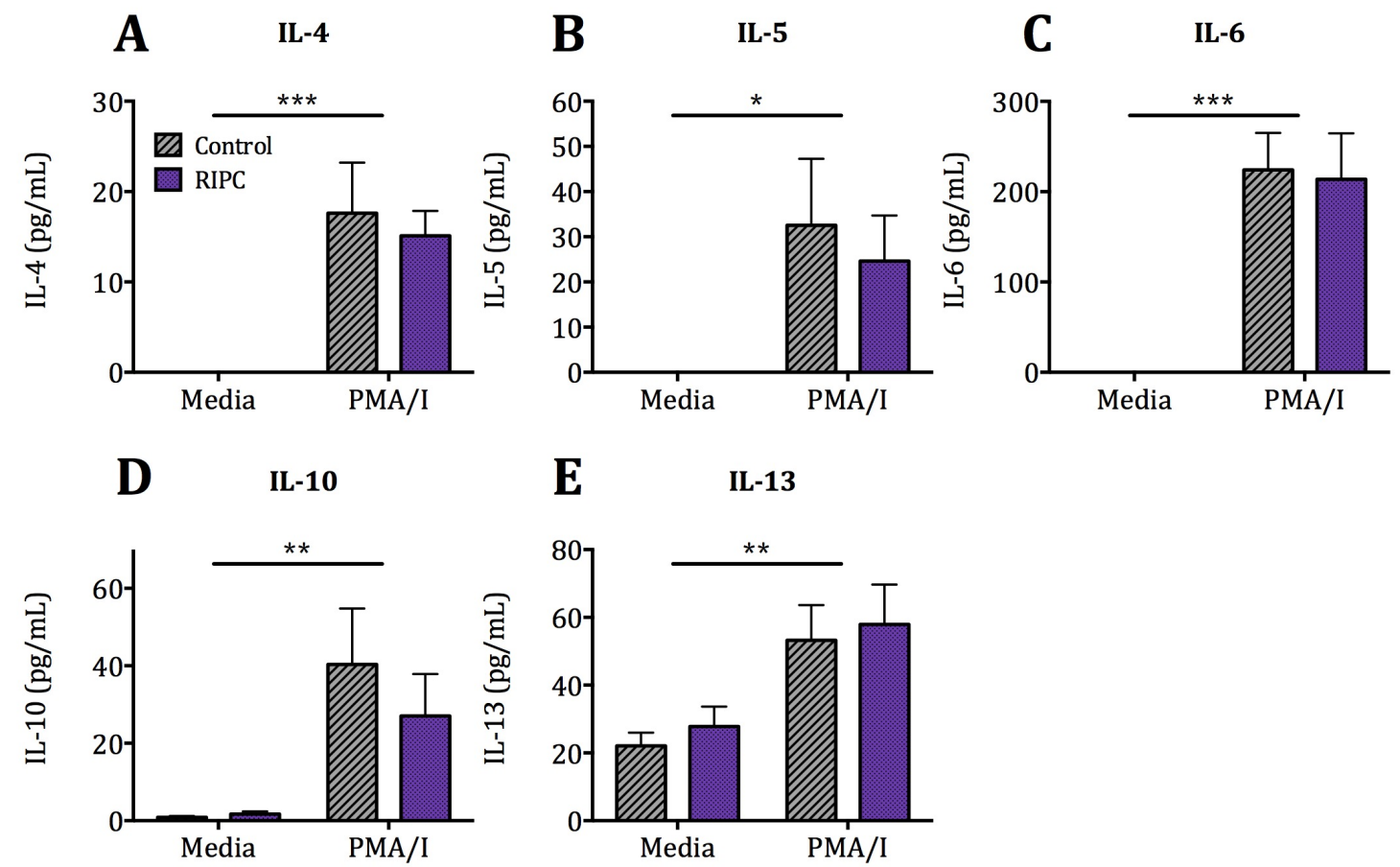

E $\quad$ IL-13

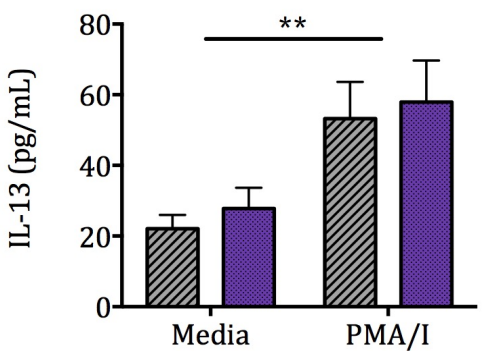

Figure 5.10 Levels of Th2 type cytokines in PMA/I stimulated PBMC cultures

PBMC were isolated from blood collected 1 hour after treatment and plated at 1 x $10^{5}$ cells/well, in triplicate, with or without $5 \mathrm{ng} / \mathrm{mL} \mathrm{PMA}$ and $200 \mathrm{ng} / \mathrm{mL}$ ionomycin, for 24 hours. Supernatant cytokine levels were assessed with a CBA and flow cytometry. (A) IL-4; (B) IL-5; (C) IL-6; (D) IL-10; (E) IL-13. Two-way repeated measures ANOVA with Bonferroni post-hoc tests were used to compare the treatments and culture conditions. ${ }^{*} \mathrm{p}<0.05,{ }^{* *} \mathrm{p}<0.01$, and ${ }^{* * *} \mathrm{p}<0.001$. Shown are the means of triplicates for $\mathrm{n}=10$ participants. 

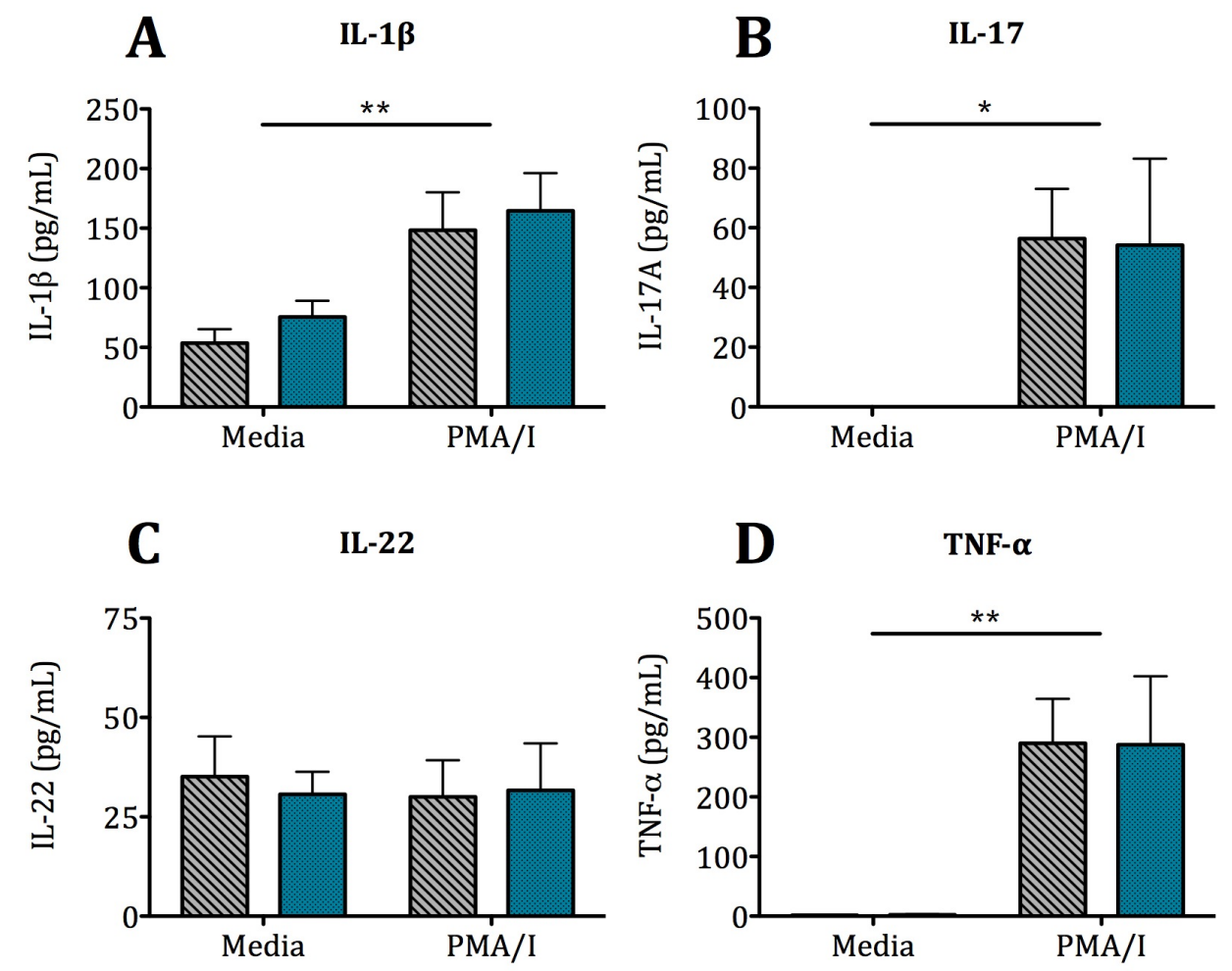

Figure 5.11 Th9/Th17/Th22 type cytokines in PMA/I stimulated PBMC cultures

PBMC were isolated from blood collected 1 hour after treatment and plated at 1 x $10^{5}$ cells/well, in triplicate, with or without $5 \mathrm{ng} / \mathrm{mL} \mathrm{PMA}$ and $200 \mathrm{ng} / \mathrm{mL}$ ionomycin, for 24 hours. Supernatant cytokine levels were assessed with a CBA and flow cytometry. (A) IL-1 ; (B) IL-17; (C) IL-22; (D) TNF- $\alpha$. Two-way repeated measures ANOVA with Bonferroni post-hoc tests were used to compare the treatments and culture conditions. ${ }^{*} \mathrm{p}<0.05$ and ${ }^{* *} \mathrm{p}<0.01$. Shown are the means of triplicates for $\mathrm{n}=10$ participants. 


\subsubsection{Response to anti-CD3/28 dynabead stimulation}

Stimulation of $\mathrm{T}$ cells with anti-CD3/28 dynabeads was associated with a substantial cytokine response. High levels of IL-2, IL-12p70, and IFN- $\gamma$ were detected following stimulation, compared to very minor or no expression in the unstimulated samples (Figure 5.12A - C). Dynabead activation also induced moderately high levels of the typical Th2 cytokines, IL-5, IL-6, IL-10, and IL-13 (Figure 5.13A - D). As was seen in the PMA/I stimulated samples, a low level of IL-13 was produced constitutively in the unstimulated cultures (Figure 5.13D). Significant levels of IL-1 $\beta$, IL-9, IL-17, IL-22, and TNF- $\alpha$ were also detected following culture with dynabeads, and lower levels of IL-1 $\beta$ and IL-22 were measured in the unstimulated samples (Figure 5.14A - E). There was no significant difference in cytokine responses between the control and RIPC treatments with anti-CD3/stimulation of T cells.

The pattern of cytokine expression was slightly different between the dynabead and PMA/I cultures. Although still substantial, the concentrations of IL-2 and IL6 measured following dynabead activation were approximately one third and one quarter of the levels detected with PMA/I stimulation, respectively. In contrast, IL-10 levels were approximately 4-fold higher, and TNF- $\alpha$ was 3-fold higher after anti-CD3/28 dynabead stimulation. Overall, the cytokine profile associated with the dynabead treatment was consistent with the more balanced Th1/Th2 type response expected towards a physiological stimulus. 

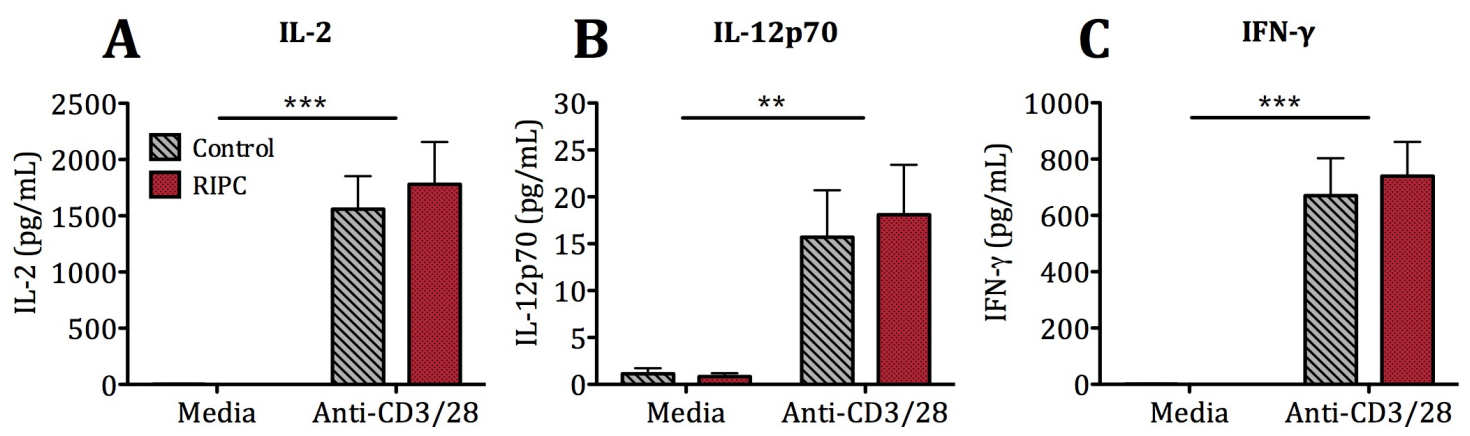

Figure 5.12 Levels of Th1 type cytokines in anti-CD3/28 dynabead stimulated PBMC

PBMC were isolated from blood collected 1 hour after treatment and plated at 8 x $10^{4}$ cells/well, in triplicate, with or without anti-CD3/28 dynabeads at a 1:1 ratio with cells, for 24 hours. Supernatant cytokine levels were assessed with a CBA and flow cytometry. (A) IL-2; (B) IL-12p70; (C) IFN- $\gamma$. Two-way repeated measures ANOVA with Bonferroni post-hoc tests were used to compare the treatments and culture conditions. ${ }^{* *} \mathrm{p}<0.01$ and ${ }^{* * *} \mathrm{p}<0.001$. Shown are the means of triplicates for $\mathrm{n}=10$ participants.
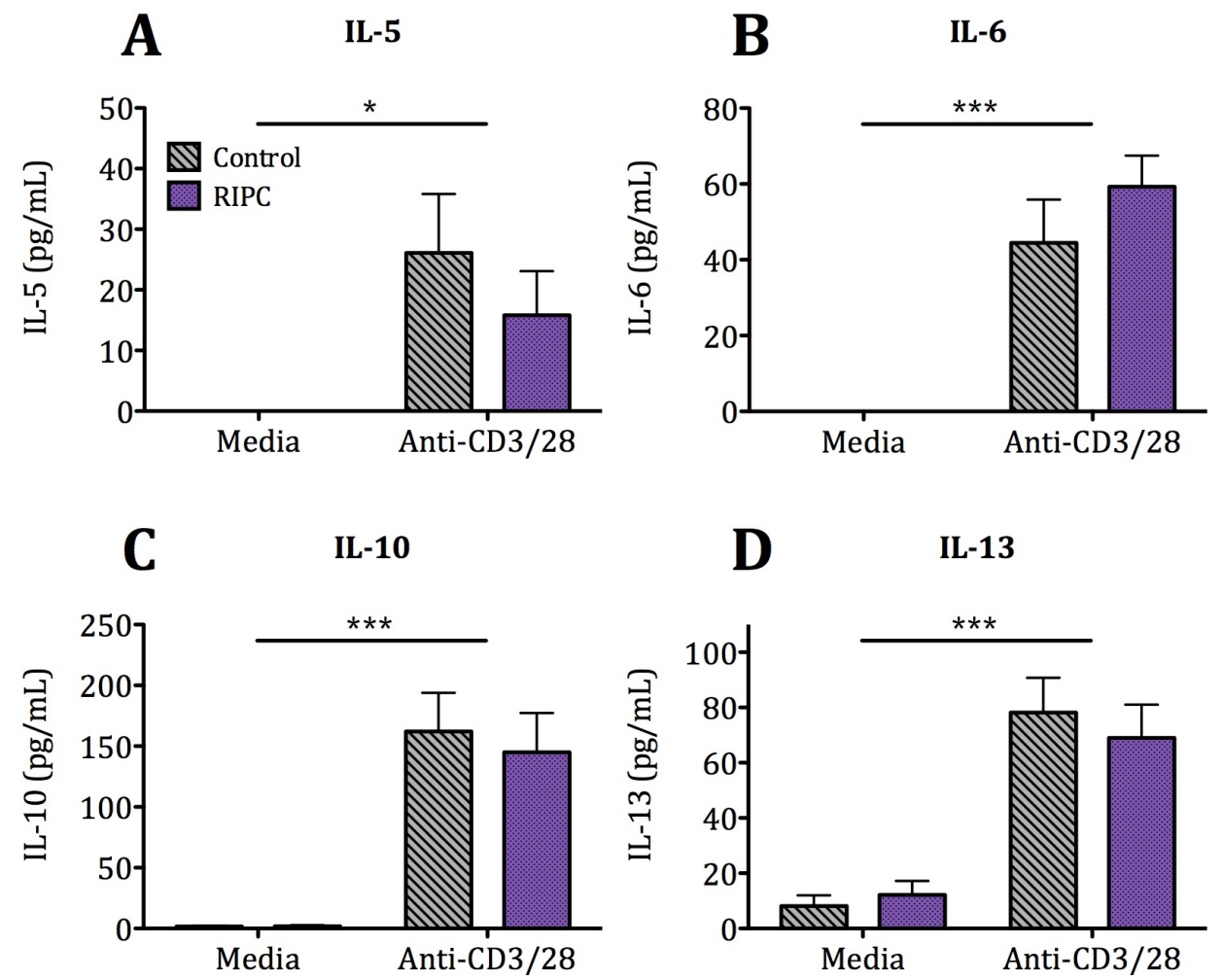

Figure 5.13 Production of Th2 type cytokines in anti-CD3/28 dynabead treated PBMC cultures

PBMC were isolated from blood collected 1 hour after treatment and plated at 8 x $10^{4}$ cells/well, in triplicate, with or without anti-CD3/28 dynabeads at a 1:1 ratio with cells, for 24 hours. Supernatant cytokine levels were assessed with a CBA and flow cytometry. (A) IL-5; (B) IL-6; (C) IL-10; (D) IL-13. Two-way repeated measures ANOVA with Bonferroni post-hoc tests were used to compare the treatments and culture conditions. ${ }^{*} \mathrm{p}<0.05$ and ${ }^{* * *} \mathrm{p}<0.001$. Shown are the means of triplicates for $\mathrm{n}=10$ participants. 
A

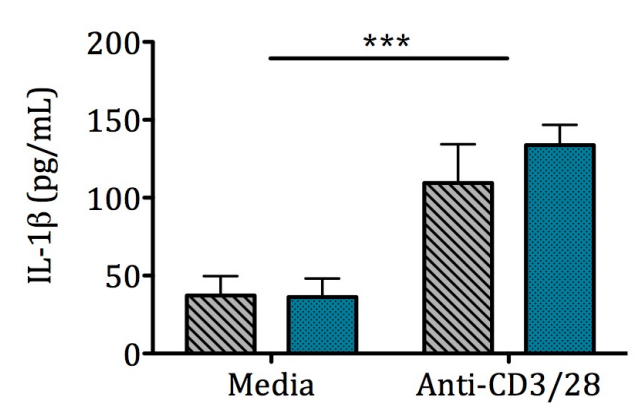

C

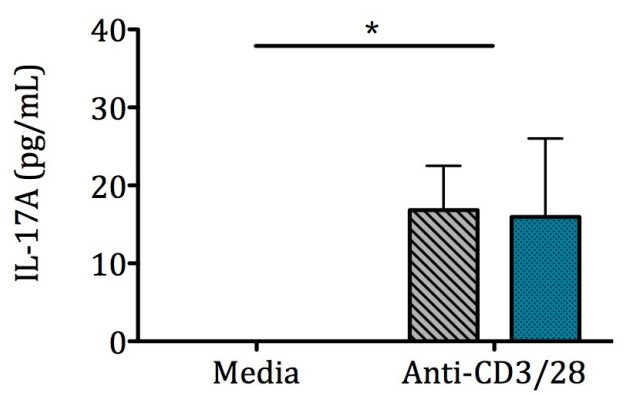

$\mathbf{E}$

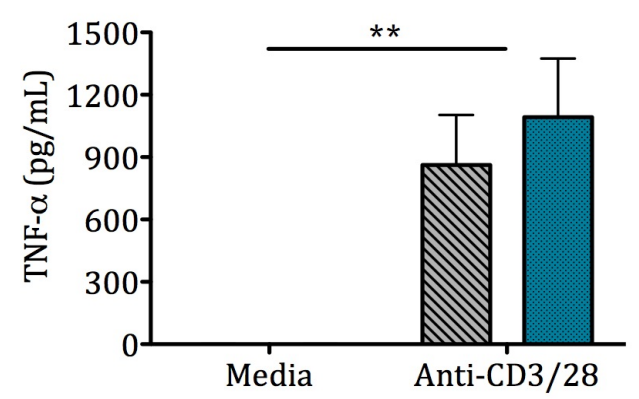

B IL-9

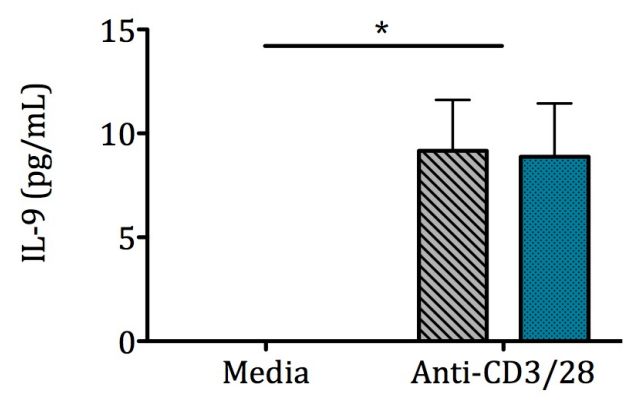

D $\quad$ IL-22

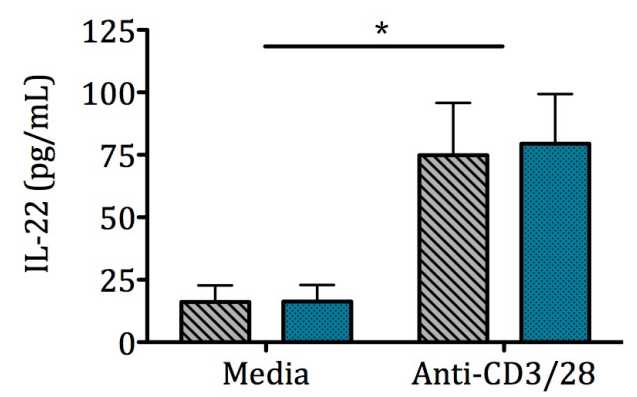

Figure 5.14 Expression of Th9/Th17/Th22 type cytokines in PBMC cultures stimulated with anti-CD3/28 dynabeads

PBMC were isolated from blood collected 1 hour after treatment and plated at 8 x $10^{4}$ cells/well, in triplicate, with or without anti-CD3/28 dynabeads at a 1:1 ratio with cells, for 24 hours. Supernatant cytokine levels were assessed with a

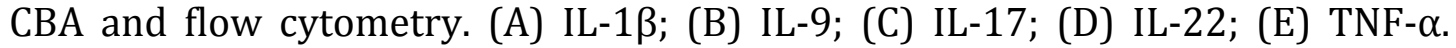
Two-way repeated measures ANOVA with Bonferroni post-hoc tests were used to compare the treatments and culture conditions. ${ }^{*} \mathrm{p}<0.05,{ }^{* *} \mathrm{p}<0.01$, and ${ }^{* * *}$ $\mathrm{p}<0.001$. Shown are the means of triplicates for $\mathrm{n}=10$ participants. 


\subsubsection{Antigen-specific T cell cytokine response}

To more specifically determine if RIPC could effect cytokine production and thus $\mathrm{T}$ cell biasing in an antigen-specific response, PBMC were cultured for 6 days with ADT Booster vaccine. Overall, the cytokine levels in these culture supernatants were pronounced, which along with the proliferative response suggests antigen-specific activation. Because the vaccine antigens only activate a small population of memory cells, the cytokine responses were significantly more modest than those seen with the 24-hour polyclonal responses. As with the previous analysis, exclusion of the two participants with high baseline levels of serum cytokines did not significantly alter the results, and so they were included in the final analysis.

There were notably higher levels of the Th1 cytokines IL- 2 and IFN- $\gamma$ in the stimulated samples, compared to the unstimulated cultures; however, due to the high level of variability between participants, the differences did not reach statistical significance (Figure 5.15A and C). Moderate levels of IL-2 and IFN- $\gamma$ were also detected in the unstimulated samples while IL-12p70 levels were, on average, low but detectable with no difference induced by stimulation (Figure $5.15 A-C)$.

The majority of the cytokines associated with a Th2 type response were below the level of detection, including IL-4, IL-5, and IL-6 (data not shown). There was, however, a significant increase in the anti-inflammatory cytokines, IL-10 and IL13, in the samples cultured with the tetanus antigen (Figure 5.16A and B). Production of these cytokines in the absence of stimulation was extremely low.

IL-1 $\beta$ levels appeared to increase following stimulation with the tetanus antigen, but did not reach statistical significance, and TNF- $\alpha$ was measurable in all the samples but highly variable between participants and conditions (Figure 5.17A and C). The levels of IL-9 and IL-17 were below the level of detection of the assay (data not shown). Interestingly, there were significantly higher levels of IL-22 detected in the samples after RIPC, compared to the control treatment (Figure 5.17B). This effect was seen in both the unstimulated and stimulated cultures, 
suggesting it was independent of the activation induced by the tetanus antigen.

As the IL-22 production was not induced as part of the antigen-specific response, it may have originated from multiple cell subsets, rather than just the $\mathrm{T}$ cell population. Preconditioning did not alter the levels of any other cytokines measured in these 6-day cultures.

A

IL-2

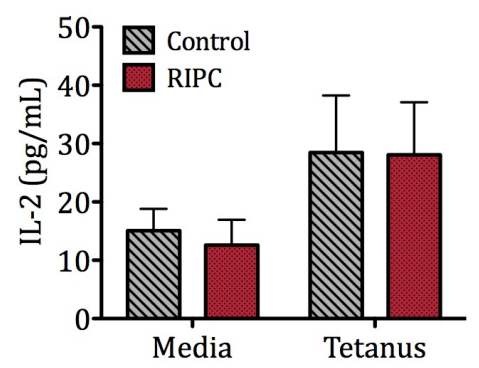

B

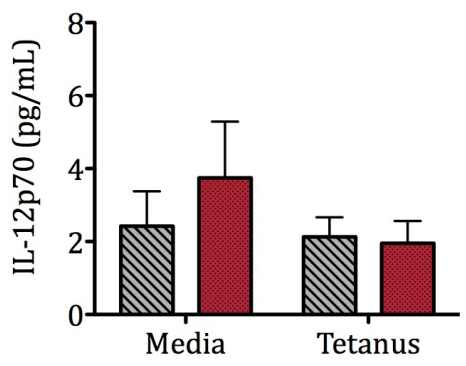

C IFN- $\gamma$

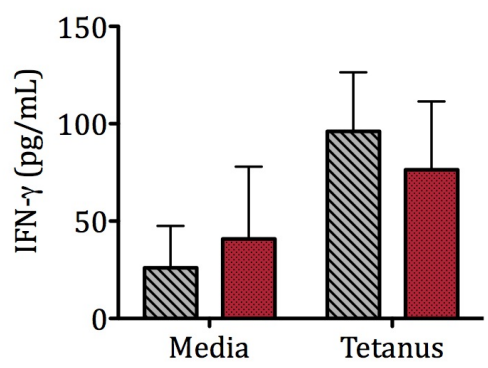

Figure 5.15 Th1 type cytokine production in tetanus stimulated PBMC PBMC were isolated from blood collected 1 hour after treatment and plated at 1 x $10^{5}$ cells/well, in triplicate, with or without the ADT vaccine diluted 1:5, for 6 days. Supernatant cytokine levels were assessed with a CBA and flow cytometry. (A) IL-2; (B) IL-12p70; (C) IFN- $\gamma$. Two-way repeated measures ANOVA with Bonferroni post-hoc tests were used to compare the treatments and culture conditions. Shown are the means of triplicates for $n=10$ participants.

A

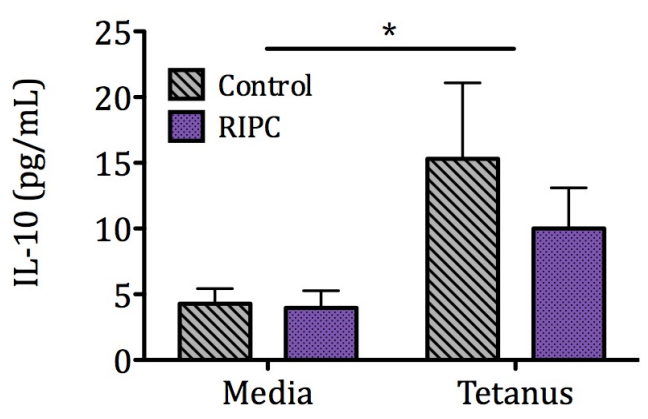

B

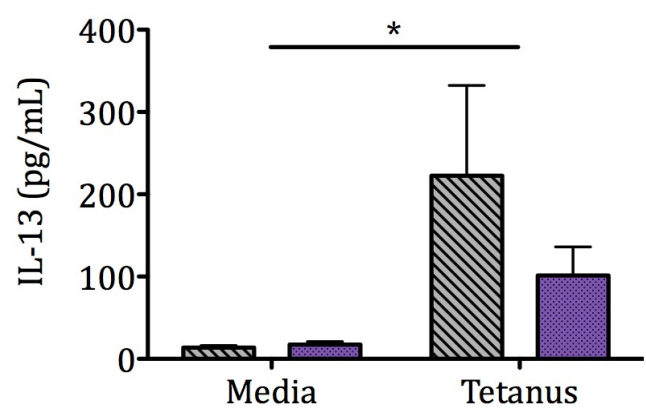

Figure 5.16 Production of Th2 type cytokines in PBMC cultured with the tetanus antigen

PBMC were isolated from blood collected 1 hour after treatment and plated at 1 x $10^{5}$ cells/well, in triplicate, with or without the ADT vaccine diluted 1:5, for 6 days. Supernatant cytokine levels were assessed with a CBA and flow cytometry. (A) IL-10; (B) IL-13. Two-way repeated measures ANOVA with Bonferroni posthoc tests were used to compare the treatments and culture conditions. ${ }^{*} \mathrm{p}<0.05$. Shown are the means of triplicates for $\mathrm{n}=10$ participants. 

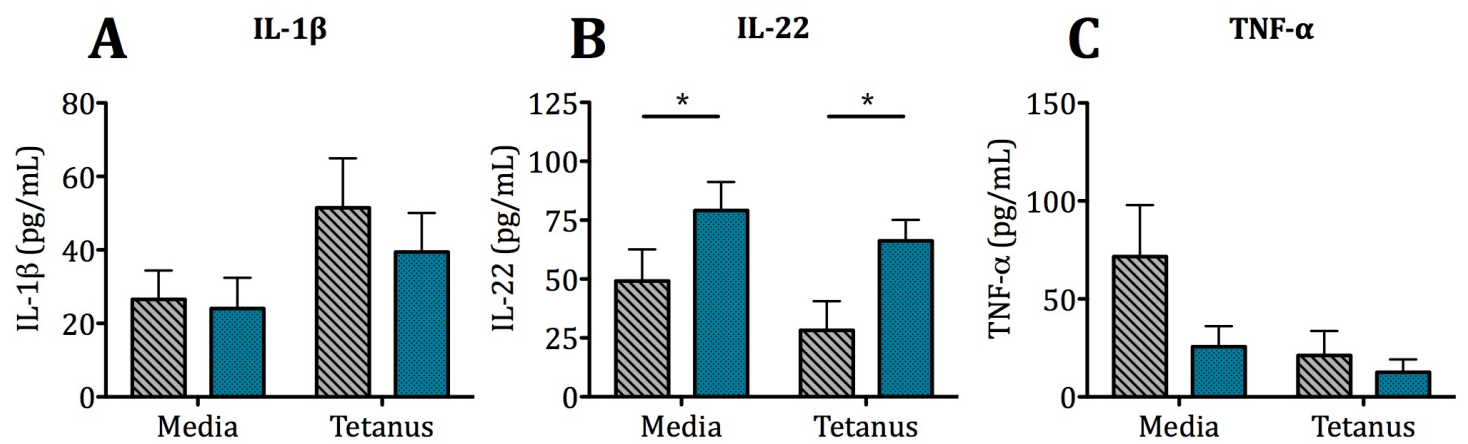

Figure 5.17 Levels of Th9/Th17/Th22 type cytokines in tetanus stimulated PBMC

PBMC were isolated from blood collected 1 hour after treatment and plated at 1 x $10^{5}$ cells/well, in triplicate, with or without the ADT vaccine diluted 1:5, for 6 days. Supernatant cytokine levels were assessed with a CBA and flow cytometry. (A) IL-1 $\beta$; (B) IL-22; (C) TNF- $\alpha$. Two-way repeated measures ANOVA with Bonferroni post-hoc tests were used to compare the treatments and culture conditions. ${ }^{*} \mathrm{p}<0.05$. Shown are the means of triplicates for $\mathrm{n}=10$ participants. 


\subsection{Discussion}

The aim of this chapter was to investigate the effect of RIPC on the functional responses of peripheral immune cells in healthy volunteers. We found a trend towards reduced expression of the activation marker, CD11b, in cultured monocytes and neutrophils after preconditioning, but no change in the intracellular production of IL-6 and IL-8 in these cells. Overall, PBMC isolated following RIPC did not show an altered proliferative response to stimulation with PMA/I, anti-CD3/28 expander beads, or the tetanus toxin. There was also no evidence that RIPC polarised the $\mathrm{T}$ helper cell response or affected $\mathrm{T}$ cell cytokine production following culture with polyclonal or antigen-specific stimuli; however, RIPC did result in a marked and significant increase in basal IL-22 production in PBMC cultured for 6 days.

RIPC has previously been associated with a decrease in CD11b expression on neutrophils in humans (Kharbanda et al., 2001; Konstantinov et al., 2004). We found a general trend towards reduced CD11b levels on both monocytes and stimulated neutrophils following culture for 4 or 27 hours, although this difference did not reach statistical significance. To the best of our knowledge, such a change in CD11b expression on monocytes following preconditioning has not previously been reported. While we cannot definitively say that RIPC decreased CD11b expression in our study, it would be equally premature to conclude that it did not. Given the hypothesis generating nature of this trial, the reduced expression of CD11b on neutrophil and monocytes following RIPC merits further investigation.

$\mathrm{CD} 11 \mathrm{~b}$ is an integrin family member, and facilitates adhesion and migration through binding to ICAM-1 on the vascular endothelium (Diamond et al., 1990). In addition, $\mathrm{CD} 11 \mathrm{~b}$ is the receptor for the complement protein, $\mathrm{C} 3 \mathrm{bi}$, which facilitates phagocytosis of bacteria and their components through opsonisation (Stossel et al., 1975; Wright et al., 1983). This process becomes relevant during cardiac surgery in response to endotoxins released into the circulation most likely due to ischaemia of the bowel (Andersen et al., 1993; Martinez-Pellús et al., 1997; Watarida et al., 1994). CD11b also binds a key component of clots, fibrin, 
and this interaction contributes to the dual role of neutrophils in both thrombus development and dissolution (Wright et al., 1988). Neutrophils enhance the early stage of clot formation through the release of tissue factor and serine proteases that degrade the key inhibitor of clot formation, tissue factor pathway inhibitor (Massberg et al., 2010; Maugeri et al., 2006). In the case of infection, this response is protective in smaller vessels by restricting the passage of bacteria; however, it is largely detrimental during cardiac surgery. In contrast, during the later stage of clot formation, neutrophils appear to degrade thrombi through phagocytosis of fibrin and the release of neutrophil elastase and cathepsin $G$ (Plow, 1986; Rábai et al., 2010; Riddle and Barnhart, 1964). CD11b, thus, mediates several important and diverse functions of neutrophils during the inflammatory response.

CD11b is regarded as a general activation marker for monocytes and neutrophils; however, the change in CD11b levels detected in our volunteers did not correspond to a change in their capacity to synthesise IL-6 and IL-8. It is therefore difficult to comment on whether this change represents an overall suppression of neutrophil activity, or a specific effect on the functions that depend on CD11b as a receptor. Saxena et al. have associated RIPC with a decrease in neutrophil kinin receptor expression, supporting the former possibility; however, further measures of neutrophil function will be required for a conclusive answer (Saxena et al., 2013, 2010b). Regardless, in the setting of cardiac surgery, a reduction in CD11b is likely to decrease tissue infiltration, complement-mediated phagocytosis by neutrophils, and thrombus formation, reducing the overall inflammatory burden.

It is interesting that we did not see a similar reduction in CD11b expression in our immunophenotyping samples collected at the same time point and assessed with and without a brief 15-minute stimulation (Chapter 4, Figure 4.9). The immunophenotyping samples were acquired on a Guava flow cytometer, which has a slightly lower level of resolution and this lower resolution may reduce the ability to measure subtle differences; however, this explanation seems unlikely given that the effect in the cultured cells was quite pronounced. It therefore 
appears that the neutrophils and monocytes required a period of time longer than 15 minutes to enact this change, and suggests the mechanism could potentially depend on an alteration in gene expression, rather than straightforward receptor endocytosis or shedding via pre-formed mediators.

Of particular note is that adenosine, which has been identified as both an initiator and mediator of RIPC, has been shown to reduce CD11b expression on neutrophils (Thiel et al., 1996; Wollner et al., 1993). Adenosine can either activate or suppress neutrophil functions depending on the concentration in the microcirculation and the receptor expression profile of the cells. More specifically, low concentrations of adenosine operate through the $A_{1}$ and $A_{3}$ receptors to stimulate chemotaxis and phagocytosis, whereas higher concentrations act through the $A_{2 A}$ and $A_{2 B}$ receptors to inhibit key neutrophil activities such as granule and inflammatory mediator release, and the oxidative burst (Chen et al., 2006; Cronstein et al., 1990; McColl et al., 2006; Salmon and Cronstein, 1990; Visser et al., 2000). It is therefore possible that a change in neutrophil CD11b expression following RIPC may be secondary to an alteration in adenosine levels and/or neutrophil adenosine receptor expression.

We detected the decrease in CD11b levels after 4 hours culture, but the effect may take place more quickly. In cardiac surgery patients this fits with the timing of the reperfusion injury relative to the administration of RIPC, but is less applicable in animal models where the injury is often induced shortly after the intervention. A change to neutrophil infiltration during I/R injury therefore may not be the primary mechanism of protection in such animal studies. Interestingly, a recent clinical trial by Albrecht et al. detected increased MPO activity in right atrial tissue samples following RIPC in cardiac surgery patients, and this was associated with a cardioprotective response. The authors noted that an initial transient increase in neutrophil infiltration of the myocardium might actually be beneficial during reperfusion (Albrecht et al., 2013). Alternatively, alterations to neutrophil extravasation shortly after preconditioning may be dependent on prompt changes to adhesion marker expression on the vascular endothelium, and the later alterations to leukocyte CD11b expression may then 
further enhance this effect (Davis et al., 1999; Linden et al., 2006). In summary, although RIPC appears to affect neutrophil and, potentially, monocyte expression of CD11b, the exact kinetics and resulting effects of this alteration are not fully understood and require further investigation in humans.

One of the most interesting findings from our study was that RIPC increased basal production of IL-22 in PBMC cultured for 6 days. IL-22 can play a dichotomous role during inflammation, but is more frequently associated with tissue protective functions, such as wound healing and tissue homeostasis and repair (Rutz et al., 2013). The majority of IL-22 receptors are present on keratinocytes, hepatocytes, and epithelial cells of the intestines and respiratory system and accordingly, IL-22 exerts some of its major functions in the liver, lung, and kidney (Ki et al., 2010; Kulkarni et al., 2014; Sonnenberg et al., 2010; Wolk et al., 2004). IL-22 has been shown to stimulate epithelial cell proliferation and endurance in numerous organs and has a particularly well-defined role in protection of the liver (Ki et al., 2010; Radaeva et al., 2004). In a murine model of hepatitis, administration of recombinant IL-22 attenuates hepatocyte damage and increases STAT3 signalling. In vitro culture of human hepatocellular carcinoma HepG2 cells with IL-22 related this increased level of STAT3 to enhanced expression of anti-apoptotic and mitogenic proteins that promote cell survival (Radaeva et al., 2004). IL-22 has also been identified as having protective functions during numerous other pathological conditions, including graft-versus-host disease, models of autoimmune myocarditis, and inflammation-induced pulmonary fibrosis (Chang et al., 2006; Kapessidou et al., 2008; Simonian et al., 2010).

The change in IL-22 detected in our study occurred in the absence of an ischaemic injury and no increase was detected in the 24-hour PBMC cultures, or in the serum shortly after preconditioning. Taken together, these results strongly imply that changes in IL-22 are specific to the later phase of preconditioning and are not connected to the biphasic mode of expression seen with many other cytokines. Nonetheless, clearly the signals required for altering IL-22 production in the PBMC occurred within the first hour following RIPC while the cells were 
still in vivo. Our study is the first to link IL-22 with RIPC and raises a number of new hypotheses regarding the delayed phase of preconditioning.

The second window of IPC is generally reported as lasting up to 72 hours, owing to a study from Baxter et al. in 1997 which reported significant cardioprotection in rabbits 24, 48, and 72 hours following preconditioning, but not after 96 hours. Subsequent studies have, however, demonstrated that hypobaric hypoxia and longer durations of IPC are associated with protective effects that persist up to 5 and 12 weeks, respectively (Neckár et al., 2004; Park et al., 2003). Accordingly, work in murine models has found that preconditioning using an initial $20-25$ minute period of ischaemia significantly improves measures of kidney damage and function following an ischaemic injury induced 4 or 7 days later (Jiang et al., 2007; Kinsey et al., 2010). The exact mechanisms involved in this extended window of protection induced by late preconditioning are unclear; however, Kinsey et al. found the renal protection observed 7 days after IPC was dependent on the induction of Tregs, which are known to induce IL-22 production through interactions with $\mathrm{CD} 4^{+} \mathrm{T}$ cells and promote Th17 cell differentiation (Kinsey et al., 2010; Pandiyan et al., 2011). Further, Xu et al. (2014) recently demonstrated for the first time that overexpression of IL-22 in mice increased survival rates following renal I/R, and lowered levels of tubular cell injury, inflammation, and apoptosis in the kidneys. It therefore seems highly likely that delayed renal protection could be, at least in part, mediated by an increase in IL-22 levels, such as was detected during the corresponding timeframe in the present work.

There are a number of possibilities for the disparity between the study suggesting that delayed preconditioning wanes after 72 hours and those reporting beneficial effects several weeks later. First, there may not be one unilateral process that evokes organ protection at both 24 hours and 12 weeks following the intervention. The underlying processes that contribute to the protective effects may shift over the course of the delayed window with periods of 'downtime' as one pathway resolves and another takes over. Second, late preconditioning may selectively protect against specific processes associated with I/R injury. For instance, delayed IPC may not reduce myocardial infarct size, 
as was the primary endpoint in Baxter's study, but help maintain the structural integrity of the kidneys, such as was seen by Jiang et al. These possibilities are not mutually exclusive and are consistent with our finding that IL-22 production was increased after 6 days but not 24 hours in culture and the literature associating IL-22 with direct renal but not myocardial protection (Rutz et al., 2013).

While IL-22 can exert both pro and anti-inflammatory effects, the choice of effects appears to be somewhat dependent on the co-expression of IL-17. For instance, in a mouse model of airway inflammation induced by bleomycin, knockout of either the IL-17 or IL-22 genes attenuates inflammation, implicating both cytokines in the pathological process; however, higher levels of IL-22 are produced in IL-17 deficient mice and are associated with resistance to airway inflammation. Further, treatment with a neutralising anti-IL-22 antibody in IL-17 knockout mice reverses this protective effect, and reinstates the inflammatory response to bleomycin (Sonnenberg et al., 2010). This work strongly suggests that IL-22 is tissue-protective in the absence of IL-17, and proinflammatory when the two cytokines are co-expressed.

Traditionally, IL-22 has been considered a cytokine of Th17 cells that is expressed in tandem with IL-17. In humans, however, IL-22 producing cells often do so independently of other cytokines; in fact, as many as $37-63 \%$ of IL-22 producing $\mathrm{CD}^{+} \mathrm{T}$ cells in human peripheral blood do not co-express IL-17 (Duhen et al., 2009). This finding is consistent with the relatively recent identification of a new subset of $\mathrm{CD}^{+} \mathrm{T}$ cells capable of producing IL-22 in the absence of IL-17, designated the Th22 subset (Eyerich et al., 2009). In addition, IL-22 can be secreted independently by a wide variety of immune cells in humans, including Th1, CD8+, and certain NK cells (Cella et al., 2009; Ortega et al., 2009; Wolk et al., 2002). No IL-17 was detected in our 6-day cultures, suggesting RIPC either enhanced the Th22 response, and/or altered IL-22 expression in multiple immune cell subsets, rather than targeting the Th17 population. Further, the lack of IL-17 in our cultures indicates that the induction of IL-22 following RIPC is more likely to be associated with anti-inflammatory effects. 
If IL-22 does provide protection during delayed RIPC, it raises the question of when is, exactly, the most beneficial time to apply preconditioning in cardiac surgery patients. While it is possible that having increased levels of IL-22 at the time of I/R may be beneficial, it is likely that the more intense effects associated with the first phase of RIPC could provide greater protection during the initial insult. A delayed increase in IL-22 production once the initial inflammatory response to surgery, which likely includes an upregulation of IL-17, has at least partially resolved may be safer and more beneficial during wound repair.

One limitation of our study was the sensitivity of the IL-22 measurements. Although the theoretical limit of detection for the assay was $2.5 \mathrm{pg} / \mathrm{mL}$, we found that the signal strength at the lower end of the standard curve was poor and therefore lacked the ideal level of sensitivity for measuring subtle changes. The use of an alternative method for determining the culture supernatant IL-22 levels, such as an ELISA, would be valuable to confirm our results. Also, as previously discussed in Chapter 4, the large number of statistical tests performed for the study is associated with an increased likelihood of type I error, therefore the results should also be interpreted in this context.

In our previous trial, we found a general trend towards higher circulating levels of IL-6, IL-8, and IL-10 in cardiac surgery patients that received the preconditioning, compared to the controls. Following on from this work, a key aim of our study in healthy volunteers was to identify whether RIPC directly impacted cytokine expression in the absence of ischaemic injury. We addressed this by not only measuring serum cytokine levels following preconditioning, but also using ICS to investigate which populations of cells might be contributing to any increased cytokine levels. We did not detect a change in intracellular IL-6 or IL-8 production in any of the peripheral leukocyte populations examined and, although we were unable to examine IL-10 intracellularly, there was no change in IL-10 production in PBMC cultured with or without PMA/I for 24 hours either. These results are consistent with our earlier assessment that RIPC either does not increase cytokine production, or else predominantly affects cytokine 
expression in cells that are not of hematopoietic origin, such as myocytes or endothelial cells.

Independently of RIPC, our ICS results did indicate that resting monocytes and stimulated neutrophils are likely to be major sources of IL-8, and that unstimulated neutrophils are notable producers of IL-6. The finding that neither population appeared to produce IL-6 and IL-8 simultaneously is interesting, particularly with respect to neutrophils which stained positively for both albeit in separate populations. This suggests that induction of IL- 6 and IL-8 synthesis is not coupled within these cells. The effector functions of neutrophils in the setting of cardiac surgery may depend on their individual cytokine expression profile.

Stimulation of PBMC with the tetanus antigen is associated with a mixed Th1/Th2 type response including production of IL-2, IFN- $\gamma$, TNF- $\beta$, IL-4, and IL10 (Larsen et al., 2000). We saw similar results in our study; however, we did not detect any IL-4 or significant levels of several other key cytokines. The ability to measure cytokine levels in culture supernatants is limited by the role of these molecules as immunoregulatory hormones, which means they are often promptly taken up or degraded (Akdiş et al., 1995). For instance, IL-4 is produced by activated Th2 and NKT cells but is rapidly consumed by cells expressing the IL-4 receptor, particularly activated T and B cells (Akbari et al., 2003; Broere et al., 2011; Ohara and Paul, 1988). Although we did detect a significant cytokine response in our 6-day cultures, especially considering we were targeting a small population of antigen-specific cells, this explains why we did not find measurable IL-4 levels. The genes for the common Th2-associated cytokines, IL-4, IL-5, and IL-13 are clustered and often subject to coordinate regulation; therefore, levels of IL-5 and IL-13 are useful as surrogate markers of IL-4 expression and were detected more consistently in our study (Kelly and Locksley, 2000). It should be noted that one weakness with our assay utilising the ADT Booster vaccine for stimulating T cells was the inability to conclusively determine that the resulting proliferation and cytokine production were antigenspecific responses. Further work to determine the specificity of this assay would be valuable to confirm our methods. 
We also only detected low levels of IL-12p70 in our PBMC culture supernatants, despite there being high levels of IL-2 and IFN- $\gamma$ present in the same samples. IL12 p70 is a heterodimer composed of the smaller p35 and p40 subunits, and is the biologically active form of the cytokine. The $\mathrm{p} 40$ subunit has been associated with IL-12 antagonism due to its ability to bind to the IL-12 receptor as a homodimer, blocking IL-12p70 binding (Gately et al., 1996; Ling et al., 1995). The p40 subunit also appears to exert some biological effects as a monomer, and forms part of the IL-23 heterodimer (Hamza et al., 2010; Khader et al., 2006). The IL-12p40 subunit is thus present in higher concentrations and more readily detectable than IL-12p70 and, despite its lower specificity compared to the active form of IL-12, it is often used as a marker of IL-12 levels (Dobreva et al., 2008). Performing an additional assay on our samples to measure IL-12p40 may provide a more sensitive indication of whether RIPC altered IL-12 serum and supernatant levels.

T cell proliferation to physiological and antigen-specific stimuli seemed largely unaffected by RIPC; however, there was a slight trend towards an increased proliferative response to stimulation with PMA/I. The pharmacological stimulants, PMA/I, activate cells in a non-specific fashion independently of the T cell receptor, thus can also affect other mononuclear leukocytes such as monocytes and B cells. However, only a small portion of circulating human monocytes retain the ability to proliferate; therefore, the vast majority of PMA/Iinduced proliferation in PBMC cultures can be attributed to lymphocyte responses (Clanchy et al., 2006). Furthermore, the predominant cell subset in isolated PBMC are the $\mathrm{CD}^{+} \mathrm{T}$ cells, which represent approximately $70-85 \%$ of the total lymphocytes. T cells are estimated to have a doubling time anywhere between 6 and 15 hours, depending on the stimulus (Boer et al., 2003; MuraliKrishna et al., 1998). Our culture period of 24 hours was primarily selected for measuring cytokine responses, and may not have allowed sufficient time for multiple doublings of the cells. A more conclusive indication of whether RIPC alters $\mathrm{T}$ cell proliferation in response to pharmacological stimuli could be achieved with a longer culture period and greater number of participants to account for inter-individual variability in responses. 
Interestingly, we detected a trend towards increased $\mathrm{T}$ cell activation in whole blood cultured for 27 hours with PMA/I following RIPC. The level of response was highly variable between individuals and therefore did not reach statistical significance; however, the effect was consistent across the study participants. Although there was significant upregulation of CD69 expression following PMA/I stimulation in cultures incubated for only 4 hours, a difference between the RIPC and control treatments was not apparent until the later time point. It therefore appears that RIPC may augment $\mathrm{T}$ cell activation during the second window of protection, but not during the early phase. Further investigation as to which subsets of T cells show enhanced CD69 expression and whether this correlates to a change in their function will be necessary to understand the impact that this change could have on the progression of I/R injury. Aside from this enhanced T cell CD69 expression, increase in IL-22 production, and potential trend towards enhanced proliferation after 24 hours stimulation in vitro, there were no further changes to T cell function detected following RIPC.

\subsection{Conclusions and future directions}

We found that in healthy volunteers, RIPC may reduce the ability of monocytes and neutrophils to respond to activation signals, as indicated by lower levels of CD11b expression, and directly increases production of IL-22 in PBMC cultured for 6 days. These alterations may reduce neutrophil tissue infiltration and inflammation during the early window of protection, and enhance tissue and wound repair several days later. RIPC did not alter the cytokine producing potential of peripheral blood leukocytes, or the proliferative and cytokine responses of PBMC to pharmacological, physiological, and antigen-specific stimuli, but a trend towards enhanced T cell activation following 27 hours of PMA/I stimulation in vitro was detected. Overall, our data strongly suggests that $\mathrm{T}$ cells do not play a major role in the initiation or mediation of the early phase of RIPC. There are, however, indications that the adaptive immune response may contribute to the delayed window of preconditioning, which warrants further investigation in human participants. 


\section{Chapter 6.}

\section{Assessing the global response to RIPC in humans}




\subsection{Introduction}

The previous two chapters explored the effect of RIPC on the immune response in healthy volunteers by assaying a series of individual endpoints. Our analysis detected a number of interesting changes following RIPC; however, the large dataset made it difficult to identify overall trends in the response to treatment and between the participants. In light of the difficulties translating RIPC into the clinical setting, it is possible that certain individuals may not respond to the treatment owing either to genetic differences or, potentially, perturbations in their baseline physiological state. We were therefore particularly interested in examining how the global response to RIPC varied amongst the healthy volunteers in our study and investigated this question through the use of a nonimmune marker of ischaemia and multivariate statistical analysis.

\subsubsection{A potential biomarker of successful preconditioning}

A particular limitation with RIPC trials to date is that no reliable marker capable of confirming the successful application of preconditioning ischaemia has been established. Identifying a positive control for RIPC in humans would be useful for a number of reasons. First, although inflation of the tourniquet in conscious healthy volunteers can be easily monitored, it is possible that the extent of the restriction of blood flow during the intervention may vary between participants. For instance, most clinical trials have inflated the tourniquet to a predetermined constant pressure of $200 \mathrm{mmHg}$; however, some trials adjust this value to ensure it sits at least $15-20 \mathrm{mmHg}$ above the systolic arterial pressure for each patient. The majority of studies, including our own, have used the former strategy, which allows for the possibility that the extent of the preconditioning may vary between participants according to their blood pressure. Second, in the absence of the full knowledge of the pathways through which RIPC operates, it is impossible to know whether all individuals are capable of responding to the intervention. Where trial participants are identified as 'non-responders' it would be useful to be able to exclude the possibility that the intervention was not successfully administered. 
In the clinical setting, ischaemia is most commonly confirmed using measures that are specific to cardiac muscle, such as troponin elevation or ECG changes (Jaffe et al., 2000; Thygesen et al., 2007). These types of endpoints are not useful for assessing ischaemia in alternative tissues, such as skeletal muscle; therefore, detecting the ischaemia elicited by RIPC requires an alternative marker. One potential candidate involves the major protein in the circulation, albumin.

Under normal conditions, the N-terminus of the human serum albumin protein is capable of binding transition metals, such as cobalt (Sadler et al., 1994). Following the observation that patients with ischaemia exhibited reduced cobalt binding to albumin in serum samples, Bar-Or et al. hypothesised that modifications to this binding site during ischaemia, most likely in the form of $\mathrm{N}$ acetylation of critical residues, decreased the metal binding capacity of albumin (Bar-Or et al., 2001a). The group went on to design a unique assay to exploit this property and quantify the extent of ischaemia-modified albumin (IMA) in serum samples (Bar-Or et al., 2000). This assay has been shown to reliably detect ischaemia in patients with acute myocardial ischaemia; however, the theory that the N-terminus metal binding ability of albumin was altered by ischaemia has since been disproved by the original authors (Bar-Or et al., 2008). Regardless of the mode of action involved the cobalt-albumin binding assay is still considered a valuable method for detecting ischaemia, as established by several trials (reviewed in Sbarouni et al., 2008), and has received approval from the US Food and Drug Administration; therefore, we believe it may also be appropriate for measuring a response to RIPC.

\subsubsection{Statistical methods for detecting patterns in the response to RIPC}

Although we found a number of our study endpoints were significantly altered by RIPC according to the mean group data, there was a high level of variability in the individual responses, with some participants showing substantial changes and others only subtle alterations. Further, with the large amount of data collected, it was difficult to discern whether the changes detected following RIPC occurred consistently within a particular subset of individuals, or fluctuated between all of the study participants. 
There are a number of statistical procedures available for determining whether study participants can be clustered into different subsets based on patterns within their respective datasets. Some of the more common methods, such as Kmeans or hierarchical clustering, are limited in their power for small sample sizes, and require a certain level of knowledge of the relationship between the variables prior to the analysis in order to specify the ideal number of target clusters or the relative importance of each variable. These methods were therefore not ideal for the present study. One available alternative method is multidimensional scaling (MDS), which is not subject to such restrictions and provides a visual representation of the overall level of similarity between datasets.

Comparisons between treatment groups and individual participants are generally investigated using common ANOVA techniques. These tests assume a normal distribution of the data and are highly vulnerable to violations of this assumption, whereas many of the non-parametric alternatives are not compatible with multi-factorial analysis (Anderson, 2001; Johnson and Field, 1993). These techniques are not well suited to many biological datasets; however, permutation-based ANOVA (PERMANOVA) is an alternative that uses a unique method to calculate a 'pseudo' test statistic that is analogous to the standard $F$-statistic, and then compares it to a distribution created by running a high number of permutations of the observations, thus does not depend on normality of the data (Anderson, 2001).

Combining MDS with PERMANOVA and similarity percentage (SIMPER) analysis provides the ability to detect differences between datasets grouped by experimental factors or the MDS analysis, and identify the relative contribution of the various endpoints to these differences, respectively (Anderson, 2001; Clarke, 1993). These methods were developed for use with ecological datasets, but are applicable to a wide range of studies and were deemed the most appropriate for our data and research question, therefore were used in the following analysis. 


\subsection{Aims}

- To evaluate the use of ischaemia-modified albumin as a marker of the successful application of RIPC.

- To determine whether the changes in inflammatory endpoints detected following RIPC appear to be related and altered consistently in certain individuals, or show a random pattern of change across the participants.

- To identify which endpoints were the main contributors to differences in the response between control and RIPC treatments.

- To investigate the level of similarity in the response to treatment between the study participants. 


\subsection{Methods}

\subsubsection{Ischaemia-modified albumin assay}

An assay to measure ischaemia-induced changes to binding of free cobalt in serum samples was performed as first described by Bar-Or et al. (2000) with minor adjustments to allow high-throughput in 96-well flat-bottom plates. Forty $\mu \mathrm{L}$ of each serum sample was gently mixed with $10 \mu \mathrm{L}$ of $0.1 \% \mathrm{CoCl}_{2}$ (Sigma Aldrich), and incubated for 10 minutes. Ten $\mu \mathrm{L}$ of $1.5 \mathrm{mg} / \mathrm{mL}$ dithiothreitol (DTT; Merck Millipore) was then added and the reaction stopped two minutes later by the addition of $200 \mu \mathrm{L}$ of $0.9 \% \mathrm{NaCl}$. The absorbance of the samples was measured at $470 \mathrm{~nm}$ on an EnSpire 2300 multilabel plate reader (PerkinElmer) and compared to blanks prepared using the same serum sample, without the addition of DTT.

As a positive control for ischaemia, serum samples were also collected from 11 patients with acute coronary syndrome (ACS) presenting at Wellington Hospital with symptoms of ischaemia lasting for more than 10 minutes and scheduled to undergo coronary angiography, with or without PCI. The incidence of ischaemia was confirmed by troponin elevation, or ECG changes such as a new ST deviation, or T wave inversion (Thygesen et al., 2007). Blood samples were collected before angiography, or in the cardiac catheterisation laboratory from the arterial sheath after insertion, and before administration of heparin. These samples were generously provided by the Wellington Cardiovascular Research Group, using a subset of patients from a larger study, which received ethics approval from the Upper South A Regional Ethics Committee (URA/11/05/016) and obtained written informed consent from all patients. An additional 5 volunteers agematched to the ACS patient cohort, but without any symptoms or history of ACS, were also recruited under the ethics approval for the trial in healthy volunteers (CEN/11/06/034) to investigate the effect of age on IMA levels.

The IMA levels were compared between treatment groups and time points using two-way repeated measures ANOVA with the Greenhouse-Geisser correction, and the levels between the ACS patients and the age-matched volunteers were 
compared using an independent samples t-test. Graphs were produced using GraphPad Prism 5 (GraphPad Software Inc) and statistical tests were performed using SPSS Statistics (IBM Corporation). Differences corresponding to $\mathrm{p}$ values < 0.05 were considered statistically significant.

\subsubsection{Multivariate analysis}

The analysis was completed with the guidance of Dr Dalice Sim, using the statistical analysis software PRIMER 6 with the PERMANOVA+ add on package (PRIMER-E Ltd, Plymouth, UK). From the complete study dataset 13 key endpoints, which showed either a significant difference between the treatment groups or a trend towards a difference, were selected for inclusion in the analysis and are presented in Table 6.1. The data expression format, such as the area under the curve (AUC), absolute levels, or percentage change from baseline, was carefully considered and chosen based on what most accurately represented the available data for each variable. A database was constructed that treated the data collected during the control and RIPC visits for each participant as independent events, meaning there was no adjustment for the repeated measures.

The multivariate analysis was performed using methods commonly employed for examining ecological data, as demonstrated in previous publications (Atalah et al., 2007; Coll et al., 2011). Prior to analysis, the data was normalised to prevent the endpoints with larger valued units from dominating the results. This was achieved by subtracting the population mean from each value, then dividing by the standard deviation. A resemblance matrix was then constructed using the Euclidean distance measure and was subsequently used to produce a dendrogram, and as the input for a non-metric multidimensional scaling (MDS) analysis. Non-parametric PERMANOVA was used to compare the differences between the treatment groups and participants, and SIMPER analysis was used to identify which factors contributed to the identified differences (Clarke, 1993; McArdle and Anderson, 2001). P values $<0.05$ were considered significant. 


\begin{tabular}{|c|c|c|}
\hline Endpoint & $\begin{array}{c}\text { Control } \\
\text { Mean (SD) }\end{array}$ & $\begin{array}{c}\text { RIPC } \\
\text { Mean (SD) }\end{array}$ \\
\hline \multicolumn{3}{|l|}{ Serum Cytokines } \\
\hline MIP- $1 \alpha$, AUC of data normalised to BL & $24693(2230)$ & $22361(2730)$ \\
\hline MIP-1 $\beta$, AUC of data normalised to BL & $24079(1604)$ & $21719(2734)$ \\
\hline \multicolumn{3}{|l|}{ Supernatant Cytokines } \\
\hline $\begin{array}{l}\text { IL- } 6 \text { from } 24 \mathrm{hr} \text { anti-CD3/28 stimulated } \\
\text { cultures }\end{array}$ & $44.46(36.07)$ & $59.28(25.84)$ \\
\hline IL-22 from 6 day unstimulated cultures & $49.19(42.31)$ & $79.14(38.25)$ \\
\hline TNF- $\alpha$ from 6 day unstimulated cultures & $71.60(83.01)$ & $25.64(33.07)$ \\
\hline $\begin{array}{l}\text { IL-1 } \beta \text { from } 6 \text { day tetanus-stimulated } \\
\text { cultures }\end{array}$ & $51.48(42.45)$ & $39.45(33.52)$ \\
\hline $\begin{array}{l}\text { IL-10 from } 6 \text { day tetanus-stimulated } \\
\text { cultures }\end{array}$ & $222.50(347.55)$ & $101.28(109.76)$ \\
\hline \multicolumn{3}{|l|}{ Intracellular Cytokines } \\
\hline $\begin{array}{l}\text { Total \% of IL- } 8^{+} \text {monocytes from } 4 \mathrm{hr} \\
\text { unstimulated cultures }\end{array}$ & $13.29(7.63)$ & $18.55(15.39)$ \\
\hline \multicolumn{3}{|l|}{ Cellular Activation Markers } \\
\hline $\begin{array}{l}\text { CD11b (\% } \% \text { from control) in neutrophils } \\
\text { from } 4 \mathrm{hr} \text { unstimulated cultures }\end{array}$ & $1691.55(205.36)$ & $1494.65(298.06)$ \\
\hline $\begin{array}{l}\text { CD11b (\% } \Delta \text { from control) in monocytes } \\
\text { from } 4 \mathrm{hr} \text { unstimulated cultures }\end{array}$ & $1136.18(484.32)$ & $916.73(290.93)$ \\
\hline $\begin{array}{l}\text { T cell CD69 MFI in PMA/I-stimulated } 27 \mathrm{hr} \\
\text { cultures }\end{array}$ & $104.82(57.61)$ & $202.97(158.61)$ \\
\hline \multicolumn{3}{|l|}{ T Cell Proliferation } \\
\hline $\begin{array}{l}\text { PMA/I-induced proliferative index in } 24 \mathrm{hr} \\
\text { cultures }\end{array}$ & $114.39(25.90)$ & $130.52(21.39)$ \\
\hline \multicolumn{3}{|l|}{ Ischaemia Marker } \\
\hline IMA ( $\%$ change from BL to $4 \mathrm{hrs}$ ) & $90.12(17.64)$ & $105.13(24.52)$ \\
\hline
\end{tabular}

Table 6.1 Variables included in the multivariate analysis

A total of 13 endpoints that showed either a statistically significant difference, or a trend towards a difference, between the control and RIPC treatment groups were selected for inclusion in the analysis. AUC = area under the curve. 


\subsection{Results}

\subsubsection{Ischaemia-modified albumin expression following RIPC}

The IMA assay was performed to determine whether the preconditioning ischaemia encountered during RIPC induced a measurable change in the circulating albumin that could be used as a positive biomarker for the intervention. There was no significant difference in IMA levels detected between the control and RIPC groups when comparing the blank-corrected $\mathrm{OD}_{470}$ measurements with a two-way repeated measures ANOVA (Figure 6.1). However, there was a notable difference in the baseline levels of IMA between the two groups and when this was accounted for by expressing the levels at 4 hours as a percentage of the pre-treatment IMA level, there did appear to be a slight trend towards a higher amount of IMA in the preconditioned participants (Figure 6.2). The majority of the participants showed a decrease in IMA levels following the control treatment, whereas the median IMA level in the RIPC participants was notably greater than the baseline measurement (Figure 6.2). To avoid the inflated type 1 error risk, this observation was not statistically tested.

Two additional sets of samples were collected from ACS patients hospitalised for a recent ischaemic episode, and from age-matched healthy volunteers. The mean age in the two groups was similar (57 years in the ACS patients vs. 55 in the agematched controls), and substantially higher than in the healthy volunteers from the RIPC study (23 years; Table 6.2). Nine of the 11 ACS patients recruited were diagnosed with ST segment elevation MI (STEMI), and 2 with non-STEMI, and all of the patients experienced troponin elevations (Table 6.3). The IMA level in the ACS patient group was significantly higher than in the age-matched controls, demonstrating that the assay was performing as expected and that IMA levels do not appear to naturally increase with age in individuals without any history of heart disease (Figure 6.1). Interestingly, the baseline $\mathrm{OD}_{470}$ values in the healthy volunteers that participated in the RIPC study were higher than the mean IMA level for the older healthy volunteers recruited as an age-matched control for the ACS patients (Figure 6.1). This most likely relates to changes in the samples resulting from different lengths of storage prior to the analysis. 


\begin{tabular}{lccc}
\hline & $\mathbf{n}$ & $\begin{array}{c}\text { Age } \\
\text { Mean (SD) }\end{array}$ & $\begin{array}{c}\text { Male sex } \\
\% \text { (number) }\end{array}$ \\
\hline RIPC study volunteers & 10 & $23(3.19)$ & $100(10)$ \\
\hline ACS patients & 11 & $57(11.21)$ & $91(10)$ \\
\hline $\begin{array}{l}\text { Non-ACS age-matched } \\
\text { volunteers }\end{array}$ & 5 & $55(14.78)$ & $80(4)$ \\
\hline
\end{tabular}

\section{Table 6.2 Baseline demographics of participant groups}

Baseline demographics for the ACS patients, age-matched healthy volunteers, and the healthy volunteers that participated in the RIPC study.

\begin{tabular}{|c|c|}
\hline & $\underset{n=11}{\text { Number (\%) }}$ \\
\hline \multicolumn{2}{|l|}{ Clinical presentation } \\
\hline STEMI & $9(82)$ \\
\hline Non-STEMI & $2(18)$ \\
\hline \multicolumn{2}{|l|}{ Ischaemic markers } \\
\hline ECG changes & $8(73)$ \\
\hline Troponin elevation & $11(100)$ \\
\hline Peak troponin (mean (SD)) & $5435(14838)$ \\
\hline \multicolumn{2}{|l|}{ Comorbidities } \\
\hline Hypertension & $3(27)$ \\
\hline Diabetes & $1(9)$ \\
\hline \multicolumn{2}{|l|}{ Smoking history } \\
\hline Current & $2(18)$ \\
\hline Former & $2(18)$ \\
\hline Never & $6(55)$ \\
\hline \multicolumn{2}{|l|}{ Medications at admission } \\
\hline ACE inhibitor & $3(27)$ \\
\hline Aspirin & $2(18)$ \\
\hline Clopidogrel & $1(9)$ \\
\hline Beta blocker & $1(9)$ \\
\hline Calcium channel blocker & $0(0)$ \\
\hline Statin & $1(9)$ \\
\hline Other lipid lowering drug & $1(9)$ \\
\hline Non-steroidal anti-inflammatory drug & $1(9)$ \\
\hline
\end{tabular}

Table 6.3 Characteristics of the ACS patient population

General demographics and clinical details for the ACS patients recruited. 


\section{Ischaemia-modified Albumin}

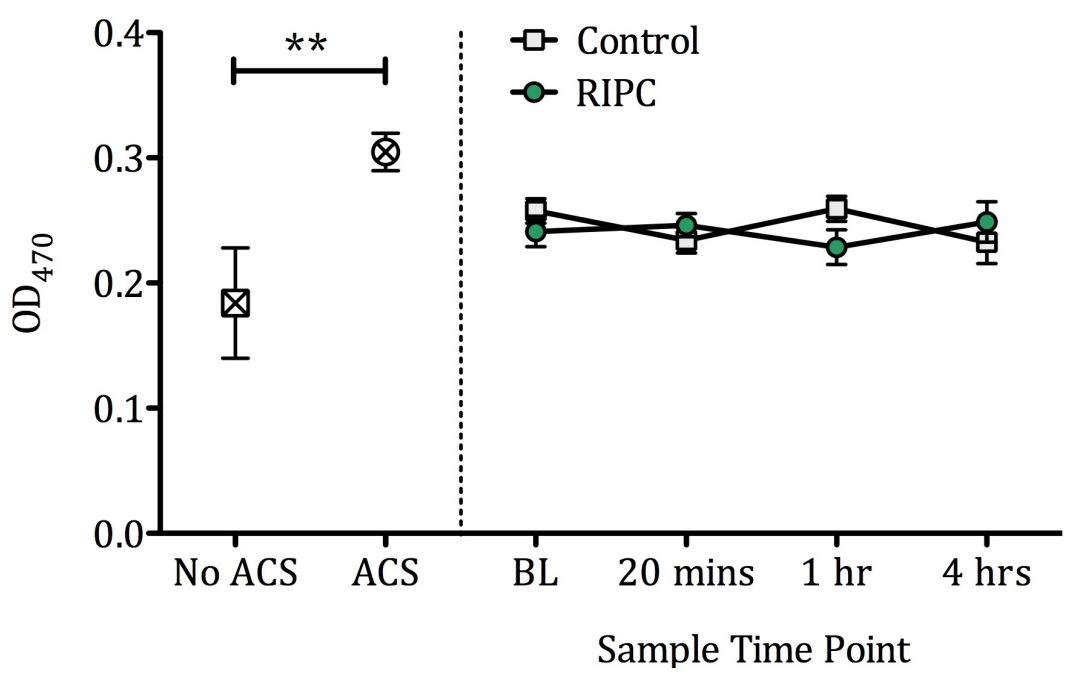

Figure 6.1 Levels of ischaemia-modified albumin in study participants

Serum samples were collected from ACS patients $(n=11)$, age-matched healthy volunteers ('No ACS'; $n=5$ ), and the healthy volunteers that participated in the RIPC study $(\mathrm{n}=10)$, and assessed for IMA. The study participants received the control and RIPC treatments on separate visits and had samples collected at BL, and 20 minutes, 1 hour, and 4 hours post-treatment. Shown are the means and SEM of the blank-corrected data. The difference between the ACS and agematched healthy volunteers was compared using a t-test, and the differences between the treatments and time points for the RIPC study participants were compared using a two-way repeated measures ANOVA. ${ }^{* *} \mathrm{p}<0.01$.

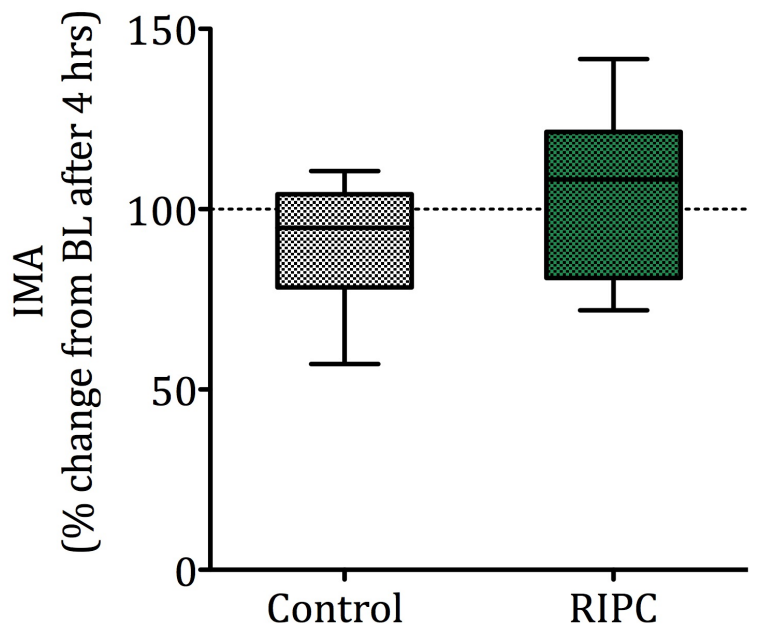

Figure 6.2 Total change in ischaemia-modified albumin from baseline to 4 hours after treatment

Serum samples were collected and assessed for IMA levels, before and after the control and RIPC treatments. Shown is the total \% change in IMA from BL to 4 hours after treatment. The box displays the median and first and third quartiles, with the minimum and maximum values shown by the whiskers. $\mathrm{N}=10$. 


\subsubsection{Multivariate analysis of the response to RIPC}

\subsubsection{Visual representation of the differences between the treatments and study participants}

The primary aim of the multivariate analysis was to determine whether common patterns of response in the endpoints that appeared to be affected by preconditioning in the univariate analysis could be detected between either the study treatments, or the participants. A resemblance matrix was constructed using the standardised Euclidean distance between the 20 datasets (10 from each of the control and RIPC visits) with our 13 selected continuous variables. The Euclidean distance value represents the overall level of 'distance' (dissimilarity) between each pair of datasets. A dendrogram was created based on the Euclidean distance measure and is presented in Figure 6.3. Interestingly, there was no clustering detected either between the treatment groups, or the paired datasets for each participant; the level of relatedness appeared to be independent of both these factors. In fact, all of the individual datasets collected during the control visit were more similar to different participants than they were to their own matching dataset obtained during the RIPC visit.

The MDS analysis revealed more clearly that the majority of participants were actually clustered together, showing similar responses; however, four sets of data from participants 2 and 4 during the control visit, and 1 and 7 during the RIPC visit were distinctly different in their response patterns, compared both to the main group and each other (Figure 6.4). At the highest level of Euclidean distance shown (5.3) the observations were grouped into 5 clusters, 4 of which contained the individual datasets for $2 \mathrm{~A}, 4 \mathrm{~A}, 1 \mathrm{~B}$, and $7 \mathrm{~B}$. The clusters identified by lower levels of distance provided no additional information, as the 4 outlying datasets remained markedly different and there was significant overlap between the clusters within the main group of remaining observations (Figure 6.4). Interestingly, a closer look at the level of distance between the datasets without the inclusion of the cluster boundaries showed a trend towards the data being loosely grouped based on the treatment visit, although there was also significant overlap between the control and RIPC visits (Figure 6.5). 


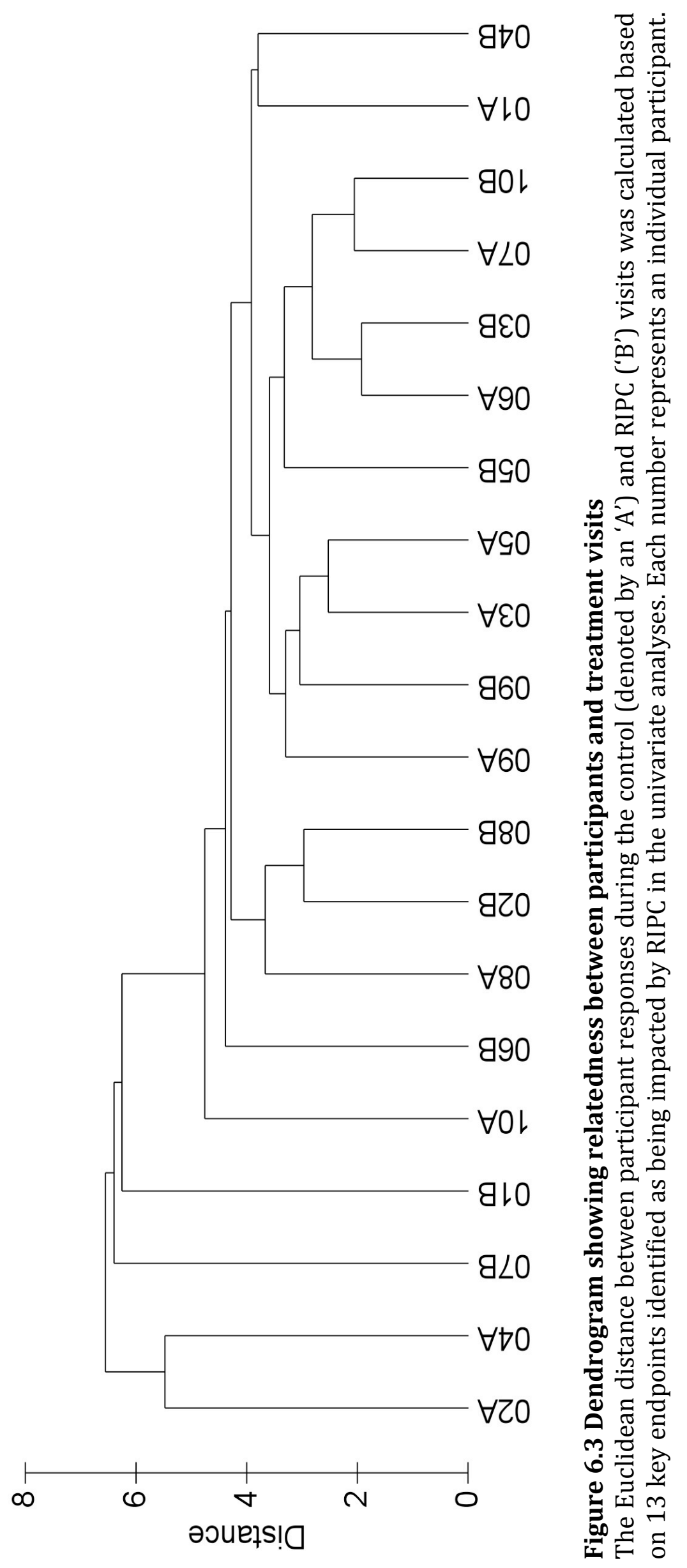




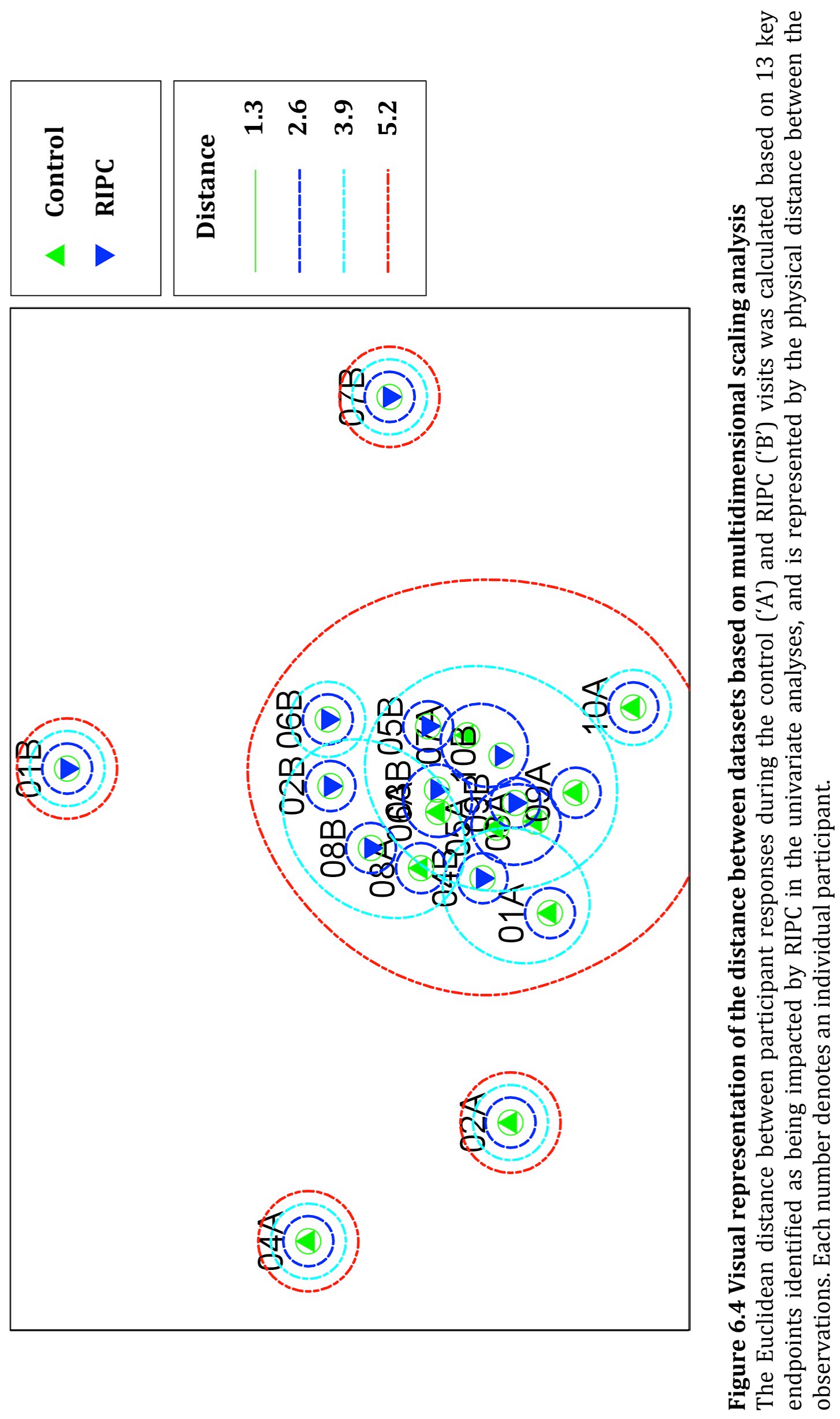




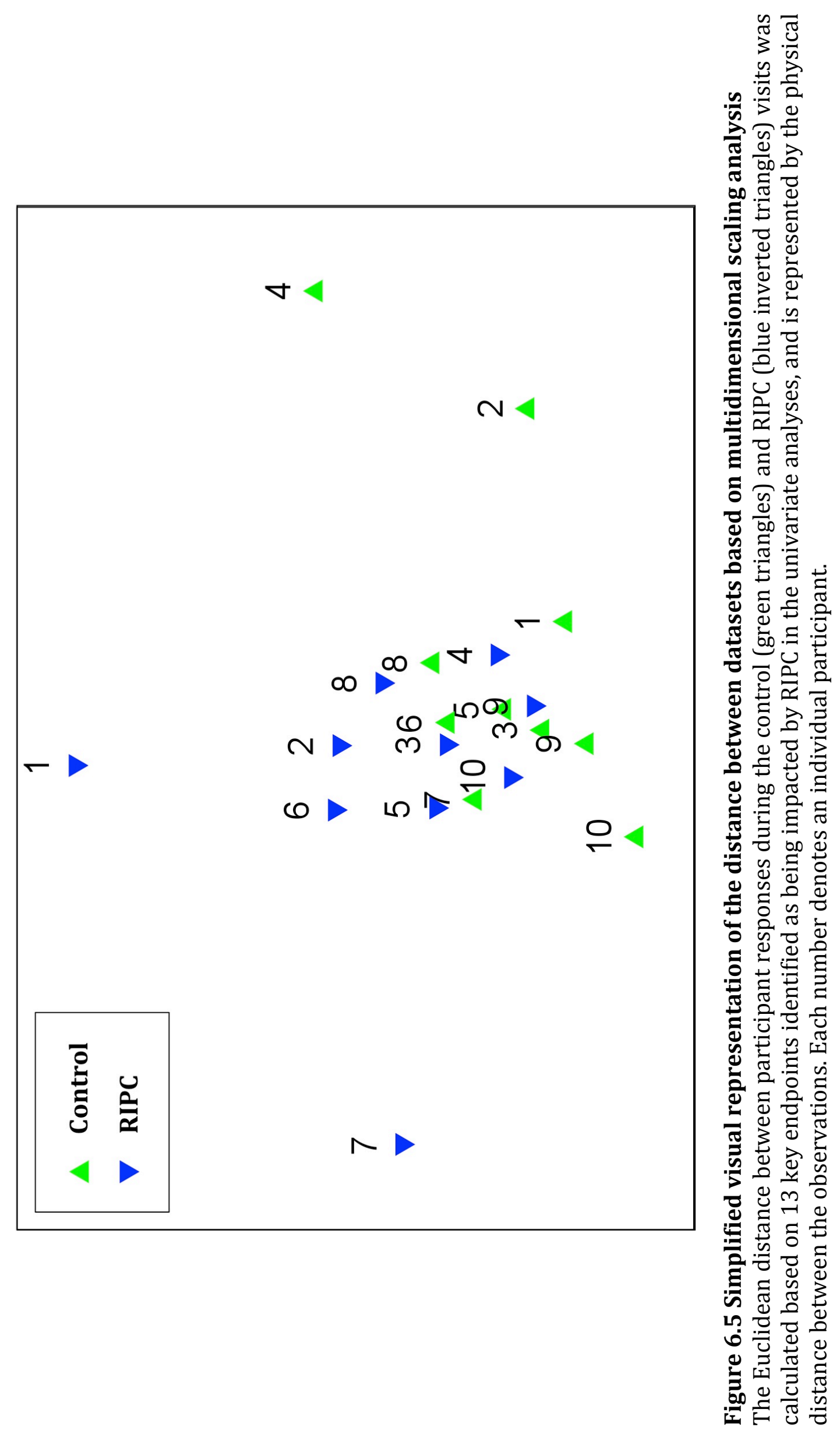




\subsubsection{Statistical analysis of the differences between the treatments and study participants}

Following on from the MDS analysis, we investigated whether the differences between the treatments and individual participants were statistically significant. A PERMANOVA run with 999 permutations determined there was a significant difference between the treatment groups $(p=0.012)$, and the individual participants $(\mathrm{p}=0.01)$, with respect to the overall changes in the 13 key endpoints included in the analysis.

In order to determine which endpoints contributed to the differences observed between the treatments and participants, we performed a SIMPER analysis. The most important variable for establishing the difference between the control and RIPC treatments was the level of CD11b expression on neutrophils stimulated with PMA/I for 4 hours of culture, which accounted for approximately $14.95 \%$ of the variation detected between the treatment groups (Table 6.4). CD69 expression on T cells cultured with PMA/I for 27 hours, and serum MIP-1 $\beta$ levels (represented by the total area under the curve for data expressed as a percentage of the baseline levels) were also important contributors, accounting for 11.6 and $11.26 \%$ of the variation, respectively. Levels of IL-22 from supernatants of 6 day unstimulated PBMC cultures, and serum MIP-1 $1 \alpha$ were the next most important variables, explaining 8.96 and $8.43 \%$ of the variation, respectively. A number of other variables, including IL-1 $\beta$, IL-10, and TNF- $\alpha$ levels from 6-day tetanus-stimulated PBMC cultures, PMA/I-induced T cell proliferation, CD11b expression in PMA/I-stimulated monocyte 4-hour cultures, and ischaemia-modified albumin (expressed as the total \% change from baseline) also contributed to the differences and individually accounted for between 4.77 and $7.84 \%$ of the detected variation (Table 6.4 ).

To investigate the factors that differed between participants, the SIMPER analysis averaged the observations collected at the control and RIPC visits for each participant and then performed pair-wise comparisons between all of the participants. Our MDS analysis indicated that participants 3, 5, 6, 8, 9, and 10 had similar responses, whereas participants 1, 2, 4, and 7 showed marked 
dissimilarities in their datasets during one of the study visits. We therefore examined the results of the pair-wise analyses between these 4 outlying participants and each of the participants in the main group cluster, and recorded the frequency with which a factor was identified as contributing to greater than $10 \%$ of the difference detected between the pair.

The endpoints that contributed to the differences between the outlying participants and the main cluster differed between each of the 4 cases, which was consistent with the MDS analysis that showed these participants were also markedly dissimilar to each other (Table 6.5). Participant 1 appeared to differ from the main cluster with respect to $\mathrm{T}$ cell and monocyte activation levels, with CD69 expression in T cells, and CD11b and intracellular IL-8 production in monocytes identified as significant contributors to the difference in 6, 5, and 3 of the 6 pair-wise comparisons, respectively. Participant 2 was separated predominantly due to CD11b expression on monocytes and neutrophils, as well as IL-1 $\beta$ and IL-6 production in 6-day tetanus-stimulated PBMC cultures, and the major source of difference for participant 4 appeared to be with the culture supernatant cytokine levels. Lastly, participant 7 stood out with respect to the level of IL- $8^{+}$monocytes and serum concentrations of MIP-1 $\alpha$ and $\beta$. Of note is that all of the outlying participants consistently differed from the main cluster participants with respect to two or three key factors, indicating that the dissimilarities were not random but attributable to specific endpoints (Table $6.5)$. 


\begin{tabular}{lccc}
\hline Variable & $\begin{array}{c}\text { Contribution to } \\
\text { the Difference (\%) }\end{array}$ & $\begin{array}{c}\text { Cumulative } \\
\text { Contributions (\%) }\end{array}$ & $\begin{array}{c}\text { RIPC } \\
\text { Effect }\end{array}$ \\
\hline Neutrophil CD11b expression & 14.95 & 14.95 & $\downarrow$ \\
\hline T cell CD69 expression & 11.60 & 26.55 & $\uparrow$ \\
\hline Serum MIP-1 $\beta$ levels & 11.26 & 37.81 & $\downarrow$ \\
\hline Culture IL-22 levels & 8.96 & 46.77 & $\uparrow$ \\
\hline Serum MIP-1 $\alpha$ levels & 8.43 & 55.20 & $\downarrow$ \\
\hline Culture IL-10 levels & 7.84 & 63.03 & $\downarrow$ \\
\hline T cell proliferation & 7.45 & 70.48 & $\uparrow$ \\
\hline Culture TNF- $\alpha$ levels & 5.88 & 76.37 & $\downarrow$ \\
\hline Culture IL-1 $\beta$ levels & 5.21 & 81.57 & $\downarrow$ \\
\hline Monocyte CD11b expression & 5.20 & 86.78 & $\downarrow$ \\
\hline Ischaemia-modified albumin & 4.77 & 91.54 & $\uparrow$ \\
\hline
\end{tabular}

Table 6.4 Variables responsible for the difference between control and RIPC treatment responses

Shown are the variables identified by SIMPER analysis as cumulatively accounting for $>90 \%$ of the difference between the treatment groups, and the effect of RIPC on each variable, relative to the control treatment. 


\begin{tabular}{|c|c|c|}
\hline Participant & $\begin{array}{l}\text { Variables Contributing } \\
\text { to the Difference }\end{array}$ & $\begin{array}{l}\text { Observed Frequency } \\
\text { (out of } 6 \text { comparisons) }\end{array}$ \\
\hline \multirow{7}{*}{1} & T cell CD69 expression & 6 \\
\hline & Monocyte CD11b expression & 5 \\
\hline & IL-8+ monocytes & 3 \\
\hline & Neutrophil CD11b expression & 2 \\
\hline & $\mathrm{T}$ cell proliferation & 2 \\
\hline & Ischaemia-modified albumin & 2 \\
\hline & Culture IL-1 $\beta$ levels & 1 \\
\hline \multirow{6}{*}{2} & Monocyte CD11b expression & 5 \\
\hline & Culture IL-6 levels & 5 \\
\hline & Neutrophil CD11b expression & 5 \\
\hline & Culture IL-1 $\beta$ levels & 4 \\
\hline & Ischaemia-modified albumin & 2 \\
\hline & Culture IL-22 levels & 1 \\
\hline \multirow{5}{*}{4} & Culture IL-10 levels & 6 \\
\hline & Culture TNF- $\alpha$ levels & 6 \\
\hline & Culture IL-6 levels & 2 \\
\hline & Monocyte CD11b expression & 1 \\
\hline & Ischaemia-modified albumin & 1 \\
\hline \multirow{5}{*}{7} & IL-8+ monocytes & 6 \\
\hline & Serum MIP- $1 \alpha$ levels & 6 \\
\hline & Serum MIP- $1 \beta$ levels & 4 \\
\hline & $\mathrm{T}$ cell proliferation & 3 \\
\hline & Culture IL-22 levels & 4 \\
\hline
\end{tabular}

Table 6.5 Variables responsible for the differences between study participants

Participants 1, 2, 4, and 7 were each compared with the datasets obtained for participants 3, 5, 6, 8, 9, and 10 using SIMPER analysis, and the frequency with which a particular endpoint was detected as contributing to greater than $10 \%$ of the observed distance between the datasets was noted. A total of 6 comparisons were made for each of the 4 individually clustered participants. 


\subsection{Discussion}

The aim of this chapter was to analyse the overall effect of RIPC by looking at a marker of ischaemia and more closely examining the pattern of changes in a set of key endpoints that appeared to be affected by RIPC. We confirmed that the IMA assay could detect changes in albumin following an acute cardiac ischaemic episode but did not find a difference in the circulating levels of IMA between the control and RIPC treatments. The multivariate analysis determined there was a significant difference in the overall responses between the treatment groups, which was largely accounted for by changes in neutrophil and $\mathrm{T}$ cell activation and the production of the cytokines, MIP-1 $\beta$ and IL-22. There was also a significant difference in the responses between the participants, the source of which varied between the individual comparisons.

The lack of change in the ischaemia-modified albumin measurements between the treatment groups suggests that the assay does not have the sensitivity to detect a response to preconditioning ischaemia. The significant increase in IMA levels seen in the ACS patients compared to the age-matched controls confirmed the assay was performing as expected; however, it was not anticipated that the $\mathrm{OD}_{470}$ levels in the RIPC study participants at baseline would be notably higher than those detected in the older healthy volunteer population. The most likely explanation for this anomaly lies in the principal reactions involved in the assay.

The IMA assay depends on a redox reaction between the transition metal, cobalt, and the two sulfhydryl groups present in the reducing agent, DTT. This reaction results in the formation of a strong brown solution that can be measured on a spectrophotometer (Bar-Or et al., 2000). Although the exact mode of action of the assay has not yet been identified, alterations to albumin and, potentially, other proteins present in the serum following ischaemia appear to reduce the amount of free cobalt left available to react with the DTT (Bar-Or et al., 2008). In normal plasma samples, a certain level of interaction between cobalt and the single sulfhydryl group present in the amino acid, cysteine, also occurs, and results in the formation of a pale yellow product. Interactions between cysteine 
and cobalt thus reduce the amount of cobalt able to interact with the DTT, and lower the IMA assay readings (Bar-Or et al., 2008).

The effect of the cysteine-cobalt interactions is relatively consistent in healthy individuals; however, following significant oxidative stress or prolonged storage of the samples there is increased oxidation of both albumin and cysteine (Bar-Or et al., 2008; Oettl and Marsche, 2010). The increased cysteine oxidation results in interactions with itself and other small molecules to form cystine and additional derivatives which contain disulfide bonds that render the sulfhydryl groups unavailable for transition metal binding (Bar-Or et al., 2008). Furthermore, both extended storage time and freeze-thawing of samples decrease the presence of free sulfhydryl groups present in plasma and serum, which may also increase the level of unbound cobalt detected during the assay (Goldblum et al., 1954). These two mechanisms thus are likely to contribute to a non-specific increase in the IMA assay readings for older samples.

We performed the IMA assay on all of our samples at the same time, in order to maintain comparability between the measurements in the absence of a defined standard. The samples from the ACS patients and age-matched controls were assayed within two weeks of their collection whereas the samples from the RIPC study were stored at $-80{ }^{\circ} \mathrm{C}$ for upwards of one year prior to the analysis. Further, there was approximately 8 months between obtaining the samples from the first and last study participants. This most likely was the cause for the higher IMA values in the RIPC study samples, compared to the fresher samples collected from the older population of healthy volunteers and may have reduced our ability to detect a difference between the treatments.

Another possible explanation is that our samples were not collected during the optimal time range for detecting an increase in IMA levels induced by preconditioning; however, this seems unlikely. Bar-Or et al. investigated the kinetics of ischaemia marker levels following percutaneous transluminal coronary angioplasty and found a significant increase in IMA immediately following the procedure, which returned back to baseline levels before the next 
time point, 6 hours later (Bar-Or et al., 2001b). We collected our first samples 20 minutes following the completion of RIPC and our last samples after 4 hours, covering a comparable time range. If the main change in IMA levels was so transient that it could not be detected as early as 20 minutes after treatment, then this assay would not be suitable for our intended purpose. The sensitivity of the IMA assay could potentially be improved with further optimisation; however, the results from the present study suggest that IMA is not appropriate as a biomarker of successful preconditioning.

It is interesting to consider that the ACS patients likely share similar characteristics to many members of the cardiac surgery patient population commonly used in RIPC clinical trials. If patients have already recently endured an ischaemic episode, it is not currently known whether RIPC can have any incremental effect. Further, if a previous exposure to ischaemia does elicit a protective effect similar to that of RIPC, inclusion of such patients in the control groups will confound trial analysis. Measurement of a non-specific marker of ischaemia prior to the intervention in future clinical trials would be very informative in these respects. It seems reasonable to speculate that innate forms of preconditioning in humans may be another key difference between the animal and clinical studies and one of the critical questions addressed in future research should be whether or not a patient with ongoing ischaemic disease can, in reality, be preconditioned against a larger, planned, acute ischaemic injury.

Our univariate analysis of the study endpoints in Chapters 4 and 5 highlighted a number of immune parameters that appeared to be affected by preconditioning; however, the large dataset and number of statistical tests performed made it difficult to comment on whether these were legitimate biological effects, or random fluctuations and type I errors. We addressed this issue by performing a multivariate analysis and limited the data that was included to address two specific research questions. The first investigated whether there was a consistent pattern of response to RIPC treatment, and the second looked for potential differences in the type of response between the participants. 
The results indicated that there was a significant difference in the overall measure of similarity in the datasets for the participants between the control and RIPC visits. This finding is extremely encouraging and suggests that although the results we measured in our study were individually quite subtle, they were part of a legitimate direct, systemic response to RIPC. Approximately 55\% of the difference in the response between the treatment groups was accounted for by changes in neutrophil and T cell activation marker expression, serum MIP-1 $\alpha$ and $\beta$ levels, and PBMC production of IL-22. While the changes in cytokine production stood out as significant in the univariate analysis, the two most important factors, neutrophil CD11b and T cell CD69 expression levels, did not reach statistical significance individually. This may be more to do with the variability in the level of response between participants, rather than a reflection of the magnitude of the effect, and highlights the usefulness of carefully considered multivariate analysis techniques in exploratory trials.

Interestingly, the multivariate analysis confirmed that in our study, RIPC was associated with suppression of neutrophil activation during the early response to preconditioning and with enhanced $\mathrm{T}$ cell activation during the delayed window of protection. Immediately following reperfusion of ischaemic tissue, the majority of leukocyte subsets play a detrimental role in the ensuing inflammatory response (Warren et al., 2009). Suppression of these cells, particularly the innate populations such as neutrophils and monocytes, which have the greatest capacity to inflict damage under these circumstances, is therefore likely to be beneficial. The effects of leukocytes during the later stages of recovery are more diverse, however, and certain subsets of $\mathrm{T}$ cells are beneficial for assisting in tissue repair and wound healing (Burzyn et al., 2013; Eyerich et al., 2009; Hofmann et al., 2012; Toulon et al., 2009). The increased level of $\mathrm{T}$ cell activation detected in cells stimulated for 27 hours ex vivo is consistent with the increased production of IL-22 detected in the PBMC culture supernatant 6 days post-RIPC, which may also exert a protective effect. Although these changes to cellular activation may seem paradoxical at first glance, they are both likely to have beneficial effects. 
The multivariate analysis also determined that there was a significant difference in the responses seen between participants. Despite attempts to recruit a fairly homogenous participant population by restricting key characteristics such as age and sex, this result was not surprising. A multitude of factors can alter the course of the immune response, ranging from polymorphisms through to lack of sleep or even the ingestion of caffeine (Born et al., 1997; Horrigan et al., 2006; Jin and Wang, 2003). Of particular interest was the finding that the four datasets that were significantly dissimilar to the main cluster of participants were also distinct from each other. Furthermore, two of these datasets were collected during a control visit, and the other two were collected from different participants during RIPC visits. Taken together, these observations strongly suggest that the differential responses were due to perturbations in the participants' immune response at the time of the corresponding study visit, rather than an alternative form of response to the treatment. The analysis did not identify a second cluster of participants that appeared to be 'non-responders' to the treatment.

Interestingly, none of the four individually clustered datasets belonged to either of the participants that were found to have particularly high serum cytokine levels, compared to the other study participants. This would suggest that the altered serum cytokine levels detected in these individuals were part of an isolated effect that did not necessarily spill over into the other immune parameters. This finding also reinforces our decision not to exclude these participants from the remaining data analyses presented in Chapters 4 and 5 .

A key limitation of the multivariate analysis was that we treated the data collected from the control and RIPC visits for each participant as though they were independent. We elected to perform the analysis this way as it enabled us to investigate whether the overall responses from each participant during their RIPC study visit were more similar to other participants receiving the RIPC treatment, or to the data collected from the same individual during the control treatment visit. Ultimately, our analysis suggested that participants were more likely to be clustered based on the treatment received, therefore the correlation between the paired datasets did not appear to significantly impact the analysis. 
The results of cluster analyses are also highly dependent on the variables included. Given the size of our full set of data relative to the number of participants, we did not have sufficient power to perform the analysis using all of our endpoints. Further, the multiple time points and conditions used to examine changes in leukocyte populations and cytokine production created a level of redundancy in our dataset that could have biased the results if included without substantial editing. Instead, we focused on a set of key endpoints that appeared to be altered by RIPC for the purpose of identifying whether these changes were consistent following treatment, or fluctuated randomly between participants. While the overall analysis both complemented and reinforced the findings of our univariate testing from Chapters 4 and 5, the accuracy of the estimates of the importance for each individual variable included in the model should be interpreted with caution.

\subsection{Conclusions and future directions}

RIPC in healthy volunteers did not elicit a measurable change in ischaemiamodified albumin in samples stored for upward of 12 months; therefore it appears this assay does not have the sensitivity required for use as a biomarker of successful preconditioning under these circumstances. Further research is needed to determine the utility of IMA testing in freshly collected samples from RIPC recipients. ACS patients had significant levels of IMA, and performing this analysis in patients participating in future RIPC clinical trials, prior to treatment, could provide useful insight as to whether people can be preconditioned if they have already recently undergone an ischaemic episode.

A multivariate analysis indicated that there was a significantly different response between the RIPC and control treatments, confirming that RIPC does have a subtle but direct and consistent effect on the innate and adaptive immune responses. These findings warrant further, more in-depth investigation of the parameters identified as being altered by RIPC in our study, particularly serum MIP-1 $\alpha$ and $\beta$ levels, neutrophil and T cell activation, and PBMC production of IL22. 
Chapter 7.

\section{General discussion}




\subsection{Research motivation}

Complications following cardiac surgery are not only detrimental to patient outcome and the incidence of mortality, but pose a significant drain on limited hospital resources. Poor post-operative recovery of myocardial contractility and the incidence of acute kidney injury are two such major factors that significantly increase the duration of stay in the ICU, at a cost of approximately $\$ 3500$ per day. Further, a lack of available space or nurses in the ICU has been identified as the most significant issue with cardiac surgery services in New Zealand, and leads to a high rate of cancellation of scheduled elective surgeries (Ministry of Health, 2008). Post-operative complications thus not only attract increased costs of care, but negatively impact the productivity of surgical units. This issue is particularly prevalent in the patient population aged 70 years and over, which is estimated to require 91\% more care and clinical resources following cardiac surgery and are increasingly presenting for these procedures (Ngaage et al., 2011).

There is a clear need for novel cardioprotective techniques beyond those currently used in clinical practice to reduce the incidence of post-operative complications and improve both patient outcome and the productivity of cardiac surgery facilities (Hausenloy et al., 2010). RIPC is one such strategy, and holds enormous potential to significantly progress clinical practice. RIPC has been shown to dramatically reduce the size of myocardial infarct sustained following an ischaemic episode in numerous animal models, and several clinical trials have also demonstrated the ability of this technique to reduce post-operative troponin release and the incidence of AKI. However, a number of clinical studies have not detected an improvement in such surrogate endpoints (Costa et al., 2013; Kaur Randhawa et al., 2014).

Repetitive studies of RIPC in similar patient populations with slight variations in trial design and methods are no longer significantly advancing our knowledge. Instead, new research needs to address the efficacy of RIPC in different patient populations and applications, and determine whether it is capable of significantly improving long-term primary clinical outcomes following cardiac surgery. The design and interpretation of such work will be greatly facilitated by 
expanding our understanding of the mechanisms through which RIPC operates in humans.

\subsection{Overview of results}

We performed the first study of RIPC in a high-risk cardiac surgery patient population, and did not detect a change in a marker of myocardial damage or clinical outcome endpoints in the preconditioned patients (Chapter 2). This study was one of the first in the RIPC field to use a double-blinded design and report a neutral clinical result, second only to a large trial by Rahman et al. (2010), but has been followed by several other trials that also failed to demonstrate a benefit from RIPC in adult cardiac surgery patients using a rigorous blinding protocol (Meybohm et al., 2013; Saxena et al., 2013; Zitta et al., 2014). Both these studies, and our own, add weight to the growing body of literature that one could reasonably argue establishes that RIPC does not consistently benefit the general cardiac surgery population. Given the positive results seen in some clinical trials and the potential of this technique demonstrated in animal studies, it seems that RIPC could work effectively in certain individuals; however, in the absence of a full understanding of the pathways through which RIPC functions, we can only speculate as to what factors may enable some patients to respond to the intervention and prevent others from being preconditioned (Hausenloy et al., 2007; Thielmann et al., 2013; Venugopal et al., 2009).

One of the key aspects of RIPC that has perplexed researchers is the identity of the humoral messenger purported to be involved in transmitting the protective signal from the preconditioned tissue to the heart and other organs of the body (Hausenloy and Yellon, 2008). Work in animal studies suggests this mediator is hydrophobic and of a low molecular weight somewhere in the range of $3.5-15$ kDa (Lang et al., 2006; Serejo et al., 2007; Shimizu et al., 2009). Based on the involvement of immune cells and changes in their gene transcription profiles in response to RIPC, we hypothesised that this factor might be a cytokine (Konstantinov et al., 2004). No significant change in the serum levels of a set of key inflammatory cytokines was detected in our high-risk cardiac surgery trial 
patients following RIPC; however, there was a slight trend towards increased levels of IL-6 and IL-8 in the preconditioned patients (Chapter 2). To separate the influence of surgery and comorbidities on cytokine expression and obtain a more conclusive result, we then examined the effect of RIPC on serum levels of a range of cytokines and chemokines critical to the inflammatory response in healthy male volunteers, and also found no significant increase in any of the 20 key analytes assessed (Chapter 4).

The findings from our studies in both cardiac surgery patients and healthy volunteers strongly suggest that a cytokine is not the missing humoral mediator. Our results are also consistent with two recently published studies that examined changes in the plasma and serum proteome of human volunteers after RIPC, which predominantly found significant alterations in the levels of proteins associated with the immune response and apoptosis, but no changes to cytokine expression (Hepponstall et al., 2012; Pang et al., 2013). Similarly, Hibert et al. (2014) found no changes in cytokine levels amongst the plasma proteome following RIPC in rats, suggesting the lack of induction of cytokines detected in humans was not a species-specific occurrence.

We did detect a significant reduction in the serum levels of MIP- $1 \alpha$ and $\beta$ following preconditioning in the healthy volunteers, and it is possible that this represents increased uptake of these cytokines by cellular targets (Chapter 4). However, this would be dependent on an increase in receptor expression, which does not explain why transfusion of plasma, coronary effluent, or blood from preconditioned animals can elicit protection in a RIPC-naïve recipient (Dickson et al., 1999a, 1999b; Shimizu et al., 2009). The changes associated with this reduction in MIP levels are thus unlikely to be related to the signalling mechanism involved in establishing RIPC.

Overall, it was determined that RIPC elicits direct but subtle effects on the immune response in healthy volunteers. In the timeframe corresponding to the early phase of protection, in addition to the decrease in serum MIP concentrations, there was a trend towards a reduction in the ability of 
monocytes and neutrophils to become activated, but no other significant changes (Chapters 4 and 5). The majority of clinical trials to date have focused on the utility of this early phase of RIPC to provide organ protection in cardiac surgery patients. Although neutrophils are a major mediator of peri-operative tissue damage and their suppression is likely to limit the level of injury sustained, it is highly unlikely that this effect solely accounts for the dramatic reduction in troponin release detected by the early clinical trials (Korthuis and Granger, 1993). This idea is supported by the particularly high levels of IL-6 and IL-8 that were detected in the high-risk cardiac surgery patients, which indicate a substantial general inflammatory response during surgery, with or without RIPC (Chapter 2). In the presence of such a strong, systemic immune response, a subtle alteration to one or two leukocyte populations is likely to translate to only a limited effect.

A marked increase in T cell activation was detected in cells cultured for 27 hours, and higher levels of basal IL-22 production in PBMC cultured for 6 days, in the preconditioned healthy volunteers (Chapter 5). Although the timing of these two endpoints corresponds to the delayed window of preconditioning, IL-22 production was not altered in 24-hour cultures; therefore these effects do not appear to overlap. This finding raises the question of whether the second window of RIPC protection can be further temporally divided based on the unique processes involved and the potentially different types of protection afforded by each stage. The late phase of preconditioning is also generally associated with changes in gene transcription; however, the proteome experiment by Hepponstall et al. (2012) found only a minor population of proteins were upregulated, whereas a substantial number of proteins were downregulated during this delayed window (Costa et al., 2013). It is therefore particularly interesting that we detected the stimulation, rather than suppression, of these immune processes.

Three clinical trials have investigated the effects of delayed RIPC in cardiac surgery, two of which were in children, and have shown mixed results (Pavione et al., 2012; Wagner et al., 2010; Zhou et al., 2010). It is possible that changes to T 
cell activation and IL-22 production may elicit protective effects, but relevant measures at time points corresponding to when these changes were observed in the healthy volunteers have not been included in most clinical trials. A beneficial contribution of IL-22 to tissue and wound repair could significantly improve the clinical course of recovery and in the future, studies that investigate IL-22 levels in tandem with more direct clinical outcome measures, such as 30-day mortality, will be better suited to determine whether this is the case.

A multivariate analysis confirmed that the subtle changes detected following RIPC in the healthy volunteers amounted to a significantly different response, compared to the control treatment (Chapter 6). Despite this clear finding that RIPC does alter the immune response, these changes may not necessarily contribute to the protective mechanisms involved with preconditioning in the setting of ischaemic injury. Our research was designed to be hypothesis generating and was performed in healthy volunteers, thus does not include any clinical outcome measures with which to correlate the changes that were detected. While these alterations may positively alter the course of I/R injury, it is also possible they might be insignificant in the face of the systemic inflammatory burden induced by the majority of cardiac surgical procedures or, alternatively, may be secondary to non-immune changes resulting from RIPC that were not measured in our study.

\subsection{Alternative mechanisms that may contribute to RIPC}

RIPC was found to have only subtle effects on both the direct immune response (Chapter 4) and the function of peripheral leukocytes in response to subsequent in vitro stimulation (Chapter 5). These small alterations may dampen the inflammatory response during I/R injury, but are unlikely to provide the level of organ protection detected during the early phase of RIPC in positive clinical trials.

Previous research has established that one of the major end effectors of RIPC is the alteration of ATP sensitive potassium channels present in the plasma membrane of cardiomyocytes and the inner mitochondrial membrane (Beavis et 
al., 1993; Loukogeorgakis et al., 2007). Opening of the channels reduces the rate of ATP depletion, decreases the intracellular calcium level, and results in vasodilation (Costa et al., 2013; Dos Santos et al., 2002; Kaur Randhawa et al., 2014). Through the intracellular loss of ATP, I/R appears to inhibit the opening of these $\mathrm{K}_{\mathrm{ATP}}$ channels, leading to accumulation of calcium and a loss of mitochondrial integrity, whereas RIPC activates the channels and restores flow mediated vasodilation (Beavis et al., 1993; Costa et al., 2013; Loukogeorgakis et al., 2007). Further, through activation of PKC, RIPC may also inhibit opening of the mPTP during reperfusion, thus preventing mitochondrial swelling, plasma membrane rupture, and cell death (Argaud et al., 2005; Baines et al., 2003; Costa et al., 2013). These combined effects are likely to have a significant direct cardioprotective effect and in the absence of a strong impact on the immune response such as is suggested by the present work, are likely the major mechanisms through which RIPC confers protection during the early phase of protection.

IPC has been associated with the activation and subsequent suppression of the major inflammatory gene transcription factor, NF- $\mathrm{BB}$; therefore it has been suggested that RIPC could exert a protective effect through suppressing inflammation (Funaki et al., 2002; Li et al., 2006; Takeshita et al., 2010). It is interesting that this mechanism did not appear to translate to a significant alteration in the function of immune cells isolated from the blood of healthy volunteers during the early phase following RIPC in our study. Previous research demonstrating this altered NF- $\mathrm{KB}$ activity has detected differences in numerous preconditioned tissues including the heart, skeletal muscle, kidneys, liver, and small intestine (Funaki et al., 2002; Jiang et al., 2007; Li et al., 2006; Takeshita et al., 2010). It is therefore possible that RIPC may still limit the inflammatory response but through mechanisms elicited in the actual organs, rather than through changes to the circulating leukocytes.

Lastly, it is possible that B cells may also respond to RIPC. B cells were not investigated as part of the present study in healthy volunteers due to practical limitations and their apparent minimal involvement in $\mathrm{I} / \mathrm{R}$ according to the 
available literature. However, administration of repetitive hypobaric preconditioning (RHP) in mice over a 2-week period was recently shown to significantly alter the B cell response to a subsequent I/R injury in the brain. RHP, which has been shown to have a neuroprotective effect, was associated with suppression of the resident B cell population and induction of a regulatory, IL-10 producing B cell subset (Monson et al., 2014; Stowe et al., 2011). Although the RHP model involves a notably stronger stimulus compared with either form of IPC, it is possible that these findings could extend to RIPC.

In summary, the early phase of protection afforded by RIPC is most likely largely independent of changes to the peripheral blood leukocytes, and dependent on changes to metabolism and gene expression in the target cells. Alterations to the immune response may be more significant during delayed preconditioning and could involve B cells, which may also facilitate longer term wound repair and recovery from surgery. The most pressing unresolved question regarding the early phase of RIPC now is how exactly the signal is transferred from the preconditioned tissue to the heart and other organs of the body.

\subsection{Comparison between animal and human models of RIPC}

Translation of RIPC from simplified and easily controlled animal models into highly variable patient populations has encountered several obstacles that have not yet been overcome. Our research highlighted a number of key areas which may contribute to the different results seen between the animal and human studies, which could help to isolate the limitations of RIPC in clinical applications.

Several cytokines, including IL-6, IL-10, and TNF- $\alpha$, have been shown to be essential for inducing IPC in animal models, yet we did not detect a significant increase in the circulating levels of the same, or other, key cytokines following RIPC in healthy volunteers (Chapter 4; Cai et al., 2013, 2012; Dawn et al., 2004; Ren et al., 2004; Smith et al., 2002). In animal studies, increased STAT3 activation, which transduces signals for the IL-6 and IL-10 cytokine family members, has accordingly been associated with the protective effects of RIPC, 
whereas deletion of the STAT3 gene abolishes RIPC-mediated protection (Goodman et al., 2011; Hattori et al., 2001; Kim et al., 2004; Schindler et al., 2007; Xuan et al., 2001). Interestingly, an increase in STAT3 phosphorylation could not be detected in human myocardial biopsies from preconditioned patients, compared to the control treatment, but STAT5 activation was significantly enhanced (Heusch et al., 2011). The role of STAT5 in animal models of preconditioning is less clear, with one study suggesting STAT5 activation is insufficient to compensate for deletion of STAT3, and another finding that IPC was ineffective in STAT5 knockout mice (Goodman et al., 2011; Yamaura et al., 2003). Overall, these studies suggest that the transcription factors activated by IPC and RIPC may differ between species, which could likely also alter the effects induced by the preconditioning ischaemia.

IL-8 is a critical mediator of the peri-operative immune response, in part due to its significant roles in neutrophil chemotaxis and propagating the inflammatory response (Kawamura et al., 1993; Larsen et al., 1989). Accordingly, we detected high levels of IL-8 in all of our high-risk cardiac surgery patients and there was a slight trend towards higher levels expressed in the preconditioned patients (Chapter 2). A subtle increase in IL-8 levels 12 hours after RIPC was also reported by a study in healthy volunteers, which corresponded to an increase in circulating progenitor CD34+ cells (Czeiger et al., 2011). Taken together, these findings allude to a possible role of IL-8 during RIPC; but it is important to note that the gene for IL-8 has been deleted from the muroid rodent lineage. Two functional homologs in mice and rats, MIP-2 and KC, have been identified but it is unclear whether these mediators perform the same roles in I/R injury and preconditioning (Modi and Yoshimura, 1999). It is therefore necessary to take particular care when comparing IL-8-mediated pathways in humans to findings from rodent models.

A wide variety of factors can influence the immune response, including genetic and environmental alterations. These elements of variation less commonly affect mice and rat models, where the animals are genetic clones maintained in regulated sterile environments, but are rife in the human population. This was 
clearly demonstrated by the multivariate analysis in our study, which indicated that four of the ten participants showed significantly different responses during one of the study visits, compared to both the remaining participants and their own data collected from the alternative treatment (Chapter 6). It is unclear whether day to day variability in the immune response has the potential to attenuate or augment the effect of RIPC, but it will almost certainly increase the variability of the inter-individual responses and necessitate the use of much larger sample sizes in human studies to achieve the statistical power required.

Transient periods of ischaemia can be encountered endogenously through a number of processes, including episodes of angina and vigorous exercise, which may induce IPC. Indeed, it has been observed that patients with unstable angina or those participating in a strenuous exercise program show a preconditioned type of protective effect in response to cardiac surgery or cardiac events (Abete et al., 1997; Rock-Willoughby et al., 2013; Wang et al., 2007). We detected a significant increase in IMA in patients with ACS, confirming a systemic exposure to ischaemia (Chapter 6). It seems likely that many of the cardiac surgery patients recruited into RIPC clinical trials are likely to overlap with this ACS patient population, and therefore may not necessarily be preconditioning naïve.

The implications of these innate forms of preconditioning on the effect of subsequently applied RIPC are unclear. A study that administrated RIPC to healthy volunteers for 10 consecutive days found significant changes in neutrophil adhesion, phagocytosis, and cytokine production, some of which appeared to be cumulative (Shimizu et al., 2010). Kharbanda et al. (2009) postulated that there must be a downside to maintaining cells in a chronically preconditioned state, otherwise it would be expected that humans would have evolved to sustain this intrinsic protection. Clearly continued suppression of the immune system would be detrimental to host defence against infection, but overlapping periods of preconditioning may also have a negative impact of the benefits afforded by the intervention itself. While the complications of innate forms of preconditioning in regards to applying RIPC are not known, it is clear that this confounding effect is not encountered in the animal models. 
A variety of additional cardioprotective strategies are utilised in the clinical setting that are unlikely to be included in the majority of animal studies. The development of improved cardioplegia strategies, and the use of heparin and volatile anaesthetics have all been established as capable of reducing myocardial dysfunction following cardiac surgery (Aldea et al., 1996; Chambers and Fallouh, 2010; Friedrichs et al., 1994; Landoni et al., 2007). Further, commonly prescribed medications, such as statins, may also enhance clinical outcome (Chopra et al., 2012). The improvement and rate of use of all of these techniques has steadily increased over the last 10 years, whereas the frequency of positive RIPC studies has declined.

There is evidence that many of these cardioprotective techniques, including RIPC, share overlapping pathways; therefore it is possible that they may competitively inhibit each other. Indeed, we were not able to detect a reduction in myocardial damage in our own RIPC clinical trial that included the use of the volatile anaesthetic, isoflurane (Chapter 2). Thus, RIPC in animals may stimulate a cardioprotective effect that is already activated, either partially or maximally, in humans by alternative interventions. If the effects of RIPC are not supplementary or synergistic with existing cardioprotective techniques, there is still potential for it to improve clinical practice. Pharmaceutical forms of cardioprotection are associated with a range of side effects and are often contraindicated in certain populations. For instance, the use of volatile anaesthetics is not recommended in patients with cardiovascular instability; therefore these patients could receive a more significant benefit from the alternative use of RIPC (Jakobsen et al., 2007).

Lastly, age and comorbidities may significantly impair or alter the pathways through which RIPC operates, limiting its effectiveness in certain patient groups (Abete et al., 1997; Hu et al., 2002; Lavi and Lavi, 2011). For instance, type 2 diabetics appear to have a natural resistance to I/R injury, which is in part due to suppression of the mPTP (Costa et al., 2013). Since RIPC partially operates through the same mechanism, there does not appear to be any additional benefit from applying the intervention in these patients (Jensen et al., 2013). Similarly, 
hypercholesterolemia, diabetes, and some treatments for comorbidities, such as the sulfonylureas used in type 2 diabetics, also inhibit another key pathway involved in RIPC that affects the mitochondrial $\mathrm{K}_{\mathrm{ATP}}$ channels (Cleveland et al., 1997; Genda et al., 2002; Smith and Wahler, 1996). Although patients who are taking sulfonylureas and other medications known to interfere with $\mathrm{K}_{\mathrm{ATP}}$ channels are commonly excluded from clinical trials, untreated type 2 diabetics are often included and many other comorbidities and medications may interfere with RIPC that have not yet been identified. Again, this source of variability is not contended with in animal models.

Overall, there are a number of critical differences between animal and patient RIPC studies, many of which have been shown to impact the ability to induce preconditioning and could explain the discrepancy in results between the preclinical and clinical work. Although animal models are a valuable resource for exploratory research, the results of such studies cannot consistently be extended to human applications and advancement of RIPC research will require more work be completed in human studies.

\subsection{Future directions}

The research presented in this thesis has highlighted a number of immune parameters that are significantly altered by RIPC which merit further investigation. Firstly, the apparent suppression of monocyte and neutrophil activation during the early window of protection, and increased activation of $\mathrm{T}$ cells during the late phase, could potentially modify the extent of I/R injury. Further work in healthy human volunteers would be beneficial to determine whether these changes in activation marker expression correspond to altered function of these cell subsets.

The neutrophil functions most relevant to their ability to inflict tissue damage during I/R are migration into tissues, and production of ROS, making them ideal parameters to assess. Kusek et al. (2014) recently described a co-culture system capable of measuring neutrophil trans-epithelial migration by establishing polarised epithelial monolayers on permeable transwell filters, and quantifying 
the number of neutrophils that migrate across the cell layer into the well below by assessing MPO levels. This method was developed to assess the migration response of neutrophils to various pathogenic bacteria; however, other stimuli such as cytokines or ROS could be substituted to mimic the activation of epithelial cells sustained during I/R. The extent of the oxidative burst in neutrophils can also be readily assessed using a tetrazolium salt, WST-1, which is reduced by superoxide to form a stable product that can be measured in a microplate reader (Tan and Berridge, 2000). Neutrophils can be quickly isolated from human blood with minimal disturbance using a density gradient medium known as Polymorphprep for use in these assays (Degel and Shokrani, 2010). Comparison of the level of neutrophil migration and superoxide production detected before and after RIPC would provide a solid indication of the ability of these cells to infiltrate the lungs and kidneys and cause damage in the setting of $\mathrm{I} / \mathrm{R}$.

While our study in healthy volunteers assessed the responses of cells using time points corresponding to the delayed window of protection (24 hours - 6 days post-RIPC), the cells were all isolated from the body during the early phase of RIPC. Whether continued signalling in vivo further alters the state of these cells, or the changes detected are established within this immediate response to RIPC is unclear. Having now identified that RIPC has a significant impact on immune cell function that comes into effect $\geq 24$ hours following treatment, subsequent studies could more appropriately characterise the effect on the late immune response by collecting samples in the days following the application of RIPC in healthy volunteers. Key parameters of interest would include serum levels of MIP-1 $\alpha$ and $\beta$, IL-22 production, and T cell activation.

More in-depth characterisation of the T cells during the late response to RIPC (> $24 \mathrm{hrs}$ ) would be worthwhile, given the increased expression of the early activation marker, $\mathrm{CD} 69$, detected in the $\mathrm{CD}^{+}$population in our study. To start, using flow cytometry with additional cell markers to identify if a particular subset of $\mathrm{T}$ cells was more affected by the RIPC than others, and correlating changes in CD69 with other activation markers, such as CD45R0, would be 
useful. Given the particular importance of CD4 T cells in the pathology of I/R injury, changes in the functional properties of this subset are particularly relevant and performing ICS to assess IFN- $\gamma$ production in samples collected within 4 hrs of RIPC, and the following day, would provide further valuable knowledge as to the effect on these cells (Linfert et al., 2009).

One of the key findings from our study was that RIPC increased basal expression of IL-22 in PBMC cultured for 6 days. Again, it would be useful to determine if this effect was maintained or potentially amplified when cells are not collected until during the delayed window of RIPC, and to identify which subset of cells were responsible for the production of IL-22 using intracellular cytokine staining. The most pressing questions regarding the increased IL-22 levels are whether this effect also occurs in cardiac surgery patients, and whether it corresponds to a benefit in terms of clinical outcome and recovery. In this respect, measurement of serum IL-22 and IL-17 levels in control and RIPC treated cardiac surgery patients in the week following the procedure would be extremely interesting. Examining the co-expression of IL-17 would be informative for determining whether increased levels of IL-22 are likely to be associated with anti or proinflammatory effects during recovery.

Lastly, identification and development of methods for measuring biomarkers of the biological effects of cardioprotective strategies was identified as a research priority at the National Heart Blood and Lung Institute Workshop on cardioprotection in 2010 (Schwartz Longacre et al., 2011). Our study has highlighted a number of potential candidates for such a marker, including MIP$1 \alpha$ and $\beta$, which warrant further investigation to see if they are correlated with a beneficial effect from RIPC. It is interesting to note that Hepponstall et al. (2012) observed incremental increases in plasma protein expression levels with each cycle of RIPC. Further, two clinical trials involving concurrent or combined upper and lower limb RIPC have both reported positive results, suggesting the standard stimulus may not reach the required threshold for protection in humans and could be improved upon (Candilio et al., 2014; Q. Wu et al., 2011). It is possible that more significant benefits and changes in the immune response could occur 
with a stronger stimulus, although it is equally possible that too many cycles or too long a period of ischaemia could have the opposite effect and negate the protective response. Identification of an easily measurable, robust and consistent marker of effective preconditioning is necessary so that the optimal application method of RIPC can be identified, and changes in the levels of MIP- $1 \alpha$ and $\beta$ may be useful in this respect.

\subsection{Clinical significance and final remarks}

Despite the challenges encountered translating RIPC into human applications, this intervention still holds promise for dramatically improving clinical practice in a number of areas. The results of recent trials have suggested that the protection afforded by RIPC is unlikely to benefit all cardiac surgery patients, but rather a specific subset of individuals with the necessary characteristics to respond to the stimulus. However, the final word on whether RIPC could be utilised as an intervention for the general cardiac surgery patient population will likely be provided by the results of two large multi-centre double-blind randomised controlled phase III trials, known as the ERICCA and RIPHeart studies, which are due in 2015. These studies not only have substantially increased statistical power with plans to recruit 3680 cardiac surgery patients between them, but also will be collecting information on long-term clinical outcomes, which represent the true real potential benefit to be gained by RIPC (Hausenloy et al., 2012; Meybohm et al., 2012). Plans for a further trial investigating the impact of RIPC on neuroprotection, called the RIPCAGE study, have also recently been described (Gasparovic et al., 2014).

Overall, the work in this thesis has extended and refined what is known about the effect of RIPC on the immune response in humans and identified a number of critical parameters to guide future mechanistic research. Whether the change in inflammation induced by RIPC will translate to a significant cardioprotective effect during I/R is unclear, but these findings do support potentially expanding the use of RIPC to investigate its efficacy for treating general inflammatory conditions. Further carefully designed research to expand our understanding of 
the pathways involved in RIPC will facilitate the translation of this promising technique into standard clinical practice. 


\section{References}

Abela, C.B., Homer-Vanniasinkham, S., 2003. Clinical implications of ischaemiareperfusion injury. Pathophysiology 9, 229-240.

Abete, P., Ferrara, N., Cacciatore, F., Madrid, A., Bianco, S., Calabrese, C., Napoli, C., Scognamiglio, P., Bollella, O., Cioppa, A., Longobardi, G., Rengo, F., 1997. Angina-induced protection against myocardial infarction in adult and elderly patients: a loss of preconditioning mechanism in the aging heart? J. Am. Coll. Cardiol. 30, 947-954.

Abu-Amara, M., Yang, S.Y., Quaglia, A., Rowley, P., de Mel, A., Tapuria, N., Seifalian, A., Davidson, B., Fuller, B., 2011. Nitric oxide is an essential mediator of the protective effects of remote ischaemic preconditioning in a mouse model of liver ischaemia/reperfusion injury. Clin. Sci. Lond. Engl. 1979 121, 257-266. doi:10.1042/CS20100598

Abu-Omar, Y., Cifelli, A., Matthews, P.M., Taggart, D.P., 2004. The role of microembolisation in cerebral injury as defined by functional magnetic resonance imaging. Eur. J. Cardiothorac. Surg. 26, 586-591. doi:10.1016/j.ejcts.2004.05.022

Adabag, A.S., Rector, T., Mithani, S., Harmala, J., Ward, H.B., Kelly, R.F., Nguyen, J.T., McFalls, E.O., Bloomfield, H.E., 2007. Prognostic significance of elevated cardiac troponin I after heart surgery. Ann. Thorac. Surg. 83, 1744-1750. doi:10.1016/j.athoracsur.2006.12.049

Addison, P.D., Neligan, P.C., Ashrafpour, H., Khan, A., Zhong, A., Moses, M., Forrest, C.R., Pang, C.Y., 2003. Noninvasive remote ischemic preconditioning for global protection of skeletal muscle against infarction. Am J Physiol Heart Circ Physiol 285, H1435-43. doi:10.1152/ajpheart.00106.2003

Akbari, O., Stock, P., Meyer, E., Kronenberg, M., Sidobre, S., Nakayama, T., Taniguchi, M., Grusby, M.J., DeKruyff, R.H., Umetsu, D.T., 2003. Essential role of NKT cells producing IL-4 and IL-13 in the development of allergen-induced $\begin{array}{llll}\text { airway hyperreactivity. } & \text { Nat. } & \text { Med. }\end{array}$ doi:http://dx.doi.org.helicon.vuw.ac.nz/10.1038/nm851

Akdiş, A.C., Towbin, H., Libsig, P., Motz, J., Alkan, Ş.Ş., 1995. Cytokine immunotrapping: an assay to study the kinetics of production and consumption or degradation of human interferon- $\gamma$. J. Immunol. Methods 182, 251-261. doi:10.1016/0022-1759(95)00055-F

Albrecht, M., Zitta, K., Bein, B., Wennemuth, G., Broch, O., Renner, J., Schuett, T., Lauer, F., Maahs, D., Hummitzsch, L., Cremer, J., Zacharowski, K., Meybohm, P., 2013. Remote ischemic preconditioning regulates HIF-1 $\alpha$ levels, apoptosis and inflammation in heart tissue of cardiosurgical patients: a pilot experimental study. Basic Res. Cardiol. 108, 314. doi:10.1007/s00395-012-0314-0

Aldea, G.S., Doursounian, M., O’Gara, P., Treanor, P., Shapira, O.M., Lazar, H.L., Shemin, R.J., 1996. Heparin-bonded circuits with a reduced anticoagulation protocol in primary CABG: A prospective, randomized study. Ann. Thorac. Surg. 62, 410-418. doi:10.1016/0003-4975(96)00324-4

Aldea, G.S., Lilly, K., Gaudiani, J.M., O’Gara, P., Stein, D., Bao, Y., Treanor, P., Osman, A., Shapira, O.M., Lazar, H.L., Shemin, R.J., 1997. Heparin-bonded 
Circuits Improve Clinical Outcomes in Emergency Coronary Artery Bypass Grafting. J. Card. Surg. 12, 389-397. doi:10.1111/j.1540-8191.1997.tb00157.x

Ali, N., Rizwi, F., Iqbal, A., Rashid, A., 2010. Induced remote ischemic preconditioning on ischemia-reperfusion injury in patients undergoing coronary artery bypass. J Coll Physicians Surg Pak 20, 427-31.

Ali, Z.A., Callaghan, C.J., Lim, E., Ali, A.A., Nouraei, S.A., Akthar, A.M., Boyle, J.R., Varty, K., Kharbanda, R.K., Dutka, D.P., Gaunt, M.E., 2007. Remote ischemic preconditioning reduces myocardial and renal injury after elective abdominal aortic aneurysm repair: a randomized controlled trial. Circulation 116, I98-105. doi:10.1161/circulationaha.106.679167

Alreja, G., Bugano, D., Lotfi, A., 2012. Effect of remote ischemic preconditioning on myocardial and renal injury: meta-analysis of randomized controlled trials. J. Invasive Cardiol. 24, 42-48.

Altay, O., Hasegawa, Y., Sherchan, P., Suzuki, H., Khatibi, N.H., Tang, J., Zhang, J.H., 2012. Isoflurane delays the development of early brain injury after subarachnoid hemorrhage through sphingosine-related pathway activation in mice. Crit. Care Med. 40, 1908-1913. doi:10.1097/CCM.0b013e3182474bc1

Amulic, B., Cazalet, C., Hayes, G.L., Metzler, K.D., Zychlinsky, A., 2012. Neutrophil Function: From Mechanisms to Disease. Annu. Rev. Immunol. 30, 459-489. doi:10.1146/annurev-immunol-020711-074942

Andersen, L.W., Landow, L., Baek, L., Jansen, E., Baker, S., 1993. Association between gastric intramucosal $\mathrm{pH}$ and splanchnic endotoxin, antibody to endotoxin, and tumor necrosis factor-alpha concentrations in patients undergoing cardiopulmonary bypass. Crit. Care Med. 21, 210-217.

Anderson, M.J., 2001. A new method for non-parametric multivariate analysis of variance. Austral Ecol. 26, 32-46. doi:10.1111/j.1442-9993.2001.01070.pp.x

Aragonés, J., Schneider, M., Van Geyte, K., Fraisl, P., Dresselaers, T., Mazzone, M., Dirkx, R., Zacchigna, S., Lemieux, H., Jeoung, N.H., Lambrechts, D., Bishop, T., Lafuste, P., Diez-Juan, A., Harten, S.K., Van Noten, P., De Bock, K., Willam, C., Tjwa, M., Grosfeld, A., Navet, R., Moons, L., Vandendriessche, T., Deroose, C., Wijeyekoon, B., Nuyts, J., Jordan, B., Silasi-Mansat, R., Lupu, F., Dewerchin, M., Pugh, C., Salmon, P., Mortelmans, L., Gallez, B., Gorus, F., Buyse, J., Sluse, F., Harris, R.A., Gnaiger, E., Hespel, P., Van Hecke, P., Schuit, F., Van Veldhoven, P., Ratcliffe, P., Baes, M., Maxwell, P., Carmeliet, P., 2008. Deficiency or inhibition of oxygen sensor Phd1 induces hypoxia tolerance by reprogramming basal metabolism. Nat. Genet. 40, 170-180. doi:10.1038/ng.2007.62

Argaud, L., Gateau-Roesch, O., Raisky, O., Loufouat, J., Robert, D., Ovize, M., 2005. Postconditioning inhibits mitochondrial permeability transition. Circulation 111, 194-197. doi:10.1161/01.CIR.0000151290.04952.3B

Arslan, F., Keogh, B., McGuirk, P., Parker, A.E., 2010. TLR2 and TLR4 in Ischemia Reperfusion Injury. Mediators Inflamm. 2010, e704202. doi:10.1155/2010/704202

Arslan, F., Smeets, M.B., O’Neill, L.A.J., Keogh, B., McGuirk, P., Timmers, L., Tersteeg, C., Hoefer, I.E., Doevendans, P.A., Pasterkamp, G., de Kleijn, D.P.V., 2010. Myocardial ischemia/reperfusion injury is mediated by leukocytic toll-like receptor- 2 and reduced by systemic administration of a novel anti-toll-like 
receptor-2 antibody. Circulation $\quad 121, \quad 80-90$. doi:10.1161/CIRCULATIONAHA.109.880187

Ascon, D.B., Lopez-Briones, S., Liu, M., Ascon, M., Savransky, V., Colvin, R.B., Soloski, M.J., Rabb, H., 2006. Phenotypic and functional characterization of kidney-infiltrating lymphocytes in renal ischemia reperfusion injury. J. Immunol. Baltim. Md 1950 177, 3380-3387.

Asimakopoulos, G., Smith, P.L., Ratnatunga, C.P., Taylor, K.M., 1999. Lung injury and acute respiratory distress syndrome after cardiopulmonary bypass. Ann Thorac Surg 68, 1107-15.

Atalah, J., Otto, S.A., Anderson, M.J., Costello, M.J., Lenz, M., Wahl, M., 2007. Temporal variance of disturbance did not affect diversity and structure of a marine fouling community in north-eastern New Zealand. Mar. Biol. 153, 199-211. doi:10.1007/s00227-007-0798-6

Auckland District Health Board, 2008. ADHB Celebrates 50th Anniversary of First Open Heart Surgery in NZ.

Auffray, C., Sieweke, M.H., Geissmann, F., 2009. Blood Monocytes: Development, Heterogeneity, and Relationship with Dendritic Cells. Annu. Rev. Immunol. 27, 669-692. doi:10.1146/annurev.immunol.021908.132557

Baines, C.P., Song, C.-X., Zheng, Y.-T., Wang, G.-W., Zhang, J., Wang, O.-L., Guo, Y., Bolli, R., Cardwell, E.M., Ping, P., 2003. Protein kinase Cepsilon interacts with and inhibits the permeability transition pore in cardiac mitochondria. Circ. Res. 92, 873-880. doi:10.1161/01.RES.0000069215.36389.8D

Barker, W.H., Mullooly, J.P., Getchell, W., 2006. Changing Incidence and Survival for Heart Failure in a Well-Defined Older Population, 1970-1974 and 1990-1994. Circulation 113, 799-805. doi:10.1161/CIRCULATIONAHA.104.492033

Bar-Or, D., Curtis, G., Rao, N., Bampos, N., Lau, E., 2001a. Characterization of the $\mathrm{Co}(2+)$ and $\mathrm{Ni}(2+)$ binding amino-acid residues of the N-terminus of human albumin. An insight into the mechanism of a new assay for myocardial ischemia. Eur. J. Biochem. FEBS 268, 42-47.

Bar-Or, D., Lau, E., Winkler, J.V., 2000. A novel assay for cobalt-albumin binding and its potential as a marker for myocardial ischemia-a preliminary report. J. Emerg. Med. 19, 311-315.

Bar-Or, D., Rael, L.T., Bar-Or, R., Slone, D.S., Mains, C.W., Rao, N.K.R., Curtis, C.G., 2008. The cobalt-albumin binding assay: insights into its mode of action. Clin. Chim. Acta Int. J. Clin. Chem. 387, 120-127. doi:10.1016/j.cca.2007.09.018

Bar-Or, D., Winkler, J.V., Vanbenthuysen, K., Harris, L., Lau, E., Hetzel, F.W., 2001b. Reduced albumin-cobalt binding with transient myocardial ischemia after elective percutaneous transluminal coronary angioplasty: a preliminary comparison to creatine kinase-MB, myoglobin, and troponin I. Am. Heart J. 141, 985-991. doi:10.1067/mhj.2001.114800

Barry, J.A.W., Barth, J.H., Howell, S.J., 2008. Cardiac troponins: their use and relevance in anaesthesia and critical care medicine. Contin. Educ. Anaesth. Crit. Care Pain 8, 62-66. doi:10.1093/bjaceaccp/mkn001 
Basu, S., Binder, R.J., Suto, R., Anderson, K.M., Srivastava, P.K., 2000. Necrotic but not apoptotic cell death releases heat shock proteins, which deliver a partial maturation signal to dendritic cells and activate the NF- $\mathrm{\kappa B}$ pathway. Int. Immunol. 12, 1539-1546. doi:10.1093/intimm/12.11.1539

Battipaglia, I., Scalone, G., Milo, M., Di Franco, A., Lanza, G.A., Crea, F., 2011. Upper arm intermittent ischaemia reduces exercise-related increase of platelet reactivity in patients with obstructive coronary artery disease. Heart Br. Card. Soc. 97, 1298-1303. doi:10.1136/hrt.2011.226415

Baufreton, C., Kirsch, M., Loisance, D.Y., 1998. Measures to control blood activation during assisted circulation. Ann Thorac Surg 66, 1837-44.

Baumgarth, N., Roederer, M., 2000. A practical approach to multicolor flow cytometry for immunophenotyping. J. Immunol. Methods, Flow Cytometry 243, 77-97. doi:10.1016/S0022-1759(00)00229-5

Baxter, G.F., Goma, F.M., Yellon, D.M., 1997. Characterisation of the infarctlimiting effect of delayed preconditioning: timecourse and dose-dependency studies in rabbit myocardium. Basic Res. Cardiol. 92, 159-167.

Bazil, V., Strominger, J.L., 1991. Shedding as a mechanism of down-modulation of CD14 on stimulated human monocytes. J. Immunol. Baltim. Md 1950 147, 15671574 .

Beavis, A.D., Lu, Y., Garlid, K.D., 1993. On the regulation of K+ uniport in intact mitochondria by adenine nucleotides and nucleotide analogs. J. Biol. Chem. 268, 997-1004.

Belhomme, D., Peynet, J., Louzy, M., Launay, J.M., Kitakaze, M., Menasche, P., 1999. Evidence for preconditioning by isoflurane in coronary artery bypass graft surgery. Circulation 100, II340-4.

Bellomo, R., Ronco, C., Kellum, J.A., Mehta, R.L., Palevsky, P., Acute Dialysis Quality Initiative workgroup, 2004. Acute renal failure - definition, outcome measures, animal models, fluid therapy and information technology needs: the Second International Consensus Conference of the Acute Dialysis Quality Initiative (ADQI) Group. Crit. Care Lond. Engl. 8, R204-212. doi:10.1186/cc2872

Biancari, F., Kangasniemi, O.P., Aliasim Mahar, M., Rasinaho, E., Satomaa, A., Tiozzo, V., Niemela, M., Lepojarvi, M., 2009. Changing risk of patients undergoing coronary artery bypass surgery. Interact Cardiovasc Thorac Surg 8, 40-4. doi:10.1510/icvts.2007.173922

Bignami, E., Landoni, G., Gerli, C., Testa, V., Mizzi, A., Fano, G., Nuzzi, M., Franco, A., Zangrillo, A., 2012. Sevoflurane vs. propofol in patients with coronary disease undergoing mitral surgery: a randomised study. Acta Anaesthesiol. Scand. 56, 482-490. doi:10.1111/j.1399-6576.2011.02570.x

Birnbaum, Y., Hale, S.L., Kloner, R.A., 1997. Ischemic preconditioning at a distance: reduction of myocardial infarct size by partial reduction of blood supply combined with rapid stimulation of the gastrocnemius muscle in the rabbit. Circulation 96, 1641-6.

Boer, R.J.D., Homann, D., Perelson, A.S., 2003. Different Dynamics of CD4+ and CD8+ T Cell Responses During and After Acute Lymphocytic Choriomeningitis Virus Infection. J. Immunol. 171, 3928-3935. doi:10.4049/jimmunol.171.8.3928 
Bonder, C.S., Norman, M.U., MacRae, T., Mangan, P.R., Weaver, C.T., Bullard, D.C., McCafferty, D.-M., Kubes, P., 2005. P-Selectin Can Support Both Th1 and Th2 Lymphocyte Rolling in the Intestinal Microvasculature. Am. J. Pathol. 167, 1647-1660.

Borgermann, J., Friedrich, I., Flohe, S., Spillner, J., Majetschak, M., Kuss, O., Sablotzki, A., Feldt, T., Reidemeister, J.C., Schade, F.U., 2002. Tumor necrosis factor-\{alpha\} production in whole blood after cardiopulmonary bypass: Downregulation caused by circulating cytokine-inhibitory activities. J Thorac Cardiovasc Surg 124, 608-617.

Born, J., Lange, T., Hansen, K., Mölle, M., Fehm, H.L., 1997. Effects of sleep and circadian rhythm on human circulating immune cells. J. Immunol. 158, 44544464.

Boyd, J.H., Mathur, S., Wang, Y., Bateman, R.M., Walley, K.R., 2006. Toll-like receptor stimulation in cardiomyoctes decreases contractility and initiates an NFkappaB dependent inflammatory response. Cardiovasc. Res. 72, 384-393. doi:10.1016/j.cardiores.2006.09.011

Boyle, E.M., Pohlman, T.H., Cornejo, C.J., Verrier, E.D., 1996a. Endothelial Cell Injury in Cardiovascular Surgery: Ischemia-Reperfusion. Ann Thorac Surg 62, $1868-1875$.

Boyle, E.M., Verrier, E.D., Spiess, B.D., 1996b. Endothelial Cell Injury in Cardiovascular Surgery: The Procoagulant Response. Ann Thorac Surg 62, 15491557.

Brevoord, D., Kranke, P., Kuijpers, M., Weber, N., Hollmann, M., Preckel, B., 2012. Remote ischemic conditioning to protect against ischemia-reperfusion injury: a systematic review and meta-analysis. PloS One 7, e42179. doi:10.1371/journal.pone.0042179

Bridgewater, B., Keogh, B., Kinsman, R., Walton, P., 2008. Sixth National Adult Cardiac Surgical Database Report 2008. The Society for Cardiothoracic Surgery in Great Britain \& Ireland.

Broere, F., Apasov, S.G., Sitkovsky, M.V., Eden, W. van, 2011. A2 T cell subsets and T cell-mediated immunity, in: Nijkamp, F.P., Parnham, M.J. (Eds.), Principles of Immunopharmacology. Birkhäuser Basel, pp. 15-27.

Brzozowski, T., Konturek, P.C., Konturek, S.J., Pajdo, R., Kwiecien, S., Pawlik, M., Drozdowicz, D., Sliwowski, Z., Pawlik, W.W., 2004. Ischemic preconditioning of remote organs attenuates gastric ischemia-reperfusion injury through involvement of prostaglandins and sensory nerves. Eur. J. Pharmacol. 499, 201-213. doi:10.1016/j.ejphar.2004.07.072

Brzozowski, T., Konturek, P.C., Pajdo, R., Kwiecień, S., Sliwowski, Z., Drozdowicz, D., Ptak-Belowska, A., Pawlik, M., Konturek, S.J., Pawlik, W.W., Hahn, G.G., 2004. Importance of brain-gut axis in the gastroprotection induced by gastric and remote preconditioning. J. Physiol. Pharmacol. Off. J. Pol. Physiol. Soc. 55, 165177.

Burne, M.J., Daniels, F., El Ghandour, A., Mauiyyedi, S., Colvin, R.B., O’Donnell, M.P., Rabb, H., 2001. Identification of the CD4(+) T cell as a major pathogenic 
factor in ischemic acute renal failure. J. Clin. Invest. 108, 1283-1290. doi:10.1172/JCI12080

Burne-Taney, M.J., Liu, M., Baldwin, W.M., Racusen, L., Rabb, H., 2006. Decreased capacity of immune cells to cause tissue injury mediates kidney ischemic preconditioning. J. Immunol. Baltim. Md 1950 176, 7015-7020.

Burzyn, D., Kuswanto, W., Kolodin, D., Shadrach, J.L., Cerletti, M., Jang, Y., Sefik, E., Tan, T.G., Wagers, A.J., Benoist, C., Mathis, D., 2013. A Special Population of Regulatory $\mathrm{T}$ Cells Potentiates Muscle Repair. Cell 155, 1282-1295. doi:10.1016/j.cell.2013.10.054

Cai, Z., Luo, W., Zhan, H., Semenza, G.L., 2013. Hypoxia-inducible factor 1 is required for remote ischemic preconditioning of the heart. Proc. Natl. Acad. Sci. U. S. A. 110, 17462-17467. doi:10.1073/pnas.1317158110

Cai, Z.P., Parajuli, N., Zheng, X., Becker, L., 2012. Remote ischemic preconditioning confers late protection against myocardial ischemia-reperfusion injury in mice by upregulating interleukin-10. Basic Res Cardiol 107, 277. doi:10.1007/s00395-0120277-1

Cai, Z., Zhong, H., Bosch-Marce, M., Fox-Talbot, K., Wang, L., Wei, C., Trush, M.A., Semenza, G.L., 2008. Complete loss of ischaemic preconditioning-induced cardioprotection in mice with partial deficiency of HIF-1 alpha. Cardiovasc. Res. 77, 463-470. doi:10.1093/cvr/cvm035

Camara-Lemarroy, C.R., 2014. Remote ischemic preconditioning as treatment for non-ischemic gastrointestinal disorders: beyond ischemia-reperfusion injury. World J. Gastroenterol. WJG 20, 3572-3581. doi:10.3748/wjg.v20.i13.3572

Candilio, L., Hausenloy, D.J., Yellon, D.M., 2011. Remote ischemic conditioning: a clinical trial's update. J. Cardiovasc. Pharmacol. Ther. 16, 304-312. doi:10.1177/1074248411411711

Candilio, L., Malik, A., Ariti, C., Barnard, M., Di Salvo, C., Lawrence, D., Hayward, M., Yap, J., Roberts, N., Sheikh, A., Kolvekar, S., Hausenloy, D.J., Yellon, D.M., 2014. Effect of remote ischaemic preconditioning on clinical outcomes in patients undergoing cardiac bypass surgery: a randomised controlled clinical trial. Heart $\mathrm{Br}$. Card. Soc. doi:10.1136/heartjnl-2014-306178

Cao, Z., Yuan, Y., Jeyabalan, G., Du, Q., Tsung, A., Geller, D.A., Billiar, T.R., 2009. Preactivation of NKT cells with alpha-GalCer protects against hepatic ischemiareperfusion injury in mouse by a mechanism involving IL-13 and adenosine A2A receptor. Am. J. Physiol. Gastrointest. Liver Physiol. 297, G249-258. doi:10.1152/ajpgi.00041.2009

Caraher, E.M., Parenteau, M., Gruber, H., Scott, F.W., 2000. Flow cytometric analysis of intracellular IFN-gamma, IL-4 and IL-10 in CD3(+)4(+) T-cells from rat spleen. J. Immunol. Methods 244, 29-40.

Cassatella, M.A., 1995. The production of cytokines by polymorphonuclear neutrophils. Immunol. Today 16, 21-26. doi:10.1016/0167-5699(95)80066-2

Cella, M., Fuchs, A., Vermi, W., Facchetti, F., Otero, K., Lennerz, J.K.M., Doherty, J.M., Mills, J.C., Colonna, M., 2009. A human natural killer cell subset provides an innate source of IL-22 for mucosal immunity. Nature 457, 722-725. doi:10.1038/nature07537 
Chambers, D.J., Fallouh, H.B., 2010. Cardioplegia and cardiac surgery: Pharmacological arrest and cardioprotection during global ischemia and reperfusion. Pharmacol. Ther. 127, 41-52. doi:10.1016/j.pharmthera.2010.04.001

Cham, B.P., Gerrard, J.M., Bainton, D.F., 1994. Granulophysin is located in the membrane of azurophilic granules in human neutrophils and mobilizes to the plasma membrane following cell stimulation. Am. J. Pathol. 144, 1369-1380.

Chang, H., Hanawa, H., Liu, H., Yoshida, T., Hayashi, M., Watanabe, R., Abe, S., Toba, K., Yoshida, K., Elnaggar, R., Minagawa, S., Okura, Y., Kato, K., Kodama, M., Maruyama, H., Miyazaki, J., Aizawa, Y., 2006. Hydrodynamic-based delivery of an interleukin-22-Ig fusion gene ameliorates experimental autoimmune myocarditis in rats. J. Immunol. Baltim. Md 1950 177, 3635-3643.

Chao, W., 2009. Toll-like receptor signaling: a critical modulator of cell survival and ischemic injury in the heart. Am. J. Physiol. - Heart Circ. Physiol. 296, H1-H12. doi:10.1152/ajpheart.00995.2008

Chen, G.Y., Nuñez, G., 2010. Sterile inflammation: sensing and reacting to damage. Nat. Rev. Immunol. 10, 826-837. doi:10.1038/nri2873

Chen, Y., Corriden, R., Inoue, Y., Yip, L., Hashiguchi, N., Zinkernagel, A., Nizet, V., Insel, P.A., Junger, W.G., 2006. ATP release guides neutrophil chemotaxis via P2Y2 and A3 receptors. Science 314, 1792-1795. doi:10.1126/science.1132559

Chen, Y.-S., Chien, C.-T., Ma, M.-C., Tseng, Y.-Z., Lin, F.-Y., Wang, S.-S., Chen, C.-F., 2005. Protection "outside the box" (skeletal remote preconditioning) in rat model is triggered by free radical pathway. J. Surg. Res. 126, 92-101. doi:10.1016/j.jss.2005.01.007

Cheung, M.M., Kharbanda, R.K., Konstantinov, I.E., Shimizu, M., Frndova, H., Li, J., Holtby, H.M., Cox, P.N., Smallhorn, J.F., Van Arsdell, G.S., Redington, A.N., 2006. Randomized controlled trial of the effects of remote ischemic preconditioning on children undergoing cardiac surgery: first clinical application in humans. J Am Coll Cardiol 47, 2277-82. doi:10.1016/j.jacc.2006.01.066

Cheung, P.Y., Sawicki, G., Wozniak, M., Wang, W., Radomski, M.W., Schulz, R., 2000. Matrix metalloproteinase-2 contributes to ischemia-reperfusion injury in the heart. Circulation 101, 1833-1839.

Choi, Y.S., Shim, J.K., Chan Kim, J., Kang, K.S., Seo, Y.H., Ahn, K.R., Kwak, Y.L., 2011. Effect of remote ischemic preconditioning on renal dysfunction after complex valvular heart surgery: A randomized controlled trial. J Thorac Cardiovasc Surg. doi:10.1016/j.jtcvs.2010.11.018

Chong, A.J., Shimamoto, A., Hampton, C.R., Takayama, H., Spring, D.J., Rothnie, C.L., Yada, M., Pohlman, T.H., Verrier, E.D., 2004. Toll-like receptor 4 mediates ischemia/reperfusion injury of the heart. J. Thorac. Cardiovasc. Surg. 128, 170179. doi:10.1016/j.jtcvs.2003.11.036

Chopra, V., Wesorick, D.H., Sussman, J.B., Greene, T., Rogers, M., Froehlich, J.B., Eagle, K.A., Saint, S., 2012. Effect of perioperative statins on death, myocardial infarction, atrial fibrillation, and length of stay: a systematic review and metaanalysis. Arch. Surg. Chic. Ill 1960 147, 181-189. doi:10.1001/archsurg.2011.897 
Cho, W.Y., Choi, H.M., Lee, S.Y., Kim, M.G., Kim, H.-K., Jo, S.-K., 2010. The role of Tregs and CD11c+ macrophages/dendritic cells in ischemic preconditioning of the kidney. Kidney Int. 78, 981-992. doi:10.1038/ki.2010.266

Christenson, J.T., 1999. Preoperative lipid-control with simvastatin reduces the risk of postoperative thrombocytosis and thrombotic complications following CABG. Eur. J. Cardio-Thorac. Surg. Off. J. Eur. Assoc. Cardio-Thorac. Surg. 15, 394-399; discussion 399-400.

Chtanova, T., Han, S.-J., Schaeffer, M., van Dooren, G.G., Herzmark, P., Striepen, B., Robey, E.A., 2009. Dynamics of T Cell, Antigen-Presenting Cell, and Pathogen Interactions during Recall Responses in the Lymph Node. Immunity 31, 342-355. doi:10.1016/j.immuni.2009.06.023

Ciofani, M., Zúñiga-Pflücker, J.C., 2010. Determining $\gamma \delta$ versus $\alpha ß \quad \mathrm{~T}$ cell development. Nat. Rev. Immunol. 10, 657-663. doi:10.1038/nri2820

Clanchy, F.I.L., Holloway, A.C., Lari, R., Cameron, P.U., Hamilton, J.A., 2006. Detection and properties of the human proliferative monocyte subpopulation. J. Leukoc. Biol. 79, 757-766. doi:10.1189/jlb.0905522

Clarke, K.R., 1993. Non-parametric multivariate analyses of changes in community structure. Aust. J. Ecol. 18, 117-143. doi:10.1111/j.1442-9993.1993.tb00438.x

Cleveland, J.C., Meldrum, D.R., Cain, B.S., Banerjee, A., Harken, A.H., 1997. Oral sulfonylurea hypoglycemic agents prevent ischemic preconditioning in human myocardium. Two paradoxes revisited. Circulation 96, 29-32.

Collard, C.D., Gelman, S., 2001. Pathophysiology, clinical manifestations, and prevention of ischemia-reperfusion injury. Anesthesiology 94, 1133-8.

Coll, M., Schmidt, A., Romanuk, T., Lotze, H.K., 2011. Food-Web Structure of Seagrass Communities across Different Spatial Scales and Human Impacts. PLoS ONE 6, e22591. doi:10.1371/journal.pone.0022591

Costa, J.F., Fontes-Carvalho, R., Leite-Moreira, A.F., 2013. Myocardial remote ischemic preconditioning: from pathophysiology to clinical application. Rev. Port. Cardiol. Orgão Of. Soc. Port. Cardiol. Port. J. Cardiol. Off. J. Port. Soc. Cardiol. 32, 893-904. doi:10.1016/j.repc.2013.02.012

Cronstein, B.N., Daguma, L., Nichols, D., Hutchison, A.J., Williams, M., 1990. The adenosine/neutrophil paradox resolved: human neutrophils possess both A1 and A2 receptors that promote chemotaxis and inhibit $\mathrm{O} 2$ generation, respectively. $\mathrm{J}$. Clin. Invest. 85, 1150-1157. doi:10.1172/JCI114547

Czeiger, D., Dukhno, O., Douvdevani, A., Porat, Y., Shimoni, D., Fulga, V., Ament, J.D., Shaked, G., 2011. Transient Extremity Ischemia Augments CD34+ Progenitor Cell Availability. Stem Cell Rev. 7, 639-45. doi:http://dx.doi.org.helicon.vuw.ac.nz/10.1007/s12015-011-9234-x

Czibik, G., Gravning, J., Martinov, V., Ishaq, B., Knudsen, E., Attramadal, H., Valen, G., 2011. Gene therapy with hypoxia-inducible factor 1 alpha in skeletal muscle is cardioprotective in vivo. Life Sci. 88, 543-550. doi:10.1016/j.lfs.2011.01.006

Dancey, J.T., Deubelbeiss, K.A., Harker, L.A., Finch, C.A., 1976. Neutrophil kinetics in man. J. Clin. Invest. 58, 705-715. doi:10.1172/JCI108517 
Danielsen, C.C., Wiggers, H., Andersen, H.R., 1998. Increased amounts of collagenase and gelatinase in porcine myocardium following ischemia and reperfusion. J. Mol. Cell. Cardiol. 30, 1431-1442.

D’Ascenzo, F., Cavallero, E., Moretti, C., Omedè, P., Sciuto, F., Rahman, I.A., Bonser, R.S., Yunseok, J., Wagner, R., Freiberger, T., Kunst, G., Marber, M.S., Thielmann, M., Ji, B., Amr, Y.M., Modena, M.G., Zoccai, G.B., Sheiban, I., Gaita, F., 2012. Remote ischaemic preconditioning in coronary artery bypass surgery: a meta-analysis. Heart Br. Card. Soc. 98, 1267-1271. doi:10.1136/heartjnl-2011301551

D’Ascenzo, F., Moretti, C., Omedè, P., Cerrato, E., Cavallero, E., Er, F., Presutti, D.G., Colombo, F., Crimi, G., Conrotto, F., Dinicolantonio, J.J., Chen, S., Prasad, A., Biondi Zoccai, G., Gaita, F., 2014. Cardiac remote ischaemic preconditioning reduces periprocedural myocardial infarction for patients undergoing percutaneous coronary interventions: a meta-analysis of randomised clinical trials. EuroIntervention J. Eur. Collab. Work. Group Interv. Cardiol. Eur. Soc. Cardiol. 9, 1463-1471. doi:10.4244/EIJV9I12A244

Davis, J.M., Gute, D.C., Jones, S., Krsmanovic, A., Korthuis, R.J., 1999. Ischemic preconditioning prevents postischemic P-selectin expression in the rat small intestine. Am. J. Physiol. 277, H2476-2481.

Dawn, B., Xuan, Y.T., Guo, Y., Rezazadeh, A., Stein, A.B., Hunt, G., Wu, W.J., Tan, W., Bolli, R., 2004. IL-6 plays an obligatory role in late preconditioning via JAKSTAT signaling and upregulation of iNOS and COX-2. Cardiovasc Res 64, 61-71. doi:10.1016/j.cardiores.2004.05.011

Day, Y.-J., Huang, L., Ye, H., Li, L., Linden, J., Okusa, M.D., 2006. Renal ischemiareperfusion injury and adenosine $2 \mathrm{~A}$ receptor-mediated tissue protection: the role of CD4+ T cells and IFN-gamma. J. Immunol. Baltim. Md 1950 176, 3108-3114.

Degel, J., Shokrani, M., 2010. Validation of the efficacy of a practical method for neutrophils isolation from peripheral blood. Clin. Lab. Sci. J. Am. Soc. Med. Technol. 23, 94-98.

De Greef, K.E., Ysebaert, D.K., Dauwe, S., Persy, V., Vercauteren, S.R., Mey, D., De Broe, M.E., 2001. Anti-B7-1 blocks mononuclear cell adherence in vasa recta after ischemia. Kidney Int. 60, 1415-1427. doi:10.1046/j.1523-1755.2001.00944.x

De Hert, S., Vlasselaers, D., Barbé, R., Ory, J.-P., Dekegel, D., Donnadonni, R., Demeere, J.-L., Mulier, J., Wouters, P., 2009. A comparison of volatile and non volatile agents for cardioprotection during on-pump coronary surgery. Anaesthesia 64, 953-960. doi:10.1111/j.1365-2044.2009.06008.x

De Jager, S.C.A., Kraaijeveld, A.O., Grauss, R.W., de Jager, W., Liem, S.-S., van der Hoeven, B.L., Prakken, B.J., Putter, H., van Berkel, T.J.C., Atsma, D.E., Schalij, M.J., Jukema, J.W., Biessen, E.A.L., 2008. CCL3 (MIP-1 alpha) levels are elevated during acute coronary syndromes and show strong prognostic power for future ischemic events. J. Mol. Cell. Cardiol. 45, 446-452. doi:10.1016/j.yjmcc.2008.06.003

Demetri, G.D., Griffin, J.D., 1991. Granulocyte colony-stimulating factor and its receptor. Blood 78, 2791-2808. 
De Ruijter, W., Musters, R.J.P., Boer, C., Stienen, G.J.M., Simonides, W.S., de Lange, J.J., 2003. The Cardioprotective Effect of Sevoflurane Depends on Protein Kinase C Activation, Opening of Mitochondrial K+ATP Channels, and the Production of Reactive Oxygen Species: Anesth. Analg. 1370-1376. doi:10.1213/01.ANE.0000081786.74722.DA

Diabetes New Zealand, 2010. National diabetes prevalence study needed [WWW Document]. https://www.diabetes.org.nz/news/press_releases/national_diabetes_prevalence_stu dy_needed (accessed 9.9.14).

Diamond, M.S., Staunton, D.E., de Fougerolles, A.R., Stacker, S.A., Garcia-Aguilar, J., Hibbs, M.L., Springer, T.A., 1990. ICAM-1 (CD54): a counter-receptor for Mac-1 (CD11b/CD18). J. Cell Biol. 111, 3129-3139.

Dickson, E.W., Lorbar, M., Porcaro, W.A., Fenton, R.A., Reinhardt, C.P., Gysembergh, A., Przyklenk, K., 1999a. Rabbit heart can be "preconditioned" via transfer of coronary effluent. Am. J. Physiol. 277, H2451-2457.

Dickson, E.W., Reinhardt, C.P., Renzi, F.P., Becker, R.C., Porcaro, W.A., Heard, S.O., 1999b. Ischemic preconditioning may be transferable via whole blood transfusion: preliminary evidence. J. Thromb. Thrombolysis 8, 123-129.

Dickson, E.W., Tubbs, R.J., Porcaro, W.A., Lee, W.J., Blehar, D.J., Carraway, R.E., Darling, C.E., Przyklenk, K., 2002. Myocardial preconditioning factors evoke mesenteric ischemic tolerance via opioid receptors and K(ATP) channels. Am. J. Physiol. Heart Circ. Physiol. 283, H22-28. doi:10.1152/ajpheart.01055.2001

Ding, Y.F., Zhang, M.M., He, R.R., 2001. Role of renal nerve in cardioprotection provided by renal ischemic preconditioning in anesthetized rabbits. Sheng Li Xue Bao 53, 7-12.

DiPietro, L.A., Burdick, M., Low, Q.E., Kunkel, S.L., Strieter, R.M., 1998. MIP1 alpha as a critical macrophage chemoattractant in murine wound repair. J. Clin. Invest. 101, 1693-1698. doi:10.1172/JCI1020

Dobreva, Z.G., Stanilova, S.A., Miteva, L.D., 2008. Differences in the inducible gene expression and protein production of IL-12p40, IL-12p70 and IL-23: involvement of p38 and JNK kinase pathways. Cytokine 43, 76-82. doi:10.1016/j.cyto.2008.04.003

Dobson, G.P., Faggian, G., Onorati, F., Vinten-Johansen, J., 2013. Hyperkalemic cardioplegia for adult and pediatric surgery: end of an era? Front. Physiol. 4. doi:10.3389/fphys.2013.00228

Domanski, M.J., Mahaffey, K., Hasselblad, V., Brener, S.J., Smith, P.K., Hillis, G., Engoren, M., Alexander, J.H., Levy, J.H., Chaitman, B.R., Broderick, S., Mack, M.J., Pieper, K.S., Farkouh, M.E., 2011. Association of myocardial enzyme elevation and survival following coronary artery bypass graft surgery. JAMA 305, 585-591. doi:10.1001/jama.2011.99

Dong, J.-H., Liu, Y.-X., Ji, E.-S., He, R.-R., 2004. [Limb ischemic preconditioning reduces infarct size following myocardial ischemia-reperfusion in rats]. Sheng Li Xue Bao 56, 41-46.

Dong, J.-W., Vallejo, J.G., Tzeng, H.-P., Thomas, J.A., Mann, D.L., 2010. Innate immunity mediates myocardial preconditioning through Toll-like receptor 2 and 
TIRAP-dependent signaling pathways. Am. J. Physiol. Heart Circ. Physiol. 298, H1079-1087. doi:10.1152/ajpheart.00306.2009

Dos Santos, P., Kowaltowski, A.J., Laclau, M.N., Seetharaman, S., Paucek, P., Boudina, S., Thambo, J.-B., Tariosse, L., Garlid, K.D., 2002. Mechanisms by which opening the mitochondrial ATP- sensitive $\mathrm{K}(+)$ channel protects the ischemic heart. Am. J. Physiol. Heart Circ. Physiol. 283, H284-295. doi:10.1152/ajpheart.00034.2002

Duhen, T., Geiger, R., Jarrossay, D., Lanzavecchia, A., Sallusto, F., 2009. Production of interleukin 22 but not interleukin 17 by a subset of human skin-homing memory T cells. Nat. Immunol. 10, 857-863. doi:10.1038/ni.1767

Durazzo, A.E.S., Machado, F.S., Ikeoka, D.T., De Bernoche, C., Monachini, M.C., Puech-Leão, P., Caramelli, B., 2004. Reduction in cardiovascular events after vascular surgery with atorvastatin: a randomized trial. J. Vasc. Surg. 39, 967-975. doi:10.1016/j.jvs.2004.01.004

Dwyer, K.M., Deaglio, S., Gao, W., Friedman, D., Strom, T.B., Robson, S.C., 2007. CD39 and control of cellular immune responses. Purinergic Signal. 3, 171-180. doi:10.1007/s11302-006-9050-y

Eckle, T., Eltzschig, H.K., 2011. Ischemia and reperfusion--from mechanism to translation. Nat. Med. 17, 1391+.

Eckle, T., Köhler, D., Lehmann, R., El Kasmi, K., Eltzschig, H.K., 2008. Hypoxiainducible factor-1 is central to cardioprotection: a new paradigm for ischemic preconditioning. Circulation $\quad 118, \quad 166-175$. doi:10.1161/CIRCULATIONAHA.107.758516

Edgerton, C., Crispín, J.C., Moratz, C.M., Bettelli, E., Oukka, M., Simovic, M., Zacharia, A., Egan, R., Chen, J., Dalle Lucca, J.J., Juang, Y.-T., Tsokos, G.C., 2009. IL-17 producing CD4+ T cells mediate accelerated ischemia/reperfusioninduced injury in autoimmunity-prone mice. Clin. Immunol. Orlando Fla 130, 313-321. doi:10.1016/j.clim.2008.09.019

Edmunds, L.H., 1998. Inflammatory response to cardiopulmonary bypass. Ann Thorac Surg 66, S12-6; discussion S25-8.

Engstad, C.S., Gutteberg, T.J., Osterud, B., 1997. Modulation of blood cell activation by four commonly used anticoagulants. Thromb. Haemost. 77, 690-696.

Er, F., Nia, A.M., Dopp, H., Hellmich, M., Dahlem, K.M., Caglayan, E., Kubacki, T., Benzing, T., Erdmann, E., Burst, V., Gassanov, N., 2012. Ischemic preconditioning for prevention of contrast medium-induced nephropathy: randomized pilot RenPro Trial (Renal Protection Trial). Circulation 126, 296-303. doi:10.1161/CIRCULATIONAHA.112.096370

Erling, N., Nakagawa, N.K., Costa Cruz, J.W.M., Zanoni, F.L., Baptista-Silva, J.C.C., Sannomiya, P., Poli-de-Figueiredo, L.F., 2010. Microcirculatory effects of local and remote ischemic preconditioning in supraceliac aortic clamping. J. Vasc. Surg. 52, 1321-1329. doi:10.1016/j.jvs.2010.05.120

Estensen, R.D., White, J.G., Holmes, B., 1974. Specific degranulation of human polymorphonuclear leukocytes. Nature 248, 347-348. 
Etzioni, D.A., Starnes, V.A., 2011. The Epidemiology and Economics of Cardiothoracic Surgery in the Elderly, in: Katlic, M.R. (Ed.), Cardiothoracic Surgery in the Elderly. Springer New York, pp. 5-24.

Eyerich, S., Eyerich, K., Pennino, D., Carbone, T., Nasorri, F., Pallotta, S., Cianfarani, F., Odorisio, T., Traidl-Hoffmann, C., Behrendt, H., Durham, S.R., Schmidt-Weber, C.B., Cavani, A., 2009. Th22 cells represent a distinct human T cell subset involved in epidermal immunity and remodeling. J. Clin. Invest. doi:10.1172/JCI40202

Fahey, T.J., Tracey, K.J., Tekamp-Olson, P., Cousens, L.S., Jones, W.G., Shires, G.T., Cerami, A., Sherry, B., 1992. Macrophage inflammatory protein 1 modulates macrophage function. J. Immunol. Baltim. Md 1950 148, 2764-2769.

Faure, E., Equils, O., Sieling, P.A., Thomas, L., Zhang, F.X., Kirschning, C.J., Polentarutti, N., Muzio, M., Arditi, M., 2000. Bacterial Lipopolysaccharide Activates NF- $\kappa$ B through Toll-like Receptor 4 (TLR-4) in Cultured Human Dermal Endothelial Cells DIFFERENTIAL EXPRESSION OF TLR-4 AND TLR-2 IN ENDOTHELIAL CELLS. J. Biol. Chem. 275, 11058-11063. doi:10.1074/jbc.275.15.11058

Favre, J., Musette, P., Douin-Echinard, V., Laude, K., Henry, J.-P., Arnal, J.-F., Thuillez, C., Richard, V., 2007. Toll-like receptors 2-deficient mice are protected against postischemic coronary endothelial dysfunction. Arterioscler. Thromb. Vasc. Biol. 27, 1064-1071. doi:10.1161/ATVBAHA.107.140723

Fearon, D.T., Collins, L.A., 1983. Increased expression of $\mathrm{C} 3 \mathrm{~b}$ receptors on polymorphonuclear leukocytes induced by chemotactic factors and by purification procedures. J. Immunol. 130, 370-375.

Feng, Y., Zhao, H., Xu, X., Buys, E.S., Raher, M.J., Bopassa, J.C., Thibault, H., Scherrer-Crosbie, M., Schmidt, U., Chao, W., 2008. Innate immune adaptor MyD88 mediates neutrophil recruitment and myocardial injury after ischemiareperfusion in mice. Am. J. Physiol. Heart Circ. Physiol. 295, H1311-H1318. doi:10.1152/ajpheart.00119.2008

Ferguson, Z., Yarborough, D.E., Jarvis, B., Sistino, J., 2014. Evidence-based medicine and myocardial protection - where is the evidence? Perfusion. doi:10.1177/0267659114551856

Field, A.P., 1998. A bluffer's guide to sphericity. Newsl. Math. Stat. Comput. Sect. Br. Psychol. Soc. 13-22.

Fitch, J.C., Rollins, S., Matis, L., Alford, B., Aranki, S., Collard, C.D., Dewar, M., Elefteriades, J., Hines, R., Kopf, G., Kraker, P., Li, L., O'Hara, R., Rinder, C., Rinder, H., Shaw, R., Smith, B., Stahl, G., Shernan, S.K., 1999. Pharmacology and biological efficacy of a recombinant, humanized, single-chain antibody C5 complement inhibitor in patients undergoing coronary artery bypass graft surgery with cardiopulmonary bypass. Circulation 100, 2499-2506.

Floess, S., Freyer, J., Siewert, C., Baron, U., Olek, S., Polansky, J., Schlawe, K., Chang, H.-D., Bopp, T., Schmitt, E., Klein-Hessling, S., Serfling, E., Hamann, A., Huehn, J., 2007. Epigenetic Control of the foxp3 Locus in Regulatory T Cells. PLoS Biol. 5. doi:10.1371/journal.pbio.0050038 
Fong, T.A., Mosmann, T.R., 1990. Alloreactive murine CD8+ T cell clones secrete the Th1 pattern of cytokines. J. Immunol. Baltim. Md 1950 144, 1744-1752.

Forsythe, J.A., Jiang, B.H., Iyer, N.V., Agani, F., Leung, S.W., Koos, R.D., Semenza, G.L., 1996. Activation of vascular endothelial growth factor gene transcription by hypoxia-inducible factor 1. Mol. Cell. Biol. 16, 4604-4613.

Freitas, M., Porto, G., Lima, J.L.F.C., Fernandes, E., 2008. Isolation and activation of human neutrophils in vitro. The importance of the anticoagulant used during blood collection. Clin. Biochem. 41, 570-575. doi:10.1016/j.clinbiochem.2007.12.021

Friedrichs, G.S., Kilgore, K.S., Manley, P.J., Gralinski, M.R., Lucchesi, B.R., 1994. Effects of heparin and $\mathrm{N}$-acetyl heparin on ischemia/reperfusion-induced alterations in myocardial function in the rabbit isolated heart. Circ. Res. 75, 701710. doi:10.1161/01.RES.75.4.701

Funaki, H., Shimizu, K., Harada, S.-I., Tsuyama, H., Fushida, S., Tani, T., Miwa, K., 2002. Essential role for nuclear factor kappaB in ischemic preconditioning for ischemia-reperfusion injury of the mouse liver. Transplantation 74, 551-556. doi:10.1097/01.TP.0000026580.51510.4E

Furchgott, R.F., Zawadzki, J.V., 1980. The obligatory role of endothelial cells in the relaxation of arterial smooth muscle by acetylcholine. Nature 288, 373-376. doi: $10.1038 / 288373 \mathrm{a} 0$

Gabrilovich, D.I., Corak, J., Ciernik, I.F., Kavanaugh, D., Carbone, D.P., 1997. Decreased antigen presentation by dendritic cells in patients with breast cancer. Clin. Cancer Res. Off. J. Am. Assoc. Cancer Res. 3, 483-490.

Gallagher, S.M., Jones, D.A., Kapur, A., Wragg, A., Harwood, S.M., Mathur, R., Archbold, R.A., Uppal, R., Yaqoob, M.M., 2014. Remote ischemic preconditioning has a neutral effect on the incidence of kidney injury after coronary artery bypass graft surgery. Kidney Int. doi:10.1038/ki.2014.259

Garab, D., Fet, N., Szabó, A., Tolba, R.H., Boros, M., Hartmann, P., 2014. Remote ischemic preconditioning differentially affects NADPH oxidase isoforms during hepatic ischemia-reperfusion. Life Sci. 105, 14-21. doi:10.1016/j.lfs.2014.04.014

Gasparovic, H., Kopjar, T., Rados, M., Anticevic, A., Rados, M., Malojcic, B., Ivancan, V., Fabijanic, T., Cikes, M., Milicic, D., Gasparovic, V., Biocina, B., 2014. Impact of remote ischemic preconditioning preceding coronary artery bypass grafting on inducing neuroprotection (RIPCAGE): study protocol for a randomized controlled trial. Trials 15, 414. doi:10.1186/1745-6215-15-414

Gately, M.K., Carvajal, D.M., Connaughton, S.E., Gillessen, S., Warrier, R.R., Kolinsky, K.D., Wilkinson, V.L., Dwyer, C.M., Higgins, G.F., Podlaski, F.J., Faherty, D.A., Familletti, P.C., Stern, A.S., Presky, D.H., 1996. Interleukin-12 antagonist activity of mouse interleukin-12 p40 homodimer in vitro and in vivo. Ann. N. Y. Acad. Sci. 795, 1-12.

Gearing, A.J., Newman, W., 1993. Circulating adhesion molecules in disease. Immunol. Today 14, 506-512. doi:10.1016/0167-5699(93)90267-O

Gedik, N., Thielmann, M., Kottenberg, E., Peters, J., Jakob, H., Heusch, G., Kleinbongard, P., 2014. No evidence for activated autophagy in left ventricular myocardium at early reperfusion with protection by remote ischemic 
preconditioning in patients undergoing coronary artery bypass grafting. PloS One 9, e96567. doi:10.1371/journal.pone.0096567

Genda, S., Miura, T., Miki, T., Ichikawa, Y., Shimamoto, K., 2002. K(ATP) channel opening is an endogenous mechanism of protection against the no-reflow phenomenon but its function is compromised by hypercholesterolemia. J. Am. Coll. Cardiol. 40, 1339-1346.

Geudens, N., Vanaudenaerde, B.M., Neyrinck, A.P., Van De Wauwer, C., Vos, R., Verleden, G.M., Verbeken, E., Lerut, T., Van Raemdonck, D.E.M., 2007. The importance of lymphocytes in lung ischemia-reperfusion injury. Transplant. Proc. 39, 2659-2662. doi:10.1016/j.transproceed.2007.08.001

Gho, B.C., Schoemaker, R.G., van den Doel, M.A., Duncker, D.J., Verdouw, P.D., 1996. Myocardial protection by brief ischemia in noncardiac tissue. Circulation 94, 2193-200.

Ginhoux, F., Jung, S., 2014. Monocytes and macrophages: developmental pathways and tissue homeostasis. Nat. Rev. Immunol. 14, 392-404. doi:10.1038/nri3671

Ginis, I., Jaiswal, R., Klimanis, D., Liu, J., Greenspon, J., Hallenbeck, J.M., 2002. TNF-alpha-induced tolerance to ischemic injury involves differential control of NF-kappaB transactivation: the role of NF-kappaB association with p300 adaptor. J. Cereb. Blood Flow Metab. Off. J. Int. Soc. Cereb. Blood Flow Metab. 22, 142152. doi:10.1097/00004647-200202000-00002

GISSI-HF investigators, 2008. Effect of rosuvastatin in patients with chronic heart failure (the GISSI-HF trial): a randomised, double-blind, placebo-controlled trial. The Lancet 372, 1231-1239. doi:10.1016/S0140-6736(08)61240-4

Givan, A.L., 2000. In Living Color - Protocols in Flow Cytometry and Cell Sorting.

Goldblum, R.W., Piper, W.N., Olsen, C.J., 1954. Studies on Sulfhydryl Groups in Sera of Normal Humans1. J. Invest. Dermatol. 22, 373-378. doi:10.1038/jid.1954.53

Goodman, M.D., Koch, S.E., Afzal, M.R., Butler, K.L., 2011. STAT subtype specificity and ischemic preconditioning in mice: is STAT-3 enough? Am. J. Physiol. Heart Circ. Physiol. 300, H522-526. doi:10.1152/ajpheart.00231.2010

Gopalakrishna, R., Anderson, W.B., 1989. Ca2+- and phospholipid-independent activation of protein kinase $\mathrm{C}$ by selective oxidative modification of the regulatory domain. Proc. Natl. Acad. Sci. U. S. A. 86, 6758-6762.

Greenhouse, S.W., Geisser, S., 1959. On methods in the analysis of profile data. Psychometrika 24, 95-112. doi:10.1007/BF02289823

Gueret, G., Lion, F., Guriec, N., Arvieux, J., Dovergne, A., Guennegan, C., Bezon, E., Baron, R., Carre, J.-L., Arvieux, C., 2009. Acute renal dysfunction after cardiac surgery with cardiopulmonary bypass is associated with plasmatic IL6 increase. Cytokine 45, 92-98.

Gunaydin, B., Cakici, I., Soncul, H., Kalaycioglu, S., Cevik, C., Sancak, B., Kanzik, I., Karadenizli, Y., 2000. Does remote organ ischaemia trigger cardiac preconditioning during coronary artery surgery? Pharmacol Res 41, 493-6. doi:10.1006/phrs.1999.0611 
Haji Mohd Yasin, N.A.B., Herbison, P., Saxena, P., Praporski, S., Konstantinov, I.E., 2014. The role of remote ischemic preconditioning in organ protection after cardiac surgery: a meta-analysis. J. Surg. Res. 186, 207-216. doi:10.1016/j.jss.2013.09.006

Halestrap, A.P., Clarke, S.J., Khaliulin, I., 2007. The role of mitochondria in protection of the heart by preconditioning. Biochim. Biophys. Acta 1767, 10071031. doi:10.1016/j.bbabio.2007.05.008

Hamza, T., Barnett, J.B., Li, B., 2010. Interleukin 12 a key immunoregulatory cytokine in infection applications. Int. J. Mol. Sci. 11, 789-806. doi:10.3390/ijms11030789

Harkin, D.W., Barros D’Sa, A.A.B., McCallion, K., Hoper, M., Campbell, F.C., 2002. Ischemic preconditioning before lower limb ischemia--reperfusion protects against acute lung injury. J. Vasc. Surg. 35, 1264-1273.

Haroun-Bizri, S., Khoury, S.S., Chehab, I.R., Kassas, C.M., Baraka, A., 2001. Does isoflurane optimize myocardial protection during cardiopulmonary bypass? J Cardiothorac Vasc Anesth 15, 418-21. doi:10.1053/jcan.2001.24954

Hart, M.L., Gorzolla, I.C., Schittenhelm, J., Robson, S.C., Eltzschig, H.K., 2010. SP1-dependent induction of CD39 facilitates hepatic ischemic preconditioning. J. Immunol. Baltim. Md 1950 184, 4017-4024. doi:10.4049/jimmunol.0901851

Hata, K., Whittaker, P., Kloner, R.A., Przyklenk, K., 1998. Brief antecedent ischemia attenuates platelet-mediated thrombosis in damaged and stenotic canine coronary arteries: role of adenosine. Circulation 97, 692-702.

Ha, T., Hu, Y., Liu, L., Lu, C., McMullen, J.R., Kelley, J., Kao, R.L., Williams, D.L., Gao, X., Li, C., 2010. TLR2 ligands induce cardioprotection against ischaemia/reperfusion injury through a PI3K/Akt-dependent mechanism. Cardiovasc. Res. 87, 694-703. doi:10.1093/cvr/cvq116

Ha, T., Liu, L., Kelley, J., Kao, R., Williams, D., Li, C., 2011. Toll-like receptors: new players in myocardial ischemia/reperfusion injury. Antioxid. Redox Signal. 15, 1875-1893. doi:10.1089/ars.2010.3723

Hattori, R., Maulik, N., Otani, H., Zhu, L., Cordis, G., Engelman, R.M., Siddiqui, M.A., Das, D.K., 2001. Role of STAT3 in ischemic preconditioning. J. Mol. Cell. Cardiol. 33, 1929-1936. doi:10.1006/jmcc.2001.1456

Hausenloy, D.J., Baxter, G., Bell, R., Bøtker, H.E., Davidson, S.M., Downey, J., Heusch, G., Kitakaze, M., Lecour, S., Mentzer, R., Mocanu, M.M., Ovize, M., Schulz, R., Shannon, R., Walker, M., Walkinshaw, G., Yellon, D.M., 2010. Translating novel strategies for cardioprotection: the Hatter Workshop Recommendations. Basic Res. Cardiol. 105, 677-686. doi:10.1007/s00395-0100121-4

Hausenloy, D.J., Candilio, L., Laing, C., Kunst, G., Pepper, J., Kolvekar, S., Evans, R., Robertson, S., Knight, R., Ariti, C., Clayton, T., Yellon, D.M., ERICCA Trial Investigators, 2012. Effect of remote ischemic preconditioning on clinical outcomes in patients undergoing coronary artery bypass graft surgery (ERICCA): rationale and study design of a multi-centre randomized double-blinded controlled clinical trial. Clin. Res. Cardiol. Off. J. Ger. Card. Soc. 101, 339-348. doi:10.1007/s00392-011-0397-x 
Hausenloy, D.J., Mwamure, P.K., Venugopal, V., Harris, J., Barnard, M., Grundy, E., Ashley, E., Vichare, S., Di Salvo, C., Kolvekar, S., Hayward, M., Keogh, B., MacAllister, R.J., Yellon, D.M., 2007. Effect of remote ischaemic preconditioning on myocardial injury in patients undergoing coronary artery bypass graft surgery: a randomised controlled trial. Lancet 370, 575-9. doi:10.1016/S01406736(07)61296-3

Hausenloy, D.J., Yellon, D.M., 2012. "Conditional Conditioning” in cardiac bypass surgery. Basic Res. Cardiol. 107, 258. doi:10.1007/s00395-012-0258-4

Hausenloy, D.J., Yellon, D.M., 2008. Remote ischaemic preconditioning: underlying mechanisms and clinical application. Cardiovasc Res 79, 377-86. doi:10.1093/cvr/cvn114

Hausenloy, D.J., Yellon, D.M., 2006. Survival kinases in ischemic preconditioning and postconditioning. Cardiovasc. Res. 70, 240-253. doi:10.1016/j.cardiores.2006.01.017

Heidbreder, M., Naumann, A., Tempel, K., Dominiak, P., Dendorfer, A., 2008. Remote vs. ischaemic preconditioning: the differential role of mitogen-activated protein kinase pathways. Cardiovasc. Res. 78, 108-115. doi:10.1093/cvr/cvm114

Hepponstall, M., Ignjatovic, V., Binos, S., Monagle, P., Jones, B., Cheung, M.H.H., d' Udekem, Y., Konstantinov, I.E., 2012. Remote ischemic preconditioning (RIPC) modifies plasma proteome in humans. PloS One 7, e48284. doi:10.1371/journal.pone.0048284

Heusch, G., 2012. HIF-1 $\alpha$ and paradoxical phenomena in cardioprotection. Cardiovasc. Res. 96, 214-215; discussion 216-219. doi:10.1093/cvr/cvs145

Heusch, G., Musiolik, J., Kottenberg, E., Peters, J., Jakob, H., Thielmann, M., 2011. STAT5 Activation and Cardioprotection by Remote Ischemic Preconditioning in Humans. Circ Res 110, 111-5. doi:10.1161/CIRCRESAHA.111.259556

Hibert, P., Prunier-Mirebeau, D., Beseme, O., Chwastyniak, M., Tamareille, S., Pinet, F., Prunier, F., 2014. Modifications in rat plasma proteome after remote ischemic preconditioning (RIPC) stimulus: identification by a SELDI-TOF-MS approach. PloS One 9, e85669. doi:10.1371/journal.pone.0085669

Hochart, H., Vincent Jenkins, P., Smith, O.P., White, B., 2006. Low-molecular weight and unfractionated heparins induce a downregulation of inflammation: decreased levels of proinflammatory cytokines and nuclear factor- $\kappa \mathrm{B}$ in LPS-stimulated human monocytes. Br. J. Haematol. 133, 62-67. doi:10.1111/j.13652141.2006.05959.x

Hochegger, K., Schätz, T., Eller, P., Tagwerker, A., Heininger, D., Mayer, G., Rosenkranz, A.R., 2007. Role of alpha/beta and gamma/delta $\mathrm{T}$ cells in renal ischemia-reperfusion injury. Am. J. Physiol. Renal Physiol. 293, F741-747. doi:10.1152/ajprenal.00486.2006

Hofmann, U., Beyersdorf, N., Weirather, J., Podolskaya, A., Bauersachs, J., Ertl, G., Kerkau, T., Frantz, S., 2012. Activation of CD4+ T Lymphocytes Improves Wound Healing and Survival After Experimental Myocardial Infarction in Mice. Circulation 125, 1652-1663. doi:10.1161/CIRCULATIONAHA.111.044164

Holmberg, F.E.O., Ottas, K.A., Andreasen, C., Perko, M.J., Møller, C.H., Engstrøm, T., Steinbrüchel, D.A., 2014. Conditioning techniques and ischemic reperfusion 
injury in relation to on-pump cardiac surgery. Scand. Cardiovasc. J. SCJ 48, 241248. doi:10.3109/14017431.2014.923930

Holmvang, L., Jurlander, B., Rasmussen, C., Thiis, J.J., Grande, P., Clemmensen, P., 2002. Use of biochemical markers of infarction for diagnosing perioperative myocardial infarction and early graft occlusion after coronary artery bypass surgery. Chest 121, 103-111.

Hong, D.M., Mint, J.J., Kim, J.H., Sohn, I.S., Lim, T.W., Lim, Y.J., Bahk, J.H., Jeon, Y., 2010. The effect of remote ischaemic preconditioning on myocardial injury in patients undergoing off-pump coronary artery bypass graft surgery. Anaesth Intensive Care 38, 924-9.

Horie, Y., Wolf, R., Chervenak, R.P., Jennings, S.R., Granger, D.N., 1999. Tlymphocytes contribute to hepatic leukostasis and hypoxic stress induced by gut ischemia-reperfusion. Microcirc. N. Y. N 1994 6, 267-280.

Hori, M., Kitakaze, M., 1991. Adenosine, the heart, and coronary circulation. Hypertension 18, 565-574.

Horrigan, L.A., Kelly, J.P., Connor, T.J., 2006. Immunomodulatory effects of caffeine: friend or foe? Pharmacol. Ther. 111, 877-892. doi:10.1016/j.pharmthera.2006.02.002

Howell, J.G., Zibari, G.B., Brown, M.F., Burney, D.L., Sawaya, D.E., Olinde, J.G., Granger, D.N., McDonald, J.C., 2000. Both ischemic and pharmacological preconditioning decrease hepatic leukocyte/endothelial cell interactions. Transplantation 69, 300-303.

Hua, F., Ha, T., Ma, J., Li, Y., Kelley, J., Gao, X., Browder, I.W., Kao, R.L., Williams, D.L., Li, C., 2007. Protection against myocardial ischemia/reperfusion injury in TLR4-deficient mice is mediated through a phosphoinositide 3-kinasedependent mechanism. J. Immunol. Baltim. Md 1950 178, 7317-7324.

Hu, C.P., Peng, J., Xiao, L., Ye, F., Deng, H.W., Li, Y.J., 2002. Effect of age on alpha-calcitonin gene-related peptide-mediated delayed cardioprotection induced by intestinal preconditioning in rats. Regul. Pept. 107, 137-143.

Huda, R., Chung, D.H., Mathru, M., 2005. Ischemic preconditioning at a distance: altered gene expression in mouse heart and other organs following brief occlusion of the mesenteric artery. Heart Lung Circ. 14, 36-43. doi:10.1016/j.hlc.2004.11.006

Huizinga, T.W., de Haas, M., Kleijer, M., Nuijens, J.H., Roos, D., von dem Borne, A.E., 1990. Soluble Fc gamma receptor III in human plasma originates from release by neutrophils. J. Clin. Invest. 86, 416-423. doi:10.1172/JCI114727

Jaffe, A.S., Ravkilde, J., Roberts, R., Naslund, U., Apple, F.S., Galvani, M., Katus, H., 2000. It's Time for a Change to a Troponin Standard. Circulation 102, 12161220. doi:10.1161/01.CIR.102.11.1216

Jakobsen, C.-J., Berg, H., Hindsholm, K.B., Faddy, N., Sloth, E., 2007. The influence of propofol versus sevoflurane anesthesia on outcome in 10,535 cardiac surgical procedures. J. Cardiothorac. Vasc. Anesth. 21, 664-671. doi:10.1053/j.jvca.2007.03.002 
Janeway, C.A., Medzhitov, R., 2002. Innate immune recognition. Annu. Rev. Immunol. 20, 197-216. doi:10.1146/annurev.immunol.20.083001.084359

Jensen, R.V., Zachara, N.E., Nielsen, P.H., Kimose, H.H., Kristiansen, S.B., Bøtker, H.E., 2013. Impact of O-GlcNAc on cardioprotection by remote ischaemic preconditioning in non-diabetic and diabetic patients. Cardiovasc. Res. 97, 369378. doi:10.1093/cvr/cvs337

Jiang, S.H., Liu, C.F., Zhang, X.L., Xu, X.H., Zou, J.Z., Fang, Y., Ding, X.Q., 2007. Renal protection by delayed ischaemic preconditioning is associated with inhibition of the inflammatory response and NF-kappaB activation. Cell Biochem. Funct. 25, 335-343. doi:10.1002/cbf.1395

Jiang, Y., Gu, X.-P., Qiu, Y.-D., Sun, X.-M., Chen, L.-L., Zhang, L.-H., Ding, Y.-T., 2003. Ischemic preconditioning decreases C-X-C chemokine expression and neutrophil accumulation early after liver transplantation in rats. World $\mathrm{J}$. Gastroenterol. WJG 9, 2025-2029.

Jin, P., Wang, E., 2003. Polymorphism in clinical immunology - From HLA typing to immunogenetic profiling. J. Transl. Med. 1, 8. doi:10.1186/1479-5876-1-8

Johnson, C.R., Field, C.A., 1993. Using fixed-effects model multivariate analysis of variance in marine biology and ecology. Oceanogr. Mar. Biol. Annu. Rev. 31, $177-221$.

Jones, B.O., Pepe, S., Sheeran, F.L., Donath, S., Hardy, P., Shekerdemian, L., Penny, D.J., McKenzie, I., Horton, S., Brizard, C.P., d' Udekem, Y., Konstantinov, I.E., Cheung, M.M.H., 2013. Remote ischemic preconditioning in cyanosed neonates undergoing cardiopulmonary bypass: a randomized controlled trial. J. Thorac. Cardiovasc. Surg. 146, 1334-1340. doi:10.1016/j.jtcvs.2013.01.003

Kaelin, W.G., Ratcliffe, P.J., 2008. Oxygen sensing by metazoans: the central role of the HIF hydroxylase pathway. Mol. Cell 30, 393-402. doi:10.1016/j.molcel.2008.04.009

Kalavrouziotis, D., Li, D., Buth, K.J., Legare, J.-F., 2009. The European System for Cardiac Operative Risk Evaluation (EuroSCORE) is not appropriate for withholding surgery in high-risk patients with aortic stenosis: a retrospective cohort study. J. Cardiothorac. Surg. 4, 32. doi:10.1186/1749-8090-4-32

Kant, R., Diwan, V., Jaggi, A.S., Singh, N., Singh, D., 2008. Remote renal preconditioning-induced cardioprotection: a key role of hypoxia inducible factorprolyl 4-hydroxylases. Mol. Cell. Biochem. 312, 25-31. doi:10.1007/s11010-0089717-5

Kapessidou, P., Poulin, L., Dumoutier, L., Goldman, M., Renauld, J.-C., Braun, M.Y., 2008. Interleukin-22 deficiency accelerates the rejection of full major histocompatibility complex-disparate heart allografts. Transplant. Proc. 40, 15931597. doi:10.1016/j.transproceed.2008.03.151

Karanicolas, P.J., Farrokhyar, F., Bhandari, M., 2010. Blinding: Who, what, when, why, how? Can. J. Surg. 53, 345-348.

Karpus, W.J., Kennedy, K.J., 1997. MIP-1alpha and MCP-1 differentially regulate acute and relapsing autoimmune encephalomyelitis as well as Th1/Th2 lymphocyte differentiation. J. Leukoc. Biol. 62, 681-687. 
Karuppasamy, P., Chaubey, S., Dew, T., Musto, R., Sherwood, R., Desai, J., John, L., Shah, A.M., Marber, M.S., Kunst, G., 2011. Remote intermittent ischemia before coronary artery bypass graft surgery: a strategy to reduce injury and inflammation? Basic Res Cardiol 106, 511-9. doi:10.1007/s00395-011-0185-9

Kaur Randhawa, P., Bali, A., Singh Jaggi, A., 2014. RIPC for multiorgan salvage in clinical settings: Evolution of concept, evidences and mechanisms. Eur. J. Pharmacol. doi:10.1016/j.ejphar.2014.08.016

Kawahito, K., Adachi, H., Ino, T., 2000. Influence of surgical procedures on interleukin- 6 and monocyte chemotactic and activating factor responses: CABG vs. valvular surgery. J Interferon Cytokine Res 20, 1-6. doi:10.1089/107999000312676

Kawamura, T., Kadosaki, M., Nara, N., Kaise, A., Suzuki, H., Endo, S., Wei, J., Inada, K., 2006. Effects of sevoflurane on cytokine balance in patients undergoing coronary artery bypass graft surgery. J Cardiothorac Vasc Anesth 20, 503-8. doi:10.1053/j.jvca.2006.01.011

Kawamura, T., Wakusawa, R., Okada, K., Inada, S., 1993. Elevation of cytokines during open heart surgery with cardiopulmonary bypass: participation of interleukin 8 and 6 in reperfusion injury. Can J Anaesth 40, 1016-21.

Kelly, B.L., Locksley, R.M., 2000. Coordinate regulation of the IL-4, IL-13, and IL-5 cytokine cluster in Th2 clones revealed by allelic expression patterns. J. Immunol. Baltim. Md 1950 165, 2982-2986.

Kelly, K.J., Williams, W.W., Colvin, R.B., Meehan, S.M., Springer, T.A., GutierrezRamos, J.C., Bonventre, J.V., 1996. Intercellular adhesion molecule-1-deficient mice are protected against ischemic renal injury. J Clin Invest 97, 1056-63. doi:10.1172/JCI118498

Ke, Q., Costa, M., 2006. Hypoxia-Inducible Factor-1 (HIF-1). Mol. Pharmacol. 70, 1469-1480. doi:10.1124/mol.106.027029

Khader, S.A., Partida-Sanchez, S., Bell, G., Jelley-Gibbs, D.M., Swain, S., Pearl, J.E., Ghilardi, N., Desauvage, F.J., Lund, F.E., Cooper, A.M., 2006. Interleukin 12p40 is required for dendritic cell migration and $\mathrm{T}$ cell priming after Mycobacterium tuberculosis infection. J. Exp. Med. 203, 1805-1815. doi:10.1084/jem.20052545

Khandoga, A., Hanschen, M., Kessler, J.S., Krombach, F., 2006. CD4+ T cells contribute to postischemic liver injury in mice by interacting with sinusoidal endothelium and platelets. Hepatol. Baltim. Md 43, 306-315. doi:10.1002/hep.21017

Kharbanda, R.K., Mortensen, U.M., White, P.A., Kristiansen, S.B., Schmidt, M.R., Hoschtitzky, J.A., Vogel, M., Sorensen, K., Redington, A.N., MacAllister, R., 2002. Transient limb ischemia induces remote ischemic preconditioning in vivo. Circulation 106, 2881-3.

Kharbanda, R.K., Nielsen, T.T., Redington, A.N., 2009. Translation of remote ischaemic preconditioning into clinical practice. Lancet 374, 1557-1565. doi:10.1016/S0140-6736(09)61421-5

Kharbanda, R.K., Peters, M., Walton, B., Kattenhorn, M., Mullen, M., Klein, N., Vallance, P., Deanfield, J., MacAllister, R., 2001. Ischemic preconditioning 
prevents endothelial injury and systemic neutrophil activation during ischemiareperfusion in humans in vivo. Circulation 103, 1624-30.

Kilgore, K.S., Tanhehco, E.J., Naylor, K.B., Lucchesi, B.R., 1999. Ex vivo reversal of heparin-mediated cardioprotection by heparinase after ischemia and reperfusion. J. Pharmacol. Exp. Ther. 290, 1041-1047.

Kim, J.-C., Shim, J.-K., Lee, S., Yoo, Y.-C., Yang, S.-Y., Kwak, Y.-L., 2012. Effect of combined remote ischemic preconditioning and postconditioning on pulmonary function in valvular heart surgery. Chest 142, 467-475. doi:10.1378/chest.11-2246

Kim, M., Kim, M., Kim, N., D’Agati, V.D., Emala, C.W., Lee, H.T., 2007. Isoflurane mediates protection from renal ischemia-reperfusion injury via sphingosine kinase and sphingosine-1-phosphate-dependent pathways. Am. J. Physiol. Renal Physiol. 293, F1827-1835. doi:10.1152/ajprenal.00290.2007

Kim, S.-C., Ghanem, A., Stapel, H., Tiemann, K., Knuefermann, P., Hoeft, A., Meyer, R., Grohé, C., Knowlton, A.A., Baumgarten, G., 2007. Toll-like receptor 4 deficiency: smaller infarcts, but no gain in function. BMC Physiol. 7, 5. doi:10.1186/1472-6793-7-5

Kim, S.Y., Park, H.-J., Choi, J.-S., Lee, J.E., Cha, J.-H., Choi, Y.-S., Cho, K.-O., Chun, M.-H., Lee, M.-Y., 2004. Ischemic preconditioning-induced expression of gp130 and STAT3 in astrocytes of the rat hippocampus. Brain Res. Mol. Brain Res. 129, 96-103. doi:10.1016/j.molbrainres.2004.06.025

Kim, T., Arnaoutakis, G.J., Bihorac, A., Martin, T.D., Hess Jr, P.J., Klodell, C.T., Tribble, C.G., Ejaz, A.A., Moldawer, L.L., Beaver, T.M., 2009. Early Blood Biomarkers Predict Organ Injury and Resource Utilization Following Complex Cardiac Surgery. J. Surg. Res. In Press, Corrected Proof.

Kim, Y.M., Kim, T.H., Chung, H.T., Talanian, R.V., Yin, X.M., Billiar, T.R., 2000. Nitric oxide prevents tumor necrosis factor alpha-induced rat hepatocyte apoptosis by the interruption of mitochondrial apoptotic signaling through S-nitrosylation of caspase-8. Hepatol. Baltim. Md 32, 770-778. doi:10.1053/jhep.2000.18291

Kinsey, G.R., Huang, L., Vergis, A.L., Li, L., Okusa, M.D., 2010. Regulatory T cells contribute to the protective effect of ischemic preconditioning in the kidney. Kidney Int 77, 771-80. doi:10.1038/ki.2010.12

Ki, S.H., Park, O., Zheng, M., Morales-Ibanez, O., Kolls, J.K., Bataller, R., Gao, B., 2010. Interleukin-22 treatment ameliorates alcoholic liver injury in a murine model of chronic-binge ethanol feeding: role of signal transducer and activator of transcription 3. Hepatol. Baltim. Md 52, 1291-1300. doi:10.1002/hep.23837

Kjekshus, J., Apetrei, E., Barrios, V., Böhm, M., Cleland, J.G.F., Cornel, J.H., Dunselman, P., Fonseca, C., Goudev, A., Grande, P., Gullestad, L., Hjalmarson, Å., Hradec, J., Jánosi, A., Kamenský, G., Komajda, M., Korewicki, J., Kuusi, T., Mach, F., Mareev, V., McMurray, J.J.V., Ranjith, N., Schaufelberger, M., Vanhaecke, J., van Veldhuisen, D.J., Waagstein, F., Wedel, H., Wikstrand, J., 2007. Rosuvastatin in Older Patients with Systolic Heart Failure. N. Engl. J. Med. 357, 2248-2261. doi:10.1056/NEJMoa0706201

Kloner, R.A., 2009. Clinical Application of Remote Ischemic Preconditioning. Circulation 119, 776-778. doi:10.1161/circulationaha.108.832832 
Koenig, A., Norgard-Sumnicht, K., Linhardt, R., Varki, A., 1998. Differential interactions of heparin and heparan sulfate glycosaminoglycans with the selectins. Implications for the use of unfractionated and low molecular weight heparins as therapeutic agents. J. Clin. Invest. 101, 877-889. doi:10.1172/JCI1509

Konstantinov, I.E., Arab, S., Kharbanda, R.K., Li, J., Cheung, M.M., Cherepanov, V., Downey, G.P., Liu, P.P., Cukerman, E., Coles, J.G., Redington, A.N., 2004. The remote ischemic preconditioning stimulus modifies inflammatory gene expression in humans. Physiol Genomics 19, 143-50. doi:10.1152/physiolgenomics.00046.2004

Konstantinov, I.E., Arab, S., Li, J., Coles, J.G., Boscarino, C., Mori, A., Cukerman, E., Dawood, F., Cheung, M.M.H., Shimizu, M., Liu, P.P., Redington, A.N., 2005. The remote ischemic preconditioning stimulus modifies gene expression in mouse myocardium. J. Thorac. Cardiovasc. Surg. 130, 1326-1332. doi:10.1016/j.jtcvs.2005.03.050

Konstantinov, I.E., Li, J., Cheung, M.M., Shimizu, M., Stokoe, J., Kharbanda, R.K., Redington, A.N., 2005. Remote ischemic preconditioning of the recipient reduces myocardial ischemia-reperfusion injury of the denervated donor heart via a Katp channel-dependent mechanism. Transplantation 79, 1691-5.

Korthuis, R.J., Granger, D.N., 1993. Reactive oxygen metabolites, neutrophils, and the pathogenesis of ischemic-tissue/reperfusion. Clin. Cardiol. 16, I19-26.

Kottenberg, E., Thielmann, M., Bergmann, L., Heine, T., Jakob, H., Heusch, G., Peters, J., 2012. Protection by remote ischemic preconditioning during coronary artery bypass graft surgery with isoflurane but not propofol - a clinical trial. Acta Anaesthesiol Scand 56, 30-8. doi:10.1111/j.1399-6576.2011.02585.x

Kouretas, P.C., Kim, Y.D., Cahill, P.A., Myers, A.K., To, L.N., Wang, Y.-N., Sitzmann, J.V., Hannan, R.L., 1999. Nonanticoagulant Heparin Prevents Coronary Endothelial Dysfunction After Brief Ischemia-Reperfusion Injury in the Dog. Circulation 99, 1062-1068. doi:10.1161/01.CIR.99.8.1062

Krishnadasan, B., Farivar, A.S., Naidu, B.V., Woolley, S.M., Byrne, K., Fraga, C.H., Mulligan, M.S., 2004. Beta-chemokine function in experimental lung ischemiareperfusion injury. Ann. Thorac. Surg. 77, 1056-1062. doi:10.1016/S00034975(03)01600-X

Kruisbeek, A.M., Shevach, E., Thornton, A.M., 2004. Proliferative Assays for T Cell Function, in: Current Protocols in Immunology. John Wiley \& Sons, Inc.

Kubes, P., Payne, D., Ostrovsky, L., 1998. Preconditioning and adenosine in I/Rinduced leukocyte-endothelial cell interactions. Am. J. Physiol. 274, H1230-1238.

Kukielka, G.L., Hawkins, H.K., Michael, L., Manning, A.M., Youker, K., Lane, C., Entman, M.L., Smith, C.W., Anderson, D.C., 1993. Regulation of intercellular adhesion molecule-1 (ICAM-1) in ischemic and reperfused canine myocardium. J. Clin. Invest. 92, 1504-1516. doi:10.1172/JCI116729

Kulkarni, O.P., Hartter, I., Mulay, S.R., Hagemann, J., Darisipudi, M.N., Kumar Vr, S., Romoli, S., Thomasova, D., Ryu, M., Kobold, S., Anders, H.-J., 2014. Toll-like receptor 4-induced IL-22 accelerates kidney regeneration. J. Am. Soc. Nephrol. JASN 25, 978-989. doi:10.1681/ASN.2013050528 
Kuschert, G.S., Coulin, F., Power, C.A., Proudfoot, A.E., Hubbard, R.E., Hoogewerf, A.J., Wells, T.N., 1999. Glycosaminoglycans interact selectively with chemokines and modulate receptor binding and cellular responses. Biochemistry (Mosc.) 38, 12959-12968.

Kusek, M.E., Pazos, M.A., Pirzai, W., Hurley, B.P., 2014. In vitro coculture assay to assess pathogen induced neutrophil trans-epithelial migration. J. Vis. Exp. JoVE e50823. doi:10.3791/50823

Kuzuya, T., Hoshida, S., Yamashita, N., Fuji, H., Oe, H., Hori, M., Kamada, T., Tada, M., 1993. Delayed effects of sublethal ischemia on the acquisition of tolerance to ischemia. Circ Res 72, 1293-9.

Landay, A., Gartland, G.L., Clement, L.T., 1983. Characterization of a phenotypically distinct subpopulation of Leu-2+ cells that suppresses $\mathrm{T}$ cell proliferative responses. J. Immunol. Baltim. Md 1950 131, 2757-2761.

Landoni, G., Biondi-Zoccai, G.G.L., Zangrillo, A., Bignami, E., D’Avolio, S., Marchetti, C., Calabrò, M.G., Fochi, O., Guarracino, F., Tritapepe, L., De Hert, S., Torri, G., 2007. Desflurane and sevoflurane in cardiac surgery: a meta-analysis of randomized clinical trials. J. Cardiothorac. Vasc. Anesth. 21, 502-511. doi:10.1053/j.jvca.2007.02.013

Lang, S.C., Elsässer, A., Scheler, C., Vetter, S., Tiefenbacher, C.P., Kübler, W., Katus, H.A., Vogt, A.M., 2006. Myocardial preconditioning and remote renal preconditioning--identifying a protective factor using proteomic methods? Basic Res. Cardiol. 101, 149-158. doi:10.1007/s00395-005-0565-0

Lappas, C.M., Day, Y.-J., Marshall, M.A., Engelhard, V.H., Linden, J., 2006. Adenosine A2A receptor activation reduces hepatic ischemia reperfusion injury by inhibiting CD1d-dependent NKT cell activation. J. Exp. Med. 203, 2639-2648. doi:10.1084/jem.20061097

Larach, D.R., Schuler, H.G., Skeehan, T.M., Peterson, C.J., 1990. Direct effects of myocardial depressant drugs on coronary vascular tone: anesthetic vasodilation by halothane and isoflurane. J. Pharmacol. Exp. Ther. 254, 58-64.

Larsen, C.E., Xu, J., Lee, S., Dubey, D.P., Uko, G., Yunis, E.J., Alper, C.A., 2000. Complex cytokine responses to hepatitis B surface antigen and tetanus toxoid in responders, nonresponders and subjects naive to hepatitis B surface antigen. Vaccine 18, 3021-3030.

Larsen, C.G., Anderson, A.O., Appella, E., Oppenheim, J.J., Matsushima, K., 1989. The neutrophil-activating protein (NAP-1) is also chemotactic for T lymphocytes. Science 243, 1464-1466.

Lavi, S., Lavi, R., 2011. Conditioning of the heart: From pharmacological interventions to local and remote protection: Possible implications for clinical practice. Int. J. Cardiol. 146, 311-318. doi:10.1016/j.ijcard.2010.08.010

Lee, J.-H., Park, Y.-H., Byon, H.-J., Kim, H.-S., Kim, C.-S., Kim, J.-T., 2012. Effect of remote ischaemic preconditioning on ischaemic-reperfusion injury in pulmonary hypertensive infants receiving ventricular septal defect repair. Br. J. Anaesth. 108, 223-228. doi:10.1093/bja/aer388

Lefer, A.M., Lefer, D.J., 1996. The role of nitric oxide and cell adhesion molecules on the microcirculation in ischaemia-reperfusion. Cardiovasc. Res. 32, 743-751. 
Lever, R., Hoult, J.R.S., Page, C.P., 2000. The effects of heparin and related molecules upon the adhesion of human polymorphonuclear leucocytes to vascular endothelium in vitro. Br. J. Pharmacol. 129, 533-540. doi:10.1038/sj.bjp.0703099

Levine, B., Kalman, J., Mayer, L., Fillit, H.M., Packer, M., 1990. Elevated circulating levels of tumor necrosis factor in severe chronic heart failure. N. Engl. J. Med. 323, 236-241. doi:10.1056/NEJM199007263230405

Liauw, S.K., Rubin, B.B., Lindsay, T.F., Romaschin, A.D., Walker, P.M., 1996. Sequential ischemia/reperfusion results in contralateral skeletal muscle salvage. Am J Physiol 270, H1407-13.

Liem, D.A., Verdouw, P.D., Ploeg, H., Kazim, S., Duncker, D.J., 2002. Sites of action of adenosine in interorgan preconditioning of the heart. Am J Physiol Heart Circ Physiol 283, H29-37. doi:10.1152/ajpheart.01031.2001

Lieschke, G.J., Grail, D., Hodgson, G., Metcalf, D., Stanley, E., Cheers, C., Fowler, K.J., Basu, S., Zhan, Y.F., Dunn, A.R., 1994. Mice lacking granulocyte colonystimulating factor have chronic neutropenia, granulocyte and macrophage progenitor cell deficiency, and impaired neutrophil mobilization. Blood 84, 17371746.

Liesz, A., Suri-Payer, E., Veltkamp, C., Doerr, H., Sommer, C., Rivest, S., Giese, T., Veltkamp, R., 2009. Regulatory $\mathrm{T}$ cells are key cerebroprotective immunomodulators in acute experimental stroke. Nat. Med. 15, 192-199. doi:10.1038/nm.1927

Li, G., Labruto, F., Sirsjö, A., Chen, F., Vaage, J., Valen, G., 2004. Myocardial protection by remote preconditioning: the role of nuclear factor kappa-B p105 and inducible nitric oxide synthase. Eur. J. Cardio-Thorac. Surg. Off. J. Eur. Assoc. Cardio-Thorac. Surg. 26, 968-973. doi:10.1016/j.ejcts.2004.06.015

Li, J., Xuan, W., Yan, R., Tropak, M.B., Jean-St-Michel, E., Liang, W., Gladstone, R., Backx, P.H., Kharbanda, R.K., Redington, A.N., 2011. Remote preconditioning provides potent cardioprotection via PI3K/Akt activation and is associated with nuclear accumulation of $\beta$-catenin. Clin. Sci. Lond. Engl. 1979 120, 451-462. doi:10.1042/CS20100466

Li, L., Li, G., Yu, C., Li, Y., 2013. The role of remote ischemic preconditioning on postoperative kidney injury in patients undergoing cardiac and vascular interventions: a meta-analysis. J. Cardiothorac. Surg. 8, 43. doi:10.1186/17498090-8-43

Li, L., Luo, W., Huang, L., Zhang, W., Gao, Y., Jiang, H., Zhang, C., Long, L., Chen, S., 2010. Remote perconditioning reduces myocardial injury in adult valve replacement: a randomized controlled trial. J. Surg. Res. 164, e21-26. doi:10.1016/j.jss.2010.06.016

Li, M., Carpio, D.F., Zheng, Y., Bruzzo, P., Singh, V., Ouaaz, F., Medzhitov, R.M., Beg, A.A., 2001. An essential role of the NF-kappa B/Toll-like receptor pathway in induction of inflammatory and tissue-repair gene expression by necrotic cells. J. Immunol. Baltim. Md 1950 166, 7128-7135.

Lim, S.Y., Yellon, D.M., Hausenloy, D.J., 2010. The neural and humoral pathways in remote limb ischemic preconditioning. Basic Res. Cardiol. 105, 651-655. doi:10.1007/s00395-010-0099-y 
Linden, M.D., Whittaker, P., Frelinger, A.L., Barnard, M.R., Michelson, A.D., Przyklenk, K., 2006. Preconditioning ischemia attenuates molecular indices of platelet activation-aggregation. J. Thromb. Haemost. 4, 2670-2677. doi:10.1111/j.1538-7836.2006.02228.x

Linfert, D., Chowdhry, T., Rabb, H., 2009. Lymphocytes and ischemia-reperfusion injury. Transplant. Rev. Orlando Fla 23, 1-10. doi:10.1016/j.trre.2008.08.003

Ling, P., Gately, M.K., Gubler, U., Stern, A.S., Lin, P., Hollfelder, K., Su, C., Pan, Y.C., Hakimi, J., 1995. Human IL-12 p40 homodimer binds to the IL-12 receptor but does not mediate biologic activity. J. Immunol. 154, 116-127.

Li, R.C., Ping, P., Zhang, J., Wead, W.B., Cao, X., Gao, J., Zheng, Y., Huang, S., Han, J., Bolli, R., 2000. PKCepsilon modulates NF-kappaB and AP-1 via mitogenactivated protein kinases in adult rabbit cardiomyocytes. Am. J. Physiol. Heart Circ. Physiol. 279, H1679-1689.

Liu, K.D., Altmann, C., Smits, G., Krawczeski, C.D., Edelstein, C.L., Devarajan, P., Faubel, S., 2009. Serum interleukin- 6 and interleukin-8 are early biomarkers of acute kidney injury and predict prolonged mechanical ventilation in children undergoing cardiac surgery: a case-control study. Crit Care 13, R104. doi:10.1186/cc7940

Li, X.-C., Ma, Y.-F., Wang, X.-H., 2006. Role of NF-kappaB as effector of IPC in donor livers before liver transplantation in rats. Transplant. Proc. 38, 1584-1587. doi:10.1016/j.transproceed.2006.02.122

Loken, M.R., Brosnan, J.M., Bach, B.A., Ault, K.A., 1990. Establishing optimal lymphocyte gates for immunophenotyping by flow cytometry. Cytometry 11, 453459. doi:10.1002/cyto.990110402

Lomivorotov, V.V., Shmyrev, V.A., Nepomnyaschih, V.A., Ponomarev, D.N., Knyazkova, L.G., Lomivorotov, V.N., Karaskov, A.M., 2012. Remote ischaemic preconditioning does not protect the heart in patients undergoing coronary artery bypass grafting. Interact Cardiovasc Thorac Surg. doi:10.1093/icvts/ivs118

Loukogeorgakis, S.P., Panagiotidou, A.T., Broadhead, M.W., Donald, A., Deanfield, J.E., MacAllister, R.J., 2005. Remote ischemic preconditioning provides early and late protection against endothelial ischemia-reperfusion injury in humans: role of the autonomic nervous system. J Am Coll Cardiol 46, 450-6. doi:10.1016/j.jacc.2005.04.044

Loukogeorgakis, S.P., Williams, R., Panagiotidou, A.T., Kolvekar, S.K., Donald, A., Cole, T.J., Yellon, D.M., Deanfield, J.E., MacAllister, R.J., 2007. Transient limb ischemia induces remote preconditioning and remote postconditioning in humans by a K(ATP)-channel dependent mechanism. Circulation 116, 1386-1395. doi:10.1161/CIRCULATIONAHA.106.653782

Louten, J., Boniface, K., de Waal Malefyt, R., 2009. Development and function of TH17 cells in health and disease. J. Allergy Clin. Immunol. 123, 1004-1011. doi:10.1016/j.jaci.2009.04.003

Lucchinetti, E., Bestmann, L., Feng, J., Freidank, H., Clanachan, A.S., Finegan, B.A., Zaugg, M., 2012. Remote ischemic preconditioning applied during isoflurane inhalation provides no benefit to the myocardium of patients undergoing on-pump coronary artery bypass graft surgery: lack of synergy or evidence of antagonism in 
cardioprotection?

Anesthesiology

116 ,

296-310.

doi:10.1097/ALN.0b013e318242349a

Lu, L., Gunja-Smith, Z., Woessner, J.F., Ursell, P.C., Nissen, T., Galardy, R.E., Xu, Y., Zhu, P., Schwartz, G.G., 2000. Matrix metalloproteinases and collagen ultrastructure in moderate myocardial ischemia and reperfusion in vivo. Am. J. Physiol. Heart Circ. Physiol. 279, H601-609.

Luo, W., Zhu, M., Huang, R., Zhang, Y., 2011. A comparison of cardiac postconditioning and remote pre-conditioning in paediatric cardiac surgery. Cardiol Young 21, 266-70. doi:10.1017/S1047951110001915

Maekawa, Y., Mizue, N., Chan, A., Shi, Y., Liu, Y., Dawood, S., Chen, M., Dawood, F., de Couto, G., Li, G.H., Suzuki, N., Yeh, W.-C., Gramolini, A., Medin, J.A., Liu, P.P., 2009. Survival and cardiac remodeling after myocardial infarction are critically dependent on the host innate immune interleukin-1 receptor-associated kinase-4 signaling: a regulator of bone marrow-derived dendritic cells. Circulation 120, 1401-1414. doi:10.1161/CIRCULATIONAHA.109.865956

Ma, F.X., Han, Z.C., 2005. Statins, nitric oxide and neovascularization. Cardiovasc. Drug Rev. 23, 281-292.

Mahnke, Y.D., Roederer, M., 2007. Optimizing a Multi-colour Immunophenotyping Assay. Clin. Lab. Med. 27, 469-v. doi:10.1016/j.cll.2007.05.002

Manduteanu, I., Voinea, M., Antohe, F., Dragomir, E., Capraru, M., Radulescu, L., Simionescu, M., 2003. Effect of enoxaparin on high glucose-induced activation of endothelial cells. Eur. J. Pharmacol. 477, 269-276.

Mannhalter, J.W., Neychev, H.O., Zlabinger, G.J., Ahmad, R., Eibl, M.M., 1985. Modulation of the human immune response by the non-toxic and non-pyrogenic adjuvant aluminium hydroxide: effect on antigen uptake and antigen presentation. Clin. Exp. Immunol. 61, 143-151.

Martinez-Pellús, A.E., Merino, P., Bru, M., Canovas, J., Seller, G., Sapiña, J., Fuentes, T., Moro, J., 1997. Endogenous endotoxemia of intestinal origin during cardiopulmonary bypass. Role of type of flow and protective effect of selective digestive decontamination. Intensive Care Med. 23, 1251-1257.

Massberg, S., Grahl, L., von Bruehl, M.-L., Manukyan, D., Pfeiler, S., Goosmann, C., Brinkmann, V., Lorenz, M., Bidzhekov, K., Khandagale, A.B., Konrad, I., Kennerknecht, E., Reges, K., Holdenrieder, S., Braun, S., Reinhardt, C., Spannagl, M., Preissner, K.T., Engelmann, B., 2010. Reciprocal coupling of coagulation and innate immunity via neutrophil serine proteases. Nat. Med. 16, 887-896. doi: $10.1038 / \mathrm{nm} .2184$

Matzinger, P., 2002. The danger model: a renewed sense of self. Science 296, 301305. doi:10.1126/science.1071059

Maugeri, N., Brambilla, M., Camera, M., Carbone, A., Tremoli, E., Donati, M.B., de Gaetano, G., Cerletti, C., 2006. Human polymorphonuclear leukocytes produce and express functional tissue factor upon stimulation. J. Thromb. Haemost. JTH 4, 1323-1330. doi:10.1111/j.1538-7836.2006.01968.x

Maulik, N., Sato, M., Price, B.D., Das, D.K., 1998. An essential role of NFkB in tyrosine kinase signaling of p38 MAP kinase regulation of myocardial adaptation to ischemia. FEBS Lett. 429, 365-369. doi:10.1016/S0014-5793(98)00632-2 
Maurer, M., von Stebut, E., 2004. Macrophage inflammatory protein-1. Int. J. Biochem. Cell Biol. 36, 1882-1886. doi:10.1016/j.biocel.2003.10.019

McArdle, B.H., Anderson, M.J., 2001. Fitting Multivariate Models to Community Data: A Comment on Distance-Based Redundancy Analysis. Ecology 82, 290297. doi: $10.2307 / 2680104$

McBride, W.T., Armstrong, M.A., Crockard, A.D., McMurray, T.J., Rea, J.M., 1995. Cytokine balance and immunosuppressive changes at cardiac surgery: contrasting response between patients and isolated CPB circuits. Br. J. Anaesth. 75, 724-733. doi:10.1093/bja/75.6.724

McColl, S.R., St-Onge, M., Dussault, A.-A., Laflamme, C., Bouchard, L., Boulanger, J., Pouliot, M., 2006. Immunomodulatory impact of the A2A adenosine receptor on the profile of chemokines produced by neutrophils. FASEB J. Off. Publ. Fed. Am. Soc. Exp. Biol. 20, 187-189. doi:10.1096/fj.05-4804fje

McCrindle, B.W., Clarizia, N.A., Khaikin, S., Holtby, H.M., Manlhiot, C., Schwartz, S.M., Caldarone, C.A., Coles, J.G., Arsdell, G.S.V., Scherer, S.W., Redington, A.N., 2014. Remote Ischemic Preconditioning in Children Undergoing Cardiac Surgery With Cardiopulmonary Bypass: A Single-Center Double-Blinded Randomized Trial. J. Am. Heart Assoc. 3, e000964. doi:10.1161/JAHA.114.000964

McGuinness, J., Bouchier-Hayes, D., Redmond, J.M., 2008. Understanding the inflammatory response to cardiac surgery. Surgeon 6, 162-71.

Mersmann, J., Berkels, R., Zacharowski, P., Tran, N., Koch, A., Iekushi, K., Dimmeler, S., Granja, T.F., Boehm, O., Claycomb, W.C., Zacharowski, K., 2010. Preconditioning by toll-like receptor 2 agonist Pam3CSK4 reduces CXCL1dependent leukocyte recruitment in murine myocardial ischemia/reperfusion injury. Crit. Care Med. 38, 903-909. doi:10.1097/CCM.0b013e3181ce50e6

Meybohm, P., Renner, J., Broch, O., Caliebe, D., Albrecht, M., Cremer, J., Haake, N., Scholz, J., Zacharowski, K., Bein, B., 2013. Postoperative neurocognitive dysfunction in patients undergoing cardiac surgery after remote ischemic preconditioning: a double-blind randomized controlled pilot study. PloS One 8, e64743. doi:10.1371/journal.pone.0064743

Meybohm, P., Zacharowski, K., Cremer, J., Roesner, J., Kletzin, F., Schaelte, G., Felzen, M., Strouhal, U., Reyher, C., Heringlake, M., Schön, J., Brandes, I., Bauer, M., Knuefermann, P., Wittmann, M., Hachenberg, T., Schilling, T., Smul, T., Maisch, S., Sander, M., Moormann, T., Boening, A., Weigand, M.A., Laufenberg, R., Werner, C., Winterhalter, M., Treschan, T., Stehr, S.N., Reinhart, K., Hasenclever, D., Brosteanu, O., Bein, B., RIP Heart-Study Investigator Group, 2012. Remote ischaemic preconditioning for heart surgery. The study design for a multi-center randomized double-blinded controlled clinical trial--the RIPHeartStudy. Eur. Heart J. 33, 1423-1426.

Miller, B.E., Levy, J.H., 1997. The inflammatory response to cardiopulmonary bypass. J. Cardiothorac. Vasc. Anesth. 11, 355-366.

Ministry of Health, 2008. Cardiac Surgery Services in New Zealand - Cardiac Surgery Service Development Working Group Report. 
Modi, W.S., Yoshimura, T., 1999. Isolation of novel GRO genes and a phylogenetic analysis of the CXC chemokine subfamily in mammals. Mol. Biol. Evol. 16, 180193.

Mojsilovic-Petrovic, J., Callaghan, D., Cui, H., Dean, C., Stanimirovic, D.B., Zhang, W., 2007. Hypoxia-inducible factor-1 (HIF-1) is involved in the regulation of hypoxia-stimulated expression of monocyte chemoattractant protein-1 (MCP1/CCL2) and MCP-5 (Ccl12) in astrocytes. J. Neuroinflammation 4, 12. doi:10.1186/1742-2094-4-12

Monson, N.L., Ortega, S.B., Ireland, S.J., Meeuwissen, A.J., Chen, D., Plautz, E.J., Shubel, E., Kong, X., Li, M.K., Freriks, L.H., Stowe, A.M., 2014. Repetitive hypoxic preconditioning induces an immunosuppressed B cell phenotype during endogenous protection from stroke. J. Neuroinflammation 11, 22. doi:10.1186/1742-2094-11-22

Morgan, E.N., Boyle, E.M., Yun, W., Griscavage-Ennis, J.M., Farr, A.L., Canty, T.G., Pohlman, T.H., Verrier, E.D., 1999. An essential role for NF- $\kappa B$ in the cardioadaptive response to ischemia. Ann. Thorac. Surg. 68, 377-382. doi:10.1016/S0003-4975(99)00646-3

Morita, S., 2011. Remote ischemic preconditioning. -Is it time to introduce it in clinical practice? Circ J 75, 1821-2.

Moskowitz, M.A., Waeber, C., 2011. Remote ischemic preconditioning: making the brain more tolerant, safely and inexpensively. Circulation 123, 709-711. doi:10.1161/CIRCULATIONAHA.110.009688

Murali-Krishna, K., Altman, J.D., Suresh, M., Sourdive, D.J.D., Zajac, A.J., Miller, J.D., Slansky, J., Ahmed, R., 1998. Counting Antigen-Specific CD8 T Cells: A Reevaluation of Bystander Activation during Viral Infection. Immunity 8, 177187. doi:10.1016/S1074-7613(00)80470-7

Muris, A.-H., Damoiseaux, J., Smolders, J., Cohen Tervaert, J.W., Hupperts, R., Thewissen, M., 2012. Intracellular IL-10 detection in T cells by flowcytometry: the use of protein transport inhibitors revisited. J. Immunol. Methods 381, 59-65. doi:10.1016/j.jim.2012.04.011

Murry, C.E., Jennings, R.B., Reimer, K.A., 1986. Preconditioning with ischemia: a delay of lethal cell injury in ischemic myocardium. Circulation 74, 1124-36.

Naamani, O., Chaimovitz, C., Douvdevani, A., 2014. Pharmacological preconditioning with adenosine $\mathrm{A}(1)$ receptor agonist suppresses cellular immune response by an $\mathrm{A}(2 \mathrm{~A})$ receptor dependent mechanism. Int. Immunopharmacol. 20, 205-212. doi:10.1016/j.intimp.2014.02.011

Nahrendorf, M., Swirski, F.K., Aikawa, E., Stangenberg, L., Wurdinger, T., Figueiredo, J.-L., Libby, P., Weissleder, R., Pittet, M.J., 2007. The healing myocardium sequentially mobilizes two monocyte subsets with divergent and complementary functions. J. Exp. Med. 204, 3037-3047. doi:10.1084/jem.20070885

Nashef, S.A., Roques, F., Michel, P., Gauducheau, E., Lemeshow, S., Salamon, R., 1999. European system for cardiac operative risk evaluation (EuroSCORE). Eur J Cardiothorac Surg 16, 9-13. 
Neckár, J., Ostádal, B., Kolár, F., 2004. Myocardial infarct size-limiting effect of chronic hypoxia persists for five weeks of normoxic recovery. Physiol. Res. Acad. Sci. Bohemoslov. 53, 621-628.

Nesher, N., Alghamdi, A.A., Singh, S.K., Sever, J.Y., Christakis, G.T., Goldman, B.S., Cohen, G.N., Moussa, F., Fremes, S.E., 2008. Troponin after Cardiac Surgery: A Predictor or a Phenomenon? Ann. Thorac. Surg. 85, 1348-1354. doi:10.1016/j.athoracsur.2007.12.077

Ngaage, D.L., Britchford, G., Cale, A.R.J., 2011. The influence of an ageing population on care and clinical resource utilisation in cardiac surgery $\mid$ The British Journal of Cardiology. Br. J. Cardiol. 18, 28-32.

Nishida, M., Maruyama, Y., Tanaka, R., Kontani, K., Nagao, T., Kurose, H., 2000. G alpha(i) and $\mathrm{G}$ alpha(o) are target proteins of reactive oxygen species. Nature 408, 492-495. doi:10.1038/35044120

Node, K., Fujita, M., Kitakaze, M., Hori, M., Liao, J.K., 2003. Short-term statin therapy improves cardiac function and symptoms in patients with idiopathic dilated cardiomyopathy. Circulation $\quad 108, \quad 839-43$. doi:10.1161/01.CIR.0000084539.58092.DE

Nomura, L., Maino, V.C., Maecker, H.T., 2008. Standardization and optimization of multiparameter intracellular cytokine staining. Cytom. Part J. Int. Soc. Anal. Cytol. 73, 984-991. doi:10.1002/cyto.a.20602

O’Brien, M.C., Bolton, W.E., 1995. Comparison of cell viability probes compatible with fixation and permeabilization for combined surface and intracellular staining in flow cytometry. Cytometry 19, 243-255. doi:10.1002/cyto.990190308

Oettl, K., Marsche, G., 2010. Chapter 11 - Redox State of Human Serum Albumin in Terms of Cysteine-34 in Health and Disease, in: Enrique Cadenas and Lester Packer (Ed.), Methods in Enzymology, Thiol Redox Transitions in Cell Signaling, Part B: Cellular Localization and Signaling. Academic Press, pp. 181-195.

Ohara, J., Paul, W.E., 1988. Up-regulation of interleukin 4/B-cell stimulatory factor 1 receptor expression. Proc. Natl. Acad. Sci. U. S. A. 85, 8221-8225.

Ohashi, K., Burkart, V., Flohé, S., Kolb, H., 2000. Cutting edge: heat shock protein 60 is a putative endogenous ligand of the toll-like receptor-4 complex. J. Immunol. Baltim. Md 1950 164, 558-561.

Ohnuma, Y., Miura, T., Miki, T., Tanno, M., Kuno, A., Tsuchida, A., Shimamoto, K., 2002. Opening of mitochondrial K(ATP) channel occurs downstream of PKCepsilon activation in the mechanism of preconditioning. Am. J. Physiol. Heart Circ. Physiol. 283, H440-447. doi:10.1152/ajpheart.00434.2001

Okamura, T., Miura, T., Iwamoto, H., Shirakawa, K., Kawamura, S., Ikeda, Y., Iwatate, M., Matsuzaki, M., 1999. Ischemic preconditioning attenuates apoptosis through protein kinase C in rat hearts. Am. J. Physiol. 277, H1997-2001.

Olguner, C., Koca, U., Kar, A., Karci, A., Islekel, H., Canyilmaz, M., Mavioglu, O., Kizildag, S., Uenlue, G., Elar, Z., 2006. Ischemic preconditioning attenuates the lipid peroxidation and remote lung injury in the rat model of unilateral lower limb ischemia reperfusion. Acta Anaesthesiol. Scand. 50, 150-155. doi:http://dx.doi.org.helicon.vuw.ac.nz/10.1111/j.1399-6576.2006.00938.x 
Ong, S.-B., Samangoueia, P., Kalkhorana, S.B., Hausenloya, D.J., 2014. The mitochondrial permeability transition pore and its role in myocardial ischemia reperfusion injury. J. Mol. Cell. Cardiol. doi:10.1016/j.yjmcc.2014.11.005

Onorati, F., Santini, F., Dandale, R., Ucci, G., Pechlivanidis, K., Menon, T., Chiominto, B., Mazzucco, A., Faggian, G., 2013. "Polarizing" microplegia improves cardiac cycle efficiency after CABG for unstable angina. Int. J. Cardiol. 167, 2739-2746. doi:10.1016/j.ijcard.2012.06.099

Ortega, C., Fernández-A, S., Carrillo, J.M., Romero, P., Molina, I.J., Moreno, J.C., Santamaría, M., 2009. IL-17-producing CD8+ T lymphocytes from psoriasis skin plaques are cytotoxic effector cells that secrete Th17-related cytokines. J. Leukoc. Biol. 86, 435-443. doi:10.1189/JLB.0109046

Oxman, T., Arad, M., Klein, R., Avazov, N., Rabinowitz, B., 1997. Limb ischemia preconditions the heart against reperfusion tachyarrhythmia. Am J Physiol 273, H1707-12.

Oyama, J., Blais, C., Liu, X., Pu, M., Kobzik, L., Kelly, R.A., Bourcier, T., 2004. Reduced Myocardial Ischemia-Reperfusion Injury in Toll-Like Receptor 4$\begin{array}{llll}\text { Deficient } & \text { Mice. } & \text { Circulation } & \text { 784-789. }\end{array}$ doi:10.1161/01.CIR.0000112575.66565.84

Pain, T., Yang, X.M., Critz, S.D., Yue, Y., Nakano, A., Liu, G.S., Heusch, G., Cohen, M.V., Downey, J.M., 2000. Opening of mitochondrial K(ATP) channels triggers the preconditioned state by generating free radicals. Circ. Res. 87, 460-466.

Pandiyan, P., Conti, H.R., Zheng, L., Peterson, A.C., Mathern, D.R., HernandezSantos, N., Edgerton, M., Gaffen, S.L., Lenardo, M.J., 2011. CD4+ CD25+ Foxp3 + regulatory $\mathrm{T}$ cells promote Th17 cells in vitro and enhance host resistance in mouse Candida albicans Th17 cell infection model. Immunity 34, 422-434. doi:10.1016/j.immuni.2011.03.002

Pangburn, M.K., Atkinson, M.A., Meri, S., 1991. Localization of the heparin-binding site on complement factor H. J. Biol. Chem. 266, 16847-16853.

Pang, T., Zhao, Y., Zhang, N.-R., Jin, S.-Q., Pan, S.-Q., 2013. Transient limb ischemia alters serum protein expression in healthy volunteers: complement $\mathrm{C} 3$ and vitronectin may be involved in organ protection induced by remote ischemic preconditioning. Oxid. Med. Cell. Longev. 2013, 859056. doi:10.1155/2013/859056

Paparella, D., Yau, T.M., Young, E., 2002. Cardiopulmonary bypass induced inflammation: pathophysiology and treatment. An update. Eur. J. Cardiothorac. Surg. 21, 232-244.

Park, J., Ann, S.H., Chung, H.C., Lee, J.S., Kim, S.-J., Garg, S., Shin, E.-S., 2014. Remote ischemic preconditioning in hemodialysis: a pilot study. Heart Vessels 29, 58-64. doi:10.1007/s00380-013-0329-y

Park, J.L., Kilgore, K.S., Naylor, K.B., Booth, E.A., Murphy, K.L., Lucchesi, B.R., 1999. N-Acetylheparin Pretreatment Reduces Infarct Size in the Rabbit. Pharmacology 58, 120-131. doi:10.1159/000028274

Park, J.S., Svetkauskaite, D., He, Q., Kim, J.-Y., Strassheim, D., Ishizaka, A., Abraham, E., 2004. Involvement of toll-like receptors 2 and 4 in cellular activation 
by high mobility group box 1 protein. J. Biol. Chem. 279, 7370-7377. doi:10.1074/jbc.M306793200

Park, K.M., Byun, J.-Y., Kramers, C., Kim, J.I., Huang, P.L., Bonventre, J.V., 2003. Inducible nitric-oxide synthase is an important contributor to prolonged protective effects of ischemic preconditioning in the mouse kidney. J. Biol. Chem. 278, 27256-27266. doi:10.1074/jbc.M301778200

Parks, W.C., Wilson, C.L., López-Boado, Y.S., 2004. Matrix metalloproteinases as modulators of inflammation and innate immunity. Nat. Rev. Immunol. 4, 617-629. doi: $10.1038 /$ nri1418

Parolari, A., Pesce, L.L., Trezzi, M., Loardi, C., Kassem, S., Brambillasca, C., Miguel, B., Tremoli, E., Biglioli, P., Alamanni, F., 2009. Performance of EuroSCORE in CABG and off-pump coronary artery bypass grafting: single institution experience and meta-analysis. Eur. Heart J. 30, 297-304. doi:10.1093/eurheartj/ehn581

Partridge, J.S.L., Harari, D., Dhesi, J.K., 2012. Frailty in the older surgical patient: a review. Age Ageing 41, 142-147. doi:10.1093/ageing/afr182

Patel, H.H., Moore, J., Hsu, A.K., Gross, G.J., 2002. Cardioprotection at a distance: mesenteric artery occlusion protects the myocardium via an opioid sensitive mechanism. J. Mol. Cell. Cardiol. 34, 1317-1323.

Pavione, M.A., Carmona, F., de Castro, M., Carlotti, A.P., 2012. Late remote ischemic preconditioning in children undergoing cardiopulmonary bypass: A randomized controlled trial. J Thorac Cardiovasc Surg. doi:10.1016/j.jtcvs.2011.12.029

Pedersen, C.M., Cruden, N.L., Schmidt, M.R., Lau, C., Botker, H.E., Kharbanda, R.K., Newby, D.E., 2011. Remote ischemic preconditioning prevents systemic platelet activation associated with ischemia-reperfusion injury in humans. J Thromb Haemost 9, 404-7. doi:10.1111/j.1538-7836.2010.04142.x

Pedersen, C.M., Schmidt, M.R., Barnes, G., Bøtker, H.E., Kharbanda, R.K., Newby, D.E., Cruden, N.L., 2011. Bradykinin does not mediate remote ischaemic preconditioning or ischaemia-reperfusion injury in vivo in man. Heart Br. Card. Soc. 97, 1857-1861. doi:10.1136/heartjnl-2011-300323

Pedersen, K.R., Ravn, H.B., Povlsen, J.V., Schmidt, M.R., Erlandsen, E.J., Hjortdal, V.E., 2012. Failure of remote ischemic preconditioning to reduce the risk of postoperative acute kidney injury in children undergoing operation for complex congenital heart disease: a randomized single-center study. J. Thorac. Cardiovasc. Surg. 143, 576-583. doi:10.1016/j.jtcvs.2011.08.044

Pell, T.J., Baxter, G.F., Yellon, D.M., Drew, G.M., 1998. Renal ischemia preconditions myocardium: role of adenosine receptors and ATP-sensitive potassium channels. Am. J. Physiol. 275, H1542-1547.

Peng, T.-C., Jan, W.-C., Tsai, P.-S., Huang, C.-J., 2011. Heme oxygenase-1 mediates the protective effects of ischemic preconditioning on mitigating lung injury induced by lower limb ischemia-reperfusion in rats. J. Surg. Res. 167, e245-253. doi:10.1016/j.jss.2010.06.010

Pepe, S., Liaw, N.Y., Hepponstall, M., Sheeran, F.L., Yong, M.S., d' Udekem, Y., Cheung, M.M., Konstantinov, I.E., 2013. Effect of remote ischemic 
preconditioning on phosphorylated protein signaling in children undergoing tetralogy of Fallot repair: a randomized controlled trial. J. Am. Heart Assoc. 2, e000095. doi:10.1161/JAHA.113.000095

Petrishchev, N.N., Vlasov, T.D., Sipovsky, V.G., Kurapeev, D.I., Galagudza, M.M., 2001. Does nitric oxide generation contribute to the mechanism of remote ischemic preconditioning? Pathophysiol. Off. J. Int. Soc. Pathophysiol. ISP 7, 271-274.

Pfister, R.R., Haddox, J.L., Dodson, R.W., Deshazo, W.F., 1984. Polymorphonuclear leukocytic inhibition by citrate, other metal chelators, and trifluoperazine. Evidence to support calcium binding protein involvement. Invest. Ophthalmol. Vis. Sci. 25, 955-970.

Pfistershammer, K., Majdic, O., Stöckl, J., Zlabinger, G., Kirchberger, S., Steinberger, P., Knapp, W., 2004. CD63 as an activation-linked T cell costimulatory element. J. Immunol. Baltim. Md 1950 173, 6000-6008.

Pigott, R., Dillon, L.P., Hemingway, I.H., Gearing, A.J.H., 1992. Soluble forms of Eselectin, ICAM-1 and VCAM-1 are present in the supernatants of cytokine activated cultured endothelial cells. Biochem. Biophys. Res. Commun. 187, 584 589. doi:10.1016/0006-291X(92)91234-H

Pilcher, J.M., Young, P., Weatherall, M., Rahman, I., Bonser, R.S., Beasley, R.W., 2012. A systematic review and meta-analysis of the cardioprotective effects of remote ischaemic preconditioning in open cardiac surgery. J. R. Soc. Med. 105, 436-445. doi:10.1258/jrsm.2012.120049

Pillay, J., den Braber, I., Vrisekoop, N., Kwast, L.M., de Boer, R.J., Borghans, J.A.M., Tesselaar, K., Koenderman, L., 2010. In vivo labeling with $2 \mathrm{H} 2 \mathrm{O}$ reveals a human neutrophil lifespan of 5.4 days. Blood 116, 625-627. doi:10.1182/blood2010-01-259028

Piriou, V., Chiari, P., Gateau-Roesch, O., Argaud, L., Muntean, D., Salles, D., Loufouat, J., Gueugniaud, P.-Y., Lehot, J.-J., Ovize, M., 2004. Desflurane-induced preconditioning alters calcium-induced mitochondrial permeability transition. Anesthesiology 100, 581-588.

Plow, E.F., 1986. The contribution of leukocyte proteases to fibrinolysis. Blut 53, 19.

Pradillo, J.M., Fernández-López, D., García-Yébenes, I., Sobrado, M., Hurtado, O., Moro, M.A., Lizasoain, I., 2009. Toll-like receptor 4 is involved in neuroprotection afforded by ischemic preconditioning. J. Neurochem. 109, 287-294. doi:10.1111/j.1471-4159.2009.05972.x

Przyklenk, K., Bauer, B., Ovize, M., Kloner, R.A., Whittaker, P., 1993. Regional ischemic "preconditioning" protects remote virgin myocardium from subsequent sustained coronary occlusion. Circulation 87, 893-9.

Rábai, G., Szilágyi, N., Sótonyi, P., Kovalszky, I., Szabó, L., Machovich, R., Kolev, K., 2010. Contribution of neutrophil elastase to the lysis of obliterative thrombi in the context of their platelet and fibrin content. Thromb. Res. 126, e94-101. doi:10.1016/j.thromres.2010.05.007

Rabb, H., Daniels, F., O’Donnell, M., Haq, M., Saba, S.R., Keane, W., Tang, W.W., 2000. Pathophysiological role of $\mathrm{T}$ lymphocytes in renal ischemia-reperfusion injury in mice. Am. J. Physiol. Renal Physiol. 279, F525-531. 
Rabb, H., Mendiola, C.C., Saba, S.R., Dietz, J.R., Smith, C.W., Bonventre, J.V., Ramirez, G., 1995. Antibodies to ICAM-1 protect kidneys in severe ischemic reperfusion injury. Biochem. Biophys. Res. Commun. 211, 67-73. doi:10.1006/bbrc.1995.1779

Radaeva, S., Sun, R., Pan, H., Hong, F., Gao, B., 2004. Interleukin 22 (IL-22) plays a protective role in T cell-mediated murine hepatitis: IL-22 is a survival factor for hepatocytes via STAT3 activation. Hepatology 39, 1332-1342. doi:10.1002/hep.20184

Rahman, I.A., Mascaro, J.G., Steeds, R.P., Frenneaux, M.P., Nightingale, P., Gosling, P., Townsend, P., Townend, J.N., Green, D., Bonser, R.S., 2010. Remote ischemic preconditioning in human coronary artery bypass surgery: from promise to disappointment? Circulation $\quad 122, \quad$ S53-9. doi:10.1161/CIRCULATIONAHA.109.926667

Rahman, I., Bonser, R.S., 2009. Remote ischaemic preconditioning: the current best hope for improved myocardial protection in cardiac surgery? Heart 95, 1553-5. doi:10.1136/hrt.2008.159988

Raphael, J., Abedat, S., Rivo, J., Meir, K., Beeri, R., Pugatsch, T., Zuo, Z., Gozal, Y., 2006. Volatile anesthetic preconditioning attenuates myocardial apoptosis in rabbits after regional ischemia and reperfusion via Akt signaling and modulation of Bcl-2 family proteins. J. Pharmacol. Exp. Ther. 318, 186-194. doi:10.1124/jpet.105.100537

Rehni, A.K., Singh, N., Jaggi, A.S., 2007. Possible involvement of insulin, endogenous opioids and calcitonin gene-related peptide in remote ischaemic preconditioning of the brain. Yakugaku Zasshi 127, 1013-1020.

Remote Preconditioning Trialists' Group, Healy, D.A., Khan, W.A., Wong, C.S., Moloney, M.C., Grace, P.A., Coffey, J.C., Dunne, C., Walsh, S.R., Sadat, U., Gaunt, M.E., Chen, S., Tehrani, S., Hausenloy, D.J., Yellon, D.M., Kramer, R.S., Zimmerman, R.F., Lomivorotov, V.V., Shmyrev, V.A., Ponomarev, D.N., Rahman, I.A., Mascaro, J.G., Bonser, R.S., Jeon, Y., Hong, D.M., Wagner, R., Thielmann, M., Heusch, G., Zacharowski, K., Meybohm, P., Bein, B., Tang, T.Y., 2014. Remote preconditioning and major clinical complications following adult cardiovascular surgery: Systematic review and meta-analysis. Int. J. Cardiol. 176, 20-31. doi:10.1016/j.ijcard.2014.06.018

Ren, X., Wang, Y., Jones, W.K., 2004. TNF-alpha is required for late ischemic preconditioning but not for remote preconditioning of trauma. J. Surg. Res. 121, 120-129. doi:10.1016/j.jss.2004.03.010

Repo, H., Jansson, S.E., Leirisalo-Repo, M., 1995. Anticoagulant selection influences flow cytometric determination of $\mathrm{CD} 11 \mathrm{~b}$ upregulation in vivo and ex vivo. J. Immunol. Methods 185, 65-79.

Rezkalla, S.H., Kloner, R.A., 2002. No-Reflow Phenomenon. Circulation 105, 656662. doi:10.1161/hc0502.102867

Riad, A., Jäger, S., Sobirey, M., Escher, F., Yaulema-Riss, A., Westermann, D., Karatas, A., Heimesaat, M.M., Bereswill, S., Dragun, D., Pauschinger, M., Schultheiss, H.P., Tschöpe, C., 2008. Toll-like receptor-4 modulates survival by induction of left ventricular remodeling after myocardial infarction in mice. J. Immunol. Baltim. Md 1950 180, 6954-6961. 
Riddle, J.M., Barnhart, M.I., 1964. ULTRASTRUCTURAL STUDY OF FIBRIN DISSOLUTION VIA EMIGRATED POLYMORPHONUCLEAR NEUTROPHILS. Am. J. Pathol. 45, 805-823.

Rimaniol, A.-C., Gras, G., Verdier, F., Capel, F., Grigoriev, V.B., Porcheray, F., Sauzeat, E., Fournier, J.-G., Clayette, P., Siegrist, C.-A., Dormont, D., 2004. Aluminum hydroxide adjuvant induces macrophage differentiation towards a specialized antigen-presenting cell type. Vaccine 22, 3127-3135. doi:10.1016/j.vaccine.2004.01.061

Risnes, I., Ueland, T., Lundblad, R., Mollnes, T.E., Baksaas, S.T., Aukrust, P., Svennevig, J.L., 2003. Changes in the cytokine network and complement parameters during open heart surgery. Interact. Cardiovasc. Thorac. Surg. 2, 19 24. doi:10.1016/S1569-9293(02)00088-9

Rizvi, A., Tang, X.L., Qiu, Y., Xuan, Y.T., Takano, H., Jadoon, A.K., Bolli, R., 1999. Increased protein synthesis is necessary for the development of late preconditioning against myocardial stunning. Am. J. Physiol. 277, H874-884.

Rock-Willoughby, J., Boardley, D., Badenhop, D.T., Tinkel, J., 2013. Vigorous Exercise Mimics Remote Ischemic Preconditioning and Provides Benefit in Cardiac Rehabilitation Patients. J. Clin. Exp. Cardiol.

Roques, F., Michel, P., Goldstone, A.R., Nashef, S. a. M., 2003. The logistic EuroSCORE. Eur. Heart J. 24, 882-882. doi:10.1016/S0195-668X(02)00799-6

Rosenson, R.S., Tangney, C.C., Casey, L.C., 1999. Inhibition of proinflammatory cytokine production by pravastatin. Lancet 353, 983-4. doi:10.1016/S01406736(98)05917-0

Rosner, M.H., Okusa, M.D., 2006. Acute kidney injury associated with cardiac surgery. Clin. J. Am. Soc. Nephrol. CJASN 1, 19-32. doi:10.2215/CJN.00240605

Rutz, S., Eidenschenk, C., Ouyang, W., 2013. IL-22, not simply a Th17 cytokine. Immunol. Rev. 252, 116-132. doi:10.1111/imr.12027

Sabroe, I., Jones, E.C., Usher, L.R., Whyte, M.K.B., Dower, S.K., 2002. Toll-like receptor (TLR) 2 and TLR4 in human peripheral blood granulocytes: a critical role for monocytes in leukocyte lipopolysaccharide responses. J. Immunol. Baltim. Md $1950168,4701-4710$.

Sadat, U., 2009. Signaling pathways of cardioprotective ischemic preconditioning. Int. J. Surg. Lond. Engl. 7, 490-498. doi:10.1016/j.ijsu.2009.06.004

Sadler, P.J., Tucker, A., Viles, J.H., 1994. Involvement of a lysine residue in the Nterminal $\mathrm{Ni} 2+$ and $\mathrm{Cu} 2+$ binding site of serum albumins. Comparison with $\mathrm{Co} 2+$, $\mathrm{Cd} 2+$ and Al3+. Eur. J. Biochem. FEBS 220, 193-200.

Salmon, J.E., Cronstein, B.N., 1990. Fc gamma receptor-mediated functions in neutrophils are modulated by adenosine receptor occupancy. A1 receptors are stimulatory and A2 receptors are inhibitory. J. Immunol. Baltim. Md 1950 145, 2235-2240.

Satpute, S.R., Park, J.M., Jang, H.R., Agreda, P., Liu, M., Gandolfo, M.T., Racusen, L., Rabb, H., 2009. The role for $\mathrm{T}$ cell repertoire/antigen-specific interactions in experimental kidney ischemia reperfusion injury. J. Immunol. Baltim. Md 1950 183, 984-992. doi:10.4049/jimmunol.0801928 
Saurim, R., Koike, M.K., Bonservizi, W.G.S., Felix, G. a. A., Silva, S.M., Taha, M.O., Montero, E.F.S., 2014. Cardiac effect of ischemic preconditioning and heparin following intestinal ischemia and reperfusion in rats. Transplant. Proc. 46, 1852-1856. doi:10.1016/j.transproceed.2014.05.055

Savransky, V., Molls, R.R., Burne-Taney, M., Chien, C.-C., Racusen, L., Rabb, H., 2006. Role of the T-cell receptor in kidney ischemia-reperfusion injury. Kidney Int. 69, 233-238. doi:10.1038/sj.ki.5000038

Saxena, P., Aggarwal, S., Misso, N.L., Passage, J., Newman, M.A.J., Thompson, P.J., d' Udekem, Y., Praporski, S., Konstantinov, I.E., 2013. Remote ischaemic preconditioning down-regulates kinin receptor expression in neutrophils of patients undergoing heart surgery. Interact. Cardiovasc. Thorac. Surg. doi:10.1093/icvts/ivt279

Saxena, P., Newman, M.A., Shehatha, J.S., Redington, A.N., Konstantinov, I.E., 2010a. Remote ischemic conditioning: evolution of the concept, mechanisms, and clinical application. J Card Surg 25, 127-34. doi:10.1111/j.15408191.2009.00820.x

Saxena, P., Shaw, O.M., Misso, N.L., Naran, A., Shehatha, J., Newman, M.A., d' Udekem, Y., Thompson, P.J., Konstantinov, I.E., 2010b. Remote ischemic preconditioning stimulus decreases the expression of kinin receptors in human neutrophils. J Surg Res 171, 311-6. doi:10.1016/j.jss.2009.11.011

Sbarouni, E., Georgiadou, P., Kremastinos, D.T., Voudris, V., 2008. Ischemia modified albumin: is this marker of ischemia ready for prime time use? Hell. J. Cardiol. HJC Hellēnikē Kardiologikē Epitheōrēsē 49, 260-266.

Scaffidi, P., Misteli, T., Bianchi, M.E., 2002. Release of chromatin protein HMGB1 by necrotic cells triggers inflammation. Nature 418, 191-195. doi:10.1038/nature00858

Schindler, C., Levy, D.E., Decker, T., 2007. JAK-STAT Signaling: From Interferons to Cytokines. J. Biol. Chem. 282, 20059-20063. doi:10.1074/jbc.R700016200

Schoemaker, R.G., van Heijningen, C.L., 2000. Bradykinin mediates cardiac preconditioning at a distance. Am J Physiol Heart Circ Physiol 278, H1571-6.

Schwartz Longacre, L., Kloner, R.A., Arai, A.E., Baines, C.P., Bolli, R., Braunwald, E., Downey, J., Gibbons, R.J., Gottlieb, R.A., Heusch, G., Jennings, R.B., Lefer, D.J., Mentzer, R.M., Murphy, E., Ovize, M., Ping, P., Przyklenk, K., Sack, M.N., Vander Heide, R.S., Vinten-Johansen, J., Yellon, D.M., National Heart, Lung, and Blood Institute, National Institutes of Health, 2011. New horizons in cardioprotection: recommendations from the 2010 National Heart, Lung, and Blood Institute Workshop. Circulation 124, 1172-1179. doi:10.1161/CIRCULATIONAHA.111.032698

Sedlmayr, P., Leitner, V., Pilz, S., Wintersteiger, R., Stewart, C.C., Dohr, G., 2001. Species-Specific Blocking of $\mathrm{Fc}$ Receptors in Indirect Immunofluorescence Assays. Lab Hematol 7, 81-4.

Sengeløv, H., Kjeldsen, L., Borregaard, N., 1993. Control of exocytosis in early neutrophil activation. J. Immunol. Baltim. Md 1950 150, 1535-1543.

Serejo, F.C., Rodrigues, L.F., da Silva Tavares, K.C., de Carvalho, A.C.C., Nascimento, J.H.M., 2007. Cardioprotective properties of humoral factors released 
from rat hearts subject to ischemic preconditioning. J. Cardiovasc. Pharmacol. 49, 214-220. doi:10.1097/FJC.0b013e3180325ad9

Sharma, A.K., LaPar, D.J., Zhao, Y., Li, L., Lau, C.L., Kron, I.L., Iwakura, Y., Okusa, M.D., Laubach, V.E., 2011. Natural killer T cell-derived IL-17 mediates lung ischemia-reperfusion injury. Am. J. Respir. Crit. Care Med. 183, 1539-1549. doi:10.1164/rccm.201007-1173OC

Shen, X.-D., Ke, B., Zhai, Y., Amersi, F., Gao, F., Anselmo, D.M., Busuttil, R.W., Kupiec-Weglinski, J.W., 2002. CD154-CD40 T-cell costimulation pathway is required in the mechanism of hepatic ischemia/reperfusion injury, and its blockade facilitates and depends on heme oxygenase-1 mediated cytoprotection. Transplantation 74, 315-319.

Shen, X., Wang, Y., Gao, F., Ren, F., Busuttil, R.W., Kupiec-Weglinski, J.W., Zhai, Y., 2009. CD4 T cells promote liver inflammation/injury by reverse signaling via CD40 without de novo activation. Hepatol. Baltim. Md 50, 1537-1546. doi:10.1002/hep.23153

Shichita, T., Sugiyama, Y., Ooboshi, H., Sugimori, H., Nakagawa, R., Takada, I., Iwaki, T., Okada, Y., Iida, M., Cua, D.J., Iwakura, Y., Yoshimura, A., 2009. Pivotal role of cerebral interleukin-17-producing gammadeltaT cells in the delayed phase of ischemic brain injury. Nat. Med. 15, 946-950. doi:10.1038/nm.1999

Shi, C., Pamer, E.G., 2011. Monocyte recruitment during infection and inflammation. Nat. Rev. Immunol. 11, 762-774. doi:10.1038/nri3070

Shimamoto, A., Chong, A.J., Yada, M., Shomura, S., Takayama, H., Fleisig, A.J., Agnew, M.L., Hampton, C.R., Rothnie, C.L., Spring, D.J., Pohlman, T.H., Shimpo, H., Verrier, E.D., 2006. Inhibition of Toll-like receptor 4 with eritoran attenuates myocardial ischemia-reperfusion injury. Circulation 114, I270-274. doi:10.1161/CIRCULATIONAHA.105.000901

Shimizu, M., Konstantinov, I.E., Kharbanda, R.K., Cheung, M.H., Redington, A.N., 2007. Effects of intermittent lower limb ischaemia on coronary blood flow and coronary resistance in pigs. Acta Physiol. Oxf. Engl. 190, 103-109. doi:10.1111/j.1748-1716.2007.01667.x

Shimizu, M., Saxena, P., Konstantinov, I.E., Cherepanov, V., Cheung, M.M., Wearden, P., Zhangdong, H., Schmidt, M., Downey, G.P., Redington, A.N., 2010. Remote ischemic preconditioning decreases adhesion and selectively modifies functional responses of human neutrophils. J Surg Res 158, 155-61. doi:10.1016/j.jss.2008.08.010

Shimizu, M., Tropak, M., Diaz, R.J., Suto, F., Surendra, H., Kuzmin, E., Li, J., Gross, G., Wilson, G.J., Callahan, J., Redington, A.N., 2009. Transient limb ischaemia remotely preconditions through a humoral mechanism acting directly on the myocardium: evidence suggesting cross-species protection. Clin. Sci. Lond. Engl. 1979 117, 191-200. doi:10.1042/CS20080523

Shishido, T., Nozaki, N., Yamaguchi, S., Shibata, Y., Nitobe, J., Miyamoto, T., Takahashi, H., Arimoto, T., Maeda, K., Yamakawa, M., Takeuchi, O., Akira, S., Takeishi, Y., Kubota, I., 2003. Toll-like receptor-2 modulates ventricular remodeling after myocardial infarction. Circulation 108, 2905-2910. doi:10.1161/01.CIR.0000101921.93016.1C 
Simard, J.-C., Girard, D., Tessier, P.A., 2010. Induction of neutrophil degranulation by S100A9 via a MAPK-dependent mechanism. J. Leukoc. Biol. 87, 905-914. doi:10.1189/jlb.1009676

Simkhovich, B.Z., Przyklenk, K., Kloner, R.A., 2013. Role of protein kinase C in ischemic "conditioning": from first evidence to current perspectives. J. Cardiovasc. Pharmacol. Ther. 18, 525-532. doi:10.1177/1074248413494814

Simón, C., Vara, E., Garutti, I., González-Casaurrán, G., Azcárate, L., Isea, J., Huerta, L., González-Aragoneses, F., 2012. Modulation of monocyte chemoattractant protein-1 expression by ischaemic preconditioning in a lung autotransplant model. Eur. J. Cardio-Thorac. Surg. Off. J. Eur. Assoc. Cardio-Thorac. Surg. 41, 933-939. doi:10.1093/ejcts/ezr049

Simonian, P.L., Wehrmann, F., Roark, C.L., Born, W.K., O’Brien, R.L., Fontenot, A.P., 2010. $\gamma \delta \mathrm{T}$ cells protect against lung fibrosis via IL-22. J. Exp. Med. 207, 2239-2253. doi:10.1084/jem.20100061

Smith, J.M., Wahler, G.M., 1996. ATP-sensitive potassium channels are altered in ventricular myocytes from diabetic rats. Mol. Cell. Biochem. 158, 43-51.

Smith, R.A., Baglioni, C., 1987. The active form of tumor necrosis factor is a trimer. J. Biol. Chem. 262, 6951-6954.

Smith, R.M., Suleman, N., McCarthy, J., Sack, M.N., 2002. Classic ischemic but not pharmacologic preconditioning is abrogated following genetic ablation of the TNFalpha gene. Cardiovasc. Res. 55, 553-560.

Song, W., Furman, B.L., Parratt, J.R., 1996. Delayed protection against ischaemiainduced ventricular arrhythmias and infarct size limitation by the prior administration of Escherichia coli endotoxin. Br. J. Pharmacol. 118, 2157-2163.

Sonnenberg, G.F., Nair, M.G., Kirn, T.J., Zaph, C., Fouser, L.A., Artis, D., 2010. Pathological versus protective functions of IL-22 in airway inflammation are regulated by IL-17A. J. Exp. Med. 207, 1293-1305. doi:10.1084/jem.20092054

Steinberg, J.B., Kapelanski, D.P., Olson, J.D., Weiler, J.M., 1993. Cytokine and complement levels in patients undergoing cardiopulmonary bypass. J. Thorac. Cardiovasc. Surg. 106, 1008-1016.

Stossel, T.P., Field, R.J., Gitlin, J.D., Alper, C.A., Rosen, F.S., 1975. The opsonic fragment of the third component of human complement (C3). J. Exp. Med. 141, 1329-1347.

Stowe, A.M., Altay, T., Freie, A.B., Gidday, J.M., 2011. Repetitive hypoxia extends endogenous neurovascular protection for stroke. Ann. Neurol. 69, 975-985. doi:10.1002/ana.22367

Strainic, M.G., Liu, J., Huang, D., An, F., Lalli, P.N., Muqim, N., Shapiro, V.S., Dubyak, G.R., Heeger, P.S., Medof, M.E., 2008. Locally produced complement fragments $\mathrm{C} 5 \mathrm{a}$ and $\mathrm{C} 3$ a provide both costimulatory and survival signals to naive CD4+ T cells. Immunity 28, 425-435. doi:10.1016/j.immuni.2008.02.001

Strohm, C., Barancík, M., von Bruehl, M., Strniskova, M., Ullmann, C., Zimmermann, R., Schaper, W., 2002. Transcription inhibitor actinomycin-D abolishes the cardioprotective effect of ischemic reconditioning. Cardiovasc. Res. $55,602-618$. 
Stroka, D.M., Burkhardt, T., Desbaillets, I., Wenger, R.H., Neil, D. a. H., Bauer, C., Gassmann, M., Candinas, D., 2001. HIF-1 is expressed in normoxic tissue and displays an organ-specific regulation under systemic hypoxia. FASEB J. 15, 24452453. doi:10.1096/fj.01-0125com

Sullivan, P.J., Sweeney, K.J., Hirpara, K.M., Malone, C.B., Curtin, W., Kerin, M.J., 2009. Cyclical ischaemic preconditioning modulates the adaptive immune response in human limb ischaemia-reperfusion injury. Br J Surg 96, 381-90. doi:10.1002/bjs.6554

Surendra, H., Diaz, R.J., Harvey, K., Tropak, M., Callahan, J., Hinek, A., Hossain, T., Redington, A., Wilson, G.J., 2013. Interaction of $\delta$ and $\kappa$ opioid receptors with adenosine A1 receptors mediates cardioprotection by remote ischemic preconditioning. J. Mol. Cell. Cardiol. 60, 142-150. doi:10.1016/j.yjmcc.2013.04.010

Swaanenburg, J.C., Loef, B.G., Volmer, M., Boonstra, P.W., Grandjean, J.G., Mariani, M.A., Epema, A.H., 2001. Creatine kinase MB, troponin I, and troponin $\mathrm{T}$ release patterns after coronary artery bypass grafting with or without cardiopulmonary bypass and after aortic and mitral valve surgery. Clin Chem 47 , $584-7$.

Swank, G.M., Deitch, E.A., 1996. Role of the gut in multiple organ failure: bacterial translocation and permeability changes. World J Surg 20, 411-7.

Tähepõld, P., Vaage, J., Starkopf, J., Valen, G., 2003. Hyperoxia elicits myocardial protection through a nuclear factor $\kappa \mathrm{B}$-dependent mechanism in the rat heart. J. Thorac. Cardiovasc. Surg. 125, 650-660. doi:10.1067/mtc.2003.36

Takada, M., Chandraker, A., Nadeau, K.C., Sayegh, M.H., Tilney, N.L., 1997. The role of the B7 costimulatory pathway in experimental cold ischemia/reperfusion injury. J. Clin. Invest. 100, 1199-1203. doi:10.1172/JCI119632

Takagi, H., Umemoto, T., 2011. Remote ischemic preconditioning for cardiovascular surgery: an updated meta-analysis of randomized trials. Vasc. Endovascular Surg. 45, 511-513. doi:10.1177/1538574410379654

Takaoka, A., Nakae, I., Mitsunami, K., Yabe, T., Morikawa, S., Inubushi, T., Kinoshita, M., 1999. Renal ischemia/reperfusion remotely improves myocardial energy metabolism during myocardial ischemia via adenosine receptors in rabbits: effects of "remote preconditioning." J Am Coll Cardiol 33, 556-64.

Takayama, H., Soltow, L.O., Chandler, W.L., Vocelka, C.R., Aldea, G.S., 2007. Does the type of surgery effect systemic response following cardiopulmonary bypass? J Card Surg 22, 307-13. doi:10.1111/j.1540-8191.2007.00413.x

Takeshita, M., Tani, T., Harada, S., Hayashi, H., Itoh, H., Tajima, H., Ohnishi, I., Takamura, H., Fushida, S., Kayahara, M., 2010. Role of transcription factors in small intestinal ischemia-reperfusion injury and tolerance induced by ischemic preconditioning. Transplant. Proc. 42, 3406-3413. doi:10.1016/j.transproceed.2010.06.038

Tan, A.S., Berridge, M.V., 2000. Superoxide produced by activated neutrophils efficiently reduces the tetrazolium salt, WST-1 to produce a soluble formazan: a simple colorimetric assay for measuring respiratory burst activation and for screening anti-inflammatory agents. J. Immunol. Methods 238, 59-68. 
Tang, Z.L., Dai, W., Li, Y.J., Deng, H.W., 1999. Involvement of capsaicin-sensitive sensory nerves in early and delayed cardioprotection induced by a brief ischaemia of the small intestine. Naunyn. Schmiedebergs Arch. Pharmacol. 359, 243-247.

Tanhehco, E.J., Yasojima, K., McGeer, P.L., McGeer, E.G., Lucchesi, B.R., 2000. Preconditioning reduces myocardial complement gene expression in vivo. Am. J. Physiol. Heart Circ. Physiol. 279, H1157-1165.

Tanhehco, E.J., Yasojima, K., McGeer, P.L., Washington, R.A., Kilgore, K.S., Homeister, J.W., Lucchesi, B.R., 1999. Preconditioning reduces tissue complement gene expression in the rabbit isolated heart. Am. J. Physiol. 277, H2373-2380.

Tao, R., de Zoeten, E.F., Ozkaynak, E., Chen, C., Wang, L., Porrett, P.M., Li, B., Turka, L.A., Olson, E.N., Greene, M.I., Wells, A.D., Hancock, W.W., 2007. Deacetylase inhibition promotes the generation and function of regulatory $\mathrm{T}$ cells. Nat. Med. 13, 1299-1307. doi:10.1038/nm1652

Tapuria, N., Junnarkar, S.P., Dutt, N., Abu-Amara, M., Fuller, B., Seifalian, A.M., Davidson, B.R., 2009. Effect of remote ischemic preconditioning on hepatic microcirculation and function in a rat model of hepatic ischemia reperfusion injury. HPB 11, 108-117. doi:10.1111/j.1477-2574.2009.00006.x

Tapuria, N., Kumar, Y., Habib, M.M., Abu Amara, M., Seifalian, A.M., Davidson, B.R., 2008. Remote ischemic preconditioning: a novel protective method from ischemia reperfusion injury--a review. J Surg Res 150, 304-30. doi:10.1016/j.jss.2007.12.747

Teoh, L.K., Grant, R., Hulf, J.A., Pugsley, W.B., Yellon, D.M., 2002. The effect of preconditioning (ischemic and pharmacological) on myocardial necrosis following coronary artery bypass graft surgery. Cardiovasc Res 53, 175-80.

Thielmann, M., Kottenberg, E., Boengler, K., Raffelsieper, C., Neuhaeuser, M., Peters, J., Jakob, H., Heusch, G., 2010. Remote ischemic preconditioning reduces myocardial injury after coronary artery bypass surgery with crystalloid cardioplegic arrest. Basic Res Cardiol 105, 657-64. doi:10.1007/s00395-010-01045

Thielmann, M., Kottenberg, E., Kleinbongard, P., Wendt, D., Gedik, N., Pasa, S., Price, V., Tsagakis, K., Neuhäuser, M., Peters, J., Jakob, H., Heusch, G., 2013. Cardioprotective and prognostic effects of remote ischaemic preconditioning in patients undergoing coronary artery bypass surgery: a single-centre randomised, double-blind, controlled trial. Lancet 382, 597-604. doi:10.1016/S01406736(13)61450-6

Thiel, M., Chambers, J.D., Chouker, A., Fischer, S., Zourelidis, C., Bardenheuer, H.J., Arfors, K.E., Peter, K., 1996. Effect of adenosine on the expression of beta(2) integrins and L-selectin of human polymorphonuclear leukocytes in vitro. J. Leukoc. Biol. 59, 671-682.

Thourani, V.H., Brar, S.S., Kennedy, T.P., Thornton, L.R., Watts, J.A., Ronson, R.S., Zhao, Z.-Q., Sturrock, A.L., Hoidal, J.R., Vinten-Johansen, J., 2000. Nonanticoagulant heparin inhibits NF- $\mathrm{KB}$ activation and attenuates myocardial reperfusion injury. Am. J. Physiol. - Heart Circ. Physiol. 278, H2084-H2093.

Thygesen, K., Alpert, J.S., White, H.D., Jaffe, A.S., Apple, F.S., Galvani, M., Katus, H.A., Newby, L.K., Ravkilde, J., Chaitman, B., Clemmensen, P.M., Dellborg, M., 
Hod, H., Porela, P., Underwood, R., Bax, J.J., Beller, G.A., Bonow, R., Wall, E.E.V.D., Bassand, J.-P., Wijns, W., Ferguson, T.B., Steg, P.G., Uretsky, B.F., Williams, D.O., Armstrong, P.W., Antman, E.M., Fox, K.A., Hamm, C.W., Ohman, E.M., Simoons, M.L., Poole-Wilson, P.A., Gurfinkel, E.P., Lopez-Sendon, J.-L., Pais, P., Mendis, S., Zhu, J.-R., Wallentin, L.C., Fernández-Avilés, F., Fox, K.M., Parkhomenko, A.N., Priori, S.G., Tendera, M., Voipio-Pulkki, L.-M., Vahanian, A., Camm, A.J., Caterina, R.D., Dean, V., Dickstein, K., Filippatos, G., Funck-Brentano, C., Hellemans, I., Kristensen, S.D., McGregor, K., Sechtem, U., Silber, S., Tendera, M., Widimsky, P., Zamorano, J.L., Morais, J., Brener, S., Harrington, R., Morrow, D., Sechtem, U., Lim, M., Martinez-Rios, M.A., Steinhubl, S., Levine, G.N., Gibler, W.B., Goff, D., Tubaro, M., Dudek, D., AlAttar, N., 2007. Universal definition of myocardial infarction Kristian Thygesen, Joseph S. Alpert and Harvey D. White on behalf of the Joint ESC/ACCF/AHA/WHF Task Force for the Redefinition of Myocardial Infarction. Eur. Heart J. 28, 2525-2538. doi:10.1093/eurheartj/ehm355

Timmers, L., Sluijter, J.P.G., van Keulen, J.K., Hoefer, I.E., Nederhoff, M.G.J., Goumans, M.-J., Doevendans, P.A., van Echteld, C.J.A., Joles, J.A., Quax, P.H., Piek, J.J., Pasterkamp, G., de Kleijn, D.P.V., 2008. Toll-like receptor 4 mediates maladaptive left ventricular remodeling and impairs cardiac function after myocardial infarction. Circ. Res. 102, 257-264. doi:10.1161/CIRCRESAHA.107.158220

Tokuno, S., Chen, F., Pernow, J., Jiang, J., Valen, G., 2002. Effects of spontaneous or induced brain ischemia on vessel reactivity: the role of inducible nitric oxide synthase. Life Sci. 71, 679-692.

Toulon, A., Breton, L., Taylor, K.R., Tenenhaus, M., Bhavsar, D., Lanigan, C., Rudolph, R., Jameson, J., Havran, W.L., 2009. A role for human skin-resident T cells in wound healing. J. Exp. Med. 206, 743-750. doi:10.1084/jem.20081787

Trickett, A., Kwan, Y.L., 2003. T cell stimulation and expansion using antiCD3/CD28 beads. J. Immunol. Methods 275, 251-255. doi:10.1016/S00221759(03)00010-3

Triplett, T.A., Curti, B.D., Bonafede, P.R., Miller, W.L., Walker, E.B., Weinberg, A.D., 2012. Defining a functionally distinct subset of human memory CD4+ T cells that are CD25POS and FOXP3NEG. Eur. J. Immunol. 42, 1893-1905. doi:10.1002/eji.201242444

Tritto, I., D’Andrea, D., Eramo, N., Scognamiglio, A., De Simone, C., Violante, A., Esposito, A., Chiariello, M., Ambrosio, G., 1997. Oxygen radicals can induce preconditioning in rabbit hearts. Circ. Res. 80, 743-748.

Ulanova, M., Tarkowski, A., Hahn-Zoric, M., Hanson, L.?., 2001. The Common Vaccine Adjuvant Aluminum Hydroxide Up-Regulates Accessory Properties of Human Monocytes via an Interleukin-4-Dependent Mechanism. Infect. Immun. 69, 1151-1159. doi:10.1128/IAI.69.2.1151-1159.2001

Unutmaz, D., Pileri, P., Abrignani, S., 1994. Antigen-independent activation of naive and memory resting T cells by a cytokine combination. J. Exp. Med. 180, 11591164.

Vahanian, A., Alfieri, O.R., Al-Attar, N., Antunes, M.J., Bax, J., Cormier, B., Cribier, A., De Jaegere, P., Fournial, G., Kappetein, A.P., Kovac, J., Ludgate, S., Maisano, 
F., Moat, N., Mohr, F.-W., Nataf, P., Pierard, L., Pomar, J.L., Schofer, J., Tornos, P., Tuzcu, M., van Hout, B., von Segesser, L.K., Walther, T., 2008. Transcatheter valve implantation for patients with aortic stenosis: a position statement from the European Association of Cardio-Thoracic Surgery (EACTS) and the European Society of Cardiology (ESC), in collaboration with the European Association of Percutaneous Cardiovascular Interventions (EAPCI). Eur. J. Cardio-Thorac. Surg. Off. J. Eur. Assoc. Cardio-Thorac. Surg. 34, 1-8. doi:10.1016/j.ejcts.2008.04.039

Vakeva, A.P., Agah, A., Rollins, S.A., Matis, L.A., Li, L., Stahl, G.L., 1998. Myocardial infarction and apoptosis after myocardial ischemia and reperfusion: role of the terminal complement components and inhibition by anti-C5 therapy. Circulation 97, 2259-2267.

Valen, G., Yan, Z., Hansson, G.K., 2001. Nuclear factor kappa-B and the heart. J. Am. Coll. Cardiol. 38, 307-314. doi:10.1016/S0735-1097(01)01377-8

Valeur, H.S., Valen, G., 2009. Innate immunity and myocardial adaptation to ischemia. Basic Res. Cardiol. 104, 22-32. doi:10.1007/s00395-008-0756-6

Van Allen, N.R., Krafft, P.R., Leitzke, A.S., Applegate, R.L., Tang, J., Zhang, J.H., 2012. The role of Volatile Anesthetics in Cardioprotection: a systematic review. Med. Gas Res. 2, 22. doi:10.1186/2045-9912-2-22

Vandervelde, S., van Luyn, M.J.A., Rozenbaum, M.H., Petersen, A.H., Tio, R.A., Harmsen, M.C., 2007. Stem cell-related cardiac gene expression early after murine myocardial infarction. Cardiovasc. Res. 73, 783-793. doi:10.1016/j.cardiores.2006.11.030

Van Eeden, S.F., Klut, M.E., Walker, B.A., Hogg, J.C., 1999. The use of flow cytometry to measure neutrophil function. J. Immunol. Methods 232, 23-43.

Van Geene, Y., van Swieten, H.A., Noyez, L., 2010. Cardiac troponin I levels after cardiac surgery as predictor for in-hospital mortality. Interact. Cardiovasc. Thorac. Surg. 10, 413-416. doi:10.1510/icvts.2009.216408

Van Hinsbergh, V.W.M., 2012. Endothelium-role in regulation of coagulation and inflammation. Semin. Immunopathol. 34, 93-106. doi:10.1007/s00281-011-0285-5

Van Straten, A.H.M., Tan, E.M.E.S.H., Hamad, M.A.S., Martens, E.J., van Zundert, A.A.J., 2010. Evaluation of the EuroSCORE risk scoring model for patients undergoing coronary artery bypass graft surgery: a word of caution. Neth. Heart J. $18,355-359$.

Venugopal, V., Hausenloy, D.J., Ludman, A., Di Salvo, C., Kolvekar, S., Yap, J., Lawrence, D., Bognolo, J., Yellon, D.M., 2009. Remote ischaemic preconditioning reduces myocardial injury in patients undergoing cardiac surgery with cold-blood cardioplegia: a randomised controlled trial. Heart 95, 1567-71. doi:10.1136/hrt.2008.155770

Verrier, E.D., Boyle, E.M., 1996. Endothelial Cell Injury in Cardiovascular Surgery. Ann Thorac Surg 62, 915-922.

Visser, S.S., Theron, A.J., Ramafi, G., Ker, J.A., Anderson, R., 2000. Apparent involvement of the $\mathrm{A}(2 \mathrm{~A})$ subtype adenosine receptor in the anti-inflammatory interactions of CGS 21680, cyclopentyladenosine, and IB-MECA with human neutrophils. Biochem. Pharmacol. 60, 993-999. 
Vivier, E., Raulet, D.H., Moretta, A., Caligiuri, M.A., Zitvogel, L., Lanier, L.L., Yokoyama, W.M., Ugolini, S., 2011. Innate or adaptive immunity? The example of natural killer cells. Science 331, 44-49. doi:10.1126/science.1198687

Vivier, E., Tomasello, E., Baratin, M., Walzer, T., Ugolini, S., 2008. Functions of natural killer cells. Nat. Immunol. 9, 503-510. doi:10.1038/ni1582

Wagner, R., Piler, P., Bedanova, H., Adamek, P., Grodecka, L., Freiberger, T., 2010. Myocardial injury is decreased by late remote ischaemic preconditioning and aggravated by tramadol in patients undergoing cardiac surgery: a randomised controlled trial. Interact Cardiovasc Thorac Surg 11, 758-62. doi:10.1510/icvts.2010.243600

Walsh, S.R., Boyle, J.R., Tang, T.Y., Sadat, U., Cooper, D.G., Lapsley, M., Norden, A.G., Varty, K., Hayes, P.D., Gaunt, M.E., 2009. Remote ischemic preconditioning for renal and cardiac protection during endovascular aneurysm repair: a randomized controlled trial. J. Endovasc. Ther. Off. J. Int. Soc. Endovasc. Spec. 16, 680-689. doi:10.1583/09-2817.1

Wang, C., Xie, H., Liu, X., Qin, Q., Wu, X., Liu, H., Liu, C., 2010. Role of nuclear factor- $\mathrm{\kappa B}$ in volatile anaesthetic preconditioning with sevoflurane during myocardial ischaemia/reperfusion. Eur. J. Anaesthesiol. 27, 747-756. doi:10.1097/EJA.0b013e32833bb3ba

Wang, E., Feng, Y., Zhang, M., Zou, L., Li, Y., Buys, E.S., Huang, P., Brouckaert, P., Chao, W., 2011. Toll-like receptor 4 signaling confers cardiac protection against ischemic injury via inducible nitric oxide synthase- and soluble guanylate cyclasedependent mechanisms. Anesthesiology 114, 603-613. doi:10.1097/ALN.0b013e31820a4d5b

Wang, Y., Xu, H., Mizoguchi, K., Oe, M., Maeta, H., 2001. Intestinal ischemia induces late preconditioning against myocardial infarction: a role for inducible nitric oxide synthase. Cardiovasc. Res. 49, 391-398.

Wang, Y., Yin, B., Liu, S., Xue, S., 2007. Cardioprotective effect by tumor necrosis factor-alpha and interleukin-6 through late preconditioning in unstable angina patients. Arch Med Res 38, 80-5. doi:10.1016/j.arcmed.2006.07.006

Wan, S., DeSmet, J.M., Barvais, L., Goldstein, M., Vincent, J.L., LeClerc, J.L., 1996. Myocardium is a major source of proinflammatory cytokines in patients undergoing cardiopulmonary bypass. J Thorac Cardiovasc Surg 112, 806-11.

Wan, S., Izzat, M.B., Lee, T.W., Wan, I.Y., Tang, N.L., Yim, A.P., 1999. Avoiding cardiopulmonary bypass in multivessel $\mathrm{CABG}$ reduces cytokine response and myocardial injury. Ann Thorac Surg 68, 52-6; discussion 56-7.

Warren, O.J., Smith, A.J., Alexiou, C., Rogers, P.L., Jawad, N., Vincent, C., Darzi, A.W., Athanasiou, T., 2009. The inflammatory response to cardiopulmonary bypass: part 1--mechanisms of pathogenesis. J Cardiothorac Vasc Anesth 23, 22331. doi:10.1053/j.jvca.2008.08.007

Warzecha, Z., Dembinski, A., Ceranowicz, P., Dembinski, M., Sendur, R., Cieszkowski, J., Sendur, P., Tomaszewska, R., 2012. Heparin inhibits protective effect of ischemic preconditioning in ischemia/reperfusion-induced acute pancreatitis. J. Physiol. Pharmacol. Off. J. Pol. Physiol. Soc. 63, 355-365. 
Watarida, S., Mori, A., Onoe, M., Tabata, R., Shiraishi, S., Sugita, T., Nojima, T., Nakajima, Y., Matsuno, S., 1994. A clinical study on the effects of pulsatile cardiopulmonary bypass on the blood endotoxin levels. J Thorac Cardiovasc Surg $108,620-5$.

Wauthy, P., Goldstein, J.P., Demanet, H., Deuvaert, F.E., 2003. Redo valve surgery nowadays: what have we learned? Acta Chir. Belg. 103, 475-480.

Weerasinghe, A., Taylor, K.M., 1998. The platelet in cardiopulmonary bypass. Ann. Thorac. Surg. 66, 2145-2152.

Wei, D., Ren, C., Chen, X., Zhao, H., 2012. The chronic protective effects of limb remote preconditioning and the underlying mechanisms involved in inflammatory factors in rat stroke. PloS One 7, e30892. doi:10.1371/journal.pone.0030892

Wei, M., Kuukasjarvi, P., Laurikka, J., Kaukinen, S., Iisalo, P., Laine, S., Laippala, P., Metsanoja, R., Tarkka, M., 2001a. Cytokine responses and myocardial injury in coronary artery bypass grafting. Scand J Clin Lab Invest 61, 161-6.

Wei, M., Kuukasjärvi, P., Laurikka, J., Pehkonen, E., Kaukinen, S., Laine, S., Tarkka, M., 2001b. Cytokine responses in patients undergoing coronary artery bypass surgery after ischemic preconditioning. Scand. Cardiovasc. J. SCJ 35, 142-146.

Weinbrenner, C., Nelles, M., Herzog, N., Sárváry, L., Strasser, R.H., 2002. Remote preconditioning by infrarenal occlusion of the aorta protects the heart from infarction: a newly identified non-neuronal but PKC-dependent pathway. Cardiovasc. Res. 55, 590-601. doi:10.1016/S0008-6363(02)00446-7

Weinbrenner, C., Schulze, F., Sárváry, L., Strasser, R.H., 2004. Remote preconditioning by infrarenal aortic occlusion is operative via deltal-opioid receptors and free radicals in vivo in the rat heart. Cardiovasc. Res. 61, 591-599. doi:10.1016/j.cardiores.2003.10.008

Weisman, H.F., Bartow, T., Leppo, M.K., Marsh, H.C., Carson, G.R., Concino, M.F., Boyle, M.P., Roux, K.H., Weisfeldt, M.L., Fearon, D.T., 1990. Soluble human complement receptor type 1: in vivo inhibitor of complement suppressing postischemic myocardial inflammation and necrosis. Science 249, 146-151.

Welters, M.J.P., Gouttefangeas, C., Ramwadhdoebe, T.H., Letsch, A., Ottensmeier, C.H., Britten, C.M., Burg, S.H. van der, 2012. Harmonization of the intracellular cytokine staining assay. Cancer Immunol. Immunother. 61, 967-978. doi:10.1007/s00262-012-1282-9

Wever, K.E., Warlé, M.C., Wagener, F.A., van der Hoorn, J.W., Masereeuw, R., van der Vliet, J.A., Rongen, G.A., 2011. Remote ischaemic preconditioning by brief hind limb ischaemia protects against renal ischaemia-reperfusion injury: the role of adenosine. Nephrol. Dial. Transplant. Off. Publ. Eur. Dial. Transpl. Assoc. - Eur. Ren. Assoc. 26, 3108-3117. doi:10.1093/ndt/gfr103

Whittaker, P., Przyklenk, K., 2011. Remote-conditioning ischemia provides a potential approach to mitigate contrast medium-induced reduction in kidney function: a retrospective observational cohort study. Cardiology 119, 145-150. doi:10.1159/000330930

Whitten, C.W., Hill, G.E., Ivy, R., Greilich, P.E., Lipton, J.M., 1998. Does the duration of cardiopulmonary bypass or aortic cross-clamp, in the absence of blood 
and/or blood product administration, influence the IL-6 response to cardiac surgery? Anesth Analg 86, 28-33.

Williams, J., 2010. Remote Ischaemic Preconditioning in High Risk Cardiac Surgery (Unpublished Honours Thesis). Victoria University of Wellington, Wellington, New Zealand.

Williams, T.M., Harken, A.H., 2008. Statins for Surgical Patients: Ann. Surg. 247, 30-37. doi:10.1097/SLA.0b013e3181492c0d

Wink, D.A., Hanbauer, I., Krishna, M.C., DeGraff, W., Gamson, J., Mitchell, J.B., 1993. Nitric oxide protects against cellular damage and cytotoxicity from reactive oxygen species. Proc. Natl. Acad. Sci. U. S. A. 90, 9813-9817.

Wolfrum, S., Nienstedt, J., Heidbreder, M., Schneider, K., Dominiak, P., Dendorfer, A., 2005. Calcitonin gene related peptide mediates cardioprotection by remote preconditioning. Regul. Pept. 127, 217-224. doi:10.1016/j.regpep.2004.12.008

Wolfrum, S., Schneider, K., Heidbreder, M., Nienstedt, J., Dominiak, P., Dendorfer, A., 2002. Remote preconditioning protects the heart by activating myocardial PKCepsilon-isoform. Cardiovasc. Res. 55, 583-589.

Wolk, K., Kunz, S., Asadullah, K., Sabat, R., 2002. Cutting edge: immune cells as sources and targets of the IL-10 family members? J. Immunol. Baltim. Md 1950 $168,5397-5402$.

Wolk, K., Kunz, S., Witte, E., Friedrich, M., Asadullah, K., Sabat, R., 2004. IL-22 increases the innate immunity of tissues. Immunity 21, 241-254. doi:10.1016/j.immuni.2004.07.007

Wollner, A., Wollner, S., Smith, J.B., 1993. Acting via A2 receptors, adenosine inhibits the upregulation of Mac-1 (Cd11b/CD18) expression on FMLP-stimulated neutrophils. Am. J. Respir. Cell Mol. Biol. 9, 179-185. doi:10.1165/ajrcmb/9.2.179

Wright, S.D., Rao, P.E., Voorhis, W.C.V., Craigmyle, L.S., Iida, K., Talle, M.A., Westberg, E.F., Goldstein, G., Silverstein, S.C., 1983. Identification of the C3bi receptor of human monocytes and macrophages by using monoclonal antibodies. Proc. Natl. Acad. Sci. 80, 5699-5703.

Wright, S.D., Weitz, J.I., Huang, A.J., Levin, S.M., Silverstein, S.C., Loike, J.D., 1988. Complement receptor type three (CD11b/CD18) of human polymorphonuclear leukocytes recognizes fibrinogen. Proc. Natl. Acad. Sci. U. S. A. $85,7734-7738$.

Wu, Q., Gui, P., Wu, J., Ding, D., Purusram, G., Dong, N., Yao, S., 2011. Effect of limb ischemic preconditioning on myocardial injury in patients undergoing mitral valve replacement surgery. -A randomized controlled trial. Circ J 75, 1885-9.

Wu, Y.-N., Yu, H., Zhu, X.-H., Yuan, H.-J., Kang, Y., Jiao, J.-J., Gao, W.-Z., Liu, Y.X., Lou, J.-S., 2011. Noninvasive delayed limb ischemic preconditioning attenuates myocardial ischemia-reperfusion injury in rats by a mitochondrial K(ATP) channel-dependent mechanism. Physiol. Res. Acad. Sci. Bohemoslov. 60, 271-279. 
Xiao, L., Lu, R., Hu, C.P., Deng, H.W., Li, Y.J., 2001. Delayed cardioprotection by intestinal preconditioning is mediated by calcitonin gene-related peptide. Eur. J. Pharmacol. 427, 131-135.

Xie, J.J., Liao, X.L., Chen, W.G., Huang, D.D., Chang, F.J., Chen, W., Luo, Z.L., Wang, Z.P., Ou, J.S., 2011. Remote ischaemic preconditioning reduces myocardial injury in patients undergoing heart valve surgery: randomised controlled trial. Heart 98, 384-8. doi:10.1136/heartjnl-2011-300860

Xiong, J., Liao, X., Xue, F.S., Yuan, Y.J., Wang, Q., Liu, J.H., 2011. Remote ischemia conditioning-an endogenous cardioprotective strategy from outside the heart. Chin Med J Engl 124, 2209-15.

Xuan, Y.T., Guo, Y., Han, H., Zhu, Y., Bolli, R., 2001. An essential role of the JAKSTAT pathway in ischemic preconditioning. Proc Natl Acad Sci U A 98, 9050-5. doi:10.1073/pnas.161283798

Xuan, Y.-T., Tang, X.-L., Banerjee, S., Takano, H., Li, R.C.X., Han, H., Qiu, Y., Li, J.-J., Bolli, R., 1999. Nuclear Factor-кB Plays an Essential Role in the Late Phase of Ischemic Preconditioning in Conscious Rabbits. Circ. Res. 84, 1095-1109. doi:10.1161/01.RES.84.9.1095

Xu, B., Gao, X., Xu, J., Lei, S., Xia, Z.-Y., Xu, Y., Xia, Z., 2011. Ischemic postconditioning attenuates lung reperfusion injury and reduces systemic proinflammatory cytokine release via heme oxygenase 1. J. Surg. Res. 166, e157164. doi:10.1016/j.jss.2010.11.902

Xu, M.-J., Feng, D., Wang, H., Guan, Y., Yan, X., Gao, B., 2014. IL-22 ameliorates renal ischemia-reperfusion injury by targeting proximal tubule epithelium. J. Am. Soc. Nephrol. JASN 25, 967-977. doi:10.1681/ASN.2013060611

Yamashita, N., Hoshida, S., Otsu, K., Taniguchi, N., Kuzuya, T., Hori, M., 2000. The involvement of cytokines in the second window of ischaemic preconditioning. $\mathrm{Br} \mathrm{J}$ Pharmacol 131, 415-22. doi:10.1038/sj.bjp.0703594

Yamaura, G., Turoczi, T., Yamamoto, F., Siddqui, M. a. Q., Maulik, N., Das, D.K., 2003. STAT signaling in ischemic heart: a role of STAT5A in ischemic preconditioning. Am. J. Physiol. Heart Circ. Physiol. 285, H476-482. doi:10.1152/ajpheart.00079.2003

Yang, L., Wang, G., Du, Y., Ji, B., Zheng, Z., 2014. Remote ischemic preconditioning reduces cardiac troponin I release in cardiac surgery: a meta-analysis. J. Cardiothorac. Vasc. Anesth. 28, 682-689. doi:10.1053/j.jvca.2013.05.035

Yang, N.-Y., Shi, L., Zhang, Y., Ding, C., Cong, X., Fu, F.-Y., Wu, L.-L., Yu, G.-Y., 2013. Ischemic preconditioning reduces transplanted submandibular gland injury. J. Surg. Res. 179, e265-273. doi:10.1016/j.jss.2012.02.066

Yang, X., Cohen, M.V., Downey, J.M., 2010. Mechanism of cardioprotection by early ischemic preconditioning. Cardiovasc. Drugs Ther. Spons. Int. Soc. Cardiovasc. Pharmacother. 24, 225-234. doi:10.1007/s10557-010-6236-X

Yang, Y., Lang, X.-B., Zhang, P., Lv, R., Wang, Y.-F., Chen, J.-H., 2014. Remote ischemic preconditioning for prevention of acute kidney injury: a meta-analysis of randomized controlled trials. Am. J. Kidney Dis. Off. J. Natl. Kidney Found. 64, 574-583. doi:10.1053/j.ajkd.2014.04.029 
Yang, Z., Day, Y.-J., Toufektsian, M.-C., Ramos, S.I., Marshall, M., Wang, X.-Q., French, B.A., Linden, J., 2005. Infarct-sparing effect of A2A-adenosine receptor activation is due primarily to its action on lymphocytes. Circulation 111, 21902197. doi:10.1161/01.CIR.0000163586.62253.A5

Yang, Z., Day, Y.-J., Toufektsian, M.-C., Xu, Y., Ramos, S.I., Marshall, M.A., French, B.A., Linden, J., 2006. Myocardial infarct-sparing effect of adenosine A2A receptor activation is due to its action on CD4+ T lymphocytes. Circulation 114, 2056-2064. doi:10.1161/CIRCULATIONAHA.106.649244

Yellon, D.M., Alkhulaifi, A.M., Pugsley, W.B., 1993. Preconditioning the human myocardium. Lancet 342, 276-7.

Yilmaz, G., Arumugam, T.V., Stokes, K.Y., Granger, D.N., 2006. Role of T lymphocytes and interferon-gamma in ischemic stroke. Circulation 113, 21052112. doi:10.1161/CIRCULATIONAHA.105.593046

Young, E., Podor, T.J., Venner, T., Hirsh, J., 1997. Induction of the Acute-Phase Reaction Increases Heparin-Binding Proteins in Plasma. Arterioscler. Thromb. Vasc. Biol. 17, 1568-1574. doi:10.1161/01.ATV.17.8.1568

Young, E., Venner, T., Ribau, J., Shaughnessy, S., Hirsh, J., Podor, T.J., 1999. The binding of unfractionated heparin and low molecular weight heparin to thrombinactivated human endothelial cells. Thromb. Res. 96, 373-381.

Young, P.J., Dalley, P., Garden, A., Horrocks, C., La Flamme, A., Mahon, B., Miller, J., Pilcher, J., Weatherall, M., Williams, J., Young, W., Beasley, R., 2012. A pilot study investigating the effects of remote ischemic preconditioning in high-risk cardiac surgery using a randomised controlled double-blind protocol. Basic Res Cardiol 107, 1-10. doi:10.1007/s00395-012-0256-6

Yu, M., Wen, N., Wenzhong, Z., Yuanchang, X., Xiaoming, D., Yongjin, L., 2010. Effect of repeated ischaemic preconditioning on TLR4 and proinflammatory cytokines TNF- $\alpha$ and IL-1 $\beta$ in myocardial ischaemia-reperfusion injury in a rat model. Arch. Med. Sci. AMS 6, 843-847. doi:10.5114/aoms.2010.19289

Zarbock, A., Ley, K., 2008. Mechanisms and consequences of neutrophil interaction with the endothelium. Am. J. Pathol. 172, 1-7. doi:10.2353/ajpath.2008.070502

Zaremba, J., Ilkowski, J., Losy, J., 2006. Serial measurements of levels of the chemokines CCL2, CCL3 and CCL5 in serum of patients with acute ischaemic stroke. Folia Neuropathol. Assoc. Pol. Neuropathol. Med. Res. Cent. Pol. Acad. Sci. 44, 282-289.

Zhang, B., Zhou, J., Li, H., Zhou, M., Chen, A., Zhao, Q., 2014. Remote ischemic preconditioning does not improve the clinical outcomes in patients undergoing coronary artery bypass grafting: a meta-analysis of randomized controlled trials. Int. J. Cardiol. 172, e36-38. doi:10.1016/j.ijcard.2013.12.086

Zhang, J., Kaupke, C.J., Yousefi, S., Cesario, T.C., Vaziri, N.D., 1995. Flow cytometric investigation of neutrophil activation pathways by n-formyl-Met-LeuPhe and phorbol myristate acetate. Biol. Cell Auspices Eur. Cell Biol. Organ. 84, $147-153$.

Zhang, J., Wang, Q., Xue, F., Li, R., Cheng, Y., Cui, X., Liao, X., Meng, F., 2013. Ischemic preconditioning produces more powerful anti-inflammatory and 
cardioprotective effects than limb remote ischemic postconditioning in rats with myocardial ischemia-reperfusion injury. Chin. Med. J. (Engl.) 126, 3949-3955.

Zhang, S., Wang, N., Xu, J., Gao, Q., Lin, G., Bruce, I.C., Xia, Q., 2006. Kappaopioid receptors mediate cardioprotection by remote preconditioning. Anesthesiology 105, 550-556.

Zhang, W., Petrovic, J.-M., Callaghan, D., Jones, A., Cui, H., Howlett, C., Stanimirovic, D., 2006. Evidence that hypoxia-inducible factor-1 (HIF-1) mediates transcriptional activation of interleukin-1beta (IL-1beta) in astrocyte cultures. J. Neuroimmunol. 174, 63-73. doi:10.1016/j.jneuroim.2006.01.014

Zhang, Y., Wallace, D.L., de Lara, C.M., Ghattas, H., Asquith, B., Worth, A., Griffin, G.E., Taylor, G.P., Tough, D.F., Beverley, P.C.L., Macallan, D.C., 2007. In vivo kinetics of human natural killer cells: the effects of ageing and acute and chronic viral infection. Immunology 121, 258-265. doi:10.1111/j.1365-2567.2007.02573.x

Zhao, P., Wang, J., He, L., Ma, H., Zhang, X., Zhu, X., Dolence, E.K., Ren, J., Li, J., 2009. Deficiency in TLR4 signal transduction ameliorates cardiac injury and cardiomyocyte contractile dysfunction during ischemia. J. Cell. Mol. Med. 13, 1513-1525. doi:10.1111/j.1582-4934.2009.00798.x

Zhou, C., Liu, Y., Yao, Y., Zhou, S., Fang, N., Wang, W., Li, L., 2013. $\beta$-blockers and volatile anesthetics may attenuate cardioprotection by remote preconditioning in adult cardiac surgery: a meta-analysis of 15 randomized trials. J. Cardiothorac. Vasc. Anesth. 27, 305-311. doi:10.1053/j.jvca.2012.09.028

Zhou, K., Yang, B., Zhou, X.-M., Tan, C.-M., Zhao, Y., Huang, C., Liao, X.-B., Xiao, H.B., 2007. Effects of remote ischemic preconditioning on the flow pattern of the left anterior descending coronary artery in normal subjects. Int. J. Cardiol. 122, 250-251. doi:10.1016/j.ijcard.2006.11.079

Zhou, W., Zeng, D., Chen, R., Liu, J., Yang, G., Liu, P., Zhou, X., 2010. Limb ischemic preconditioning reduces heart and lung injury after an open heart operation in infants. Pediatr Cardiol 31, 22-9. doi:10.1007/s00246-009-9536-9

Zhu, J., Yamane, H., Cote-Sierra, J., Guo, L., Paul, W.E., 2006. GATA-3 promotes Th2 responses through three different mechanisms: induction of Th2 cytokine production, selective growth of Th2 cells and inhibition of Th1 cell-specific factors. Cell Res. 16, 3-10. doi:10.1038/sj.cr.7310002

Zhu, X., Zhao, H., Graveline, A.R., Buys, E.S., Schmidt, U., Bloch, K.D., Rosenzweig, A., Chao, W., 2006. MyD88 and NOS2 are essential for toll-like receptor 4-mediated survival effect in cardiomyocytes. Am. J. Physiol. Heart Circ. Physiol. 291, H1900-1909. doi:10.1152/ajpheart.00112.2006

Zimmerman, R.F., Ezeanuna, P.U., Kane, J.C., Cleland, C.D., Kempananjappa, T.J., Lucas, F.L., Kramer, R.S., 2011. Ischemic preconditioning at a remote site prevents acute kidney injury in patients following cardiac surgery. Kidney Int 80, 861-7. doi:10.1038/ki.2011.156

Zitta, K., Meybohm, P., Bein, B., Gruenewald, M., Lauer, F., Steinfath, M., Cremer, J., Zacharowski, K., Albrecht, M., 2014. Activities of cardiac tissue matrix metalloproteinases 2 and 9 are reduced by remote ischemic preconditioning in cardiosurgical patients with cardiopulmonary bypass. J. Transl. Med. 12, 94. doi:10.1186/1479-5876-12-94 
Zitta, K., Meybohm, P., Bein, B., Heinrich, C., Renner, J., Cremer, J., Steinfath, M., Scholz, J., Albrecht, M., 2012. Serum from patients undergoing remote ischemic preconditioning protects cultured human intestinal cells from hypoxia-induced damage: involvement of matrixmetalloproteinase-2 and -9. Mol. Med. Camb. Mass 18, 29-37. doi:10.2119/molmed.2011.00278

Zografos, T.A., Katritsis, G.D., Katritsis, D.G., 2014. Remote ischemic preconditioning reduces peri-procedural myocardial injury in elective percutaneous coronary intervention: a meta-analysis. Int. J. Cardiol. 173, 530-532. doi:10.1016/j.ijcard.2014.03.026

Zwacka, R.M., Zhang, Y., Halldorson, J., Schlossberg, H., Dudus, L., Engelhardt, J.F., 1997. CD4(+) T-lymphocytes mediate ischemia/reperfusion-induced inflammatory responses in mouse liver. J. Clin. Invest. 100, 279-289. doi:10.1172/JCI119533 


\section{Appendices}




\section{Appendix A. Standardised trial protocols for peri-operative management of cardiac surgery patients}

The following anaesthesia protocol was developed by Dr Paul Dalley, and the haemodynamic protocols by Dr Paul Young.

\section{Pre-operative Care}

ACE inhibitors, diuretics and alpha blockers were withheld on the day of surgery except in cases of severe diastolic hypertension or poorly controlled left ventricular failure. All other cardiac medications, including beta blockers, calcium channel blockers and statins, were given as usual. Half the usual morning beta blocker dose was administered if heart rate (HR) was below 55 and for those patients taking warfarin, treatment was ceased 5 days prior to surgery and a baseline international normalised ratio measure of coagulation was checked pre-operatively. Clopidogrel and, in patients without recent symptoms of angina, aspirin were similarly withheld for 5 days before surgery wherever possible. Low molecular weight heparin was stopped a minimum of 24 hours pre-operatively and, in patients with ongoing unstable angina, was substituted with unfractioned heparin to maintain an activated partial thromboplastin time (ACT) between 60 - 80 seconds. Metformin and other oral hypoglycaemic agents were stopped the night before and on the day of surgery, respectively. Patients with diabetes mellitus were treated with glucose-insulinpotassium therapy as necessary to maintain a target blood sugar level of $4-10$ mmol/L. Sildenafil (50 mg) was administered pre-operatively to patients with chronic pulmonary hypertension, defined as pulmonary artery systolic pressure $>60 \mathrm{mmHg}$.

\section{Anaesthesia}

Pre-medications consisted of zopiclone (7.5 - $15 \mathrm{mg}$ ) the night before surgery with or without midazolam (3.75 - $15 \mathrm{mg}$ ) and oxygen $(6 \mathrm{~L} / \mathrm{min}$ via face mask) on the morning of the operation, depending on the patient's age and physical state. Anaesthesia was induced with midazolam $(0.05-0.15 \mathrm{mg} / \mathrm{kg})$ and fentanyl (5 - $15 \mathrm{mcg} / \mathrm{kg})$, with vecuronium $(0.1-0.2 \mathrm{mg} / \mathrm{kg})$ or rocuronium $(0.5-1.5$ $\mathrm{mg} / \mathrm{kg}$ ). Before cardiopulmonary bypass (CPB), anaesthesia was maintained with isoflurane $(0.3-2.0 \%)$ to keep the bispectral index $\leq 60$. Propofol infusion $(2 \mathrm{mg} / \mathrm{kg} / \mathrm{h})$ was given with additional boluses of fentanyl $(100-250 \mathrm{mcg})$ as required, up to a total dose of $2000 \mathrm{mcg}$. At commencement of $\mathrm{CPB}$, prior to aortic cross-clamping, morphine $(20 \mathrm{mg})$ was administered and further doses given (10 - $20 \mathrm{mg}$ ) if necessary. Anaesthesia while on CPB consisted of isoflurane $(0.3-3.0 \%)$ and propofol infusion $(2 \mathrm{mg} / \mathrm{kg} / \mathrm{h})$. In addition to routine monitoring, a Swan-Ganz catheter (Edwards Lifesciences, CA, USA) was inserted into the internal jugular vein to continuously monitor cardiac output and haemodynamic pressures. Cefazolin ( 2 g) was administered on induction, with further doses in the perfusion pump prime $(1 \mathrm{~g})$, and the earlier of $4 \mathrm{~h}$ post induction or separation from CPB. Subsequent doses were 4 hourly while the sternum remained open, then 8 hourly for 2 final doses. In patients with 
penicillin allergy, antibiotic treatment consisted of vancomycin (1 g over $30 \mathrm{~min}$ ) and gentamicin ( $4 \mathrm{mg} / \mathrm{kg}$ over $30 \mathrm{~min}$ ). A bolus of tranexamic acid (20 mg/kg), was given on induction, followed by smaller doses at the start of CPB and for each hour of CPB $(10 \mathrm{mg} / \mathrm{kg})$. Heparin $(350 \mathrm{U} / \mathrm{kg})$ was given and supplemented as needed to maintain an ACT $\geq 480 \mathrm{~s}$ until reversal with protamine $(1 \mathrm{mg} / 100 \mathrm{U}$ of estimated residual heparin). Actrapid insulin was infused as necessary to keep blood sugar level $\leq 10 \mathrm{mmol} / \mathrm{L}$. Glucocorticoids were not administered unless there was evidence of adrenal suppression. Before CPB, PlasmaLyte 148 was used to replace pre-operative fluid deficits and ongoing insensible losses with additional boluses in cases of hypovolemia. Post CPB, PlasmaLyte was administered as necessary to assist with optimal ventricular filling.

\section{Perfusion}

Non-pulsatile perfusion was performed using a VHK Venous Hardshell Reservoir, Quadrox oxygenator system, Jostra Rotaflow centrifugal pump head (Macquet, Rastatt, Germany), Affinity 38 micron in-line arterial filter (Carmeda, AB, Sweden) and Softline coated biocompatible tubing (Macquet). The pump was primed with PlasmaLyte 148 (2 L) and heparin (10,000 U) without mannitol, $\mathrm{HCO}_{3}{ }^{-}$, or colloid. Tepid bypass (32 - $34^{\circ} \mathrm{C}$ nasopharyngeal) was performed, with a pump flow of $2.4 \mathrm{~L} / \mathrm{min} / \mathrm{m}^{2}$. ACT was kept $\geq 480 \mathrm{~s}$, and checked at $30 \mathrm{~min}$ intervals. Alpha-stat management was used to evaluate pH levels. Cardiotomy suction was reserved for fresh bleeding directly from the heart or aorta, and a cell saver was used for pooled blood collected from the mediastinum and pleura which, together with the remaining pump blood, was washed and reinfused following CPB. A "hotshot" of normothermic blood was given immediately before removal of the aortic cross-clamp, and rewarming to $36.5^{\circ} \mathrm{C}$ was induced with the application of a sterile forced air blanket to the patient's lower body prior to separation from CPB. Metabolic acidosis was treated by $\mathrm{HCO}_{3}{ }^{-}$, or haemofiltration. Haemoglobin was kept at $\geq 70 \mathrm{~g} / \mathrm{L}$ while on CPB, and anaemia was treated with packed red blood cells or haemoconcentration, as appropriate. The cardioplegia solution contained potassium chloride $(\mathrm{KCl} ; 50 \mathrm{mmol} / \mathrm{L})$, $\mathrm{MgSO}_{4}(20 \mathrm{mmol} / \mathrm{L}), 8.4 \% \mathrm{NaHCO}_{3}(20 \mathrm{mmol} / \mathrm{L})$ with $5 \%$ dextrose and was infused at $400 \mathrm{ml} / \mathrm{h}$ into tepid blood flowing at $300 \mathrm{ml} / \mathrm{min}$. Induction cardioplegia consisted of $4 \mathrm{~min}$ antegrade, then retrograde, perfusion with an additional 1 - $2 \mathrm{~min}$ if required to achieve electrical silence. Maintenance cardioplegia was administered every 15 - 25 min for 2 min to reach a coronary sinus pressure of $20-60 \mathrm{mmHg}$.

\section{Haemodynamic Management}

The haemodynamic aims during surgery of maintaining a mean arterial pressure (MAP) $\geq 65 \mathrm{mmHg}, \mathrm{CI} \geq 2 \mathrm{~L} / \mathrm{min} / \mathrm{m}^{2}, \mathrm{SVO}_{2} \geq 65 \%$ and $\mathrm{Hb} \geq 70 \mathrm{~g} / \mathrm{L}$ were considered violated in the event of failure to achieve acceptable values despite 5 min of maximal appropriate therapy. The nature and duration of any violations were documented in the patient data. Briefly, MAP was increased in cases of hypotension prior to CPB using phenylephrine $(0.01-1 \mathrm{mcg} / \mathrm{kg} / \mathrm{min})$. During and following $\mathrm{CPB}$, phenylephrine $(250 \mathrm{mcg}$ boluses up to every $2 \mathrm{~min}$ ), noradrenaline infusion $(0.05-0.4 \mathrm{mcg} / \mathrm{kg} / \mathrm{min})$, vasopressin $(2-3 \mathrm{U} / \mathrm{h})$, or 
methylene blue ( $2 \mathrm{mg} / \mathrm{kg}$ over $10 \mathrm{~min}$ ) were used as necessary to raise MAP. In situations of hypertension (MAP $>80$ ), isoflurane was increased $(\leq 3.0 \%)$ and glycerol trinitrate $(0.1-2.5 \mathrm{mcg} / \mathrm{kg} / \mathrm{min})$, administered. Milrinone was infused at $0.375-0.75 \mathrm{mcg} / \mathrm{kg} / \mathrm{min}$ to treat reduced cardiac index, ventricular dysfunction or pulmonary hypertension (PHT). In cases of persistently low CI despite milrinone and noradrenaline dosing, adrenaline $(0.05-0.5 \mathrm{mcg} / \mathrm{kg} / \mathrm{min})$ or inhaled nitric oxide (40 ppm) was administered, with the latter also used for severe PHT with right ventricular dysfunction. Sub-optimal $\mathrm{SVO}_{2}(<65 \%)$ in the presence of target pump flow and normal arterial carbon dioxide pressure was treated by setting the sweep gas at $100 \%$ and increasing the pump speed. In the first 12 hours following surgery, the haemodynamic goals were CI $\geq 1.7$ $\mathrm{L} / \mathrm{min} / \mathrm{m}^{2}$, mixed venous $\geq 55 \%$, and MAP $60-75 \mathrm{mmHg}$. These goals were altered from $\mathrm{CI} \geq 2 \mathrm{~L} / \mathrm{min} / \mathrm{m}^{2}$, mixed venous $\geq 60 \%$ and MAP $65-75 \mathrm{mmHg}$ after the first 40 patients after data comparing these targets with historical non-PA catheter driven in the study unit. However, equal numbers of RIPC and control group patients received each fluid protocol due to block randomization in groups of 8. If any of the goals were not met within the first 12 hours, the patients were managed according to the protocol shown in Figure A.1. 
Step 1: Assessment of volume responsiveness (Omit step 1 if MAP>70) ${ }^{1}$

$5 \mathrm{ml} / \mathrm{kg}$ of Hartmann's (rounded nearest $250 \mathrm{ml}$ ) as rapidly as possible via large bore peripheral line

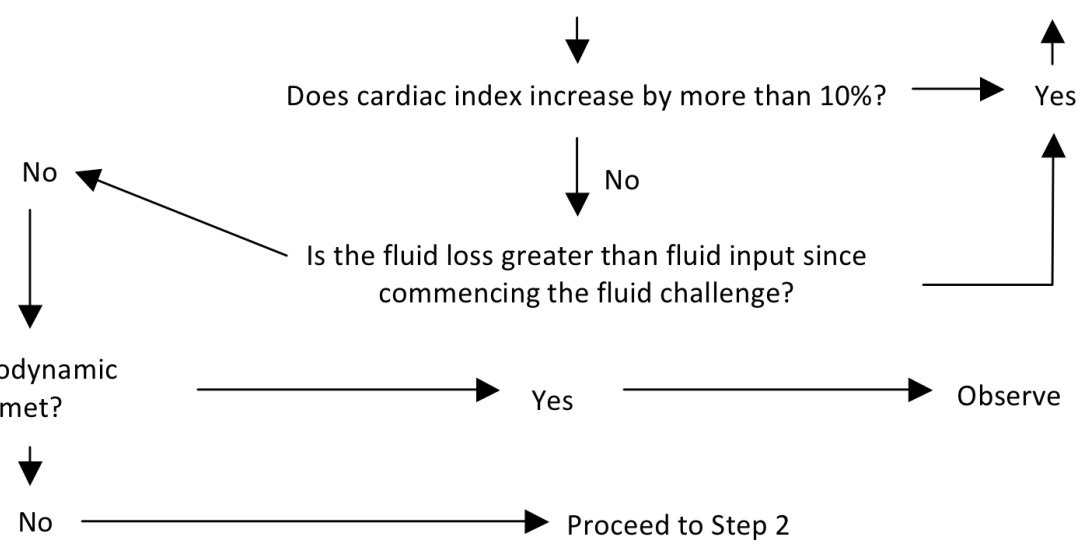

Step 2: Afterload reduction

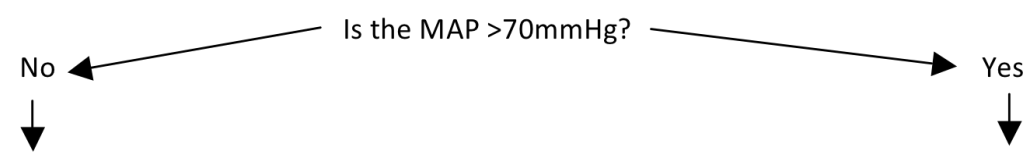

Are haemodynamic goals met?

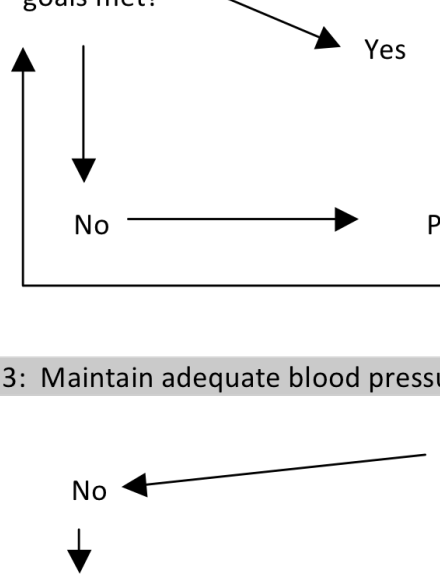

Are haemodynamic goals met?

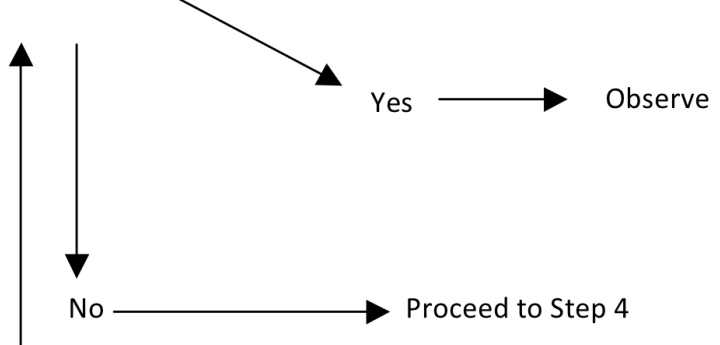

If noradrenaline is running, decrease the dose. Otherwise, commence GTN at $0-5 \mathrm{mcg} / \mathrm{kg} / \mathrm{min}$ aiming for MAP 60 70; if the MAP is target not achieved, add sodium nitroprusside at 0 -

\section{$2.5 \mathrm{mcg} / \mathrm{kg} / \mathrm{min}$}

Proceed to Step 3
Is MAP $<60 \mathrm{mmHg}$ ?

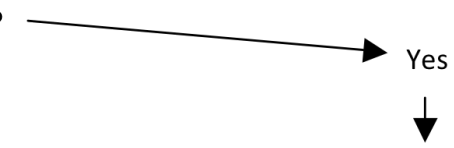

Call the ICU Consultant prior to commencing noradrenaline at 0 $0.5 \mathrm{mcg} / \mathrm{kg} / \mathrm{min}$; if the MAP target is not achieved at the upper end of the dose range, call ICU Consultant and add vasopressin at $2 \mathrm{U} / \mathrm{hr}$. Continue to titrate noradrenaline up to $0.75 \mathrm{mcg} / \mathrm{kg} / \mathrm{min}$ 


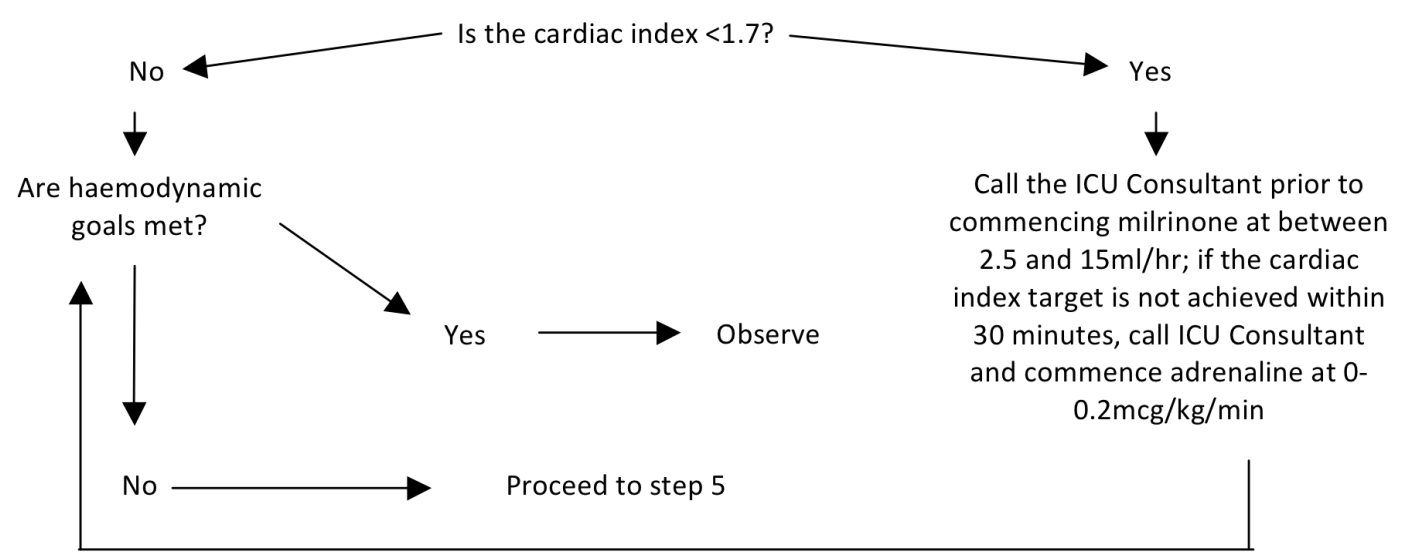

Step 5: Maintain adequate mixed venous

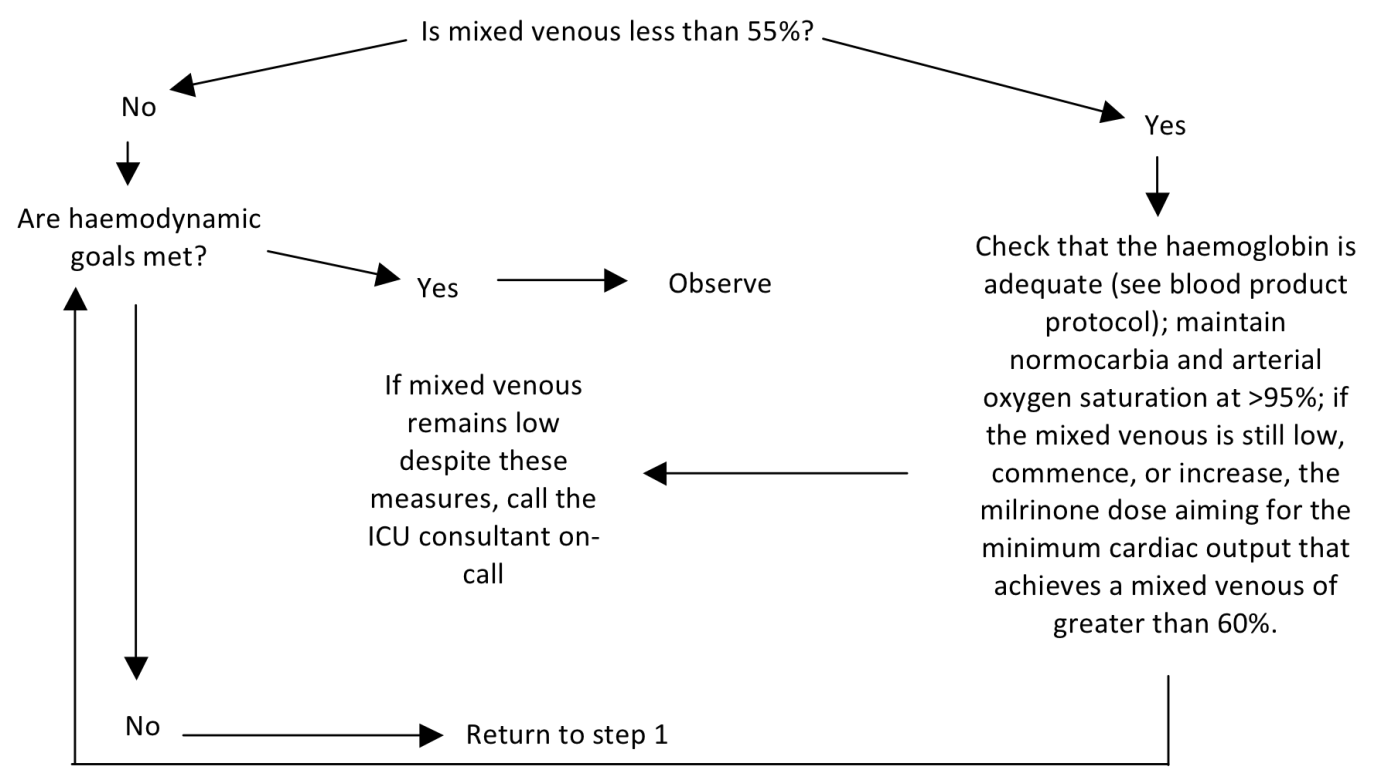

Figure A.1 Protocol for haemodynamic management of patients in the first 12 hours following cardiac surgery

Patients should be maintained in sinus rhythm at $80-110 \mathrm{bpm}$. If the rate is $<80$ then DDD pacing is the preferred mode. If $\mathrm{HR}<80$, sinus rhythm may still be preferred to VVI if there are no atrial wires.

Maintenance fluid is dextrose $5 \%$ in water at $0.8 \mathrm{~mL} / \mathrm{kg} / \mathrm{hr}$ (rounded to the nearest $10 \mathrm{mLs}$ ).

All dosing on the protocol is based on the patient's actual pre-operative body weight.

${ }^{1}$ A sustained rise in CVP or PA diastolic in response to fluid boluses usually indicates adequate volume resuscitation. If the patient develops new 'instability' and filling pressures have not dropped, it may be appropriate to omit step 1 of the algorithm. A straight leg raise may give you an idea of the patient's volume responsiveness and may be performed at step 1 if the patient is felt to be 'well 
filled'. If the BLOOD PRESSURE does not rise in response to a straight leg raise the patient is unlikely to be volume responsive.

2 If the cardiac index is $>3.5$ and the mixed venous is adequate, positive inotropes (milrinone, adrenaline, dobutamine, dopamine) should be weaned. Milrinone can generally be ceased without weaning due to its long half life. An intra-aortic balloon pump should be considered if adequate cardiac index is not achieved with adequate fluid resuscitation and milrinone at $0.75 \mathrm{mcg} / \mathrm{kg} / \mathrm{min}$. Unexpected low cardiac output should be evaluated by echocardiography. 


\section{Appendix B. Criteria for EuroSCORE and RIFLE Evaluations}

\section{EuroSCORE}

The logistic EuroSCORE was used to evaluate the operative risk of patients recruited to the trial of RIPC in high-risk cardiac surgery patients, according to the following formula and the variables presented in Table B.1 (Nashef et al., 1999; Roques et al., 2003).

Predicted mortality $=\frac{\mathrm{e}^{(\beta o+\Sigma \beta i X i)}}{1+\mathrm{e}^{(\beta o+\Sigma \beta i X i)}}$

Where: $\quad$ e is the natural logarithm

$\beta o$ is the constant of the logistic regression equation (-4.789594)

$\beta i$ is the coefficient of the variable $X i$ in the logistic regression equation provided in Table B.1

$X i=1$ if a categorical risk factor is present and 0 if it is absent

For age, $X i=1$ if patient age $<60 ; X i$ increases by one point per

year thereafter 


\begin{tabular}{|c|c|c|}
\hline \multicolumn{2}{|c|}{ Patient-related factors } & \multirow{2}{*}{$\frac{\boldsymbol{\beta}}{0.0666354}$} \\
\hline Age & Continuous & \\
\hline Sex & Female & 0.3304052 \\
\hline $\begin{array}{l}\text { Chronic pulmonary } \\
\text { disease }\end{array}$ & $\begin{array}{l}\text { Longterm use of bronchodilators or steroids for } \\
\text { lung disease }\end{array}$ & 0.4931341 \\
\hline $\begin{array}{l}\text { Extracardiac } \\
\text { arteriopathy }\end{array}$ & $\begin{array}{l}\text { Any one or more of the following: claudication, } \\
\text { carotid occlusion or }>50 \% \text { stenosis, previous or } \\
\text { planned intervention on the abdominal aorta,limb } \\
\text { arteries or carotids }\end{array}$ & 0.6558917 \\
\hline $\begin{array}{l}\text { Neurological } \\
\text { dysfunction disease }\end{array}$ & $\begin{array}{l}\text { Severely affecting ambulation or day-to-day } \\
\text { functioning }\end{array}$ & 0.841626 \\
\hline $\begin{array}{l}\text { Previous cardiac } \\
\text { surgery }\end{array}$ & Requiring opening of the pericardium & 1.002625 \\
\hline Serum creatinine & $>200 \mathrm{~m}$ micromol/L preoperatively & 0.6521653 \\
\hline Active endocarditis & $\begin{array}{l}\text { Patient still under antibiotic treatment for } \\
\text { endocarditis at the time of surgery }\end{array}$ & 1.101265 \\
\hline $\begin{array}{l}\text { Critical } \\
\text { preoperative state }\end{array}$ & $\begin{array}{l}\text { Any one or more of the following: ventricular } \\
\text { tachycardia or fibrillation or aborted sudden death, } \\
\text { preoperative cardiac massage, preoperative } \\
\text { ventilation before arrival in the anaesthetic } \\
\text { room,preoperative inotropic support, intraaortic } \\
\text { balloon counterpulsation or preoperative acute } \\
\text { renal failure (anuria or oliguria }<10 \mathrm{ml} / \text { hour) }\end{array}$ & 0.9058132 \\
\hline \multicolumn{3}{|c|}{ Cardiac-related factors } \\
\hline Unstable angina & $\begin{array}{l}\text { Rest angina requiring iv nitrates until arrival in the } \\
\text { anaesthetic room }\end{array}$ & 0.5677075 \\
\hline \multirow[t]{2}{*}{ LV dysfunction } & Moderate or LVEF $30-50 \%$ & 0.4191643 \\
\hline & Poor or LVEF $<30$ & 1.094443 \\
\hline $\begin{array}{l}\text { Recent myocardial } \\
\text { infarct }\end{array}$ & (<90 days) & 0.5460218 \\
\hline $\begin{array}{l}\text { Pulmonary } \\
\text { hypertension }\end{array}$ & Systolic PA pressure $>60 \mathrm{mmHg}$ & 0.7676924 \\
\hline \multicolumn{3}{|c|}{ Operation-related factors } \\
\hline Emergency & $\begin{array}{l}\text { Carried out on referral before the beginning of the } \\
\text { next working day }\end{array}$ & 0.7127953 \\
\hline $\begin{array}{l}\text { Other than isolated } \\
\text { CABG }\end{array}$ & $\begin{array}{l}\text { Major cardiac procedure other than or in addition } \\
\text { to CABG }\end{array}$ & 0.5420364 \\
\hline $\begin{array}{l}\text { Surgery on thoracic } \\
\text { aorta }\end{array}$ & For disorder of ascending, arch or descending aorta & 1.159787 \\
\hline $\begin{array}{l}\text { Postinfarct septal } \\
\text { rupture }\end{array}$ & & 1.462009 \\
\hline
\end{tabular}

Table B.1 Beta coefficients for the logistic regression model of EuroSCORE 


\section{RIFLE}

The RIFLE classification of acute kidney injury was used to evaluate postoperative renal damage in the trial of RIPC in high-risk cardiac surgery patients, as detailed in Table B.2 (Bellomo et al., 2004).

\begin{tabular}{|c|c|c|}
\hline Class & GFR & UO \\
\hline Risk & $\uparrow \mathrm{SCr} \times 1.5$ or $\downarrow$ GFR $>25 \%$ & $<0.5 \mathrm{~mL} / \mathrm{kg} / \mathrm{h} \times 6 \mathrm{~h}$ \\
\hline Injury & $\uparrow \mathrm{SCr} \times 2$ or $\downarrow$ GFR $>50 \%$ & $<0.5 \mathrm{~mL} / \mathrm{kg} / \mathrm{h} \times 12 \mathrm{~h}$ \\
\hline Failure & $\begin{array}{l}\uparrow \mathrm{SCr} \times 3 \text { or } \downarrow \mathrm{GFR}>75 \% \text { or if } \\
\text { baseline } \mathrm{SCr} \geq 353.6 \mu \mathrm{mol} / \mathrm{L}(\geq 4 \\
\mathrm{mg} / \mathrm{dL}) \uparrow \mathrm{SCr}>44.2 \mu \mathrm{mol} / \mathrm{L}(>0.5 \\
\mathrm{mg} / \mathrm{dL})\end{array}$ & $\begin{array}{l}<0.3 \mathrm{~mL} / \mathrm{kg} / \mathrm{h} \times 24 \mathrm{~h} \\
\text { or anuria } \times 12 \mathrm{~h}\end{array}$ \\
\hline Loss of kidney function & $\begin{array}{l}\text { Complete loss of kidney function } \\
>4 \text { weeks }\end{array}$ & \\
\hline $\begin{array}{l}\text { End-stage kidney } \\
\text { disease }\end{array}$ & $\begin{array}{l}\text { Complete loss of kidney function } \\
>3 \text { months }\end{array}$ & \\
\hline
\end{tabular}

Table B.2 RIFLE classification criteria

$\mathrm{GFR}=$ glomerular filtration rate, $\mathrm{UO}=$ urine output, $\mathrm{SCr}=$ serum creatinine. Note: a patient can fulfil the criteria through changes in SCr or changes in UO, or both. The criteria that lead to the worst possible classification should be used. Note that the F component of RIFLE is present even if the increase in $\mathrm{SCr}$ is under 3fold, as long as the new SCr is greater than $4.0 \mathrm{mg} / \mathrm{dL}$ (350 mol/L) in the setting of an acute increase of at least $0.5 \mathrm{mg} / \mathrm{dL}(44 \mathrm{~mol} / \mathrm{L})$. The designation RIFLE-F should be used in this case to denote 'acute-on-chronic' disease. Similarly, when the RIFLE-F classification is achieved by UO criteria, a designation of RIFLE-Fo should be used to denote oliguria. There is a decrease in sensitivity and increase in specificity with each progressive RIFLE stage. 


\section{Appendix C. Recipes}

\section{PBS (10X)}

$\mathrm{NaCl}$

$\mathrm{Na}_{2} \mathrm{HPO}_{4} \cdot 12 \mathrm{H}_{2} \mathrm{O}$

$\mathrm{NaH}_{2} \mathrm{PO}_{4} .2 \mathrm{H}_{2} \mathrm{O}$

$\mathrm{ddH}_{2} \mathrm{O}$

Solution was sterilised by autoclaving prior to use.

\section{FACS Buffer (v/v)}

FCS

Sodium azide

PBS

\section{2\% Saponin Buffer}

Bovine serum albumin

$0.5 \mathrm{~g}$

Saponin

$1 \mathrm{~g}$

Hepes $(10 \mathrm{mM})$

Sodium azide

$\mathrm{ddH}_{2} \mathrm{O}$

4\% Paraformaldehyde (w/v)

Paraformaldehyde

1X PBS

$96 \%$

Once dissolved, $\mathrm{pH}$ adjusted to 7.4.

\section{ELISA Capture Buffer}

$\mathrm{Na}_{2} \mathrm{HPO}_{4}$

Solution was dissolved in $\mathrm{ddH}_{2} 0$ and adjusted to $\mathrm{pH} 6.0$ or 9.0.

\section{ELISA Stop Solution}

$\mathrm{H}_{2} \mathrm{SO}_{4}$

Prepared in $\mathrm{ddH}_{2} \mathrm{O}$.

\section{Complete T Cell Media (CTCM; v/v)}

Dulbecco's Modified Eagle Medium

Fetal calf serum

L-glutamate $(200 \mathrm{mM})$

$1 \%$

Penicillin/Streptomycin $(10,000 \mathrm{U} / \mathrm{mL} / 10 \mathrm{mg} / \mathrm{mL}) \quad 1 \%$

HEPES buffer $(1 \mathrm{M}) \quad 1 \%$

B-Mercaptoethanol

$0.1 \%$

Non-essential amino acids (10 nM)

$0.1 \%$ 


\title{
Appendix D. Manuscripts resulting from the trial of RIPC in high-risk cardiac surgery patients
}

Basic Res Cardiol (2012) 107:256

DOI $10.1007 / \mathrm{s} 00395-012-0256-6$

ORIGINAL CONTRIBUTION

\section{A pilot study investigating the effects of remote ischemic preconditioning in high-risk cardiac surgery using a randomised controlled double-blind protocol}

\author{
Paul Jeffrey Young · Paul Dalley · Alexander Garden · Christopher Horrocks • \\ Anne La Flamme - Barry Mahon · John Miller · Janine Pilcher · \\ Mark Weatherall $\cdot$ Jenni Williams $\cdot$ William Young $\cdot$ Richard Beasley
}

Received: 21 November 2011/Revised: 10 February 2012/Accepted: 13 February 2012

(c) Springer-Verlag 2012

\begin{abstract}
The efficacy of remote ischemic preconditioning (RIPC) in high-risk cardiac surgery is uncertain. In this study, 96 adults undergoing high-risk cardiac surgery were randomised to RIPC ( 3 cycles of 5 min of upper-limb ischemia induced by inflating a blood pressure cuff to $200 \mathrm{mmHg}$ with $5 \mathrm{~min}$ of reperfusion) or control. Main endpoints were plasma high-sensitivity troponin T (hsTNT) levels at 6 and $12 \mathrm{~h}$, worst post-operative acute kidney injury (AKI) based on RIFLE criteria, and noradrenaline duration. hsTNT levels were log-normally distributed and higher with RIPC than control at 6-h post cross-clamp removal $[810 \mathrm{ng} / \mathrm{ml}$ (IQR $527-1,724)$ vs. $634 \mathrm{ng} / \mathrm{ml}$ (429-1,012); ratio of means 1.41 (99.17\% CI 0.92-2.17); $P=0.04]$ and $12 \mathrm{~h}[742 \mathrm{ng} / \mathrm{ml}(\mathrm{IQR} 427-1,700)$ vs. $514 \mathrm{ng} / \mathrm{ml}$
\end{abstract}

To this article two Invited Editorials are available at doi: 10.1007/s00395-012-0258-4 and doi:10.1007/s00395-012-0259-3.

Electronic supplementary material The online version of this article (doi:10.1007/s00395-012-0256-6) contains supplementary material, which is available to authorized users.

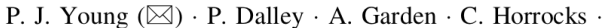
B. Mahon - W. Young $\cdot$ R. Beasley

Wellington Hospital, Capital and Coast District Health Board,

Private Bag 7902, Wellington 6242, New Zealand

e-mail: paul.young@ccdhb.org.nz

P. J. Young $\cdot$ J. Pilcher $\cdot$ M. Weatherall $\cdot$ R. Beasley

Medical Research Institute of New Zealand, Private Bag 7902,

Wellington 6242, New Zealand

A. Garden · A. La Flamme $\cdot$ J. Miller · J. Williams

School of Biological Sciences, Victoria University

of Wellington, PO Box 600, Wellington 6140, New Zealand

M. Weatherall

University of Otago, Wellington, PO Box 7343,

Wellington South, Wellington 6021, New Zealand
(IQR 356-833); ratio of means 1.56 (99.17\% CI 0.97-2.53); $P=0.01]$. After adjustment for baseline confounders, the ratio of means of hsTNT at $6 \mathrm{~h}$ was $1.23(99.17 \% \mathrm{CI} 0.88-1.72$; $P=0.10)$ and at $12 \mathrm{~h}$ was $1.30(99.17 \% \mathrm{CI} 0.92-1.84 ; P=0.05)$. In the RIPC group, 35/48 (72.9\%) had no AKI, 5/48 (10.4\%) had AKI risk, and $8 / 48$ (16.7\%) had either renal injury or failure compared to the control group where $34 / 48$ (70.8\%) had no AKI, 7/48 (14.6\%) had AKI risk, and 7/48 (14.6\%) had renal injury or failure (Chi-squared 0.41 ; two degrees of freedom; $P=0.82$ ). RIPC increased post-operative duration of noradrenaline support [21 h (IQR 7-45) vs. $9 \mathrm{~h}$ (IQR $3-19)$; ratio of means 1.70 (99.17\% CI 0.86-3.34); $P=0.04]$. RIPC does not reduce hsTNT, AKI, or ICU-support requirements in high-risk cardiac surgery.

Keywords Cardioprotection - Cardiac surgery - Remote ischemic preconditioning $\cdot$ Reperfusion

\section{Introduction}

There is considerable research interest in potential methods of cardioprotection against ischemia and reperfusion injury [29]. One such method is remote ischemic preconditioning (RIPC) which is a phenomenon by which brief periods of ischemia in one organ can condition other organs to tolerate subsequent prolonged ischemic insults. The ability to precondition the heart through periods of ischemia in other organs has been convincingly demonstrated in animals $[2,8,25]$. The first trial in humans was a small pilot trial of eight patients which suggested that RIPC might increase lactate dehydrogenase production by the heart in response to cardiopulmonary bypass [9]. This trial was followed by a trial in 37 children having surgical correction of congenital cardiac anomalies [5]. In this study, troponin I levels, 
inotrope scores, and airway resistance were all lower in children who received RIPC than in those who received standard care. Initial studies in adults having coronary artery bypass graft surgery $(\mathrm{CABG})$ also demonstrated that RIPC reduced post-operative troponin $\mathrm{T}$ levels compared with standard care [12, 32]. Subsequently, RIPC has been shown to increase myocardial salvage when used as an adjunct to primary angioplasty in patients with acute myocardial infarction [3]. Despite this early promise, the largest randomised trial of RIPC in patients having CABG surgery, showed that RIPC did not reduce post-operative troponin $\mathrm{T}$ levels, ventilation, inotrope or dialysis requirements [27]. Overall, there remains uncertainty about the efficacy of RIPC in patients undergoing cardiac surgery and, in particular, there are limited data specifically evaluating RIPC in the high-risk cardiac surgery population $[6,20]$.

Our hypothesis was that RIPC would reduce postoperative troponin levels, vasopressor requirements and the incidence of acute kidney injury (AKI) compared to standard care in patients undergoing high-risk cardiac surgery. As well as evaluating our primary hypotheses, we also aimed to explore the effects of RIPC on post-operative cardiac function and respiratory support requirements.

\section{Methods}

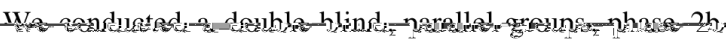
randomised controlled trial of RIPC in adults undergoing high-risk cardiac surgery with cardiopulmonary bypass. Participants were randomised in a 1:1 fashion to either RIPC or control.

This study was performed at Wellington Regional Hospital which provides tertiary referral cardiothoracic surgery for central New Zealand and performs approximately 600 cardiac operations per year. All data were collected in the cardiothoracic theatre and in the Intensive Care Unit in the early post-operative period.

Patients were eligible for inclusion if they were having one of the following 'high-risk' cardiac surgical procedures with cardiopulmonary bypass: double-valve or triple-valve surgery, mitral valve surgery, CABG plus valve(s), CABG with documented pre-operative ejection fraction of less than $50 \%$, or any 'redo' operation. These procedures were deemed high risk because they typically involve prolonged bypass times or are performed in patients with significantly impaired cardiac function. Patients were not considered for participation if they were less than 18 years of age, had known peripheral vascular disease affecting the upper limbs, required deep hypothermic circulatory arrest, or were being considered for radial artery conduit harvesting.

All patients received pre-medication, volatile-based cardiac anaesthesia, tepid cardiopulmonary bypass including blood cardioplegia and post-operative haemodynamic management in accordance with a standardised study protocol described in the electronic supplementary material. Patients received zopiclone $(7.5-15 \mathrm{mg})$ the night before their operation, if required. Midazolam (3.75-15 mg orally) was administered on the morning of surgery with the dose administered dependent on the patient's age and physical status. All patients had a 14 or 16 gauge peripheral cannula, a radial arterial line in the same arm as the peripheral cannula, a 4-lumen central venous line, and a continuous cardiac output pulmonary artery catheter inserted. Trans-oesophageal echocardiography was used in all cases. Patients were induced with midazolam $0.05-0.15 \mathrm{mg} / \mathrm{kg}$ and fentanyl $5-15 \mu \mathrm{g} / \mathrm{kg}$. Paralysis was achieved either with vecuronium or rocuronium. Patients received a propofol infusion at $2 \mathrm{mg} / \mathrm{kg} / \mathrm{h}$ and isoflurane $0.3-2.0 \%$ to maintain a Bispectral Index ${ }^{\mathrm{TM}}$ (BIS) of less than or equal to 60 throughout the surgery including whilst the patient was on cardiopulmonary bypass. Additional boluses of $100-250 \mu \mathrm{g}$ of fentanyl were administered throughout the operation as required. Morphine $(20 \mathrm{mg})$ was administered on commencement of cardiopulmonary bypass prior to aortic cross-clamping and an additional morphine (10-20 mg) was administered if required subsequently. Tranexamic acid was administered by intermittent bolus dosing: $20 \mathrm{mg} / \mathrm{kg}$ on induction, $10 \mathrm{mg} / \mathrm{kg}$ at the start of cardiopulmonary bypass, $10 \mathrm{mg} / \mathrm{kg}$ each hour of

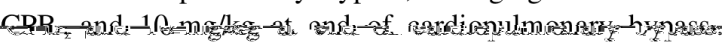
Heparin $350 \mathrm{U} / \mathrm{kg}$ was administered prior to bypass, and subsequent doses were administered as necessary to achieve an activated clotting time (ACT) of at least $480 \mathrm{~s}$ Protamine was administered after weaning from bypass in a dose of $1 \mathrm{mg}$ per $100 \mathrm{U}$ of estimated residual heparin. Additional boluses of $50 \mathrm{mg}$ were administered if the ACT remained more than $10 \%$ above baseline, or if there was evidence of residual heparin effect on a thromboelastogram. Insulin was administered as required to maintain a blood sugar level of less than or equal to $10 \mathrm{mmol} / \mathrm{L}$.

The active treatment of RIPC was applied with an automatic pneumatic tourniquet (ATS 3000, Zimmer, OH, USA) applied to an upper limb and inflated to $200 \mathrm{mmHg}$ for $5 \mathrm{~min}$, followed by $5 \mathrm{~min}$ of deflation. This process began with the first surgical incision and was repeated three times. In the control group, the same cycles were performed using a tourniquet wrapped around a towel lying next to the patient on the operating table.

The random allocation sequence was generated by a third party using an online randomisation sequence generator (http://www.randomization.com) using block randomisation with a block size of eight. No stratification was undertaken. The allocation to RIPC or control was concealed in sequentially numbered opaque envelopes until the intervention was applied by an anaesthetic technician 
without loss of masking. Each subject had a pneumatic tourniquet around their upper limb and a second pneumatic tourniquet wrapped around a towel next to them on the operating table. Once the patient was anaesthetised and draped, the anaesthetic technician attached an automatic pneumatic tourniquet device to the appropriate tourniquet under the drapes depending on whether the patient was assigned to the RIPC group or the control group.

Participants, all theatre staff (with the exception of a technician), ICU staff and those assessing outcomes were masked as to treatment allocation. Concealment of allocation was maintained until data collection was completed for all participants. Because the automatic pneumatic tourniquet was inflated on all participants, either on the arm or next to the participant, the treatment of participants in theatre appeared identical to theatre staff irrespective of whether the patients were allocated to the RIPC group or the control group.

There were four main end points: post-operative plasma high-sensitivity troponin T (hsTNT) levels measured at $6 \mathrm{~h}$ following aortic cross-clamp removal, hsTNT measured $12 \mathrm{~h}$ following aortic cross-clamp removal, duration of noradrenaline use amongst ICU survivors, and worst postoperative renal injury determined by RIFLE criteria [1]. The RIFLE criteria categorise AKI into levels of severity of 'risk', 'injury', 'failure', 'loss' and 'end stage renal failure' based on urine output and serum creatinine criteria. We had originally intended to measure troponin levels at 6,12 and $24 \mathrm{~h}$; however, due to funding constraints we were only able to measure two post-operative levels. We chose to measure troponin levels at 6 and $12 \mathrm{~h}$ because troponin levels usually peak early after cardiac surgery, [34] and early elevations in troponin predict major adverse cardiac events [24].

Other outcome measures were the duration of mechanical ventilation amongst ICU survivors; haemodynamic parameters of cardiac index, mixed venous oxygen saturation and mean arterial pressure recorded at 3,6, and $12 \mathrm{~h}$ from the time of ICU admission. Duration of noradrenaline use was defined as the number of hours from ICU admission until noradrenaline was permanently ceased during the index ICU admission. If a noradrenaline infusion was stopped and then re-started within the index ICU admission, the time when noradrenaline was ceased permanently was recorded. For the purposes of determining the duration of mechanical ventilation, extubation time was based on the final time a patient was extubated if more than one period of mechanical ventilation was required. In-hospital and 30-day mortality were recorded.

The power calculation was based on a previous study of RIPC in CABG [12, 32]. This study showed a mean (SD) troponin $\mathrm{T}$ in the control group at $12 \mathrm{~h}$ after surgery of $0.69 \mu \mathrm{g} / \mathrm{L}(0.48)$. Our sample size of 96 subjects, with equal sized groups could detect an absolute difference of troponin $\mathrm{T}$ of $0.28 \mu \mathrm{g} / \mathrm{L}$, a difference of about $40 \%$, which is less than the difference in troponin $\mathrm{T}$ between RIPC and control groups previously demonstrated in studies of RIPC in adults undergoing coronary artery bypass graft surgery $[12,32]$, with $80 \%$ power and an alpha of $5 \%$. No interim analyses were undertaken.

The primary statistical analysis plan was to compare post-operative troponin levels and vasopressor support by a $t$-test. In the event, the data distribution for both variables did not meet normality assumptions as they were skewed to the right and analysis was carried out on the natural-logarithm scale. Exponentiation of the difference on the logarithm scale gives the ratio of mean levels in the two groups. The incidence of post-operative renal injury based on RIFLE criteria [1] was analysed by contingency table analysis expressed as a Chi-square test. Pre-specified secondary analyses were carried out to account for potential confounding variables affecting post-operative troponin levels by analysis of co-variance (ANCOVA). The potential confounding variables were: sulphonylurea use, statin use, Euroscore predicted mortality [23], cross-clamp time, total bypass time, and the number of procedures performed with patients categorised as having one, two, or more than two procedures. Secondary outcomes were analysed by $t$ tests and contingency table analysis as relative risk differences or absolute risk differences. A $p$-value of 0.05 was used for statistical significance. For the six analyses of the main outcome variables $(6 \mathrm{~h}$ troponin, $12 \mathrm{~h}$ troponin, $6 \mathrm{~h}$ adjusted troponin, $12 \mathrm{~h}$ adjusted troponin, noradrenaline duration, and incidence of renal injury), we performed a Bonferroni correction to adjust for multiple comparisons while preserving the overall $p$-value of 0.05 . The adjusted $p$-value for accepting statistical significance for the main outcome variables after this adjustment was 0.0083 and the appropriate corresponding confidence intervals for estimates of $99.17 \%$ are presented in the results.

The study was registered on the Australian New Zealand Clinical Trials Registry (ACTRN 12609000965202) and received ethics approval from the Health Research Council of New Zealand Central Regional Ethics Committee (CEN/ 09/12/096). All participants provided written informed consent. The entry in the Clinical Trials Registry was amended after study completion to indicate that only two post-operative troponin levels were measured rather than three as originally intended.

\section{Results}

Ninety-six patients were recruited between 18 May 2010 and 23 June 2011. The participant flow for the study is shown in Fig. 1. Demographics are described in Table 1. Although participant characteristics were similar between 


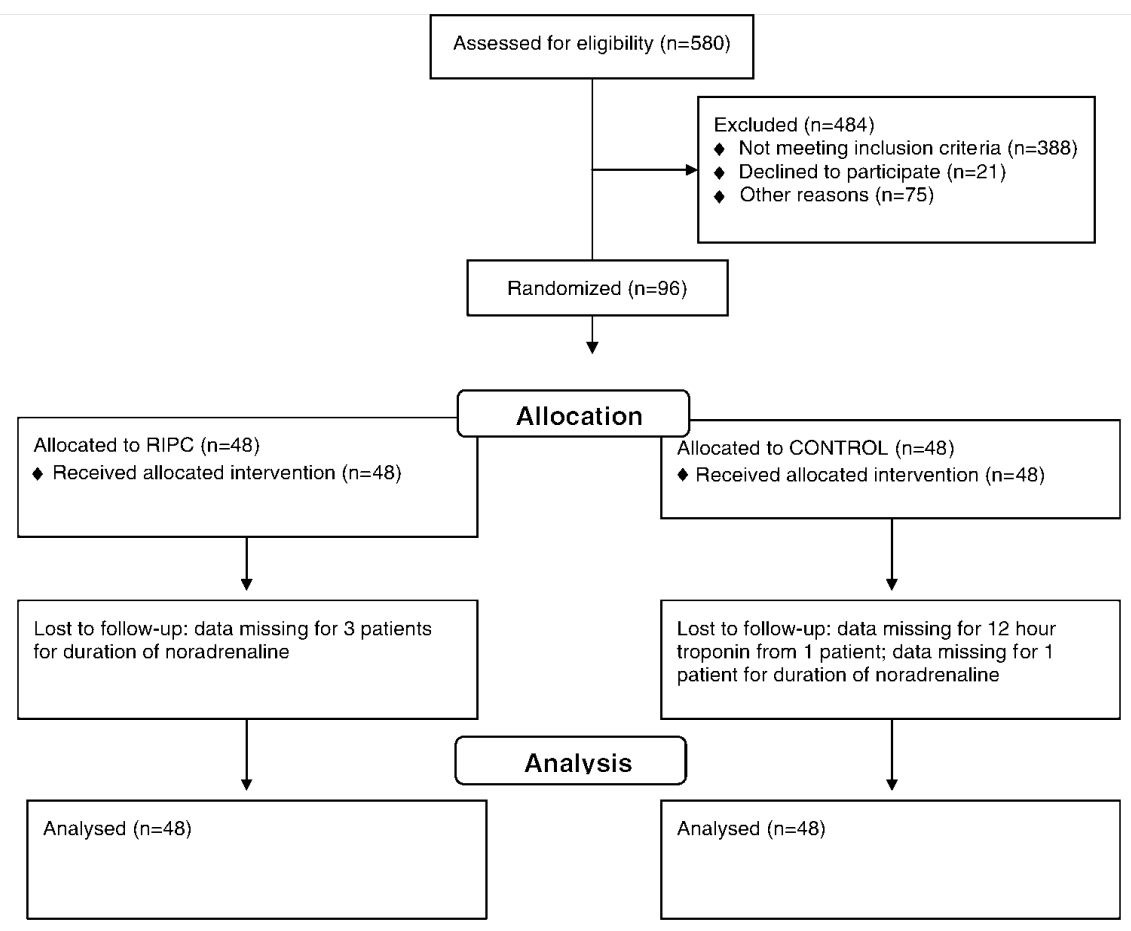

Fig. 1 CONSORT diagram

the randomised groups, there were some differences in the operation details (Table 2). 5/48 (10.4\%) of the patients assigned to the RIPC group had surgery involving three valves or two valves and a coronary artery bypass compared to $0 / 48(0 \%)$ of the participants in the control group.

The main outcome variables are shown in Table 3. The primary analysis showed point estimates consistent with higher hsTNT levels in the RIPC group than the control group at both 6 and $12 \mathrm{~h}$ after removal of the aortic crossclamp (see Table 3). In the secondary analysis after adjustment for baseline hsTNT, sulphonylurea use, statin use, number of surgery types, Euroscore, cross-clamp and total bypass time, the magnitude of the difference and statistical significance at both 6 and $12 \mathrm{~h}$ were reduced. Of the confounding variables, number of types of surgery $(P<0.001)$, absence of statin use $(P=0.02$ and $P=0.012$ for 6 and $12 \mathrm{~h}$, respectively), cross-clamp time $(P<0.001)$ and total bypass time $(P<0.001)$ were statistically significant predictors of greater absolute hsTNT.

Patients assigned to the RIPC group generally had higher requirements for post-operative ICU support than those assigned to the control group. In particular, they required a longer duration of noradrenaline support $[21 \mathrm{~h}$ (IQR 7-45) vs. $9 \mathrm{~h}$ (IQR 3-19); ratio of means 1.70 (99.17\% CI $0.86-3.34) ; P=0.04]$, were more likely to require post-operative vasopressin [17 vs. $0 \%$, absolute risk difference $17 \%$ (95\% CI 6-28); $P=0.006$ ], and generally had a longer duration of post-operative mechanical ventilation [11.7 h (IQR 6.2-22.2) vs. 7.6 h [IQR 5.1-15.8), ratio of means 1.67 (95\% CI 1.13-2.44); $P=0.01$ ]. Despite the RIPC group receiving more vasopressor support, cardiac physiology was generally similar between the two groups. RIPC did not affect the incidence of AKI based on RIFLE criteria (see Table 4) [1]. Despite the high-risk nature of the patients studied, the mortality rate was low with only $1 / 48(2.1 \%)$ patients in each group dying in hospital and no further deaths occurring by 30 days.

\section{Discussion}

This double-blind, randomised, controlled trial of RIPC in high-risk cardiac surgery found that, contrary to our a priori hypothesis, RIPC does not appear to lead to improved outcomes in this population. RIPC did not reduce postoperative hsTNT levels, renal injury or ICU support requirements.

This study has markedly different findings to the previous trials of RIPC in cardiac surgery that show either reduced troponin levels or no effect attributable to RIPC. 
Table 1 Demographics of study patients

ACE Angiotensin converting enzyme, no number

${ }^{\text {a }}$ Mean \pm SD

${ }^{\mathrm{b}}$ Median (IQR)

\begin{tabular}{|c|c|c|}
\hline Variable & Remote ischemic preconditioning & Control \\
\hline \multicolumn{3}{|l|}{ Demographics } \\
\hline Age (years) ${ }^{\mathrm{a}}$ & $65.5 \pm 12.6$ & $64.4 \pm 14.5$ \\
\hline Female sex, no./total no. (\%) & $19 / 48(39.6)$ & $17 / 48(35.4)$ \\
\hline \multicolumn{3}{|l|}{ Ethnicity } \\
\hline European, no./total no. (\%) & $32 / 48(66.7)$ & $38 / 48(79.2)$ \\
\hline Maori, no./total no. (\%) & $7 / 48(14.6)$ & $7 / 48(14.6)$ \\
\hline Other, no./total no. (\%) & $9 / 48(18.8)$ & $3 / 48(6.3)$ \\
\hline \multicolumn{3}{|l|}{ Pre-operative medications } \\
\hline Beta blockers, no./total no. (\%) & $35 / 48(72.9)$ & $29 / 48(60.4)$ \\
\hline ACE inhibitors, no./total no. (\%) & $25 / 48(52.1)$ & $25 / 48(52.1)$ \\
\hline Digoxin, no./total no. (\%) & $5 / 48(10.4)$ & $10 / 48(20.8)$ \\
\hline Frusemide, no./total no. (\%) & $20 / 48(41.7)$ & $17 / 48(35.4)$ \\
\hline Statins, no./total no. $(\%)$ & $28 / 48(58.3)$ & $30 / 48(62.5)$ \\
\hline Sulfonylureas, no./total no. (\%) & $4 / 48(8.3)$ & $1 / 48(2.1)$ \\
\hline \multicolumn{3}{|l|}{ Euroscore variables } \\
\hline Chronic pulmonary disease, no./total no. (\%) & $7 / 48(14.6)$ & $4 / 48(8.3)$ \\
\hline Extracardiac arteriopathy, no./total no. (\%) & $3 / 48(6.3)$ & $3 / 48(6.3)$ \\
\hline Nervous system dysfunction, no./total no. (\%) & $1 / 48(2.1)$ & $0 / 48(0)$ \\
\hline Previous cardiac surgery, no./total no. (\%) & $7 / 48(14.6)$ & $4 / 48(8.3)$ \\
\hline Baseline creatinine $(\mu \mathrm{mol} / \mathrm{L})^{\mathrm{a}}$ & $102.0 \pm 29.8$ & $95.0 \pm 21.8$ \\
\hline Baseline high-sensitivity troponin $\mathrm{T}(\mathrm{ng} / \mathrm{ml})^{\mathrm{b}}$ & $10(6.5-21)$ & $10(6-20)$ \\
\hline Logarithm baseline high-sensitivity troponin $\mathrm{T}$ & $2.5 \pm 0.8$ & $2.5 \pm 0.8$ \\
\hline Active endocarditis, no./total no. (\%) & $1 / 48(2.1)$ & $2 / 48(4.2)$ \\
\hline Critical pre-operative state; no./total no. (\%) & $1 / 48(2.1)$ & $1 / 48(2.1)$ \\
\hline Unstable angina, no/total no. (\%) & $0 / 48(0)$ & $0 / 48(0)$ \\
\hline Left ventricular function $>50 \%$, no./total no. $(\%)$ & $28 / 48(58.3)$ & $30 / 48(62.5)$ \\
\hline Left ventricular function $30-50 \%$, no./total no. (\%) & $20 / 48(41.7)$ & $14 / 48(29.2)$ \\
\hline Left ventricular function $<30 \%$, no./total no. $(\%)$ & $0 / 48(0)$ & $4 / 48(8.3)$ \\
\hline Recent myocardial infarction, no./total no. (\%) & $11 / 48(22.9)$ & $15 / 48(31.3)$ \\
\hline Pulmonary hypertension, no./total no. (\%) & $6 / 48(12.5)$ & $5 / 48(10.4)$ \\
\hline Euroscore & $7.1 \pm 6.1$ & $6.6 \pm 6.1$ \\
\hline
\end{tabular}

The reason for our findings is not clear. Previous positive trials in adults have generally focussed on patients having uncomplicated cardiac surgery [12, 20, 31, 32, 37]. Only two of these trials included patients having valvular surgery. In one, patients who had valve surgery made up only $8 / 55(14.5 \%)$ of the patients enrolled in the trial [32], and in the other, post-operative troponin levels were not reported [37]. In two other trials [12, 32], a post hoc analysis of the effect of RIPC on the relative risk of AKI defined by AKI Network (AKIN) criteria in non-diabetic patients revealed that RIPC significantly reduced post-operative AKI [33]. The two largest trials of RIPC in cardiac surgery to date, which were limited to 'off-pump' coronary artery bypass graft surgery [14] and CABG with cold-blood cardioplegia bypass, respectively [27], both failed to demonstrate a significant effect of RIPC on troponin levels or postoperative ICU-support requirements. The latter also reported that RIPC did not reduce renal injury although a standardised definition of AKI was not used [27]. Unlike the previous positive trials, both these negative trials, like ours, induced remote ischemic preconditioning as surgery began, rather than before it. One previous trial limited to adults having complex valvular surgery used three $10 \mathrm{~min}$ cycles of lower-limb ischaemia followed by $10 \mathrm{~min}$ of reperfusion and demonstrated that RIPC reduced CK-MB levels $24 \mathrm{~h}$ after surgery [6]. However, that trial was primarily designed to assess the effect of RIPC on postoperative renal injury and, like our trial, failed to demonstrate a significant effect of RIPC on AKI [6]. Another study limited to valvular surgery patients failed to demonstrate an effect of preconditioning on troponin levels; although, only 53 patients were included in the study [30]. One recently published trial focussing on patients having valvular surgery demonstrated significant reductions in post-operative troponin levels [36]. A recent paper has also reported that RIPC reduced the incidence of AKI in adult 
Table 2 Operative history of study patients
$A V R$ Aortic valve replacement,

$M V R$ mitral valve repair or replacement, $T A$ tricuspid annuloplasty, $C A B G$ coronary artery bypass graft, no. number, $E F$ ejection fraction, $A S D$ atrial septal defect, min minutes

${ }^{\text {a }}$ Mean \pm SD

\begin{tabular}{lll}
\hline Variable & $\begin{array}{l}\text { Remote Ischemic } \\
\text { preconditioning }\end{array}$ & Control \\
\hline Operation performed & & \\
AVR, MVR, TA, CABG, no./total no. (\%) & $1 / 48(2.1)$ & $0 / 48(0)$ \\
CABG with pre-op EF <50\%, no./total no. (\%) & $9 / 48(18.8)$ & $9 / 48(18.8)$ \\
AVR, MVR, CABG, no./total no. (\%) & $1 / 48(2.1)$ & $0 / 48(0)$ \\
MVR, no./total no. (\%) & $8 / 48(16.7)$ & $10 / 48(20.1)$ \\
AVR, MVR, TA, no./total no. (\%) & $2 / 48(4.2)$ & $0 / 48(0)$ \\
AVR, CABG, no./total no. (\%) & $10 / 48(20.8)$ & $14 / 48(29.2)$ \\
MVR, CABG, no./total no. (\%) & $7 / 48(14.6)$ & $2 / 48(4.2)$ \\
AVR, MVR, no./total no. (\%) & $4 / 48(8.3)$ & $6 / 48(12.5)$ \\
AVR, MVR, TA, ASD REPAIR, no./total no. (\%) & $1 / 48(2.1)$ & $0 / 48(0)$ \\
AVR, no./total no. (\%) & $3 / 48(6.3)$ & $2 / 48(4.2)$ \\
MVR, TA, no./total no. (\%) & $2 / 48(4.2)$ & $5 / 48(10.4)$ \\
Number of procedures & & $21 / 48(43.8)$ \\
One, no./total no. (\%) & $20 / 48(41.7)$ & $27 / 48(56.3)$ \\
Two, no./total no. (\%) & $23 / 48(47.9)$ & $0 / 48(0)$ \\
More than two, no./total no. $(\%)$ & $5 / 48(10.4)$ & $104.7 \pm 40.6$ \\
Bypass details & & $2 / 48(4.2)$ \\
Cross-clamp time ${ }^{a}$ (min) & $117.4 \pm 50.3$ &
\end{tabular}

patients following various types of cardiac surgery from 47 to $20 \%(P=0.004)$ [37]; however, the incidence of AKI in their control arm was much higher than in either group in our trial.

Our trial differs from the previous trials in a number of respects. A standardised protocol was used and included the use of generous doses of opioids as well as propofol and volatile anaesthesia throughout the operation including whilst on cardiopulmonary bypass. There are now significant inconsistencies in the literature regarding the efficacy of RIPC and it is possible that in beneficial effects are only seen when particular anaesthesia regimes are used [26]. There is evidence that volatile anaesthesia itself has ischemic preconditioning effects [15]. Many previous negative trials in adults having cardiac surgery used volatile anesthesia $[14,16,27]$ and positive trials have used volatile anaesthesia inconsistently $[32,36]$ or did not use volatile anaesthesia at all $[12,20]$. However, only one trial has specifically evaluated the efficacy of RIPC with isoflurane anaesthesia compared to propofol anaesthesia [17]. Paradoxically, this trial demonstrated that RIPC was effective when isoflurane was used, but not when propofol was used [17]. Opioids have been suggested to exhibit preconditioning effects when tested in animals [28] and our anaesthetic protocol mandated the administration of generous doses of morphine including a $20 \mathrm{mg}$ bolus immediately prior to aortic cross-clamping.

Beta blockers have been demonstrated to attenuate anaesthesia-induced preconditioning $[10,18,19]$ and the fact that beta blocker use was somewhat more common in the RIPC group than in the control group may help explain our findings. Similarly, the higher use of sulphonylureas in the RIPC group may also be of significance as the sulphonylurea, glibenclamide, may interfere with signal transduction of ischemic preconditioning and render it ineffective [22]. Whilst no patients in our study were receiving glibenclamide, this may be a class effect and is an important confounding factor to consider.

Our trial includes patients having a range of high-risk cardiac operations encompassing both valvular and nonvalvular surgery. There is no accepted definition of what constitutes high-risk cardiac surgery and we chose to define this pragmatically as outlined by our inclusion criteria. The patients enrolled in our trial had much longer average bypass times than those enrolled in most previous trials $[12,14,27,32,35]$. By choosing to study a heterogenous group of high-risk patients with generally long cross-clamp times, we selected a population that could be expected to have a high risk of needing prolonged ICU support and a higher risk of myocardial ischaemia because of extended cross-clamp times. Choosing a heterogenous group increases the generalisability of our findings compared to the previous studies using a more limited range of cardiac surgeries. However, because we analysed a number of outcome variables, there is an increased risk of type I error which may be an explanation for our unexpected findings suggesting the possibility of harm attributable to RIPC. Furthermore, our heterogenous study population meant our 
Table 3 Main outcome variables

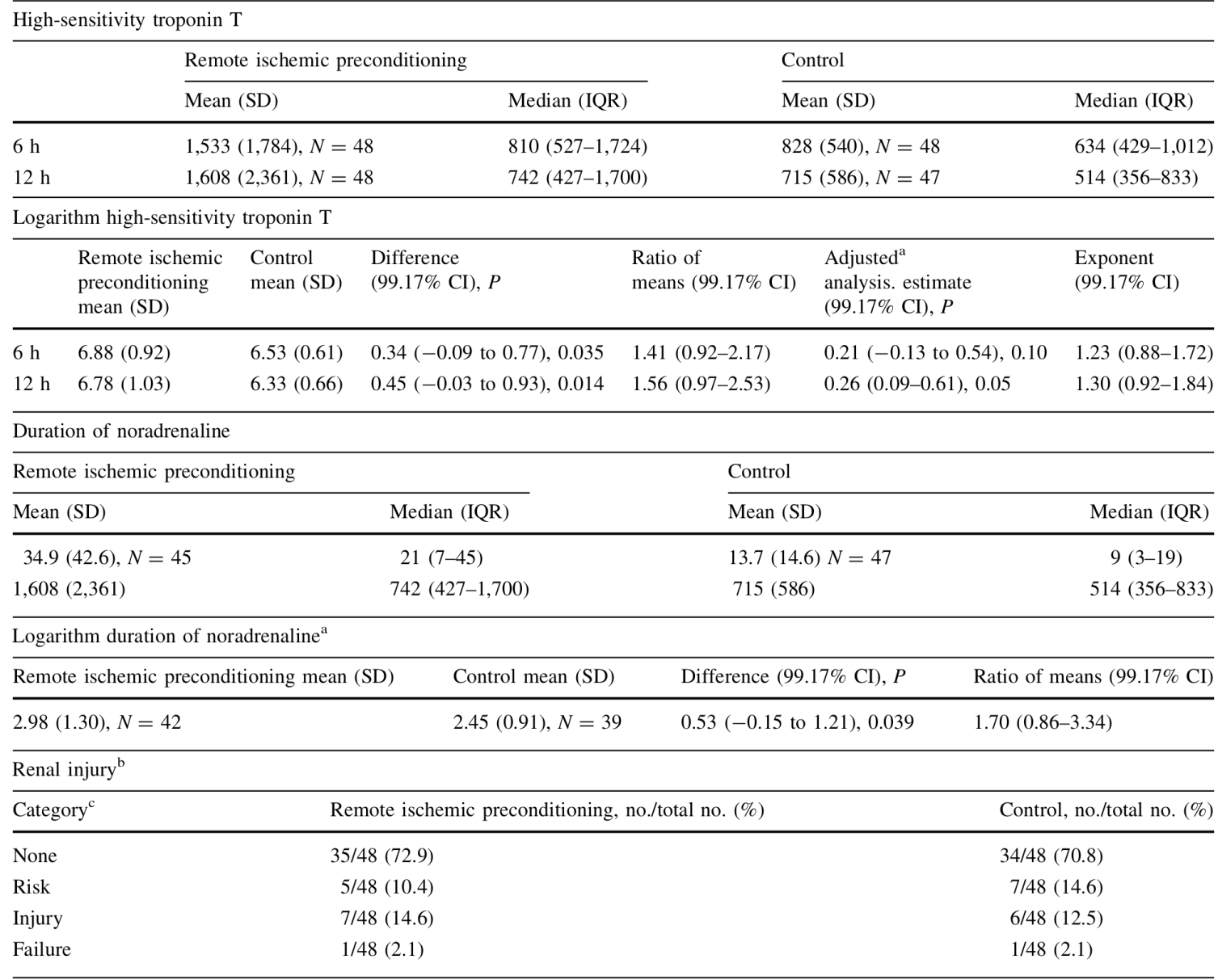

$S D$ Standard deviation, $I Q R$ interquartile range

a Adjusted for baseline high-sensitivity troponin, sulphonylurea use, statin use, number of surgery types $(1,2$ or $>2)$, Euroscore predicted mortality, cross-clamp time, total bypass time

b Three patients in the RIPC group and eight patients in the control group did not receive noradrenaline. Because logarithm of zero is undefined, these patients were excluded from the analysis based on logarithm duration of noradrenaline

${ }^{c}$ For the purposes of statistical comparison, injury and failure categories were merged giving a Chi-squared value of 0.41 on 2 degrees of freedom, $P=0.82$

study lacked statistical power to provide definitive results. However, to attempt to offset this, we controlled perioperative confounding factors to a greater extent than any previous study. Specifically, we standardised cardiac anaesthesia, cardiopulmonary bypass and haemodynamic management in the post-operative period as outlined in the electronic supplementary material. Controlling these factors served to reduce confounding as far as possible. Moreover, unlike the previous trials in the field we conducted a multivariate analysis to account for observed baseline differences. Nevertheless, the heterogeneity of our study population, small sample size, and the number of statistical analyses we carried out, mean our results should be viewed as hypothesis generating. Whilst we measured post operative troponin at fewer time points than in previous RIPC studies, there are data demonstrating that kinetic analyses are no more accurate than single measures in predicting major adverse cardiac events [7].

Our finding of point estimates suggesting an increase in hsTNT levels and ICU support requirements in subjects receiving RIPC makes a beneficial effect of this technique in the setting of high-risk cardiac surgery with volatile 
Table 4 Other outcome variables

\begin{tabular}{|c|c|c|c|c|}
\hline \multicolumn{5}{|c|}{ Duration of ventilation } \\
\hline \multicolumn{2}{|c|}{ Remote ischemic preconditioning } & & \multicolumn{2}{|l|}{ Control } \\
\hline Mean (SD) & Median (IQR) & \multicolumn{2}{|r|}{ Mean (SD) } & Median (IQR) \\
\hline $30.2(61.1), N=48$ & $11.7(6.2-22.2)$ & \multicolumn{2}{|r|}{$12.1(11.8), N=48$} & $7.6(5.1-15.8)$ \\
\hline \multicolumn{5}{|c|}{ Logarithm duration of ventilation } \\
\hline RIPC mean (SD) & Control mean (SD) & \multicolumn{2}{|c|}{ Difference $(95 \% \mathrm{CI}), P$} & Ratio of means $(95 \% \mathrm{CI})$ \\
\hline $2.70(1.08), N=47$ & $2.19(0.78), N=47$ & \multicolumn{2}{|c|}{$0.51(0.12-0.89), 0.01$} & $1.67(1.13-2.44)$ \\
\hline \multicolumn{5}{|l|}{ Re-intubation rate } \\
\hline \multicolumn{2}{|c|}{ Remote ischemic preconditioning mean (SD) } & \multicolumn{2}{|c|}{ Control mean (SD) } & Relative risk $(95 \% \mathrm{CI}), P$ \\
\hline \multicolumn{2}{|l|}{$5 / 47(10.6)$} & \multicolumn{2}{|l|}{$1 / 46(2.2)$} & $4.9(0.59-40.3), 0.20$ \\
\hline \multicolumn{5}{|l|}{ Cardiac physiology } \\
\hline Variable & Remote ischemic preconditioning & \multicolumn{2}{|c|}{ Control } & Difference $(95 \% \mathrm{CI}), P$ \\
\hline \multicolumn{5}{|l|}{ Cardiac index } \\
\hline $3 \mathrm{~h}$ & $2.46(0.51), N=47$ & \multicolumn{2}{|c|}{$2.48(0.57), N=47$} & $-0.02(-0.25$ to 0.21$), 0.84$ \\
\hline $6 \mathrm{~h}$ & $2.58(0.47), N=47$ & \multicolumn{2}{|c|}{$2.57(0.61), N=42$} & $0.005(-0.23$ to 0.24$), 0.97$ \\
\hline $12 \mathrm{~h}$ & $2.54(0.52), N=44$ & \multicolumn{2}{|c|}{$2.67(0.66), N=40$} & $-0.14(-0.40$ to 0.12$), 0.29$ \\
\hline \multicolumn{5}{|l|}{ Mean arterial pressure } \\
\hline $3 \mathrm{~h}$ & $68.2(7.6), N=47$ & \multicolumn{2}{|c|}{$70.8(6.3), N=43$} & $-2.6(-5.5$ to 0.32$), 0.08$ \\
\hline $6 \mathrm{~h}$ & $68.9(6.6), N=46$ & \multicolumn{2}{|c|}{$71.3(7.2), N=44$} & $-2.4(-5.3$ to 0.5$), 0.10$ \\
\hline $12 \mathrm{~h}$ & $68.2(9.0), N=44$ & \multicolumn{2}{|c|}{$72.4(6.9), N=43$} & -4.2 ( -7.6 to -0.8$), 0.017$ \\
\hline \multicolumn{5}{|c|}{ Mixed venous oxygen saturation } \\
\hline $3 \mathrm{~h}$ & $63.9(7.3), N=47$ & \multicolumn{2}{|c|}{$64.0(8.7), N=47$} & -0.04 ( -3.4 to 3.4$), 0.98$ \\
\hline $6 \mathrm{~h}$ & $65.9(8.1), N=47$ & \multicolumn{2}{|c|}{$64.8(7.1), N=42$} & $1.1(-2.1$ to 4.4$), 0.49$ \\
\hline $12 \mathrm{~h}$ & $64.2(9.9), N=44$ & \multicolumn{2}{|c|}{$63.4(7.6), N=40$} & $0.8(-3.1$ to 4.6$), 0.70$ \\
\hline \multicolumn{5}{|l|}{ Cardiac support } \\
\hline Variable & Remote ischemic preconditioning & \multicolumn{2}{|l|}{ Control } & r risk difference ${ }^{\mathrm{a}}(95 \% \mathrm{CI}), P$ \\
\hline Milrinone use & $20 / 48(41.7)$ & $18 / 48$ & $1.11(0$ & 2), 0.83 \\
\hline Vasopressin use & $8 / 47(17.0)$ & $0 / 47(C$ & $17.0(6$ & .006 \\
\hline
\end{tabular}

SD Standard deviation, $I Q R$ interquartile range

${ }^{a}$ Risk difference used for 'vasopressin use because relative risk cannot be estimated with a zero cell count for the control group

anaesthesia and propofol administration less likely than the previous research suggests.

A particular strength of our study is that, unlike some previous trials that found beneficial effects of RIPC in cardiac surgery $[12,31,32,36,37]$, we maintained rigorous blinding of the staff involved in the operative and postoperative care of study participants. Our inclusion of patients undergoing having complex valvular procedures may mean that troponin rises were partly attributable to direct myocardial trauma rather than myocardial ischemia in some patients; however, our patient group would also have been expected to show significant myocardial ischemia due to their long cross-clamp times and RIPC might have been expected to be useful in these circumstances [11].

Overall, we consider that RIPC probably does not reduce hsTNT levels, renal injury, or ICU-support requirements in high-risk cardiac surgery in patients receiving anaesthesia in the manner that we have described. However, our data should be regarded as exploratory with further studies needed in high-risk patients. We would recommend that such studies stratify participants by operation type. Whilst it appears that RIPC involves both neural and humoral mechanisms in animal models $[4,21]$, research evaluating the potential mechanisms by which RIPC exerts its effects in humans is currently in its infancy 
$[13,16]$. However, given the current uncertainty regarding the confounding influences of various anaesthesia regimens, determining the interactions between RIPC and other preconditioning pathways is now an important priority.

Acknowledgments This study was funded by unrestricted grants from the New Zealand Lotteries Commission and the National Heart Foundation of New Zealand.

Conflicts of interest None.

\section{References}

1. Bellomo R, Ronco C, Kellum JA, Mehta RL, Palevsky P (2004) Acute renal failure: definition, outcome measures, animal models, fluid therapy and information technology needs: the Second International Consensus Conference of the Acute Dialysis Quality Initiative (ADQI) Group. Crit Care 8:R204-R212. doi: $10.1186 / \mathrm{cc} 2872$

2. Birnbaum Y, Hale SL, Kloner RA (1997) Ischemic preconditioning at a distance: reduction of myocardial infarct size by partial reduction of blood supply combined with rapid stimulation of the gastrocnemius muscle in the rabbit. Circulation 96:1641-1646. doi:10.1161/01.CIR.96.5.1641

3. Botker HE, Kharbanda R, Schmidt MR, Bottcher M, Kaltoft AK, Terkelsen CJ, Munk K, Andersen NH, Hansen TM, Trautner S, Lassen JF, Christiansen EH, Krusell LR, Kristensen SD, Thuesen L, Nielsen SS, Rehling M, Sorensen HT, Redington AN, Nielsen TT (2010) Remote ischaemic conditioning before hospital admission, as a complement to angioplasty, and effect on myocardial salvage in patients with acute myocardial infarction: a randomised trial. Lancet 375:727-734. doi:10.1016/S0140-6736 (09)62001-8

4. Breivik L, Helgeland E, Aarnes EK, Mrdalj J, Jonassen AK (2011) Remote postconditioning by humoral factors in effluent from ischemic preconditioned rat hearts is mediated via PI3 K/ Akt-dependent cell-survival signaling at reperfusion. Basic Res Cardiol 106:135-145. doi:10.1007/s00395-010-0133-0

5. Cheung MM, Kharbanda RK, Konstantinov IE, Shimizu M, Frndova H, Li J, Holtby HM, Cox PN, Smallhorn JF, Van Arsdell GS, Redington AN (2006) Randomized controlled trial of the effects of remote ischemic preconditioning on children undergoing cardiac surgery: first clinical application in humans. J Am Coll Cardiol 47:2277-2282. doi:10.1016/j.jacc.2006.01.066

6. Choi YS, Shim JK, Kim JC, Kang KS, Seo YH, Ahn KR, Kwak YL (2011) Effect of remote ischemic preconditioning on renal dysfunction after complex valvular heart surgery: a randomized controlled trial. J Thorac Cardiovasc Surg 142:148-154. doi: 10.1016/j.jtcvs.2010.11.018

7. Fellahi JL, Hanouz JL, Gue X, Monier E, Guillou L, Riou B (2008) Kinetic analysis of cardiac troponin I release is no more accurate than a single 24-h measurement in predicting in-hospital outcome after cardiac surgery. Eur J Anaesthesiol 25:490-497. doi:10.1017/S0265021508003827

8. Gho BC, Schoemaker RG, van den Doel MA, Duncker DJ, Verdouw PD (1996) Myocardial protection by brief ischemia in noncardiac tissue. Circulation 94:2193-2200

9. Gunaydin B, Cakici I, Soncul H, Kalaycioglu S, Cevik C, Sancak B, Kanzik I, Karadenizli Y (2000) Does remote organ ischaemia trigger cardiac preconditioning during coronary artery surgery? Pharmacol Res 41:493-496. doi:10.1006/phrs.1999.0611
10. Hanouz JL, Yvon A, Massetti M, Lepage O, Babatasi G, Khayat A, Bricard H, Gerard JL (2002) Mechanisms of desfluraneinduced preconditioning in isolated human right atria in vitro. Anesthesiology 97:33-41

11. Hausenloy DJ, Baxter G, Bell R, Botker HE, Davidson SM, Downey J, Heusch G, Kitakaze M, Lecour S, Mentzer R, Mocanu MM, Ovize M, Schulz R, Shannon R, Walker M, Walkinshaw G, Yellon DM (2010) Translating novel strategies for cardioprotection: the Hatter workshop recommendations. Basic Res Cardiol 105:677-686. doi:10.1007/s00395-010-0121-4

12. Hausenloy DJ, Mwamure PK, Venugopal V, Harris J, Barnard M, Grundy E, Ashley E, Vichare S, Di Salvo C, Kolvekar S, Hayward M, Keogh B, MacAllister RJ, Yellon DM (2007) Effect of remote ischaemic preconditioning on myocardial injury in patients undergoing coronary artery bypass graft surgery: a randomised controlled trial. Lancet 370:575-579. doi:10.1016/ S0140-6736(07)61296-3

13. Heusch G, Musiolik J, Kottenberg E, Peters J, Jakob H, Thielmann M (2012) STAT5 activation and cardioprotection by remote ischemic preconditioning in humans. Circ Res 110:111115. doi:10.1161/CIRCRESAHA.111.259556

14. Hong DM, Mint JJ, Kim JH, Sohn IS, Lim TW, Lim YJ, Bahk JH, Jeon Y (2010) The effect of remote ischaemic preconditioning on myocardial injury in patients undergoing off-pump coronary artery bypass graft surgery. Anaesth Intensive Care 38:924-929

15. Hu ZY, Liu J (2009) Mechanism of cardiac preconditioning with volatile anaesthetics. Anaesth Intensive Care 37:532-538

16. Karuppasamy P, Chaubey S, Dew T, Musto R, Sherwood R, Desai J, John L, Shah AM, Marber MS, Kunst G (2011) Remote intermittent ischemia before coronary artery bypass graft surgery: a strategy to reduce injury and inflammation? Basic Res Cardiol 106:511-519. doi:10.1007/s00395-011-0185-9

17. Kottenberg E, Thielmann M, Bergmann L, Heine T, Jakob H, Heusch G, Peters J (2012) Protection by remote ischemic preconditioning during coronary artery bypass graft surgery with isoflurane but not propofol: a clinical trial. Acta Anaesthesiol Scand 56:30-38. doi:10.1111/j.1399-6576.2011.02585.x

18. Lange M, Redel A, Smul TM, Lotz C, Nefzger T, Stumpner J, Blomeyer C, Gao F, Roewer N, Kehl F (2009) Desfluraneinduced preconditioning has a threshold that is lowered by repetitive application and is mediated by beta 2-adrenergic receptors. J Cardiothorac Vasc Anesth 23:607-613. doi: 10.1053/j.jvca.2009.01.016

19. Lange M, Smul TM, Blomeyer CA, Redel A, Klotz KN, Roewer N, Kehl F (2006) Role of the betal-adrenergic pathway in anesthetic and ischemic preconditioning against myocardial infarction in the rabbit heart in vivo. Anesthesiology 105:503-510

20. Li L, Luo W, Huang L, Zhang W, Gao Y, Jiang H, Zhang C, Long L, Chen S (2010) Remote perconditioning reduces myocardial injury in adult valve replacement: a randomized controlled trial. J Surg Res 164:e21-e26. doi:10.1016/j.jss.2010.06.016

21. Lim SY, Yellon DM, Hausenloy DJ (2010) The neural and humoral pathways in remote limb ischemic preconditioning. Basic Res Cardiol 105:651-655. doi:10.1007/s00395-010-0099-y

22. Liu Y, Sato T, O'Rourke B, Marban E (1998) Mitochondrial ATP-dependent potassium channels: novel effectors of cardioprotection? Circulation 97:2463-2469. doi:10.1161/01.CIR. 97.24.2463

23. Nashef SA, Roques F, Michel P, Gauducheau E, Lemeshow S, Salamon R (1999) European system for cardiac operative risk evaluation (EuroSCORE). Eur J Cardiothorac Surg 16:9-13. doi: 10.1016/S1010-7940(99)00134-7

24. Nesher N, Alghamdi AA, Singh SK, Sever JY, Christakis GT, Goldman BS, Cohen GN, Moussa F, Fremes SE (2008) Troponin after cardiac surgery: a predictor or a phenomenon? Ann Thorac Surg 85:1348-1354. doi:10.1016/j.athoracsur.2007.12.077 
25. Oxman T, Arad M, Klein R, Avazov N, Rabinowitz B (1997) Limb ischemia preconditions the heart against reperfusion tachyarrhythmia. Am J Physiol 273:H1707-H1712

26. Peters J (2011) Remote ischaemic preconditioning of the heart: remote questions, remote importance, or remote preconditions? Basic Res Cardiol 106:507-509. doi:10.1007/s00395-011-0187-7

27. Rahman IA, Mascaro JG, Steeds RP, Frenneaux MP, Nightingale $\mathrm{P}$, Gosling P, Townsend P, Townend JN, Green D, Bonser RS (2010) Remote ischemic preconditioning in human coronary artery bypass surgery: from promise to disappointment? Circulation 122:S53-S59. doi:10.1161/circulationaha.109.926667

28. Schultz JE, Hsu AK, Gross GJ (1996) Morphine mimics the cardioprotective effect of ischemic preconditioning via a glibenclamide-sensitive mechanism in the rat heart. Circ Res 78:1100-1104

29. Longacre LS, Kloner RA, Arai AE, Baines CP, Bolli R, Braunwald E, Downey J, Gibbons RJ, Gottlieb RA, Heusch G, Jennings RB, Lefer DJ, Mentzer RM, Murphy E, Ovize M, Ping P, Przyklenk K, Sack MN, Vander Heide RS, Vinten-Johansen J, Yellon DM (2011) New horizons in cardioprotection: recommendations from the 2010 National Heart, Lung, and Blood Institute Workshop. Circulation 124:1172-1179. doi:10.1161/ CIRCULATIONAHA.111.032698

30. Siami S, Bailly-Salin J, Polito A, Porcher R, Blanchard A, Haymann JP, Laborde K, Maxime V, Boucly C, Carlier R, Annane D, Sharshar T (2010) Osmoregulation of vasopressin secretion is altered in the postacute phase of septic shock. Crit Care Med 38:1962-1969. doi:10.1097/CCM.0b013e3181eb9acf

31. Thielmann M, Kottenberg E, Boengler K, Raffelsieper C, Neuhaeuser M, Peters J, Jakob H, Heusch G (2010) Remote ischemic preconditioning reduces myocardial injury after coronary artery bypass surgery with crystalloid cardioplegic arrest. Basic Res Cardiol 105:657-664. doi:10.1007/s00395-010-0104-5
32. Venugopal V, Hausenloy DJ, Ludman A, Di Salvo C, Kolvekar S, Yap J, Lawrence D, Bognolo J, Yellon DM (2009) Remote ischaemic preconditioning reduces myocardial injury in patients undergoing cardiac surgery with cold-blood cardioplegia: a randomised controlled trial. Heart 95:1567-1571. doi:10.1136/hrt. 2008.155770

33. Venugopal V, Laing CM, Ludman A, Yellon DM, Hausenloy D (2010) Effect of remote ischemic preconditioning on acute kidney injury in nondiabetic patients undergoing coronary artery bypass graft surgery: a secondary analysis of 2 small randomized trials. Am J Kidney Dis 56:1043-1049. doi:10.1053/j.ajkd. 2010.07.014

34. Vermes E, Mesguich M, Houel R, Soustelle C, Le Besnerais P, Hillion ML, Loisance D (2000) Cardiac troponin I release after open heart surgery: a marker of myocardial protection? Ann Thorac Surg 70:2087-2090

35. Wagner R, Piler P, Bedanova H, Adamek P, Grodecka L, Freiberger T (2010) Myocardial injury is decreased by late remote ischaemic preconditioning and aggravated by tramadol in patients undergoing cardiac surgery: a randomised controlled trial. Interact Cardiovasc Thorac Surg 11:758-762. doi:10.1510/icvts. 2010.243600

36. Xie JJ, Liao XL, Chen WG, Huang DD, Chang FJ, Chen W, Luo ZL, Wang ZP, Ou JS (2011) Remote ischaemic preconditioning reduces myocardial injury in patients undergoing heart valve surgery: randomised controlled trial. Heart. doi:10.1136/heartjnl2011-300860

37. Zimmerman RF, Ezeanuna PU, Kane JC, Cleland CD, Kempananjappa TJ, Lucas FL, Kramer RS (2011) Ischemic preconditioning at a remote site prevents acute kidney injury in patients following cardiac surgery. Kidney Int 80:861-867. doi:10.1038/ ki. 2011.156 


\title{
Remote ischaemic preconditioning does not alter perioperative cytokine production in high-risk cardiac surgery
}

\author{
Jenni M Williams, ${ }^{1}$ Paul Young, ${ }^{2,3}$ Janine Pilcher, ${ }^{3}$ Mark Weatherall, ${ }^{2-4}$
} John Holmes Miller, ${ }^{1}$ Richard Beasley, ${ }^{2,3}$ Anne Camille La Flamme ${ }^{1}$

\begin{abstract}
- Additional data are published online only. To view these files please visit the journal online (http://dx doi rorg/10.1136/ heartasia-2012-0101221

${ }^{1}$ School of Biological Sciences, Victoria University of Wellington Wellington, New Zealand ${ }^{2}$ Capital and Coast Distric Health Board, Wellington, New Health Board
Zealand Zealand
${ }^{3}$ Medical Research Institute of New Zealand, Wellington, New Zealand

${ }^{4}$ Department of Medicine Otago University, Wellington, New Zealand

Correspondence to Dr Anne Camille La Flamme School of Biological Sciences, Victoria University of Wellington, PO Box 600, Wellington 6140, New Zealand; anne.laflamme@ vuw.ac.nz
\end{abstract}

$\overline{\text { Accepted } 16}$ July 2012

\section{ABSTRACT}

Rationale Remote ischaemic preconditioning (RIPC) is a novel cardioprotective strategy that uses brief intermittent limb ischaemia to protect the myocardium and other organs from perioperative ischaemic damage. The precise mechanism through which this protective effect occurs is unknown, but potentially could be related to changes in blood-borne mediators such as cytokines.

Objective To determine whether RIPC alters inflammatory cytokine expression in a double-blind, randomised, controlled trial of patients undergoing highrisk cardiac surgery.

Methods and results Serum interleukin (IL)-6, IL-8, and IL-10 levels from 95 patients randomised to RIPC $(n=47)$ or control treatment $(n=48)$ were measured preoperatively, and 1, 2, 3, 6 and $12 \mathrm{~h}$ after cross-clamp removal. Systemic concentrations of all cytokines were increased from baseline following surgery, and, compared with simple procedures, complex surgeries were associated with significantly higher release of IL-6 (ratio of mean area under the curves $1.54(95 \% \mathrm{Cl} 1.02$ to 2.34), $p=0.04$ ) and IL-10 (1.97 (1.16 to 3.35),

$\mathrm{p}=0.012$ ). No significant difference in mean cytokine levels between the RIPC and control groups was detected at any time point, irrespective of the type of surgery undergone.

Conclusions High levels of IL-6, IL-8 and IL-10 are produced during high-risk cardiac surgery, and RIPC does not alter these elevated perioperative cytokine concentrations. Identification of factors that influence the ability to induce RIPC-mediated cardioprotection should be the priority of future research.

Trial registration is in the Australian New Zealand Clinical Trials Registry (http://www.anzctr.org.au; ACTRN12609000965202)

\section{INTRODUCTION}

Ischaemia-reperfusion (I/R) injury is a major cause of myocardial and renal damage following cardiac surgery with cardiopulmonary bypass. Remote ischaemic preconditioning (RIPC) is a novel cytoprotective strategy capable of attenuating $\mathrm{I} / \mathrm{R}$ injury by utilising brief periods of ischaemia in one tissue to elicit protection from subsequent prolonged ischaemic insults in other organs. Animal studies have repeatedly demonstrated the ability of this technique to reduce myocardial infarct size by up to $50 \%$ in cardiac I/R injury ${ }^{12}$; however, trials of RIPC in humans undergoing cardiac surgery have not shown such reproducible results. ${ }^{3}{ }^{4}$ These inconsistencies have prompted a call for further research investigating the mechanisms of RIPC in order to define its clinical indication and limitations. ${ }^{5}$

There is mounting evidence that RIPC modulates the inflammatory response, suppressing pro-inflammatory gene expression in human leukocytes ${ }^{6}$ and activation of the key effector cells of postoperative tissue damage, neutrophils.' Furthermore, the inflammatory cytokine, interleukin (IL)-6, is essential for preconditioning-induced cardioprotection in mice. ${ }^{8}$ In cardiac surgery, high levels of IL- 6 and IL- 8 have been associated with numerous postoperative complications, including increased myocardial damage 9 and acute kidney injury, ${ }^{10}$ yet the impact of RIPC on early expression of these biomarkers has not been previously characterised. IL- 6 , IL- 8 , and other cytokines may have a direct role in the initiation of RIPC or, alternatively, function as indirect markers of preconditioning. Higher systemic levels of these mediators are associated with increasing duration and invasiveness of surgery. ${ }^{11}{ }^{12}$ In this study, we therefore aimed to determine whether RIPC alters cytokine expression in the perioperative period in patients undergoing high-risk cardiac surgery.

\section{METHODS}

We completed a double-blind, randomised, controlled trial of RIPC in 96 adult high-risk cardiac surgery patients recruited between May 2010 and June 2011. The study was registered on the Australian New Zealand Clinical Trials Registry (ACTRN 12609000965202) and received ethics approval from the Central Regional Ethics Committee (CEN/09/12/096).

Patients over 18 years of age were invited to participate if they were undergoing high-risk cardiac surgery, defined as double, triple or mitral valve replacement, coronary artery bypass graft surgery (CABG) with ejection fraction $<50 \%$, CABG +valve(s), or any redo cardiac operation. These surgeries were considered high-risk because they are generally associated with extended bypass times, or are performed in patients with significantly impaired cardiac function. For the study overall, patients with peripheral vascular disease affecting the upper limbs, or requiring deep hypothermic circulatory arrest or radial artery conduit harvesting were excluded. Additionally, for the cytokine analyses, patients receiving systemic immunosuppressives were also excluded. Written informed consent was obtained from all patients. 


\section{Original research}

Patients were permuted-block randomised in groups of eight by a third party using an online randomisation sequence generator with an allocation ratio of $1: 1$ to either RIPC or control. Treatment group allocation was concealed in sequentially numbered opaque envelopes until an anaesthetic technician applied the intervention. Each participant had one tourniquet placed on their upper limb and a second tourniquet wrapped around a towel next to them on the operating table. RIPC was applied beginning with the first surgical incision by inflating the cuff to $200 \mathrm{~mm} \mathrm{Hg}$ for $5 \mathrm{~min}$, followed by $5 \mathrm{~min}$ of deflation. This process was repeated three times. For the control group, the same intervention was applied to the tourniquet wrapped around the towel. Patients, theatre staff (with the exception of the anaesthetic technician), Intensive Care Unit (ICU) staff, and investigators were masked as to treatment allocation until data collection was completed.

All patients received premedication, cardiac anaesthesia involving the use of isoflurane, tepid cardiopulmonary bypass with blood cardioplegia, and postoperative hemodynamic management, according to standardised study protocols, as described in the online data supplement.

The main clinical outcome variables were high-sensitivity troponin-T, postoperative renal injury, and ICU-support requirements, and have been reported elsewhere. ${ }^{13}$ Blood samples were collected from the radial artery preoperatively, and 1, 2, 3, 6 and $12 \mathrm{~h}$ after cross-clamp removal. The samples were incubated at room temperature for $30 \mathrm{~min}$ then centrifuged, and the serum collected for storage at $-80^{\circ} \mathrm{C}$ until analysis. Serum concentrations of IL-1B, IL-6, IL-8, IL-10, IL-12p70 and tumour necrosis factor- $\alpha$ (TNF- $\alpha$ ) were quantified using a human inflammatory cytokine cytometric bead array (CBA) kit with an upper range limit of $5000 \mathrm{pg} / \mathrm{ml}$, and sensitivities of $7.2,2.5$ 3.6, 3.3, 1.9 and $3.7 \mathrm{pg} / \mathrm{ml}$, respectively (BD Biosciences, San Jose, California, USA). According to the manufacturer, the inter-assay coefficients of variation for samples at $2500 \mathrm{pg} / \mathrm{m}$ were $11,11,10,7,9$ and $13 \%$ for IL-1ß, IL-10, IL-6, IL-8, IL-12p70 and TNF- $\alpha$, respectively. Patient samples were run in singlet, and standards as well as a serum sample used as a known positive control were run in duplicate for each assay. The cytokine data distribution was skewed and normality assumptions were better met with logarithm transformation, thus cytokine concentrations were log transformed and compared using t tests and analysis of covariance (ANCOVA). The exponent of the difference in logarithms shown in the results is equivalent to the ratio of mean values for the two groups. These exploratory analyses were each carried out with a type I error rate of 5\%, and no adjustment was used for multiple comparisons.

Our sample size was based on power calculations performed for the main clinical end points of our study, which are described elsewhere. ${ }^{13}$ At the time our study protocol was written, there was no relevant published data on which to base a power calculation for the cytokine measurements.

\section{RESULTS}

Perioperative serum samples from 95 patients were analysed ( $n=47$ RIPC and $n=48$ control; figure 1). Randomisation appeared successful with similar distribution of baseline charac teristics in the treatment groups, although there were some differences in the operative details. Five patients in the RIPC group underwent three or more procedures, whereas, no patients in the control group had more than two procedures (table 1). The main clinical outcome variables were not different between treatment groups. ${ }^{13}$ Serum IL-6, IL- 8 and IL-10 levels increased considerably from baseline following surgery (see supplementary table S1). By contrast, IL-1ß, IL-12p70 and
TNF- $\alpha$ concentrations did not increase, and were below the level of detection of the CBA $(7.2,1.9$ and $3.7 \mathrm{pg} / \mathrm{ml}$, respectively; data not shown). The inflammatory cytokines, IL-6 and IL-8, peaked between 1 and $3 \mathrm{~h}$ postreperfusion, and remained increased at $12 \mathrm{~h}$. To reduce the incidence of type I error, statistical testing of the cytokine data was limited to the 1 and $12 \mathrm{~h}$ time points, which were deemed the most informative. The point estimates were consistent with the hypothesis that there are higher levels of the proinflammatory mediators present in the RIPC patients, compared with controls. However, the CIs were wide and there were no statistically significant differences in mean log IL- 6 and IL-8 levels between the RIPC and control groups at 1 or $12 \mathrm{~h}$ (table 2), or in the total area under the curve (AUC; IL-6: ratio of means 1.32 (95\% CI 0.84 to 2.05), $p=0.22$; IL-8: 1.17 (0.79 to 1.72$), p=0.43)$. ANCOVA was used to determine if accounting for potential confounding variables altered the difference between the treatment groups. Adjustment for baseline interleukin levels, age, EuroSCORE-predicted mortality, ${ }^{14}$ bypass and cross-clamp times, surgery type (CABG only vs all other procedures), and statin use, did not alter the absence of a statistically significant difference in the univariate analysis (table 2). Levels of the immunosuppressive cytokine, IL-10, peaked in all patients $1 \mathrm{~h}$ postreperfusion and then steadily declined. IL-10 levels were similar in the RIPC and control groups (AUC: ratio of means $1.13(0.70$ to 1.80$), p=0.62)$, and this was not affected by adjustment for confounding variables (table 2 ).

A posthoc analysis investigated whether RIPC differentially modulates cytokine expression depending on the complexity of the surgery undergone. In this study and in previous work, ${ }^{15}$ we observed that troponin release and bypass duration varied with surgical procedure. Participants were, therefore, categorised as having either simple surgery: $C A B G$ with ejection fraction $(\mathrm{EF})<50 \%$ or $\mathrm{CABG} \pm$ aortic valve surgery; or complex surgery: Mitral valve repair with or without any other procedure. ANCOVA with baseline interleukin levels and treatment randomisation as covariates was used to compare cytokine levels. Complex surgery was associated with significantly higher levels of IL-6 (ratio of means 2.14 (95\% CI 1.45 to 3.16 ); $p<0.001)$ and IL-10 (1.82 (1.05 to 3.19); $p=0.033)$ at $1 \mathrm{~h}$ postbypass, and in the total AUC (IL-6: ratio of means 1.54 (95\% CI 1.02 to 2.34 ), $p=0.04$; IL-10: 1.97 (1.16 to 3.35 ), $p=0.012$; figure $1 \mathrm{~A}, \mathrm{~B})$. By $12 \mathrm{~h}$, there was no evidence of a difference in the estimates (IL-6: 0.91 (0.64 to 1.31 ), $p=0.62$; IL-10: 1.08 (0.72 to 1.63$), p=0.69)$. RIPC did not alter expression of these cytokines in either simple or complex surgeries (figure $2 \mathrm{C}-\mathrm{F}$ ).

\section{DISCUSSION}

This study is the first to show that RIPC does not alter expression of a panel of inflammatory cytokines in high-risk cardiac surgery patients. Although the point estimates favoured the hypothesis that there were higher levels of IL- 6 and IL-8 in the RIPC group, the differences were not statistically significant even after adjustment for confounding variables, such as statin use, EuroSCORE and total bypass time. Consistent with previous research, ${ }^{16}$ we confirmed that perioperative IL- 6 levels are higher with complex valvular surgery compared with straightforward $C A B G$ and aortic valve replacement (AVR) procedures. In addition, we found that the release of the anti-inflammatory cytokine, IL-10, is also enhanced. A posthoc analysis indicated that RIPC did not alter IL- 6 and IL-10 expression in patients undergoing simple procedures, or the more complex surgeries that are associated with higher cytokine levels. As this was an exploratory study, one limitation is that multiple comparisons 


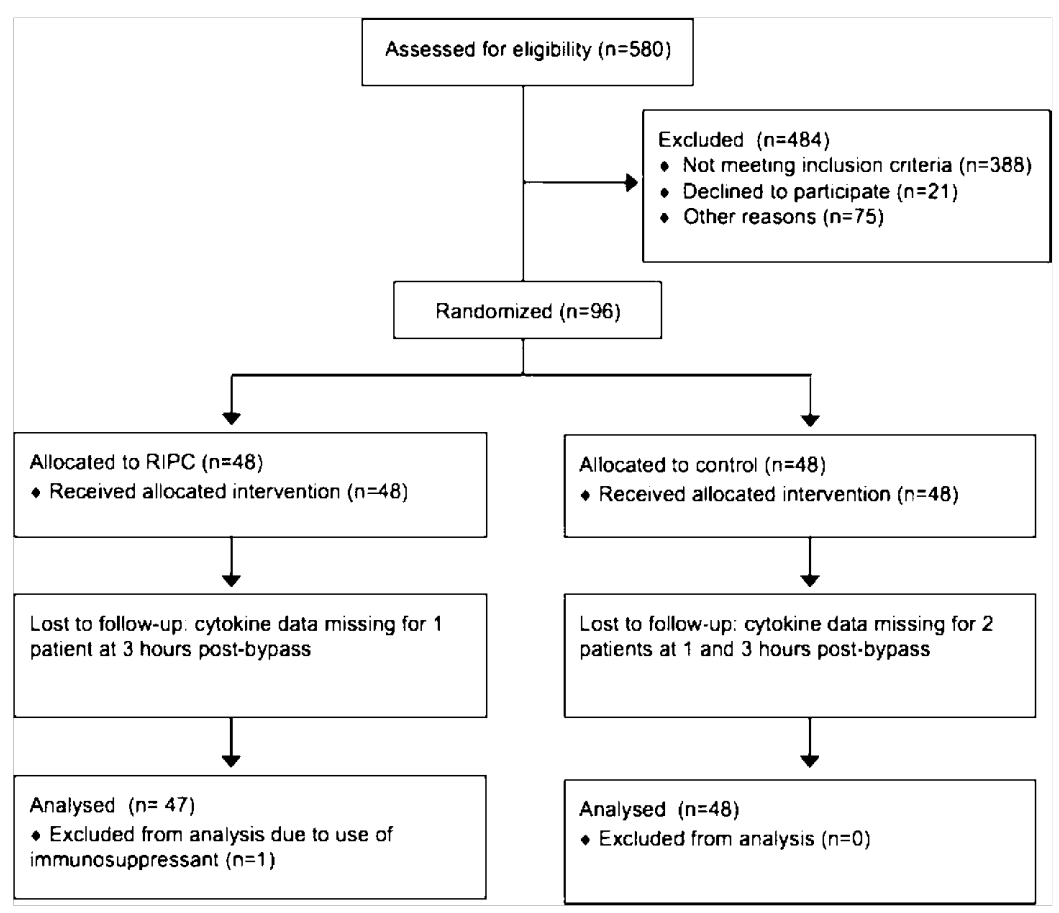

Figure 1 CONSORT diagram showing the flow of participants through the trial.

were performed, and therefore it is possible that some of the statistically significant differences may represent type I error.

The precise mechanism of RIPC has yet to be fully elucidated; however, there are data supporting the hypothesis that

Table 1 Demographic, clinical and operative data

\begin{tabular}{lcc}
\hline & RIPC $\mathbf{n = 4 7}$ & Control $\mathbf{n = 4 8}$ \\
\hline Demographics & & \\
Age (years) & $65.3 \pm 12.8$ & $64.3 \pm 14.5$ \\
Females & $18(38)$ & $17(35)$ \\
BMI (kg/m ${ }^{2}$ ) & $28.5 \pm 5.2$ & $28.6 \pm 5.9$ \\
Ethnicity & & \\
European & $31(66)$ & $38(79)$ \\
Maori & $7(15)$ & $7(15)$ \\
Other & $9(19)$ & $3(6)$ \\
Medications & & \\
B blockers & $34(72)$ & $29(60)$ \\
ACE inhibitors & $24(51)$ & $25(52)$ \\
Statins & $27(57)$ & $30(63)$ \\
Digoxin & $5(10)$ & $10(21)$ \\
Frusemide & $19(40)$ & $17(35)$ \\
Sulfonylureas & $4(9)$ & $1(2)$ \\
EuroSCORE (total) & $7.07 \pm 6.10$ & $6.58 \pm 6.08$ \\
Operative details & & \\
Number of procedures & & \\
One & $21(45)$ & $21(44)$ \\
Two & $21(45)$ & $0(0)$ \\
Three or more & $5(11)$ & $135 \pm 49.1$ \\
Bypass time (min) & $151 \pm 59.0$ & $104 \pm 41$ \\
Cross-clamp time (min) & $117 \pm 51$ & \\
\hline Val ar ing &
\end{tabular}

Values are reported as mean \pm SD or number $(\%)$

BMI, body mass index; RIPC, remote ischaemic preconditioning. an unidentified humoral factor transmits the protective signal from the remote preconditioned tissue to the myocardium. ${ }^{17}$ Cytokines are a prime candidate for such a factor given their function as endocrine-signalling peptides; however, our finding that RIPC does not modify expression of the key cytokines, IL-6, IL-8 and IL-10, suggests this mechanism is unlikely. This confirms previous research by Karuppasamy et al ${ }^{4}$ who showed that systemic concentrations of these cytokines were not altered following each cycle of ischaemia during RIPC application and postoperatively. One possible explanation for our findings is that the systemic cytokine levels induced by high-risk cardiac procedures are too high to be significantly altered by the RIPC treatment in this setting.

Two previous studies have reported on cytokine expression following RIPC in children undergoing repair of congenital heart defects. The first applied lower limb RIPC and reported IL-6, IL-8, IL-10 and TNF- $\alpha$ levels were not significantly different from the controls. ${ }^{18}$ The second, which applied RIPC both 1 day and $1 \mathrm{~h}$ preoperatively, found that levels of proinflammatory and anti-inflammatory cytokines were weakly attenuated postoperatively. ${ }^{19}$ Both studies differ substantially from our own in that bypass times were dramatically shorter, the earliest samples corresponded to time points after detection of the peak period of cytokine expression in our study, and the RIPC interventions were fundamentally different to the standard stimulus applied in the majority of trials, including our own, making it difficult to relate this earlier work to the present findings.

RIPC appears to afford two windows of protection that may involve different pathways. The first occurs immediately, lasting less than $4 \mathrm{~h}$; whereas, the second presents $24 \mathrm{~h}$ after the initial conditioning. ${ }^{20}$ RIPC is most feasibly applied in 


\section{Original research}

Table 2 Perioperative cytokine concentrations

\begin{tabular}{|c|c|c|c|c|c|c|}
\hline & \multicolumn{2}{|c|}{ Mean concentration, $\mathrm{pg} / \mathrm{ml}$ (SD) } & \multicolumn{2}{|c|}{ Mean log concentration (SD) } & \multirow[t]{2}{*}{ Difference $(95 \% \mathrm{Cl}), \mathrm{p}$} & \multirow[t]{2}{*}{ Adjusted* estimate $(95 \% \mathrm{Cl}), \mathrm{p}$} \\
\hline & $\operatorname{RIPC}(n=47)$ & Control $(n=48)$ & RIPC $(n=47)$ & Control $(n=48)$ & & \\
\hline \multicolumn{7}{|l|}{ IL-6 } \\
\hline Preop & $7.17(8.14)$ & $5.75(6.36)$ & $1.62(0.79)$ & $1.41(0.74)$ & & \\
\hline $1 \mathrm{~h}$ & $865.7(1573)$ & $391.3(620.0)$ & $5.90(1.23)$ & $5.52(0.81)$ & $0.37(-0.05$ to 0.80$), 0.09$ & $0.23(-0.15$ to 0.62$), 0.23$ \\
\hline $12 \mathrm{~h}$ & $508.3(865.5)$ & $273.2(273.0)$ & $5.57(1.11)$ & $5.28(0.78)$ & $0.29(-0.10$ to 0.68$), 0.15$ & $0.21(-0.18$ to 0.60$), 0.28$ \\
\hline \multicolumn{7}{|l|}{ IL-8 } \\
\hline Preop & $18.2(14.5)$ & $13.6(8.25)$ & $2.66(0.69)$ & $2.47(0.54)$ & & \\
\hline $1 \mathrm{~h}$ & $239.8(423.1)$ & $130.8(113.4)$ & $4.89(1.01)$ & $4.56(0.76)$ & $0.31(-0.06$ to 0.68$), 0.10$ & $0.22(-0.13$ to 0.58$), 0.22$ \\
\hline $12 \mathrm{~h}$ & $164.0(233.6)$ & $96.5(99.3)$ & $4.60(0.91)$ & $4.29(0.70)$ & $0.32(-0.02$ to 0.65$), 0.06$ & $0.20(-0.11$ to 0.50$), 0.20$ \\
\hline \multicolumn{7}{|l|}{ IL-10 } \\
\hline Preop & $1.92(1.71)$ & $2.58(2.88)$ & $0.02(1.44) \dagger$ & $0.44(1.25) \dagger$ & & \\
\hline $1 \mathrm{~h}$ & $228.8(218.1)$ & $231.2(242.6)$ & $4.89(1.21)$ & $4.88(1.20)$ & $0.01(-0.48$ to 0.51$), 0.97$ & $0.00(-0.51$ to 0.51$), 0.99$ \\
\hline $12 \mathrm{~h}$ & $13.7(12.5)$ & $20.7(47.7)$ & $2.25(0.86)$ & $2.26(1.03)$ & $-0.01(-0.39$ to 0.38$), 0.98$ & $-0.03(-0.43$ to 0.37$), 0.89$ \\
\hline
\end{tabular}

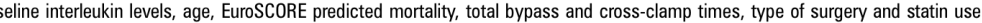
$+12 / 47 \mathrm{RIPC}$ and $7 / 48$ control patients had concentrations of zero, therefore, these were given a value of 0.1 to allow for logarithm transformation. RIPC, Remote ischaemic preconditioning
Rif

surgery patients postanaesthesia, therefore, most trials, to date, including our own, have investigated the efficacy of early phase RIPC. Biomarkers of myocardial damage peak $6-8 \mathrm{~h}$ postbypass, ${ }^{15}$ rendering the first $12 \mathrm{~h}$ following surgery the most relevant period for examining factors that may modify or mediate myocardial injury. Our time points were thus carefully selected to allow us to determine whether an RIPC-induced change in cytokine expression could contribute to the protection against myocardial damage previously described in the literature. We were able to capture the period of peak perioperative cytokine release, and have shown no change in systemic cytokine levels following RIPC in the pathophysiologically relevant period.
A related study by Karuppasamy et $a l^{4}$ determined that RIPC does not alter levels of IL-6, IL-8 or IL-10 at 24 and $48 \mathrm{~h}$ following uncomplicated CABG. This study, and our own, demonstrates that IL- 6 levels are not altered during early or late-phase RIPC in humans, which is intriguing given that IL-6 is considered obligatory for preconditioning-induced cardioprotection in mice. ${ }^{8}$ Furthermore, in humans there is evidence that upregulation of proinflammatory cytokines in unstable angina patients is sufficient to induce a late preconditioning effect. ${ }^{21}$ The results of these studies have two key implications. First, it is possible that the mechanism of RIPC may differ between species, and to that of classic local ischaemic preconditioning. This is
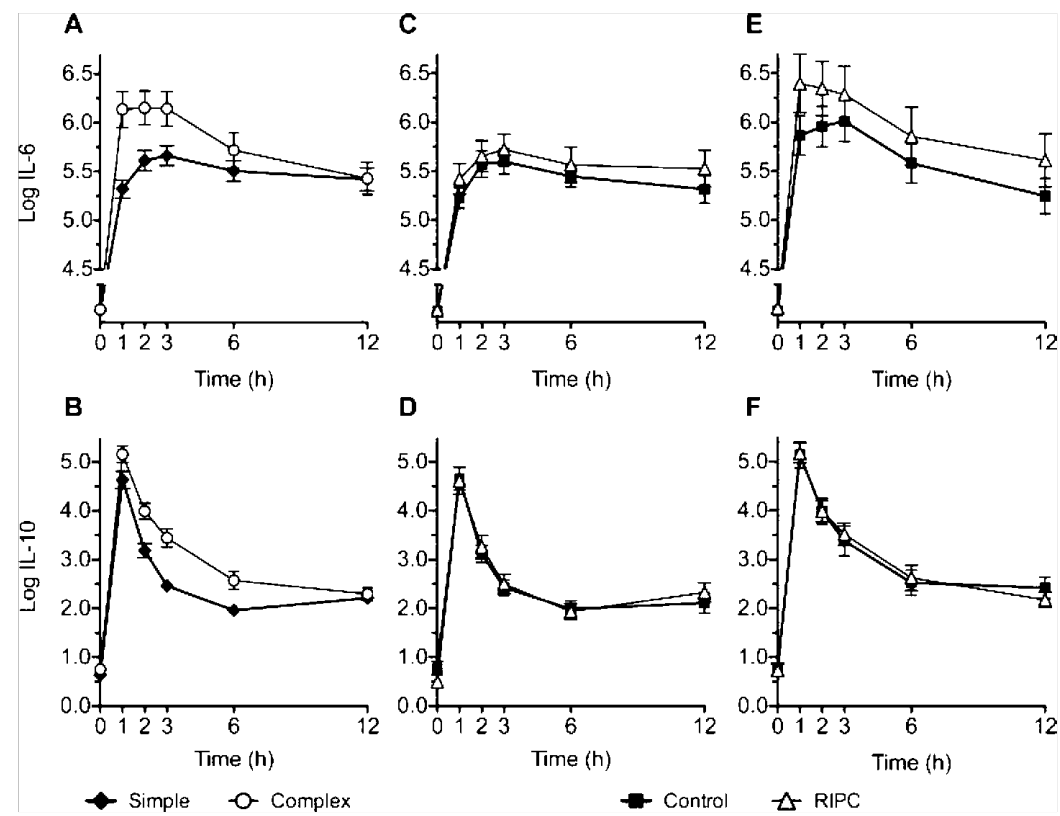

Figure 2 Effect of remote ischaemic preconditioning (RIPC) on perioperative cytokine expression in simple versus complex surgery. Compared with simple surgery $(n=49)$, complex procedures $(n=46)$ significantly increase IL-6 (A) and IL-10 (B) release in the early postbypass period. (C, E) RIPC has no impact on IL-6 expression in simple or complex procedures, respectively. (D, F) IL-10 levels are not altered by RIPC in simple or complex surgery, respectively. Data expressed as mean \pm SEM. 
supported by the recent suggestion that the cytokine-activated transcription factors associated with cardioprotection may differ between animals and humans. ${ }^{22}$ Second, contrary to the hypotheses of previous research, they suggest preconditioning would be expected to induce protection through an increase in IL- 6 and IL-8 levels, rather than a decrease. Indeed, while RIPC may not alter systemic IL- 6 and IL-8 concentrations, it is well established that the reperfused myocardium is a major source of inflammatory cytokines. ${ }^{23}$ The possibility that the local inflammatory milieu in the myocardium is modulated by RIPC cannot be excluded. Sampling blood from the coronary sinus may be of interest in this regard for future studies.

Our trial adds to a growing number that have not shown a clinical benefit from RIPC in adult cardiac surgery patients. ${ }^{3} 424$ It is possible that the absence of a clinical effect in our trial was due to the lack of change in the cytokine levels. If an increase in cytokines is essential to induce RIPC, factors that alter cytokine production, such as the use of anti-inflammatory or statin therapies, ${ }^{25}{ }^{26}$ may influence the ability to precondition patients. Alternatively, a preconditioning effect may have been activated in all patients through secondary pathways. Our study used a standardised anaesthesia protocol that included the use of volatile anaesthetics, which have attracted attention for their ability to induce cardiac preconditioning ${ }^{27} 28$ and decrease inflammatory cytokine expression. ${ }^{29}$ Thus, our control group may also have been, to some extent, preconditioned. This interpretation would suggest that the marginal effect of RIPC in patients already treated with volatile anaesthetics as per our study protocol is negligible. Given the widespread adoption of these anaesthetic agents, future research is essential to clarify their relationship with RIPC.

In conclusion, we provide evidence that RIPC does not alter perioperative cytokine concentrations in patients treated with the volatile anaesthetic, isoflurane, irrespective of whether the surgeries performed are simple or complex. The priority for future research should be to identify and characterise potential factors that may alter the ability to induce RIPC-mediated cardioprotection in order to facilitate the design and interpretation of further clinical trial work.

Contributors JW conducted the research and wrote the manuscript, PY designed and conducted the clinical trial, JP contributed to the research, MW contributed to the statistical analyses, JHM contributed to the experimental design, RB contributed to the design of the clinical trial, and ACL (the guarantor) oversaw the design and realisation of the experimental work and the final manuscript.

Funding This study was funded by grants from the New Zealand Lotteries Commission and the National Heart Foundation of New Zealand.

Competing interests None

Ethics approval New Zealand Central Regional Ethics Committee

Provenance and peer review Not commissioned; externally peer reviewed.

\section{REFERENCES}

Kharbanda RK, Mortensen UM, White PA, et al. Transient limb ischemia induces remote ischemic preconditioning in vivo. Circulation 2002;106:2881-3.

2. Gho BC, Schoemaker RG, van den Doel MA, et al. Myocardial protection by brief ischemia in noncardiac tissue. Circulation 1996;94:2193-200.

3. Rahman IA, Mascaro JG, Steeds RP, et al. Remote ischemic preconditioning in human coronary artery bypass surgery: from promise to disappointment? Circulation 2010;122: $553-9$.
4. Karuppasamy P, Chaubey S, Dew T, et al. Remote intermittent ischemia before coronary artery bypass graft surgery: a strategy to reduce injury and inflammation? Basic Res Cardiol 2011;106:511-19.

5. Morita S. Remote ischemic preconditioning. Is it time to introduce it in clinical practice? Circ J 2011;75:1821-2.

6. Konstantinov IE, Arab S, Kharbanda RK, et al. The remote ischemic preconditioning stimulus modifies inflammatory gene expression in humans. Physiol Genomics 2004:19:143-50.

7. Kharbanda RK, Peters M, Walton B, et al. Ischemic preconditioning prevents endothelial injury and systemic neutrophil activation during ischemia-reperfusion in humans in vivo. Circulation 2001:103:1624-30.

8. Dawn B, Xuan YT, Guo Y, et al. IL-6 plays an obligatory role in late preconditioning via JAK-STAT signaling and upregulation of iNOS and COX-2. Cardiovasc Res 2004:64:61-71.

9. Wei M, Kuukasjarvi P, Laurikka J, et al. Cytokine responses and myocardial injury in coronary artery bypass grafting. Scand J Clin Lab Invest 2001:61:161-6.

10. Liu KD, Altmann C, Smits G, et al. Serum interleukin-6 and interleukin-8 are early biomarkers of acute kidney injury and predict prolonged mechanical ventilation in children undergoing cardiac surgery: a case-control study. Crit Care 2009;13:R104.

11. Takayama $\mathbf{H}$, Soltow $L O$, Chandler WL, et al. Does the type of surgery effect systemic response following cardiopulmonary bypass? J Card Surg 2007:22:307-13.

12. Whitten CW, Hill GE, Ivy R, et al. Does the duration of cardiopulmonary bypass or aortic cross-clamp, in the absence of blood and/or blood product administration, influence the IL-6 response to cardiac surgery? Anesth Analg 1998;86:28-33.

13. Young PJ, Dalley P, Garden A, et al. A pilot study investigating the effects of remote ischemic preconditioning in high risk cardiac surgery using a randomised controlled double-blind protocol. Basic Res Cardiol 2012;107:256-65.

14. Nashef SA, Roques F, Michel $P$, et al. European system for cardiac operative risk evaluation (EuroSCORE). Eur J Cardiothorac Surg 1999;16:9-13.

15. Swaanenburg JC, Loef BG, Volmer M, et al. Creatine kinase MB, troponin I, and troponin T release patterns after coronary artery bypass grafting with or without cardiopulmonary bypass and after aortic and mitral valve surgery. Clin Chem cardiopulmonary

16. Kawahito K, Adachi H, Ino T. Influence of surgical procedures on interleukin-6 and monocyte chemotactic and activating factor responses: CABG vs. valvular surgery. $J$ Interferon Cytokine Res 2000;20:1-6.

17. Hausenloy DJ, Yellon DM. Remote ischaemic preconditioning: underlying mechanisms and clinical application. Cardiovasc Res 2008;79:377-86.

18. Cheung MM, Kharbanda RK, Konstantinov IE, et al. Randomized controlled trial of the effects of remote ischemic preconditioning on children undergoing cardiac surgery: first clinical application in humans. J Am Coll Cardiol 2006;47:2277-82.

19. Zhou W, Zeng D, Chen R, et al. Limb ischemic preconditioning reduces heart and lung injury after an open heart operation in infants. Pediatr Cardiol 2010;31:22-9.

20. Loukogeorgakis SP, Panagiotidou AT, Broadhead MW, et al. Remote ischemic preconditioning provides early and late protection against endothelial ischemia-reperfusion injury in humans: role of the autonomic nervous system. $J$ Am Coll Cardiol 2005:46:450-6.

21. Wang $\mathbf{Y}$, Yin B, Liu S, et al. Cardioprotective effect by tumor necrosis factor-alpha and interleukin-6 through late preconditioning in unstable angina patients. Arch Med Res 2007:38:80-5.

22. Heusch G, Musiolik J, Kottenberg E, et al. STAT5 activation and cardioprotection by remote ischemic preconditioning in humans. Circ Res 2011:110:111-15.

23. Wan S, DeSmet JM, Barvais $L$, et al. Myocardium is a major source of proinflammatory cytokines in patients undergoing cardiopulmonary bypass. $J$ Thorac Cardiovasc Surg 1996;112:806-1

24. Hong DM, Mint JJ, Kim JH, et al. The effect of remote ischaemic preconditioning on myocardial injury in patients undergoing off-pump coronary artery bypass graft surgery. Anaesth Intensive Care 2010;38:924-9.

25. Node K, Fujita M, Kitakaze M, et al. Short-term statin therapy improves cardiac function and symptoms in patients with idiopathic dilated cardiomyopathy. Circulation 2003;108:839-43.

26. Rosenson RS, Tangney CC, Casey LC. Inhibition of proinflammatory cytokine production by pravastatin. Lancet 1999:353:983-4.

27. Belhomme D, Peynet J, Louzy $\mathrm{M}$, et al. Evidence for preconditioning by isoflurane in coronary artery bypass graft surgery. Circulation 1999;100:II340-4.

28. Haroun-Bizri S, Khoury SS, Chehab IR, et al. Does isoflurane optimize myocardial protection during cardiopulmonary bypass? J Cardiothorac Vasc Anesth 2001:15:418-21.

29. Kawamura T, Kadosaki M, Nara N, et al. Effects of sevoflurane on cytokine balance in patients undergoing coronary artery bypass graft surgery. $J$ Cardiothorac Vasc Anesth 2006:20:503-8. 


\section{Appendix E. Complete list of flow cytometry antibodies}

\begin{tabular}{|c|c|c|c|c|c|}
\hline Specificity & Fluorophore & Clone & Isotype & Manufacturer & $\begin{array}{c}\text { Optimal } \\
\text { Final } \\
\text { Dilution }\end{array}$ \\
\hline CD3 & $\mathrm{PE}$ & UCHT1 & Mouse IgG1 & BD Pharmingen & $1: 100$ \\
\hline CD3 & PE/Cy7 & UCHT1 & Mouse IgG1 & Biolegend & $1: 100$ \\
\hline CD3 & APC/H7 & SK7 & Mouse IgG1 & BD Pharmingen & $1: 100$ \\
\hline $\mathrm{CD} 4$ & APC/H7 & RPA-T4 & Mouse IgG1 & BD Pharmingen & $1: 100$ \\
\hline $\mathrm{CD} 4$ & V500 & RPA-T4 & Mouse IgG1 & BD Horizon & $1: 50$ \\
\hline CD8a & AF488 & HIT8a & Mouse IgG1 & Biolegend & $1: 800$ \\
\hline CD11b & AF488 & ICRF44 & Mouse IgG1 & Biolegend & $1: 400$ \\
\hline CD14 & APC & M5E2 & Mouse IgG2a & Biolegend & $1: 200$ \\
\hline CD14 & PerCP & MOP9 & Mouse IgG2b & BD Biosciences & $1: 25$ \\
\hline CD14 & PerCP/Сy5.5 & M5E2 & Mouse IgG2a & BD Pharmingen & $1: 200$ \\
\hline CD16 & PE/Cy7 & 3G8 & Mouse IgG1 & Biolegend & $\begin{array}{c}1: 800 \\
\text { (PBMC) or } \\
1: 3000 \\
\text { (Neutrophils) }\end{array}$ \\
\hline CD16 & V450 & 3G8 & Mouse IgG1 & BD Horizon & $1: 800$ \\
\hline CD25 & AF488 & BC96 & Mouse IgG1 & Biolegend & $1: 100$ \\
\hline CD45 & PE/Cy7 & HI30 & Mouse IgG1 & Biolegend & $1: 200$ \\
\hline CD56 & PerCP/Cy5.5 & HCD-56 & Mouse IgG1 & Biolegend & $1: 150$ \\
\hline CD62L & APC & DREG-56 & Mouse IgG1 & Biolegend & $1: 40$ \\
\hline CD63 & PE & MEM-259 & Mouse IgG1 & Biolegend & $1: 10$ \\
\hline CD69 & PE & FN50 & Mouse IgG1 & Biolegend & $1: 100$ \\
\hline IL-6 & APC & MQ2-13A5 & Rat IgG1 & Biolegend & $1: 25$ \\
\hline IL-8 & PE & E8N1 & Mouse IgG1 & Biolegend & $1: 25$ \\
\hline IL-10 & BV421 & JES3-9D7 & Rat IgG1 & Biolegend & $1: 25$ \\
\hline \multirow{13}{*}{$\begin{array}{l}\text { Isotype } \\
\text { Controls }\end{array}$} & AF488 & MOPC-21 & Mouse IgG1 & Biolegend & \multirow{13}{*}{-} \\
\hline & APC & MOPC-21 & Mouse IgG1 & BD Pharmingen & \\
\hline & APC & G155-178 & Mouse IgG2a & BD Pharmingen & \\
\hline & APC & RTK2071 & Rat IgG1 & Biolegend & \\
\hline & APC-H7 & MOPC-21 & Mouse IgG1 & BD Pharmingen & \\
\hline & BV421 & RTK2071 & Rat IgG1 & Biolegend & \\
\hline & PE & MOPC-21 & Mouse IgG1 & BD Pharmingen & \\
\hline & $\mathrm{PE}$ & MOPC-21 & Mouse IgG1 & Biolegend & \\
\hline & PE/Cy7 & MOPC-21 & Mouse IgG1 & Biolegend & \\
\hline & PerCP & MPC-11 & Mouse IgG2b & Biolegend & \\
\hline & PerCP-Cy5.5 & MOPC-21 & Mouse IgG1 & Biolegend & \\
\hline & V450 & MOPC-21 & Mouse IgG1 & BD Horizon & \\
\hline & V500 & $\mathrm{X} 40$ & Mouse IgG1 & BD Horizon & \\
\hline
\end{tabular}




\section{Appendix F. Flow cytometry data analysis}

\begin{tabular}{|c|c|c|c|c|c|}
\hline \multirow[b]{2}{*}{ Laser } & \multirow{2}{*}{$\begin{array}{l}\text { Channel / } \\
\text { Fluorophore }\end{array}$} & Panel 1 & Panel 2 & Panel 3 & Panel 4 \\
\hline & & $\begin{array}{l}\text { CD4 + CD25 } \\
\text { T Cells }\end{array}$ & $\begin{array}{c}\text { CD8 T Cells + } \\
\text { NKT/NKs }\end{array}$ & $\begin{array}{l}\text { Broad T Cell } \\
\text { Subsets }\end{array}$ & $\begin{array}{c}\text { Monos + } \\
\text { Neuts }\end{array}$ \\
\hline \multirow{4}{*}{ Blue (488) } & Green/AF488 & CD25 & CD8 & CD8 & CD11b \\
\hline & Yellow/PE & CD69 & CD69 & CD3 & CD63 \\
\hline & $\begin{array}{l}\text { Red/PerCP or } \\
\text { PerCP/Cy5.5 }\end{array}$ & CD56 & CD56 & CD56 & CD14 \\
\hline & $\begin{array}{c}\text { Infrared/PE- } \\
\text { Cy7 }\end{array}$ & CD3 & CD16 & CD16 & CD16 \\
\hline \multirow{2}{*}{ Red (633) } & Red/APC & CD62L & CD62L & - & - \\
\hline & $\begin{array}{c}\text { Infrared/APC- } \\
\text { H7 }\end{array}$ & CD4 & CD3 & CD4 & CD3 \\
\hline
\end{tabular}

Table F.1 Immunophenotyping antibody panels for a Guava easyCyte 8HT

\begin{tabular}{|c|c|c|c|c|}
\hline \multirow[b]{2}{*}{ Laser } & \multirow{2}{*}{$\begin{array}{c}\text { Channel / } \\
\text { Fluorophore }\end{array}$} & Panel 5 & Panel 6 & Panel 7 \\
\hline & & $\begin{array}{c}\text { CD4 + CD25 T } \\
\text { Cells } \\
\end{array}$ & $\begin{array}{c}\text { CD8 T Cells + } \\
\text { NKT } / N K s \\
\end{array}$ & $\begin{array}{c}\text { Monos + } \\
\text { Neuts } \\
\end{array}$ \\
\hline \multirow{2}{*}{ Violet (405) } & Blue/BV421 & IL-10 & IL-10 & IL-10 \\
\hline & Green/V500 & CD4 & - & - \\
\hline \multirow{4}{*}{ Blue (488) } & Green/AF488 & CD25 & CD8 & CD11b \\
\hline & Yellow/PE & IL-8 & IL-8 & IL-8 \\
\hline & $\begin{array}{l}\text { Red/PerCP or } \\
\text { PerCP/Cy5.5 }\end{array}$ & CD56 & CD56 & CD14 \\
\hline & $\begin{array}{l}\text { Infrared/PE- } \\
\quad \text { Cy7 }\end{array}$ & CD3 & CD3 & CD16 \\
\hline \multirow{2}{*}{ Red (633) } & Red/APC & IL-6 & IL-6 & IL-6 \\
\hline & $\begin{array}{c}\text { Infrared/APC- } \\
\text { H7 }\end{array}$ & Viability & Viability & Viability \\
\hline
\end{tabular}

Table F.2 Overview of antibody panels used for ICS on the FACSCanto ${ }^{\mathrm{TM}}$ II 


\section{Immunophenotyping data gating strategies:}

Unstimulated

\section{A}
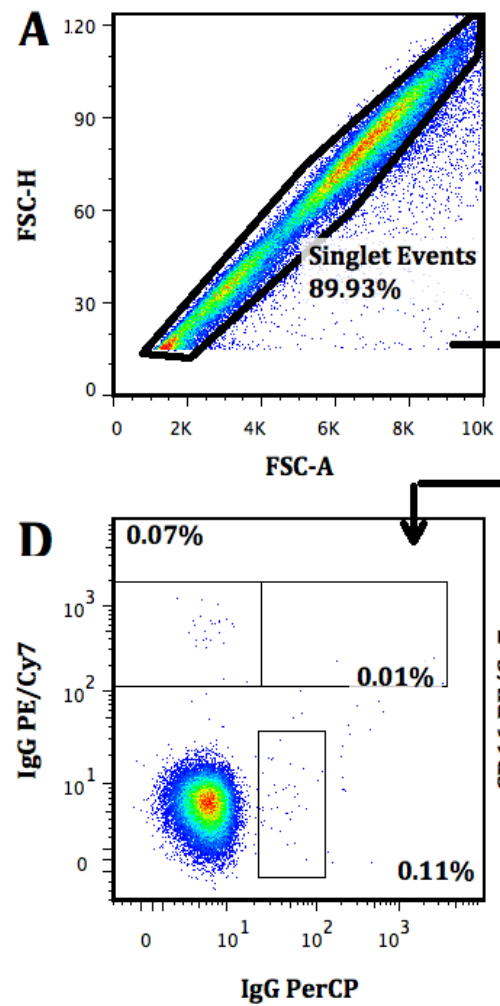

$\mathbf{B}$

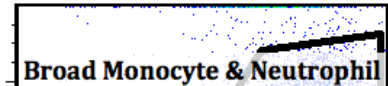

C

PMA/I Stimulated

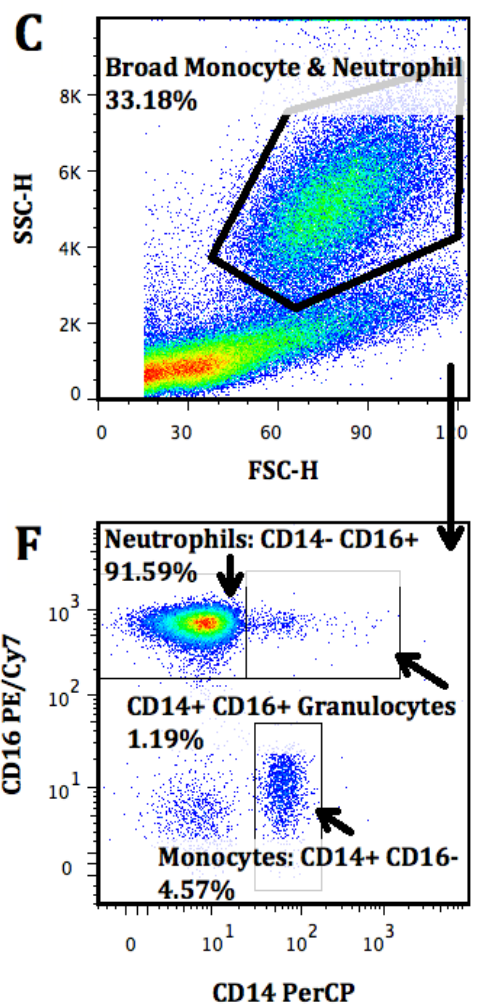

Figure F.1 Gating of monocytes and neutrophils in panel 4

Whole blood was prepared as described in section 4.3.4. (A) Singlet events were first isolated using FSC-A vs. FSC-H; a broad gate was used to separate the monocyte and neutrophil populations with FSC-H vs. SSC-H in unstimulated (B) and PMA/I stimulated (C) samples; the CD14-CD16 ${ }^{\text {high }}$ neutrophils and $\mathrm{CD} 14^{+} \mathrm{CD} 16^{-}$monocytes were gated in the unstimulated (E) and PMA/I stimulated (F) cells, and compared to a sample stained with isotype control antibodies (D). Shown is representative data taken from one participant following the control treatment. 


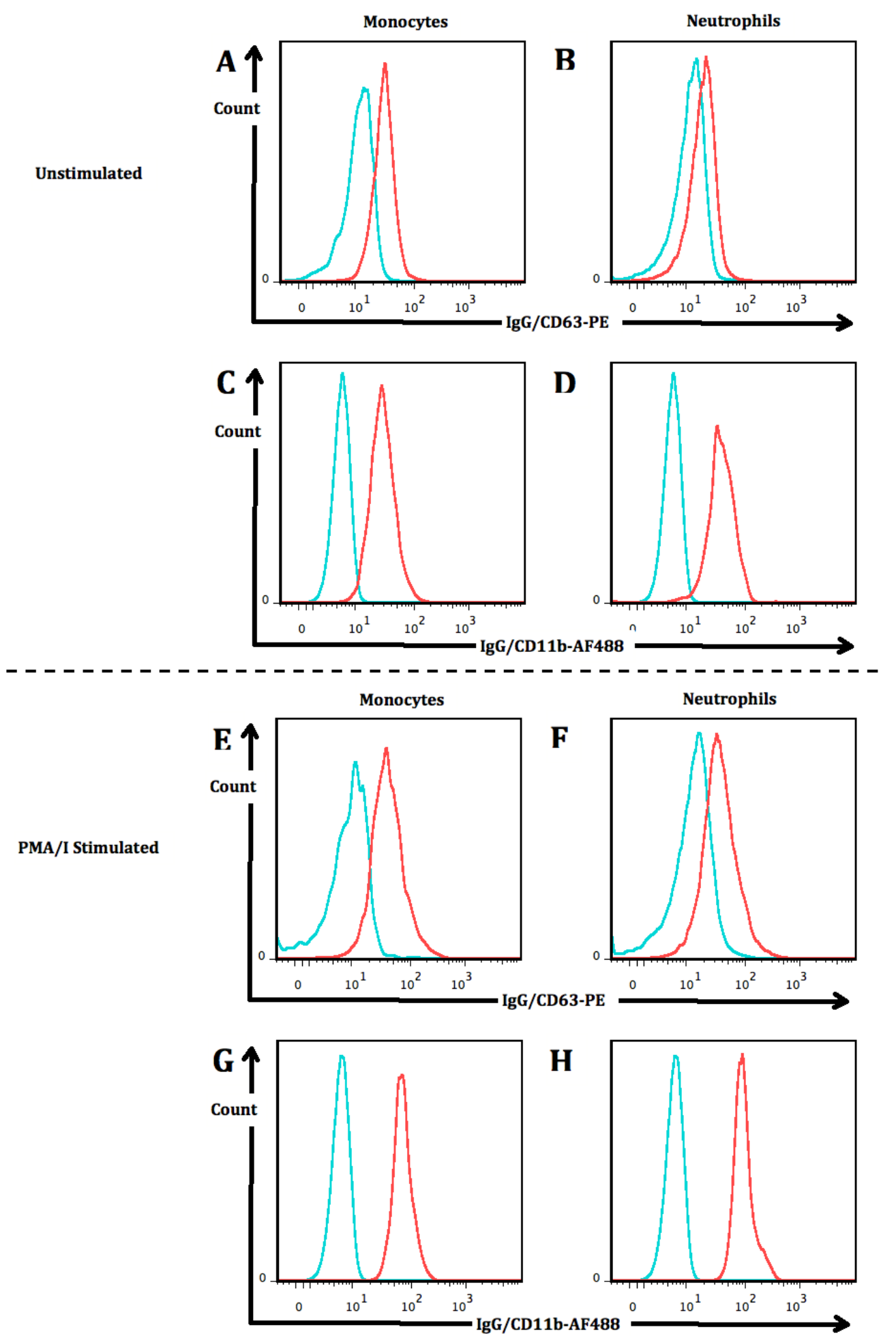

Figure F.2 Representative plots of CD63 and CD11b staining in monocytes and neutrophils during immunophenotyping

Whole blood was prepared as described in section 4.3.4. Staining is shown for IgG-PE (blue) vs. CD63-PE (red) in monocytes (A and E) and neutrophils (B and F), in unstimulated (A and B) and PMA/I stimulated (E and F) samples; and IgGAF488 (blue) vs. CD11b-AF488 (red) in monocytes (C and G) and neutrophils (D and $\mathrm{H}$ ), in unstimulated ( $\mathrm{C}$ and $\mathrm{D}$ ) and PMA/I stimulated (G and H) samples. Shown is representative data taken from one participant following the control treatment. 

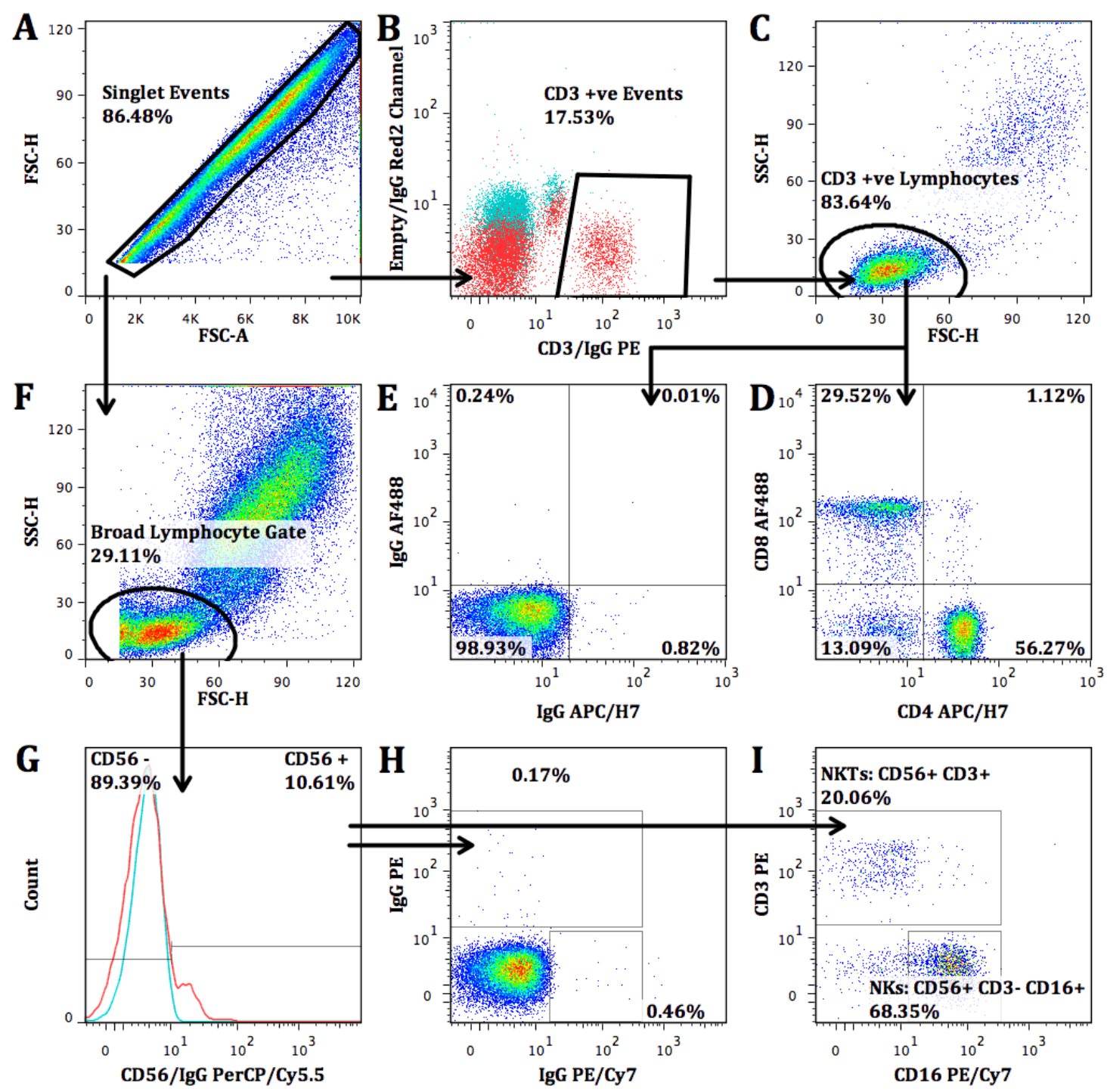

Figure F.3 Gating of total T cell populations and $\mathrm{CD56}^{+}$cells in panel 3

Whole blood was prepared as described in section 4.3.4. (A) Singlet events were first isolated using FSC-A vs. FSC-H; (B) cells staining positively for CD3 were gated (red) and compared to the isotype control (blue); (C) the $\mathrm{CD}^{+}$ lymphocytes were gated using FSC-H vs. SSC-H; (D) the $\mathrm{CD}^{+} \mathrm{T}$ cells were sorted into CD4, CD8, and double positive populations, compared to the isotype control stained samples (E); (F) a broad gate was used to isolate the lymphocyte subset of singlet events on FSC-H vs. SSC-H; (G) CD56 ${ }^{+}$stained lymphocytes (red) were gated using the isotype control (blue); (I) the $\mathrm{CD}^{2} 6^{+}$lymphocyte population was further separated into $\mathrm{CD}^{+} \mathrm{CD} 16^{-/+} \mathrm{NKT}$ cells and CD3-CD16 ${ }^{+} \mathrm{NK}$ cells, compared to isotype control stained samples $(\mathrm{H})$. Shown is representative data taken from one participant following the control treatment. 

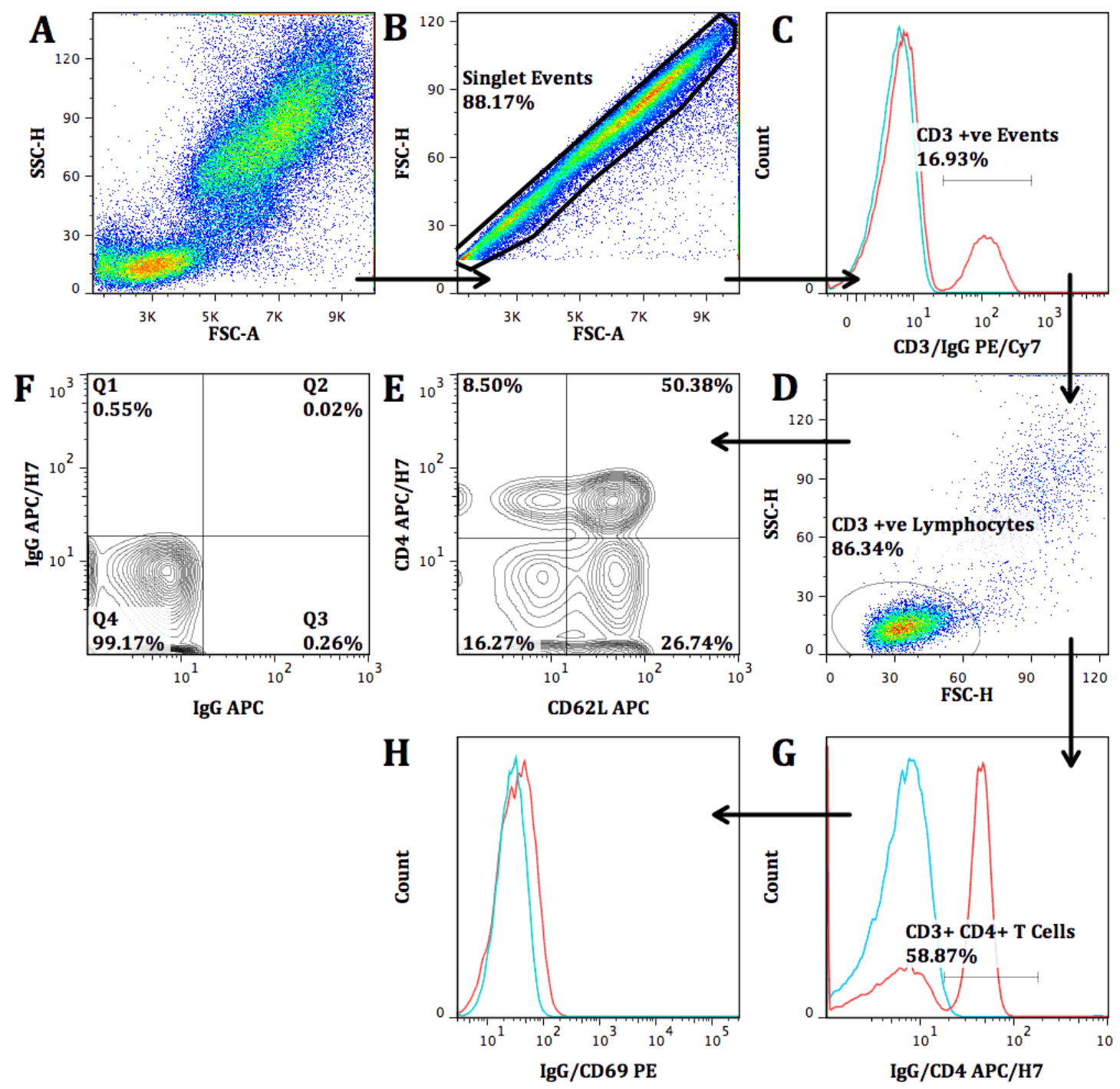

Figure F.4 Gating of CD4 T cells in panel 1

Whole blood was prepared as described in section 4.3.4. (A) Ungated leukocytes; (B) singlet events were isolated using FSC-A vs. FSC-H; (C) cells staining positively for CD3 were gated (red) using the isotype control (blue); (D) the $\mathrm{CD}^{+}$ lymphocytes were gated using FSC-H vs. SSC-H; (E) the $\mathrm{CD}^{+}$lymphocytes were separated based on expression of CD4 and CD62L using a contour plot, and an isotype control stained sample (F); (G) total CD4 T cells (red) were gated from the lymphocyte gate, compared to the isotype control (blue); (H) CD69 levels (red) compared to the isotype control (blue) in the CD4 T cells. Shown is representative data taken from one participant. 


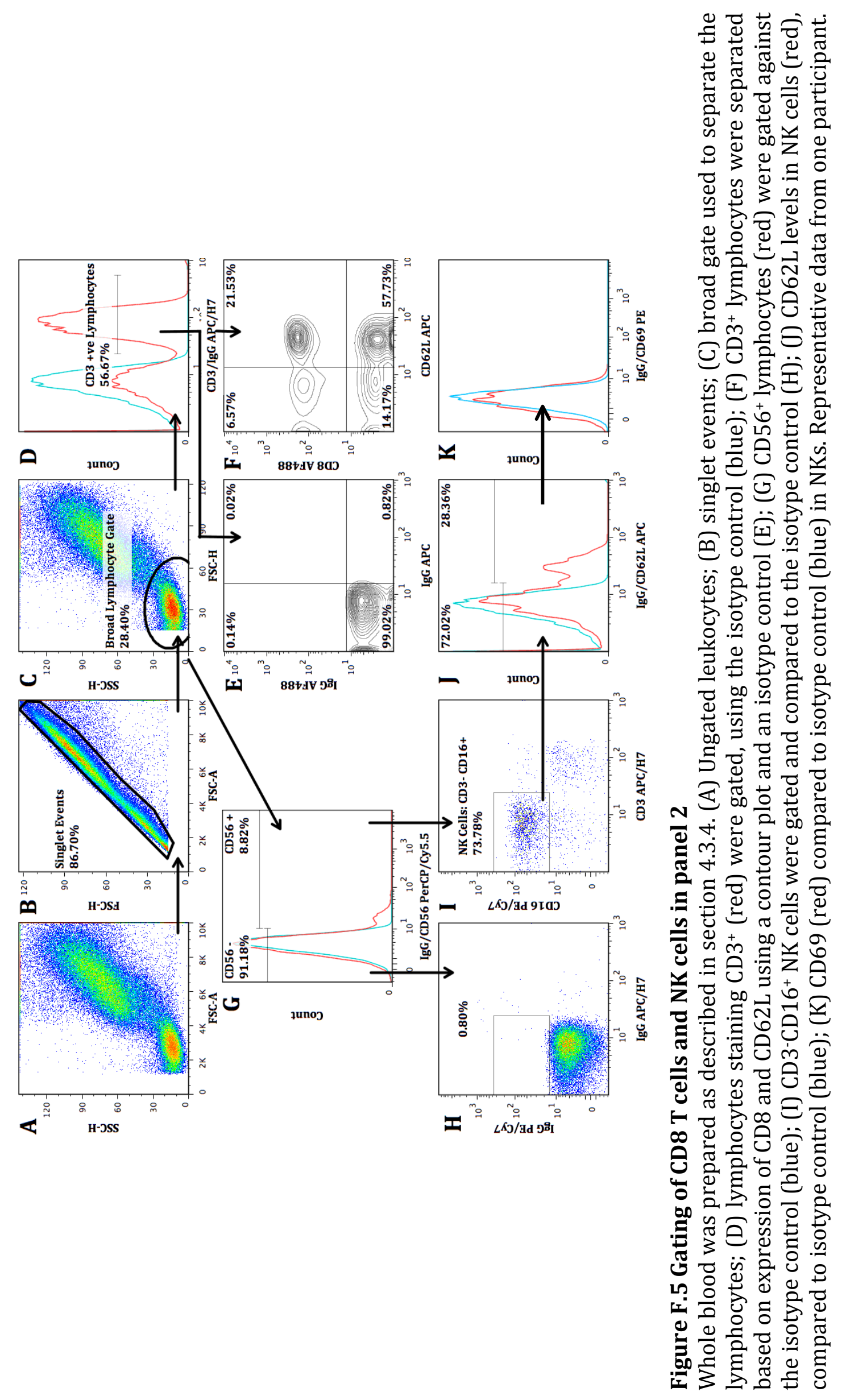


Intracellular cytokine staining data gating strategies:

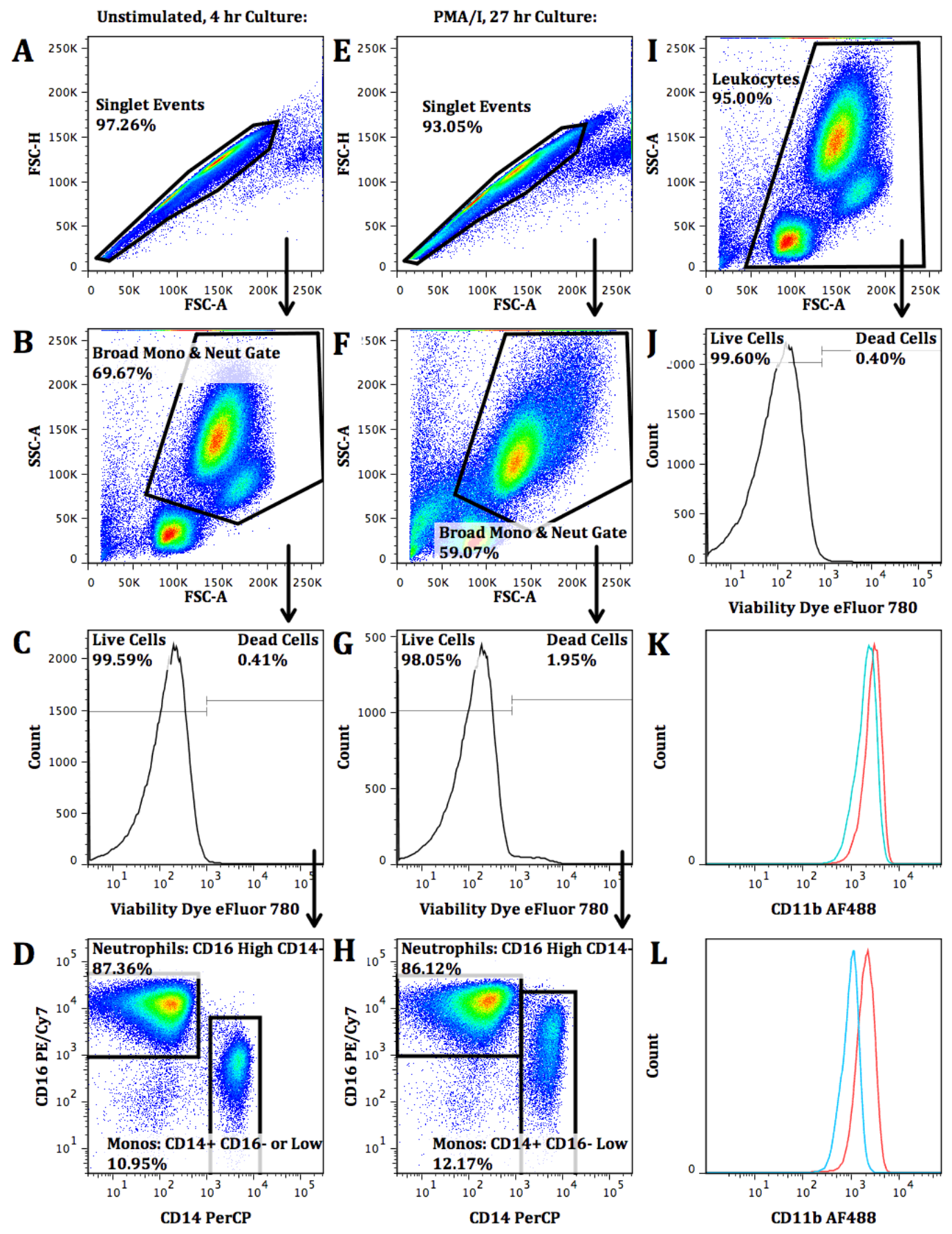

Figure F.6 Gating of total live leukocytes, monocytes, and neutrophils in panel 7 during ICS

Whole blood was prepared as described in section 5.3.1. In samples cultured for $4 \mathrm{hrs}$ in media (A - D) or $27 \mathrm{hrs}$ with PMA/I (E - H): (A and E) singlet leukocyte events were gated; ( $B$ and $F$ ) a broad gate was used to separate the monocytes and neutrophils; (C and G) dead cells were excluded; (D and H) CD14-CD16 ${ }^{+}$ neutrophils and $\mathrm{CD} 14^{+} \mathrm{CD} 16^{-/ \text {low }}$ monocytes were gated. Total leukocytes were also gated (I) and assessed for viability (J). Representative plots of CD11b staining in $4 \mathrm{hr}$ unstimulated (blue) and stimulated (red) monocytes (K) and neutrophils (L). Shown is representative data from one participant during a control visit. 


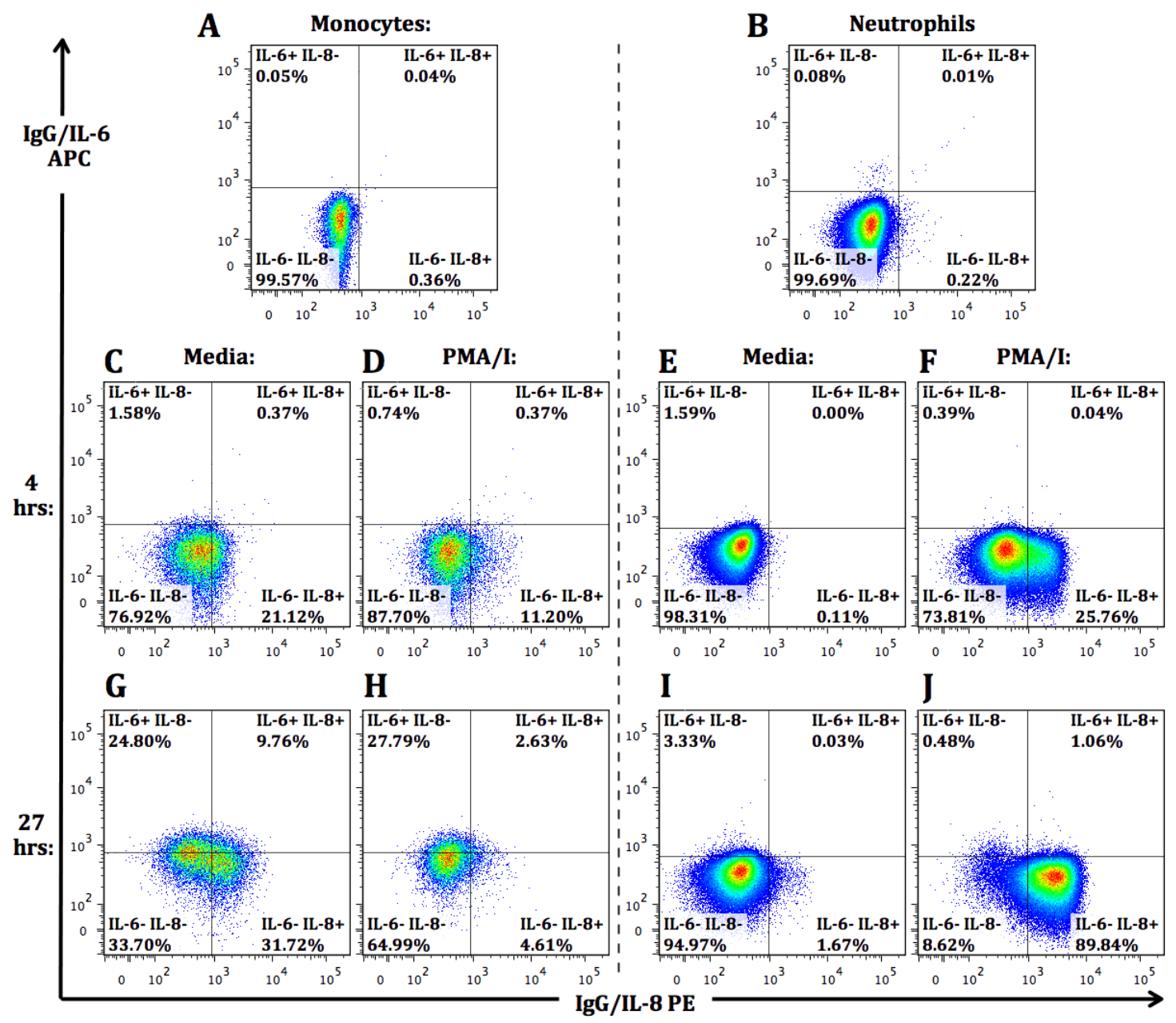

Figure F.7 Gating of intracellular cytokines in monocytes and neutrophils

Whole blood was prepared as described in section 5.3.1. Monocytes (A) and neutrophils (B) were gated in a stimulated intracellular PE and APC isotype control sample with CD14 and CD16 staining, and used to set quadrant gates. Shown is IL-6 vs. IL-8 staining in samples cultured for 4 hours $(\mathrm{C}-\mathrm{F})$ or 27 hours $(G-J)$, in monocytes (C, D, G, and H) and neutrophils (E, F, I, and J), in media (C, $G$, E, and I) or with PMA/I (D, H, F, and J). Shown is representative data from one participant during a control visit. 

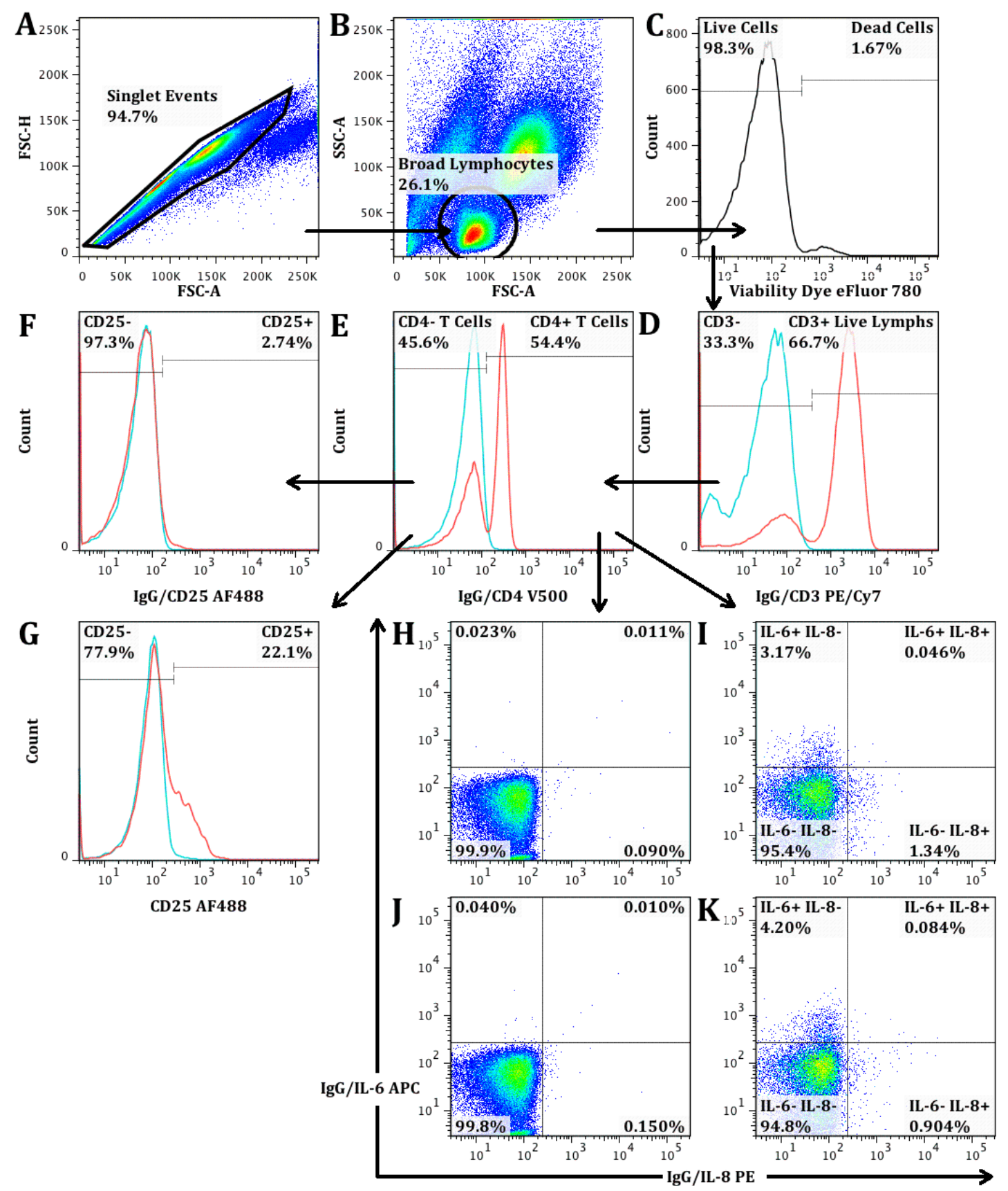

Figure F.8 Gating of intracellular cytokines in CD4 T cells in panel 5

Whole blood was prepared as described in section 5.3.1. (A) Singlet leukocyte events were gated; (B) a broad gate was used to separate the lymphocyte population; (C) dead cells were excluded; (D) $\mathrm{CD}^{+}$lymphocytes (red) were gated against the isotype control (blue); (E) $\mathrm{CD}^{+} \mathrm{T}$ cells (red) were gated using an isotype control (blue); (F) $\mathrm{CD} 4^{+} \mathrm{CD} 25^{+}$cells were identified (red) by gating against an isotype control sample (blue); (H) representative plot of $\mathrm{CD} 4{ }^{+} \mathrm{CD} 25^{+} \mathrm{T}$ cells in unstimulated (blue) and stimulated (red) cells; IL-6 and IL-8 levels in the CD4 T cell population (I and K) were gated using the isotype control samples for reference ( $\mathrm{H}$ and $\mathrm{J}$ ) in unstimulated $(\mathrm{H}$ and $\mathrm{I}$ ) and stimulated ( $\mathrm{J}$ and $\mathrm{K}$ ) samples. Shown are representative plots from one participant following the control treatment (no RIPC) in cells cultured for 27 hours. The samples shown in plots A - F were unstimulated. 

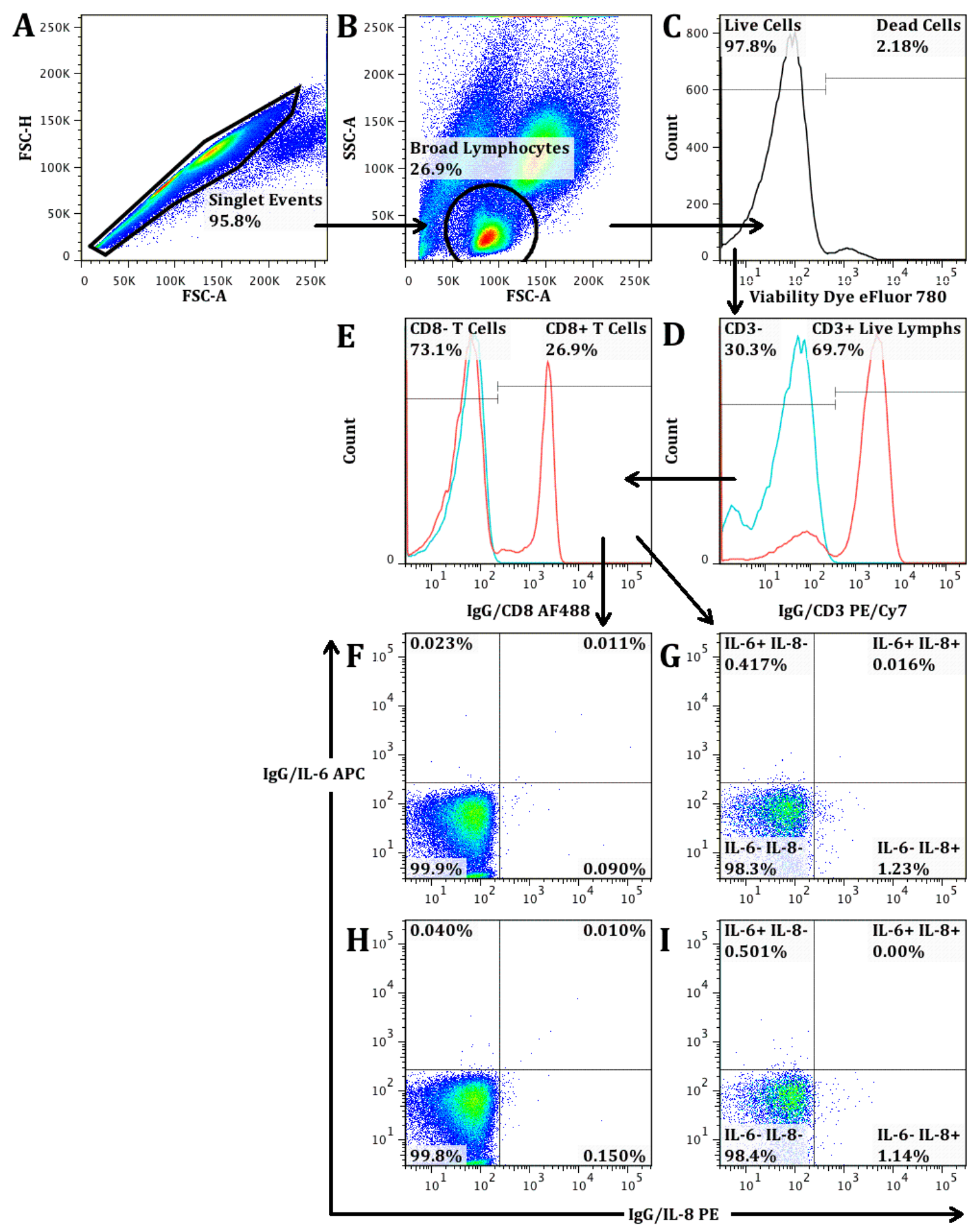

Figure F.9 Gating of intracellular cytokines in CD8 T cells in panel 6

Whole blood was prepared as described in section 5.3.1. (A) Singlet leukocyte events were gated; (B) a broad gate was used to separate the lymphocyte population; (C) dead cells were excluded; (D) $\mathrm{CD}^{+}$lymphocytes (red) were gated against the isotype control (blue); (E) CD8 ${ }^{+} \mathrm{T}$ cells (red) were gated using an isotype control (blue); IL-6 and IL-8 levels in the CD8 T cell population (G and I) were gated using the isotype control samples for reference ( $\mathrm{F}$ and $\mathrm{H}$ ) in unstimulated (F and G) and stimulated ( $\mathrm{H}$ and $\mathrm{I}$ ) samples. Shown are representative plots from one participant following the control treatment (no RIPC) in cells cultured for 27 hours. The samples shown in plots A - E were unstimulated. 


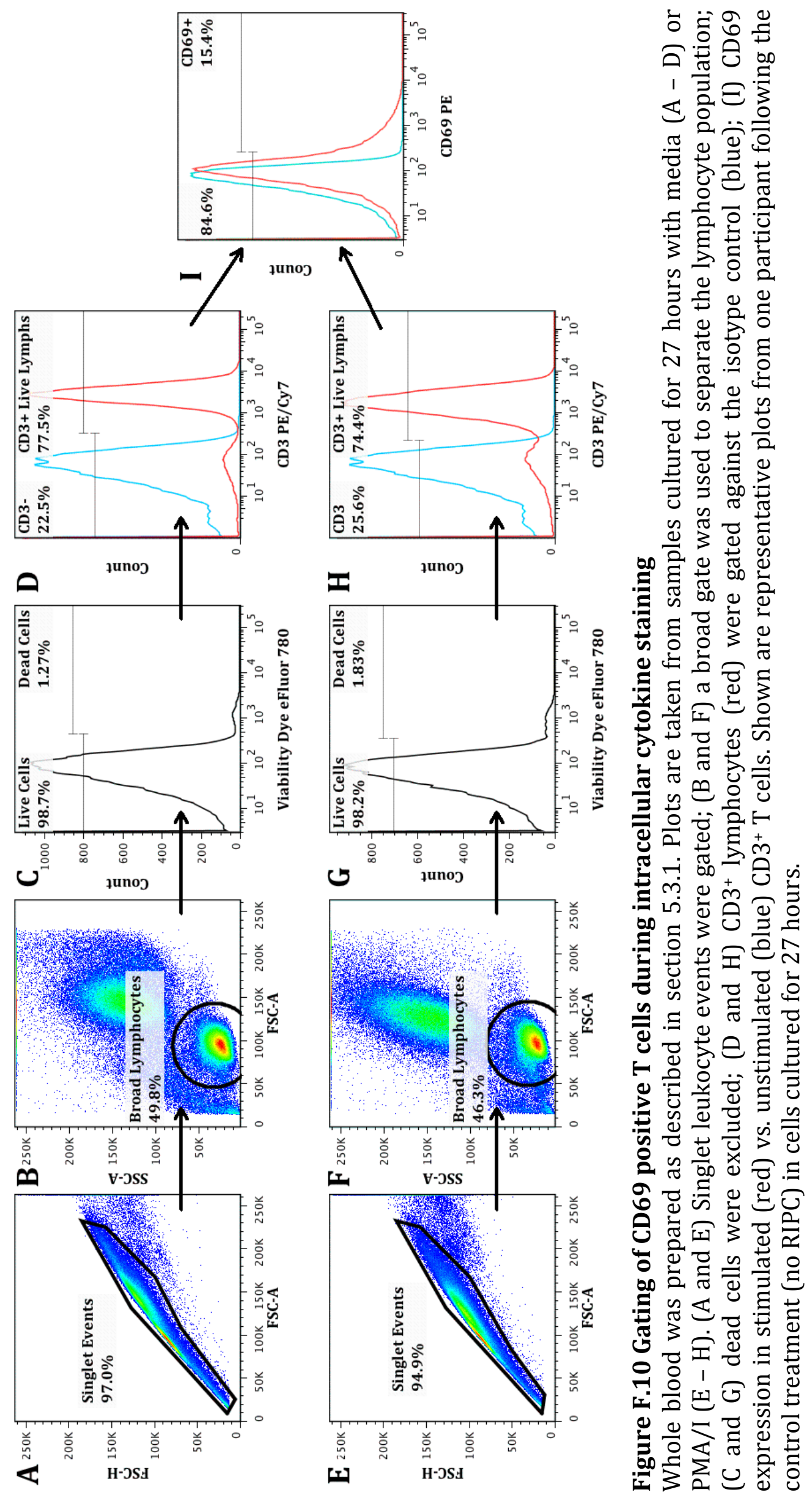




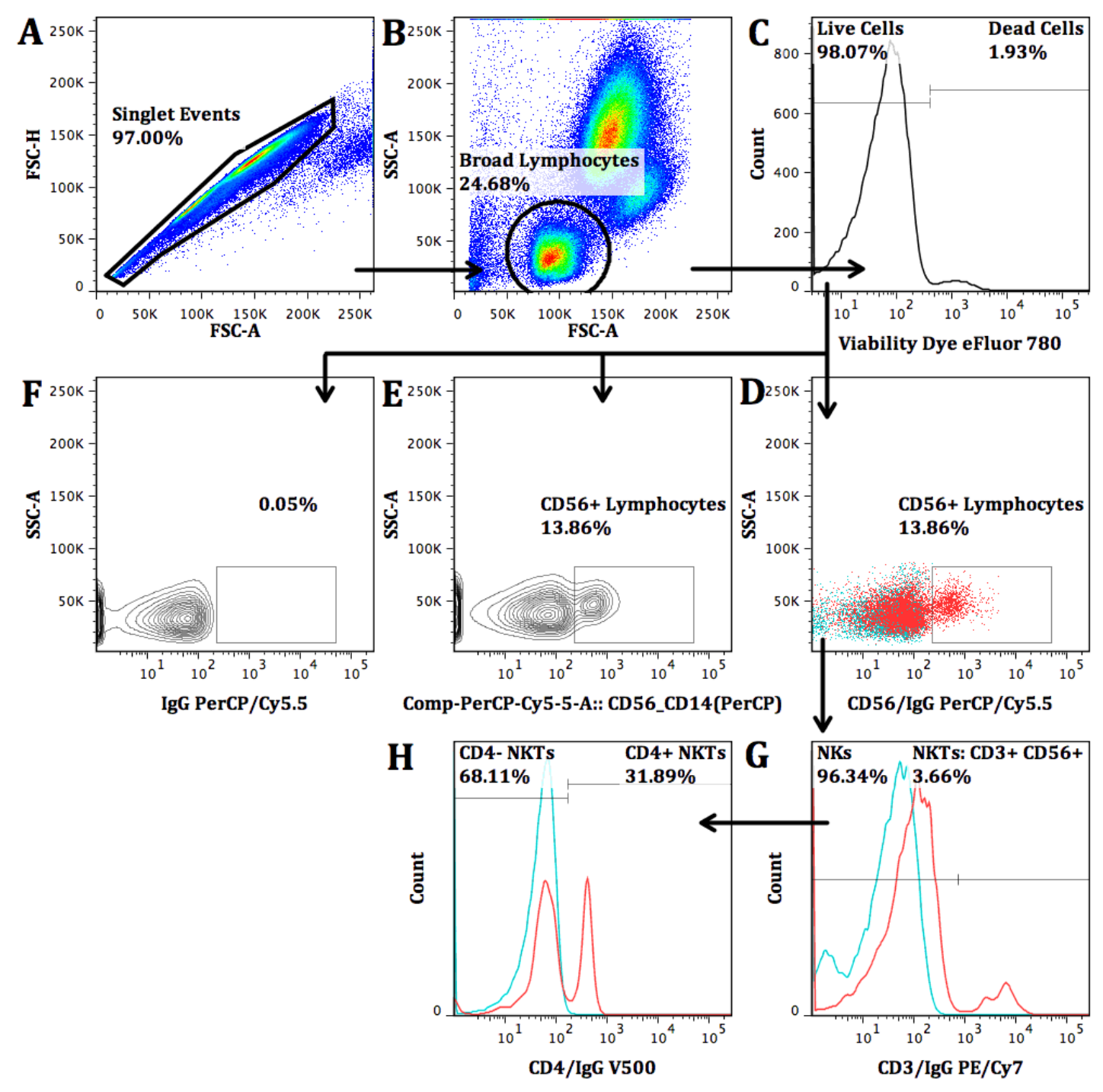

Figure F.11 Gating of NK and CD4+ NKT cells in panel 5

Whole blood was prepared as described in section 5.3.1. (A) Singlet leukocyte events were gated using FSC-A vs. FSC-H; (B) a broad gate was used to separate the lymphocyte population; (C) dead cells were excluded; (D) CD56 ${ }^{+}$ lymphocytes (red) were gated against the isotype control (blue; F), using contour graphs (E \& F); (G) NKT cells were gated using CD3 (red) compared to an isotype control (blue); (H) $\mathrm{CD}^{+}$NKTs were identified (red) by gating against an isotype control sample (blue). Shown is representative data from unstimulated samples of one participant. 

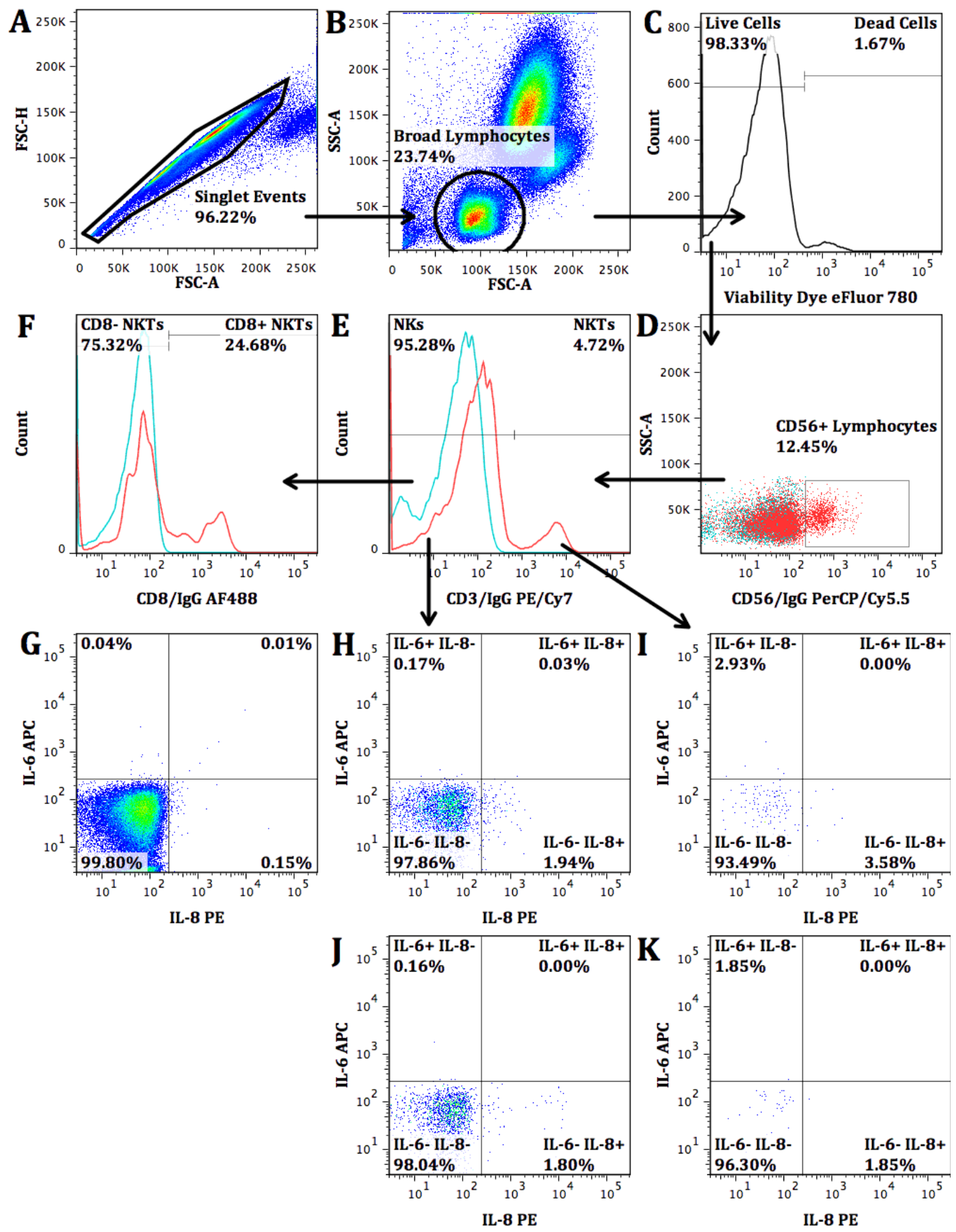

Figure F.12 Gating of CD8+ NKT cells and intracellular cytokines in NK and NKT cells in panel 6

Whole blood was prepared as described in section 5.3.1. (A) Singlet leukocyte events were gated; (B) a broad gate was used to separate the lymphocyte population; (C) dead cells were excluded; (D) CD56 lymphocytes (red) were gated against the isotype control (blue); (E) NKT cells were gated using CD3 (red) compared to an isotype control (blue); (F) CD8 ${ }^{+}$NKTs were identified (red) by gating against an isotype control sample (blue). IL-6 and IL-8 levels in the NK ( $\mathrm{H}$ and $\mathrm{J}$ ) and NKT (I and K) populations were gated using the isotype control sample for reference $(G)$ in 27 hour cultures with media (H and I) or PMA/I (J and $\mathrm{K}$ ). Shown is representative data from one participant. 


\section{Appendix G. Supplementary figures for Chapter 4}

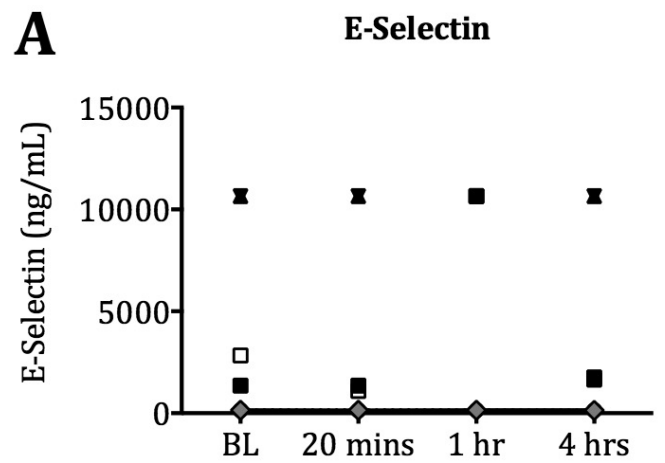

B ICAM-1

C

IL-12p70
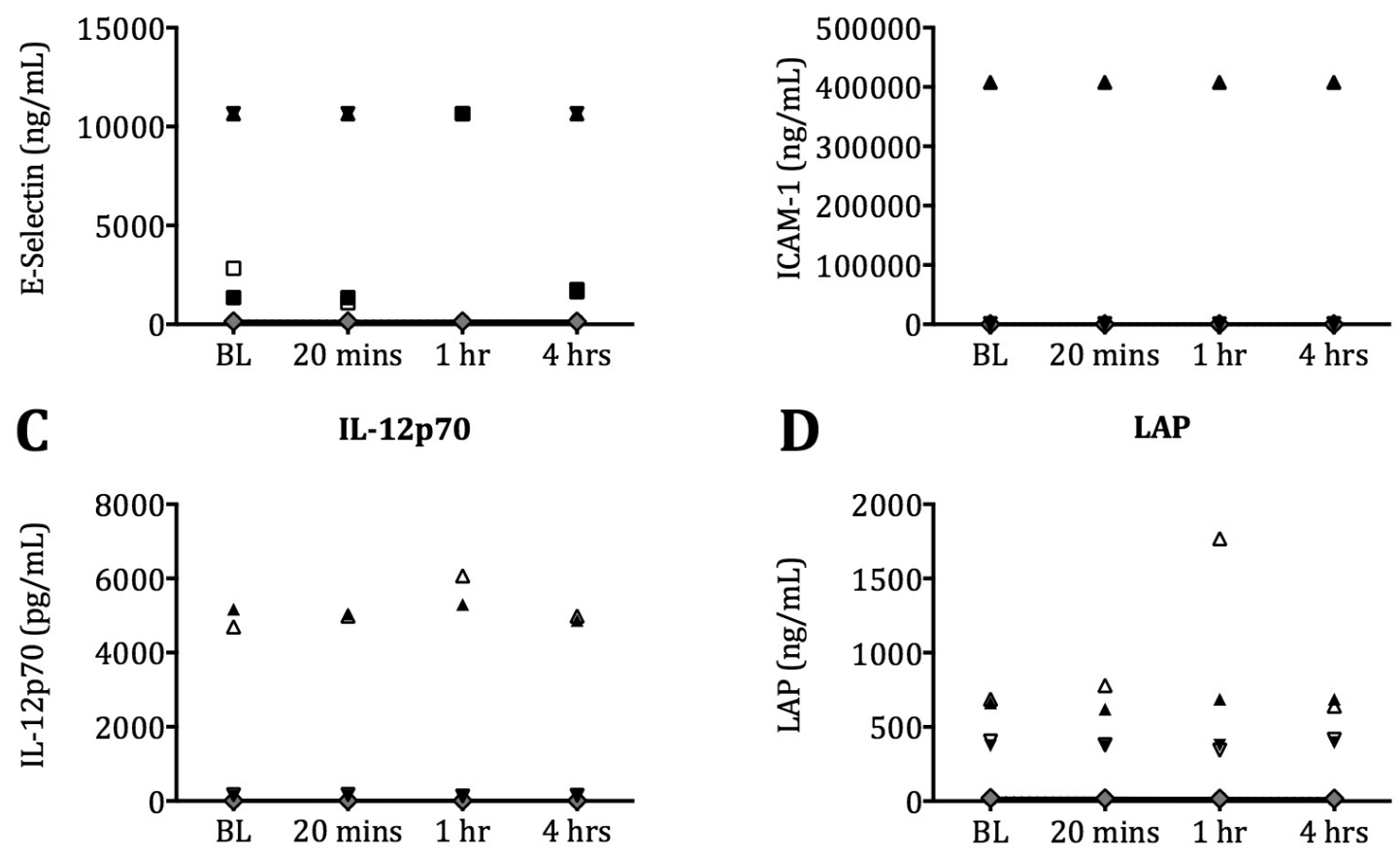

D LAP

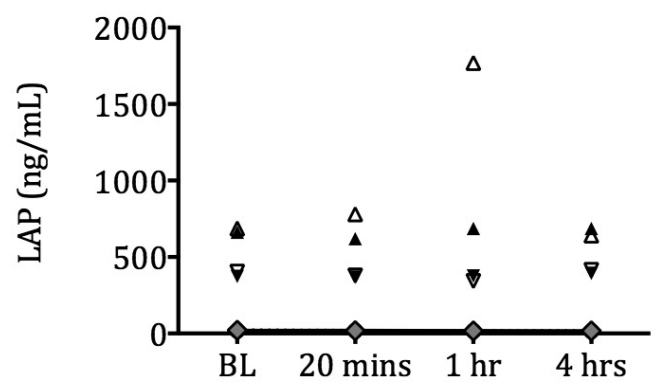

$\mathbf{E}$

IFN- $\gamma$

F

MIP-1及
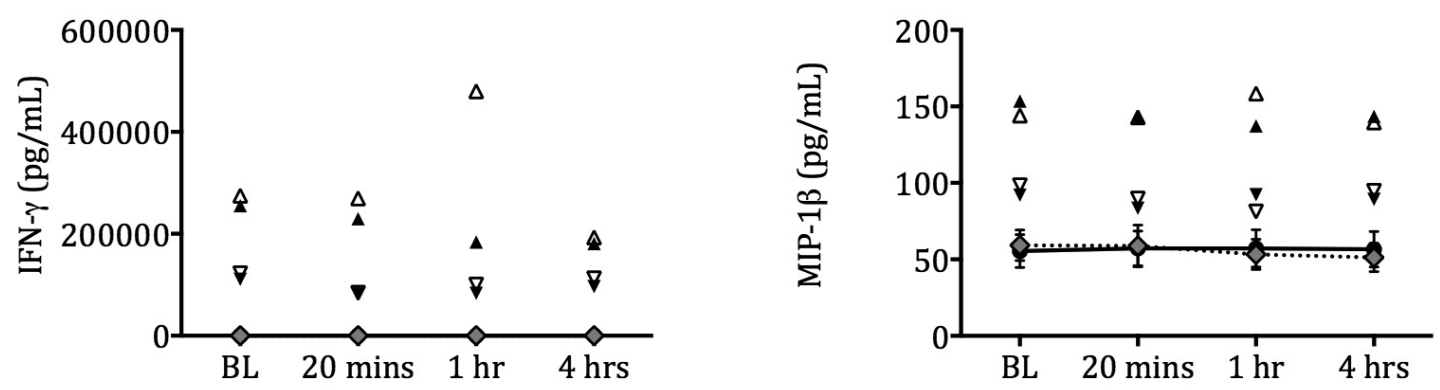

G

G-CSF

H

MCP-1
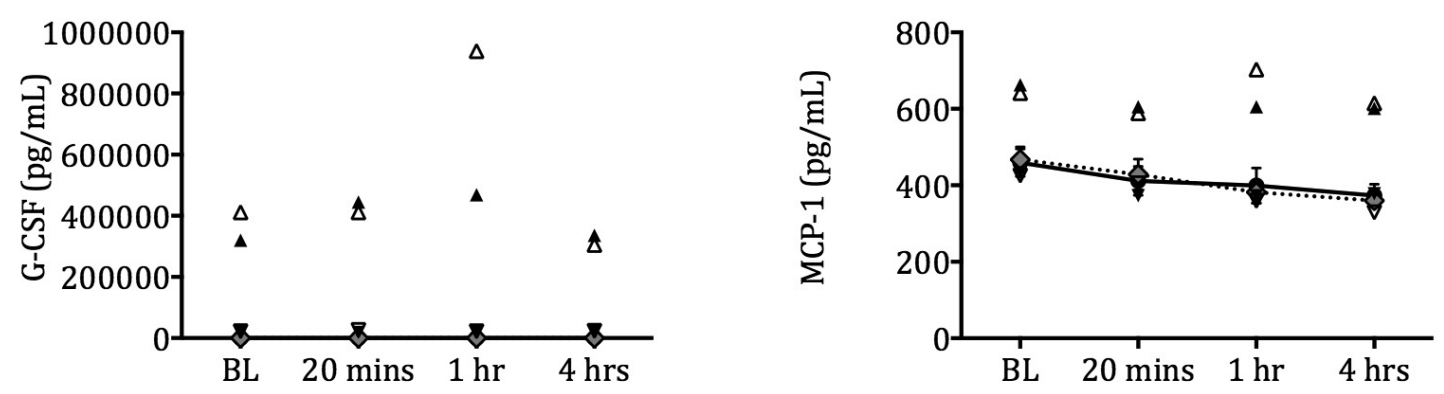

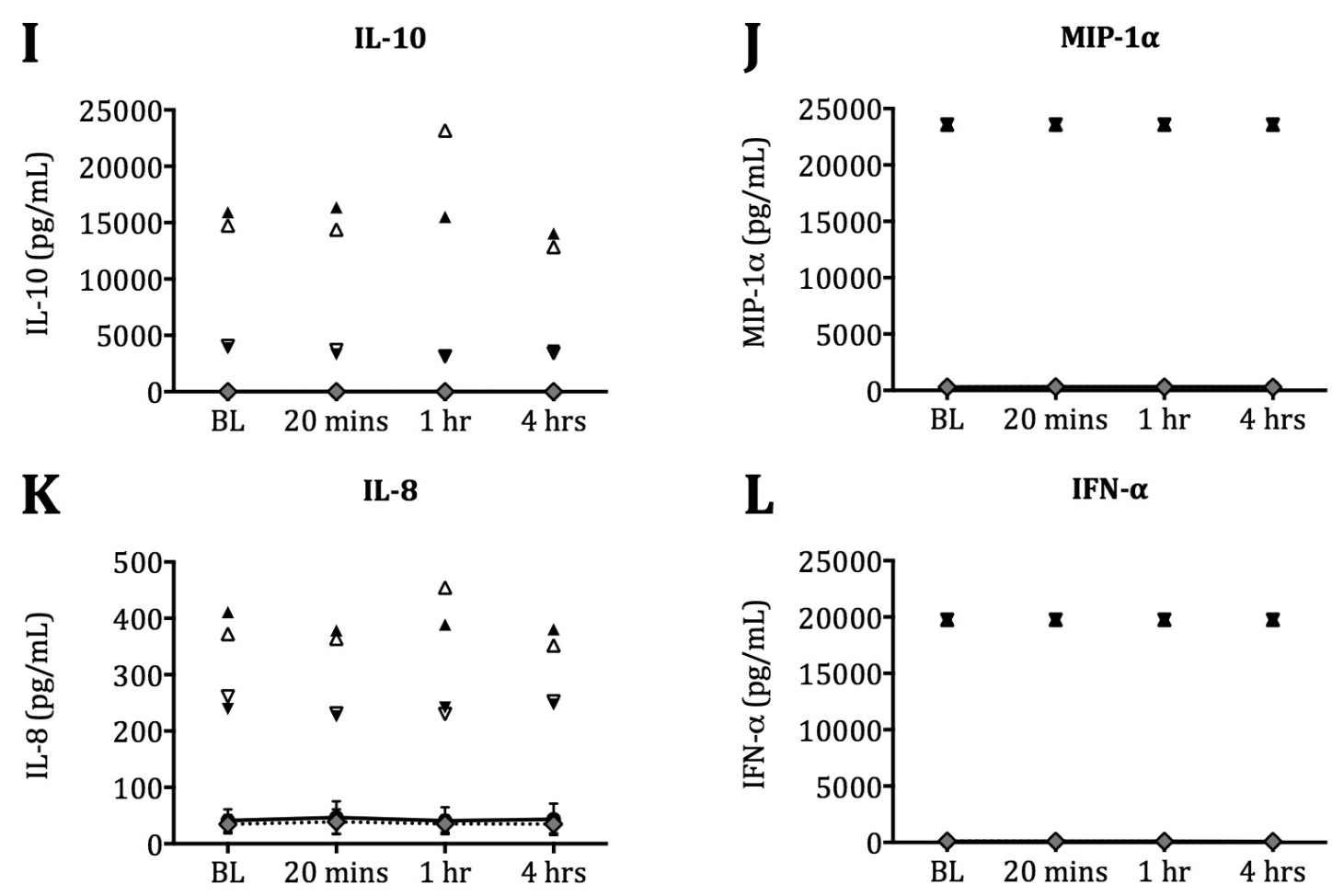

L

IFN- $\alpha$

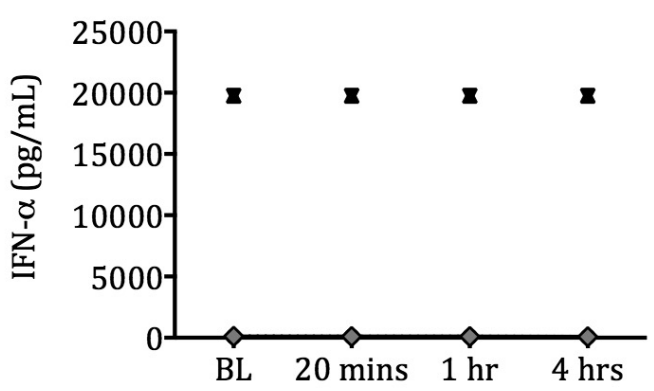

M

IL-13

$\mathbf{N}$

IP-10
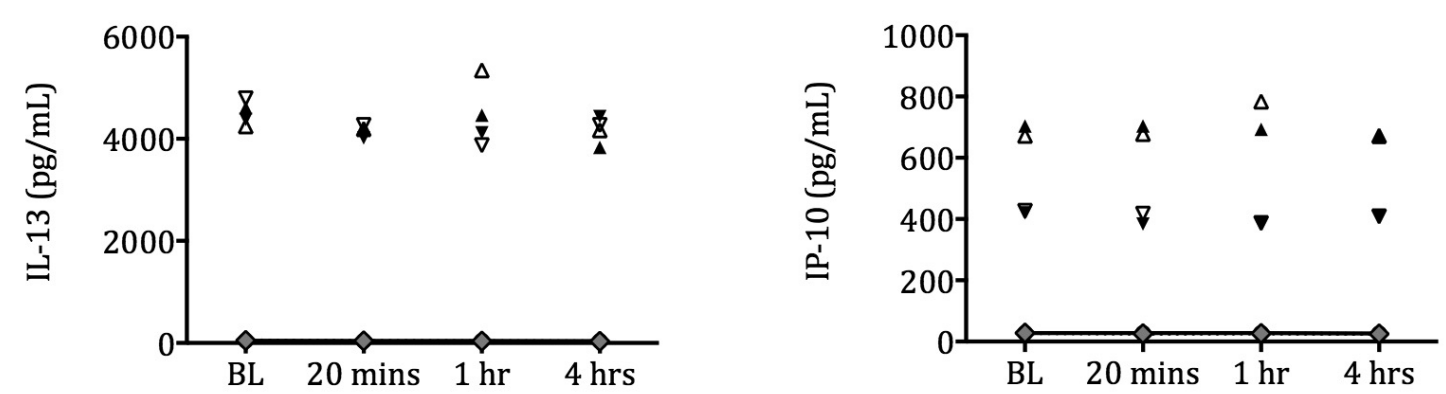

0

IL-17A

P

IL-1及
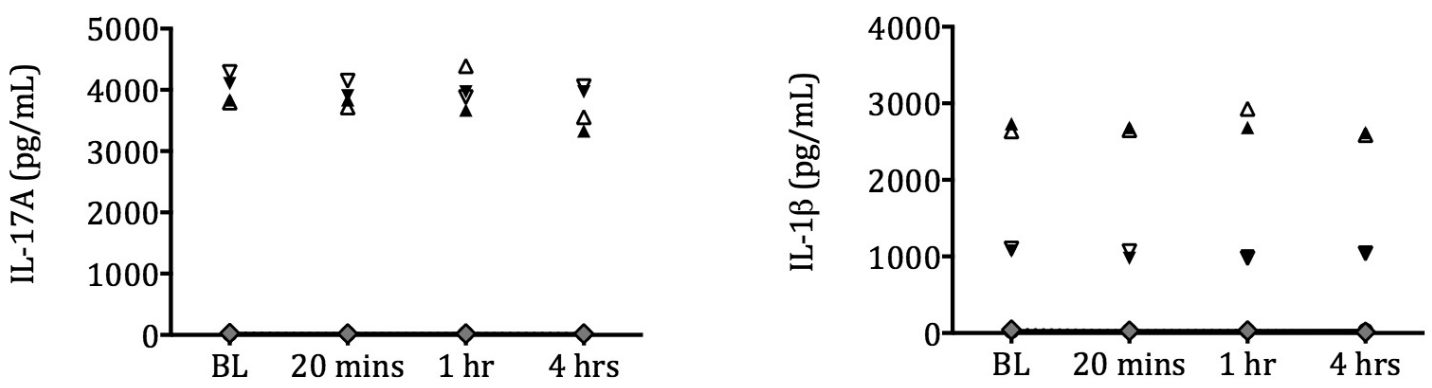

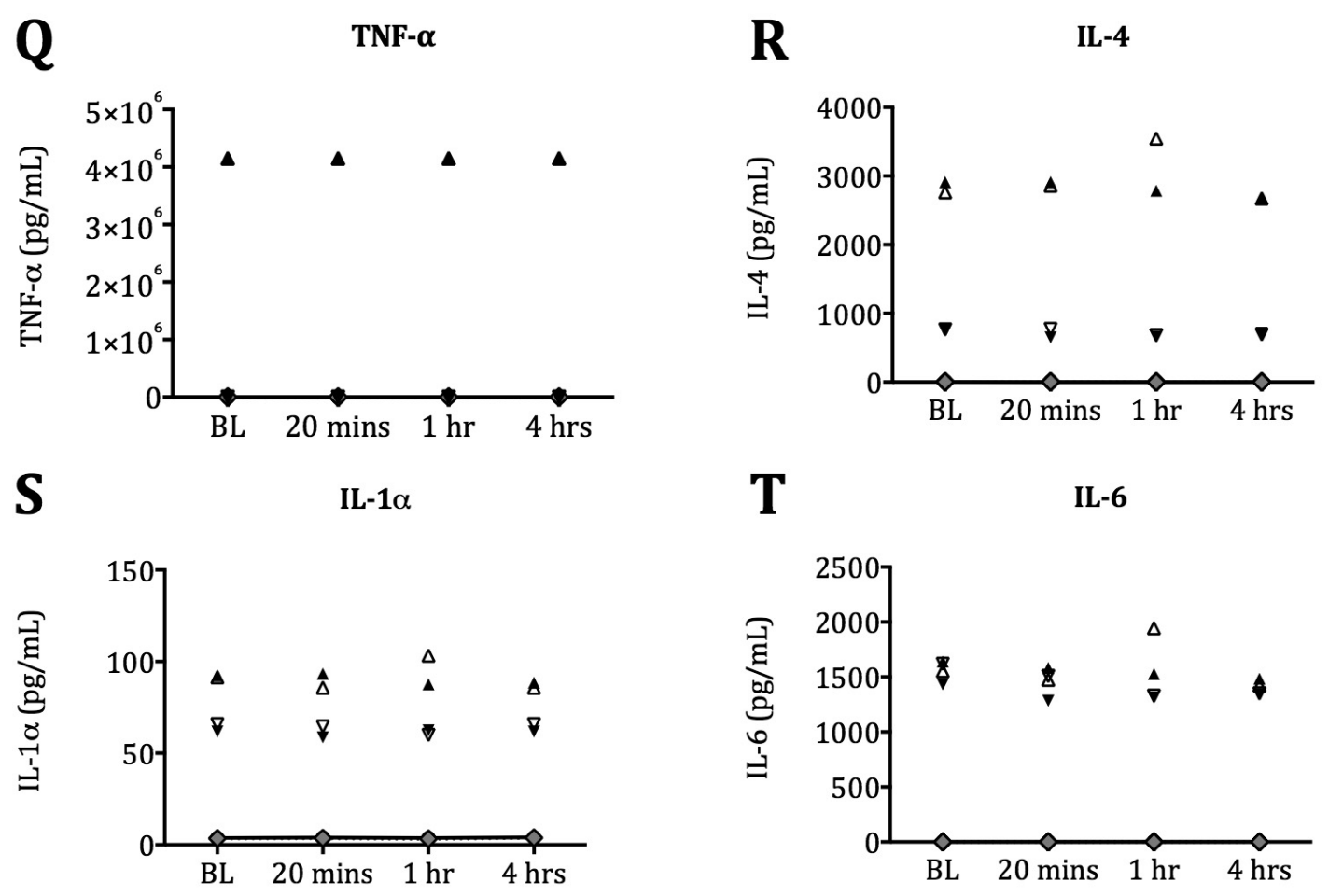

\begin{tabular}{|llllllll|}
\hline$\sim$ & Control Group & $\boldsymbol{\Delta}$ & 03-Control & $\boldsymbol{\square}$ & 06-RIPC & $\boldsymbol{\nabla}$ & 08-Control \\
$\bullet$ & RIPC Group & $\Delta$ & 03-RIPC & $\boldsymbol{\square}$ & 06-Control & $\boldsymbol{\nabla}$ & 08-RIPC \\
\hline
\end{tabular}

\section{Figure G.1 Serum cytokine levels for all study participants}

Blood samples were collected from healthy volunteers at baseline, and 20 minutes, 1 hour, and 4 hours after control or RIPC treatment and serum biomarker levels were quantified using a CBA and flow cytometry. Shown are the absolute concentrations for the participants with high levels of cytokines before and after treatment that were excluded from the main analysis $(03$ and 08; represented by the normal and inverted triangles), compared to the mean levels for the remaining 8 participants. In the case of E-selectin (A), one additional participant who had levels that exceeded the detection range of the assay and was excluded from the analysis is also shown separately from the remaining participants (06, represented by the squares). 
A

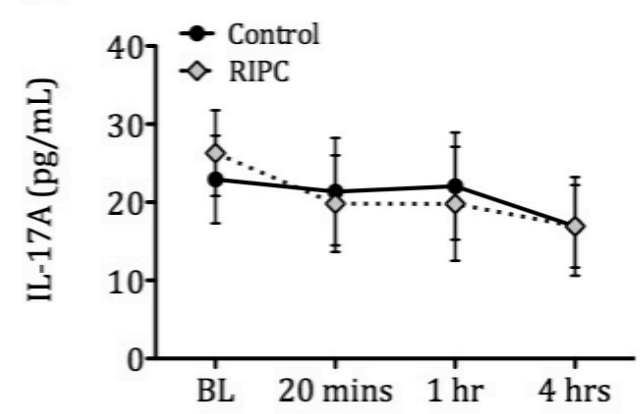

B

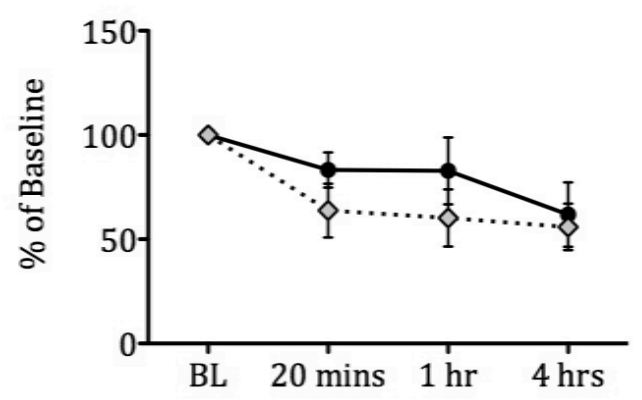

Figure G.2 Serum IL-17 concentrations with outliers removed

Blood samples were collected from healthy volunteers at baseline, and 20 minutes, 1 hour, and 4 hours after control or RIPC treatment and serum biomarker levels were quantified using a CBA and flow cytometry. (A) Absolute concentrations of IL-17; (B) IL-17 levels normalised to baseline expression levels. Shown are the mean and SEM for $n=7$ participants. Participants with unusually high or inconsistent expression levels were excluded. 


\section{Appendix H. Supplementary figures for Chapter 5}

A CD3 T Cells
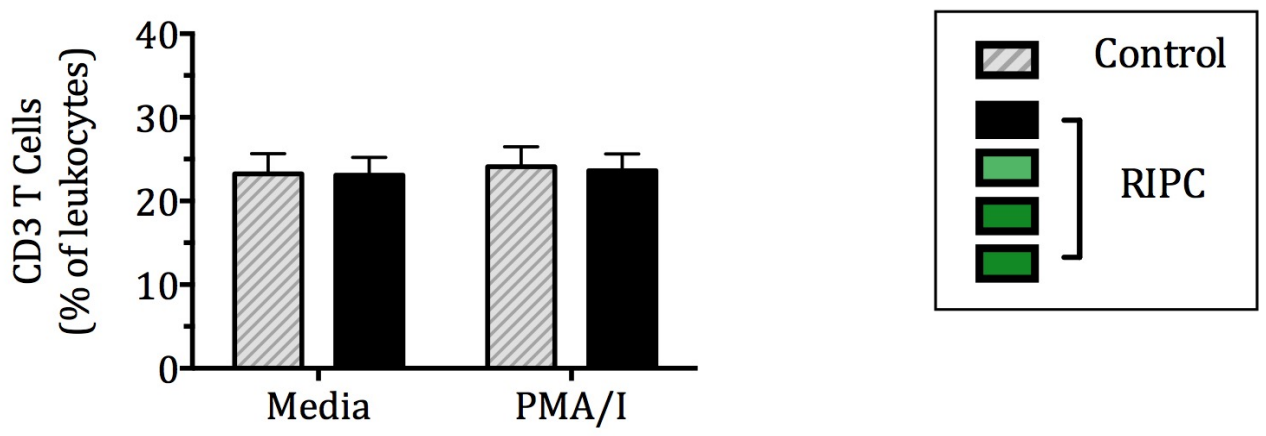

B

CD4 T Cells

C CD8 T Cells
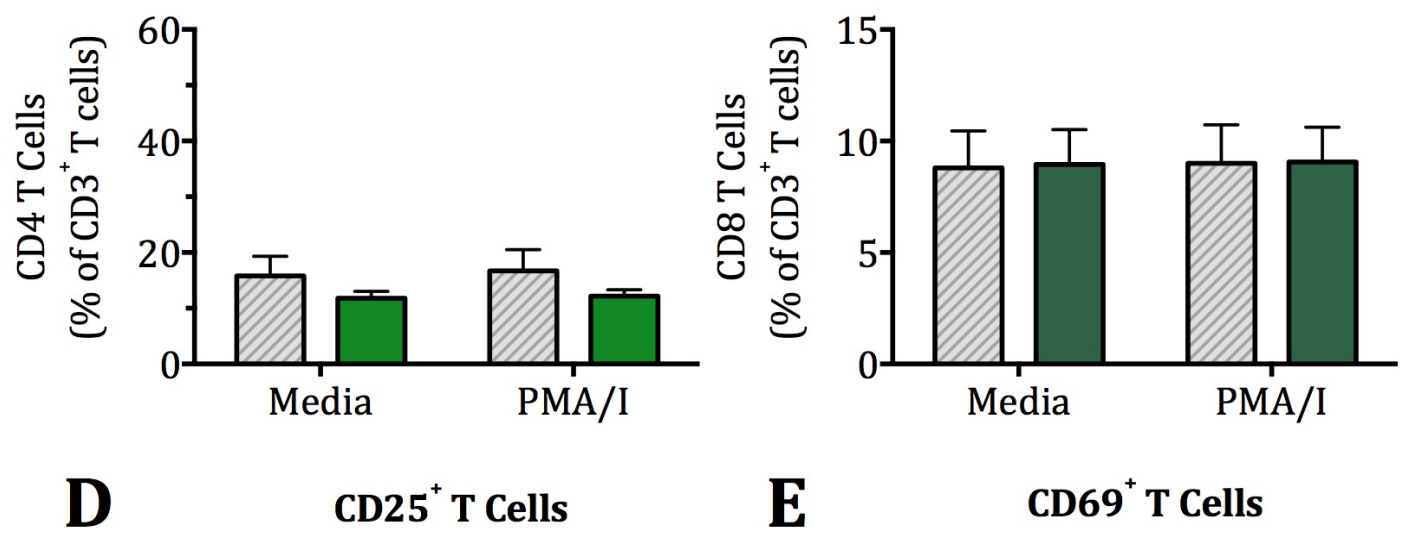

D

$\operatorname{CD25}^{+} \mathrm{T}$ Cells

$\mathbf{E}$

CD69 $^{+}$T Cells
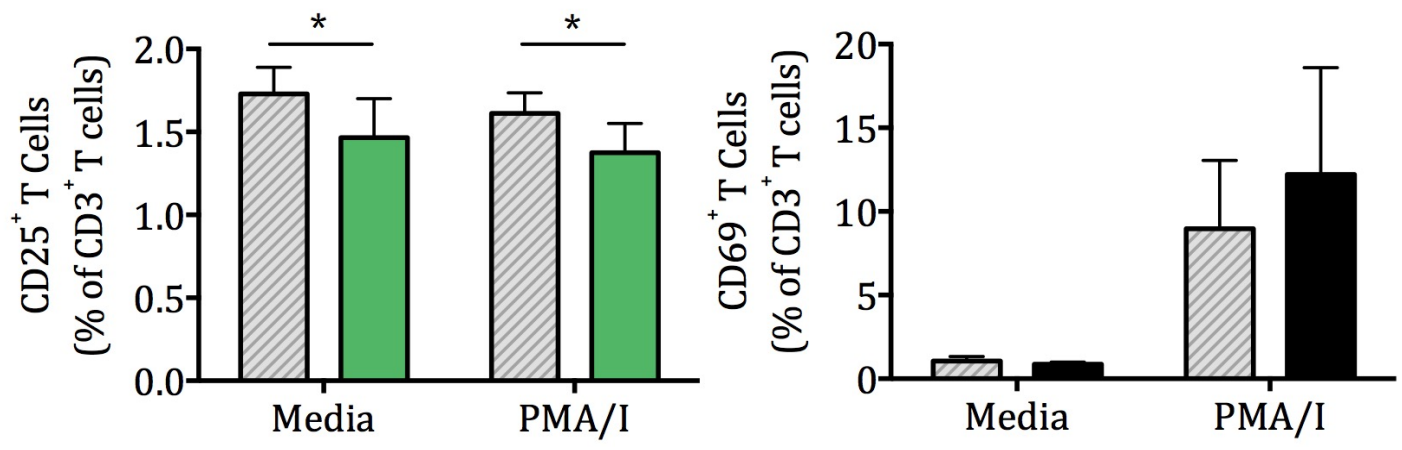

Figure H.1 T cell subset proportions and activation state after 4 hours culture

Blood was collected 1 hour after treatment; cultured for 4 hours with or without $50 \mathrm{ng} / \mathrm{mL}$ PMA, $500 \mathrm{ng} / \mathrm{mL}$ ionomycin, and GolgiStop (1:1000); then assessed for intracellular cytokines with flow cytometry. Proportions of cell populations are shown for (A) $\mathrm{CD}^{+}$cells; (B) CD4 ${ }^{+} \mathrm{T}$ cells; (C) $\mathrm{CD}^{+} \mathrm{T}$ cells; (D) $\mathrm{CD} 4{ }^{+} \mathrm{CD} 25^{+} \mathrm{T}$ cells; (E) CD69+ $\mathrm{T}^{+}$cells. $\mathrm{CD}^{+}$cells are expressed as the percentage of the total live leukocytes, and the remaining subsets as a percentage of the $\mathrm{CD}^{+}$parent population. Samples were prepared in duplicate and shown are the means and SEM for $\mathrm{n}=10$ for $\mathrm{A}, \mathrm{C}$, and $\mathrm{E}$, and $\mathrm{n}=9$ for $\mathrm{B}$ and $\mathrm{D} .{ }^{*} \mathrm{p}<0.05$. 


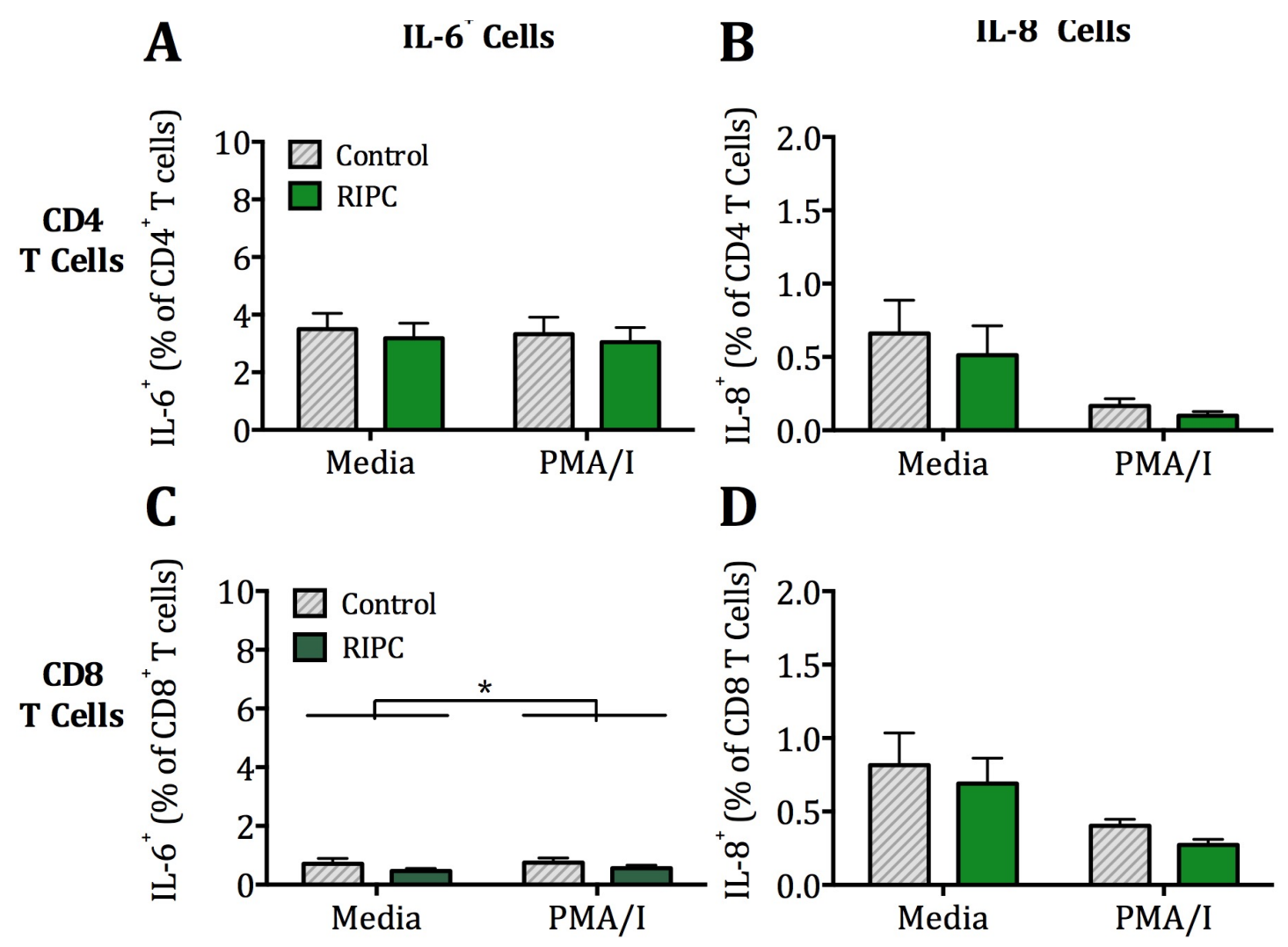

Figure H.2 Intracellular IL-6 and IL-8 production in T cells cultured for 4 hours

Blood was collected 1 hour after treatment; cultured for 4 hours with or without $50 \mathrm{ng} / \mathrm{mL}$ PMA, $500 \mathrm{ng} / \mathrm{mL}$ ionomycin, and GolgiStop (1:1000); then assessed for intracellular cytokines with flow cytometry. Percentage of IL- $6^{+}$cells in CD4 (A) and CD8 (C) T cells; percentage of IL-8+ CD4 (B) and CD8 (D) T cells. Cells are expressed as the percentage of the total live parent population. Samples were prepared in duplicate and shown are the group means and SEM for $\mathrm{n}=9$ for $\mathrm{A}$ and $B$, and $n=10$ for $C$ and D. $* p<0.05$. 

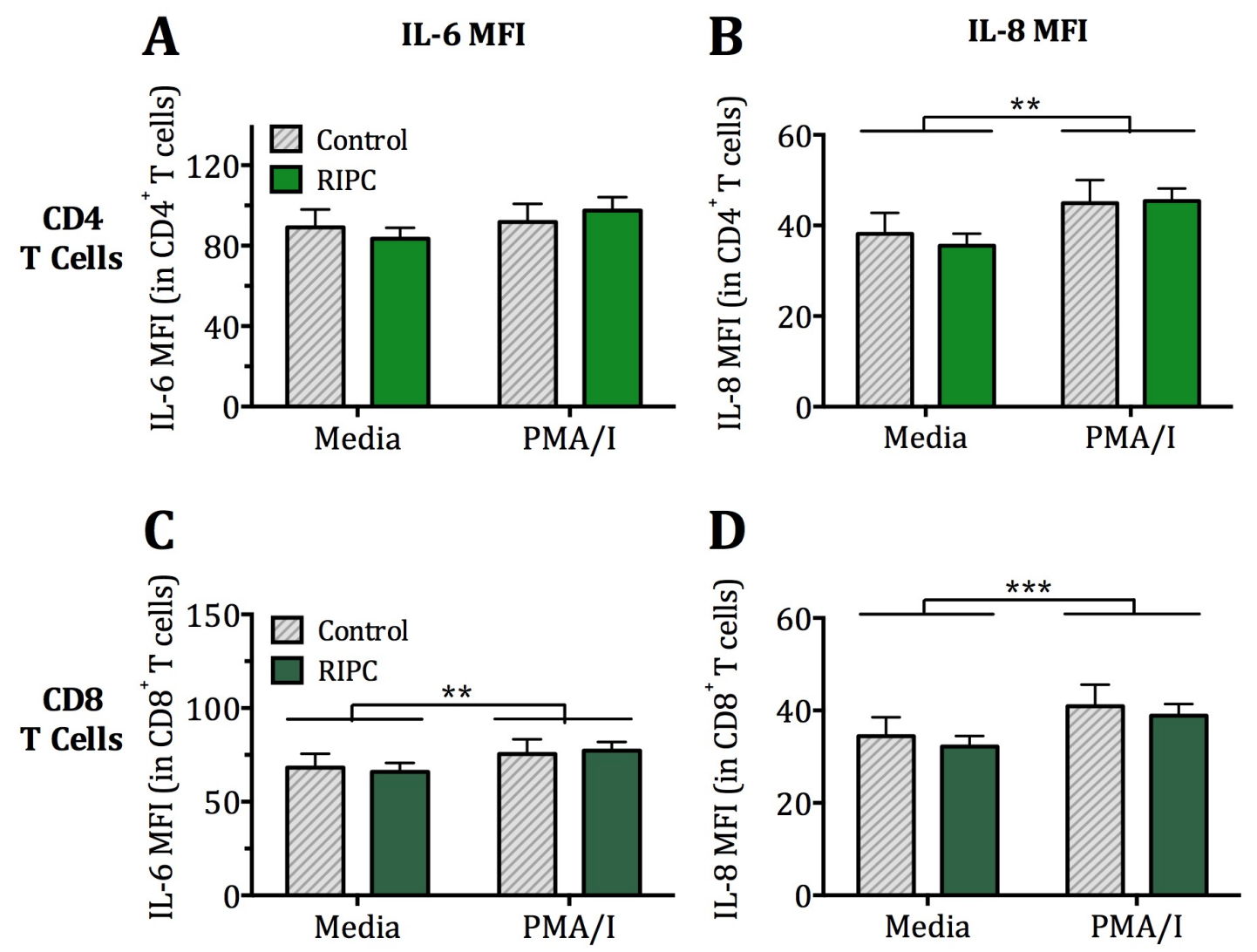

Figure H.3 Alternative expressions of intracellular cytokine data in $\mathrm{T}$ cells cultured for 27 hours

Blood was collected 1 hour after treatment; cultured for 27 hours with or without $10 \mathrm{ng} / \mathrm{mL}$ PMA, $500 \mathrm{ng} / \mathrm{mL}$ ionomycin, and GolgiStop (1:1000) for the final 4 hours; then assessed for intracellular cytokines with flow cytometry. IL-6 MFI in CD4 (A) and CD8 (C) T cells; IL-8 MFI in CD4 (B) and CD8 (D) T cells. Cells are expressed as the percentage of the total live parent population. Samples were prepared in duplicate and shown are the group means and SEM for $\mathrm{n}=9$ for $\mathrm{A}$ and $\mathrm{B}$, and $\mathrm{n}=10$ for $\mathrm{C}$ and $\mathrm{D} .{ }^{* *} \mathrm{p}<0.01$ and ${ }^{* * *} \mathrm{p}<0.001$. 

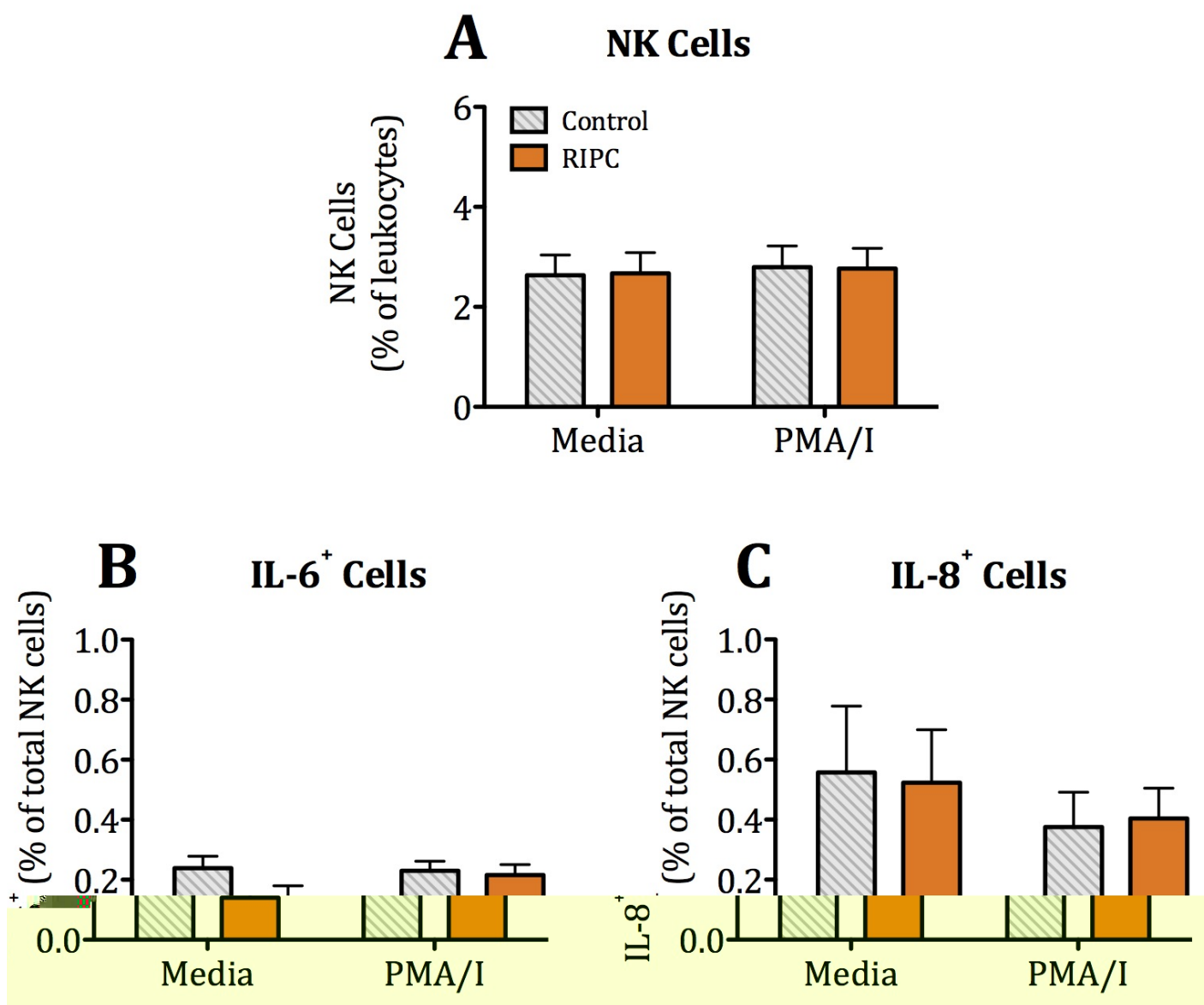

Figure H.4 Intracellular cytokine production in NK cells cultured for 4 hours

Blood was collected 1 hour after treatment; cultured for 4 hours with or without $50 \mathrm{ng} / \mathrm{mL}$ PMA, $500 \mathrm{ng} / \mathrm{mL}$ ionomycin, and GolgiStop (1:1000); then assessed for intracellular cytokines with flow cytometry. (A) Proportion of CD3-CD56 ${ }^{+} \mathrm{NK}$ cells; (B) percentage of IL- $6^{+}$NK cells; (C) percentage of IL- $8^{+}$NK cells. Cells are expressed as the percentage of the total live parent population. Samples were prepared in duplicate and shown are the group means and SEM for $n=10$. 


\section{A NKT Cells}
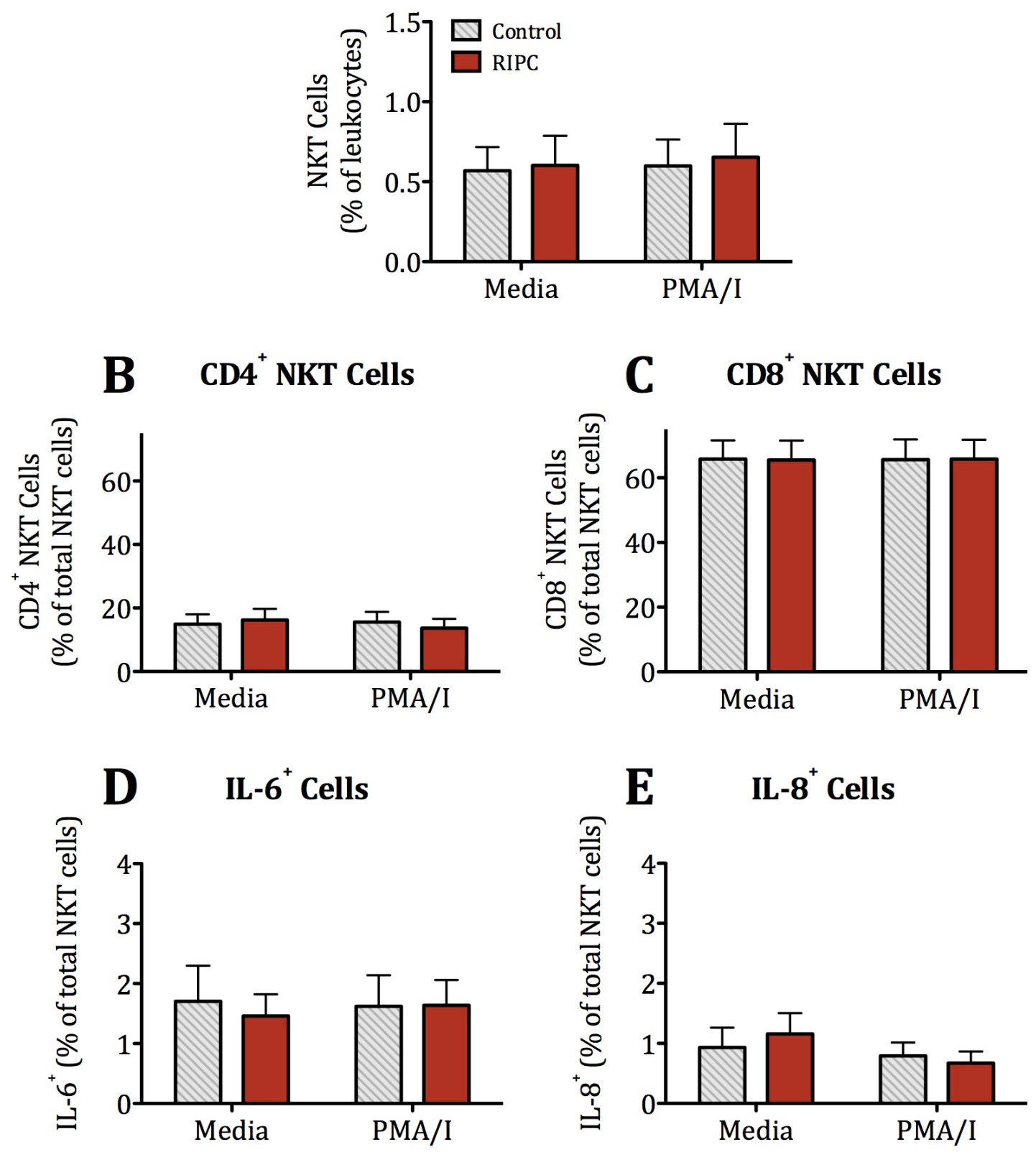

Figure H.5 Cytokine production in NKT cells after 4 hours of culture

Blood was collected 1 hour after treatment; cultured for 4 hours with or without $50 \mathrm{ng} / \mathrm{mL}$ PMA, $500 \mathrm{ng} / \mathrm{mL}$ ionomycin, and GolgiStop (1:1000); then assessed for intracellular cytokines with flow cytometry. (A) Proportion of $\mathrm{CD}^{+}{ }^{+} \mathrm{CD} 56^{+}$ NKT cells; (B) CD4+ NKT cells; (C) CD8 ${ }^{+}$NKT cells; (D) percentage of IL- $6^{+}$NKT cells; (E) percentage of IL-8 $8^{+}$NKT cells. Cells are expressed as the percentage of the total live parent population. Samples were prepared in duplicate and shown are the group means and SEM for $\mathrm{n}=10$. 